University of Rhode Island

DigitalCommons@URI

Open Access Dissertations

2017

\title{
Assessing Hand Hygiene Compliance in Healthcare Workers to Reduce Infectious Disease
}

\author{
Ahmed Salem Alhasani \\ University of Rhode Island, ahmed_alhasani@my.uri.edu
}

Follow this and additional works at: https://digitalcommons.uri.edu/oa_diss

\section{Recommended Citation}

Alhasani, Ahmed Salem, "Assessing Hand Hygiene Compliance in Healthcare Workers to Reduce Infectious Disease" (2017). Open Access Dissertations. Paper 613.

https://digitalcommons.uri.edu/oa_diss/613

This Dissertation is brought to you for free and open access by DigitalCommons@URI. It has been accepted for inclusion in Open Access Dissertations by an authorized administrator of DigitalCommons@URI. For more information, please contact digitalcommons-group@uri.edu. 
ASSESSING HAND HYGIENE COMPLIANCE IN HEALTHCARE

WORKERS TO REDUCE INFECTIOUS DISEASE

BY

AHMED SALEM ALHASANI

A DISSERTATION SUBMITTED IN PARTIAL FULFILLMENT OF THE REQUIREMENTS FOR THE DEGREE OF

DOCTOR OF PHILOSOPHY

IN

INDUSTRIAL AND SYSTEMS ENGINEERING

UNIVERSITY OF RHODE ISLAND

2017 
DOCTOR OF PHILOSOPHY DISSERTATION

OF

AHMED SALEM ALHASANI

APPROVED:

Dissertation Committee:

Major Professor Valerie Maier-Speredelozzi

Jyh-Hone Wang

Gavino Puggioni

Nasser H. Zawia

DEAN OF THE GRADUATE SCHOOL

UNIVERSITY OF RHODE ISLAND

2017 


\begin{abstract}
Hand hygiene is the first line of defense against the prevalence of infectious diseases in healthcare settings. Therefore, healthcare costs can be reduced. However, having rare incidents of healthcare-associated infections (HAI) does not always mean that hand hygiene compliance is high and at its desired level. This research study aims to develop multi-statistical measurements to assess hand hygiene compliance of the medical and nursing groups at the inpatient wards, 5B, 6B and ICU at the Providence Veterans Affairs Medical Center (PVAMC). The PVAMC was trying to identify whether the few cases or rare incidents of HAI that have been reported in the past few years was caused by or linked to poor hand hygiene practices. Healthcare worker (HCWs) subgroups of nurses and hospitalist doctors were asked to self report their patient contact over one complete week. The URI research team and 25 other secret observers were asked to directly observe the medical and nursing groups' hand hygiene behavior over two complete months including all working shifts: night, day and evening. These two months were overlapped with the one complete week of selfreporting patient contact. The results indicated that the monthly hand hygiene compliance mean estimation was not as expected by the PVAMC. The monthly hand hygiene compliance mean estimation was around 50\%. The results also indicated that as bed days of care (BDOC) increased, hand hygiene compliance decreased. In contrast, the results did not indicate any strong correlation between hand hygiene compliance and HAIs. However, the possibility that the PVAMC has been adopting other infection prevention methods that are associated with the rare HAI incident (for example zero MRSA for the past five years) cannot be eliminated or ignored. Hand
\end{abstract}


hygiene compliance was higher after touching a patient than before, even though both are recommended in the World Health Organization's 5 moments of hand hygiene. Risk factors for poor adherence to recommended hand-hygiene practices were observed and found to be statistically significant, including being a male patient, working in a step-down unit (5B) and working on weekdays and working in night shift. In addition, an attempt was made to indirectly estimate hand hygiene compliance over a 10 month period by measuring how many times Purell and Soap cartridges were replaced at inpatient wards. Similarly, an attempt was made to indirectly estimate personal protective equipment (PPE) compliance over a three year period using PPE inventory data. In the indirect methods, patient contact data was used to average how many times a patient was seen by the medical and nursing groups. This estimation was used to indirectly estimate the hand hygiene compliance. The indirect hand hygiene compliance via measuring product use (Purell and Soap) was very low compared to the hand hygiene compliance estimated via the direct hand hygiene observation method when the same two months were compared in all inpatient wards. The actual Purell and Soap replacement was not equal to or close to the targeted replacement at any of the inpatient wards. The research study did not find any correlation between BDOC and hand hygiene compliance under such a method. The source of error on the indirect PPE compliance method forced the compliance to go beyond $100 \%$ in several months. The research study did not find any correlation between BDOC and PPE compliance. Such methods need more validation, but is an interesting first step for a new proposed method. 


\section{ACKNOWLEDGMENTS}

In the name of Allah the Most Gracious the Most Merciful, and Peace and Blessings be upon the most honorable prophets and messengers Muhammad and his family and companions. Praise and thanks be to Allah first and foremost, and praise be to Allah for his kindness and thankfulness for his kindness and gratitude. Without his conciliation I was not able to achieve and complete what was accomplished.

I would like to sincerely thank my academic advisor, Prof. Valerie MaierSperedelozzi for her full support and guidance throughout my Ph.D journey. And I would like to express my gratitude to my dissertation committee, Prof. Jay Wang and Prof. Gavino Puggioni for their academic consultation. I have learned a lot from them and their classes and courses. On top of that, I would like to express my thankfulness to the University of Rhode Island (URI) for accepting me as one of its students. I am so proud to have my Ph.D degree awarded from URI. I would like to thank Prof. Carol Thornber for being the chair of the doctoral comprehensive examination. I also would like to thank Prof. Liliana Gonzalez for being the chair of the oral examination in defense of the doctoral dissertation.

Special thanks and gratitude to the Providence Veterans Affairs Medical Center (PVAMC) for allowing me to conduct my Ph.D research at their facility. Thanks as big as the size of the sky to Mr. Robert Harris for his mentoring and full support as my supervisor at the PVAMC. His friendship is an achievement that goes along with my Ph.D achievement. Truly his friendship is accolade on my chest. Special thanks to Dr. Bonnie Charland, the head of the Quality Management, and Dr. Melissa Gaitanis, the Chief of Infectious Disease and Control. I cannot and do not know how to thank 
Brigida Cedeno, the Infection Preventionist at the PVAMC, for her full support and assistance through out my Ph.D research. Without her support, my project at the PVAMC would literally have failed without any doubt. I would like to thank Mr. Edward Robertson for his support and motivation during the two month of experiment. I would like to thank everyone at the PVAMC who have participated in my study, supported me, mentored me and guided me throughout the four years of my research. I express my regret and apologies for anyone I have not been able to thank personally or mention here in my dissertation. But for sure you all are in my heart and good memories. I will not forget the PVAMC, the floors and my office there. I have already missed all of that.

As Allah says in the holy Quran, Chapter 14 entitled "The Night Journey" verse 24: "And lower to them the wing of humility out of mercy and say, "My Lord, have mercy upon them as they brought me up [when I was] small." All my thanks and gratitude goes to my parents, Mr. Salem Alhasani and Mrs. Wedad Tayeb, indeed without them I am not who I am. The same goes to my uncle, Mr. Abdulmuti Alhasani, and my aunt, Mrs. Salma Alhasani, my parents in law and my lovely wife's parents for their full support and prayers day and night for the whole seven years that my family and I have been away from home. Earning my Ph.D degree is just a simple gift that goes back to my biological parents and my parents in law.

With true feelings, I cannot and do not know how to thank my wife, Mrs. Mona Alhasani, but I can say thanks sweetheart for being in my heart and in my life. I do not want to forget thanking my two little knights, Faris and Feras for cheering me up days and nights when I come back from my office at URI. Once I see their smiley faces I 
totally forget being exhausted. A tiny tiny thanks goes to the unborn baby boy Yousef and through this platform I loudly say welcome to the world my little beloved son. I would like to thank my dear sister, Mrs. Ola Alhasani, and my dear brothers, Mr. Emad Alhasani, Mr. Abdulaziz Alhasani and Mr. Faisal Alhasani for their prayers days and nights.

And last but not least, I would like to thank my government for their custodian of the two holy mosques program for external scholarship along with the full financial support. My thanks and gratitude also goes to the unknown soldiers who for sure have worked days and nights to ensure the full and superior success of the two holy mosques program for external scholarship, which for sure and without any doubt its fruits will be harvested soon for present and future generations. 


\section{PREFACE}

The following research is presented in standard format according to the guidelines provided by the Graduate School of the University of Rhode Island. The dissertation is composed of eight chapters.

The first chapter is entitled "Introduction" and briefly introduces the research field and topic "Assessing Hand Hygiene Compliance in Healthcare Workers to Reduce Infectious Disease". In addition, it talks about the motivation of the study and how important it is to both the PVAMC and URI research team. It also points out the primary and secondary objectives of the research.

The second chapter is entitled "Review of Literature" and talks in-depth about hand hygiene, healthcare-associated infections and direct and indirect methods of measuring hand hygiene compliance.

The third chapter is entitled "Methodology" and talks about the methodology of the research, from study design, study hypotheses, study methods, data required, evaluation and study population.

The fourth chapter is entitled "Patient Contact Analysis" and talks about selfreported patient contact data by healthcare workers (HCWs), doctors and nurses at the PVAMC over one complete week in October 2016. The three working shifts are included and the three inpatient wards are included as well. The primary objective of such a study is to estimate on average how many times a patient is seen by all HCWs. This information is used in chapter six and chapter seven for an estimation of hand hygiene opportunities and an estimation of personal protective equipment (PPE) opportunities. 
The fifth chapter is entitled "Direct Hand Hygiene Observation Method" and talks about a retrospective analysis using the PVAMC historical data. It also analyzes a hand hygiene compliance study conducted by the URI research team in September and October 2016. This chapter answers the main and the primary objectives of the study, which is to precisely and accurately estimate hand hygiene compliance at the PVAMC and perform hypothesis testing to determine risk factors for poor adherence to recommended hand-hygiene practices.

The sixth chapter is entitled "Indirect Hand Hygiene Compliance Method" and talks about how hand hygiene compliance can be estimated based on counting the replacements of Purell and Soap cartridges at each inpatient ward. It also reports a breakdown use of Purell and Soap use per inpatient ward by making a comparison with facility-wide sales data of Purell and Soap.

The seventh chapter is entitled "Indirect Personal Protective Equipment (PPE) Compliance Method" and talks about how PPE compliance can be estimated based on inventory data of Gloves, Gowns and Masks ordered at each inpatient ward.

The eight and last chapter is entitled "Conclusions" and talks about recommendations that the PVAMC can adopt to improve hand hygiene compliance, and any future work that could lead to better hand hygiene compliance outcomes. Of note, the sixth and the seventh chapters answer the secondary objective of the research study, which is to indirectly measure hand hygiene and PPE compliance. The fourth, fifth, sixth and the seventh chapters represent the research findings. 


\section{TABLE OF CONTENTS}

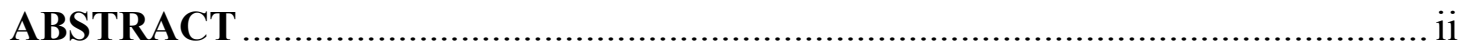

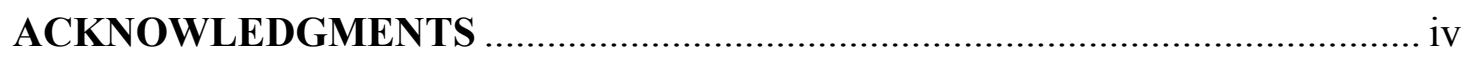

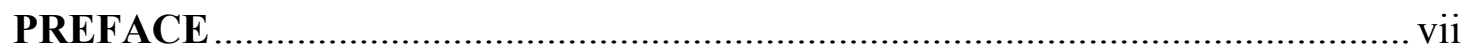

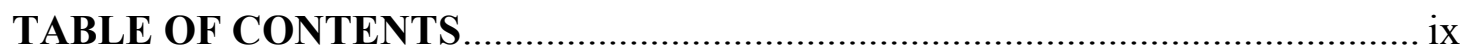

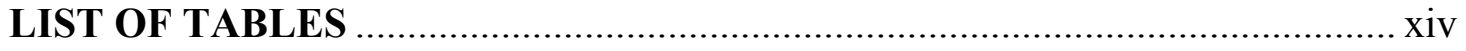

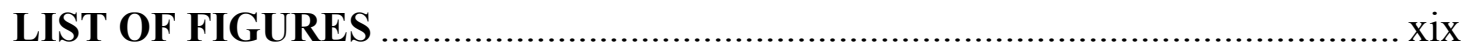

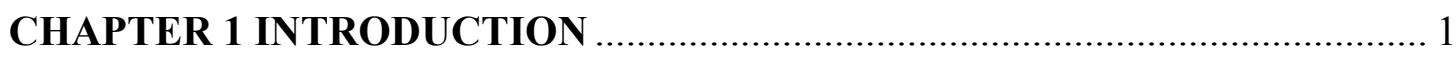

CHAPTER 2 REVIEW OF LITERATURE ........................................... 8

2.1 Healthcare and Epidemic Infectious Diseases ................................. 9

2.1.1 Hospital-Onset Infections Caused by Specific Pathogens ............. 9

2.1.2 Device Invasive Infections ................................................ 16

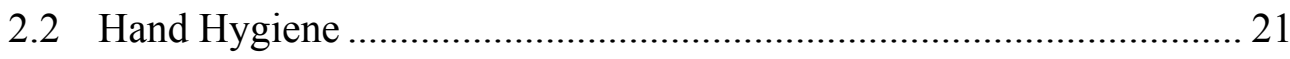

2.3 Hand Hygiene Adherence Measurement Methods ........................... 26

2.3.1 Direct Hand Hygiene Monitoring Methods................................ 30

2.3.2 Indirect Hand Hygiene Monitoring Methods ............................. 36

2.3.3 Radio Frequency Identification (RFID) Method........................ 44

2.4 RFID and Hand Hygiene .......................................................... 49

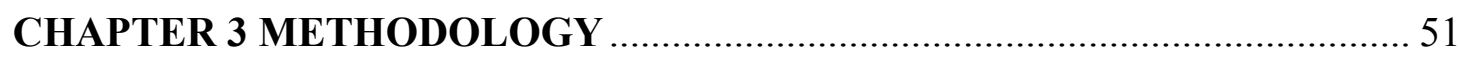

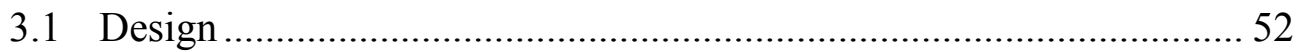

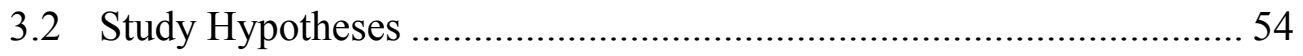

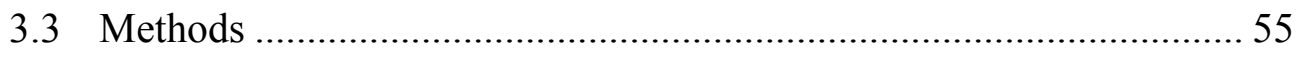

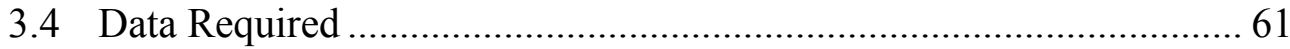

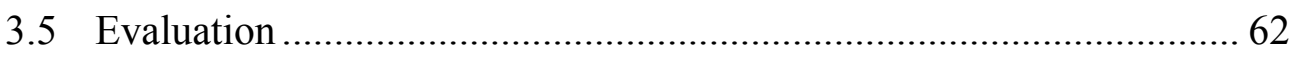

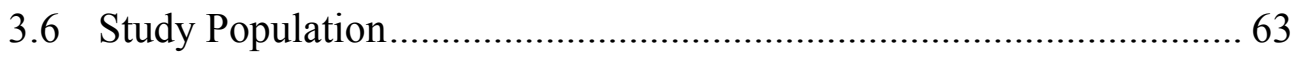




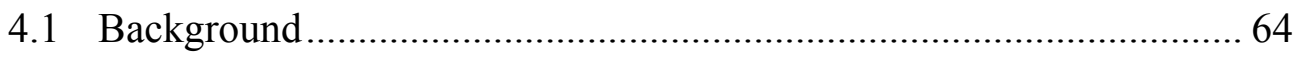

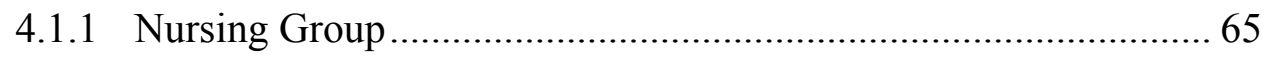

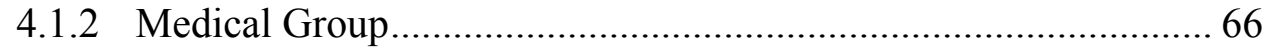

4.1.3 Health Administration Service ................................................... 67

4.1.4 Environmental Management Service (EMS) .......................... 70

4.2 Patient Contact Participants Summary Statistics ............................. 71

4.3 Patient Contact Objectives........................................................ 75

CHAPTER 5 DIRECT HAND HYGIENE OBSERVATION METHOD ........... 95

5.1 The Providence Veterans Affairs Medical Center (PVAMC) ............ 95

5.1.1 Inpatient Wards ............................................................... 95

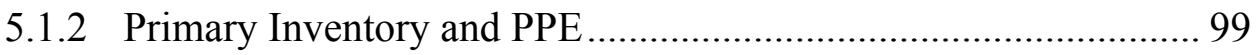

5.1.3 Isolation Precautions .................................................................. 100

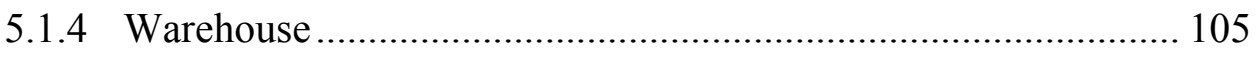

5.1.5 Physical Contact with Patients ............................................... 106

5.2 Retrospective Analysis of Hand Hygiene Compliance..................... 108

5.2.1 VISN 1 Hand Hygiene Management Tool ................................ 108

5.2.2 PVAMC Direct Hand Hygiene Observation Method................. 114

5.2.3 Facility-Wide Retrospective Hand Hygiene Compliance ........... 116

5.2.4 Inpatient-Wards Retrospective Hand Hygiene Compliance ....... 133

5.2.5 Retrospective Hand Hygiene and PPE Compliance................... 158

5.2.6 Retrospective PPE Compliance............................................ 177

5.3 Observational Study of Hand Hygiene Compliance.......................... 191

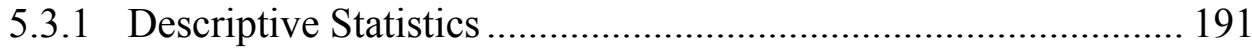

5.3.2 Hand Hygiene Population and Compliance ............................ 197

5.3.3 Hypothesis Testing ........................................................ 212

5.4 The Correlation of HAI and Hand Hygiene Compliance ................. 225

CHAPTER 6 INDIRECT HAND HYGIENE COMPLIANCE METHOD ....... 227

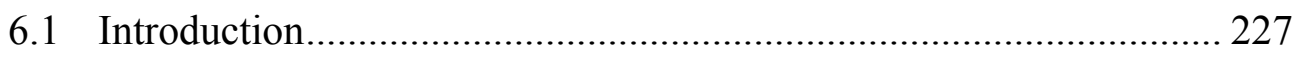

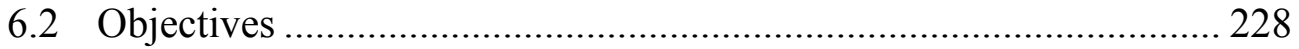




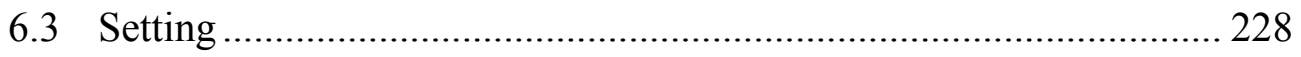

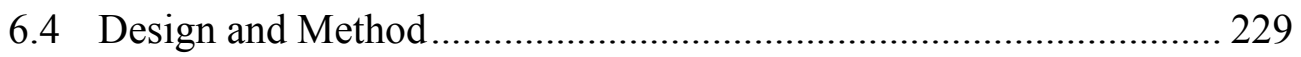

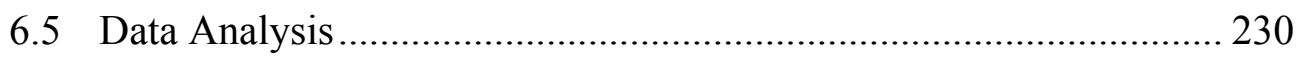

6.5.1 Inpatient-Wards Bed Days of Care vs. Patient Admission ......... 230

6.5.2 Soap-and-Purell Cartridge Replacement BDOC .......................... 234

6.5.3 Actual vs. Target Replacement of Soap and Purell..................... 249

6.5.4 Inpatient Ward Sales Data and Hygiene Products Use ............... 252

CHAPTER 7 INDIRECT PPE COMPLIANCE METHOD ............................... 254

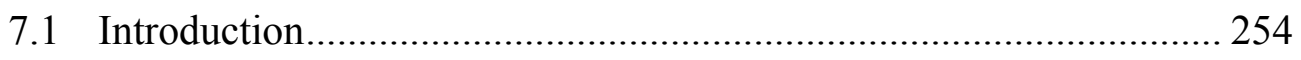

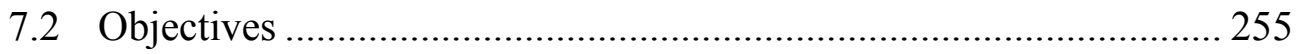

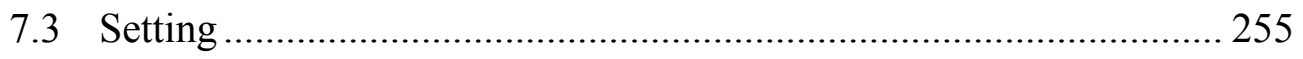

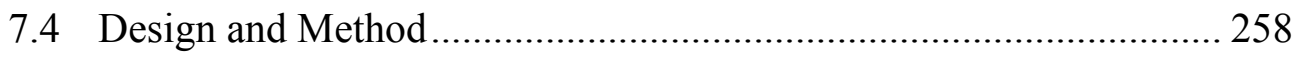

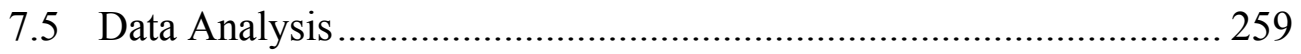

7.5.1 Inpatient-Wards Bed Days of Care vs. PPE Use......................... 259

7.5.2 PPE Ordering and PPE True Moments ....................................... 284

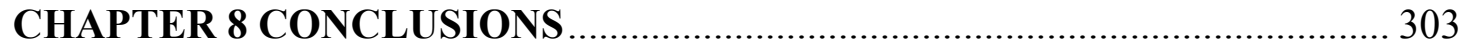

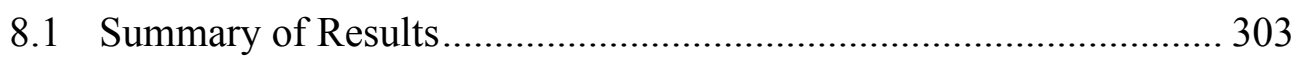

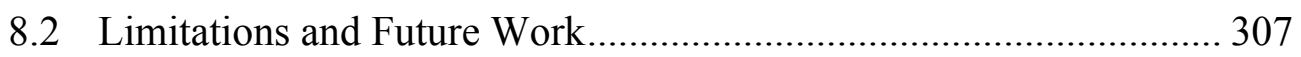

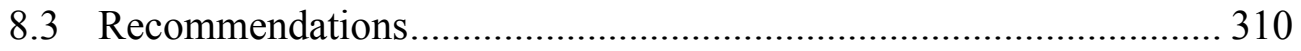

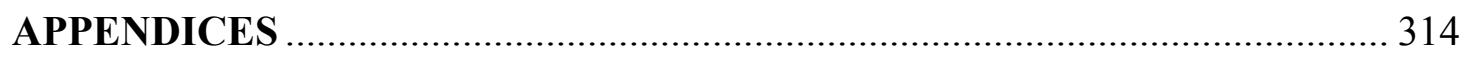

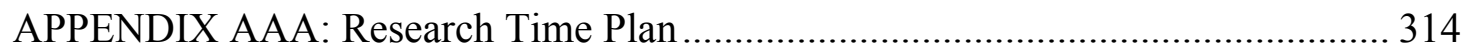

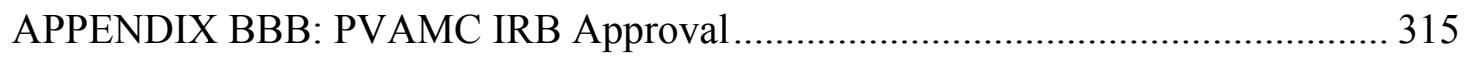

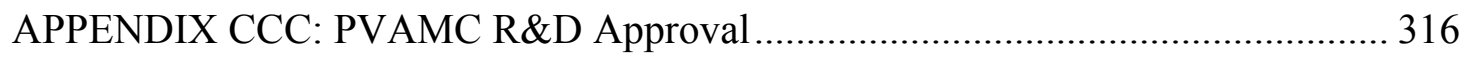

APPENDIX A: Employee Class at PVAMC …………………………………..... 317

APPENDIX B: Location and Service Type at PVAMC ............................................ 318

APPENDIX C: 6B Patient-Contact Day Shift Data Sheet........................................... 319

APPENDIX D: 6B Patient-Contact Evening Shift Data Sheet................................... 320

APPENDIX E: 6B Patient-Contact Night Shift Data Sheet ………………………... 321 
APPENDIX F: 5B Patient-Contact Day Shift Data Sheet

APPENDIX G: 5B Patient-Contact Evening Shift Data Sheet 323

APPENDIX H: 5B Patient-Contact Night Shift Data Sheet 324

APPENDIX I: ICU Patient-Contact Day Shift Data Sheet. 325

APPENDIX J: ICU Patient-Contact Evening Shift Data Sheet 326

APPENDIX K: ICU Patient-Contact Night Shift Data Sheet. 327

APPENDIX L: Patient Contact Consent Form 328

APPENDIX M: 6B Hand-Hygiene Compliance Monitoring Tool.

APPENDIX N: 5B Hand-Hygiene Compliance Monitoring Tool 332

APPENDIX O: ICU Hand-Hygiene Compliance Monitoring Tool ....... 333

APPENDIX P: Hand Hygiene and Precautions Compliance Monitoring Tool 334

APPENDIX Q: Hand Hygiene and Precautions Compliance Monitoring Tool with

Stethoscope 335

APPENDIX R: Hand Hygiene Management Site 336

APPENDIX S: Hand Hygiene Event Data Input Form 337

APPENDIX T: Secret Observer Consent Form 346

APPENDIX U: 6B Purell \& Soap Log Sheet 349 APPENDIX V: 5B Purell \& Soap Log Sheet 350

APPENDIX W: ICU Purell \& Soap Log Sheet 351

APPENDIX X: 6B HAIs per Operating Bed per Inpatient Unit Sheet. 352

APPENDIX Y: 5B HAIs per Operating Bed per Inpatient Unit Sheet. 353

APPENDIX Z: ICU HAIs per Operating Bed per Inpatient Unit Sheet. 354 APPENDIX AA: 5B and 6B Inpatient Ward Assignment Sheet 355 
APPENDIX BB: ICU Inpatient Ward Assignment Sheet

APPENDIX CC: 2-Step Checklist Sheet Page One. 357

APPENDIX CC: 2-Step Checklist Sheet Page Two 358

APPENDIX DD: Contact Precautions Sign Page One 359

APPENDIX DD: Contact Precautions Sign Page Two 360

APPENDIX EE: Droplet Precautions Sign Page One 361

APPENDIX EE: Droplet Precautions Sign Page Two 362

APPENDIX FF: Airborne Precautions Sign Page One 363

APPENDIX FF: Airborne Precautions Sign Page Two. 364

APPENDIX GG: Protective Environment Sign. 365

APPENDIX HH: Crown Program Sign 366

APPENDIX II: Strict I\&O’s Sign . 367

BIBLIOGRAPHY 368 


\section{LIST OF TABLES}

Table 2.1 Factors Influencing AdHerence to Hand Hygiene Practices (CDC, 2002) 25

Table 2.2 Advantages and Disadvantages of Different Types of AIDC

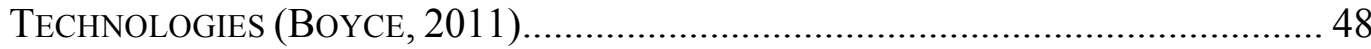

TABle 4.1 InPatient Wards AND Their Operating-Licensed Bed CAPACity ....... 64

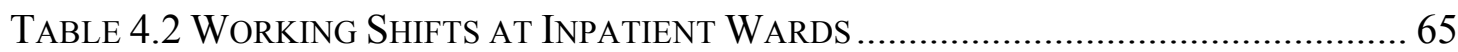

Table 4.3 Medical Doctor Participation Tracking Matrix ................................ 72

TABle 4.4 Patient Contact Frequency Per InPatient Wards Summarized by HEALTHCARE CATEGORY AND SUBCATEGORY .................................................. 80

Table 4.5 Patient Contact Frequency for InPatient Wards Combined SUMMARIZED BY HEALTHCARE CATEGORY AND SUBCATEGORY 81

Table 4.6 ICU InPatient Ward Patient Contact Frequency Per Shift SUMMARIZED By HEALTHCARE CATEGORY AND SUBCATEGORY 82

Table 4.7 5B InPatient Ward Patient Contact Frequency Per Shift SUMMARIZED By HEALTHCARE CATEGORY AND SUBCATEGORY 83

Table 4.8 6B InPatient Ward Patient Contact Frequency Per Shift SUMMARIZED BY HEALTHCARE CATEGORY AND SUBCATEGORY

Table 4.9 InPatient Wards Combined Patient Contact Frequency Per Shift SuMMARIZED By HEALTHCARE CATEGORY AND SUBCATEGORY 85

TABLE 4.10 ICU INPATIENT WARd PATIENT CONTACT FREQUENCY PER ISOLATION Status Summarized by HealthCARE CATEGORY AND SubCATEGORY 91

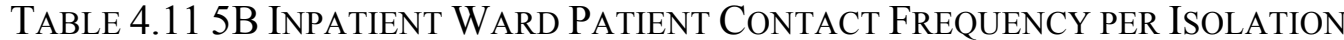
Status Summarized By HealthCARE CATEGORY AND SUBCATEGORY 92

TABLE 4.12 6B InPATIENT WARD PATIENT CONTACT FREQUENCY PER ISOLATION Status Summarized By HealthCARE CATEGORY AND SUBCATEGORY 93

TABle 4.13 InPatient Wards Combined Patient Contact Frequency PeR ISOLATION STATUS SUMMARIZED BY HEALTHCARE CATEGORY AND SUBCATEGORY 
TABle 5.1 Standard Precautions (VAMHCS, 2012) 102

Table 5.2 My Five Moments for Hand Hygiene Summarized by Hygiene PRODUCTS.

Table 5.3 (A) Facility-Wide Hand Hygiene Rate Summarized by My Five MOMENTS AND EMPLOYEE CLASS

Table 5.4 (B) Facility-Wide Hand Hygiene Rate Summarized by My Five MOMENTS AND EMPLOYEE CLASS

TABle 5.5 InPatient-Wards Hand Hygiene AdHEREnCe Rates Summarized by My Five Moments AND Hygiene Products 138

Table 5.6 InPatient-Wards Hand Hygiene Observations Converted to Purell AND SOAP CARTRIDGES

TABle 5.7 (A) 5B InPatient-Ward Hand Hygiene Rate Summarized by EMPLOYEE Class AND My FIVE MOMENTS 145

TABle 5.8 (B) 5B InPATIENT-WARd HAND Hygiene Rate SumMARIZEd by EMPLOYeE Class AND My Five MOMENTS 146

Table 5.9 (A) 6B InPatient-Ward Hand Hygiene Rate Summarized by EMPLOYeE Class and My Five MOMENTS

TABLE 5.10 (B) 6B InPatient-Ward Hand Hygiene Rate Summarized By EMPLOYEe Class AND My Five MOMENTS 148

TABle 5.11 (A) ICU InPatient-WARD Hand Hygiene Rate Summarized by EMPLOYEe Class AND MY Five MOMENTS

TABLE 5.12 (B) ICU InPATIENT-WARd HAND Hygiene RATE SumMaRIZED BY EMPLOYeE Class AND My Five MOMENTS 150

TABLE 5.13 (A) 5B InPatient-WARd Hand Hygiene Rate Summarized By Employee Class, My Five Moments and Hygiene Products 152

TABLE 5.14 (B) 5B InPATIENT-WARd HAND Hygiene RATE SumMARIZED BY Employee Class, My Five Moments and Hygiene Products 153

TABle 5.15 (A) 6B InPatient-WARd Hand Hygiene Rate Summarized By Employee Class, My Five Moments and Hygiene Products 154

TABLE 5.16 (B) 6B InPATIENT-WARd HAND Hygiene RATE Summarized BY Employee Class, My Five Moments and Hygiene Products 155 
TABle 5.17 (A) ICU InPatient-Ward Hand Hygiene Rate Summarized By Employee Class, My Five Moments and Hygiene Products 156

TABLE 5.18 (B) ICU InPaTient-Ward Hand Hygiene RATE Summarized By Employee Class, My Five Moments and Hygiene Products. 157

TABLE 5.19 FaCiLITY-Wide HAND HygiENE AND PPE OBSERVATIONS SUMmaRIZED BY PRECAUTION TYPES AND YEAR. 160

TABLE 5.20 InPatient-WARdS HAND HygIENE ANd PPE OBSERVATIONS SUMMARIZED By PRECAUTION TYPES 161

TABle 5.21 Facility-Wide PPE ObSeRVATIONS SumMarized by PreCaution TyPeS PER YEAR 178

TABle 5.22 InPatient-Wards PPE ObSERVATIONS Summarized By PreCaUtion TYPES PER YEAR. 179

TABle 5.23 Monthly Mean, Standard DeViation and 95\% Confidence InTERVAL FOR HAND HYGIENE COMPLIANCE PER INPATIENT WARD AND ALL WARDS COMBINED 205

TABle 5.24 One Sample Hypotheses Test (90\%) For Monthly Hand Hygiene COMPLiance MEAN For All InPaTIENT Wards COMBINED ..... 207

TABle 5.25 One Sample Hypotheses Test (50\%) For Monthly Hand Hygiene COMPLiance Mean for All InPatient Wards COMBined .... 208

TABle 5.26 First Hypothesis Test: Proportion of HAND Hygiene Compliance by HCWS GENDER 212

Table 5.27 Second Hypothesis Test: Proportion of Hand Hygiene Compliance BY HCWS' JOB CATEGORY

TABle 5.28 Third Hypothesis Test: Proportion of Hand Hygiene Compliance BY INPATIENT WARDS

Table 5.29 Hypothesis Three: The Marascuilo Procedure for Multiple PROPORTIONS COMPARISONS OF INPATIENT WARDS 215

Table 5.30 Fourth Hypothesis Test: Proportion of Hand Hygiene Compliance

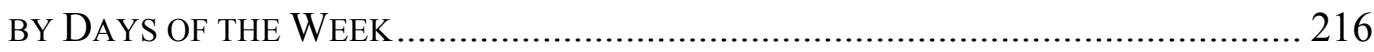

Table 5.31 Hypothesis Four: The Marascuilo Procedure for Multiple PROPORTIONS COMPARISONS OF DAYS OF THE WEEK 
Table 5.32 Fifth Hypothesis Test: Proportion of Hand Hygiene Compliance by HAND HYGIENE MOMENTS ............................................................................ 218

Table 5.33 Sixth Hypothesis Test: Proportion of Hand Hygiene Compliance by PATIENT'S GENDER ......................................................................................... 219

Table 5.34 Seventh Hypothesis Test: Proportion of Hand Hygiene Complance

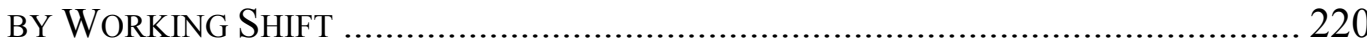

Table 5.35 Hypothesis Seven: The Marascuilo Procedure for Multiple PROPORTIONS COMPARISONS OF WORKING SHIFT

Table 5.36 Eighth Hypothesis Test: Proportion of Hand Hygiene Compliance BY PATIENT’S ISOLATION STATUS............................................................. 221

TABle 5.37 Hypothesis Test for Two Proportions (Hypothesis 1, 2, 5, 6 And 8), 95\% CONFIDENCE INTERVAL AND P-VALUE. 223

Table 5.38 Hypothesis Test for SeVeral Proportions (Hypothesis 3,4 ANd 7) AND P-VALUE

TABLE 5.39 HEALTHCARE-ASSOCIATED INFECTIONS (HAI) AT THE PVAMC FOR YEAR 2012-2015

Table 6.1 Pros and Cons of Indirect Monitoring of Hand Hygiene Performance by Measuring the Consumption of Product Use (WHO, 2009, PAGE 161 AND 162).............................................................................. 228

TABle 6.2 Aggregated SoAP And Purell for InPAtient WARds Combined ...... 237

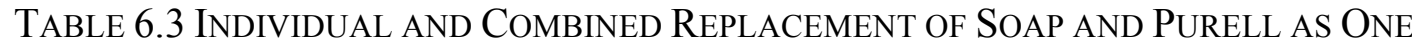
HYGIENE PRODUCT PER INPATIENT WARD........................................................ 238

Table 6.4 Total Purell and Soap Replacement and the Associated Total COST OF EACH HYGIENE PRODUCT PER INPATIENT WARD 239

TABle 6.5 CORRElation COEFFICIENTS OF BDOC VERSus HygIENE PRODUCTS REPLACEMENT 241

Table 6.6 The Likelihood Ratio Test for Negative Binomial GLM Model ... 242

TABle 6.7 Hand Hygiene AdHerence Rates Per InPATIENT Ward PER MONTH BASED ON INDIRECT METHOD 246 
Table 6.8 Descriptive Statistics of Hand Hygiene Adherence Rate Based on Indirect Method of Hygiene Products RePlacement PER INPATIENT WARD PER MONTH 2015

Table 6.9 Actual versus Target Replacement of Hygiene Products Per Ward PER MONTH 251

Table 7.1 Pros and Cons of Indirect Manual Monitoring of PPE Performance BY MEASURING INVENTORY CONSUMPTION .................................................... 255

TABle 7.2 MEAn, VARIANCE AND STANDARd DEVIATION OF PPE PER INPATIENT WARD AND INPATIENT WARDS COMBINED FOR YEAR 2014 .......................................... 261

TABLE 7.3 CORRELATION COEFFICIENTS OF BDOC VERSUS PPE PER INPATIENT WARD

TABLE 7.4 PPE TRUe AND PRediction VALUes of YeAr 2016 PER INPATIENT WARD

TABLE 7.5 PPE PREDICTION VALUES PER WARD FOR YEAR 2015 271

TABle 7.6 MeAn, VARIANCE AND StANDARd DEVIATION OF PPE PER WARD AND WARDS COMBINED 274

TABle 7.7 MeAn AND StANDARd DEVIATION OF PPE PER WARD PER YeAR 274

TABLE 7.8 DESCRIPTIVE STATISTICS OF BDOC PER WARD AND WARDS COMBINED PER YEAR 279

TABLE 7.9 CORRELATION COEFFICIENTS OF BDOC VERSUS PPE PER WARD AND WARDS COMBINED 281

TABle 7.10 Summary Of Descriptive Statistic of Gloves COMPliance PER WARD AND WARD COMBINED (2014-2016) 298

TABle 7.11 Summary of Descriptive Statistic OF GOWNS COMPLIANCE PER WARD AND WARD COMBINED (2014-2016) 300 


\section{LIST OF FIGURES}

Figure 2.1 My 5 Moments for Hand Hygiene (WHO, 2009) .................................. 26

Figure 2.2 RFID TeChNOLOGY AND OTHER TYPeS OF AIDC TECHNOLOGIES (WAMBA,

LEFEBRVE, BENDAVID AND LEFEBVRE, 2008) ................................................... 46

Figure 4.1 Hierarchical Structure of Patient Contact Participants Per INPATIENT WARD PER WORKING SHIFT .............................................................. 73

Figure 4.2 Matrix Structure of Patient Contact Nursing Participants Per INPATIENT WARD PER WORKING SHIFT BY DATE OF PARTICIPATION ..................... 74

Figure 4.3 Before and After Touching a Patient and Total Patient Contact POPULATION PER INPATIENT WARD AND WARDS COMBINED ................................. 79

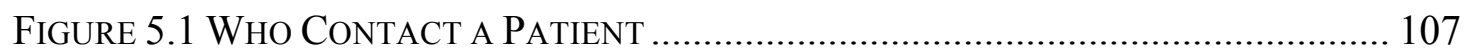

Figure 5.2 RoAdmap Retrosepctive ANALysis of Hand Hygiene Direct

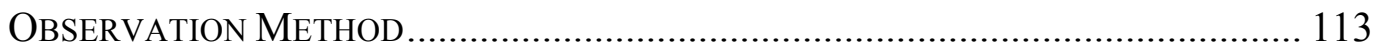

Figure 5.3 Facility-Wide Hand Hygiene Adherence Rate................................ 117

Figure 5.4 ISOLATION (Option THREE) VS. ISOLATION AND NON-ISOLATION (OPTION One) Facility-Wide Hand Hygiene Adherence Rate ................................... 119

Figure 5.5 Facility-Wide Hand Hygiene ObServations Summarized By Hygiene PRODUCTS YEARS COMBINED ........................................................................ 121

Figure 5.6 Facility-Wide Hand Hygiene ObServations Summarized by Hygiene PRODUCTS PER YEAR ……………………………………………………..... 122

Figure 5.7 Facility-Wide Hand Hygiene ObServations Summarized By My Five

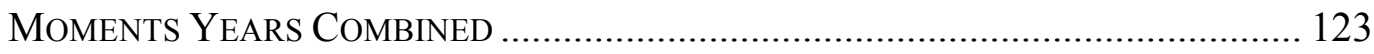

Figure 5.8 Facility-Wide Hand Hygiene ObServations Summarized By My Five MOMENTS AND BY HYGIENE PRODUCTS .......................................................... 124

Figure 5.9 FaCiLity-Wide Hand Hygiene ObSERVATIONS SUMmarized by

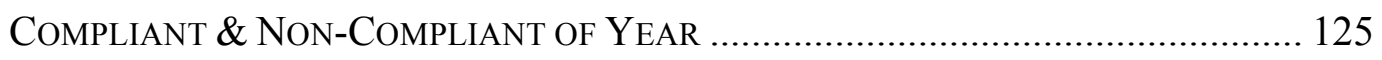

Figure 5.10 Facility-Wide Hand Hygiene ObServations Summarized by Division, COMPLIANT AND NON-COMPLIANT OF STATION 126 
Figure 5.11 Facility-Wide Hand Hygiene ObSERVATIONS SumMarized by COMPLIANT \& NON-COMPLIANT OF LOCATION

Figure 5.12 FaCility-Wide Hand Hygiene OBSERVATIONS SuMmarized By LOCATION PER MONTH PER YEAR.............................................................. 128

Figure 5.13 Facility-Wide Hand Hygiene ObSERVATIONS SumMarized By COMPLIANT AND NON-COMPLIANT OF EMPLOYEE CLASS .................................. 130

Figure 5.14 InPatient-WaRds Hand Hygiene Rate ............................................. 133

Figure 5.15 InPatient-Wards Isolation vs. Non-Isolation Hand Hygiene Rate

Figure 5.16 InPATIENT-WARDS HAND HYGIENE OBSERVATIONS SUMMARIZED BY HYGIENE PRODUCTS AND YEARS COMBINED................................................... 135

Figure 5.17 InPATIENT-WARDS HAND HygIENE OBSERVATIONS SUMMARIZED BY

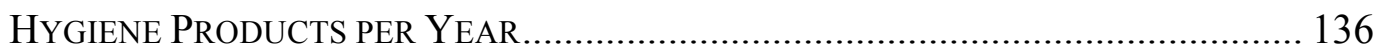

Figure 5.18 InPatient-Wards Hand Hygiene Rate Summarized by My Five MOMENTS

Figure 5.19 InPatient-Wards Hand Hygiene ObServations Summarized by My Five MOMENTS AND Hygiene PROducts

Figure 5.20 InPATIENT-WARdS HAND Hygiene OBSERVATIONS AND COMPLIANCE

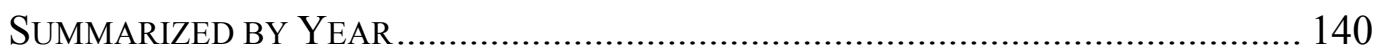

Figure 5.21 InPatient-Wards Hand Hygiene AdHERENCE RATE Summarized by EMPLOYEE CLASS

Figure 5.22 InPATIENT-WARdS HANd Hygiene OBSERVATIONS AND COMPLiANCE SumMarized by EMPLOYEe Class AND My Five MOMENTS 144

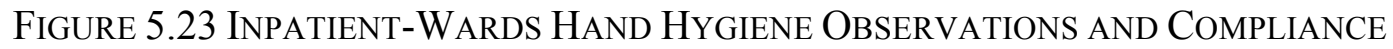
Summarized by Employee Class, My Five Moments and Hygiene Products

Figure 5.24 Facility Wide Hand Hygiene and PPE ObSeRvations by Precaution TYPES AND YEAR 160

Figure 5.25 InPATIENT-WARdS HAND HygIENE AND PPE OBSERVATIONS AND COMPLIANCE FOR CONTACT PRECAUTIONS INCLUDING MASKS 165 
FiguRE 5.26 INPATIENT-WARDS HAND HYGIENE AND PPE OBSERVATIONS AND

Figure 5.27 INPATIENT-WARdS HAND HygIENE AND PPE OBSERVATIONS AND

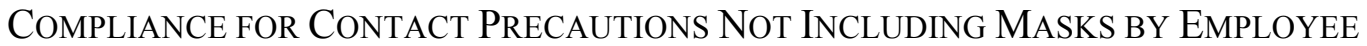
CLASS

Figure 5.28 INPATIENT-WARDS HAND HyGIENE AND PPE OBSERVATIONS FOR Droplet PRECAUTIONS. 173

FigURE 5.29 INPATIENT-WARDS HAND HYGIENE AND PPE OBSERVATIONS FOR Droplet PRECAutions by EMPloyee Class

Figure 5.30 InPATIENT-WARDS HAND Hygiene AND PPE OBSERVATIONS FOR AIRBORNE PRECAUTIONS 176

Figure 5.31 InPATIENT-WARdS HAND HygIENE AND PPE OBSERVATIONS FOR Airborne PRECAUTIONS By EMPLOYEe Class

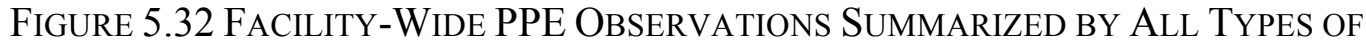
PRECAUTION PER MONTH AND YEAR. 178

Figure 5.33 InPATIENT-WARds PPE ObSERVATIONS For CONTACT PReCAUtions

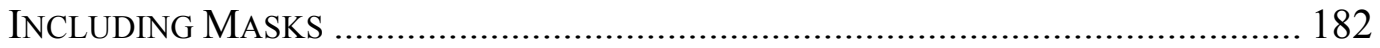

Figure 5.34 InPATIENT-Wards PPE ObSERVATIONS FOR Contact PRECAUTIONS Not INCLUDING MASKS 184

Figure 5.35 InPatient-Wards PPE ObSerVations for Contact Precautions Not INCLUDING MASKS BY EMPLOYEE CLASS 185

Figure 5.36 InPatient-Wards PPE OBSERVATIONS For Droplet PreCAUTIONS .. 187

Figure 5.37 InPATIENT-WARdS PPE ObSERVATIONS For Droplet Precautions By EMPLOYEE CLASS 188

Figure 5.38 InPatient-Wards PPE ObSERVATIONS FOR Airborne PreCaUtions By EMPLOYEE CLASS 190

Figure 5.39 Summary STATISTiC OF Hand Hygiene ObServations (Total SAMPLES, HAND Hygiene COMPLIANCE AND NON-COMPLIANCE AND Percentages)

Figure 5.40 Patient Contact ANd BDOC PER WeEkday PER InPATIENT WaRd AND INPATIENT WARDS COMBINED..... 
Figure 5.41 PATIENT CONTACT AND BDOC (IsOlation AND NON-IsOlation) PER MONTH PER INPATIENT WARD AND INPATIENT WARDS COMBINED 201

Figure 5.42 Weekly and Monthly Hand Hygiene Compliance Mean, Standard DEVIATION AND SAMPLE SIZE PER INPATIENT W ARD AND COMBINED FOR SEPTEMBER AND OCTOBER 2016 202

Figure 5.43 Monthly Hand Hygiene Compliance Mean and 95\% Confidence INTERVAL OF SEPTEMBER AND OCTOBER PER INPATIENT WARDS AND ALL WARDS COMBINED 210

Figure 5.44 Correlation of Weekly Hand Hygiene Mean with Weekly BDOC PER INPATIENT WARD AND ALL WARDS COMBINED. 211

FigURE 5.45 CORRELATION OF HEALTHCARE-AsSOCIATED INFECTION (HAI) AND HAND HYGIENE COMPLIANCE (\%) FOR YEAR 2012-2015 AT THE PVAMC. 226

FigURE 6.1 HisTOGRAM OF BDOC OF INPATIENT WARDS. 231

Figure 6.2 AdMisSion VERSUS BDOC AGgREGATED (2011-2016) PER INPATIENT WARD 232

Figure 6.3 Modeling AdMission Versus BDOC with Negative BinOMial DisTRIBUTION AND A LINEAR DETERMINISTIC FUNCTION …………………....... 233

Figure 6.4 CONSUMPTION OF Hygiene PRoducts PER INPATIENT WARD ................. 235

Figure 6.5 Histograms of PURELl AND SoAP RePLACEMENT InPatient WARDS COMBINED 236

Figure 6.6 CONSUMPTION OF Hygiene PRODUCts PeR InPATIENT WARD 237

FiguRe 6.7 BDOC VERSUS PURELL AND SoAP REPLACEMENT................................... 240

Figure 6.8 BDOC VERSUS PURELL AND SOAP REPLACEMENT DURING 2015.............. 241

Figure 6.9 BDOC VERSus SoAP AND PuRell RePlacement At ICU........................ 242

Figure 6.10 BDOC Versus Hand Hygiene Compliance Rate Per InPATIENT Ward BASED ON INDIRECT METHOD 245

Figure 6.11 Hand Hygiene AdHerence Rate Per InPatient Ward Per Month (2015) 247

Figure 6.12 Actual (Purple) versus Target (Green) Replacement of Hygiene PRODUCTS PER INPATIENT WARD PER MONTH (2015) 250 
Figure 6.13 Total Number of SoAP AND PuREll Cartridges RePlaced During 2015 At 5B, 6B AND ICU COMPARED With SAles Data FOR OVERALl FaCility AT THE PVAMC.

Figure 6.14 Breakdown of Product Use of Soap and Purell Cartridges COMPARED TO THE REPLACED UNITS DURING 2015. 253

Figure 7.1 Primary Inventory PPE Products AND Their Stock AND ReORDER LEVELS

Figure 7.2 BDOC versus PPE (Gloves, Gowns AND Masks) PER WARd FOR Year 2014 262

Figure 7.3 Negative Binomial Distribution with a Power Deterministic FunCtion FitTED MOdel FOR BDOC VERSUS GLOVES PER WARD FOR YEAR 2014

Figure 7.4 Negative Binomial Distribution with a Power Deterministic FUNCTION FITTED MODEL FOR BDOC VERSUS GOWNS PER WARD FOR YEAR 2014

Figure 7.5 Negative BinOMial Distribution with a Linear Deterministic FUNCTION FITTED MODEL FOR BDOC VERSUS MASKS PER WARD FOR YEAR 2014

Figure 7.6 Gloves True (Green) ANd Prediction (Red) Values Per Month Per YEAR 2016 PER WARD WITH MSPE AND MAPE VALUES 268

Figure 7.7 Gowns True (Green) and Prediction (Red) Values Per Month Per YEAR 2016 PER WARD WITH MSPE AND MAPE VALUES 269

Figure 7.8 Masks True (Green) and Prediction (Red) Values Per Month Per YEAR 2016 PER WARD WITH MSPE AND MAPE VALUES 270

Figure 7.9 Quantity of Gloves Ordered Per Ward Per Month PER Year ....... 273

Figure 7.10 Quantity of Gowns ORdered PER Ward PER Month PER Year...... 275

Figure 7.11 Quantity of MASKs ORdered PER WARD PER Month PER YeAr ...... 276

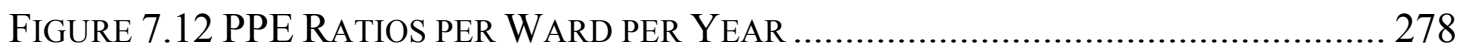

FigURE 7.13 BDOC PER WARD PER MONTH PER YEAR ............................................... 279

FigURE 7.14 BDOC VERSUS PPE PER WARD FOR YEAR 2014-2016 ............................ 281 
Figure 7.15 Negative BinOMial Distribution with a Linear Deterministic FUNCTION FITTED MOdEL FOR BDOC VERSUS GLOVES PER WARD FOR YEAR 2014-2016

Figure 7.16 Negative BinOMial Distribution With a Linear Deterministic FUNCTION FITTED MOdEL FOR BDOC VERSUS GOWNS PER WARD FOR YEAR 2014-2016

Figure 7.17 Negative Binomial Distribution With a Linear Deterministic FUNCTION FITTED MODEL FOR BDOC VERSUS MASKS PER WARD FOR YEAR 2014-2016

Figure 7.18 Standard (PuRPLE) BDOC Versus TRANSMission (Red) BDOC Ratio PER WARD PER YEAR

Figure 7.19 Standard (Purple) BDOC Versus Transmission (Green) BDOC PER WARD PER MONTH PER YEAR 293

Figure 7.20 Gloves Moments versus Gloves Quantity Ordered Per Ward (2014-2016)

Figure 7.21 Gowns Moments Versus Gowns QuANTITY ORDERED PER WARD (2014-2016)

Figure 7.22 Negative Binomial Distribution with a Power Deterministic FunCtion FitTED MODEl FOR Gloves MOMENTS VERsus Gloves QuANTITY ORDERED PER WARD (2014-2016). 295

Figure 7.23 Negative Binomial Distribution with a Power Deterministic FunCtion FitTED MOdel FOR GOWNS MOMENTS VERSUS Gowns QuANTITY ORDERED PER WARD (2014-2016) 296

Figure 7.24 Gloves Compliance (\%) PER Ward (2014-2016) ............................... 298

Figure 7.25 GownS Compliance (\%) PER WARD (2014-2016) ................................. 300

Figure 7.26 PPE Non-CoMPLIANCE (\%) PER WARD (2014-2016)............................ 302 


\section{CHAPTER 1}

\section{INTRODUCTION}

Saving lives, whether human beings or other types of creatures, is a noble action. One of the best opportunities to save lives exists in healthcare settings. People visit hospitals seeking cures or treatment for all kinds of healthcare issues. Yet, healthcare settings can endanger their lives by exposing patients to infections.

According to Horan, Andrus and Dudeck (2008), colonization in healthcare means the presence of a bacteria or a microorganism inside or outside a body but with no presence or symptoms of an illness such as the presence of a bacteria on healthcare worker's hands. However, those colonized people, whether healthcare workers (HCWs) or other patients or their visitors could transmit and transfer that microorganism to patients or to medical objects like catheters and inanimate objects that the patients use and may cause infection (Centers for Disease Control and Prevention (CDC, 2002).

The Joint Commission Center for Transforming Healthcare (TJC) (2009) states that according to CDC (2002) and the World Health Organization (WHO) (2009), ignoring or neglecting to comply with hand hygiene for whatever reasons is one of the critical factors to the spread of Healthcare-Associated Infections (HAIs). CDC (2014) states "in the United States, hospital patients get an estimated 722,000 infections each year" and "that's about 1 infection for every 25 patients" (CDC, 2014). CDC (2002) mentions that "hand hygiene is a general term that applies to either handwashing, antiseptic handwash, antiseptic hand rub, or surgical hand antisepsis" (CDC, 2002, p. 3). 
According to TJC (2009) "Other factors include such things as patient severity of illness, equipment and environmental sanitation practices, and adherence to recommended practices" (TJC, 2009, xvi). Thus, according to Pittet (2003) and CDC (2002), hand hygiene is one of the most important intervention tools to prevent and control the spreading of HAIs. Advanced technologies have been widely used in healthcare to reduce cost of care and improve quality of care. Yet, controlling the transmission of healthcare-associated infections (HAIs) between healthcare workers (HCWs) and patients, and preventing disease from spreading within the hospital environment remain unsolved (Pittet, 2003).

At the Providence Veterans Affairs Medical Center (PVAMC), one way of saving the veterans' lives, besides providing them with high quality care, is by preventing and controlling infectious diseases. As an additional systematic approach to the direct observation method, the indirect manual monitoring of product use and indirect method of measuring personal protective equipment (PPE) are needed to monitor hand hygiene practices. These methods are intended to have a better estimate of hand hygiene compliance.

The facility size of a medical center, the authorized operating beds and the number of bed days of care influence the infections rate at the facility level when comparisons are conducted to rank facilities. As the total number of operating beds and bed days of care increases, the rate of infections starts to diminish though the number of infections keeps growing. One of the motivations to conduct this study is that the Medical Center at Providence is considered a medium medical facility compared to other Veterans Affairs facilities nationwide with 73 operating beds 
(licensed), in which 56 operating beds are in three inpatient-units 23 beds in Ward 5B, 27 beds in Ward 6B and 8 beds in the Intensive Care Unit (ICU) are associated with a monthly average of 319 admissions and a monthly average of 1200 bed days of care based on 4-fiscal-years worth of data (2012-2015). Because the PVAMC has low numbers of healthcare-associated infections (HAIs) each year, the hospital is sometimes ranked low when compared to other Veterans Affairs facilities in the same categories. These inpatient wards are considered acute care wards. However, the 5B ward is considered a step-down unit.

The aim of this research is to study hand hygiene compliance by directly observing healthcare workers (HCWs) at the Providence Veterans Affairs Medical Center (PVAMC) and to obtain a more accurate estimate of the total number of times per week that a doctor or nurse contacts different patients in an effort to identify the best estimate of the true hand hygiene compliance rate at PVAMC. The estimate of the true hand hygiene compliance rate will be measured by first using descriptive or summary statistics of hand hygiene compliance per inpatient ward and all wards combined. Hypotheses testing of selected risk factors for poor adherence to recommended hand hygiene practices will be conducted and correlations of hand hygiene compliance with BDOC and HAIs will be made. Self-reporting data of patient-contacts by doctors and nurses who work at the inpatient units, 5B, $6 \mathrm{~B}$ and ICU at PVAMC for all shifts (Night, Day and Evening) will be collected. Then, recruited secret observers and the University of Rhode Island (URI) research team member will observe healthcare workers (doctors and nurses) at the inpatient units during hand hygiene critical moments (Before and After Patient-Contact). Patient- 
contact data collection will be done for one complete week, and secret observers data collection will be conducted one month prior to self-reporting patient-contact data and one month overlapping with the week of patient-contact data collection. One month prior, and one month overlapping the one week self-reporting patient-contact data collection, the URI research member will also do hallway observations to augment the self-report data on patient contacts that comes from the doctors and nurses randomly in the inpatient units, 5B, 6B and ICU at PVAMC. Hand hygiene will then be compared with the total number of Healthcare Associated-Infections (HAIs) of interest, individually and collectively, to look for correlations and trends. In addition, the aim of this research is to study hand hygiene compliance by indirectly monitoring hand hygiene of healthcare workers (HCWs) at the Providence Veterans Affairs Medical Center (PVAMC) by monitoring the consumption of product use by counting how many times products have been used and replaced in each inpatient-units $(5 \mathrm{~B}, 6 \mathrm{~B}$ and ICU). As a secondary measurement, a new indirect hand hygiene manual monitoring of product use method and a new indirect method of measuring personal protective equipment (PPE) compliance will be proposed to compare inpatient-units (5B, 6B and ICU) at PVMAC against each other because they are of different sizes in terms of number of operating beds (OB) and bed days of care (BDOC). The indirect method of measuring personal protective equipment (PPE) compliance is not found in the literature.

Historical data of key infectious diseases at PVAMC will be used to assess appropriate statistical analysis methods and recommend improved methods of tracking and reporting data for hand hygiene compliance of HCWs. In the healthcare industry, 
the challenge is to prevent and control the prevalence and transmission of HAIs to patients inside the hospitals who were admitted for medical needs rather than the treatment of infectious diseases.

According to TJC (2009), "measurement is only the beginning" (TJC, 2009, p.107). Thus, after measuring the hand hygiene rate precisely and accurately, barriers that hinder HCWs from practicing hand hygiene accordingly can be identified and tackled for further hand hygiene compliance improvement. However, tracking and measuring hand hygiene practices of HCWs is no easy task since it involves privacy issues of HWCs as well as privacy issues of patients. Before exploring each method and its strengths and limitations, hospital-associated infections (HAIs) of interest are first explored and defined.

There is always room for improvement in hand hygiene compliance measurement. In this study, a more reliable direct hand hygiene observation method is conducted at a Veterans Affairs Medical Center by recruiting secret observers. Reliability of the method is met by having a larger sample size of hand hygiene observations that ensures an error size of about 3\% from the population hand hygiene compliance parameter, by focusing on two important healthcare worker groups, medical and nursing and by exploring total patient contact of each group to see whether confidence interval of hand hygiene compliance could be corrected using the finite population correction (fpc) factor to reduce the standard error by a value equal to $\sqrt{\frac{N-n}{N-1}}$ where $\mathrm{N}$ is the population size and $\mathrm{n}$ is the sample size. Exploring variations in hand hygiene practices between acute care units and examining risk factors that contribute to poor hand hygiene compliance in a healthcare setting belonging to a 
Veterans Affairs Medical Center have not been attempted before. Risk factors such as HCWs gender, healthcare profession, acute care units, weekdays, hand hygiene moments, patient isolation status, and working shift have been already studied in different healthcare settings (Pittet, 2000; WHO, 2009; Duggan, Hensley, Khuder, Papadimos and Jacobs, 2008; Arenas et al., 2005). However, the Veterans Affairs Medical Center still is considered having a very unique healthcare setting in terms of the way HCWs interact with patients and in terms of gender imbalance of HCWs and of patients. Thus, gender imbalance is a unique risk factor that fits the Veterans Affairs Medical Center and has not been statistically studied before. So, it is interesting to examine and explore how hand hygiene compliance varies by such a factor. Exploring the variations of hand hygiene practices between HCWs and Patient by gender is very interesting under such a healthcare setting.

In this study, the indirect hand hygiene manual monitoring of product use is improved mostly by having better average patient contact data based on self-report data for one complete week and also encompassing all device-associated moments (device-invasive days). Such a method is a good alternative for hand hygiene compliance monitoring if the direct hand hygiene observation method is not an option. In addition, a brand new indirect method of measuring personal protective equipment (PPE) compliance is developed based on an assumption that states PPE quantity ordered is PPE quantity consumed.

Radio Frequency Identification (RFID) technology for hand hygiene measurement and intervention was considered, however, the request was not accepted by the PVAMC because of privacy issues. RFID was also suggested for patient 
contact data collection, which could be used over at least 6 months to have a better estimate of average patient contact per day, however, the request was also not accepted.

The study has several limitations common to research studies in the healthcare field and in hand hygiene compliance. The major limitation was that the research methods, (patient contact, direct hand hygiene observation, indirect hand hygiene manual monitoring of product use and indirect method of measuring personal protective equipment (PPE) compliance) did not overlap and were not conducted over one timeline. In addition, patient contact and the direct hand hygiene observation method data collection were conducted once and not three times as planned. In the indirect hand hygiene manual monitoring of product use, residues of Soap and Purell cartridges were not measured, which could impact the hand hygiene compliance estimate. In the indirect method of measuring personal protective equipment (PPE) compliance, the assumption that states PPE quantity ordered is PPE consumed on the floor has to be validated more.

Nevertheless, this study is beneficial to the Providence Veterans Affairs Medical Center and could also help 168 other Veterans Affairs Medical Centers nationwide in the United States of America and other hospitals in general that are of similar healthcare settings to the Veterans Affairs Medical Center. 


\section{CHAPTER 2}

\section{REVIEW OF LITERATURE}

There are several aspects of hand hygiene in the literature when it comes to tracking and observing healthcare workers (HCWs). According to CDC (2002), before proceeding with tracking, observing and measuring the hand hygiene compliance of HCWs, one should determine which aspect or aspects to measure and observe. Consequently, one can determine types of products used to cleanse hands and monitor the use of the appropriate product type after contacting patients. For example, Clostridium Difficile Infection (CDI) requires washing hands with soap and water, thoroughness of cleansing, catching key moments of washing hands such as "My five moments for hand hygiene" introduced by WHO (2009), and wearing gloves (CDC, 2002), which is part of the use of personal protective equipment (PPE) when it comes to adhering to the right precautions before entering a patient room.

There are several hand hygiene adherence measurement methods defined in the literature to remind healthcare workers to cleanse and wash hands during key moments or what WHO (2009) calls "My 5 moments for hand hygiene", and to track, measure and report hand hygiene compliance rate per individual, per healthcare professional category and per division. Some are technology-based such as electronic counting devices and radio frequency identification number (RFID) based on real time locating system (RTLS), and some are not such as direct observation (Secret Observers or Shoppers) and product usage. Each has strengths and limitations and leads to different rates of hand hygiene compliance. Each has a different sample size, whom and how frequently to observe, and which opportunities of five moments to 
observe. Some studies have combined multiple methods and some others have just applied one method to measure hand hygiene practice among HCWs.

\subsection{Healthcare and Epidemic Infectious Diseases}

Over the past 15 years, several new virus strains have emerged in various areas of the world that have challenged healthcare systems. They have been similar because each of them began in a specific region of the world, and caused concern of an epidemic as people traveled to and from those areas. They are also similar because many of them had a link with transmission hosts in animals (for example the bird flu, H1N1 in the United States, swine flu, SARS in China, MERS and Coronavirus which may spread through camels or bats, etc.). Healthcare-Associated Infections (HAIs) are adverse events that can be spread or contracted while a patient is in the hospital for treatment of unrelated health conditions. In contrast, Community-Acquired Infections (CAI) are adverse events that can be spread or contracted in a community settings. Unfortunately, some infectious disease cases have risen in community settings as well, which causes epidemics such as Ebola in Sierra Leone and other African countries, which has recently become the first concern of the World Health Organization, WHO. Preventing and controlling the spread of any kind of infectious diseases would not only result in a significant reduction in costs, but also result in better patient outcomes.

\subsubsection{Hospital-Onset Infections Caused by Specific Pathogens}

According to Magill, Edwards, Bamberg, et al. (2014), Hospital-Onset infections are those infections caused by specific pathogens such as Clostridium Difficile Infection (C.Diff or CDI) and Methicillin-Resistant Staphylococcus Aureus 
Bacteremia (MRSA), and develop "onset symptoms on or after the third day of hospitalization with the day of admission counted as the first day" (Magill, Edwards, Bamberg, et al., 2014, p.1206).

CDI and MRSA have been the two most major contributor pathogens to the occurrence of healthcare-associated infections HAIs in healthcare settings in the United Estates (McDonald, Owings, Jernigan, 2006). CDI and MRSA are associated with significant morbidity, mortality and burden cost of healthcare in the United States.

Healthcare settings are required to isolate patients who acquire diseases including, but not limited to, C.Diff, MRSA or MRSA colonization on Contact Precautions rooms because they are contagious (Siegel, Rhinehart, Jackson and Chiarello, 2007). "Contact Precautions are intended to prevent transmission of infectious agents, including epidemiologically important microorganisms, which are spread by direct or indirect contact with the patient or the patient's environment" (Siegel, Rhinehart, Jackson and Chiarello, 2007, p.70).

\subsubsection{Clostridium Difficile Infection (C.Diff or CDI)}

According to Gerding and Johnson (2012), Clostridium Difficile Infection (C.Diff or CDI) is an infection caused by a harmful bacterium that dominates the intestine when the harmless bacteria were killed due to the excessive use of antibiotics. Once the spores of Clostridium Difficile start to ingest and overgrow inside the intestine and release toxins, the lining of the intestine gets affected and the infection takes place. Traugott (2013) mentions several risk factors that might contribute to CDI incidence including but not limited to "advanced age, duration of 
hospitalization, antimicrobial use" (Traugott, 2013, p.1). However, according to Gloud and McDonlad (2009) and Dubberke, Gerding, Classen, et al. (2008), the two main risk factors of acquiring CDI are the exposure of antimicrobial overdose and the acquisition of Clostridium Difficile germs.

The increase in morbidity and mortality of CDI makes the healthcare industry worldwide worried (Gerding, Muto and Owens, 2008). Consequently, the burden cost of treating CDI increases as well. Fekety, Kim, Brown, Batts, Cudmore and Silva (1981) state that the environment plays an important role in the transmission of CDI because its spores can live for prolonged periods on hard surfaces. According to Gerding, Muto and Owens (2008), the spores also cannot be removed from the forehands of healthcare workers and the forehands of healthcare workers cannot be disinfected with the use of alcohol-based sanitizers. So, antiseptic handwash or antiseptic hand-rub is no use in removing the spores physically from the hands of healthcare workers (Larson and Morton, 1991; Denton, 1991; Gershenfeld, 1962; Russell, 1991). Thus, healthcare workers must practice hand hygiene with antimicrobial soap and water to ensure CDI spore removal after contacting a CDI incidence case (Larson and Morton, 1991; Denton, 1991; Gershenfeld, 1962; Russell, 1991).

As the hands of healthcare workers are considered a main source of transmission of the bacterium (Gerding, Johnson, Peterson, Mulligan and Silva, 1995; National Clostridium difficile Standards Group, 2004; and McFarland, Mulligan, Kwok, Stamm, 1989), the contaminated surfaces of healthcare environments with the spores 
of CDI can be a source-carrier of the bacterium too (Hughes, Tunney and Bradley, 2013; National Clostridium Difficile Standards Group, 2004).

The special characteristics of Clostridium Difficile spores make CDI one of the highest contagious bacterial infections in healthcare settings. One special characteristic of the spores is that, according to Fekety, Kim, Brown, Batts, Cudmore and Silva (1981), Fordtran (2006) and Owens (2006), the spores can survive on dry and hard surfaces for months. Thus, special care and attention has to be present when cleaning and disinfecting the environments of healthcare settings during the presence of CDI. However, according to Owens (2006), Mayfield, Leet, Miller and Mundy (2000), Underwood, et al. (2005), some of the cleaning detergent agents are not meant to be used for CDI cases because they might make the situation worse by being a good source for sporulation and then increase the prevalence of CDI. Subsequently, it is highly recommended to use sporicidal chemical cleaning detergent agents especially during outbreaks of CDI (Gerding, Muto and Owens, 2008; CDC, 2002). Yet, more attention should be present when using such agents because, according to Mayfield, Leet, Miller and Mundy (2000), overuse of these detergents for prolonged time puts equipment and inanimate surfaces of healthcare at risk of being deteriorated and puts floor workers such as housekeepers at risk of developing respiratory illness though those agents were found to be effective in controlling and preventing CDI.

According to Dubberke, Gerding, Classen, et al. (2008), some of the CDI prevention strategies are that healthcare personnel including housekeeping, families and visitors have to be on contact precautions every time they enter patients' rooms with CDI, and they have to adhere to hand hygiene compliance by washing hands with 
soap and water when exiting patients rooms with CDI and when taking gloves off hands.

According to Dubberke and Olsen (2012), and Dubberke, Butler, Yokoe, et al. (2000), CDI was found to contribute the most to the overall cost burden of healthcareassociated infections in acute care hospitals in the United States of America. According to Magill, Edwards, Bamberg, et al. (2014), CDI was also found to be the highest morbidity pathogen in a recent national survey with $12 \%$ of HAIs and an estimated 80,400 hospital-onset infections. According to Lessa, Mu, Bamberg, et al. (2015), about half a million CDI cases occurred in 2011 in the United States.

According to Loo, Poirier, Miller, et al. (2005), Pepin, Valiquette and Cossette (2005) and Dubberke, Butler, Reske, et al. (2008), the mortality rate attributable to $\mathrm{CDI}$ at 30 days after diagnosis was estimated to be around $6.9 \%$ and the cumulative was estimated to be around 16.7\%. According to Lessa, Mu, Bamberg, et al. (2015), in a recent study, it was found that 29,000 infected patients with CDI died within the first 30 days of the initial diagnosis in 2011 in the United States.

According to Scott (2009), the range of the estimated cost of CDI per infection was between $\$ 5,000$ and $\$ 7,000$ dollars and the estimation was based on 2003 dollars. Scott (2009) used an estimate from a study conducted by Dubberke, Reske, Olsen, et al. (2008). This estimate was used to calculate the estimated total cost of CDI per year, which ranges between $\$ 1.01$ billion and $\$ 1.62$ billion for inpatient hospital services (Scott, 2009). 


\subsubsection{Methicillin-Resistant Staphylococcus Aureus (MRSA)}

According to Cosgrove, Qi, Kaye, Harbarth, Karchmer and Carmeli (2005) and McCoy (2013), a Methicillin-Resistant Staphylococcus Aureu (MRSA) is a multidrug resistant bacterial pathogen. According to Jevons (1961), MRSA was first reported in 1961. According to Cosgrove et al. (2005), a multidrug resistant pathogen is a nightmare and a concern to healthcare worldwide. It affects the economy because its burden cost is high due to its resistance feature against many traditional antibiotics. It also affects the economy because it increases hospitalization time and has a high rate in morbidity and mortality.

According to CDC (2015), "Most MRSA infections are skin infections." According to McCoy (2013), MRSA can affect other parts of the human body such as "blood, bones, or lungs" (McCoy, 2013, p.1). Thus, according to CDC (2015) and Marshall, Wesselingh, McDonald and Spelman (2004), MRSA can lead to lifethreatening subsequent infections in healthcare settings such as bloodstream infections, which is referred to as MRSA bacteremia, pneumonia and surgical site infections (SSI).

According to McMaster, Booth, Smith and Hamilton (2015), the longer the hospitalization, the higher the risk of MRSA infection. Hospitalization was identified as an independent risk factor for nasal colonization of MRSA (Hidron et al., 2005). Hidron et al. (2005) also mentions other independent risk factors for nasal colonization of MRSA such as history of antibiotic use. A colonized person with MRSA might get himself infected by transmitting MRSA from his skin to his body through, for example, a wound, and also to others (McCoy, 2013). 
According to Hughes, Tunney and Bradley (2013), a recommended intervention strategy to prevent and control MRSA in healthcare settings is that those who are infected with MRSA should be isolated from other patients in a cohorting room. According to CDC (2002), HCWs should show a high level of hand hygienic practice and compliance by washing their hands with antimicrobial soap or waterless antiseptic agent before and after contacting MRSA patients. Standard precautions such as wearing gloves, gowns, masks and hats can be of helpful in preventing and controlling MRSA transmission (Hughes, Tunney and Bradley, 2013).

According to Sumitani and Kobayashi (2009), in 2006, the Centers for Disease Control and Prevention (CDC) has recommended to apply a new prevention and control strategy for Multidrug-Resistant Organisms (MDRO), such as MRSA, in addition to the existing intervention strategies based on an active surveillance culture (ASC) to detect asymptomatic colonization. According to Siegel, Rhinehart, Jackson and Chiarello (2006), for MRSA detection, culture of nares, wounds or urinary for those patients who are admitted with indwelling foley catheters are recommended to detect asymptomatic colonization upon admission. The conventional culture methods (Culture-based) for ASC might take up to 2-3 days to obtain results of MRSA colonies in screening cultures from the microbiology laboratory. Unfortunately, the conventional detection methods for MRSA colonies may delay the use of the desired infection control measures such as Contact Precautions until results are obtained. Thus, efforts were made to come up with methods for obtaining results of ASC in less time. Commercially available detection methods for ASC might take a minimum of 16 hours to obtain results of MRSA colonies in screening cultures from the microbiology 
laboratory. On the other hand, the rapid detection methods for ASC (realtime polymerase chain reaction PCR-based) might take 1-2 hours to obtain results of MRSA colonies in culture swabs. Luteijn, Hubben, Pechlivanoglou, Bonten and Postma (2011) list nine different commercially available methods to obtain ASC to detect asymptomatic colonization.

The Providence Veterans Administration Medical Center (PVAMC) applies two detection methods, which are realtime PCR-based method and Chromogenic Media (culture-based) method. Any patient who needs to occupy a bed in acute care units (inpatient units) or in an intensive care units (ICU), except the inpatient Mental Health unit, has to be swabbed within 24 hours of admission (I. Straub and B. Cedeno, Personal Communication, November 24, 2015). Realtime PCR-based method costs about $\$ 29.42$ per nares culture swab while Chromogenic Media (culture-based) method costs about \$2.67 per nares culture swab (G. Hawthorne, Personal Communication, November 24, 2015). In addition, upon discharge, patients are nares culture swabbed to ensure they left the VA without MRSA acquired at the hospital, but the result of that swab is not required soon. The result of the test will be mailed or given via the phone to the patient (I. Straub and B. Cedeno, Personal Communication, November 9, 2015).

\subsubsection{Device Invasive Infections}

Device Invasive (DI) infections in non-neonatal intensive care and acute care units are caused by specific pathogens which may occur if a patient's health status requires an insertion of some type of medical device. They cause the patients to stay longer under treatment. Catheter-Associated Urinary Tract Infection (CAUTI), Central 
Line-Associated Bloodstream Infection (CLABSI) and Ventilator-Associated Pneumonia (VAP) are the most common non-neonatal intensive care units and acute care infections in healthcare settings. Unfortunately, such infection cases are highly unlikely to disappear as long as some patients are in a need of these medical devices and tools that save their lives, such as invasive urinary catheter devices for CAUTI, central lines for CLABSI and ventilator tubes for VAP.

\subsubsection{Catheter-Associated Urinary Tract Infection (CAUTI)}

According to Gould et al. (2009), Urinary Tract Infection (UTI) is an infection "caused by instrumentation of the urinary tract" (Gould et al., 2009, p.22). CatheterAssociated Urinary Tract Infections (CAUTI) are those infections specifically "associated with indwelling urinary catheters" (Gould et al., 2009). According to Gould et al., (2009), though CAUTI has not been associated with high morbidity or mortality compared to other HAIs, subsequent secondary nosocomial bloodstream infections are mainly caused by CAUTI.

Short-term indwelling urinary catheters are required for about $15 \%$ to $25 \%$ of patients who require hospitalization (Gould et al., 2009). On the other hand, long-term indwelling urinary catheters are required for about $5 \%$ to $10 \%$ of patients who are in need of prolonged hospitalization care (Nicole, 2000). In general, the use of urinary catheters is considered the main risk factor for developing UTIs (Richard, Edwards, Culver and Gaynes, 2000; Orgeas et al., 2008). The unnecessary use of urinary catheters for prolonged time (Gould et al., 2009; Shuman and Chenoweth, 2010) and the unawareness of nurses of their patients who are on use of indwelling urinary 
catheters are among the important risk factors for developing CAUTI (Gould et al., 2009).

The best intervention to prevent and control CAUTI is to use urinary catheters (UC) as needed following the recommended guidelines for catheter insertion, and to routinely check for replacing the used or the unnecessary ones, accompanied with the adherence to hand hygiene compliance before and after the insertion of indwelling urinary catheters (Gould et al., 2009).

\subsubsection{Central Line-Associated Bloodstream Infection (CLABSI)}

According to CDC and NHSN (2015), Central Line-Associated Bloodstream Infection (CLABSI) is an infection that occurs when germs transmit through a central line (CL) or umbilical catheter (UC) into a patient's bloodstream. A central line or a umbilical catheter has to be "in place for $>2$ calendar days on the date of events, with day of device placement being Day 1 AND a CL or UC was in place on the date of event or the day before" (CDC and NHSN, 2015, p.2), to classify the infection as CLABSI and not as a Bloodstream Infection (BSI). According to TJC (2012) and US Department of Health and Human Services (2009), the four HAIs that account for more than $75 \%$ of all HAI cases in hospitals are Urinary Tract Infection (UTI), Surgical Site Infection (SSI), Bloodstream Infection (BSI) and Pneumonia. BSI accounts for about 17\% (TJC, 2012; US Department of Health and Human Services, 2009).

According to Chopra, Krein, Olmsted, et al. (2013), sometimes a patient's status requires the insertion of a central line (CL) or central vascular catheter (CVC) to draw blood or to provide the patient with medications, though CVC is considered as the 
main risk factor for developing CLABSI. According to O'Grady, Alexander, et al. (2011), "in the United States, 15 million central vascular catheter days (i.e., the total number of days of exposure to CVCs among all patients in the selected population during the selected time period) occur in intensive care units (ICUs) each year" (O'Grady, Alexander, et al., 2011, p.8-9). Though CVC is more frequently used in ICUs, it is unknown how frequently CVC is used in non-ICUs units (Chopra, Krein, Olmsted, et al., 2013). Like other HAIs, CLABSI is associated with high mortality and high morbidity rates (TJC, 2012). According to TJC (2012) and Chopra, Krein, Olmsted, et al. (2013), there are about 250,000 CLABSI cases detected in hospitals in the United States annually. About $32 \%$ of that is detected in ICUs only, which is about 80,000 CLABSI cases. Moreover, a survey of major medical centers found that about $24.4 \%$ of CLABSI cases occurred outside ICUs. CLABSI is also associated with high burden cost of treatment as well as extension of hospitalization length of stay (Chopra, Krein, Olmsted, et al., 2013; Edgeworth, 2009; Rosenthal, Guzman, Migone and Crnich, 2003; Higuera et al., 2007; Pittet, Tarara and Wenzel, 1994; Warren et al., 2006; Blot et al., 2005). In a recent estimate set by CDC, CLABSI costs about $\$ 16,550$ per infection (CDC, 2011). In addition, CLABSI was found to be related to an increase in hospitalization length of stay from one week to almost three weeks (Chopra, Krein, Olmsted, et al., 2013; Stone, Braccia and Larson, 2005).

Though those numbers and percentages seem high, CLABSI can be prevented and controlled using the correct prevention strategies. According to Chopra, Krein, Olmsted, et al. (2013), Alonso-Echanove, Edwards, Richards, et al. (2003) and Safdar, Kluger and Maki (2002), valuable strategies have been implemented in order to 
prevent and control CLABSI by first identifying individual risk factors causing the infection and then targeting them. Some of the identified risk factors causing CLABSI include, but are not limited to, how long a patient has been hospitalized before the use of CVC, how long CVC has been inserted in the body and finally types of CVC. Some of the strategies and practices to reduce, prevent and control CLABSI include adhering to hand hygiene practice before inserting $\mathrm{CVC}$, and educating healthcare workers about best practices of insertion and replacement of CVC (Chopra, Krein, Olmsted, et al., 2013; O’Grady, Alexander, et al., 2011).

\subsubsection{Ventilator-Associated Pneumonia (VAP)}

According to CDC (2012), Ventilator-Associated Pneumonia (VAP) is a pneumonia infection where the germs transmit through a ventilator tube into a lung of a patient. The infection has to develop 48-72 hours after the insertion of the ventilator tube to be classified as VAP (Craven, et al., 1986; Tablan, Anderson, Besser, Bridges and Hajjeh, 2004).

According to Horan et al., (1984) and Emori and Gaynes (1993), VAP is considered the second highest HAI while UTI is considered the first highest HAI. According to Jarvis, Edwards, Culver, et al. (1991), The use of ventilating tube is considered the most important risk factor for developing VAP. VAP is associated with high attributable mortality rate of about $20 \%$ to $33 \%$ (Tablan et al., 2004). VAP is also associated with increasing days of hospitalization by about 4-9 days (Tablan et al., 2004), which was found to lead to an excess direct cost of hospital stay due to VAP of about $\$ 40,000$ per patient (Rello, Ollendorf, Osler et al., 2002). 
Prevention strategies for VAP are to disinfect hands with hand rub or wash hands with antimicrobial soap and water before and after contacting a patient with VAP. It is also recommended to adhere to standard precautions such as wearing gloves before the insertion of ventilating tube, washing hands before and after wearing gloves, and wearing gowns especially when a patient with VAP is expected to be soiling with respiratory secretions (Tablan et al., 2004).

\subsection{Hand Hygiene}

Healthcare-associated infections (HAIs) are adverse events that not just endanger patients' lives but also add extra burden costs to the economy of countries. Extended time of hospitalizations, extra diagnosis and the required use of antibiotics and other medications are all extra costs that are incurred to deal with HAIs and add to the regular costs of care. In the United States, healthcare-associated infections (HAIs) were associated with healthcare costs of $\$ 28.4$ to $\$ 33.8$ billion in 2007 U.S. dollars (Scott, 2009).

In order for healthcare-associated infections to transmit in hospitals, microorganisms have to exist in a susceptible host that can be transmitted via other carriers (Collins, 2008). Gastmeier, Stamm-Balderjahn, Hansen, et al. (2005) list the five most common transmission sources of healthcare-associated infections in descending order in their analysis of 1,022 outbreaks, which are "the individual patient, medical equipment or devices, the hospital environment, the health care

personnel, contaminated drugs, contaminated food, and contaminated patient care equipment" (Collins, 2008, p.549). 
According to Collins (2008), there are several intrinsic and extrinsic risk factors that endanger patients at hospitals. For example, patients with severe illness and elderly patients are prone to develop HAIs. Severity of illness and aging are listed as intrinsic risk factors. On the other hand, examples of extrinsic risk factors are patients who are required to have organ-transplant surgery or who are in need of deviceinvasive procedures.

Over the past century, practicing hand hygiene at critical moments was found to be one of the main interventions to reduce, prevent and control infectious diseases from occurring and spreading (CDC, 2002; Collins, 2008). Yet, the simple behavior of hand washing with antimicrobial soap and water and the simple behavior of hand cleansing with alcohol-based rub sanitizer at critical moments in healthcare settings by healthcare workers (HCWs) have been found to be poor (Collins, 2008). In 34 observational studies conducted from 1981 to 2000 in different settings (ICU, All Wards, ER, Medical Wards, SICU, PICU, MICU, Pedi OPDs, Nursery and NICU, Pediatric Wards) the minimum and maximum hand hygiene adherence baseline were found to be $5 \%$ and $81 \%$ respectively. Many of these studies only focused on one or two of the five moments for hand hygiene, such as before and after patient contact. On average, the hand hygiene adherence baseline was found to be $40 \%$, which is considered poor (CDC, 2002).

Adhering to proper hand hygiene practices at critical moments can ensure patient safety and superior quality of care by preventing and reducing the prevalence of healthcare-associated infections (TJC, 2009). Consequently, unnecessary costs of healthcare-associated infection treatment are avoided (Berild, Ringertz, Lelek and 
Fosse, 2001; Mille et al., 2000; Nathwani, Rubinstein, Barlow and Davey, 2001) and extra-extended days of stay for HAI hospitalizations are shortened and reduced (Nguyen, Nguyen and Jones, 2008).

Though hand hygiene practice is a simple exercise and is encountered on a daily basis in people's lives, according to Pittet (2000), there are factors that are considered barriers, which can influence the quality of hand hygiene practices. Some are related to the patients, the HCWs, the environment and some are self-reported by HCWs such as the quality and availability of hand hygiene products (Purell, Soap dispensers, paper towels) when hand hygiene opportunities occur (WHO, 2009). According to Pittet (2000), some of these factors were reported by healthcare workers (HCWs) who sometimes forget to wash hands or have hands affected by complications such as skin irritation and dryness. Other risk factors for poor adherence to hand hygiene practices include job status, location and time of hand hygiene opportunities, days of the week (Pittet et al., 1999) and "high number of opportunities for hand hygiene per hour of patient care" (WHO, 2009, p.73). Additionally, according to Haley and Bregman (1982), Pittet et al. (1999), Harbarth et al., (1999), Pittet (2000), O’Boyle, Henly and Duckett (2001), Kuzu et al.(2005), understaffing and overcrowding are important risk factors. An example of a self-reported risk factor for poor adherence to hand hygiene by HCWs is prioritizing patients' needs above hand hygiene (Kretzer and Larson, 1998; Pittet, 2000; Patarakul, Tan-Khum, Kanha, Padungpean, and Jaichaiyapum, 2005). For a complete list of factors and barriers to poor hand hygiene practices, CDC (2002) has adapted these factors from Pittet (2000) as seen in Table 2.1. 
Many hand hygiene measurement interventions and methods have been proposed and applied (WHO, 2009; TJC, 2009, Bittner and Rich, 1998; Pittet, 2000; Pittet, 2002; Pittet, Allegranzi, Sax, Dharan, Pessoa-Silva, Donaldson and Boyce, 2006), yet, assessing and measuring healthcare workers' hand hygiene compliance is still a challenging task. TJC (2009) lists some of the specific challenges that hinder hand hygiene compliance measurement. The interactions between healthcare workers and patients and the environment of hospitals are countless. Thus, hand hygiene opportunities occur on an hourly basis. Also, opportunities for hand hygiene vary by level of care and by wards and divisions. It is time consuming, costly and an intensive use of staff resources to track, assess and measure opportunities for hand hygiene practices (TJC, 2009). 


\section{Observed Risk Factors for Poor Adherence to Recommended Hand-Hygiene Practices}

- Physician status (rather than a nurse)

- Nursing assistant status (rather than a nurse)

- Male sex

- Working in an intensive-care unit

- Working during the week (versus the weekend)

- Wearing gowns/gloves

- Automated sink

- Activities with high risk of cross-transmission

- High number of opportunities for hand hygiene per hour of patient care

\section{Self-Reported Factors for Poor Adherence with Hand Hygiene}

- Handwashing agents cause irritation and dryness

- Sinks are inconveniently located/shortage of sinks

- Lack of soap and paper towels

- Often too busy/insufficient time

- Understaffing/overcrowding

- Patient needs take priority

- Hand hygiene interferes with health-care worker relationships with patients

- Low risk of acquiring infection from patients

- Wearing of gloves/beliefs that glove use obviates the need for hand hygiene

- Lack of knowledge of guidelines/protocols

- Not thinking about it/forgetfulness

- No role model from colleagues or superiors

- Skepticism regarding the value of hand hygiene

- Disagreement with the recommendations

- Lack of scientific information of definitive impact of improved hand hygiene on health-care-associated infection rates

\section{Additional Perceived Barriers to Appropriate Hand Hygiene}

- Lack of active participation in hand-hygiene promotion at individual or institutional level

- Lack of role model for hand hygiene

- Lack of institutional priority for hand hygiene

- Lack of administrative sanction of noncompliers/rewarding compliers

- Lack of institutional safety climate

Table 2.1 Factors Influencing Adherence to Hand Hygiene Practices (CDC, 2002) 


\subsection{Hand Hygiene Adherence Measurement Methods}

The occurrence of healthcare-associated infections HAIs at healthcare settings is proportionally related to how compliant HCWs are to hand hygiene practices. Thus, assessing and evaluating the adherence to hand hygiene compliance of healthcare workers (HCWs) is vital. It is hard to assess hand hygiene behavior of healthcare workers completely and to capture all critical and correct moments of washing hands which were defined as "My 5 Moments for Hand Hygiene" by the World Health Organization WHO (2009) as depicted in Figure 2.1.

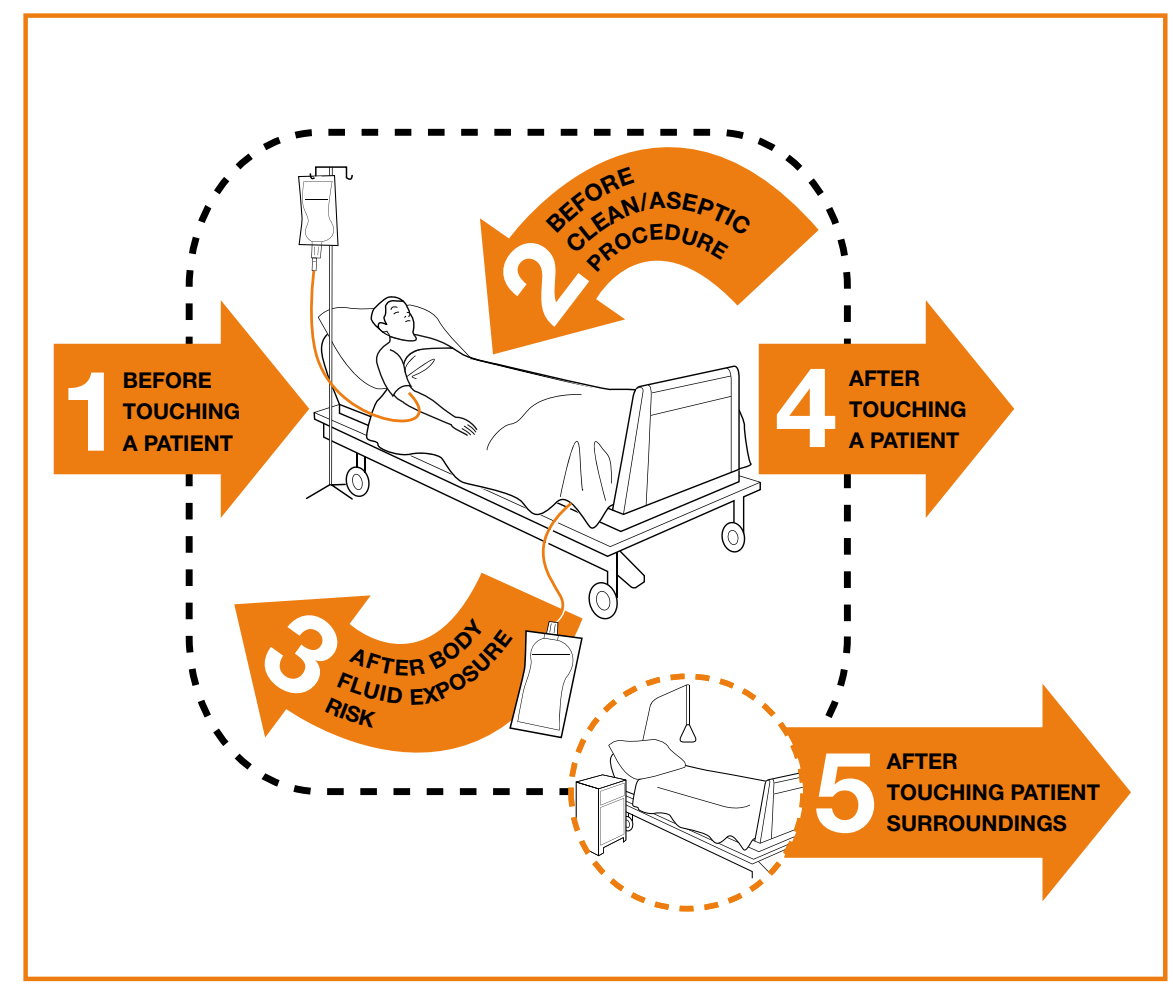

Figure 2.1 My 5 Moments for Hand Hygiene (WHO, 2009)

"The correct moment of hand hygiene is usually termed opportunity" (WHO, 2009, p.158). On the other hand, "actions" are those required moments for hand hygiene practiced by healthcare workers. However, not every moment requires hand 
hygiene (WHO, 2009). According to Sax et al. (2007) and Pittet et al. (2006), the opportunity is defined as the time interval between touching a contaminated surface or patient and touching an uncontaminated surface or patients. This definition was based on "an evidence-based model of hand transmission" (WHO, 2009, p.158), conducted by Sax et al. (2007) and Pittet et al. (2006). An infectious disease might occur as a result of not practicing hand hygiene in such an opportunity (WHO, 2009).

In calculation of the rate of hand hygiene compliance, opportunity is used in the denominator of the equation. However, not all hand hygiene compliance rate measurement methods are capable of quantifying all true and correct opportunities for the denominator such as measuring the consumption of Soap and Purell Volume (WHO, 2009).

The ideal measurement method for hand hygiene performance has to be unbiased and has to generate a true rate that reflects the true hand hygiene behavior of healthcare workers. Thus, the World Health Organization recommends that the ideal method has to be technology-based with no influence on the behavior of the ones who are being observed, and has to capture all true moments of hand hygiene in real time. More importantly, unnecessary extra costs and extra staffing time have to be avoided in such a method. Extra costs and extra staffing time may provide a large enough dataset for hand hygiene performance that leads to more meaningful analysis by eliminating underpowered sampling size and by eliminating sampling bias in the study (WHO, 2009).

"Bias and insufficient sample size represent the two major threats to meaningful monitoring outputs" (WHO, 2009, p.158). Statistically, selection bias is defined as 
"The error introduced when the study population does not represent the target population" (Delgado-Rodríguez, Llorca, 2004, p.635), while underpowered sample size means that the number of participants involved in the study was below the required sample size to capture and detect the true effect of the study (McCrumGardner, 2010). Thus, drawing conclusions and inferences in an underpowered sampling size study may be misleading (Button et al., 2013). On the other hand, overpowered sample size means that the numbers of participants involved in the study was excessive and considered above the required sample size (McCrum-Gardner, 2010). If an overpowered sampling size study were carried out, this is considered unethical, especially when the study involves human- and animal-subjects (Lenth, 2001), because it is considered as a waste of the study resources (McCrum-Gardner, 2010). "The power is the probability of correctly rejecting $\mathrm{H}_{0}$ " (Montgomery, 2012, p.113), "Power $=1-\beta=\mathrm{P}\left\{\right.$ reject $\mathrm{H}_{0} \mid \mathrm{H}_{0}$ is false $\}$ " (Montgomery, 2012, p.113). In contrast, type I error is called (Alpha) “ $\alpha=\mathrm{P}\{$ type I error $\}=\mathrm{P}\left\{\right.$ reject $\mathrm{H}_{0} \mid \mathrm{H}_{0}$ is true $\}$ ” (Montgomery, 2012, p.113), and Type II Error is called (Beta) “ $\beta=\mathrm{P}\{$ type II error $\}=$ $\mathrm{P}\left\{\right.$ fail to reject $\mathrm{H}_{0} \mid \mathrm{H}_{0}$ is false\}" (Montgomery, 2012, p.113). Thus, a balanced power value between both over- and underpowered sampling size has to be used to minimize the effects of Type I Error and/or Type II Error (Lieberman and Cunningham, 2009). Usually, "A target value of .80 is fairly common and also somewhat minimal" (Lenth, 2001, p.188); however, this value depends on the type of the study to be carried out (Sullivan and Feinn, 2012).

According to WHO (2009), all existing measuring methods for hand hygiene performance do not meet such ideal measuring criteria (WHO, 2009). Fortunately, 
there are existing methods that vary in terms of sharing some of the criteria of the ideal measuring method. According to WHO (2009) and TJC (2009), monitoring hand hygiene performance of healthcare workers can be done directly or indirectly. "Direct methods include direct observation, patient assessment or HCW self-reporting" (WHO, 2009, p.158). TJC (2009) gives a different name for "patient assessment or HCW self-reporting" (WHO, 2009, p.158) and names it the "Conducting Survey" method (TJC, 2009, p.63); however, TJC (2009) does not classify it as an indirect or direct method. "Indirect methods include monitoring consumption of products, such as soap or handrub, and automated monitoring of the use of sinks and handrub dispensers" (WHO, 2009, p.158). In addition, TJC (2009) states that, "Directly observing, Measuring product use and Conducting surveys" are considered the three top hand hygiene performance monitoring methods (TJC, 2009, p.xviii).

In addition to these lists of methods, healthcare sectors recently have started using technology-based real-time locating systems (RTLS) to track and capture opportunities for hand hygiene. According to $\mathrm{Wu}$, Ranasinghe, Sheng, Zeadally and Yu (2011) and Ajami and Akbari (2012), real-time locating systems (RTLS) are wireless-based systems that are used to identify the location of objects or human beings, for example, assets or healthcare workers HCWs within defined areas and zones. Historically, according to Landt (2005), RTLS was first implemented during the Second World War in the 1940s in the USA. Its application at that time was merely used in the identification of allied airplanes. According to Krohn (2015), RTLS can optimize processes, remove waste of duplicated hospital operations and increase the quality of care and thus reduce healthcare costs. 
RTLS is not only deployed in monitoring hand hygiene practices for healthcare workers performance. According to Krohn (2015), RTLS is also deployed in optimizing the workflows of hospital staffing and in managing Emergency Rooms (ER) and Operation Rooms (OR), to name few. According to Vakili, Pandit, Singman, Appelbaum, and Boland (2015), RTLS can be in different forms of technologies such as "Blue-tooth, iBeacon, Wi-Fi, camera vision, ultrasound, radio frequency identification (RFID), infrared (IR), global positioning systems (GPS), and cellular signals" (Vakili, Pandit, Singman, Appelbaum, and Boland, 2015, p.2).

Each proposed method to track and measure hand hygiene performance of healthcare workers has its advantages and disadvantages. Some healthcare sectors have applied bundles of hand hygiene performance tracking methods together to ensure a superior level of hand hygiene compliance rate by capturing most of the critical and required moments for hand hygiene practices.

\subsubsection{Direct Hand Hygiene Monitoring Methods}

Healthcare sectors have sought direct monitoring of HCWs' hand hygiene performance either by recruiting secret observers or patient observers or by asking HCWs for self-report. Though direct observation methods are valuable and are "considered the gold standard" (WHO, 2009, p.158), large efforts are made to train the observers and to validate their observations (WHO, 2009). In addition, the direct methods encounter potential biased such as observation, observer and selection bias and they are time consuming (WHO, 2009). 


\subsubsection{Direct Observation Methods}

WHO (2009) states that the direct observation method is the "gold standard" (WHO, 2009, p.158), because, according to WHO (2009) and TJC (2009), it allows the observer to identify whether healthcare workers adhere to hand hygiene compliance at critical moments of the "five moments for hand hygiene" WHO (2009), and which product is used to practice hand hygiene. It also allows the observer to evaluate how rigorously healthcare workers disinfect and clean their hands. In addition, it allows the observer to identify how hands were dried after washing with soap and water. It also allows the observer to identify whether HCWs wear gloves at critical moments and clean hands before and after wearing gloves. It also allows the observer to evaluate healthcare units and healthcare workers' behavior toward hand hygiene compliance so recommended improvements can be suggested (WHO, 2009; TJC, 2009). Perhaps the most valuable advantage of directly observing hand hygiene performance of healthcare workers is that, according to WHO (2009), direct observation methods enable the observer to capture all critical and true moments of the "five moments for hand hygiene", and no other existing methods are capable of doing that (WHO, 2009).

On the other hand, according to WHO (2009) and TJC (2009), one of the main disadvantages of the direct observation method is that it requires excessive time and large numbers of observers to capture enough true moments of hand hygiene. It also

requires selection of the right observers and training them for consistency to avoid inter-observer variability. Another major disadvantage of this method is that the hand 
hygiene behavior of healthcare workers who are being observed may be altered because they are being observed (WHO, 2009; TJC, 2009).

WHO (2009) lists three observational effects or bias, which is generated when the direct observation hand hygiene performance method is applied. The first bias is observation bias, which is described as an increase in the quality of hand hygiene performance for healthcare workers as seen by an observer. According to Hugonnet, Perneger and Pittet (2002), Pittet (2002), Landsberger (1958) and Bittner and Rich (1998), this is what it is referred to as the "Hawthorne Effect". Its name was adapted from the Hawthorne Factory of Western Electrics after conducting several ergonomics studies in the USA at the beginning of the $20^{\text {th }}$ century (WHO, 2009). Observation bias can be reduced or eliminated if the observer's identity is unrevealed. However, if the observer's identity were revealed, trust might disappear. Unrevealing the observer's identity is also hard for long-term observing of hand hygiene opportunities (WHO, 2009).

The second bias is observer bias. Such bias is defined as "the systematic error introduced by inter-observer variation in the observation method" (WHO, 2009, p.159). Each observer has his own systematic understanding and interpretations of the direct observation methods for hand hygiene performance. This type of bias results in different outputs based on the observer who conducts the method. Validating each observer's method consistently over time can reduce observer bias (WHO, 2009). According to the WHO (2009), validation of the observers can be done in two ways. First, two observers can be evaluated during a real-life hand hygiene scenario where both observe the same sequence of healthcare and the same healthcare workers. Then, 
both observers are asked to complete a separate observation form. This process is recommended to be repeated several times till validation of the observers is reached. It is also recommended that the trainer who is selected for validating the observers under the same settings remain the same. It is also recommended to perform validation of the observers for any future selected observers under any care setting. Second, a trainer can provide a training film provided by the WHO. Visual examples are provided in the film that present different scenarios for five moments for hand hygiene for healthcare workers where hand hygiene is necessary during points of care. The examples show observers observing healthcare workers during different scenarios and then completing an observation form. During the film, the trainee-observers are tested by completing observation forms about the provided examples in the film. Then, their results are compared with the results of the examples shown in the film to evaluate their performance.

The third bias is selection bias. Such bias is generated as the direct observation methods are systematically conducted by non-randomly selecting the observed HCWs, units, type of services, and opportunities for hand hygiene, just to name few. Such bias leads to outputs that do not explain or represent the true hand hygiene performance of healthcare workers. Randomized selection of direct observation settings can eliminate or reduce such bias (WHO, 2009).

\subsubsection{Patient Assessment Method}

Hand hygiene performance could be assessed and measured by patients. In two studies conducted by McGuckin et al. (2001) and (2004), patients were involved in an educational program for behavioral change called "Partner in Your Care" (McGuckin 
et al., 2001, p.222; McGuckin et al., 2004, p.235). Such a program empowers patients to be part of hand hygiene performance intervention and thus to be part of their program of care. In both studies, patients were asked to be part of assessing hand hygiene performance of their healthcare workers before a contact has taken place. Consequently, the hand hygiene compliance rate increased by at least $50 \%$. However, small sample size was an issue for both studies.

Though the patient assessment method requires less healthcare staffing and may reduce cost for hospitals (McGuckin et al., 2001) and may provide "the framework for a synergistic healthcare experience" between healthcare workers and patients (Williams, 2002, p.104), involving and empowering patients in assessing and measuring hand hygiene performance of their healthcare workers has some challenges and difficulties. According to Pittet, Mourouga and Perneger (1999), and Wade, (1995), patients' health status might hinder the accomplishment of such a task. Patients also might find it inconvenient to observe their healthcare workers and evaluate them. In general, the patient assessment method "is not well documented" (WHO, 2009, p.159), and an objective evaluation of the method has not been conducted yet (Williams, 2002).

\subsubsection{Healthcare Workers Self-Reporting Method (Conducting Surveys and Questionnaires)}

Conducting surveys and questionnaires or self-reporting hand hygiene performance monitoring could reveal important information regarding healthcare workers' perceptions about hand hygiene practices and adherence rates of compliance at hospitals. The self-reporting method also determines whether the perceptions of 
both healthcare workers and their patients and families of patients match (TJC, 2009). According to a study conducted by Simmons et al. (1990) where nurses were the focus of the study, the self-assessment questionnaire and surveys did reveal the true perceptions of the nurses about their hand hygiene practices and adherence rate of compliance did not match that of their patients' and families' though the main objective of the study was to find any correlation between handwashing frequency and infectious disease rate. Nurses thought that they were doing a great job in practicing hand hygiene and their compliance rate was above $90 \%$.

The perceptions of the nurses were positively corrected after the infection control nurses critiqued them during the intervention. In the same study, a secret observer was covertly conducting direct observations (Simmons et al., 1990). Interestingly, data of the self-assessment method did not correlate with that of the direct observation methods (Simmons et al., 1990; WHO, 2009). Simmons et al. (1990) lists some limitations that might have hindered preventing any cross-infections. The hospital where the study was conducted did not have any intervention program for colonization identifications for those patients who were colonized, which might have been the source of transmission from patient to patient. Also, limiting the study to nurses only might have been another reason for non-determining any cross-infections.

In general, conducting surveys for hand hygiene performance monitoring sometimes provides unreliable outcomes because healthcare workers' perceptions for their hand hygiene practices are overestimated (WHO, 2009; TJC, 2009). To approach accurate results, surveys and questionnaires have to be well planned and designed. In addition, guidelines for surveys and questionnaires have to be shared with people of 
interest such as patients and their families and healthcare workers involved in the study. Most importantly, conducting surveys and questionnaires must be well managed, led and controlled (TJC, 2009).

\subsubsection{Indirect Hand Hygiene Monitoring Methods}

Healthcare sectors have sought monitoring hand hygiene performance indirectly either manually by measuring the volume of product use (soap, alcohol-based handrub and paper towel) or electronically by measuring the frequency of occurrence or counts associated with the consumption of product use (soap, alcohol-based handrub and paper towel) using electronic counting devices or electronic monitoring systems (TJC, 2009). Manual monitoring is cost-effective; but does not provide accurate and precise qualitative outcomes. On the other hand, electronic monitoring is associated with cost of installation and maintenance but provides better qualitative outcomes. Both provide hand hygiene performance feedback over time (WHO, 2009).

Of note, adherence rates of indirectly monitoring hand hygiene performance is estimated and not directly calculated because of the absence of the denominator, or total number of opportunities (TJC, 2009). Larson, Albrecht and O'Keefe (2005) proposed more reliable methods to calculate the number of indications and the number of actual episodes for hand hygiene based on consumption of product use. These two numbers are used in the estimation of hand hygiene adherence rate.

\subsubsection{Manual Monitoring of Product Use}

Healthcare sectors have sought monitoring hand hygiene practices via the measurement of product use consumption such as soap, handrub (Purell) and paper 
towels as a cost-effective method (WHO, 2009). One reason for seeking such an approach to measure hand hygiene performance is that, according to Bittner et al. (2002), sometimes an intervention that is based on feedback collection is better than an intervention that is based on cultural and organizational changes.

Healthcare sectors can measure the consumption of product use by weighing what is left of the product to measure what has been used over a specific period of time. They also can measure the consumption of product use by counting how many units of product have been used and replaced in a ward or a unit at hospitals. These data can also be compared with sales data of product purchased for the entire hospitals (TJC, 2009). One major drawback of such a method is that since it is an indirect type of method to monitor hand hygiene performance, opportunities vary between wards and units at the hospitals, and also varies by sales data. Thus, hand hygiene compliance comparison between wards and units based on sales data of product use is unreliable (TJC, 2009).

Recall that, according to WHO (2009), indirect methods for measuring hand hygiene performance miss the denominator "opportunity" in the calculation of hand hygiene compliance. Thus, to develop and apply a more significant method for monitoring consumption of products for hand hygiene performance, the denominator issue has to be overcome (WHO, 2009). In one study conducted by Bittner et al. (2002) at the Omaha Veterans Affairs Medical Center, soap and paper towel consumptions were recorded every weekday for about 5 months. These data were used to monitor hand hygiene performance by estimating handwashing performance. Bittner et al. (2002) obviated the denominator issue by dividing the consumption of 
soap and paper towels by occupied bed per hour, and named the result as "Estimated hand washing episodes (EHWEs)" (Bittner et al., 2002, p.120).

In the same study conducted by Bittner et al. (2002) and another study conducted by Lankford et al. (2003), the intervention monitoring method of measuring the consumption of soap and paper towel use did not improve hand hygiene compliance. In other studies, correlation was found between the consumption of soap and alcohol-based handrub with observed hand hygiene compliance. However, in other studies, correlation between the consumption of product use and observed hand hygiene compliance did not exist (Pittet, 2002; Hugonnet, Perneger and Pittet, 2002; Bischoff et al., 2000). Though consistent findings varied between different conducted studies (TJC, 2009), according to Haas and Larson (2007), monitoring consumption of product use requires more validation.

Some limitations of monitoring hand hygiene performance by measuring the consumption of product use are as follows. Such a method lacks the capability of capturing the true moment for hand hygiene (WHO, 2009). In addition, it is not possible to distinguish between healthcare professionals or their patients and their families and visitors, who might practice hand hygiene upon visiting the hospital (Mortel, Francisca and Murgo, 2006). It is also hard to indicate and identify the missed opportunity to hand hygiene and the reason(s) behind missing it (TJC, 2009). Additionally, it is hard to accurately and precisely measure the consumption of the product; thus, right improvement actions can be mislead by the results (TJC, 2009). On the other hand, using consumption of product can reduce observation bias and observation selection (WHO, 2009; TJC, 2009). Such a monitoring method is also 
easy to implement (WHO, 2009). It also requires less labor resources and less training (TJC, 2009). Thus, it is inexpensive compared to other monitoring methods (WHO, 2009).

\subsubsection{Electronically Monitoring of Product Use via Electronic Counting Devices}

According to Swoboda et al. (2007), Venkatesh et al. (2008), Swoboda et al. (2004), Kinsella, Thomas and Taylor (2007), monitoring hand hygiene performance electronically via automatically tracking the use of sinks and handrub dispensers can be carried out.

Larson, Albrecht and O'Keefe (2005) made a comparison between two different dispensing systems: manual dispensers of alcohol and touch-free alcohol sanitizers. They directly observed the frequency of product use and electronically monitored product use via electronic counters installed in dispensers. Larson et al. (2005) found that the total counts of uses per manual dispenser per day significantly differ from the total counts of uses per touch-free dispenser per day with a p-value of 0.02 in favor with touch-free dispensers. Larson et al. (2005) state that though touch-free dispensers are quite similar in cost to those manual ones; touch-free dispensers operate on batteries that last for about 2 years and a warning light on the dispensers flashes if they run out of batteries.

However, monitoring hand hygiene via electronic counting devices did not significantly affect hand hygiene compliance. One limitation of the study was the high cost of electronic counting devices. In addition, touch-free dispensers might have dispensed during high traffic time. Also, healthcare workers might have been using 
multiple hits of touch-free dispensers for a single moment for hand hygiene (Larson et al., 2005).

In another study conducted at the Hospital of Saint Raphael in New Haven, CT by Boyce, Cooper and Dolan (2008), electronic counting devices were integrated with alcohol-based hand rub dispensers to record the frequency of alcohol use. Data recorded at each dispenser were extracted using a handheld data transfer unit. The data was uploaded into a secure website that was used to conduct a periodic hand hygiene compliance analysis. The way the study was conducted and the types of data that were collected enabled the hospital to estimate how many hand hygiene episodes were done per patient day and break that down by units. The researchers also mapped the location of each dispenser included in the study to determine which dispenser was used more frequently at each unit.

In another study conducted at the Veterans Affairs Medical Center in Omaha, NE, electronic counting devices were also installed inside soap and alcohol dispensers. Each time the dispenser was used (hit), electronic counters recorded hand hygiene episodes. Data were compared every two weeks by subtracting the current-week dataset from the previous-week dataset to generate a total number of how frequently each dispenser was used at each unit. This number was then divided by the number of patient care days (TJC, 2009).

In general, using electronic counting devices to monitor hand hygiene performance has some drawbacks. According to TJC (2009), electronic counting devices are expensive and might fall onto the floor and get damaged. Both dispensers 
and counters operate on batteries. These devices are not self-time devices. Healthcare workers have to read and record the time of hand hygiene practices.

In contrast, electronic counting devices may reduce observation bias because observers are not present. Mapping out the location of each dispenser by unit and patient rooms may provide valuable information about which daily time or hourly time hand hygiene episodes occurred the most. It may also reveal valuable information about the location of patient rooms with precaution signage, where episodes occurred more frequently (WHO, 2009).

\subsubsection{Electronically Monitoring of Product Use via Electronic Monitoring Systems}

Healthcare sectors have sought advanced technological devices to electronically track specific events of hand hygiene. These devices can be used to distinguish between the consumption of product use and track each healthcare worker's activities individually. They also can remind healthcare workers to practice handwashing at critical moments (Boyce, 2011; TJC, 2009). However, based on studies conducted by Larson et al. (2005) and Wurtz, Moye and Jovanovic (1994), these systems are expensive and required more validation in terms of hand hygiene improvement.

These advanced technological devices vary in terms of their shapes, functionalities and how they are used. According to TJC (2009), a new tracking system has been used by Dartmouth-Hitchcock Medical Center in Lebanon, New

Hampshire in late 2007. This system is a dispenser that "is worn at the waist or from a lanyard and can be operated with one hand" (TJC, 2009, p.57). The system is capable of electronically recording each time healthcare workers perform hand hygiene. The 
system is also capable of emitting a sound to remind healthcare workers to practice handwashing if the dispenser has not been used for a while. The recorded data can be downloaded into a computer for further analysis for hand hygiene performance.

Another hospital in Canada has used a tracking system based on infrared sensors to track hand hygiene performance of their healthcare workers. The system consists of two devices. One is placed beside patient's beds and healthcare workers wear the other one. Every time healthcare workers enter or leave a patient room, the worn device beeps when hand hygiene is not practiced as needed. The system is capable of tracking both soap and alcohol-based handrub dispensers. The system is also capable of recording the last time hand hygiene was performed (TJC, 2009).

In another study conducted by Venkatesh et al. (2008), electronic monitoring devices were installed in the entrances of 12 patient beds beside alcohol-based hand rub dispensers. These devices are capable of beeping three times if hand hygiene was not practiced upon entering or leaving patient rooms. The devices are also capable of emitting a sound message that says "Please wash your hands" (Venkatesh et al., 2008, p.200). Results were promising when these devices were used to monitor and improve hand hygiene compliance (Venkatesh et al., 2008).

An interesting study was conducted by Polgreen et al. (2010) using a device that does not rely on radio frequency identification (RFID) technology installed outside patients' rooms. Healthcare workers are not required to wear these devices. These devices operate on WiFi spectrum and do not interrupt the workflow of patient care by interfering with the medical devices. Polgreen et al. (2010) were able to develop such a device and named it as "mote" (Polgreen et al., 2010, p.1), which consists of four 
main components: "Badges, Beacons, Triggers and Recorder" (Polgreen et al., 2010, p.2). These devices were used to track hand hygiene performance upon entering patients' rooms. Results were promising by accurately measuring and capturing hand hygiene moments (entering and leaving). Hand hygiene compliance was also improved. However, mote devices were unable to identify whether a contact between healthcare workers and their patients occurred or not. On the other hand, the presence of multiple badges in one patient room does not interrupt the whole monitoring system.

Some studies have assessed the use of a video monitoring system to track hand hygiene compliance and the consumption of products (Boyce, 2011). In a study that was conducted by Davis (2010) to prevent and control MRSA infection at a surgical ward, a hidden video camera was used to monitor hand hygiene activities of healthcare workers upon entering and leaving the ward. The study also involved a healthcare direct intervention to hand rubs with an alcohol using a red tape that pointed out the location of an alcohol-based dispenser. Hand hygiene events of entering and leaving patients' rooms in the surgical ward were documented after watching the recorded video from the hidden camera. Hand hygiene compliance results were not shared with anyone in the study. Consequently, adherence of hand hygiene compliance jumped up from $24 \%$ to about $62 \%$.

In a similar study that was conducted by Armellino et al. (2013), a hidden camera was used to monitor entering and exiting intensive care unit (ICU) patients' rooms for hand hygiene activities using both soap and alcohol hand rub dispensers. Some of the patients' rooms are double bed. To maintain privacy, the cameras were 
only filming the location of the dispensers. What is different in this study is that hand hygiene compliance feedback was publicly updated every 10 minutes inside ICU using electronic boards. Also, a validation of the video monitoring system method was conducted by sometimes applying a direct observation method. On average, an $80 \%$ hand hygiene compliance rate was achieved compared to $30.42 \%$ rate before the intervention.

Though these technological systems seem promising, still they have some limitations. The use of automatic devices in monitoring hand hygiene performance of healthcare workers may interfere in healthcare workers' and patients' privacy (WHO, 2009; Boyce, Cooper and Dolan, 2008; Boscart et al., 2008), and might cause ethical issues (WHO, 2009). Also, these devices are expensive to get and are vulnerable to wear out (WHO, 2009). The level of acceptance of use for these devices varies among healthcare workers (Boyce, 2011). Also, these devices were only able to capture moment 1 (before touching a patient), moment 4 (after touching a patient) and moment 5 (after touching patient's surroundings) (Boyce, 2011) of "my five moments for hand hygiene" defined by WHO (2009). However, some studies have concluded that capturing the entrance and exiting events of patients' rooms are not considered similar to capturing the moments when patients or their surroundings were contacted and touched by healthcare workers as in the study conducted by Polgreen et al. (2010).

\subsubsection{Radio Frequency Identification (RFID) Method}

Recently, advanced information technologies (ITs) have been effectively used in the healthcare industry to increase the quality of patient care and to increase efficiency of healthcare service levels (Payton, Pare, LeRouge, and Reddy, 2011). The adoption 
of advanced information technologies (ITs) enables healthcare sectors to remove the waste of reentering the data again and to electronically access patients' records in realtime (PCAST, 2010). In addition, the adoption of advanced information technologies (ITs) enables healthcare sectors, to increase the quality of patient safety and to improve inter-communication between healthcare workers (Burkhard, Schooley, Dawson, and Horan, 2010).

One interesting wireless technology that has recently been implemented in the healthcare industry by integrating it with advanced information technologies (ITs) is Radio Frequency Identification (RFID) technology. Though RFID was first used as radar during the Second World War to identify the allied aircraft from those aircraft that belonged to the enemies, the year of 2003 was considered to be a booming year for RFID in healthcare (Wamba, Lefebvre, Bendavid, and Lefebvre, 2008).

"The RFID is a technology that uses radio waves to transfer data from an electronic tag, called RFID tag attached to an object, through a reader for the purpose of identifying and tracking the object" (Ajami, and Rajabzadeh, 2013, p.2). It is "classified as a wireless automatic identification and data capture (AIDC) technology" (Wamba, Lefebvre, Bendavid, and Lefebvre, 2008, p.615). There are other types of AIDC technologies that are similar to RFID, (Wamba, Lefebvre, Bendavid, and Lefebvre, 2008), as depicted in Figure 2.2. 


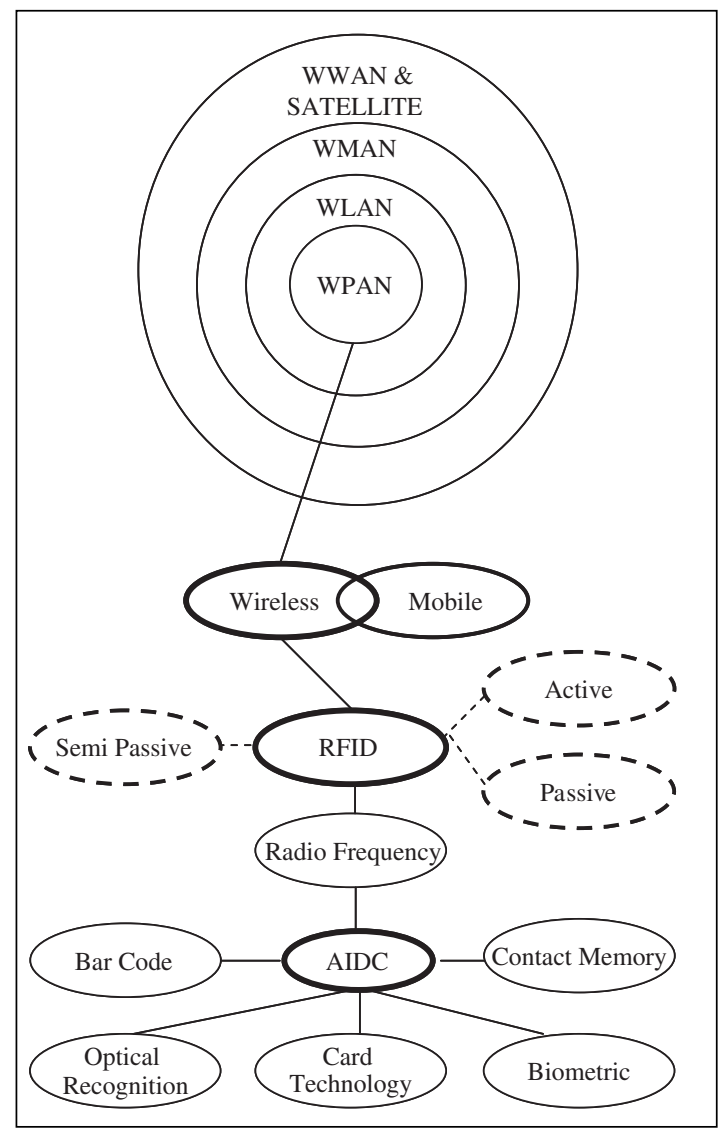

Figure 2.2 RFID Technology and other Types of AIDC Technologies (Wamba, Lefebrve, Bendavid and Lefebvre, 2008)

However, according to Asif and Mandviwalla (2005), RFID carries a wide range of benefits and values to the user more than other types of AIDC. First, RFID does not interrupt the workflow environment with the connection of lines because it works wirelessly. RFID also is able to read and write multiple tags at the same time (Asif and Mandviwalla, 2005). In contrast, RFID still is very costly compared to other AIDC types (Bensel, Gunther, Tribowski, and Vogeler, 2008). Boyce (2011) lists a summarized comprehensive comparison of advantages and disadvantages between different AIDC technologies shown in Table 2.2. 
A simple RFID technology is comprised of three main components, which are a tag, a reader and a host computer with application software. A tag (chip or transponder) can either be passive, semi passive and active. A tag stores a digital identification number and information about the object to which it is attached or a human being that wears it. A reader's role is to read out the digital data stored in a tag and send it to the host computer (Wamba, Lefebvre, Bendavid, and Lefebvre, 2008; Asif, and Mandviwalla, 2005).

With its valuable capabilities and features compared to other technologies, according to Wamba, Anand, and Carter (2013), RFID has been recently implemented in several pilot studies in healthcare for more validation. Hospitals have been using RFID for tracking their valuable tagged assets such as wheelchairs, and for improving the workflows of healthcare workers and patients, and for assessing the adherence of hand hygiene compliance among healthcare workers (Wamba, Anand, and Carter, 2013), just to name few. The Veterans Medical Center at Providence (PVAMC) is about to implement RFID technology for "Asset (equipment) tracking, Catheter laboratory supply tracking, Sterilization Department workflow, and Temperature monitoring (medication refrigerator, etc.)" (D. Pillittere, Chief Clinical Engineer, Personal Communication, May 18, 2015). Using RFID at PVAMC to track and monitor hand hygiene compliance of healthcare workers is neither a topic that is on the table to discuss nor to approve. High cost of hand hygiene RFID application and union regulations are the main two reasons for such delay (D. Pillittere, Chief Clinical Engineer, Personal Communication, May 18, 2015). 


\begin{tabular}{|c|c|c|}
\hline Technology & Advantages & Disadvantages \\
\hline $\mathrm{WiFi}$ & $\begin{array}{l}\text { Hospitals may have existing WiFi } \\
\text { infrastructure, reducing initial costs; } \\
\text { long communication range; can be } \\
\text { used in conjunction with other } \\
\text { technologies. }\end{array}$ & $\begin{array}{l}\text { Some systems may not } \\
\text { provide bed-level location } \\
\text { accuracy unless additional } \\
\text { beacons are used; badges may } \\
\text { be large and relatively } \\
\text { expensive; may have higher } \\
\text { power consumption. }\end{array}$ \\
\hline RFID & $\begin{array}{l}\text { Better location accuracy than WiFi } \\
\text { alone. }\end{array}$ & $\begin{array}{l}\text { Requires parallel wireless } \\
\text { infrastructure; goes through } \\
\text { walls, so location accuracy } \\
\text { may not be ideal. }\end{array}$ \\
\hline ZigBee & $\begin{array}{l}\text { Low power consumption; can be } \\
\text { portable; less ex-pensive than some } \\
\text { other wireless systems. }\end{array}$ & $\begin{array}{l}\text { Accurate location may require } \\
\text { multiple beacons in an area, } \\
\text { or combination with other } \\
\text { technology; some systems } \\
\text { may credit two HCWs with } \\
\text { hand hygiene event if HCWs } \\
\text { are very close together. }\end{array}$ \\
\hline ANT & $\begin{array}{l}\text { Very low power consumption; faster } \\
\text { transmission speeds than ZigBee; } \\
\text { low system cost; long battery life. }\end{array}$ & $\begin{array}{l}\text { Not designed for complex } \\
\text { high-frequency events. }\end{array}$ \\
\hline Infrared (IR) & $\begin{array}{l}\text { Does not penetrate walls, so location } \\
\text { accuracy good to subroom level; IR } \\
\text { room ID devices can be battery- } \\
\text { operated, so wiring not needed. }\end{array}$ & $\begin{array}{l}\text { Requires IR detectors and } \\
\text { other technology such as } \\
\text { RFID for communication. }\end{array}$ \\
\hline Ultrasound & $\begin{array}{l}\text { Does not penetrate walls, so high- } \\
\text { level room and subroom accuracy; } \\
\text { no electromagnetic interference with } \\
\text { other equipment; does not require } \\
\text { line of sight between tag and } \\
\text { detector. }\end{array}$ & $\begin{array}{l}\text { Requires ultrasound sensor on } \\
\text { RFID or other tag; requires } \\
\text { integration with WiFi, RFID, } \\
\text { or local area network to } \\
\text { transmit signals to central } \\
\text { server. }\end{array}$ \\
\hline $\begin{array}{l}\text { IR/RFID } \\
\text { Combination }\end{array}$ & Provides good location accuracy. & $\begin{array}{l}\text { Required both IR and RFID } \\
\text { infrastructure. }\end{array}$ \\
\hline $\begin{array}{l}\text { Ultrawide } \\
\text { Band }\end{array}$ & $\begin{array}{l}\text { Low power, resistant to interference; } \\
\text { good location accuracy. }\end{array}$ & $\begin{array}{l}\text { Shorter range than } \\
\text { narrowband frequency; } \\
\text { variable standards. }\end{array}$ \\
\hline
\end{tabular}

Table 2.2 Advantages and Disadvantages of Different Types of AIDC Technologies (Boyce, 2011) 


\subsection{RFID and Hand Hygiene}

Recently, different hand hygiene approaches and systems that are integrated with the use of RFID technology have been proposed and assessed in several hospitals to prevent and control cross-contamination healthcare-associated infections by improving hand hygiene compliance. However, some barriers have been hindering the early use of RFID in hand hygiene tracking application. According to Boyce (2011), it would be more reasonable to use RFID in tracking hand hygiene compliance at a hospital that has already installed RFID infrastructure to be used in other applications such as asset-tracking applications. In this case, the cost factor would be lowered. Additionally, quality of patient care and healthcare level of service need to be furthered assisted under the use of such technology.

In 2015, one interesting system that has been applied and implemented, according to Wittrup and Burba (2015), at one of the US Midwest hospitals to prevent and control Clostridium Difficile Infection (CDI) is developed by Biovigil Healthcare Systems. Biovigil healthcare systems developed their own hand hygiene compliance tracking system that is integrated with RFID technology. The system consists of four simple functions "the four R's" as described by Wittrup and Burba (2015, p.8), "the ability to Record data, Report it, Remind health-care workers to sanitize hands, and Reassure patients and their family members that proper hand hygiene has been observed" (Wittrup and Burba, 2015, p.8). According to Wittrup and Burba (2015), the system consists of a badge to wear by each healthcare worker that has a sensor that is capable of soap and alcohol detection, a virtual wall that is installed inside and outside each patient room to identify the location of the badge, and a base-station 
device that works as a badge charger and a data-storage hard disk. At the end of each shift, healthcare workers plug in their badges in the base-station device for charging and the base-station device transfers the hand hygiene compliance data from the badges into the device itself. The base-station device makes the data available for analysis by using a webpage software application or smart-phone application (Wittrup and Burba, 2015). Upon entering or leaving a patient room, the badge will emit a sound and turn yellow after a few seconds if hand hygiene is not practiced. Once healthcare workers practice hand hygiene, whether soap or alcohol, and place their hand over their badges, the badge turns green. If, however, hand hygiene is not practiced for more than the set duration time, the badge emits sounds and turns red. Though the system is limited to capturing hand hygiene compliance upon entering or leaving a patient room, the adherence rate for hand hygiene compliance has been reported to be above 95\% (Wittrup and Burba, 2015). 


\section{CHAPTER 3}

\section{METHODOLOGY}

A direct method will be used to monitor the overall hand hygiene compliance at PVAMC during routine patient care at three inpatient units, 5B, $6 \mathrm{~B}$ and intensive care unit (ICU). The data will be compared to the current hand hygiene monitoring direct methods conducted by PVAMC, which will be used as a baseline for the study. The collected data from the designated two-month study will be compared to the same two months from the current method for the past four fiscal years, 2012 to 2015 . This proposed hand hygiene direct method would not only report the estimate of the best hand hygiene compliance rate of HCWs at PVAMC during the time of the study, but also will identify several risk factors for poor adherence. Thus, the researchable questions of interest for this study are as follows:

- What is a better estimate of hand hygiene compliance rate?

- Is there any correlation between hand hygiene opportunities and HAI incidents, MRSA colonization and community-acquired infections (CAI)?

- What are the risk factors for poor adherence (low compliance)?

As a secondary measurement, new indirect hand hygiene monitoring methods

of measuring product use and personal protective equipment (PPE) compliance will be proposed to compare each inpatient ward at the PVAMC against each other because they are of similar and different sizes in terms of number of operating beds $(\mathrm{OB})$ and bed days of care (BDOC). 
Historical data on HAIs, admission numbers and bed days of care, are all aggregated and summary report data do not involve patient medical records.

\subsection{Design}

In the literature, there are several causes to infectious diseases. However, poor adherence to hand hygiene compliance is a leading cause of HAIs (WHO, 2009). Effective and efficient hand hygiene compliance monitoring will not only control and prevent HAIs, but also helps to serve "multiple functions: system monitoring, incentive for performance improvement, outbreak investigation, staffing management, and infrastructure design" (WHO, 2009, p.158). There are leading risk factors for poor adherence to hand hygiene compliance (see Table 2.1). In this study, seven risk factors, which are reported in Table 2.1 and by WHO (2009), are chosen to be studied, which are:

- HCWs gender.

- Job status.

- Type of unit.

- Weekdays.

- Hand hygiene moments.

- Working shift.

- Patient isolation status.

These seven factors are chosen to be studied under Veterans Affairs healthcare settings. HCWs gender is an important factor because of the imbalance where more female HCWs than male are typically expected in healthcare. Job status is also an important factor to see how hand hygiene compliance varies between the medical and 
nursing groups though the nursing group is expected to have higher patient contact than the medical group. Type of unit is selected to see how hand hygiene compliance varies between inpatient wards, where each is different in terms of the level of patient care. Weekday is selected to see whether hand hygiene compliance is different between days of the week and weekends. Hand hygiene moment is also an important factor because it is crucial to explore how hand hygiene compliance varies between before touching a patient and after touching a patient moment. Working shift is also important because it tells more about how hand hygiene compliance varies between Night, Day and Evening shift where HCWs' hand hygiene behavior changes over time based on patients' behavior and care needs. Patient isolation status is another important factor that leads to explore whether HCWs' hand hygiene compliance is different when providing care to two different patients based on their isolation status when, for example, gloves are required to be worn. Another important factor that has not been explored in the literature before and is of an interest to be studied in Veterans Affairs medical healthcare settings is patient gender imbalance. It is expected that there are more male patients than female patients. It is interesting to see how hand hygiene compliance varies by such a factor especially there are more female HCWs than male HCWs at the Veterans Affairs Medical Centers.

A best estimation of the true rate of hand hygiene compliance and its confidence interval will be measured using the sample distribution of a proportion. A power analysis will be applied to determine the effective sample size of the group study and the total number of observations required for meaningful hypothesis testing. 


\subsection{Study Hypotheses}

Next, several hypotheses will be tested, to study the risk factors for poor compliance.

Hypothesis 1: There is no difference in hand hygiene compliance rate by employee gender.

Hypothesis 2: There is no difference in hand hygiene compliance rate by professional category, doctors vs. nurses.

Hypothesis 3: There is no difference in hand hygiene compliance rate by type of unit, $5 \mathrm{~B}$ vs. $6 \mathrm{~B}$ vs. ICU.

Hypothesis 4: There is no difference in hand hygiene compliance rate by days of the week, Monday vs. Friday.

Hypothesis 5: There is no difference in hand hygiene compliance rate by "My 5 Moments for Hand Hygiene" (WHO, 2009), such as Before and After Contacting a Patient.

Hypothesis 6: There is no difference in hand hygiene compliance rate by patient gender.

Hypothesis 7: There is no difference in hand hygiene compliance rate by working shift, Night, Day and Evening.

Hypothesis 8: There is no difference in hand hygiene compliance rate by patient status, such as patients who are on isolation vs. those who are not on isolation.

Conclusions are drawn based on the p-value of tests for two samples proportions for hypothesis $1,2,5,6$ and 8 and based on the p-value of tests for several proportions for hypothesis 3, 4 and 7. For hypothesis 3, 4 and 7, multiple comparisons of several 
proportions are conducted using the Marascuilo Procedure, which is a nonparametric test that is similar to Tukey-Kramer.

\subsection{Methods}

In order to estimate the true magnitude of patient contact, or the correct number of opportunities for hand hygiene before and after contacting a patient by HCWs, a Patient-Contact data collection form will be used by several healthcare professional categories at PVAMC at inpatient units, 5B, 6B and ICU. These professional categories and their subcategories include only Doctors and Nurses. In total, there will be 100 nurses and 50 doctors broken down by units, 5B, 6B and ICU, and broken down by shifts, day, evening and night. These numbers are not uniformly distributed per unit per shift. These sample sizes are driven by including all nurses and doctors who work at the inpatient units, 5B, 6B and ICU. Other healthcare professional categories will be excluded in this study, because medicine (doctors) and nursing (nurses) are among the most important and critical professions in healthcare who have patient contact (Appendices A and B).

Each HCW will self-report the total number of patient contacts for one complete week starting on a Monday night shift and ending on a Sunday evening shift for all shifts, day, evening and night. This will be completed once during the year. One month prior, and one month overlapping the one week of self-reporting patient-contact data collection, a URI research team member will also be present on the hallway of inpatient wards to assess the participants during the self-report data on patient contacts that comes from the doctors and nurses in the inpatient units, 5B, 6B and ICU at PVAMC. The Patient-Contact form (Appendices C, D, E, F, G, H, I, J and K) is 
designed to capture the following information: employee discipline and gender (i.e. a female attending doctor), date, day, time and shift of patient contact, patient gender, inpatient unit (5B, 6B and ICU), patient room and which bed (bed $\mathrm{A}$ or $\mathrm{B}$, left or right, door to window). There will be a unique form for each inpatient unit and for each of the 3 shifts, for a total of 9 sub-forms (Appendices C, D, E, F, G, H, I, J and K). To protect employee and patient privacy, names are not included on the form and will not be reported. Each form will be coded by a letter that refers to the employee discipline (D for Doctor, $\mathrm{N}$ for Nurse, etc.) followed by a unique number (D001, N005). No one would know that code except the URI research team. A spreadsheet will be created to only include names and unique assigned codes of doctors and nurses that will participate in the study to self-report their patient-contacts. This spreadsheet will be electronically stored in a restricted folder on the secure research server at the PVAMC that cannot be accessed by anyone except the URI research team. This spreadsheet and its contents will be created after the consent form (Appendix L) is signed by the participants. The participant nurses will be approached individually by the URI research team before the start or after the end of their work shift at each inpatient unit for recruitment and for obtaining the informed consent and their names and unique codes will be recorded in the spreadsheet at the PVAMC. The participant doctors will be approached by the URI research team individually at their offices for recruitment and for obtaining the informed consent and their names and unique codes will be recorded in the spreadsheet at the PVAMC. Then, a printout copy of the patientcontact form will be distributed to each participant. There will be a labeled and locked mailbox at each inpatient unit (5B, 6B and ICU) where participants can drop their 
patient-contact forms at the end of each shift or day. This will ensure the privacy of participants. The URI research team will be the only one who has access to the locked mailboxes. The URI research team will remotely access the secured folder via the VA Citrix Access Gate (CAG) from URI campus to conduct the required analysis and write up the $\mathrm{PhD}$ dissertation on a URI computer.

For two months, one month prior to self-reporting patient-contact data collection and one month overlapping with the week of patient-contact data collection, secret observers, who are HCWs at PVAMC, will be asked to observe hand hygiene compliance at inpatient units $(5 \mathrm{~B}, 6 \mathrm{~B}$ and ICU). During the same months, the URI research team member will also participate in observing hand hygiene compliance at inpatient units (5B, 6B and ICU). In order to capture an effective sample size of hand hygiene indications, 40 secret observers will be recruited. This number is not uniformly distributed per unit per shift. An updated hand hygiene monitoring data collection form (Appendices M, N and O) will be used. The pilot study will focus on three inpatients units, two healthcare professional categories, and two out of five moments for hand hygiene, so the updated hand hygiene monitoring data collection form will capture partial information of the online hand hygiene compliance form (Appendices P and Q) that has been used by the PVMAC, and add more. However, the partial information is valid to be entered in the online hand hygiene management tool (Appendices $\mathrm{R}$ and $\mathrm{S}$ ), which will remove a waste of using the online hand hygiene management tool form in parallel with the updated hand hygiene monitoring data collection form during the time of the study. The updated hand hygiene monitoring data collection form (Appendices M, N and O) includes: healthcare discipline of the 
observed (Doctors, Nurses) and their subcategories, if any; before/after patient-contact hand hygiene with waterless (alcohol-based hand rub or Purell dispenser), soap and water, or none; patient gender (male/female) and health status (in isolation or not), date, day of the week and shift of the observation; and the unit (5B, 6B and ICU), room number of the observation and which bed the observed patient is utilizing. Patient gender, health status, shift, room and bed numbers are new pieces of information that is not provided in the online hand hygiene monitoring tool used by the PVAMC for hand hygiene. Each updated hand hygiene monitoring data collection form will be coded by a letter that refers to the secret observer (S) and will be followed by a unique number (S001), which only the URI research team will know. A spreadsheet will be created to only include names and unique assigned codes of the secret observers that will participate in the study using the updated hand hygiene monitoring data collection form. This spreadsheet will be electronically stored in a restricted folder on the secure research server at the PVAMC that cannot be accessed by anyone except the URI research team. This spreadsheet and its contents will be created after the consent form (Appendix $\mathrm{T}$ ) is signed by each participant. The participant secret observers will be approached by the URI research team individually at their offices for recruitment and for obtaining informed consent and their names and unique codes will be recorded in the spreadsheet at the PVAMC. Then, a printout copy of the updated hand hygiene monitoring data collection form will be distributed to each secret observer. There will be a labeled and locked mailbox in the Infectious Disease Department at the PVAMC where each secret observer will drop hand hygiene monitoring data collection forms at the end of each shift or day. This will 
ensure privacy of both the secret observers and the observed HCWs. The URI research team will remotely access this secured folder via the VA Citrix Access Gate (CAG) from URI campus to conduct the required analysis and write up the PhD dissertation on a URI computer.

Indirect hand hygiene monitoring of product use is a method that will be conducted by tracking Purell and Soap cartridge replacement at inpatient units 5B, $6 \mathrm{~B}$ and ICU by asking housekeepers to mark an (x) on a log-tracking sheet (Appendices U, V and W) each time they replace a cartridge of Purell or Soap. The time of replacement and location of dispenser will be indicated as well. Each Soap-Purell dispenser at each inpatient ward will be marked with a unique identification number to be used on the tracking sheet. The purchasing and re-stocking sales data of Purell and Soap will be assessed as well. The tracking will be conducted one time for several months of a year.

An indirect PPE (Gloves, Gowns and Masks) compliance method will be developed by using the PPE inventory data of three years 2014, 2015 and 2016 at the PVAMC. The PVAMC's inventory system reports PPE orders per department and inpatient ward. The PPE quantities ordered will be assumed equal to the PPE that are consumed by HCWs on the floor during patient care.

The PVAMC does not track or report infections by operating bed at each inpatient unit, 5B, 6B and ICU. Conversely, the infections and bed days of care dataset are reported together monthly either aggregated by unit level such as $5 \mathrm{~B}, 6 \mathrm{~B}$ or ICU, or by facility level for VISN 1 using the IPEC Data Management System. Statistically, it is worth tracking and reporting infections by operating beds by bed days of care for 
each unit during the same period of time. Additional data will need to be collected to track and report infections (MRSA, CDI, CAUTI, CLABSI, VAP/VAE) by operating beds by bed days of care at the inpatient units for the designated months of the study. A track of HAIs per operating bed per inpatient unit will be used during the same month of conducting secret observers hand hygiene monitoring data collection (Appendices $\mathrm{X}, \mathrm{Y}$ and $\mathrm{Z}$ ). This form will be filled out by a volunteer infection preventionist from the PVAMC Infectious Disease Department for the two months of the study. MRSA and CDI rates are calculated based on 1000 and 10,000 bed days of care respectively, while CAUTI is calculated based on 1000 catheter days, CLABSI is calculated based on 1000 line days, and VAP/VAE is calculated based on 1000 vent days. Reporting BDOC per bed per inpatient ward will be used in evaluating patient contact frequency per inpatient ward and also in evaluating the direct and indirect hand hygiene compliance and the indirect PPE compliance methods.

The URI research team will work closely with the PVAMC Infectious Disease Department to gather relevant historical data for statistical analysis of HAIs and hand hygiene such as:

- Analyzing the occurrence of infectious disease records to see which statistical models fit best and are most applicable.

- Reviewing procedures for collection, recording, and reporting of HAIs and hand-hygiene.

- Assessing any correlations between HAIs data and hand-hygiene compliance records.

- Reviewing calculations of HAIs rates conducted by the VA. 


\subsection{Data Required}

The following data is requested from the PVAMC:

- 5 years of data of occurrences of identified HAIs of interest (preferably daily, but weekly or monthly data could be acceptable if that is all that is available).

- 3 years of data of purchasing or re-stocking data on hand-hygiene, Purell and Soap.

- 4 years of data of "Secret Observers" hand-hygiene compliance records and dataset.

- Performance metrics, The Strategic Analytics for Improvement and Learning (SAIL) Value Model data, used by VISN 1 and the national VA organization for rating PVAMC on matters related to HAIs and hand hygiene.

- 3 years of data of personal protective equipment (PPE) (Gloves, Gowns and Masks) inventory data.

- Data related to treatment costs of key HAIs, and hospitalization or length of stay LOS estimates.

The data on HAI occurrences, costs, and LOS are all aggregate summary data in existing reports and do not involve specific patient medical records. These required data will be electronically sent to and stored in a restricted folder on the secure research server at the PVAMC. The PVAMC infectious disease preventionist at the Infectious Disease Department will be asked to send these required data to the secured folder. The URI research team will be the only one who have access to the secured folder. The URI research team will remotely access this secured folder via the VA 
Citrix Access Gate (CAG) from URI campus to conduct the required analysis and write up the $\mathrm{PhD}$ dissertation on a URI computer.

\subsection{Evaluation}

After collecting the data for patient-contact, secret observers for hand hygiene, Log-tracking, purchasing, and restocking of Purell and Soap, PPE (Gloves, Gowns and Masks) inventory data, tracking HAIs per operating bed per inpatient unit, and other requested dataset from the PVAMC such as HAIs and SAIL, the following statistical analysis will be conducted using RStudio Version 1.0.1.136 on Mac, which is a freeware open source tool that is used to statistically analyze data because it has no cost and runs on both Mac and Windows Operating Systems.

1. Patient-Contact and secret-observer datasets will be analyzed to identify the bestfit statistical distributions that explain the data.

2. Mean, median and mode and other descriptive statistics.

3. The true hand hygiene compliance rate will be estimated.

4. Hand hygiene compliance rate and its trends will be broken down by shifts (Day, Evening and Night), professional category (Doctor vs. Nurse), inpatient unit (5B, 6B and ICU), days of the week (weekdays vs. weekend), and healthcare workers' gender (male vs. female).

5. Hand hygiene compliance rate will be analyzed over time to identify trends.

The research team will submit a report on hand hygiene analysis as well as a new proposed indirect hand hygiene manual monitoring of product use method and a new proposed indirect method of measuring PPE compliance at the end of the research to the PVAMC quality management and infectious disease department. In addition, the 
research team plans to present and publish the research findings in conferences and journals related to infectious diseases, hand hygiene, ranking systems in healthcare, performance improvement, and industrial engineering.

\subsection{Study Population}

All VA doctors and nurses who work at the inpatient units, 5B, 6B and ICU, and within the age group of $18-90$ will be asked to self-report their patient-contacts. The patient contact data represents the target population of the study. There are no additional criteria to select the participants for the patient-contact self-reporting. Recruited secret observers will be any VA employees who agree to be a secret observer for the study, but are not part of the patient-contact self-reporting group, who will be asked to randomly observe the patient-contact self-reporting group during the time of the study. The collected observations represent the study population. The estimated maximum numbers of VA employees that can be requested to participate in the patient-contact self-reporting and as secret observers are 190 (50 doctors, 100 nurses and 40 secret observers). The patient contact group is sampled based on a universal (convenience) sampling method since the objectives of such a sampling method are to find an approximation of patient contact population per inpatient ward, though hand hygiene opportunities are immeasurable, and to find average patient visits per inpatient ward. The convenience sampling generally is used when the study population is either small or not easily obtainable. The latter is the case in the research study. In the literature (Etikan, Musa and Alkassim, 2016) convenience sampling is categorized as a nonrandom sampling method where the target population is, for example, bounded by time interval. 


\section{CHAPTER 4}

\section{PATIENT CONTACT ANALYSIS}

This chapter provides a descriptive statistics summary about patient contact data and also answers the two main objectives for which patient contact data collection is conducted at the first place:

- An approximation of patient contact population per inpatient ward.

- Average patient visits per inpatient ward.

Patient contact is conducted for one complete week starting on Night Shift on Monday Oct $3^{\text {rd }}$ and ending on Evening Shift on Sunday Oct $9^{\text {th }}$ of 2016 . Night Shift starts on 12:00 am and ends on 8:00 am. Day Shift starts on 8:00 am and ends on 4:00 pm. Evening Shift starts on 4:00 pm and ends on 12:00 am.

\subsection{Background}

The Providence Veterans Affairs Medical Center (PVAMC) is a medium medical center that serves veterans in the state of Rhode Island, USA, and the surrounding areas. PVAMC is licensed to 73 operating beds, which are distributed to three inpatient units $(5 \mathrm{~B}, 6 \mathrm{~B}$ and $\mathrm{ICU})$, a mental health ward and an emergency department. The inpatient wards, $(5 \mathrm{~B}, 6 \mathrm{~B}$ and $\mathrm{ICU})$ are acute care units and they utilize most of the operating beds at the PVAMC as shown in Table 4.1:

\begin{tabular}{|c|c|c|}
\hline Ward & Operating-Bed Capacity & Licensed Operating Bed \\
\hline 5B & 25 & 23 \\
\hline 6B & 27 & 27 \\
\hline ICU & 8 & 8 \\
\hline
\end{tabular}

Table 4.1 Inpatient Wards and Their Operating-Licensed Bed Capacity 
There are two main factors that influence the utilization of the true capacity (CAP) of the operating beds at each inpatient unit, especially at 5B and 6B wards. First, how many patients need isolation. Second, how many staff is on the floor for each shift. The three-inpatient units at the PVAMC follow a three-shift period per day as seen in Table 4.2 .

\begin{tabular}{|c|c|c|}
\hline Shift & Start Time & End Time \\
\hline Night & $12: 00$ a.m. & $8: 00$ a.m. \\
\hline Day & $8: 00$ a.m. & $4: 00$ p.m. \\
\hline Evening & $4: 00$ p.m. & $12: 00$ a.m. \\
\hline
\end{tabular}

Table 4.2 Working Shifts at Inpatient Wards

In terms of staffing, each ward during each shift has various types of healthcare employees.

\subsubsection{Nursing Group}

- Registered Nurse (RN)

- Licensed Practical Nursing (LPN)

- $\quad$ Certified Nursing Assistant (CNA)

- Nursing Student (NS)

Each ward has several RNs assigned to several patients per shift. The RNs are the ones who take care of patients medically. Usually, the $\mathrm{RN}$ who is in charge does the job of assigning patients to the RNs and CNAs for the next shift before the current shift ends. 5B and 6B use the same assignment sheet template (Appendix AA), while ICU uses a different assignment sheet template (Appendix BB). The assignment sheet can only include the patient's last name. There should be no full name or the last four digits of the SSN. 
Each $\mathrm{RN}$ is provided with a medication cart, a laptop computer and a scanner that are attached to the medication cart. There is a sharps container (biohazardous trash bin) that is attached to the medication cart for safety to discard sharp items. This sharp container is part of the standard precautions guidelines. RNs usually hang a trash bag for their trash items during patient care.

Each shift is occupied by several CNAs who help the RNs in their caregiving services such as supplying snacks and water to patients plus helping housekeepers in making beds. Usually there is one LPN that either is available to $5 \mathrm{~B}$ or $6 \mathrm{~B}$ but not to ICU. The LPN's main job is to sit with patients who need supervision all the time. The NS is usually on the floor to finish the nursing degree and get trained. There is usually one NS at each ward except ICU, which has none.

\subsubsection{Medical Group}

- Attending

- Fellow

- Resident

- Intern

- Medical Student

The medical group usually is assigned to patients all over the three inpatient wards. Attending and fellow are usually permanent employees at the PVAMC; however, residents, interns and medical students are floating in and out on a monthly basis for practicing and training. There are several medical doctorate groups, pulmonary, cardiology and GI, that these medical subgroups belong to. Basically, after finishing medical school, medical students spend one full year of training to be 
transitioned into what is medically called a post-graduate year (PGY) of 1 to 10 years at most divided as follows:

- $1^{\text {st }}$ Year: Intern.

- $2^{\text {nd }}-3^{\text {rd }}$ Years: Resident.

- $4^{\text {th }}-6^{\text {th }}$ Years: Fellow.

Practicing medicine and contacting patients unsupervised cannot be permitted until experience is gained by going through these years. Generally, Pulmonary requires finishing the first two years; Cardiology and GI require finishing the third year.

\subsubsection{Health Administration Service}

- Secretary

There is a secretary at each ward during each shift who takes care of entering the newly admitted patient's information into VistA, which is then entered on the BedBoard Management System (BMS) manually.

VistA is "The Veterans Health Information Systems and Technology Architecture Information System - is a Health Information Technology (HIT) system created and used by the Veterans Health Administration (VHA) of the U.S. Department of Veterans Affairs (VA) in serving America's Veterans through the provision of exceptional-quality health care which enhances our Veterans' health and well-being." (U.S. Department of Veterans Affairs, 2013, Page 13).

Bed-Board Management System (BMS) "provides real-time, user friendly, web-based VistA interface to track patient movement and determine bed availability." (U.S. Department of Veterans Affairs, 2013, Page 29). At each inpatient ward, there is 
a BMS (it is called whiteboard) screen that shows patient-room and bed number and bed letter with phone extension to the telephone for each bed, patient's name and gender, assigned staff (RN), assigned attending, comments on isolation and nonisolation, discharge status, bed status, ward, and length of stay (LOS) at the ward in time unit. The whiteboard also shows the ward census and the facility census. The whiteboard uses five different colors to mark each bed status as follows:

- Green: used for unoccupied bed/room.

- Blue: used for occupied bed/room by male patient.

- Pink: used for occupied bed/room by female patient.

- Orange: used for booked or reserved bed/room for transferred or admitted patient who is on the way to the ward.

- Red: used for bed/room with environmental issues (water leak or no heat).

The BMS is live all the time and unfortunately does not save data over time. The infection preventionist from the infectious disease team at the PVAMC takes the patient information from the BMS on a daily basis, for those patients who are on isolation, and tracks them back in VistA to identify any HAIs incidents and report them into the InPatient Evaluation Center (IPEC) System. IPEC is a system that contains all infections information related to patients broken down by inpatient ward and by overall facility. IPEC, unfortunately, does not report how many BDOC are associated with those patients who are on precautions. Admission numbers and BDOC in total are reported monthly per inpatient ward (5B, 6B and ICU) and overall facility. Thus, BDOC has to be reported separately for those patients who are on precautions with the types of precautions, Contact for MRSA or C.Diff, Droplet for Flu for 
example or Airborne for TB. In addition, healthcare-device-associated infections such as CATUI, CLABSI or VAE, which do not require isolation since they are not MultiDrug Resistant Organisms (MDOR) infections which means they are not contagious, are also not reported by isolation or non-isolation. A patient with CAUTI who does not require transmission signage on the door could have MRSA or C.Diff or both, which requires contact precaution signage. Distinguishing between BDOC for nonisolated patients from BDOC for isolated patients indicates the required PPE use at the time of providing care. For example, when patients are on contact precautions HCWs are required to wear gloves and gowns every time they go in the room even if there is no physical contact. However, if folly catheter, central line, or ventilator is required and a patient is not on any precautions, HCWs are only required to wear gloves but gowns are not required unless patients are on precaution. In terms of who installs the folly catheter, central line or ventilator, according to (B. Cedeno, Personal Communication, December 23, 2016), central line is always done by doctors but not medical students, and it is used, for example, for food, blood draw or providing medication. Both doctors, including medical students, and nurses do catheter. Ventilator is done by doctors and sometimes by medical students but under the attending/fellow supervision. Catheter, ventilator and central line days are calculated based on how many days the patients are on those devices. They are not based on how many times a folly catheter, ventilator or line are used or replaced. IPEC also does not tell how many patients are on catheter, central line, or ventilator or their associated BDOC. Of note, all HAIs are reported per inpatient ward and facility-wide except for C.Diff, which is only reported facility-wide. 


\subsubsection{Environmental Management Service (EMS)}

- Housekeepers

Each inpatient ward has several EMS employees whose job it is to clean the hallway, clean patients' rooms and make beds upon discharging patients. During day and evening shifts, rooms with non-isolation and with isolation signs, except for C.Diff, are regularly cleaned once with bleach with or without the existence of patients in the rooms. However, rooms with C.Diff are cleaned twice, with regular bleach first and secondly with special cleaning chemicals. Sometimes UV equipment is used to disinfect the rooms with C.Diff but it is not required. It takes about 5 minutes to clean a bathroom, and 5 minutes to clean one side of the room for each bed, which in total takes 15 minutes for the whole room. The EMS uses a two-step Checklist form (Appendix CC) that is kept at each nursing station at each inpatient ward to follow up with room cleaning. This 2-step checklist form includes date, room number, bed (A [left or door] or B [right or window]), isolation yes or no or C.Diff, posted and completed time and by whom housekeeper. Isolation signs are kept on doors of the rooms after discharging patients to notify housekeepers and CNA to comply with the isolation precautious by wearing the appropriate PPE when cleaning the rooms and making beds. The VA at Providence has patient rooms with either a feet-to-feet type, which means the bed to the left is $\mathrm{A}$ and the bed to the right is B, or a door-to-window type, which means the bed by the door is A and the bed by the window is B. 


\subsection{Patient Contact Participants Summary Statistics}

Healthcare groups of interest to the research study are:

- Medical Group: Attending Physician, Resident, Intern and Medical Student.

- Nursing Group: Registered Nurse Practitioner (RNP), Registered Nurse (RN), Licensed Practical Nurse (LPN) and Certified Nursing Assistant (CNA).

Medical and nursing groups work in all inpatient wards; however, not all subcategories work in all inpatient wards. For example, LPN works only in 6B and NP sometimes visits all wards and sometimes not. Medical group is not assigned per inpatient ward. They are assigned by patients. So, Medical subcategories sometimes are on and off each inpatient ward. Nursing group differs between inpatient ward based on the ward capacity and how many patients are on the floor. Normally, the highest workforce is available at $6 \mathrm{~B}$ because $6 \mathrm{~B}$ has the highest capacity; then, $5 \mathrm{~B}$ and ICU come next.

As explained in Chapter 3, Methodology, all doctors and all nurses at the threeinpatient wards were asked to participate in the study. In the literature, this is called Universal Sampling. For patient contact, 50 doctors and 100 nurses were requested to participate. The study included 28 doctors and 93 nurses voluntarily participating, which was most of the nurses in these units. Two CNAs withdrew from the study, one in the middle and one at the beginning, due to their intense work. Among all doctors, a resident and a medical student decided not to participate at all. In addition, another resident quit in the middle of the study. A matrix is created to track the medical participants during the one week of the study as shown in Table 4.3. The URI research team was asked not to approach other medical groups such as Fellows, Surgeons, 
Psychiatrists and Psychologists. A hierarchical structure is depicted in Figure 4.1 showing all participants per inpatient ward per working shift. A matrix structure is depicted in Figure 4.2 showing nursing participants and BDOC per inpatient ward per working shift by date of participation.

\begin{tabular}{|c|c|c|c|c|c|c|c|c|}
\hline$\#$ & Participant & 3-Oct & 4-Oct & 5-Oct & 6-Oct & 7-Oct & 8-Oct & 9-Oct \\
\hline 1 & Attending & & & & & & & \\
\hline 2 & Attending & & & & & & & \\
\hline 3 & Attending & & & & & & & \\
\hline 4 & Attending & & & & & & & \\
\hline 5 & Attending & & & & & & & \\
\hline 6 & Attending & & & & & & & \\
\hline 7 & Attending & & & & & & & \\
\hline 8 & Attending & & & & & & & \\
\hline 1 & Interns & & & & & & & \\
\hline 2 & Interns & & & & & & & \\
\hline 3 & Interns & & & & & & & \\
\hline 4 & Interns & & & & & & & \\
\hline 5 & Interns & & & & & & & \\
\hline 1 & MS & & & & & & & \\
\hline 2 & $\mathrm{MS}$ & & & & & & & \\
\hline 3 & MS & & & & & & & \\
\hline 4 & MS & & & & & & & \\
\hline 1 & Resident & & & & & & & \\
\hline 2 & Resident & & & & & & & \\
\hline 3 & Resident & & & & & & & \\
\hline 4 & Resident & & & & & & & \\
\hline 5 & Resident & & & & & & & \\
\hline 6 & Resident & & & & & & & \\
\hline 7 & Resident & & & & & & & \\
\hline 8 & Resident & & & & & & & \\
\hline 9 & Resident & & & & & & & \\
\hline 10 & Resident & & & & & & & \\
\hline 11 & Resident & & & & & & & \\
\hline 1 & RNP & & & & & & & \\
\hline
\end{tabular}

\begin{tabular}{|c|}
\hline Criteria \\
\hline Received \\
\hline Missing \\
\hline NA \\
\hline No Patient \\
\hline
\end{tabular}

Table 4.3 Medical Doctor Participation Tracking Matrix 


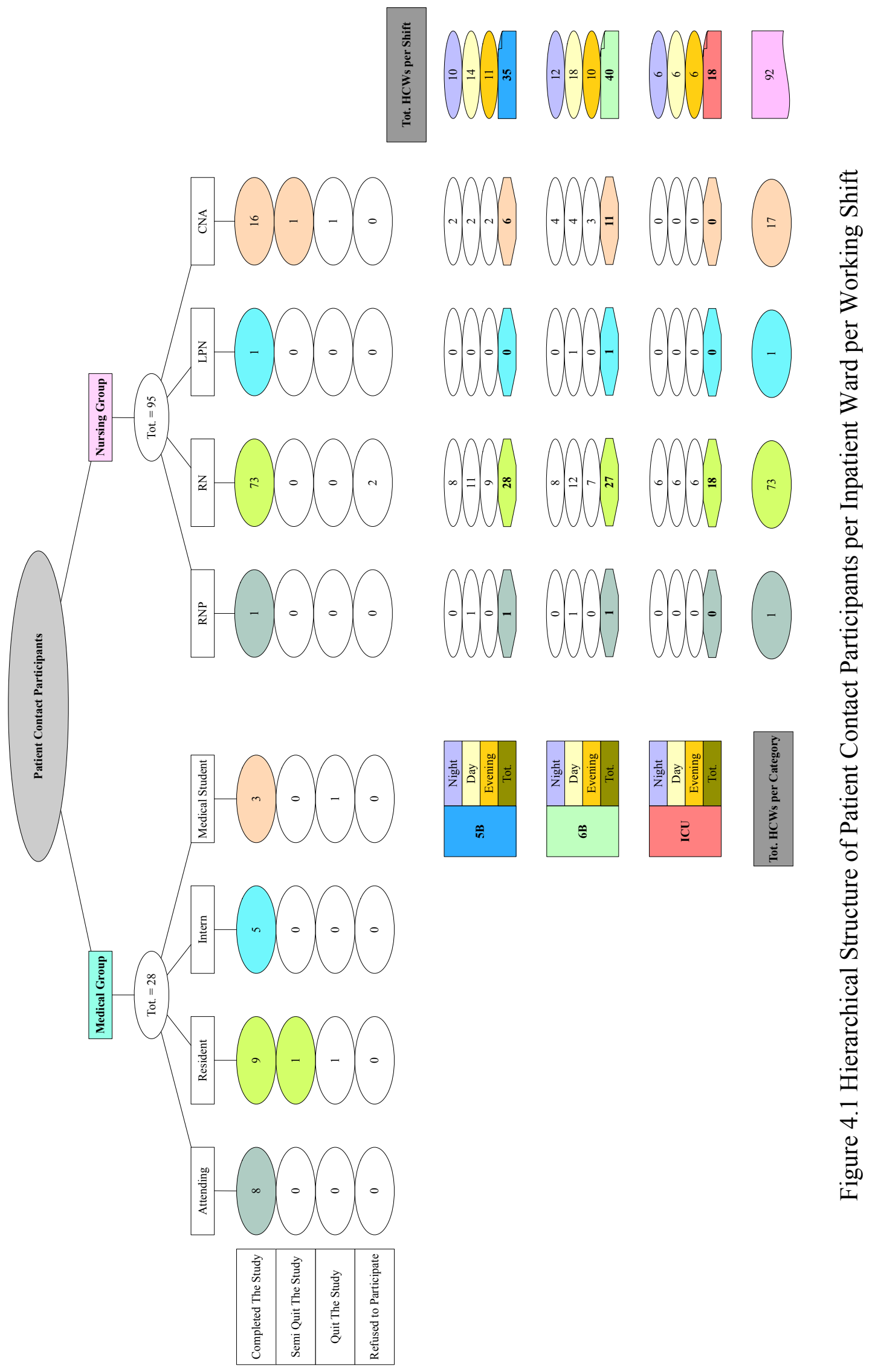




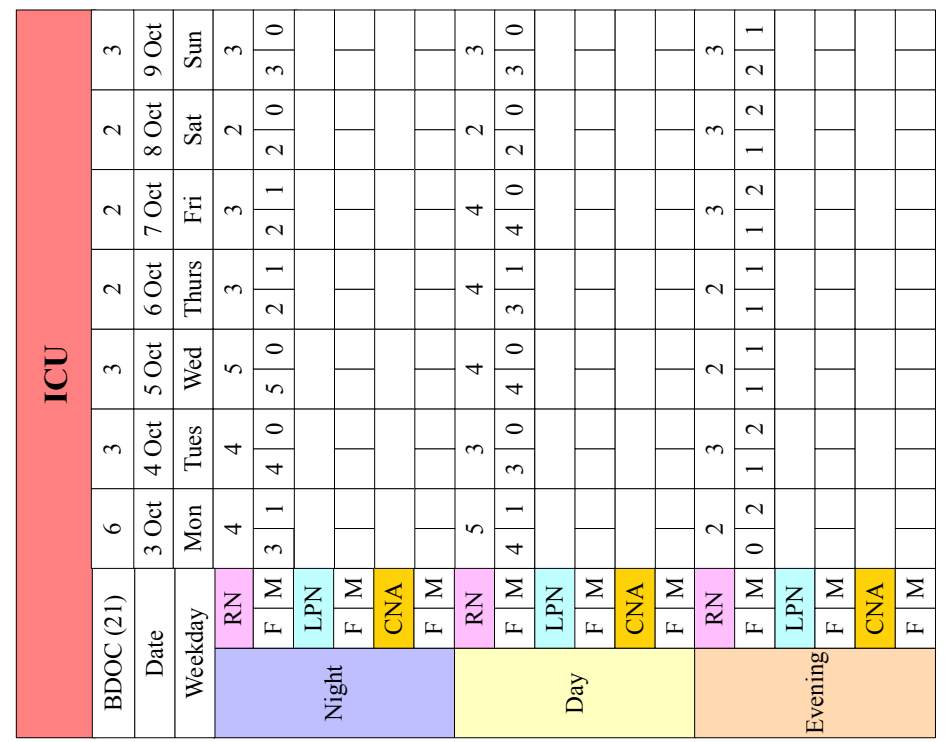

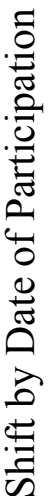

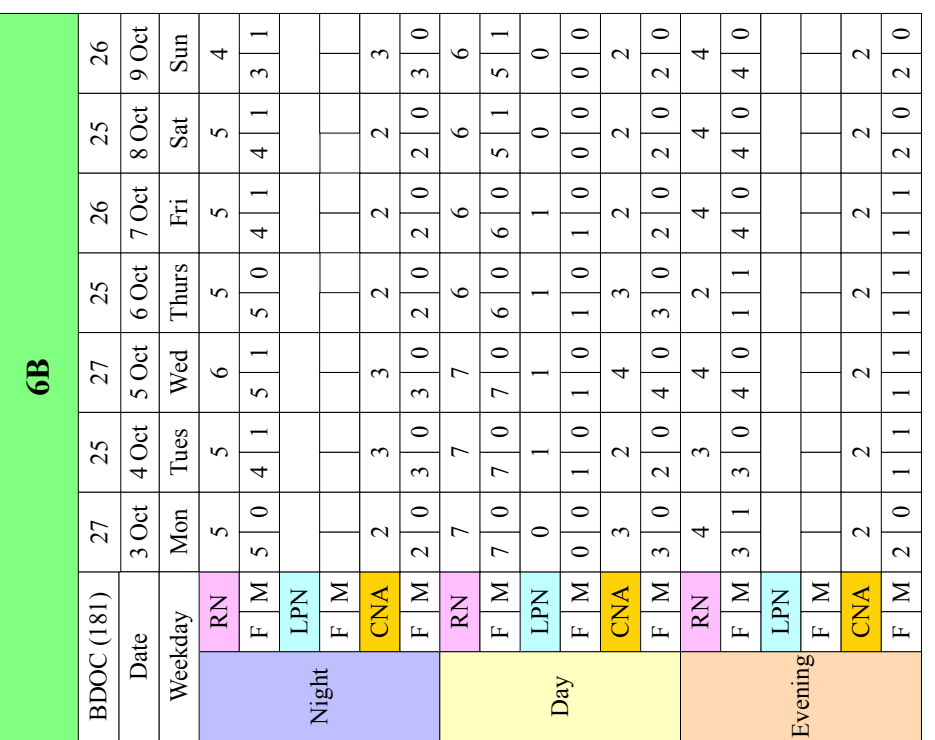

\begin{tabular}{|c|c|c|c|c|c|c|c|c|c|c|c|c|c|c|c|c|c|}
\hline \multirow{14}{*}{ है } & $=$ & & $\bar{\Sigma}$ & & $\begin{array}{l}0 \\
m\end{array}$ & & - & \begin{tabular}{|l}
0 \\
-
\end{tabular} & $i n \frac{0}{n}$ & $\frac{0}{n}$ & & $0 \frac{0}{0}$ & to & \begin{tabular}{l|} 
\\
$m$
\end{tabular} & & - & \begin{tabular}{|l}
0 \\
-1
\end{tabular} \\
\hline & & & & & 0 & & & 0 & & 0 & & 0 & & - & & & 0 \\
\hline & 9 & $\infty_{\infty}^{\infty}$ & 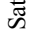 & & $m$ & & - & - & & $n$ & & 0 & 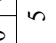 & A & & - & - \\
\hline & N & 8 & $=$ & t & - & & & 0 & & - & & -10 & 0 & 0 & & $\ldots$ & 0 \\
\hline & d & - & I & 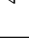 & $m$ & & & - & 7 & r & & - & & 0 & & & - \\
\hline & d & 8 & 当 & n & - & & - & 0 & & - & & $\mathrm{N}^{\circ}$ & 0 & - & & - & 0 \\
\hline & & 6 & 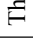 & & $\nabla$ & & & - & & in & & $\sim$ & & in & & & - \\
\hline & $\vec{N}$ & ¿ & 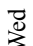 & in & - & & - & 0 & 6 & - & & . 0 & 0 & - & & c & - \\
\hline & & & & & t & & & - & & & & - & - & $n$ & & _ & -1 \\
\hline & $\tilde{\curvearrowright}$ & $g$ & 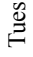 & & in & . & - & \begin{tabular}{|l} 
\\
-
\end{tabular} & & $\frac{0}{6}$ & & -10 & 0 & \begin{tabular}{|l|} 
\\
$n$
\end{tabular} & & 4 & - \\
\hline & $\approx$ & 8 & పే & 寸 & - & & 0 & 0 & & - & & -0 & & - & & - & 0 \\
\hline & 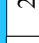 & & $\Sigma$ & & $m$ & & & 0 & & 6 & & - & & in & & & - \\
\hline & \pm & & 害 & & $\Sigma$ & $\frac{\Sigma}{I}$ & $\overleftrightarrow{z}$ & \begin{tabular}{|l}
$\Sigma$ \\
\end{tabular} & $z^{2}$ & & $\Sigma$ & $\begin{array}{ll}\overleftarrow{z} & \Sigma \\
\end{array}$ & & & $z_{a} \frac{\Sigma}{x}$ & $\Sigma \vec{z}$ & $\Sigma$ \\
\hline & लि & $\mathbb{J}$ & & & & & & & & & ब्ञ & & & & & & \\
\hline
\end{tabular}

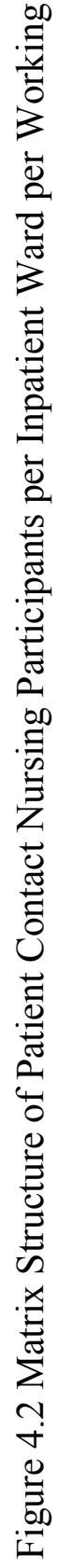




\subsection{Patient Contact Objectives}

After receiving all the patient contact responses, data are entered in Excel, analyzed in $\mathrm{R}$, and the patient contact frequency is reported per inpatient ward by healthcare category and subcategory, medical (attending, resident, intern and medical student) versus nursing groups (RNP, RN, LPN and CNA). Patient contact frequency per inpatient ward and wards combined is summarized in Table 4.4 and Table 4.5. At first glance and as expected, the nursing group has higher patient contact frequency than medical group on average. On average, medical versus nursing patient contact frequency is 7 and 43 (14\% versus 86\%), 7 and 51 (12\% versus 88\%), and 8 and 103 (7\% versus $93 \%$ ) for $5 \mathrm{~B}, 6 \mathrm{~B}$ and ICU respectively as seen in Table 4.4 . On average, inpatient wards combined, medical versus nursing patient contact frequency is 7 and $54(12 \%$ versus $88 \%)$ as seen in Table 4.5 . Interestingly, ICU nursing group patient contact frequency is significantly higher than that of $5 \mathrm{~B}$ and $6 \mathrm{~B}$ even though $\mathrm{RN}$ is the only nursing subcategory that is available at ICU. ICU patients' health status could be one significant reason. ICU nurses need to visit patients frequently.

Over one complete week, BDOC is 147 (10 female and 137 male), 181 (7 female and 174 male) and 21 (5 female and 16 male) for 5B, 6B and ICU respectively. At 5B, the medical group visits 56 patients with a patient contact frequency of 67,46 patients with a patient contact frequency of 93, 71 patients with a patient contact frequency of 169 and 35 patients with a patient contact frequency of 48 out of the total 147 BDOC for attending, resident, intern and MS respectively. On the other hand, the nursing group visits 15 patients with a patient contact frequency of 24, 133 patients with a patient contact frequency of 3859,129 patients with a patient contact frequency 
of 1602 and 0 patients with a patient contact frequency of 0 out of the total 147 BDOC for RNP, RN, CNA and LPN respectively. In total, the medical group visits 208 patients versus 277 visits for nursing group collectively and mutually inclusively. At $6 \mathrm{~B}$, the medical group visits 76 patients with a patient contact frequency of 96,51 patients with a patient contact frequency of 90,77 patients with a patient contact frequency of 204 and 48 patients with a patient contact frequency of 73 out of the total 181 BDOC for attending, resident, intern and MS respectively. On the other hand, the nursing group visits 9 patients with a patient contact frequency of 15, 158 patients with a patient contact frequency of 4026,166 patients with a patient contact frequency of 3033 and 8 patients with a patient contact frequency of 42 out of the total 181 BDOC for RNP, RN, CNA and LPN respectively. In total, medical group visits 252 patients versus 341 visits for nursing group collectively and mutually inclusively. At ICU, the medical group visits 9 patients with a patient contact frequency of 9,10 patients with a patient contact frequency of 16,6 patients with a patient contact frequency of 25 and 5 patients with a patient contact frequency of 5 out of the total 21 BDOC for attending, resident, intern and MS respectively. On the other hand, the nursing group visits 0 patients with a patient contact frequency of 0,19 patients with a patient contact frequency of 1959,0 patients with a patient contact frequency of 0 and 0 patients with a patient contact frequency of 0 out of the total 21 BDOC for RNP, RN, CNA and LPN respectively. In total, medical group visits 30 patients versus 19 visits for nursing group collectively and mutually inclusively. Inpatient wards combined, the medical group visits 141 patients with a patient contact frequency of 172,107 patients with a patient contact frequency of 199,154 patients with a patient 
contact frequency of 398 and 88 patients with a patient contact frequency of 126 out of the total $349 \mathrm{BDOC}$ for attending, resident, intern and MS respectively. On the other hand, the nursing group visits 24 patients with a patient contact frequency of 39,310 patients with a patient contact frequency of 9844,295 patients with a patient contact frequency of 4635 and 8 patients with a patient contact frequency of 42 out of the total 349 BDOC for RNP, RN, CNA and LPN respectively. In total, medical group visits 490 patients versus 637 visits for nursing group collectively and mutually inclusively.

In summary, to answer the two main objectives for which patient contact data collection is conducted in the first place the following calculations are taken place. Of note, patient contact is conducted over one complete week. Thus, all calculations and averages are done per week.

\section{An Approximation of Patient Contact Population per Inpatient Ward:}

To identify how large the Before or After patient contact population is, total patient contact frequency, neglecting healthcare category or subcategory, is summed per inpatient ward and inpatient wards combined. Based on Table 4.4 and Table 4.5, the Before or After patient contact population is 5863, 7578 and 2014 hand hygiene opportunities for 5B, 6B and ICU respectively and 15455 hand hygiene opportunities for inpatient wards combined collectively. To account for both hand hygiene moments, the Before and After patient contact moments, the total population is then $11726(2 * 5863), 15156(2 * 7578)$ and $4028(2 * 2014)$ hand hygiene opportunities for 5B, 6B and ICU respectively and $30910(2 * 15455)$ hand hygiene opportunities for inpatient wards combined collectively. Patient contact population is summarized in 
Figure 4.3. These numbers are used in direct hand hygiene observation method in Chapter 5 to have a better estimate of hand hygiene adherence rate per inpatient ward. Average Patient Visits per Inpatient Ward:

To identify the average patient visits per inpatient ward over one complete week, for each healthcare subcategory the average patient contact frequency is divided by total patients seen and is summed per healthcare category and summed per inpatient ward as seen in Table 4.4 and Table 4.5. Patient contact is neither summarized by working shift nor by isolation status for the moment.

Average patient contact frequency visit is summarized in Table 4.5. The total HCWs average patient contact frequency visits, 50 for $5 \mathrm{~B}, 58$ for $6 \mathrm{~B}$ and 111 for ICU, are used in indirect hand hygiene manual monitoring of product use method in Chapter 6 and in indirect method of measuring personal protective equipment (PPE) compliance in Chapter 7 to estimate the denominator.

To identify the average patient visits per working shift per inpatient ward over one complete week, for each healthcare subcategory the average patient contact frequency is divided by total patients seen and is summed up per healthcare category and summed up per working shift per inpatient ward as seen in Table 4.6, Table 4.7, Table 4.8 and Table 4.9 for ICU, 5B, 6B and inpatient wards combined respectively. 


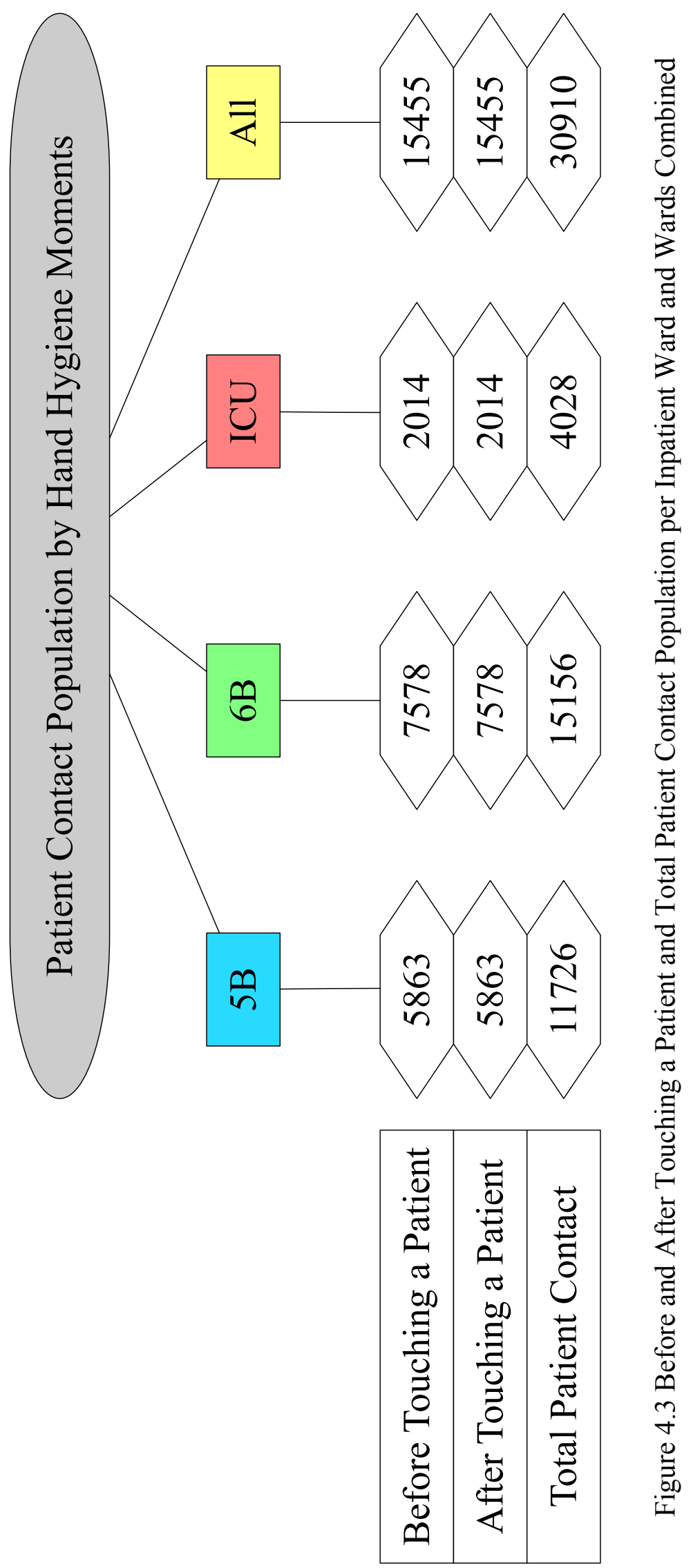




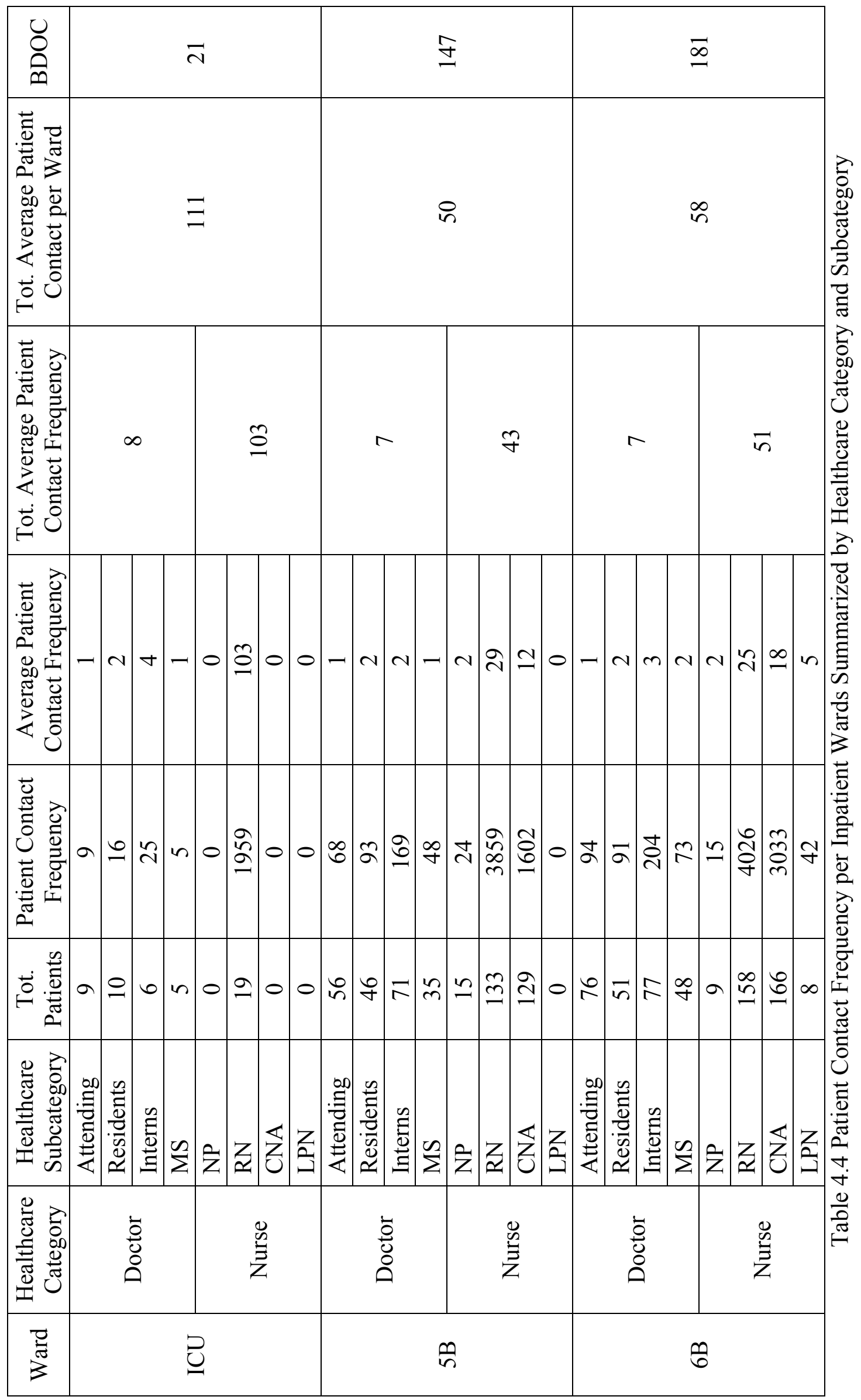




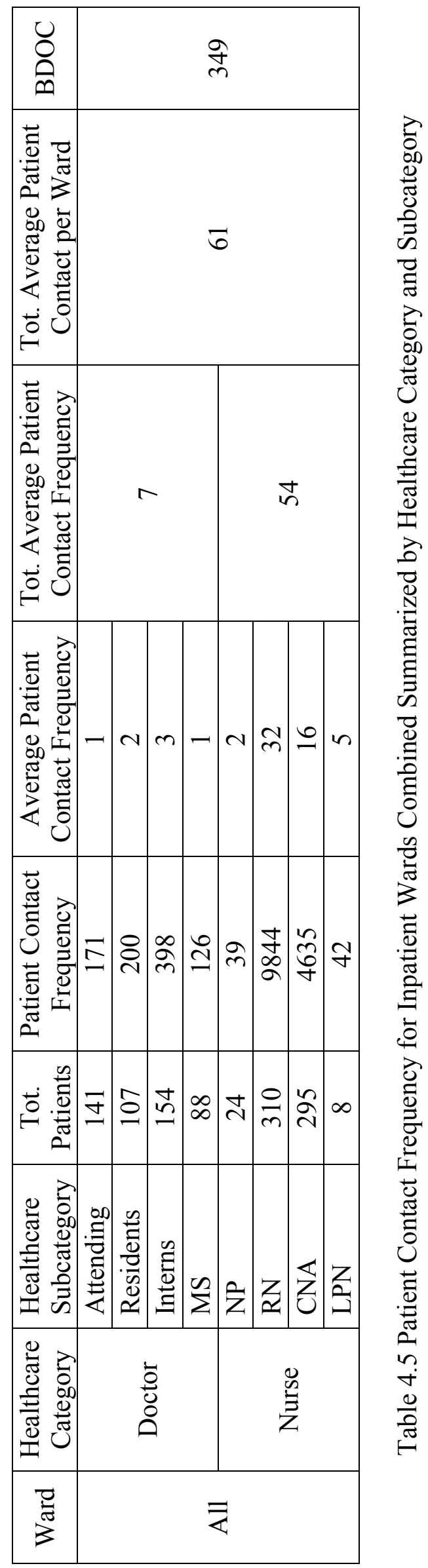




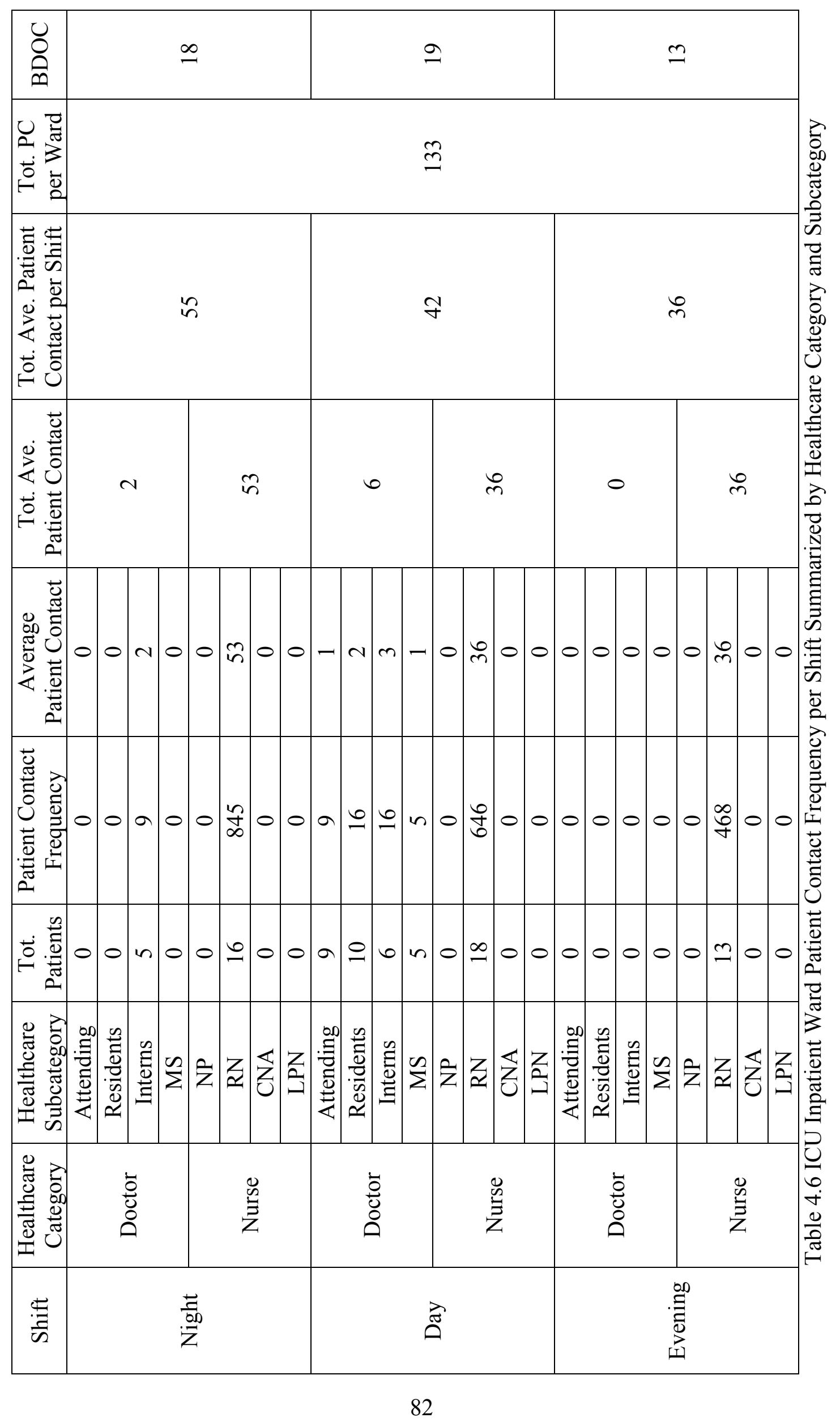




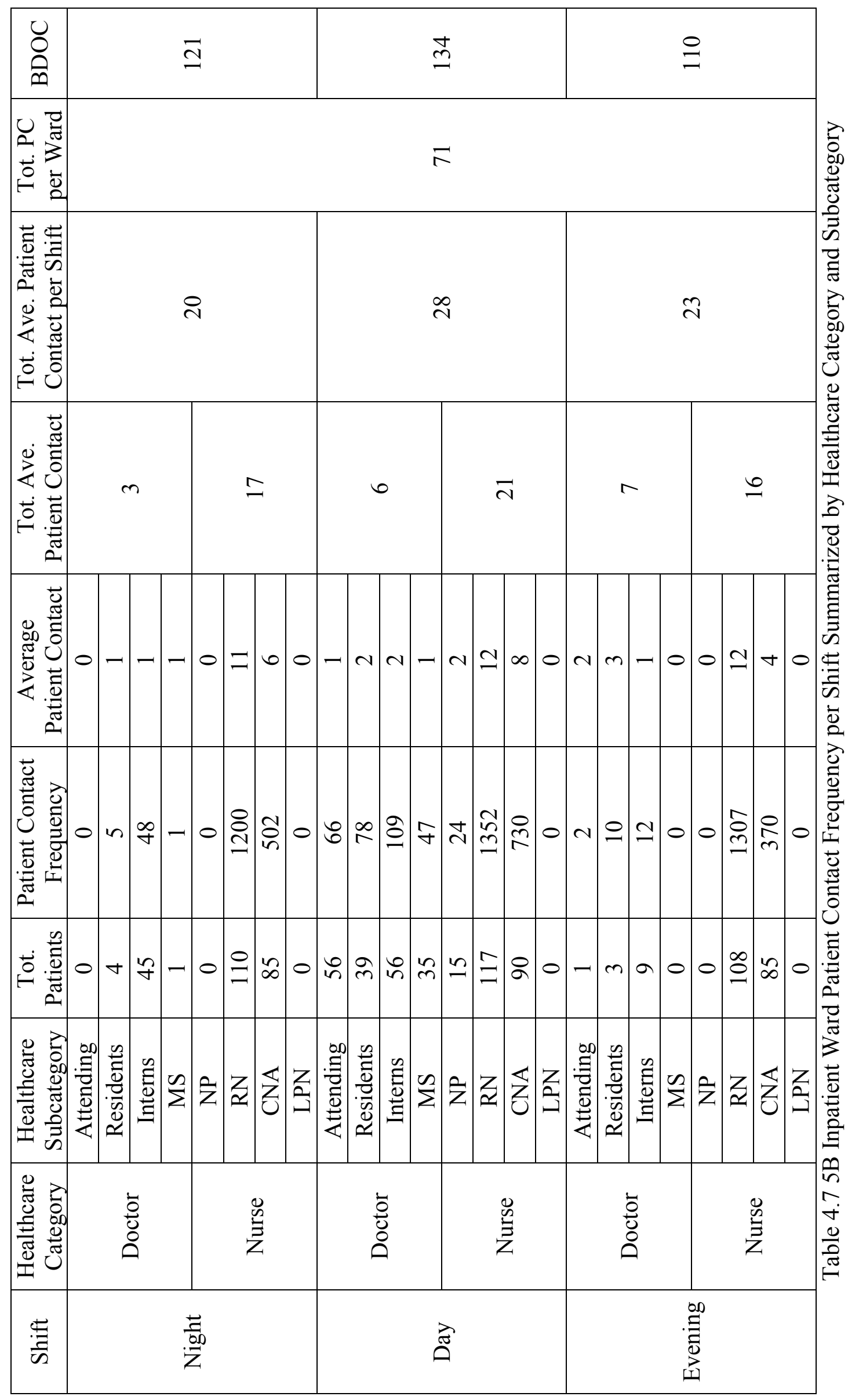




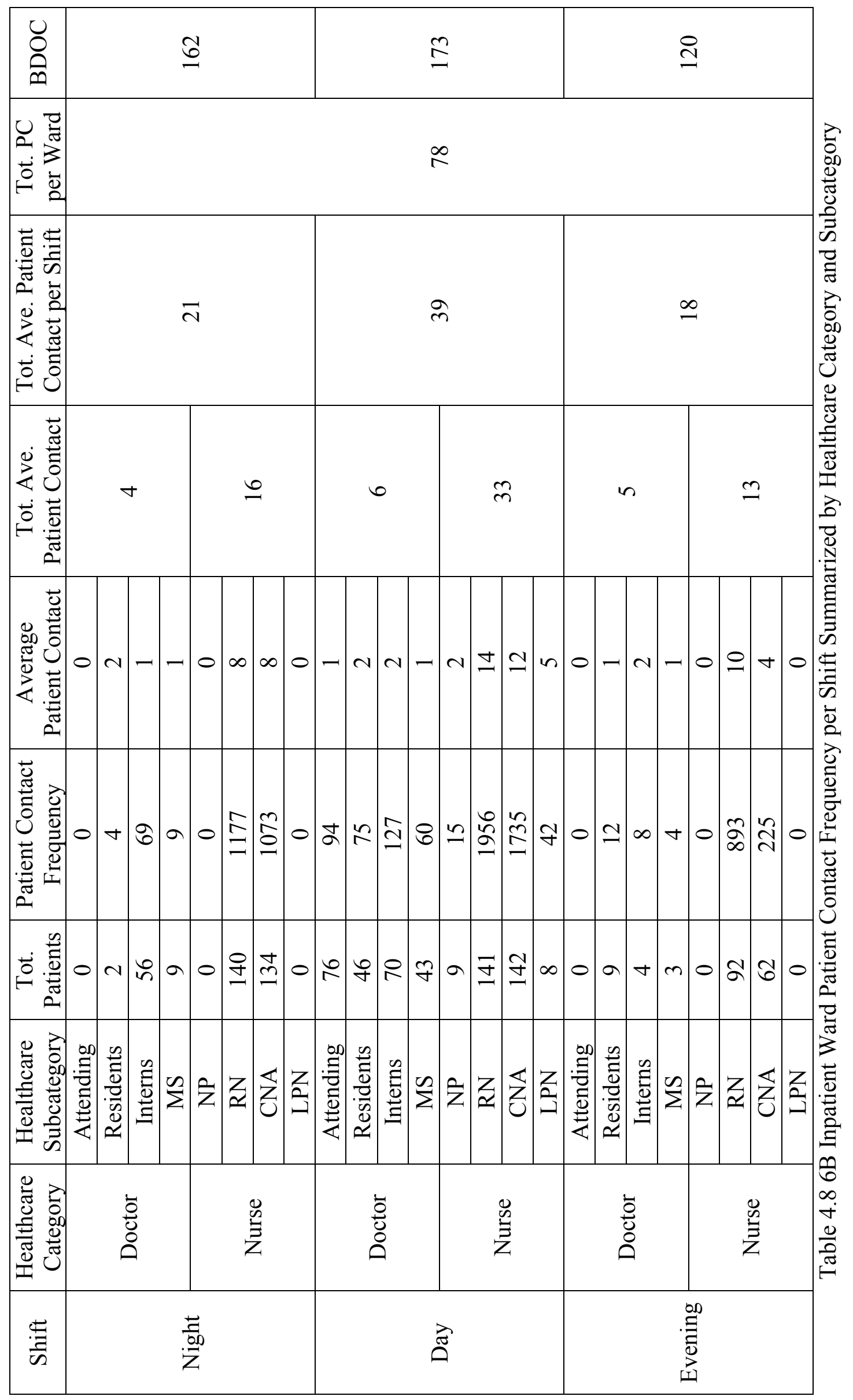




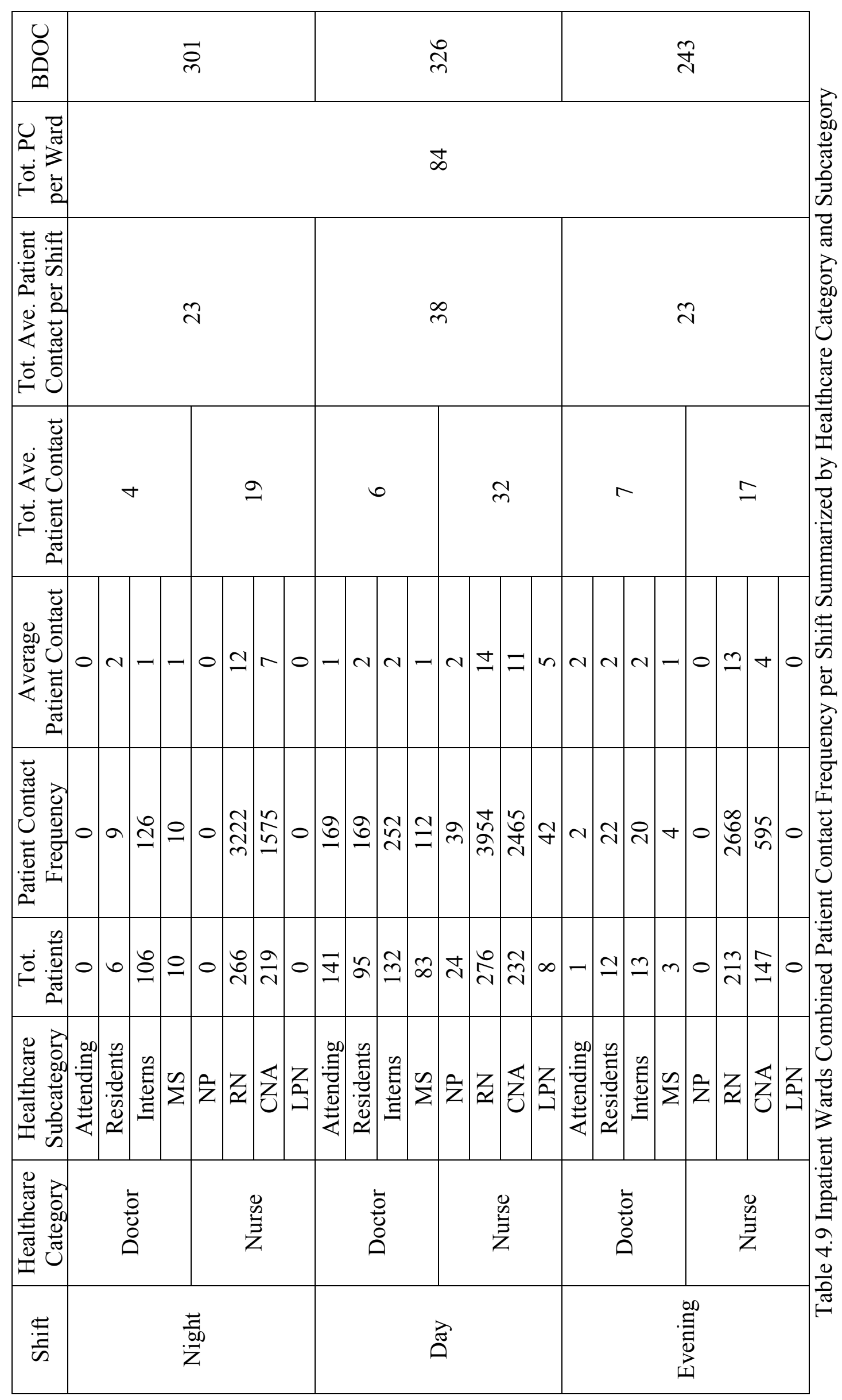


The total HCWs average patient contact frequency visit combining all working shifts at $5 \mathrm{~B}$ based on working shift grouping is 71 . This number, which is based on working shift grouping, is significantly different from 50 , which is based inpatient ward grouping ignoring working shift and isolation status, and 102, which is based on inpatient ward grouping per isolation status. The total HCWs average patient contact frequency visit combining all working shifts at $6 \mathrm{~B}$ based on working shift grouping is 78. This number, which is based on working shift grouping, is significantly different from 58, which is based inpatient ward grouping ignoring working shift and isolation status, and 114, which is based on inpatient ward grouping per isolation status. The total HCWs average patient contact frequency visit combining all working shifts at ICU based on working shift grouping is 133 . This number, which is based on working shift grouping, is significantly different from 111, which is based inpatient ward grouping ignoring working shift and isolation status, and is exactly equal to 111, which is based on inpatient ward grouping per isolation status. The total HCWs average patient contact frequency visit combining all working shifts when all wards are combined based on working shift grouping is 84 . This number, which is based on working shift grouping, is significantly different from 61 , which is based on inpatient ward grouping ignoring working shift and isolation status, and 119, which is based on inpatient ward grouping per isolation status.

At 5B, BDOC is 121,134 and 110 for night, day and evening shift respectively. At $6 \mathrm{~B}, \mathrm{BDOC}$ is 162,173 and 120 for night, day and evening shift respectively. At ICU, BDOC is 18,19 and 13 for night, day and evening shift respectively. Inpatient wards combined, BDOC is 301,326 and 243 for night, day and evening shift 
respectively. It is noticeable that $\mathrm{RN}$ and $\mathrm{CNA}$ are the only $\mathrm{HCW}$ sho see and contact all patients during all working shifts. Ranking average patient contact frequency per shift across all inpatient wards by ascending order is day shift, night shift and evening shift.

To identify the average patient visits per isolation status per inpatient ward over one complete week, for each healthcare subcategory the average patient contact frequency is divided by total patients seen and is summed up per healthcare category and summed up per isolation status per inpatient ward as seen in Table 4.10, Table 4.11, Table 4.12 and Table 4.13 for ICU, 5B, 6B and inpatient wards combined respectively.

At 5B, the medical group total average patient contact frequency visits is 7 and 7 for isolation and non-isolation status respectively. On the other hand, the nursing group total average patient contact frequency visits is 47 and 42 for isolation and nonisolation status respectively. The total HCWs average patient contact frequency visit per isolation status neglecting healthcare category is 53 and 49 for isolation and nonisolation status respectively. The total HCWs average patient contact frequency visit at $5 \mathrm{~B}$ based on isolation status grouping is 102 . This number, which is based on isolation status grouping, is significantly different from 50, which is based on inpatient ward grouping ignoring working shift and isolation status, and 71, which is based on inpatient ward grouping per working shift.

At $6 \mathrm{~B}$, the medical group total average patient contact frequency visits is 7 and 7 for isolation and non-isolation status respectively. On the other hand, the nursing group total average patient contact frequency visits is 53 and 46 for isolation and non- 
isolation status respectively. The total HCWs average patient contact frequency visit per isolation status neglecting healthcare category is 61 and 53 for isolation and nonisolation status respectively. The total HCWs average patient contact frequency visit at $6 \mathrm{~B}$ based on isolation status grouping is 114 . This number, which is based on isolation status grouping, is significantly different from 58 , which is based on inpatient ward grouping ignoring working shift and isolation status, and 78, which is based on inpatient ward grouping per working shift.

At ICU, the medical group total average patient contact frequency visits is 0 and 8 for isolation and non-isolation status respectively. On the other hand, the nursing group total average patient contact frequency visits is 0 and 103 for isolation and nonisolation status respectively. The total HCWs average patient contact frequency visit per isolation status neglecting healthcare category is 0 and 111 for isolation and nonisolation status respectively. The total HCWs average patient contact frequency visit at ICU based on isolation status grouping is 111. This number, which is based on isolation status grouping, is exactly equal to 111 , which is based inpatient ward grouping ignoring working shift and isolation status, and is not significantly different from 133, which is based on inpatient ward grouping per working shift. ICU has 0 isolation BDOC during the week of patient contact data collection.

Combining all inpatient wards, the medical group total average patient contact frequency visits is 7 and 7 for isolation and non-isolation status respectively. On the other hand, the nursing group total average patient contact frequency visits is 51 and 53 for isolation and non-isolation status respectively. The total HCWs average patient contact frequency visit per isolation status neglecting healthcare category is 58 and 60 
for isolation and non-isolation status respectively. The total HCWs average patient contact frequency visit when all wards are combined based on isolation status grouping is 119. This number, which is based on isolation status grouping, is significantly different from 61 , which is based inpatient ward grouping ignoring working shift and isolation status, and 84 , which is based on inpatient ward grouping per working shift.

At 5B, BDOC is 41 and 106 for isolation and non-isolation status respectively. At $6 \mathrm{~B}, \mathrm{BDOC}$ is 66 and 115 for isolation and non-isolation status respectively. At ICU, BDOC is 0 and 21 for isolation and non-isolation status respectively. Inpatient wards combined, BDOC is 107 and 241 for isolation and non-isolation status respectively. It is noticeable that, in general, non-isolation BDOC significantly outweighs isolation $\mathrm{BDOC}$ across all inpatient wards, 5B and 6B, and when wards are combined. However, the previous statement cannot be said toward ICU since ICU has 0 isolation BDOC. Though non-isolation BDOC significantly outweighs isolation $\mathrm{BDOC}$ at $5 \mathrm{~B}$ and $6 \mathrm{~B}$ and when all wards are combined, the total average patient contact per isolation status is not significantly different. Sadly, this result cannot be conclusive because patients who are reported as being on non-isolation could be on isolation because of different infections that are not part of the study like Flu, TB or Vancomycin-Resistant Enterococci (VRE). But, generally speaking that could be said since normally patients who are on isolation are sicker and required more attention in terms of providing care. In addition, if all patients are categorized by isolation status with more fair, isolation BDOC could at least equal to or outweigh non-isolation BDOC and then higher patient contact frequency could be the result. 
Of note, the total HCWs average patient contact frequency visits differ by how patient contact frequency is grouped. Various ways of patient contact grouping generate different divisor (denominator) and dividend (numerator).

- Grouping by inpatient wards only and ignoring working shift or isolation status.

- Grouping by inpatient wards and by working shift and ignoring isolation status.

- Grouping by inpatient wards and by ignoring isolation status and working shift.

Thus, it would be better if total average patient contact visits is generated by grouping inpatient wards and by isolation status and the results are used in estimating the denominator in indirect hand hygiene manual monitoring of product use method in Chapter 6 and in indirect method of measuring personal protective equipment (PPE) compliance in Chapter 7. However, ICU has zero isolation BDOC, which would be misleading since the developed equations in Chapter 6 and Chapter 7 are based on isolation status. Ultimately, it would be at best if grouping were made by inpatient ward, working shift and isolation status. This would ensure a more accurate and precise estimate of the denominator in indirect hand hygiene manual monitoring of product use method in Chapter 6 and in indirect method of measuring personal protective equipment (PPE) compliance in Chapter 7. However, neither indirect hand hygiene manual monitoring of product use method nor indirect method of measuring personal protective equipment (PPE) compliance are conducted per working shift. An attempt is made to define the formal method but due to some limitations such as the difficulty of one participant to track all Purell and Soap dispensers at all inpatient wards during all three shifts are impossible. An attempt is not made to the latter because PPE inventory data is not reported per working shift per inpatient wards. 


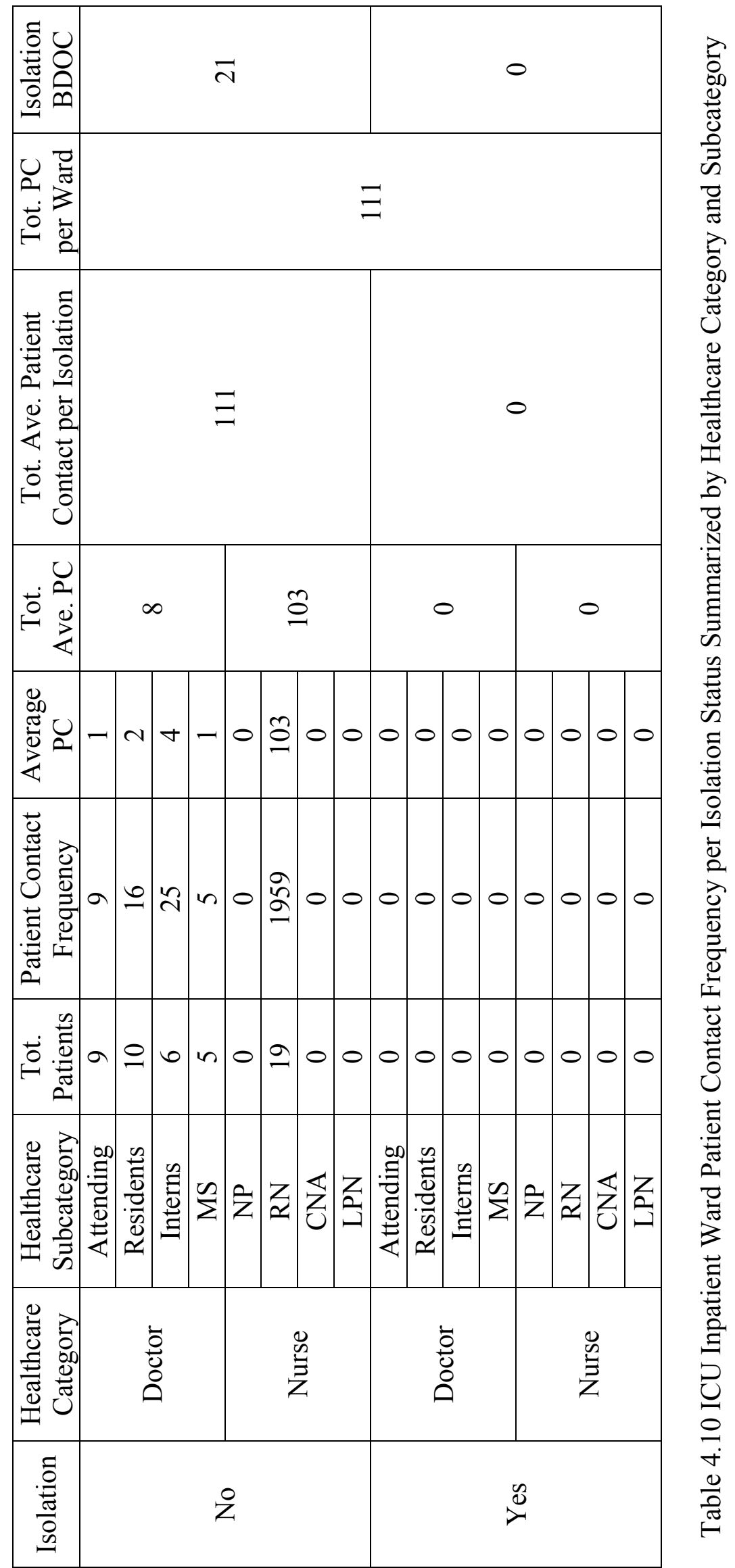




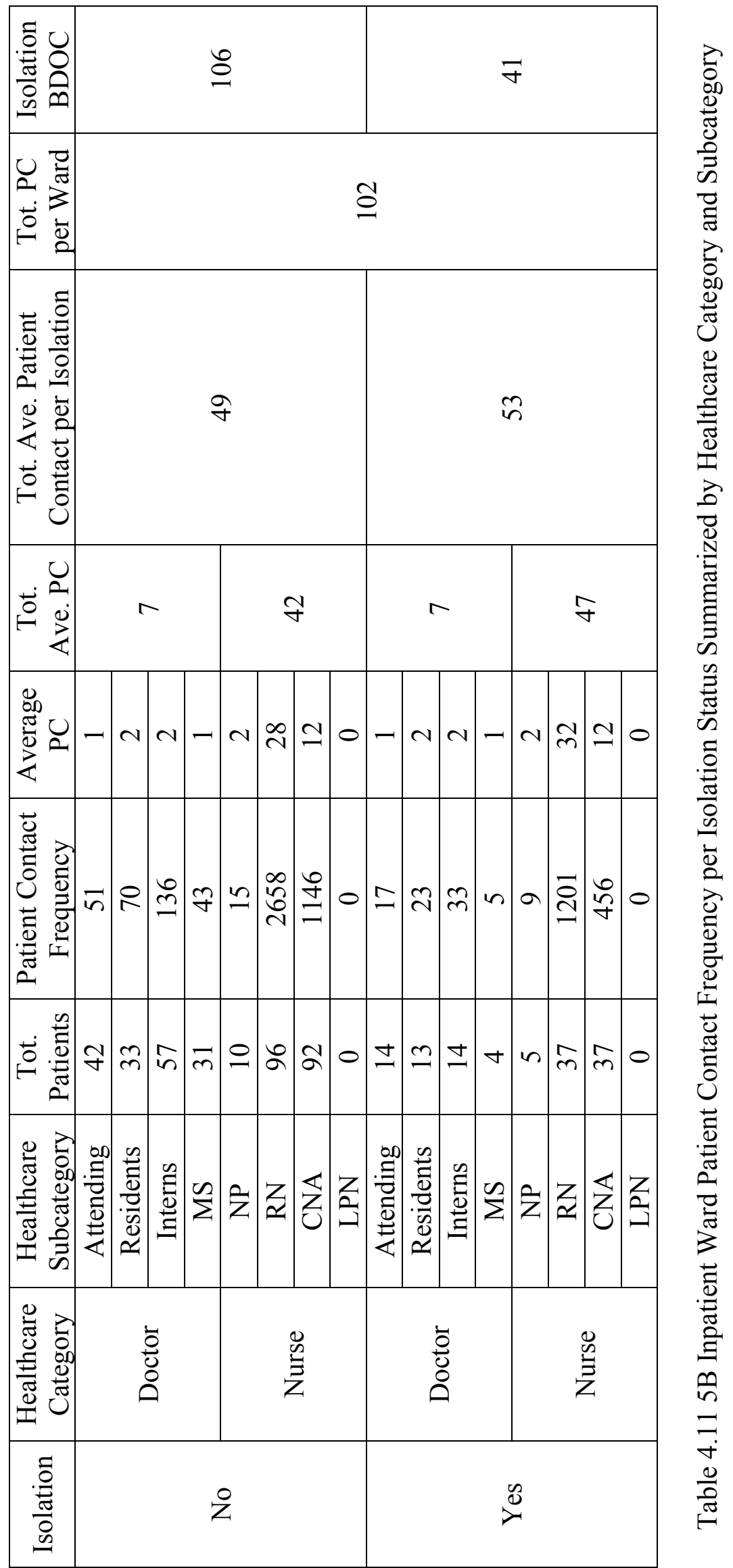




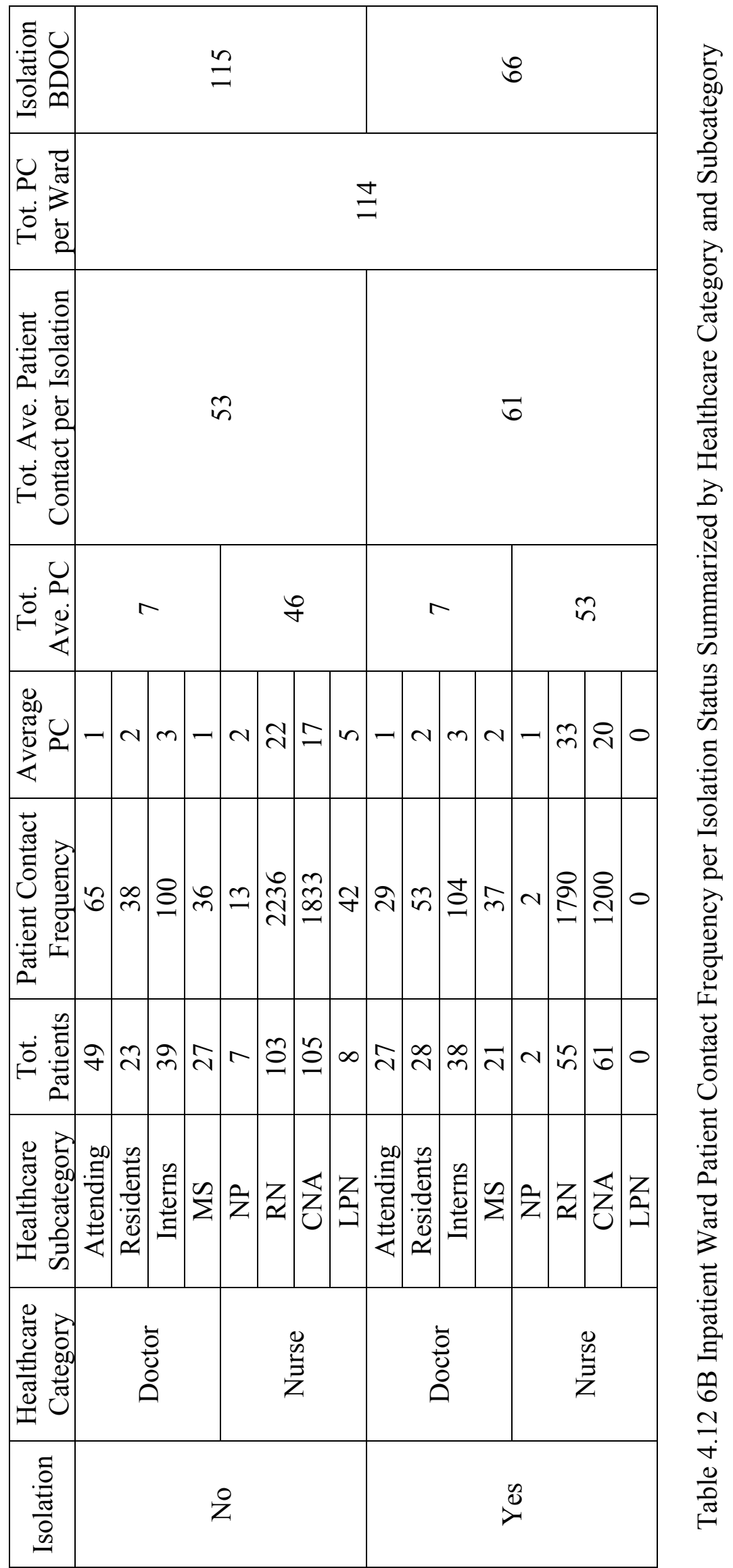




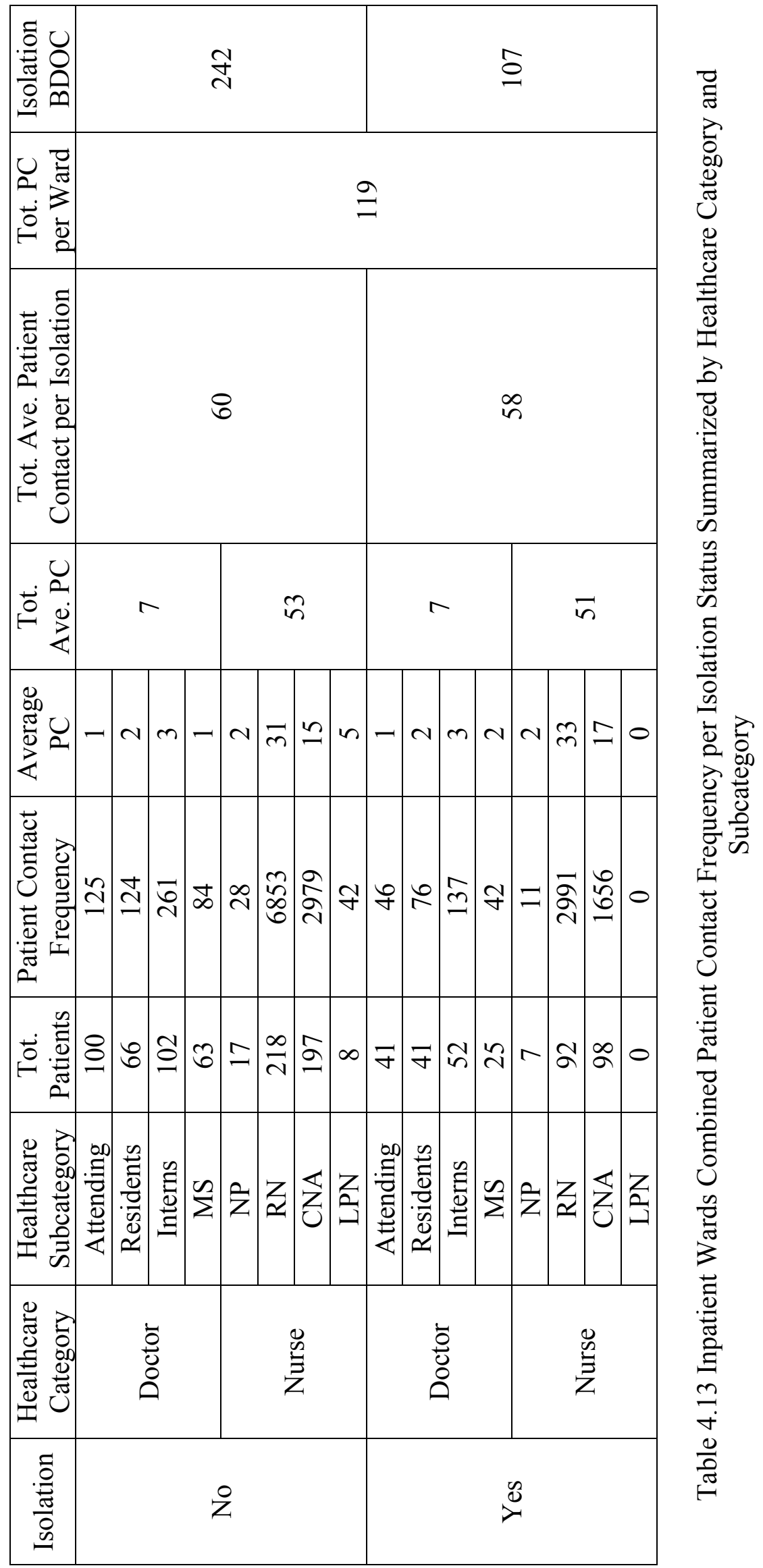




\section{CHAPTER 5}

\section{DIRECT HAND HYGIENE OBSERVATION METHOD}

This chapter provides a retrospective and an observational study analysis for assessing the hand hygiene $(\mathrm{HH})$ direct observation method at the Veterans Affairs Medical Center at Providence (PVAMC). Purchase and sales data of Purell and Soap are accompanied with the analysis. Moreover, an indirect Personal Protective Equipment (PPE) compliance method is developed. Before proceeding to the preliminary analysis, a background about the PVAMC is presented.

\subsection{The Providence Veterans Affairs Medical Center (PVAMC)}

\subsubsection{Inpatient Wards}

Each inpatient ward is explored individually in terms of locations and how many Soap-Purell dispensers are available, how many bed(s) exist at each patient-room and locations of personal protective equipment, which is (PPE) "protective equipment, such as approved head and hair coverings, face shields, safety glasses/goggles, long cuffed rubber/vinyl decontamination gloves, impervious gowns, and shoe covers that are utilized to protect the employee from the environment." (The VHA Handbook, 2016, Page 4).

The three-inpatient wards (5B, 6B and ICU) utilize about $80 \%\left(\frac{[23+27+8]}{73} * 100\right)$ of the total 73 operating beds. 5B and 6B has a floor shape of $\mathrm{Y}$ letter, where ICU has a rectangular floor shape. At 5B and 6B, there are about 90 Vinyl Composition Tile (VCT) tiles of 10 by 10 inches from the nurse station to the end of each hallway (right and left of the nursing station) that takes about 28 seconds to cross from the nursing 
station till the end of each right or left hallway. At ICU, there are about $60 \mathrm{VCT}$ tiles that take about 16 seconds to cross. The pillar of the $\mathrm{Y}$ letter at $5 \mathrm{~B}$ and $6 \mathrm{~B}$, the corridor, has no patient rooms; there is an office for the nurse manager of the ward, dermatology, orthopedics, medical resident and chief medical, inpatient case management, nurse practitioners offices, staff lounge, family meeting room, utility room and EMS housekeeping closets, and electrical and linen closets. 5B and $6 \mathrm{~B}$ are identical in shape and floor size. However, ICU is much smaller and most of the ward is used for patient rooms and beds. There is a nurse station at each ward, which is located at the center of the end of corridor of $5 \mathrm{~B}$ and $6 \mathrm{~B}$ and to the right middle of the ICU ward. All Purell-Soap dispensers are wall-mounted. Purell dispensers are automated touch-free and are powered by batteries that need to be replaced every three years. On the other hand, Soap dispensers are manual. All hallway sinks are foot-pedal to prevent the transmission of germs to the hands of HCWs. However, soap dispensers are manual and HCWs will need to touch the soap dispenser for soap aliquot. Purell dispensers outnumber Soap dispensers because soap dispensers are always accompanied with a sink and it is a waste to have sinks all over the floor. The VA is restricted on what to attach to the ward walls. However, the inpatient wards are designed to have a Bed-Site Chart on the wall of the ward where the patient's folder is hung on beside his/her room door to ease access health record. Each inpatient ward is explored in details below.

At the three inpatient wards, there are wall-mounted supplies on the wall of the ward that contain personal protective equipment such as gloves (small, medium, large and x-large sizes) each size comes in a separate case of 100 gloves and there are four 
slots for each size case, gowns which comes in 10 per case and there is one slot that fits up to about 30 cases of gowns, between most of the rooms. There are mask hungers or hoods between most of the rooms as well, which comes in a case of 100 masks.

\subsubsection{Inpatient Ward 5B}

$5 \mathrm{~B}$ ward is a step-down unit that is licensed to 23 beds and has a capacity of 25 beds. There are 10 rooms with double beds (A and B) and 3 rooms with a single bed. Usually single bedrooms in $5 \mathrm{~B}$ or $6 \mathrm{~B}$ are reserved for female patients and/or patients in general with airborne precautions or a patient with C.Diff if there is no other patient with C.Diff to share a room. A list of patient's room numbers and bed type A or B of the ward are found on Appendix F. There is a Purell dispenser in and out of each patient room. There is a bathroom inside each patient room equipped with a sink and Soap dispenser. On the hallway, there are five sinks equipped with Soap dispensers. Totally, there are 26 Soap dispensers, 5 located on the hallway and the remaining located inside bathrooms of patients' rooms. There are 29 Purell dispensers, 12 located on the hallway and 17 located inside patients' rooms but outside of the bathrooms. Each side of the Y letter consists of an equal numbers of patients' rooms. In addition there are two Anterooms and one Linen room and a conference room inside the ward. 


\subsubsection{Inpatient Ward 6B}

$6 \mathrm{~B}$ ward is an acute care unit that is licensed to 27 beds and has a capacity of 27 beds. There are 12 rooms with double beds (A and B) and 3 rooms with a single bed. A list of patient's room numbers and bed type A or B of the ward is found in Appendix C. There is a Purell dispenser outside of each patient room; however, there are no Purell dispensers inside each patient's room. There is a bathroom inside each patient room equipped with a sink and Soap dispenser. On the hallway, there are five sinks equipped with Soap dispensers. Totally, there are 27 Soap dispensers, 5 located on the hallway and the remaining located inside bathrooms of patients' room. There are 22 Purell dispensers, all located on the hallway. Each side of the Y letter consists of an equal numbers of patients' rooms. In addition there are two Anterooms and one Linen room and a conference room inside the ward.

\subsubsection{Inpatient Ward ICU}

The ICU ward is an acute care unit that is licensed to 8 beds and has a capacity of 8 beds. There are 5 rooms with a single bed and 3 beds located on the bay of the unit. Two patient's rooms and the nurses' station of the ward are located to the right of the corridor and three patient's rooms and the three bay beds are located to the left of the corridor. The three beds on the bay are not used for isolation. A list of patient's room numbers and beds of the ward are found in Appendix I. There is a Purell dispenser inside and outside of three patient's rooms, outside of two patient's rooms and some on the hallway and at the bay of the unit. There is a bathroom inside each patient room equipped with a sink and Soap dispenser. On the hallway, there are two sinks equipped with Soap dispensers. Totally, there are 11 Soap dispensers, 2 located 
on hallway and 9 located inside patients' rooms. And there are 12 Purell dispensers, 9 located on the hallway and 3 located inside three patients' rooms with two rooms with no dispensers. In addition there is a conference room inside the ward.

\subsubsection{Primary Inventory and PPE}

The PVAMC has one primary inventory for medical and surgical items that is located on the third floor, C Wing of the main building, Building \#1. This inventory has two different groups of supplies:

1. Stocked inventory in the Primary locations.

The stocked inventory in the Primary areas is set up on a Generic Inventory Package (G.I.P.). This system is uploaded with all of the item information such as description of product, vendor to purchase product, pricing, and unit of issue, storage location, and levels. The GIP Supply Techs are responsible for the inventory and ordering of these supplies based on an auto-generated order through the GIP system (L. Renaud, Personal Communication, September 26, 2016; The VHA Handbook, 2016).

The Personal Protective Equipment (PPE) is a Medical-Surgical item that is considered an example of an auto-generated ordered item stocked from inventory in the primary locations. PPE includes, but is not limited to:

- Nitrile Examination Gloves: comes in four different sizes (Small, Medium, Large and X-Large) and is powder-\&-latex free and textured and for single use only.

- Mask, Face and Earloop.

- Gowns. 
2. Special ordered items, or non-stocked.

The non-stocked or special ordered items are requested from the service technicians or nursing staff with a Special Order form. The form is emailed to the GIP Supply Techs to enter a request in the system and then place the order. The order will ship and be delivered directly to the service that requested the products (L. Renaud, Personal Communication, September 26, 2016; The VHA Handbook, 2016).

At the PVAMC, an inventory employee takes care of filling in the supply closet at each inpatient unit on a daily basis with gloves, masks and gowns. An inventory cart is used to deliver inventory items to ease the movement of large boxes. Two large elevators are designated for such job besides patient transfer and bed movement from to the room and the required care such as X-rays. It is CNA's job to fill in wallmounted supplies and supply drawers beside each patient room on the floor with gloves, gowns and mask.

\subsubsection{Isolation Precautions}

There are two types of isolation precautions. The first type is the standard precautions. Regardless of patient's health status, caregivers have to comply with Standard Precautions when patients receive care. The goal of complying with the standard precaution is to prevent and control the transmission of diseases from/to identified source of infection and unidentified source of infection (VAMHCS, 2012). In other words, though a patient is not on isolation they could get an infection if the caregiver was recently giving care to a patient who is on isolation. Thus, standard precautions are "the primary and most important strategy for health care associated infection control." (VAMHCS, 2012, Page 2). However, according to VAMHCS 
(2012), it is not required to hang standard precautions signage on the patient room door because there is no signage for it. Table 5.1 includes standard precautions in details.

Second type is transmission-based precautions. "Transmission-Based Precautions are for patients known or clinically suspected to be colonized or infected by epidemiologically important pathogens spread by airborne or droplet transmission or by contact with dry skin or contaminated surfaces." (VAMHCS, 2012, Page 1). Each type of transmission has a unique colored signage that has to be placed on room door of patients with the associated infectious or colonization status. At the PVAMC, the following colors are used for each type of transmission based precautions signage:

- Contact Precautions: Orange Color, Gloves and Gowns are required and masks as needed, as can be seen in Appendix DD.

- Droplet Precautions: Pink Color, masks is required and gloves and gowns as needed, as can be seen in Appendix EE.

- Airborne Precautions: Blue Color, N95 masks, gloves and gowns are required, as can be seen in Appendix FF.

Standard precautions are accompanied with each transmission-based precautions, for example wearing gloves, gowns and mask and complying with hand hygiene before/after wearing gloves and before/after touching patients. According to VAMHCS (2012), caregivers such as RNs can initiate the use of transmission-based precautions during patient admission or while patient being provided with care. However, discontinuing the use of transmission-based precautions for any reasons such as upon patient discharge or after curing or ruling out his health status from 


\begin{tabular}{|c|c|}
\hline రె & 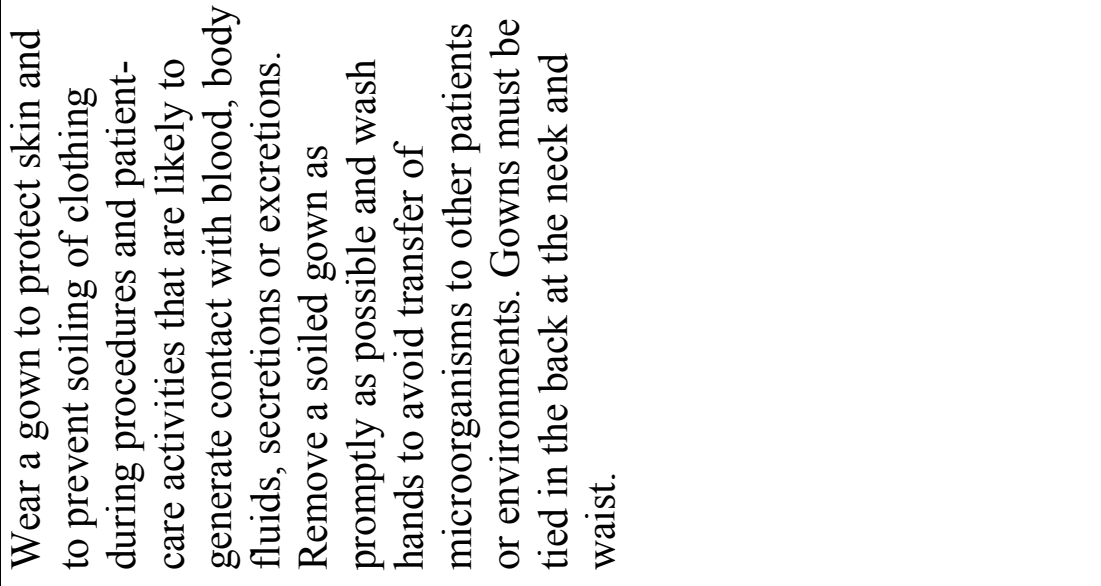 \\
\hline 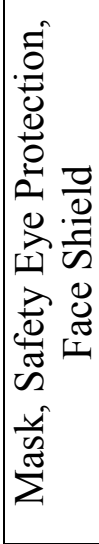 & 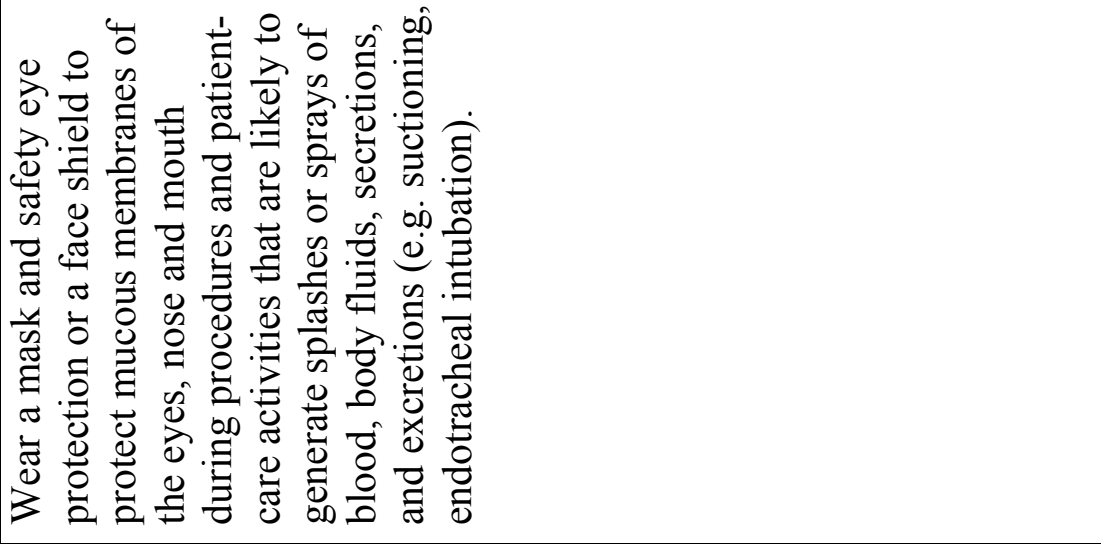 \\
\hline$\frac{\sum_{0}^{\infty}}{0}$ & 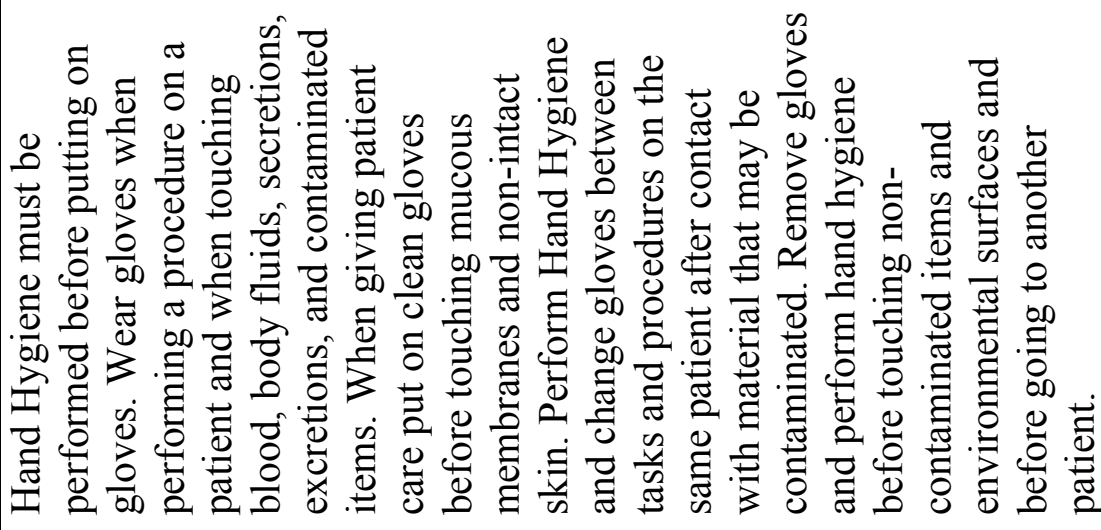 \\
\hline 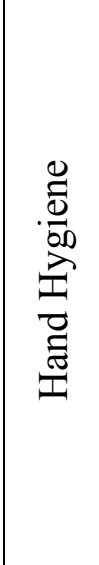 & 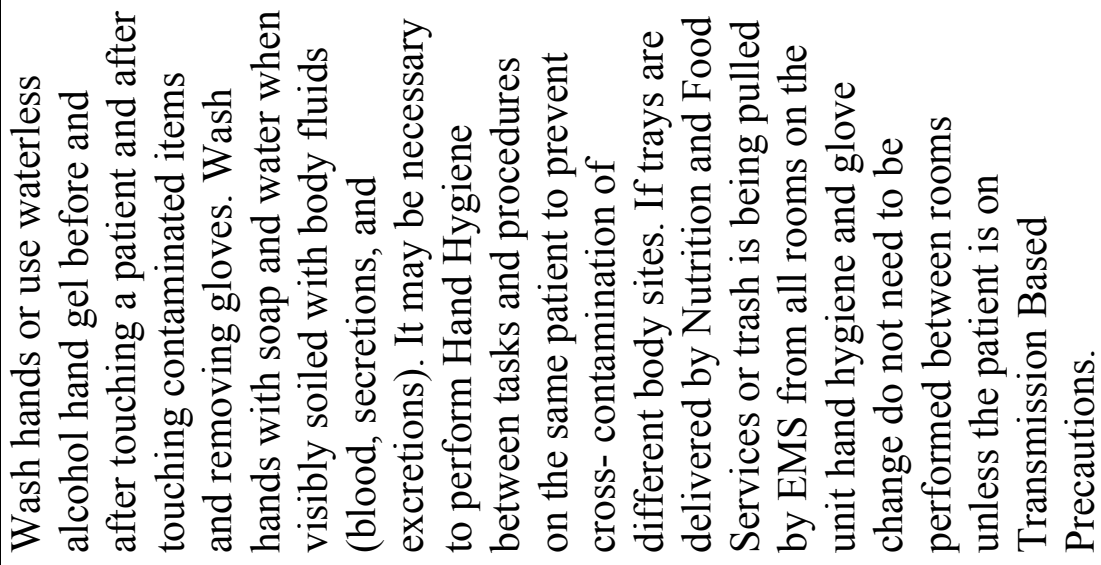 \\
\hline
\end{tabular}


infection or colonization during providing care cannot be done by RNs before receiving the approval from the infectious disease and control department.

At the PVAMC, transmission-based precautions signage are kept on rooms after the discharge of patients to notify EMS and CNA to comply with such precautions when entering and leaving the room for cleaning and making beds. Nutrition and food service at the PVAMC also has to comply with these two types of precautions when providing food trays, plates and utensils to patients in rooms with and without precautions signage. However, the nutrition and food service employees are not allowed to going inside isolation rooms. RNs or housekeepers usually leave a table by the door or help in delivering food trays. After use, trays are collected and kept in the dirty utility room inside the ward. Usually nutrition and food service visits the ward twice a day around 7:00 a.m. and 3:00 p.m. A food cart is used to deliver food trays to patients and it can fit up to 24 trays and each tray is labeled with patient information. However, there is no tag or label used for those isolation rooms before delivery or after use. Consequently, food trays, plates and utensils could get contaminated. However, if there is blood or fluid on trays or plates, they will be thrown away for no further use. Other hospitals, based on RNs at the PVAMC, bag trays and plates that are used by isolation rooms to notify the nutrition and food service for special wash and clean.

There are other precautions signage used at the PVAMC for patients with neither infections nor colonization but who require careful attention when entering or leaving and when providing care such as: 
- Protective Environment (Neutropenic Precautions): patients with very low white cells, such signage requires wearing all PPE including N95 masks, Appendix GG.

- Crown Program (Risk Fall) Signage: patients who could fall if standing up and need assistant when going to the bathroom, for example, Appendix HH.

- Strict I\&O's Signage: patients who have Congestive Heart Failure (CHF) and need to be in body liquid balance in terms of what goes in (I) the body, for example through mouth, and what goes out $(\mathrm{O})$ of the body, for example blood draw, as the two letters indicated in the signage (I\&O), because CHF holds liquid in the body, Appendix II.

Crown Program and Strict I\&O's signage indicate there are physical interactions between HCWs and patients. Thus, hand hygiene opportunities occur regularly and PPE may or may not be required, specifically gloves and/or gowns and masks.

Hand hygiene has to be practiced no matter what the precautions types are, whether patients are on transmission precautions or on standard precautions. However, wearing gloves during patient care on standard precautions is still justified by the following. HCWs are at risk of blood or other body fluid exposure from patients. HCWs' hands could be contaminated with germs that could be transferred to patients or patients' surroundings. Patients on standard precautions could have infections without showing any symptoms. Thus, wearing gloves could protect HCWs at this time. Thus, wearing gloves and practicing hand hygiene before and after wearing gloves could stop the infections transmission between patients to patients and HCWs to patients and vice versa (WHO, 2009). 
The types of care provided by HCWs at the inpatient wards based on the regular health status of VA patients necessitate wearing gloves. Patients at the PVAMC, in general, on a daily basis require $\mathrm{HCWs}$ to, but is not limited to, (HCWs at the PVAMC, Personal Communication, December 23, 2016):

- Administer medications,

- Perform skin assessment,

- Draw blood,

- Assist in the Activities of Daily Living (ADLs) such as eating, bathing, dressing, toileting and walking,

- Assist in incontinent and human excreta.

\subsubsection{Warehouse}

The Warehouse at the PVAMC is located in building 6, which is a maintenance building. Purell and Soap cartridges are shipped from vendors to the warehouse and picked up by EMS employees and stored at the basement of the main building, building 1. From building 1, they are delivered to EMS supply closets at each inpatient unit. An EMS employee is required to replace Purell and Soap cartridges from each dispenser at the wards.

The PVAMC provides to the whole facility and specifically to the inpatient units a Green-Certified Foam type of Purell of a $1200 \mathrm{~mL}$ size (40.5 FL OZ) and a Steris Medicated hand Soap of a $1000 \mathrm{~mL}$ size (33.8 FL OZ). These types have been ordered and used starting in fiscal year 2015. However, different types of Purell and Soap were used before then. Starting in November 2016, there was a different type of hand Soap provided to the floor. Purell comes in a case of two cartridges (2/CS) and Soap comes 
in one cartridge each (1/EA). At the PVAMC, VistA does not have a break down of Purell-Soap demands or use per ward or unit. It only tracks the orders per facility wide on a monthly basis.

\subsubsection{Physical Contact with Patients}

Patients, in general, get exposed to different people who physically have contact with them and others who have to be present inside the room either for medical reasons or non-medical reasons. In the tree diagram shown in Figure 5.1, two different groups, who have contact and have no contact with patients, are identified with their subgroups, if any exists. 


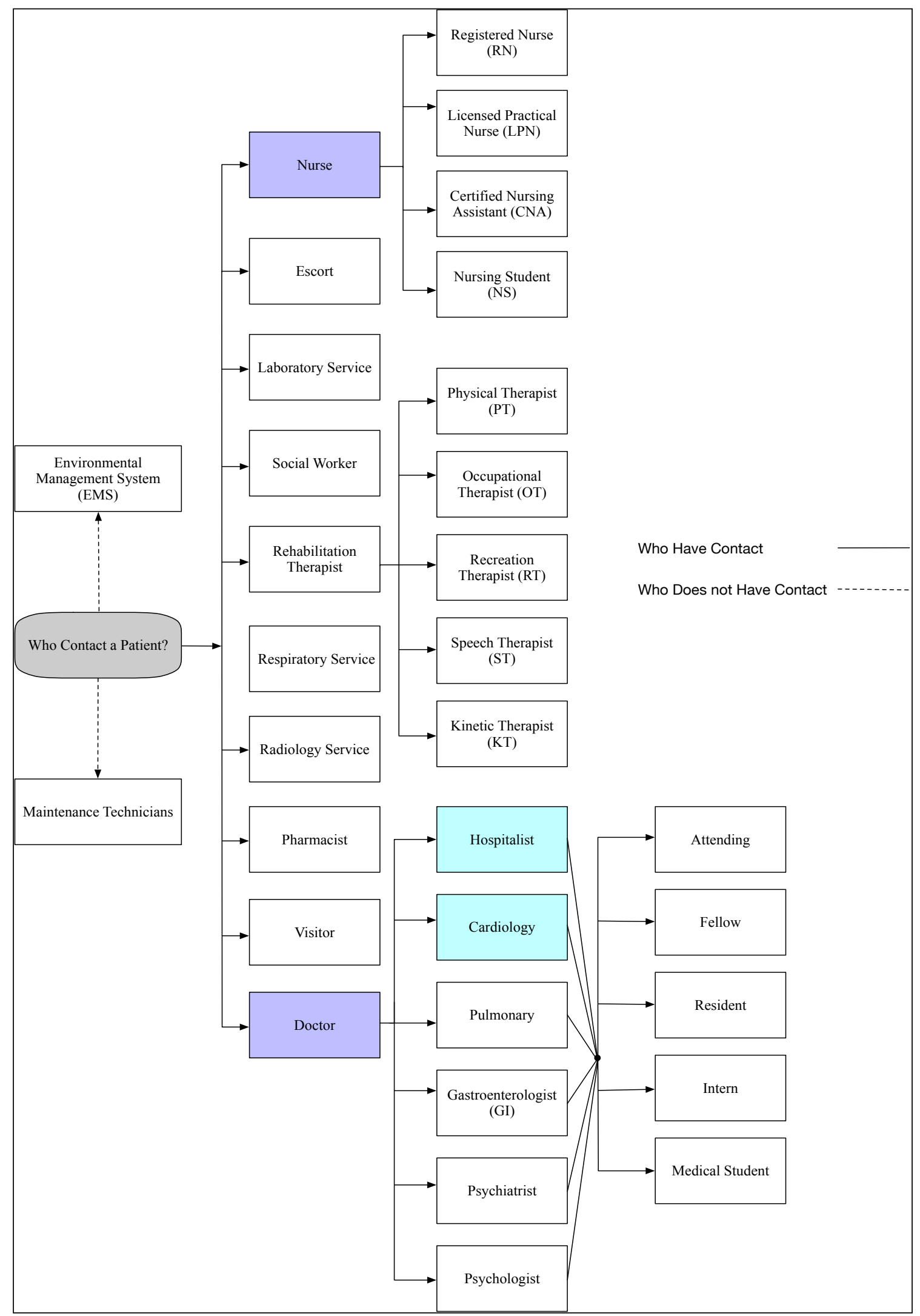

Figure 5.1 Who Contact a Patient 


\subsection{Retrospective Analysis of Hand Hygiene Compliance}

In this section, a retrospective study analysis is carried out to analyze and assess the hand hygiene direct observation method conducted by the PVAMC. The collected data is pulled form the Veterans Integrated Service Network (VISN) 1 Hand Hygiene Management Tool. The data analysis is carried out by analyzing the overall hand hygiene compliance rate by combining isolation versus non-isolation moments and ignoring PPE compliance, by analyzing hand hygiene and PPE compliance rate for isolation moments only, and by analyzing PPE compliance rate by itself for isolation moments only too. Such analysis is carried out at all levels, for the overall facility and per inpatient wards. Each level is broken down by healthcare employee class, my five moments for hand hygiene and hygiene products per month per year. At the end, a comparison analysis between hand hygiene compliance rates when patients are in isolation versus non-isolation is carried out.

\subsubsection{VISN 1 Hand Hygiene Management Tool}

"VA Boston Healthcare System (VABHS) Infection Control (IC) in collaboration with New England Veterans Engineering Resource Center partnered to create, pilot, and implement a standardized electronic monitoring and reporting tool for HH observations within all VISN 1 facilities.” (Strymish and Gupta, 2012).

According to Strymish and Gupta (2012), all Veterans Integrated Service Network (VISN) 1 facilities are connected via the Hand Hygiene Management Tool Page SharePoint site where each "hand hygiene site is set up under a parent Infection Control SharePoint." (Strymish and Gupta, 2012). The hand hygiene management tool page has several options for library views and system functions (Appendix R) 
(Strymish and Gupta, 2012). Microsoft InfoPath is used to design and create a friendly interface user for Hand Hygiene Event Data Input Form (Appendix S).

"The System has the ability to be used on tablets or personal electronic devices; however, this has not yet been piloted on a significant scale." (Strymish \& Gupta, 2012). At the PVAMC, it is more convenient for the observers to use a printout template for the Hand Hygiene and Precautions Compliance Monitoring Tool (Appendix P) that is filled out by an observer and then the data is entered into the system manually by the same observer from their VA computer, if he or she is registered on the system, or by an infection preventionist who has access to the online form. Any HCWs can get permission to be a direct observer (Secret observer) and can get an access to the Hand Hygiene Management Tool. The infection preventionist recommend adding an option for stethoscope dedicated in patient room and stethoscope cleaning with sanitizing wipe post contacting a patient into the system, as seen in (Appendix Q).

To extract and export data and outputs, there is an option called Run Report, (Strymish and Gupta, 2012). The starting date for run report goes back to April 2012. Strymish and Gupta (2012) recommended extracting and exporting data into an Excel spreadsheet and run macros to generate some analysis by employee class, location or service type quarterly or fiscally. An Excel expert has to make some minor changes for each new fiscal year to show up on the generated report since the tool is set up through fiscal year 2013.

There are 8 Veterans Affairs Medical Centers in VISN 1, which are Manchester, Western Central Mass, Boston, Bedford, Togus, Connecticut, Providence and White 
River Junction (PVAMC, 2015). Some of the VAMC uses the same hand hygiene template for hand hygiene input, and some use a different form that is improved. The Connecticut hand hygiene input form does not work, and it gives an error message when trying to click the online link.

Currently, directly observing hand hygiene performance at the PVAMC consists of three main steps. First, an observer has to directly observe an opportunity for hand hygiene performance. The time duration for observing the opportunity varies by the types of moments for hand hygiene, location of the observation, the person's experience making observations, the service type and the professional category of the observed employee. Second, an observer has to fill out the hand hygiene and precautions compliance monitoring form (Appendix P) or the hand hygiene and precautions compliance monitoring form with Stethoscope (Appendix Q). The time duration for filling out such form(s) depends on the employee's experience as an observer. Filling out the hand hygiene management form per opportunity might take 12 minutes for an experienced observer such as those who are infection preventionist (I. Straub, Personal Communication, 2015). In contrast, it might take 3-5 minutes to fill in the form for those who are quite new observers (I. Straub, Personal Communication, 2015). Third, an observer or anyone who has access to the hand hygiene event data input form must transfer the written data from the hand hygiene and precautions compliance monitoring form.

The hand hygiene online management tool has a three-option form when entering the collected data online: 
- Option One is Only Hand Hygiene Compliance Observations: There is no classification between isolation and non-isolation observations. Observations could belong to one or the other.

- Option Two is Only Isolation Precautions: There is no classification between isolation and non-isolation observations. Observations could belong to one or the other since gloves, for example, are sometimes required during nonisolation care.

- Option Three is Both Hand Hygiene Compliance Observations and Isolation Precautions: There is no classification between isolation and non-isolation observations since observing both hand hygiene and PPE compliance together could also occur during non-isolation events.

The first option, only hand hygiene compliance observations, is designated for hand hygiene observations for both isolation and non-isolation patients where PPE is not observed. The first option is the most common option evaluated at the PVAMC. The second option, only isolation precautions, is designated for observing PPE compliance only for isolation patients. However, it does not tell whether hand hygiene is practiced before or after wearing gloves. Thus, hand hygiene is neither observed nor reported. The third option, where both hand hygiene and isolation precautions are observed and reported at the same time, is designated for observing hand hygiene practices with PPE on isolation patients.

Hand hygiene observations from option one and three are combined to evaluate the overall hand hygiene adherence rate. Then, option three is analyzed separately to enable a comparison between the rates when patients are in isolation (only hand 
hygiene observations from option three) versus the combination of non-isolation and isolation (hand hygiene observations from option one). Option two is analyzed to evaluate PPE compliance adherence in terms of how many times gloves, gowns and masks are used during patient care in isolation rooms.

There are several drawbacks about how the hand hygiene management tool is designed. In all options, it is better to have an option that distinguishes between isolation and non-isolation observations whether for hand hygiene only, PPE only or both. It would be interesting to compare hand hygiene compliance during isolation versus non-isolation. In addition, in option two and three, a drawback is that there is no column for N-95 mask for observations that are associated with airborne precautions. Instead, surgical mask, which is associated with droplet precautions, is used for both types of isolations, droplet and airborne. Gloves and gowns are not required to be worn all the time during droplet precautions. However, for airborne precautions, gloves and gowns plus N-95 mask are required. Sometimes gloves are required to be used for care of patients who are on non-precautions (standard precautions); however, the hand hygiene management tool under option two does not have an option for such a scenario.

Figure 5.2 summarizes the existing options for hand hygiene direct observation method at PVAMC. 


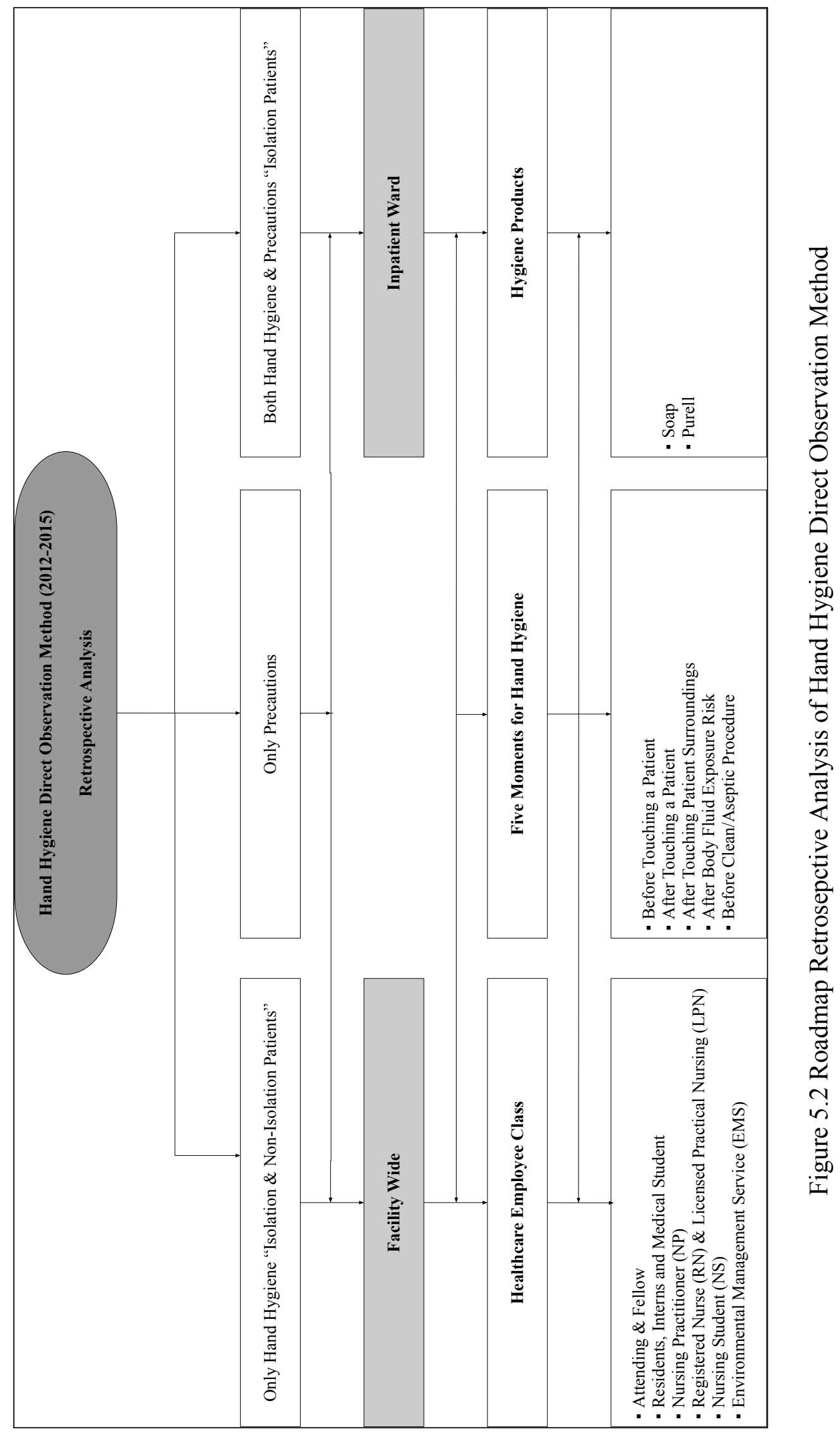




\subsubsection{PVAMC Direct Hand Hygiene Observation Method}

At the PVAMC, the only available method to track, assess and measure the hand hygiene compliance of HCWs is via conducting direct observations (Secret Shoppers or Secret Observers). There are about 1038 full-time employees with 32 subspecialty clinics and 119 authorized beds with 73 operating beds (PVAMC, 2015). Compared with other VA medical centers, the PVAMC is considered to be a medium VA medical center in terms of facility size. The direct observation method is now described, including areas for improvement.

The direct observation method is conducted as follows. An observer, assuming the secret observer is covert and who can be any employee at the PVAMC, takes a round at anytime in any healthcare unit (inpatient or outpatient wards), and observes other healthcare professional categories to see if they, the observed ones, practice hand hygiene at key hand hygiene moments, for example, before or after touching a patient. The observation might capture whether the observed ones comply to precautions signage by, for example, wearing personal protective equipment (PPE) such as gloves, gowns and masks before going into an isolation room. The secret observer has to have an excuse to enter patient rooms and be there with the medical teams. Typically, each observer carries a hand hygiene and precautions compliance monitoring tool form (Appendix P) to fill out as an opportunity occurs. However, observers at the PVAMC sometimes prefer to memorize what happens and then fill in the form either at their offices or while heading to observe more.

If a hand hygiene opportunity is detected and not practiced, it is recorded as none (non-compliance). On the other hand, if an opportunity is detected and practiced, 
it is recorded as waterless, referring to alcohol-based hand rub (Purell), or as wash, referring to soap and water (compliance). Sometimes if an opportunity is detected and the observer is able to notify and remind the observed one to practice hand hygiene, the observed one does not get credit whether he/she practices or not, this opportunity is recorded as none (non-compliance). However, it is better to report that as no compliance and report what hygiene product is used. In contrast, sometimes observers do not notify or remind the observed ones to practice and they also record the event as non-compliance. In the first case, when an observed one is notified and does practice hand hygiene, there is no option on the form to tell which product is used because the opportunity is recorded non-compliance. In the latter case, it does not matter since the observed one does not actually practice hand hygiene.

The physical hand hygiene and precaution compliance monitoring form includes options for correctly using personal protective equipment (PPE) and isolation; however, there is no subheading showing what type(s) of PPE is used or should be used. In addition, the form only captures two moments for hand hygiene, which are before and after touching a patient. There is a space for comments on the form to record other captured moments and fill them out on the online hand hygiene event data input form. The physical hand hygiene and precaution compliance monitoring form has space to record only 13 hand hygiene events to record. No personal information of the observed ones is included on the form. Basically, the physical hand hygiene and precaution compliance monitoring form does not resemble the online hand hygiene event data input form at the PVAMC. 


\subsubsection{Facility-Wide Retrospective Hand Hygiene Compliance}

Direct observations data is provided and extracted by an infection preventionist at the PVAMC from the hand hygiene management tool system. The data goes back to April $4^{\text {th }} 2012$, the first collected data, until Nov $4^{\text {th }} 2015$, which is about 1309 days including weekends and holidays. The data consists of a complete dataset for calendar year 2013 and 2014, and partial dataset for calendar year 2012 (missing the first 3 months) and calendar year 2015 (missing the last month). There are 5 different divisions at RI VAMC (Providence, New Bedford Primary Care Center, Hyannis Primary Care Center and Middletown, and Nantucket), 38 different units (5B, 6B, ICU, etc.), and 4 different service types (Inpatient Ward, Outpatient Clinic, NonClinic Support and Clinical Support).

The dataset shows that there are 63 secret observers who directly conduct and observe hand hygiene opportunities at 4 out of 5 different divisions of the PVAMC (Providence, New Bedford Primary Care Center, and Hyannis Primary Care Center and Middletown), 22 out of 38 different locations (ICU, 6A, etc.), 4 different service types (inpatient ward, outpatient clinic, ... etc.), and on 26 different employee classes (registered nurses, doctors, etc.). Interestingly, 48 out of 63 secret observers enter the collected data into the online form for themselves or for others.

The four-years of hand hygiene observation data for isolation and non-isolation combined from option one and isolation from option three, generated 6249 total observations, of which 5522 are compliant and 727 are non-compliant as depicted in Figure 5.3. The hand hygiene rate is $88 \%$ and $12 \%$ for compliant and non-compliant respectively. 


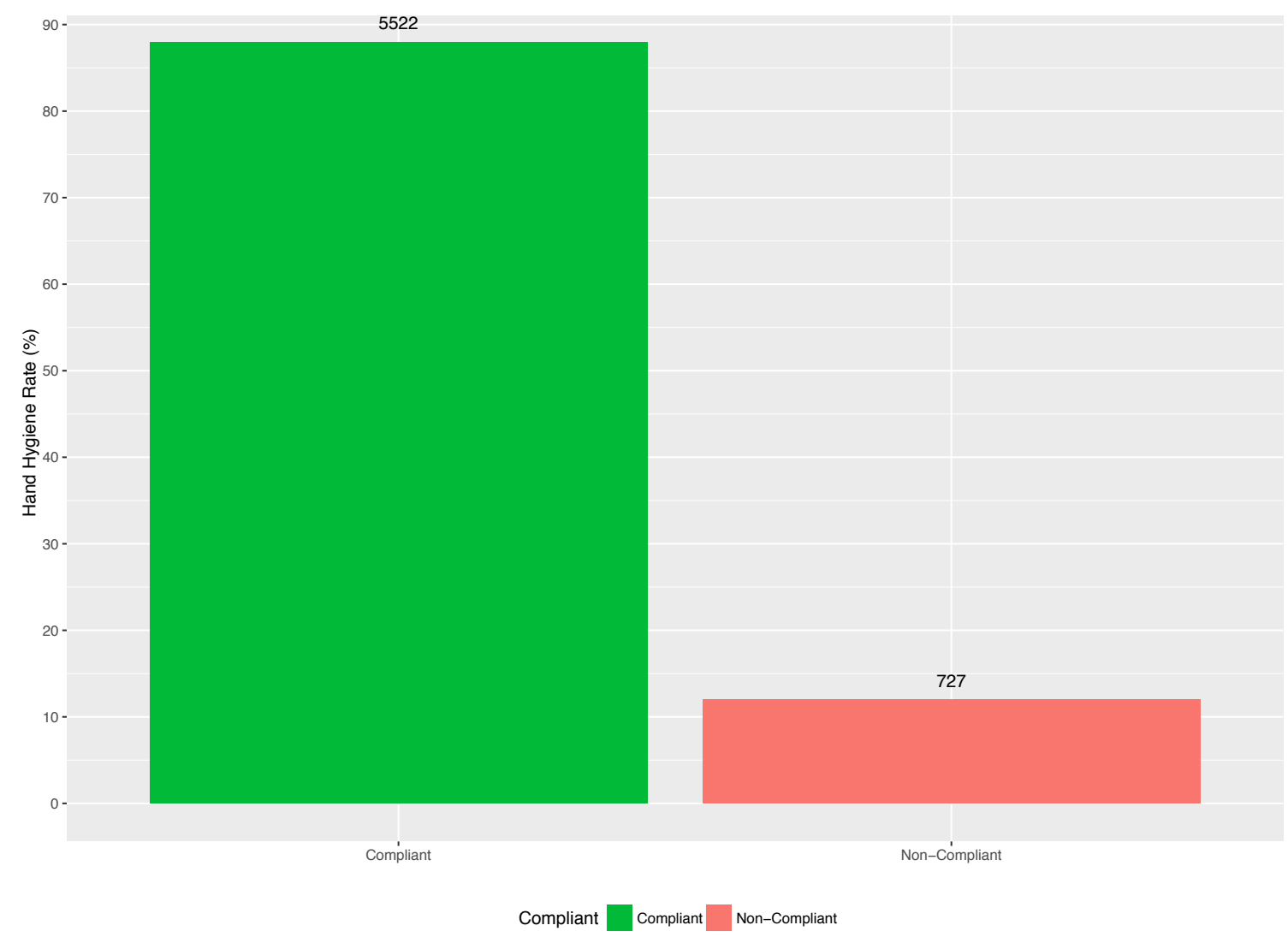

Figure 5.3 Facility-Wide Hand Hygiene Adherence Rate

There are 5817 isolation and non-isolation observations from option one versus 432 isolations observations from option three facility wide. There are 5161 compliant versus 656 non-compliant for isolation and non-isolation observations from option one. On the other hand, there are 361 compliant versus 71 non-compliant isolations observation from option three. The breakdown of isolation from option three versus isolation and non-isolation from option one compliant and non-compliant observations are depicted in Figure 5.4. The hand hygiene adherence rates are $89 \%$ and $84 \%$ for isolation and non-isolation from option one and isolation from option three respectively as depicted in Figure 5.4. Isolation and non-isolation hand hygiene adherence rate from option one is higher than the isolation rate from option three. 
However, it is hard to judge the lower hand hygiene adherence rate of isolation observations from option three because that cannot be compared to the higher hand hygiene adherence rate from isolation and non-isolation observations from option one. Nevertheless, it is expected that HCWs give more attention to their hand hygiene practices during patient care in isolation rooms. Sometimes HCWs do not feel the need to use hygiene products before wearing gloves for patient contact. Additionally, the hand hygiene adherence rates were found to be equivalent for before touching a patient moment for both isolation (81\%) and non-isolation rooms (82\%); however, the rate for after patient contact for non-isolation rooms (92\%) was higher than for isolation rooms $(86 \%)$. This could imply that HCWs at the PVAMC believe or have faith on PPE to prevent HAIs transmissions and were not necessarily need to practice hand hygiene while wearing gloves. 


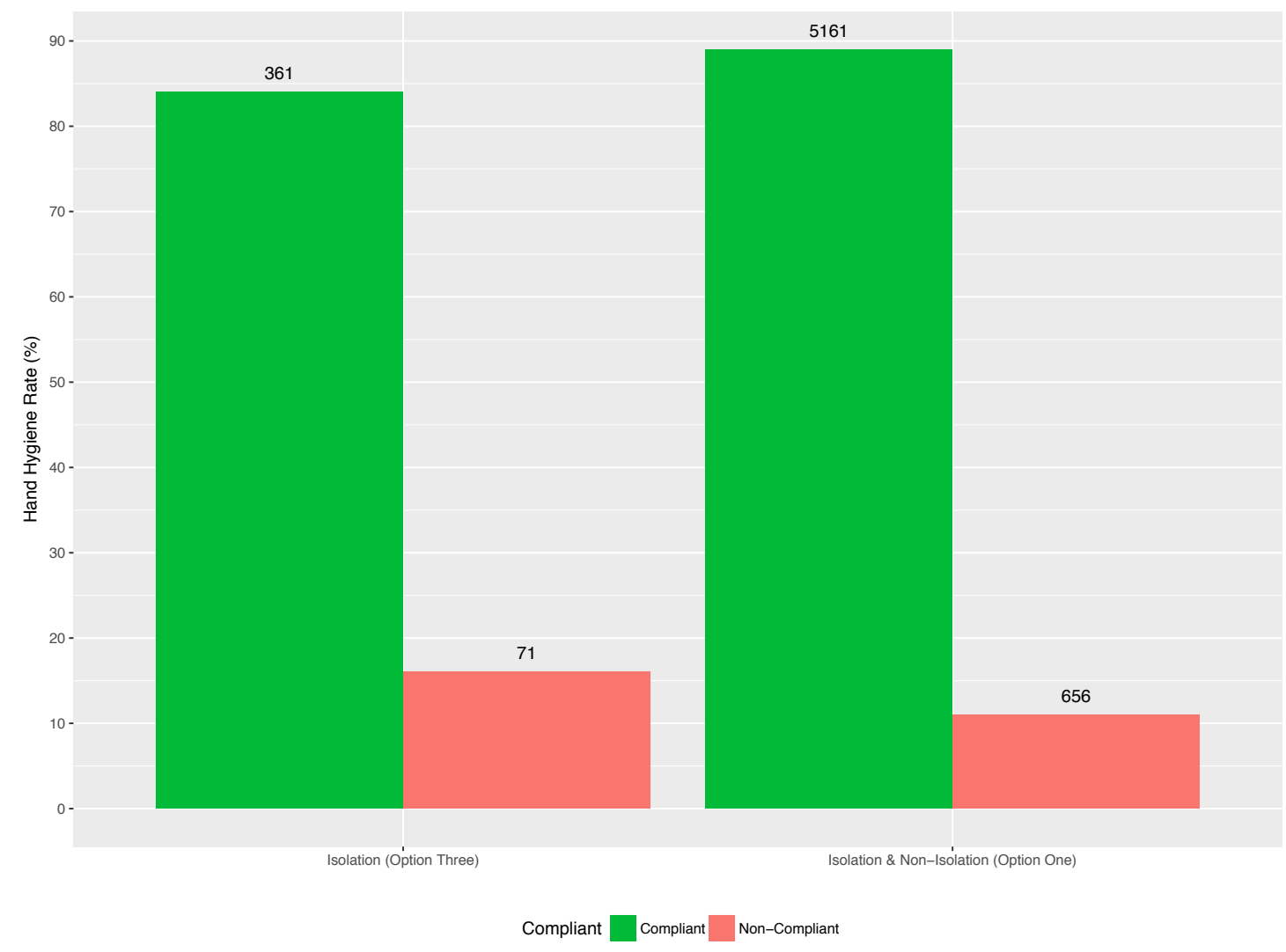

Figure 5.4 Isolation (Option Three) vs. Isolation and Non-Isolation (Option One) Facility-Wide Hand Hygiene Adherence Rate

According to TJC (2009) there are about $1.5 \mathrm{~mL}$ Soap or Purell per hit or aliquot generated by Purell or Soap dispenser. Based on this information, the total number of cartridges of soap or Purell is calculated. A cartridge of soap is $1000 \mathrm{~mL}$ and provides about 667 hits (1000/1.5). A cartridge of Purell is $1200 \mathrm{~mL}$ and provides about 800 hits $(1200 / 1.5)$.

Based on the information above, observations that are associated to either Soap or Purell hygiene product are converted to hygiene product cartridges as depicted in Figure 5.5. Figure 5.5 shows the aggregated observations that are associated with Soap, Purell and none for all years combined. There are 2321 out of 5522 total compliant observations for handwashing with soap and water, which is about 3.5 
cartridges of Soap. In addition, 3201 out of 5522 total compliant observations represent hand rubbing with alcohol (Purell), which is about 4 cartridges of Purell. There are 727 non-compliant observations captured, which represents about 0.8 Purell cartridge or about 1.0 Soap cartridge if hygiene products had been used during these moments of non-compliance. Figure 5.6 shows the breakdown of hygiene product observations per year. Purell observations are captured than Soap observations for each single year.

A drawback of the way the data is reported by the PVAMC is that if an observer observes a HCW not practicing hand hygiene during a hand hygiene opportunity and if the observer is able to remind the $\mathrm{HCW}$ before the event occurs and the $\mathrm{HCW}$ practices hand hygiene based on the reminder, the $\mathrm{HCW}$ does not get credit for practicing hand hygiene and the event is reported as non-compliant. In this instance, the dataset does not tell which hygiene product is used. Consequently, some of the 727 non-compliant are actually compliant but with no which hygiene product is used. 


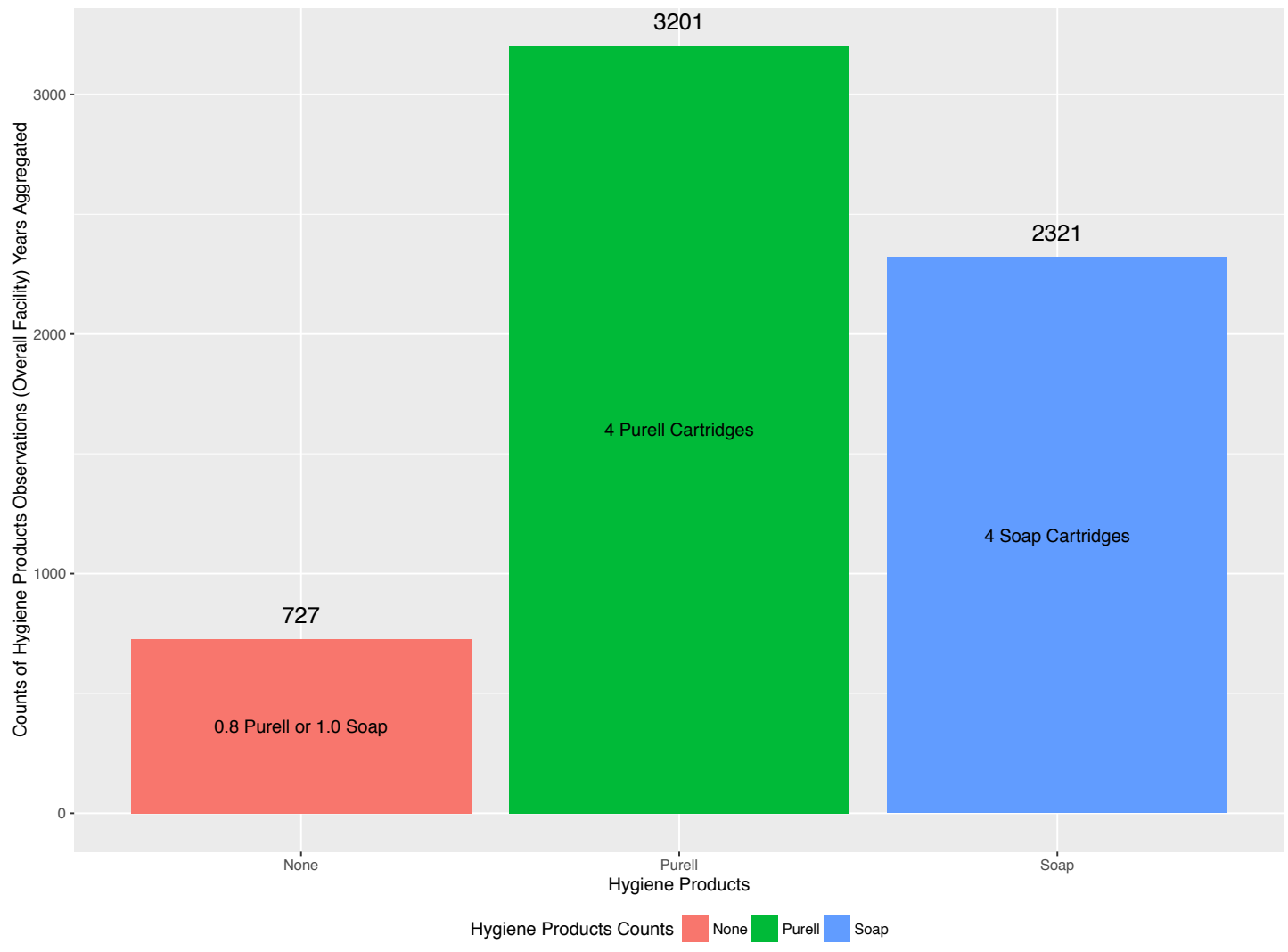

Figure 5.5 Facility-Wide Hand Hygiene Observations Summarized by Hygiene Products Years Combined 


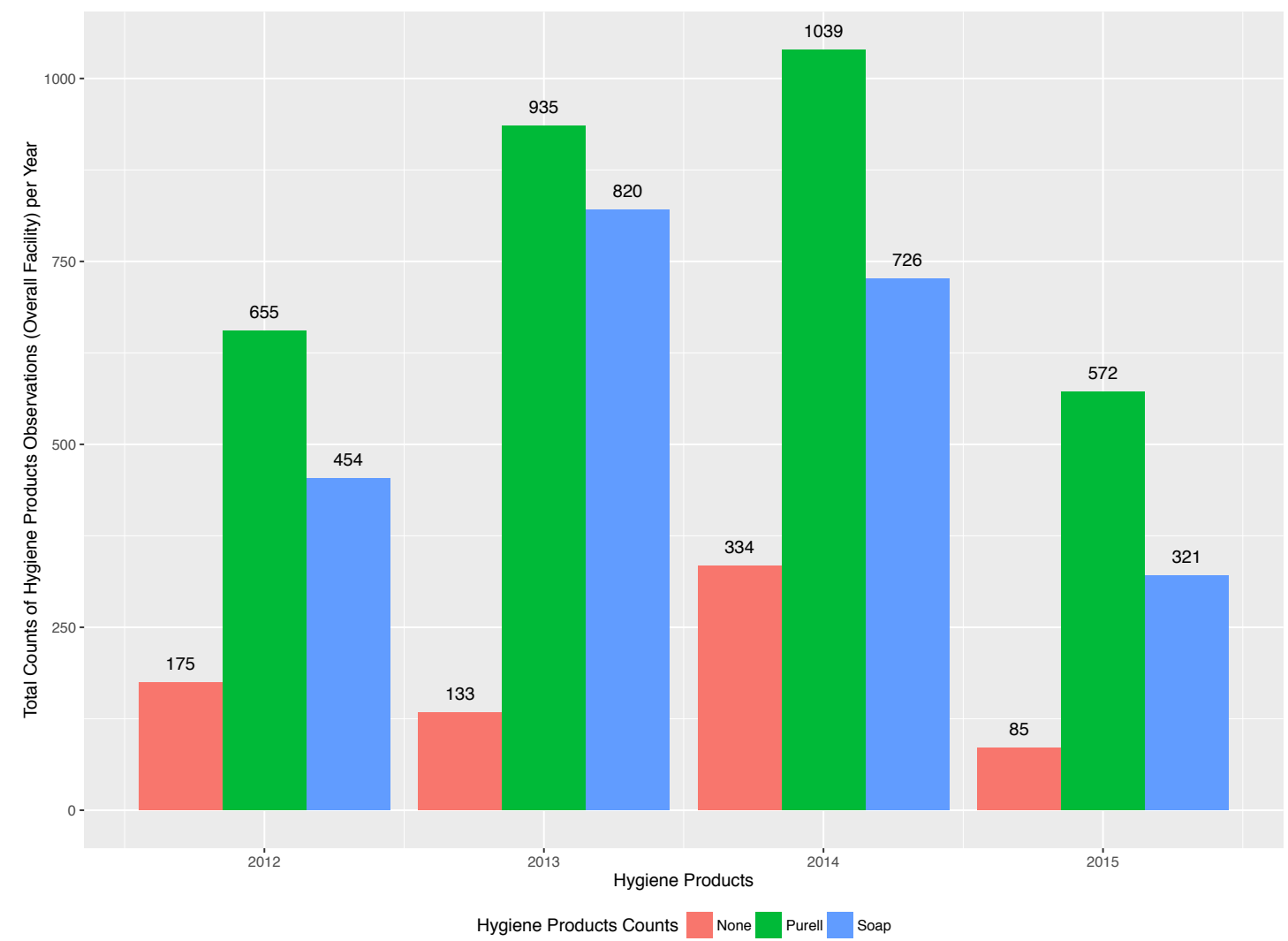

Figure 5.6 Facility-Wide Hand Hygiene Observations Summarized by Hygiene Products per Year

Figure 5.7, with aggregated observations per hand hygiene moments, shows that the two most frequent hand hygiene moments captured are before and after touching a patient, as expected. The overall hand hygiene adherence rate for before touching a patient is about $82 \%$ while the rate after touching a patient is about $92 \%$ as seen in Table 5.2. This could imply that HCWs at the PVAMC could slightly underestimate practicing hand hygiene before touching a patient and value practicing hand hygiene after touching a patient. As expected, the numbers of observations captured for the other three moments for hand hygiene are quite small especially for after blood/body fluid exposure risk and before clean/aseptic procedures. In general, the compliance rate is above $80 \%$ for all moments, as seen in Table 5.2 and Figure 5.7. 


\begin{tabular}{|c|l|c|c|c|c|c|c|}
\hline \multirow{2}{*}{\multicolumn{2}{|c|}{ Five Moments for Hand Hygiene }} & \multicolumn{3}{|c|}{ Hygiene Products } & \multirow{2}{*}{ Total } & \multicolumn{2}{|c|}{$\begin{array}{c}\text { Compliance } \\
\%\end{array}$} \\
\cline { 3 - 5 } \cline { 3 - 5 } & No & Purell & Soap & & Yes & No \\
\hline 1 & Before Touching a Patient & 407 & 1206 & 639 & 2252 & $82 \%$ & $18 \%$ \\
\hline 2 & Before Clean/Aseptic Procedures & 25 & 70 & 53 & 148 & $83 \%$ & $17 \%$ \\
\hline 3 & After Body Fluid Exposure/Risk & 4 & 56 & 125 & 185 & $98 \%$ & $2 \%$ \\
\hline 4 & After Touching a Patient & 223 & 1288 & 1181 & 2692 & $92 \%$ & $8 \%$ \\
\hline 5 & After Touching Patient Surroundings & 68 & 581 & 323 & 972 & $93 \%$ & $7 \%$ \\
\hline
\end{tabular}

Table 5.2 My Five Moments for Hand Hygiene Summarized by Hygiene Products

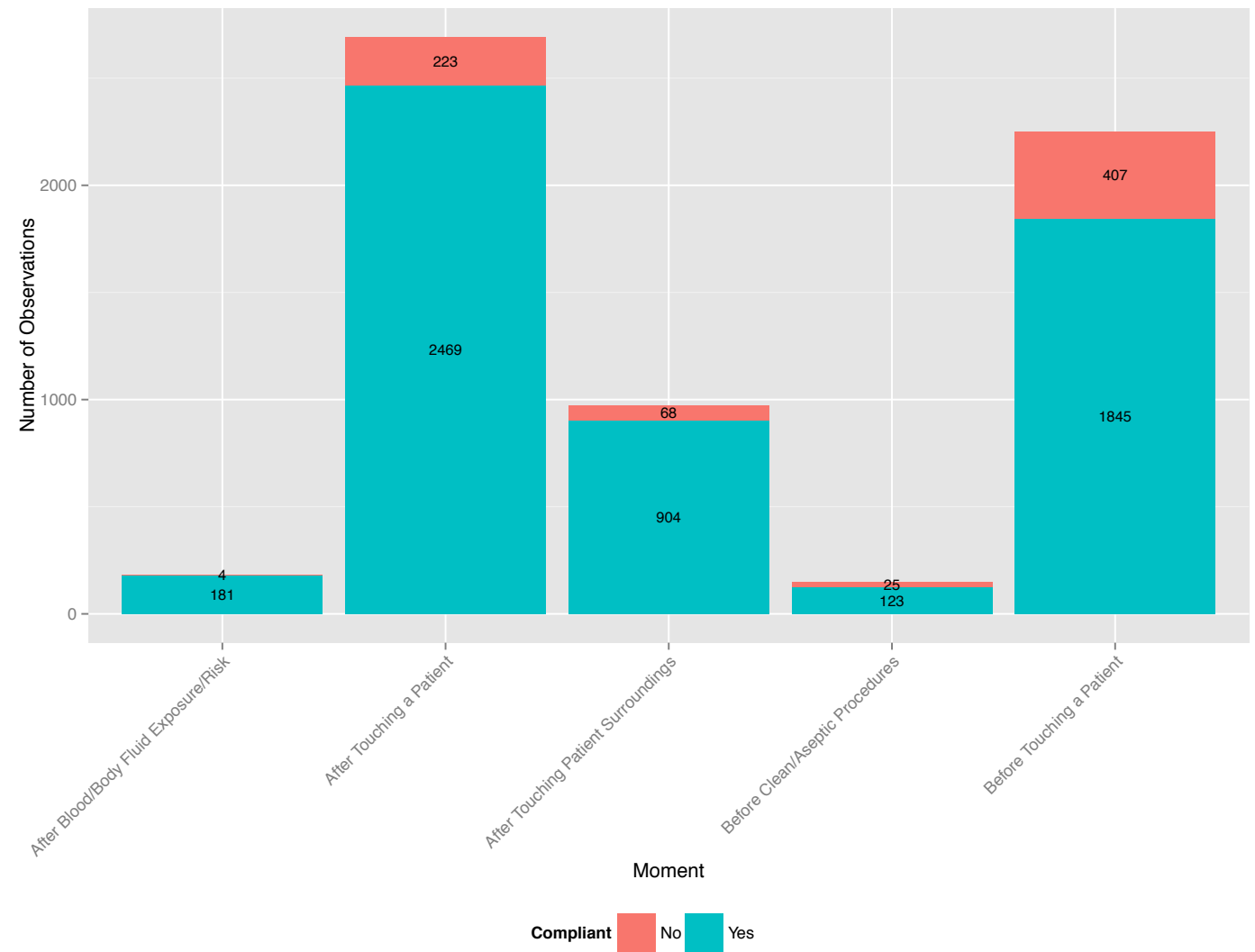

Figure 5.7 Facility-Wide Hand Hygiene Observations Summarized by My Five Moments Years Combined

Figure 5.8 shows that observations that are associated with Purell outweigh those that are associated with Soap for before and after touching a patient and after touching patient surroundings hand hygiene moments. Purell is almost $47 \%$ higher for 
before touching a patient, $8 \%$ higher for after touching a patient and $44 \%$ higher for after touching patient surroundings.

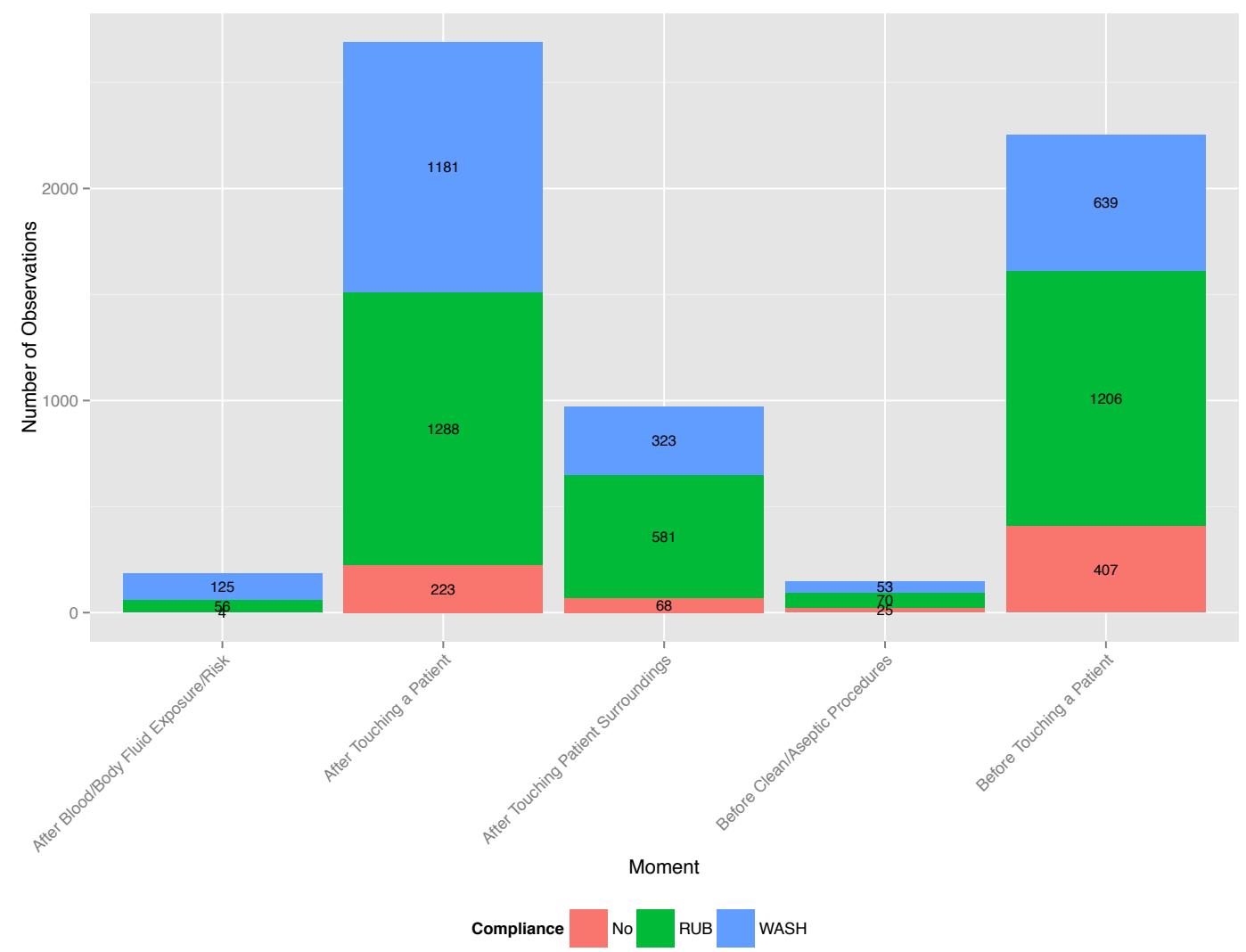

Figure 5.8 Facility-Wide Hand Hygiene Observations Summarized by My Five Moments and by Hygiene Products

Facility-wide hand hygiene compliance is also summarized by month per year as depicted in Figure 5.9. The first month of conducting direct observations method is April 2012 with 17 observations in total with 5 non-compliant and 12 compliant. The low number of observations could be due to the fact that the hand hygiene direct observations method was at the beginning and the few secret observers who voluntarily participated needed time to master data collection. Then, an increase can be seen in the total collected observations from April through August of 2012. Then, a fluctuation of up and down can be seen for September through the end of year 2012. 
On the other hand, there is a decrease in the total number of collected observations in year 2013. In 2014, a peak can be seen in March. In 2015, it seems that there is a drop in the data collected and in performing direct observation at the PVAMC. The hand hygiene adherence rates per year are 86\% (1284 samples), 93\% (1888 samples), $84 \%$ (2099 samples), and 91\% (978 samples) for 2012, 2013, 2014 and 2015 respectively.
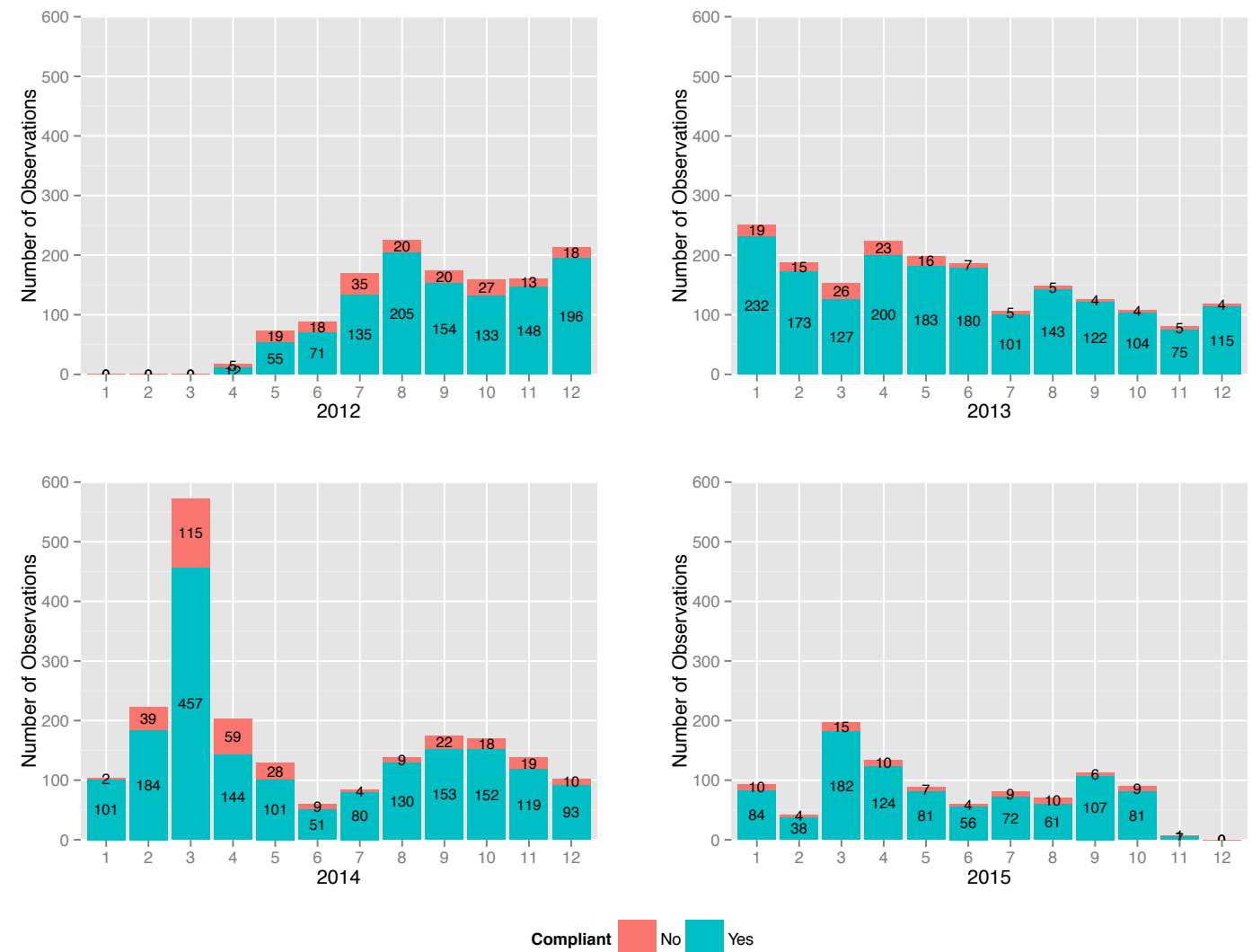

Figure 5.9 Facility-Wide Hand Hygiene Observations Summarized by Compliant \& Non-Compliant of Year

Direct observations are also summarized by division as seen in Figure 5.10. Most of the observations are conducted at Providence medical center with 4873 observations in total with $17 \%$ of non-compliant. Middletown comes next with 925 observations, Hyannis Primary Care Center comes third with 274 observations with 0\% non-compliant and New Bedford Primary Care Center comes fourth with 177 
observations. Nantucket has no information regarding whether direct observations method is conducted there or not.

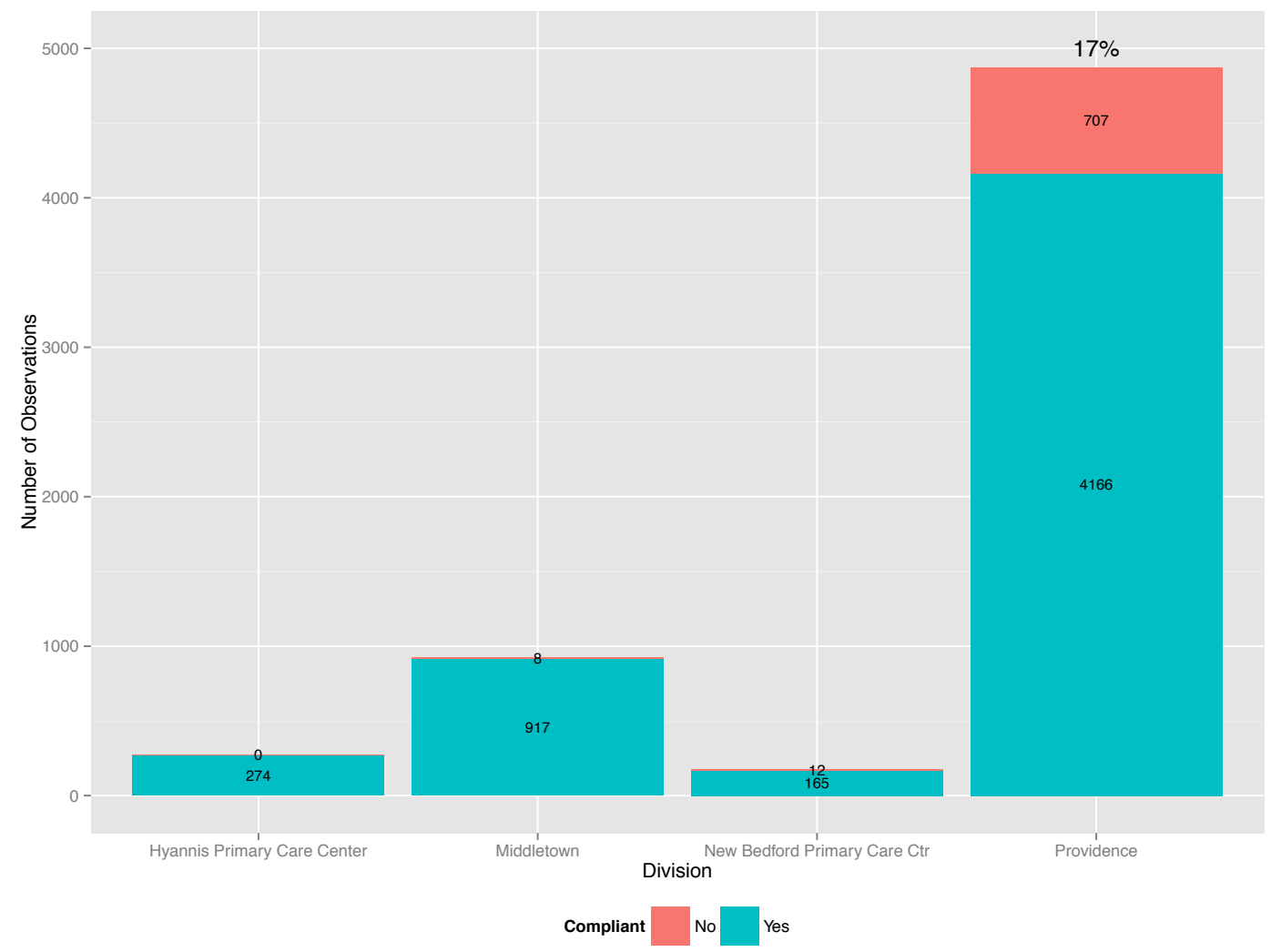

Figure 5.10 Facility-Wide Hand Hygiene Observations Summarized by Division, Compliant and Non-Compliant of Station

Hand hygiene compliance is summarized by locations per month per year as seen in Figure 5.11 and Figure 5.12. The main three locations of inpatient units are 5B, $6 \mathrm{~B}$ and ICU. ICU has a hand hygiene adherence rate of about $86 \%$ based on a sample size of 725 observations. $6 \mathrm{~B}$ has a hand hygiene adherence rate of about $75 \%$ based on a sample size of 645 observations. 5B has a hand hygiene adherence rate of about $83 \%$ based on a sample size of 1030 observations. Of note, the percentages shown in Figure 5.11 are non-compliant percentages. 

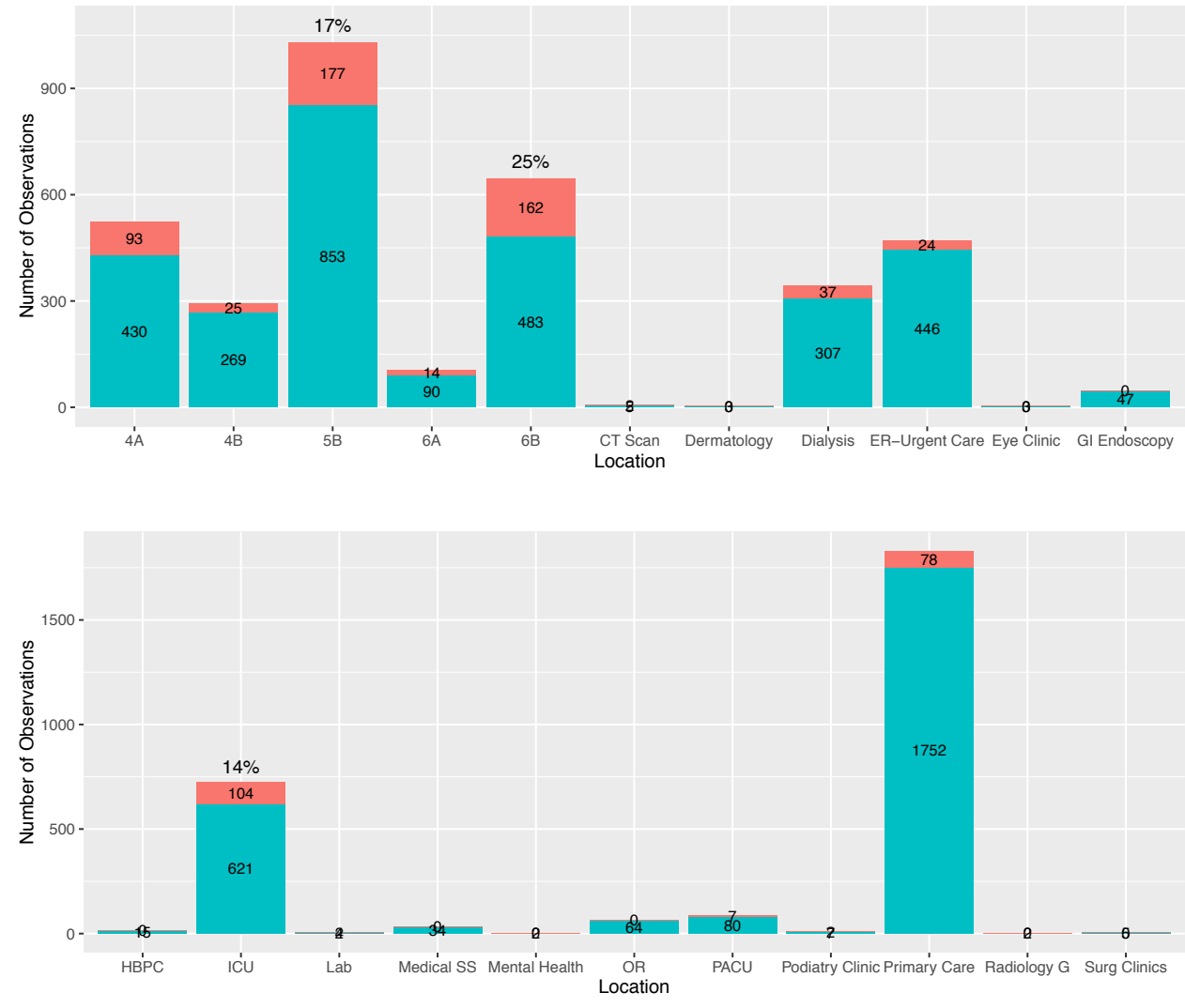

Figure 5.11 Facility-Wide Hand Hygiene Observations Summarized by Compliant \& Non-Compliant of Location 


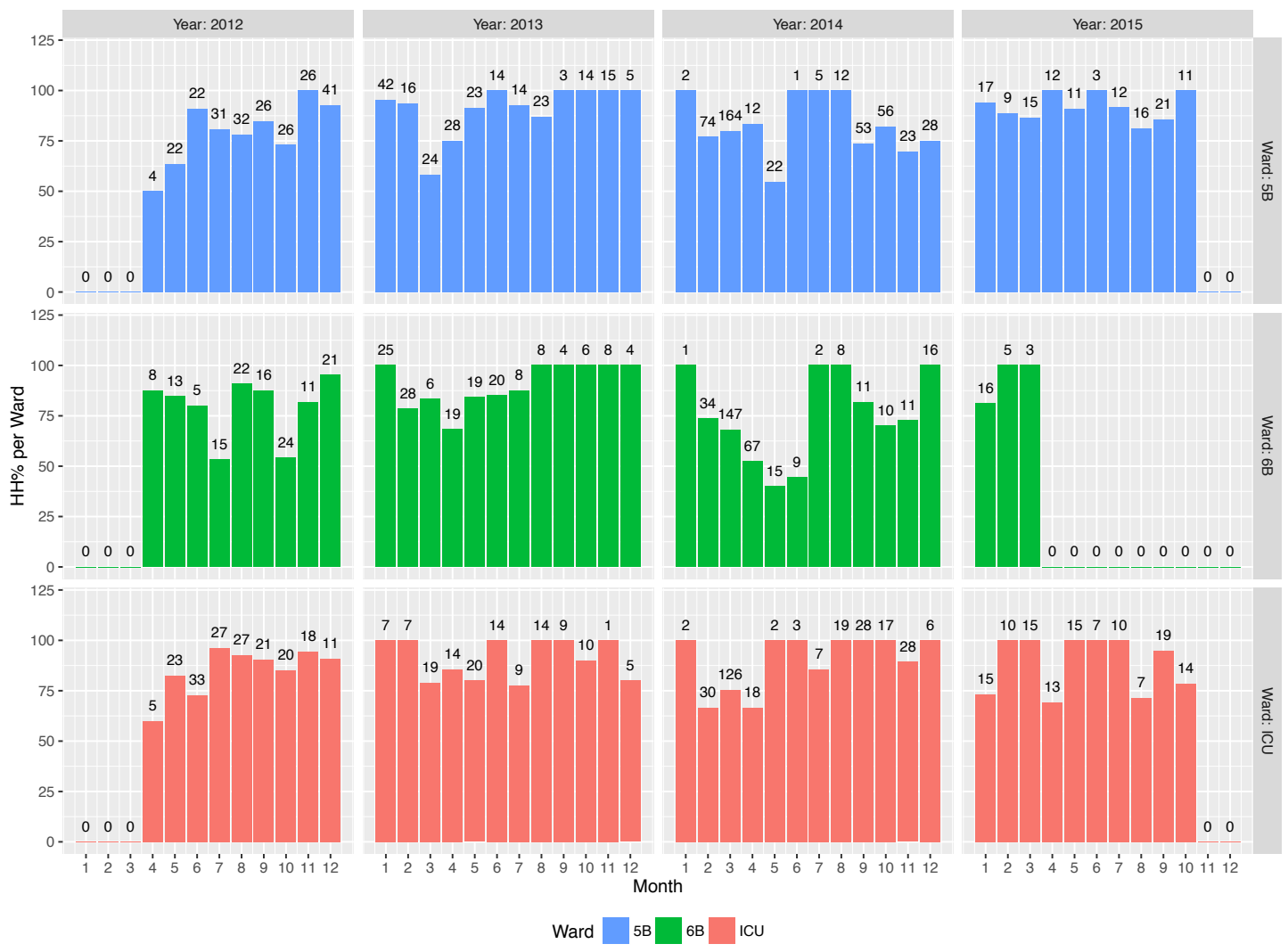

Figure 5.12 Facility-Wide Hand Hygiene Observations Summarized by Location per Month per Year

Direct observations are also summarized by employee class as can be seen in Figure 5.13. Out of the different employee classes, doctor (Attendant/Fellow), doctors (Medical Student/Interns/Resident), nursing practitioner, nurses (Registered Nurses/Licensed Practiced Nurses), nursing students and environmental management service (EMS) are only analyzed. Figure 5.13 shows that higher numbers of observations are associated with employee classes such as registered nurses and licensed practical nurses, attending and fellow and residents, interns and medical students. This makes sense because they have higher patient contact. Figure 5.13 shows that the rate for hand hygiene compliance of doctors (Attendant/Fellow) is about $87 \%$ based on a sample size of 793 observations. The rate for hand hygiene 
compliance of doctors (Medical Student/Intern/Resident) is about $78 \%$ based on a sample size of 294 observations. The rate for hand hygiene compliance of nurses practitioner is about $92.5 \%$ based on a sample size of 40 observations. The rate for hand hygiene compliance of nurses (Registered Nurses/Licensed Practiced Nurses) is about $92 \%$ based on a sample size of 3416 observations. The 3416 samples represent about 55\% (3416/6249) of the total observations captured during the four years. This is not surprising because registered nurses and licensed practiced nurses are considered to be the largest groups of HCW at the PVAMC with the highest patient contact frequency. The rate for hand hygiene compliance of nursing students is about $83 \%$ based on a sample size of 30 observations, which is a very small sample. Finally, the rate for hand hygiene compliance of environmental management service (EMS) is about $34 \%$ based on a sample size of 113 observations, which is very low compared to the role of EMS staff where they take care of all patient room and floor cleaning. Of note, the percentage shown in Figure 5.13 is the non-compliant percentage. 

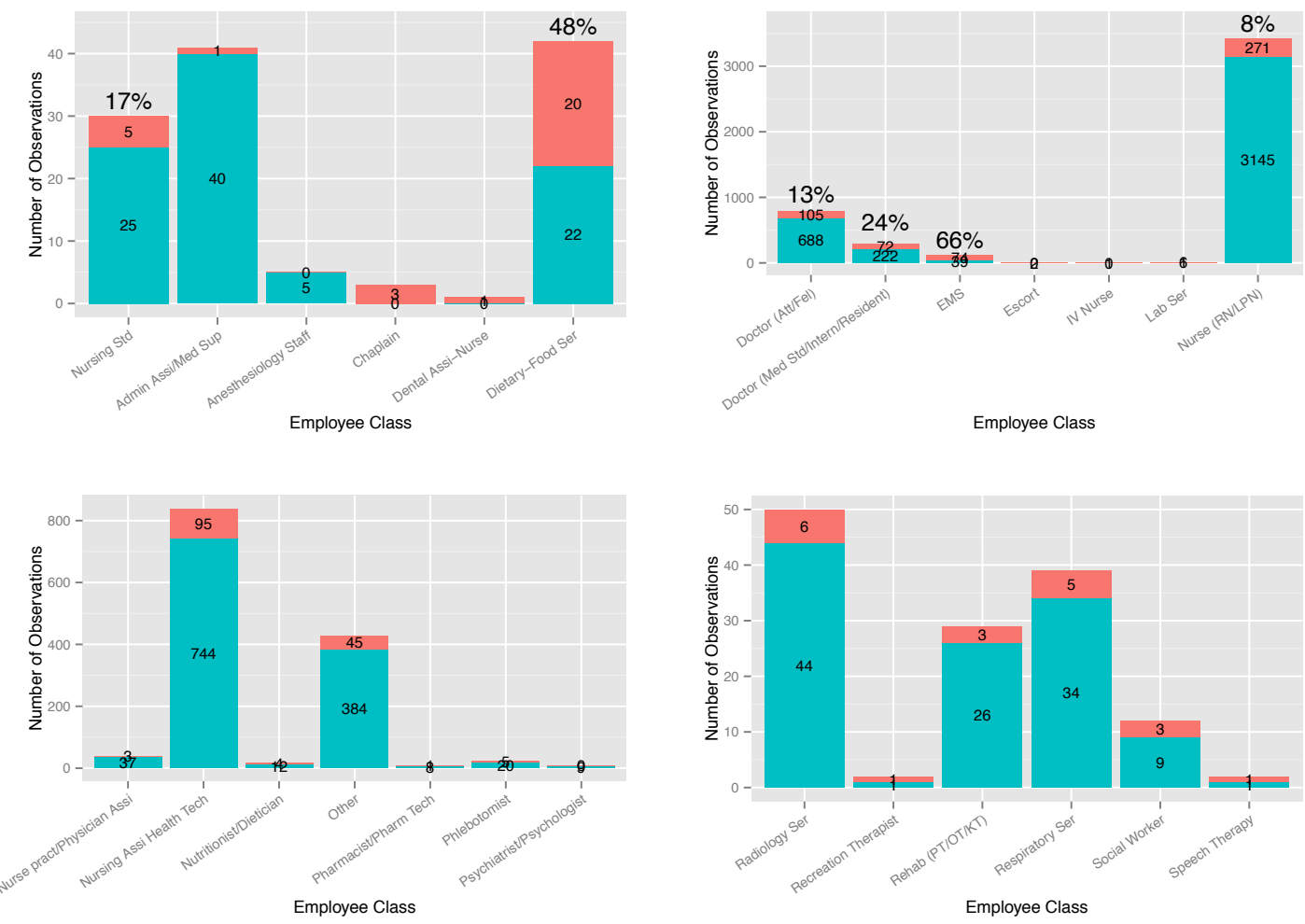

Compliant

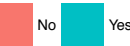

Figure 5.13 Facility-Wide Hand Hygiene Observations Summarized by Compliant and Non-Compliant of Employee Class

Facility-wide summarizing hand hygiene adherence rate by moments and employee class together reveals important facts as can be seen in Table 5.3 and Table 5.4. The medical group (attending, fellow) hand hygiene rate before touching a patient is moderate, $80 \%$ and high for after touching a patient $94 \%$. On the other hand, the medical group (residents, interns and medical students) hand hygiene rate before and after touching a patient are disappointingly, $74 \%$ and $76 \%$ respectively. The nursing group (RN and LPN) hand hygiene rate before and after touching a patient are high, $85 \%$ and $96 \%$ respectively. Again, HCWs seem to slightly underestimate practicing hand hygiene before touching a patient and highly value that after touching a patient. 
Interestingly, Table 5.4 shows that the most frequent hand hygiene moments observed by EMS are after touching patient surroundings, before touching a patient and after touching a patient. However, data analysis shows that there is only two events occurred for Before Clean/Aseptic Procedures moment and EMS does not have after body fluid exposure risk moment though their role is to clean patient room and to make bed with or without the existence of a patient. In general, rates are very low for all three moments. In general, EMS has to be considered for further hand hygiene observations and analysis. Of note, there is an employee class category labeled as "Others" with no definition of whom they might be, with 429 observations shown on Table 5.4.

\begin{tabular}{|c|c|c|c|c|c|c|}
\hline \multirow{3}{*}{\multicolumn{2}{|c|}{ Five Moments for Hand Hygiene }} & \multirow{2}{*}{\multicolumn{3}{|c|}{$\begin{array}{c}\text { Employee Class } \\
\text { Doctor } \\
\text { (Attending/Fellow) } \\
\end{array}$}} & \multirow{2}{*}{\multicolumn{2}{|c|}{ Compliance $\%$}} \\
\hline & & & & & & \\
\hline & & \multirow{2}{*}{$\begin{array}{l}\text { Yes } \\
316\end{array}$} & \multirow{2}{*}{$\frac{\text { No }}{81}$} & \multirow{2}{*}{$\begin{array}{c}\text { Total } \\
397\end{array}$} & \multirow{2}{*}{$\frac{\text { Yes }}{79.60 \%}$} & \multirow{2}{*}{$\frac{\mathrm{No}}{20.40 \%}$} \\
\hline 1 & Before Touching a Patient & & & & & \\
\hline 2 & Before Clean/Aseptic Procedures & 2 & 0 & 2 & $100.00 \%$ & $0.00 \%$ \\
\hline 3 & After Body Fluid Exposure/Risk & 7 & 0 & 7 & $100.00 \%$ & $0.00 \%$ \\
\hline 4 & After Touching a Patient & 360 & 23 & 383 & $93.99 \%$ & $6.01 \%$ \\
\hline 5 & $\begin{array}{l}\text { After Touching Patient } \\
\text { Surroundings }\end{array}$ & 3 & 1 & 4 & $75 \%$ & $25 \%$ \\
\hline \multirow{2}{*}{\multicolumn{2}{|c|}{ Five Moments for Hand Hygiene }} & \multicolumn{3}{|c|}{$\begin{array}{c}\text { Doctor (Medical } \\
\text { Student/Intern/Resident) }\end{array}$} & \multicolumn{2}{|c|}{ Compliance \% } \\
\hline & & Yes & No & Total & Yes & No \\
\hline 1 & Before Touching a Patient & 67 & 24 & 91 & $73.63 \%$ & $26.37 \%$ \\
\hline 2 & Before Clean/Aseptic Procedures & 7 & 1 & 8 & $87.50 \%$ & $12.50 \%$ \\
\hline 3 & After Body Fluid Exposure/Risk & 2 & 0 & 2 & $100.00 \%$ & $0.00 \%$ \\
\hline 4 & After Touching a Patient & 139 & 44 & 183 & $75.96 \%$ & $24.04 \%$ \\
\hline 5 & $\begin{array}{l}\text { After Touching Patient } \\
\text { Surroundings }\end{array}$ & 7 & 3 & 10 & $70.00 \%$ & $30.00 \%$ \\
\hline
\end{tabular}

Table 5.3 (A) Facility-Wide Hand Hygiene Rate Summarized by My Five Moments and Employee Class 


\begin{tabular}{|c|c|c|c|c|c|c|}
\hline & \multirow{2}{*}{ Five Moments for Hand Hygiene } & \multicolumn{3}{|c|}{$\begin{array}{l}\text { Nurse Practitioner } \\
\text { (NP) }\end{array}$} & \multicolumn{2}{|c|}{ Compliance \% } \\
\hline & & Yes & No & Total & Yes & No \\
\hline 1 & efore Touching a Patient & 14 & 2 & 16 & $87.50 \%$ & $12.50 \%$ \\
\hline 2 & efore Clean/Aseptic Procedures & 0 & 0 & 0 & - & - \\
\hline 3 & After Body Fluid Exposure/Risk & 1 & 0 & 1 & $100.00 \%$ & $0.00 \%$ \\
\hline 4 & After Touching a Patient & 20 & 1 & 21 & $95.24 \%$ & $4.76 \%$ \\
\hline \multirow[t]{3}{*}{5} & After Touching Patient $\mathrm{Su}$ & 2 & 0 & - & $100.00 \%$ & $0.00 \%$ \\
\hline & \multirow{2}{*}{ Five Moments for Hand Hygiene } & & \multicolumn{2}{|c|}{ Compliance \% } \\
\hline & & \begin{tabular}{l|l|l}
\multicolumn{2}{c}{ Nurse (RN/LPN) } \\
Yec
\end{tabular} & No & Total & Yes & No \\
\hline 1 & Before To & 934 & 171 & 1105 & $84.52 \%$ & $15.48 \%$ \\
\hline 2 & Before Clean/Aseptic Procedures & 85 & 19 & 104 & $81.73 \%$ & $18.27 \%$ \\
\hline 3 & After B & 98 & 0 & 98 & $100.00 \%$ & $0.00 \%$ \\
\hline 4 & After Touching a Patient & 1324 & 62 & 1386 & $95.53 \%$ & $4.47 \%$ \\
\hline \multirow[t]{3}{*}{5} & After To & 704 & 19 & 723 & $97.37 \%$ & $2.63 \%$ \\
\hline & \multirow{2}{*}{ Five Moments for Hand Hygiene } & \multicolumn{3}{|c|}{ Nurse (Students) } & \multicolumn{2}{|c|}{ Compliance $\%$} \\
\hline & & \multicolumn{2}{|c|}{ Total } & Tota & Yes & No \\
\hline 1 & Before Touching a Patient & 5 & 2 & 7 & $71.43 \%$ & $28.57 \%$ \\
\hline 2 & Before Clean/Aseptic Procedures & 3 & 2 & 5 & $60.00 \%$ & $40.00 \%$ \\
\hline 3 & After Body Flu & 0 & 0 & 0 & - & - \\
\hline 4 & After Touch & 10 & 0 & 10 & $100.00 \%$ & $0.00 \%$ \\
\hline 5 & A & 7 & 1 & 8 & $87.50 \%$ & $12.50 \%$ \\
\hline \multirow{2}{*}{\multicolumn{2}{|c|}{ Five Moments for Hand Hygiene }} & \multicolumn{3}{|c|}{ EMS } & \multicolumn{2}{|c|}{ Compliance $\%$} \\
\hline & & Yes & No & Total & Yes & No \\
\hline 1 & Before Touching a Patient & 6 & 30 & 36 & $16.67 \%$ & $83.33 \%$ \\
\hline 2 & Before Clean/Aseptic Proce & 2 & 0 & 2 & $100.00 \%$ & $0.00 \%$ \\
\hline 3 & After Body Fluid Exposure/Risk & 0 & 0 & 0 & - & - \\
\hline 4 & After Touching a Patient & 6 & 19 & 25 & $24.00 \%$ & $76.00 \%$ \\
\hline \multirow[t]{3}{*}{5} & Patient Surroundin & 25 & 25 & 50 & $50.00 \%$ & $50.00 \%$ \\
\hline & \multirow{2}{*}{ Five Moments for Hand Hygiene } & \multicolumn{3}{|c|}{ Others } & \multicolumn{2}{|c|}{ Compliance \% } \\
\hline & & Yes & No & Total & Yes & No \\
\hline 1 & Before Touching a Patient & 196 & 22 & 218 & $90.00 \%$ & $10.00 \%$ \\
\hline 2 & Before $\mathrm{Cl}$ & 1 & 0 & 1 & $100.00 \%$ & $0.00 \%$ \\
\hline 3 & Aft & 0 & 3 & 3 & $0.00 \%$ & $100.0 \%$ \\
\hline 4 & After & 182 & 17 & 199 & $91.50 \%$ & $8.50 \%$ \\
\hline 5 & After Touching Patient Surroundings & 5 & 3 & 8 & $62.50 \%$ & $37.50 \%$ \\
\hline
\end{tabular}

Table 5.4 (B) Facility-Wide Hand Hygiene Rate Summarized by My Five Moments and Employee Class 


\subsubsection{Inpatient-Wards Retrospective Hand Hygiene Compliance}

After analyzing hand hygiene compliance facility-wide, the analysis is carried out per inpatient ward. The four years of hand hygiene observations per inpatient ward, isolations (option three) and isolations and non-isolations (option one) combined, is 2400 observations out of 6249 total observations in which 1957 are compliant and 443 are non-compliant. The breakdown of compliant versus noncompliant observations per inpatient ward is depicted in Figure 5.14. The hand hygiene adherence rates per ward are $87 \%, 75 \%$ and $86 \%$ for $5 \mathrm{~B}, 6 \mathrm{~B}$ and ICU respectively.

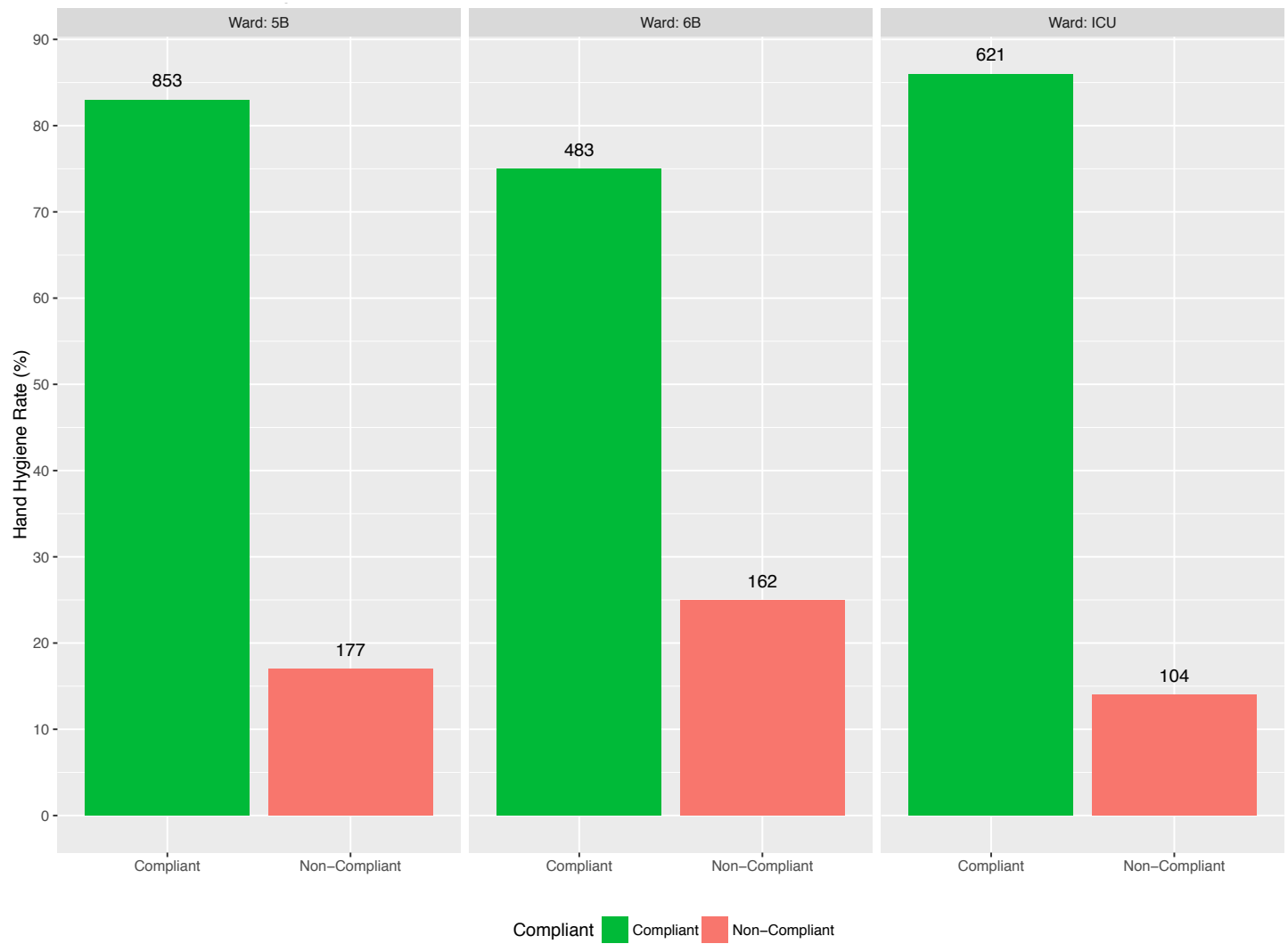

Figure 5.14 Inpatient-Wards Hand Hygiene Rate 
There are 2047 isolation and non-isolation (option one) versus 353 isolation (option three) hand hygiene observations in inpatient wards combined. There are 1668 compliant versus 379 non-compliant isolation and non-isolation (option one) observations in inpatient wards combined. On the other hand, there are 289 compliant versus 64 non-compliant isolation (option three) observations in inpatient wards combined. The breakdown of isolation (option three) versus isolation and nonisolation (option one) for compliant and non-compliant observations per inpatient ward is depicted in Figure 5.15. The isolation and non-isolation (option one) hand hygiene adherence rates per ward are $83 \%, 75 \%$ and $86 \%$ for $5 \mathrm{~B}, 6 \mathrm{~B}$ and ICU respectively. The isolation (option three) hand hygiene adherence rates per ward are $80 \%, 82 \%$ and $84 \%$ for $5 \mathrm{~B}, 6 \mathrm{~B}$ and ICU respectively.

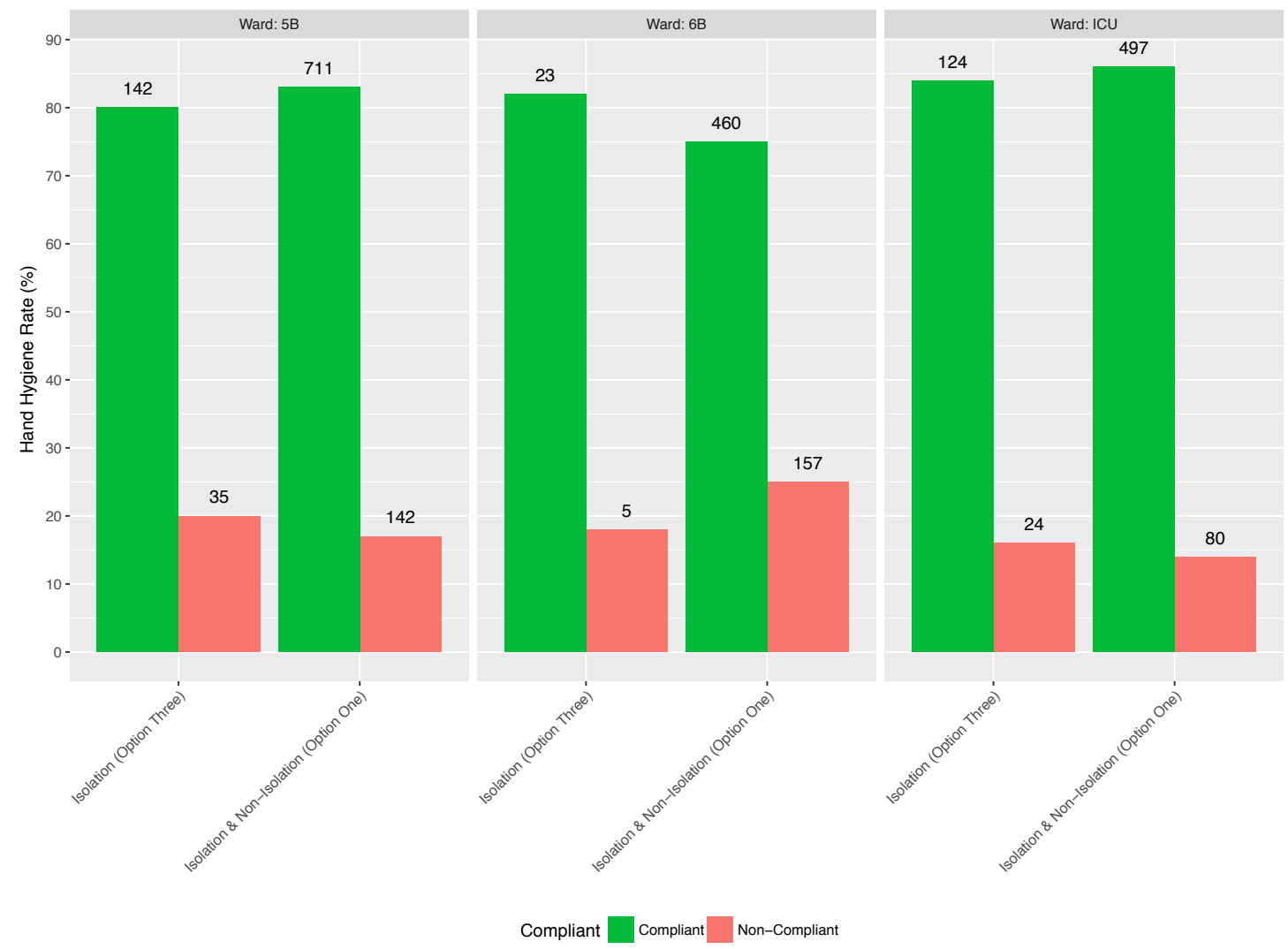

Figure 5.15 Inpatient-Wards Isolation vs. Non-Isolation Hand Hygiene Rate 
The hygiene products analysis per inpatient ward, as depicted in Figure 5.16 and Figure 5.17, show that 610 out of 1941 total compliant observations represent total handwashing with soap and water, which is worth about 0.9 cartridge of Soap for all three inpatient wards combined. On the other hand, 1331 out of 1941 total compliant observations represent hand rubbing with alcohol (Purell), which is worth about 1.67 cartridges of Purell. Generally speaking, there are more Purell observations captured than soap observations. It would be inconclusive, based on the hygiene products observations counts, to conclude that HCWs prefer to use Purell than Soap when disinfecting their hands.

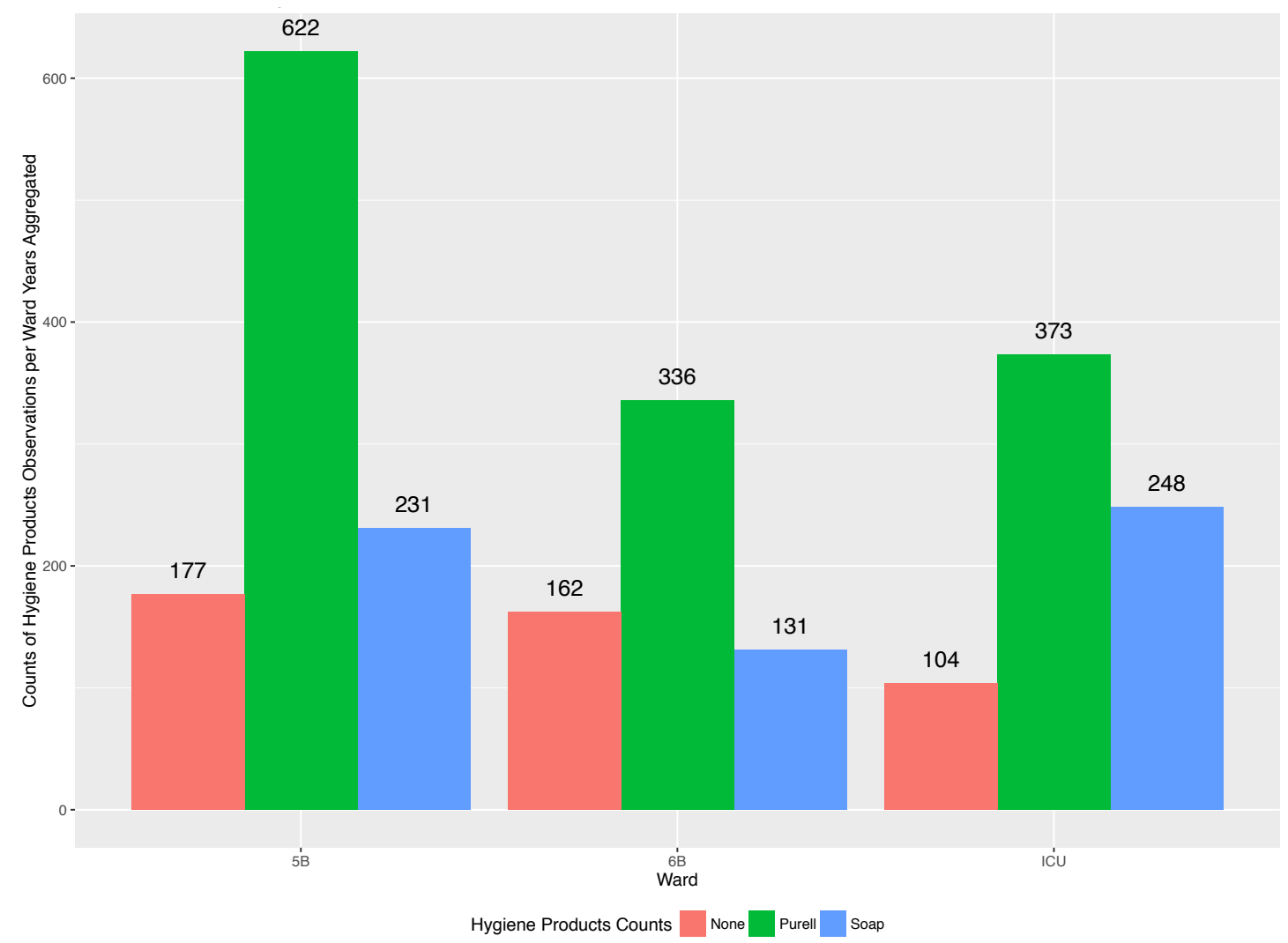

Figure 5.16 Inpatient-Wards Hand Hygiene Observations Summarized by Hygiene Products and Years Combined 

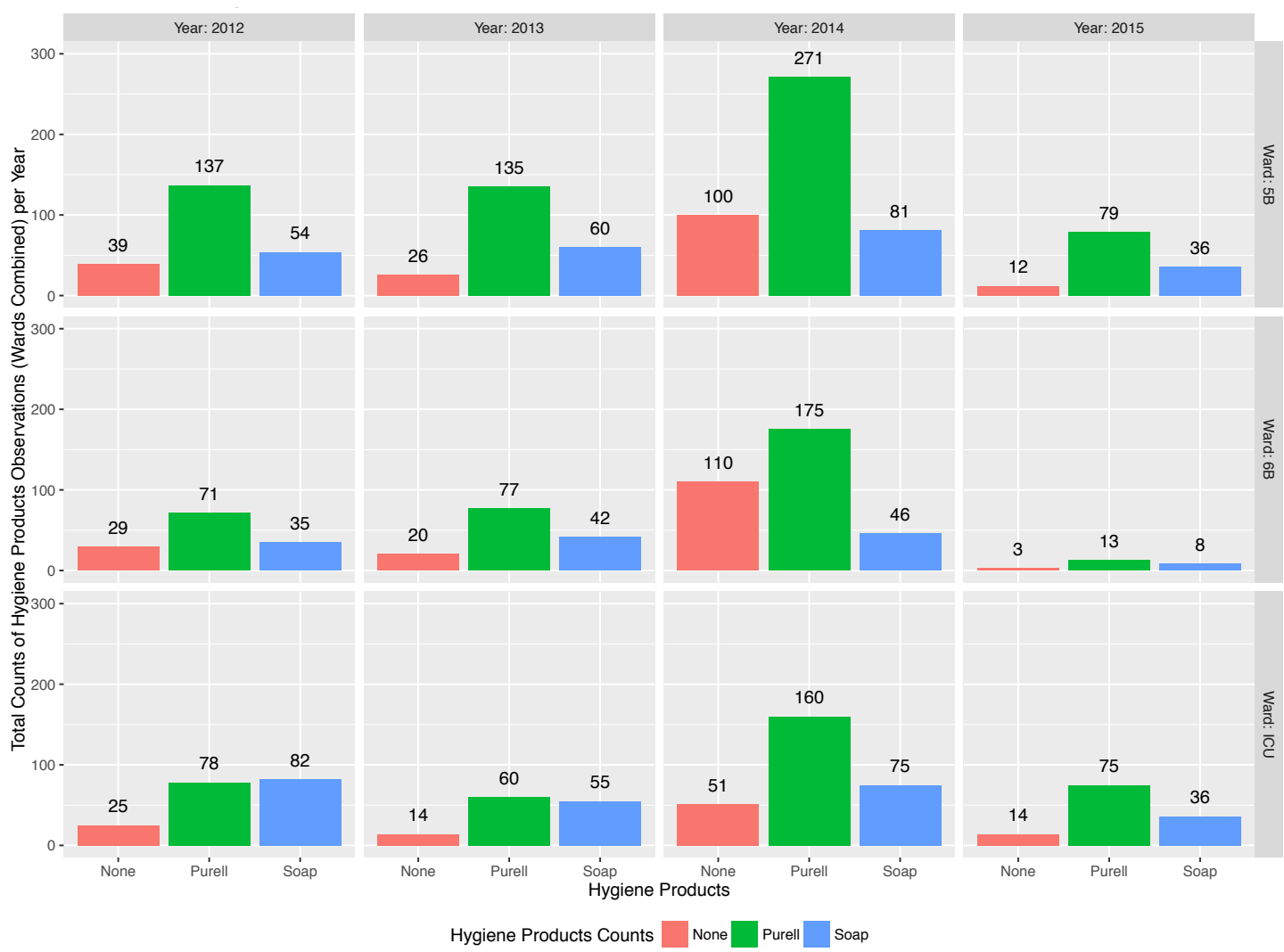

Figure 5.17 Inpatient-Wards Hand Hygiene Observations Summarized by Hygiene Products per Year

Hand hygiene observations are also summarized by hand hygiene moments per inpatient ward as depicted in Figure 5.18. Figure 5.18 shows that the two most frequent hand hygiene captured moments are before and after touching a patient, as expected. The hand hygiene adherence rates for before touching a patient are $73 \%$, $55 \%$, and $76 \%$ for $5 \mathrm{~B}, 6 \mathrm{~B}$ and ICU respectively as seen in Table 5.5 . It is considered very low for all inpatient wards especially for $6 \mathrm{~B}$. The hand hygiene adherence rates for after touching a patient are $88 \%, 83 \%$, and $90 \%$ for $5 \mathrm{~B}, 6 \mathrm{~B}$ and ICU respectively as seen in Table 5.5. Rates after touching a patient outweigh rates before touching a patient across all inpatient wards. Again, this could imply that HCWs at the PVAMC could slightly underestimate practicing hand hygiene before touching a patient and 
value practicing hand hygiene after touching a patient. As expected, the observations captured for the other three moments for hand hygiene are quite small, especially for after blood/body fluid exposure risk and before clean/aseptic procedures.
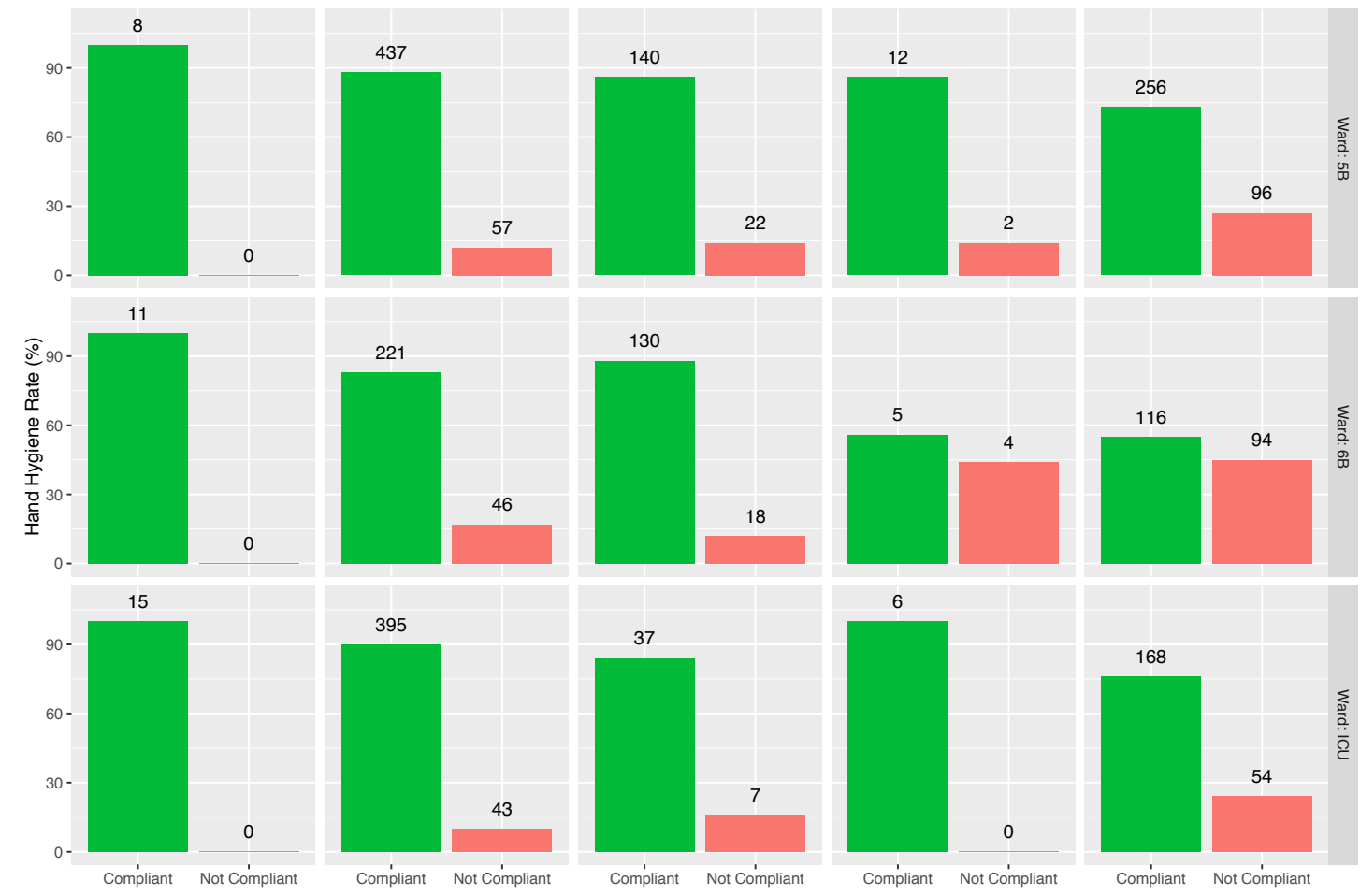

Compliance Compliant Not Compliant

Figure 5.18 Inpatient-Wards Hand Hygiene Rate Summarized by My Five Moments

Combining the inpatient wards summary of "My five moments for hand hygiene" (WHO, 2009) is as follows. Purell outweigh Soap for all moments at 5B and 6B as can be seen in Figure 5.19 and Table 5.6. It seems that HCWs at ICU prefer to disinfect their hands with soap and water more than with alcohol-based handrub for before clean/aseptic procedures and after body fluid exposure risk.

Aggregating separately all Purell and Soap observations per ward reveals that more observations are collected at $5 \mathrm{~B}, \mathrm{ICU}$ and then $6 \mathrm{~B}$. Though these data are aggregated for four years, 2012 till 2015, the data only represents about 0.78 worth of 
Purell cartridge and 0.35 of Soap cartridge at 5B, 0.42 worth of Purell cartridge and 0.22 of Soap cartridge at $6 \mathrm{~B}$ and 0.47 worth of Purell cartridge and 0.37 of Soap cartridge at ICU as seen in Table 5.6.

\begin{tabular}{|c|c|c|c|c|c|c|c|c|}
\hline \multicolumn{9}{|c|}{$5 \mathrm{~B}$} \\
\hline & \multirow{2}{*}{$\begin{array}{c}\text { Five Moments for Hand } \\
\text { Hygiene }\end{array}$} & \multicolumn{3}{|c|}{ Product of Use } & \multirow[b]{2}{*}{ Total } & \multicolumn{3}{|c|}{ Compliance $\%$} \\
\hline & & \multirow{2}{*}{$\begin{array}{c}\text { No } \\
96 \\
\end{array}$} & \multirow{2}{*}{$\begin{array}{c}\text { Purell } \\
229\end{array}$} & \multirow{2}{*}{$\begin{array}{c}\text { Soap } \\
27\end{array}$} & & \multirow{2}{*}{$\begin{array}{c}\begin{array}{c}\text { No } \\
\%\end{array} \\
27 \% \\
\end{array}$} & \multirow{2}{*}{$\begin{array}{c}\text { Purell } \\
\% \\
65 \%\end{array}$} & \multirow{2}{*}{$\begin{array}{c}\text { Soap } \\
\%\end{array}$} \\
\hline 1 & Before Touching a Patient & & & & 352 & & & \\
\hline 2 & $\begin{array}{l}\text { Before Clean/Aseptic } \\
\text { Procedures }\end{array}$ & 2 & 9 & 3 & 14 & $14 \%$ & $64 \%$ & $21 \%$ \\
\hline 3 & $\begin{array}{l}\text { After Body Fluid } \\
\text { Exposure/Risk }\end{array}$ & 0 & 4 & 4 & 8 & $0 \%$ & $50 \%$ & $50 \%$ \\
\hline 4 & After Touc & 57 & 283 & 154 & 494 & $12 \%$ & $57 \%$ & $31 \%$ \\
\hline 5 & $\begin{array}{l}\text { After Tou } \\
\text { Surroundi }\end{array}$ & 22 & 97 & 43 & 162 & $14 \%$ & $60 \%$ & $27 \%$ \\
\hline \multicolumn{9}{|c|}{$6 \mathrm{~B}$} \\
\hline \multirow{2}{*}{\multicolumn{2}{|c|}{$\begin{array}{c}\text { Five Moments for Hand } \\
\text { Hygiene }\end{array}$}} & \multicolumn{3}{|c|}{ Product of Use } & & \multicolumn{3}{|c|}{ Compliance $\%$} \\
\hline & & No & Purell & Soap & Total & $\begin{array}{c}\text { No } \\
\%\end{array}$ & $\begin{array}{l}\text { Purell } \\
\%\end{array}$ & $\begin{array}{c}\text { Soap } \\
\%\end{array}$ \\
\hline 1 & Before Touch & 94 & 106 & 10 & 210 & $45 \%$ & $50 \%$ & $5 \%$ \\
\hline 2 & $\begin{array}{l}\text { Before Cle } \\
\text { Procedures }\end{array}$ & 4 & 4 & 1 & 9 & $44 \%$ & $44 \%$ & $11 \%$ \\
\hline 3 & $\begin{array}{l}\text { After Body } \\
\text { Exposure/Ri }\end{array}$ & 0 & 6 & 5 & 11 & $0 \%$ & $55 \%$ & $45 \%$ \\
\hline 4 & After & 46 & 138 & 83 & 267 & $17 \%$ & $52 \%$ & $31 \%$ \\
\hline 5 & $\begin{array}{l}\text { After Touching Patient } \\
\text { Surroundings }\end{array}$ & 18 & 82 & 48 & 148 & $12 \%$ & $55 \%$ & $32 \%$ \\
\hline \multicolumn{9}{|c|}{ ICU } \\
\hline \multirow{2}{*}{\multicolumn{2}{|c|}{$\begin{array}{c}\text { Five Moments for Hand } \\
\text { Hygiene }\end{array}$}} & \multicolumn{3}{|c|}{ Product of Use } & & \multicolumn{3}{|c|}{ Compliance $\%$} \\
\hline & & No & Purell & Soap & Total & $\begin{array}{l}\text { No } \\
\%\end{array}$ & $\begin{array}{c}\text { Purell } \\
\%\end{array}$ & $\begin{array}{c}\text { Soap } \\
\%\end{array}$ \\
\hline 1 & Before To & 54 & 149 & 19 & 222 & $24 \%$ & $67 \%$ & $9 \%$ \\
\hline 2 & $\begin{array}{l}\text { Before Clean/Aseptic } \\
\text { Procedures }\end{array}$ & 0 & 1 & 5 & 6 & $0 \%$ & $17 \%$ & $83 \%$ \\
\hline 3 & $\begin{array}{l}\text { After Body Fluid } \\
\text { Exposure/Risk }\end{array}$ & 0 & 6 & 9 & 15 & $0 \%$ & $40 \%$ & $60 \%$ \\
\hline 4 & After Touching a Patien & 43 & 197 & 198 & 438 & $10 \%$ & $45 \%$ & $45 \%$ \\
\hline 5 & $\begin{array}{l}\text { After Touching Patient } \\
\text { Surroundings }\end{array}$ & 7 & 20 & 17 & 44 & $16 \%$ & $45 \%$ & $39 \%$ \\
\hline
\end{tabular}

Table 5.5 Inpatient-Wards Hand Hygiene Adherence Rates Summarized by My Five Moments and Hygiene Products 


\begin{tabular}{|c|c|c|}
\hline \multirow{2}{*}{ Ward } & \multicolumn{2}{|c|}{ Hygiene Products Cartridges } \\
\cline { 2 - 3 } & Purell & Soap \\
\hline 5B & 0.78 & 0.35 \\
\hline 6B & 0.42 & 0.22 \\
\hline ICU & 0.47 & 0.37 \\
\hline
\end{tabular}

Table 5.6 Inpatient-Wards Hand Hygiene Observations Converted to Purell and Soap Cartridges

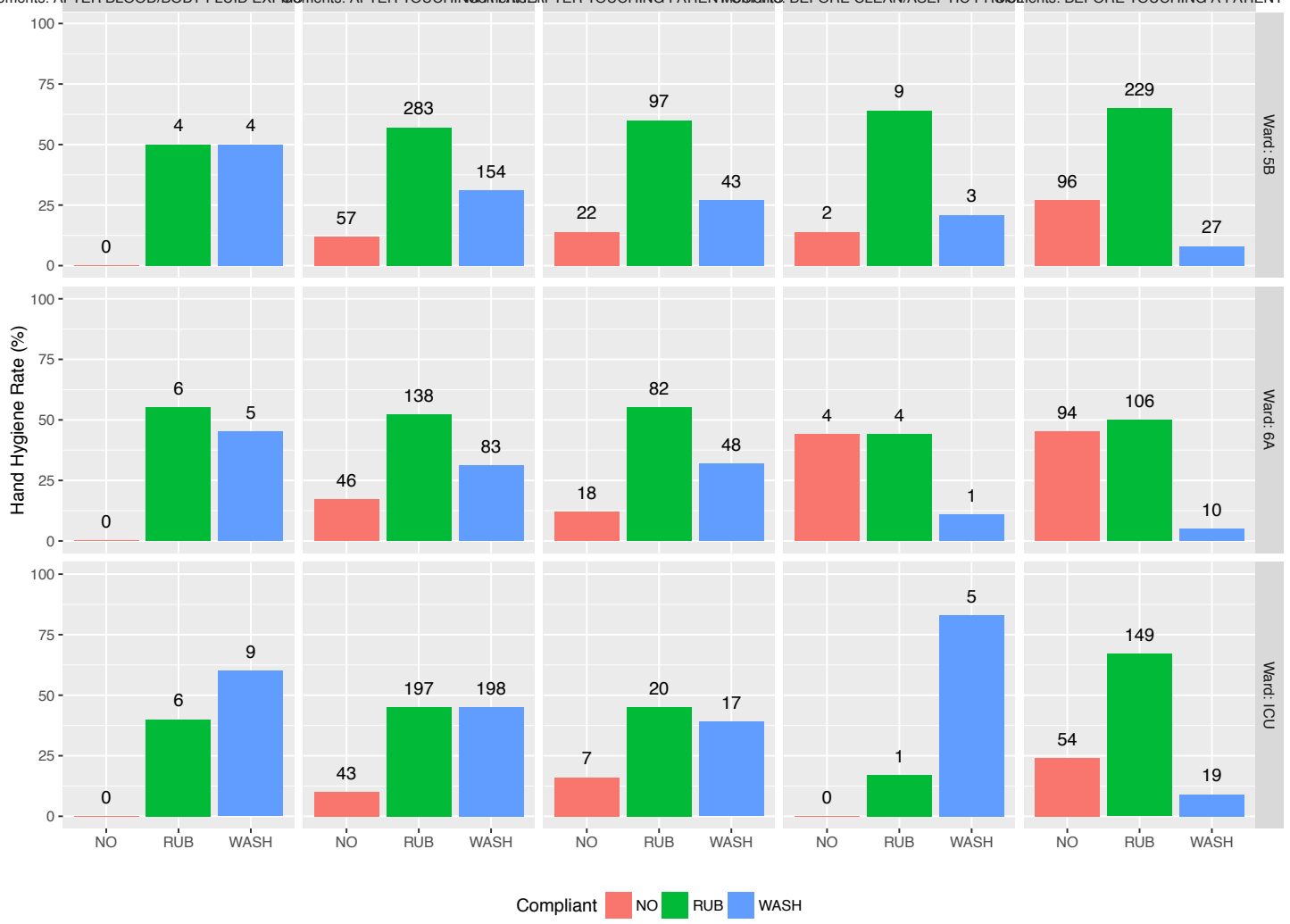

Figure 5.19 Inpatient-Wards Hand Hygiene Observations Summarized by My Five Moments and Hygiene Products

Hand hygiene compliance per inpatient wards is also summarized by year as depicted in Figure 5.20. The hand hygiene adherence rates for 5B are 83\% (230 samples), $88 \%$ (221 samples), 78\% (452 samples), and 91\% (127 samples) for 2012, 2013, 2014 and 2015 respectively. The hand hygiene adherence rates for $6 \mathrm{~B}$ are $79 \%$ (135 samples), $87 \%$ (155 samples), 67\% (331 samples), and 88\% (24 samples) for 
2012, 2013, 2014 and 2015 respectively. The hand hygiene adherence rates for ICU are $86 \%$ (185 samples), $89 \%$ (129 samples), 82\% (286 samples), and 89\% (125 samples) for 2012, 2013, 2014 and 2015 respectively. In general, there is an indication that the larger the samples, the smaller the rates. This could lead to the fact that if very large samples are collected, the hand hygiene rate of the PVAMC could be low.

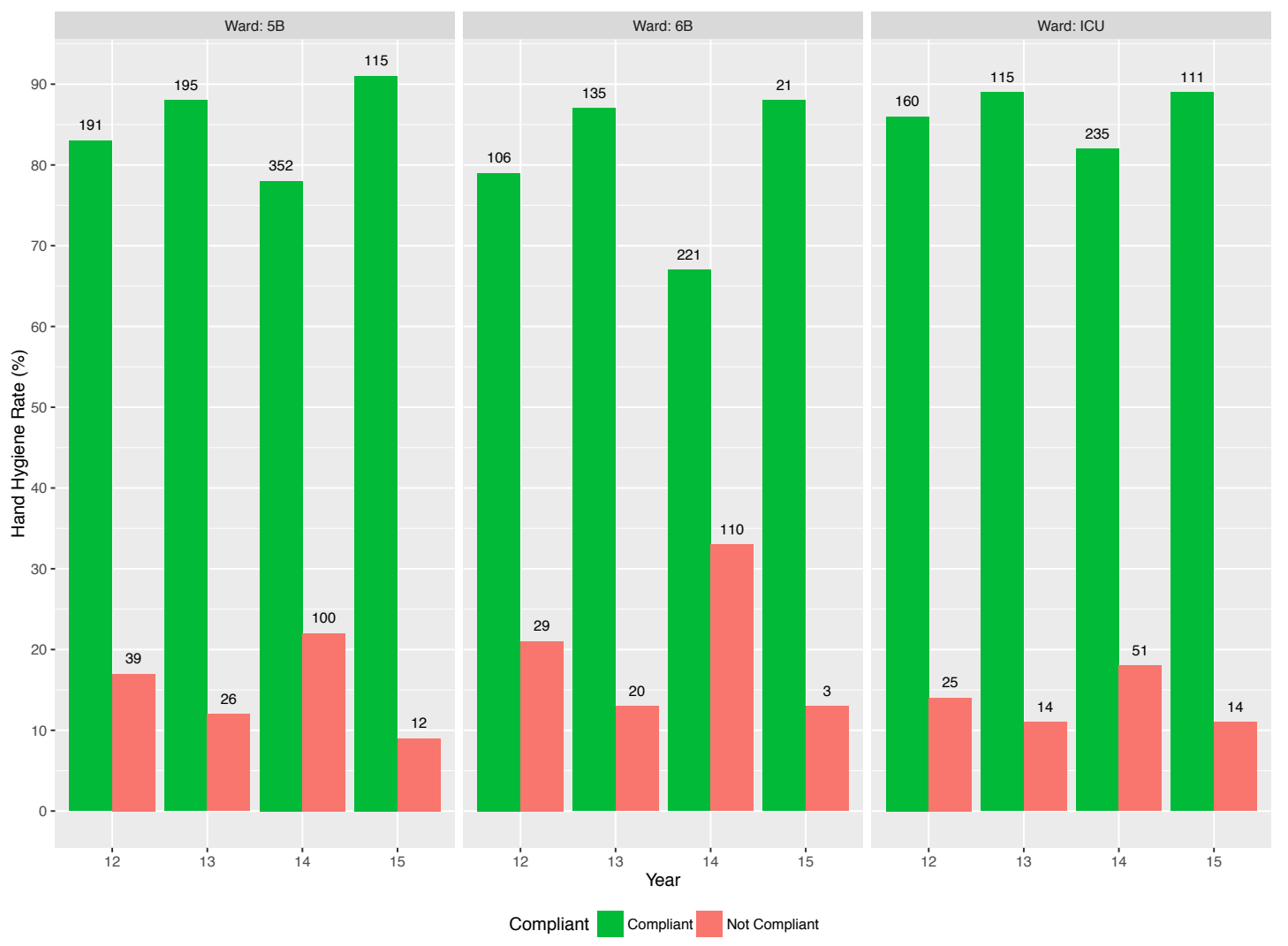

Figure 5.20 Inpatient-Wards Hand Hygiene Observations and Compliance Summarized by Year

Hand hygiene compliance per inpatient ward is summarized by employee class in Figure 5.21. Only the Medical group (Attending, Fellow, Resident, Interns and Medical Student), Nursing group (NP, RN, LPN and NS) and EMS employee classes are analyzed. Figure 5.21 shows that higher numbers of observations are captured for RN and LPN, attending and fellow, and residents, interns and medical students, 
because these groups of healthcare workers have higher patient contact. Comparing rates of employee class across inpatient wards reveals that, in general, RN and LPN has an adherence rate that is above $85 \%$, and they are considered to be the group with the highest patient contact. The rates are $89 \%, 85 \%$ and $95 \%$ for $5 \mathrm{~B}, 6 \mathrm{~B}$ and ICU respectively. Disappointingly, Medical group (attending and fellow, residents, interns and medical students) has a rate that is below $80 \%$; yet, their patient contact is not comparable with RN and LPN. The rates of attending and fellow are $73 \%, 59 \%$ and $74 \%$ for $5 \mathrm{~B}, 6 \mathrm{~B}$ and ICU respectively. The rates of residents, interns and medical students are $78 \%, 61 \%$ and $68 \%$ for $5 \mathrm{~B}, 6 \mathrm{~B}$ and ICU respectively. A very critical group of interest is EMS because of the role they play on the floor of each inpatient ward, which is taking care of patient room and floor cleaning. The rates are $31 \%, 29 \%$ and $29 \%$ for $5 \mathrm{~B}, 6 \mathrm{~B}$ and ICU, respectively, based on very small samples. However, based on such small samples this conclusion could be misleading. This sample size is for four year worth of data. 


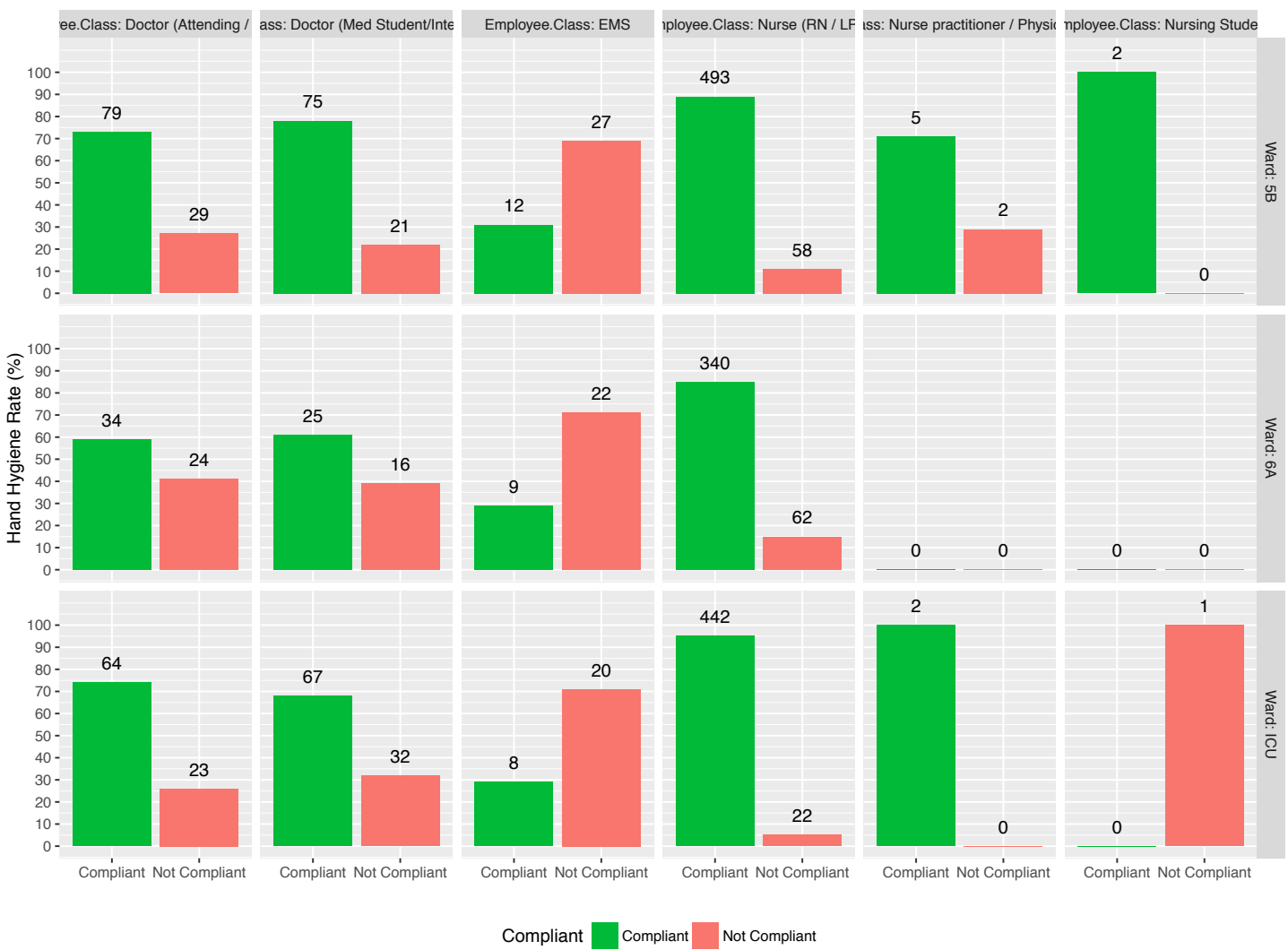

Figure 5.21 Inpatient-Wards Hand Hygiene Adherence Rate Summarized by Employee Class

Summarizing hand hygiene adherence rate per inpatient-wards by hand hygiene moments and employee class together reveals important facts as can be seen in Figure 5.22 and Table 5.7, Table 5.8, Table 5.9, Table 5.10, Table 5.11 and Table 5.12. The medical group (attending, fellow) hand hygiene rate before touching a patient is very low, $56 \%, 37 \%$ and $56 \%$ for $5 \mathrm{~B}, 6 \mathrm{~B}$ and ICU respectively. On the other hand, the medical group (attending, fellow) hand hygiene rate after touching a patient is the opposite, $89 \%, 77 \%$ and $89 \%$ for $5 \mathrm{~B}, 6 \mathrm{~B}$ and ICU respectively. The medical group (residents, interns and medical students) hand hygiene rate before touching a patient is very low, $75 \%, 64 \%$ and $61 \%$ for $5 \mathrm{~B}, 6 \mathrm{~B}$ and ICU respectively. On the other hand, the medical group (residents, interns and medical students) hand hygiene rate after 
touching a patient is $81 \%, 54 \%$ and $70 \%$ for $5 \mathrm{~B}, 6 \mathrm{~B}$ and ICU respectively. The nursing group ( $\mathrm{RN}$ and LPN) hand hygiene rate before touching a patient is better than the medical groups but still is low for $6 \mathrm{~B}, 82 \%, 67 \%$ and $91 \%$ for $5 \mathrm{~B}, 6 \mathrm{~B}$ and ICU respectively. On the other hand, the nursing group (RN and LPN) hand hygiene rate after touching a patient is very high, $93 \%, 87 \%$ and $98 \%$ for $5 \mathrm{~B}, 6 \mathrm{~B}$ and ICU respectively. Again, $\mathrm{HCWs}$ seem to underestimate practicing hand hygiene before touching a patient and value that after touching a patient.

Interestingly, Figure 5.22 shows that the most frequent hand hygiene moments conducted by EMS are after touching patient surroundings, before touching a patient and after touching a patient. However, rates are very low for all three moments across inpatient wards. Additionally, data analysis shows that there is only one observation corresponding to Before Clean/Aseptic Procedures moment for 5B and 6B and EMS has no observations after body fluid exposure risk moment though their role is to clean patient rooms and to make beds with or without the existence of a patient. In general, EMS has to be considered for further hand hygiene observations and analysis. 


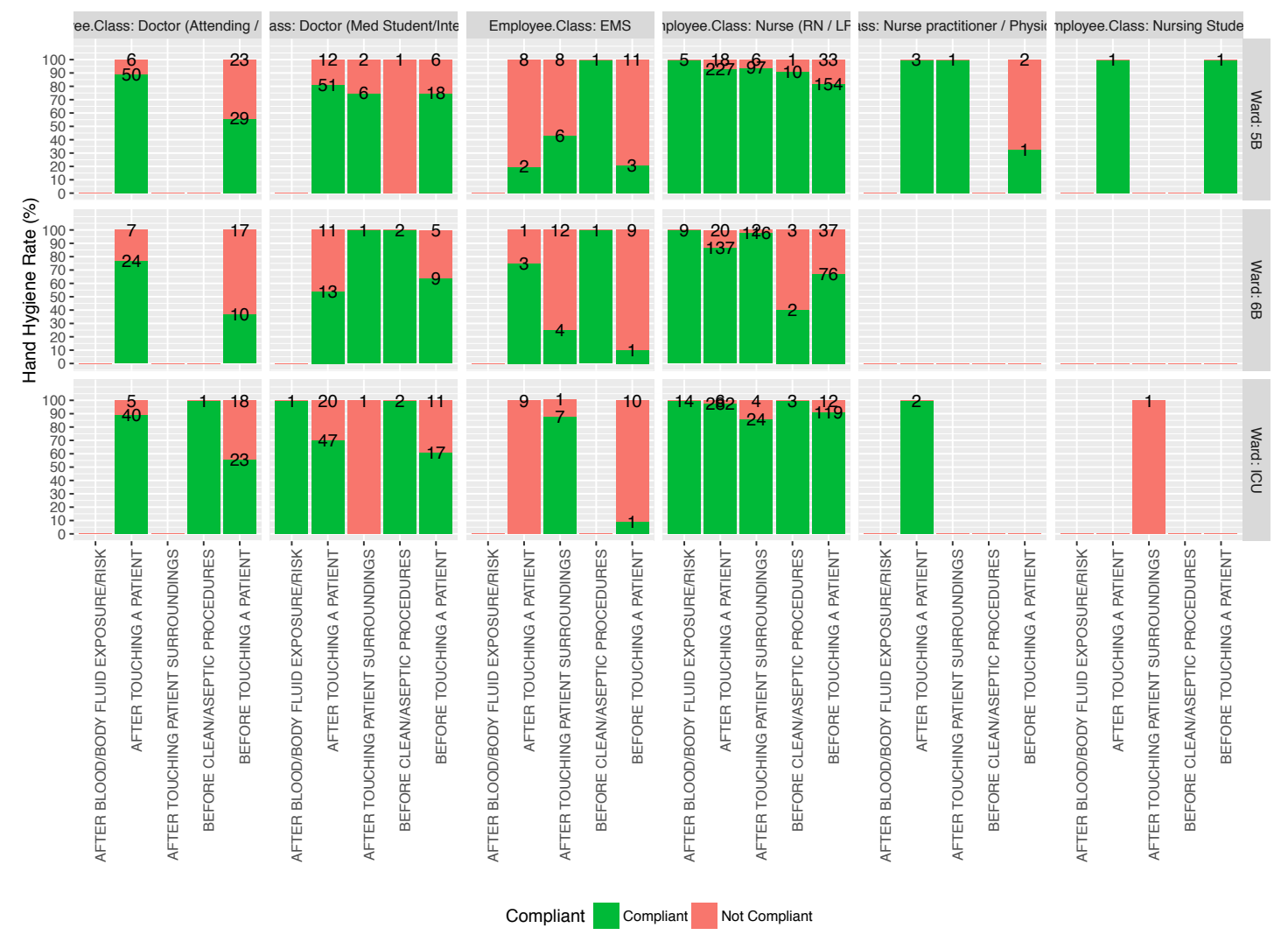

Figure 5.22 Inpatient-Wards Hand Hygiene Observations and Compliance Summarized by Employee Class and My Five Moments 


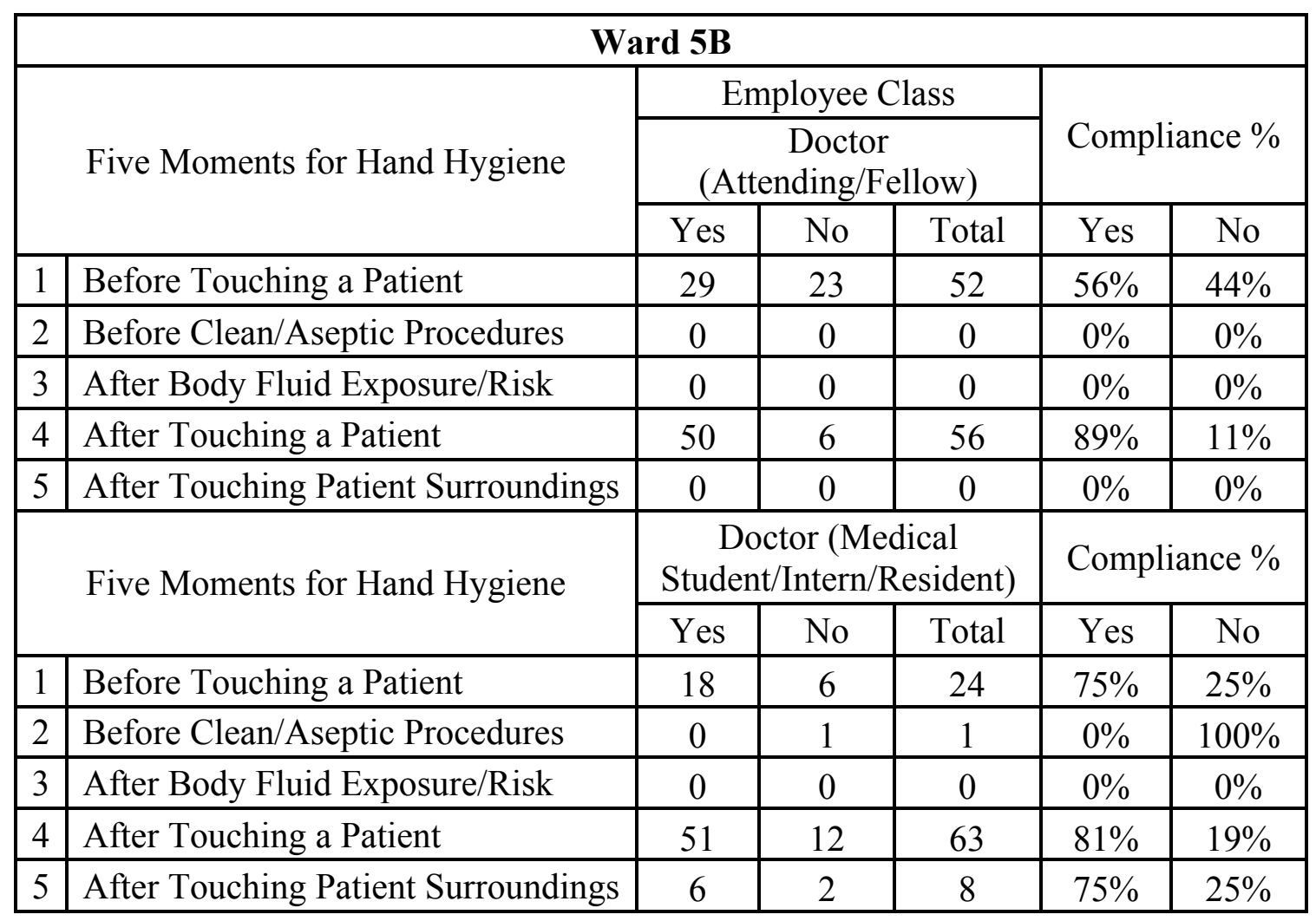

Table 5.7 (A) 5B Inpatient-Ward Hand Hygiene Rate Summarized by Employee Class and My Five Moments 


\begin{tabular}{|c|c|c|c|c|c|c|}
\hline & \multirow{2}{*}{ Five Moments for Hand Hygiene } & \multicolumn{3}{|c|}{ Nurse Practitioner (NP) } & \multicolumn{2}{|c|}{ Compliance $\%$} \\
\hline & & Yes & No & Total & Yes & No \\
\hline 1 & Before Touching a Patient & 1 & 2 & 3 & $33 \%$ & $67 \%$ \\
\hline 2 & Before Clean/Aseptic Procedures & 0 & 0 & 0 & $0 \%$ & $0 \%$ \\
\hline 3 & After Body Fluid Exposure/Risk & 0 & 0 & 0 & $0 \%$ & $0 \%$ \\
\hline 4 & After Touching a Patient & 3 & 0 & 3 & $100 \%$ & $0 \%$ \\
\hline \multirow[t]{3}{*}{5} & After Touching Patient Surro & 1 & 0 & 1 & $100 \%$ & $0 \%$ \\
\hline & \multirow{2}{*}{ Five Moments for Hand Hygiene } & \multicolumn{3}{|c|}{ Nurse (RN/LPN) } & \multicolumn{2}{|c|}{ Compliance $\%$} \\
\hline & & Yes & No & Total & \multicolumn{2}{|l|}{ Yes } \\
\hline 1 & Before Touching a Patient & 154 & 33 & 187 & $82 \%$ & $18 \%$ \\
\hline 2 & Before Clean/Aseptic Procedures & 10 & 1 & 11 & $91 \%$ & $9 \%$ \\
\hline 3 & After Body Fluid Exposure/Risk & 5 & 0 & 5 & $100 \%$ & $0 \%$ \\
\hline 4 & After Touching a Patient & 227 & 18 & 245 & $93 \%$ & $7 \%$ \\
\hline 5 & After Touching Patient Surroundings & 97 & 6 & 103 & $94 \%$ & $6 \%$ \\
\hline \multirow{2}{*}{\multicolumn{2}{|c|}{ Five Moments for Hand Hygiene }} & \multicolumn{3}{|c|}{ Nurse (Students) } & \multicolumn{2}{|c|}{ Compliance $\%$} \\
\hline & & Yes & No & Total & Yes & No \\
\hline 1 & Before Touching a Patient & 1 & 0 & 1 & $100 \%$ & $0 \%$ \\
\hline 2 & Before Clean/Aseptic Procedures & 0 & 0 & 0 & $0 \%$ & $0 \%$ \\
\hline 3 & After Body Fluid Exposure/Risk & 0 & 0 & 0 & $0 \%$ & $0 \%$ \\
\hline 4 & After Touching a Patient & 1 & 0 & 1 & $100 \%$ & $0 \%$ \\
\hline \multirow[t]{3}{*}{5} & After Touching Patient Surroundings & 0 & 0 & 0 & $0 \%$ & $0 \%$ \\
\hline & \multirow{2}{*}{ Five Moments for Hand Hygiene } & \multicolumn{3}{|c|}{ EMS } & \multicolumn{2}{|c|}{ Compliance $\%$} \\
\hline & & Yes & No & Total & Yes & No \\
\hline 1 & Before Touching a Patient & 3 & 11 & 14 & $21 \%$ & $79 \%$ \\
\hline 2 & Before Clean/Aseptic Procedures & 1 & 0 & 1 & $100 \%$ & $0 \%$ \\
\hline 3 & After Body Fluid Exposure/Risk & 0 & 0 & 0 & $0 \%$ & $0 \%$ \\
\hline 4 & After Touching a Patient & 2 & 8 & 10 & $20 \%$ & $80 \%$ \\
\hline 5 & After Touching Patient Surroundings & 6 & 8 & 14 & $43 \%$ & $57 \%$ \\
\hline
\end{tabular}

Table 5.8 (B) 5B Inpatient-Ward Hand Hygiene Rate Summarized by Employee Class and My Five Moments 


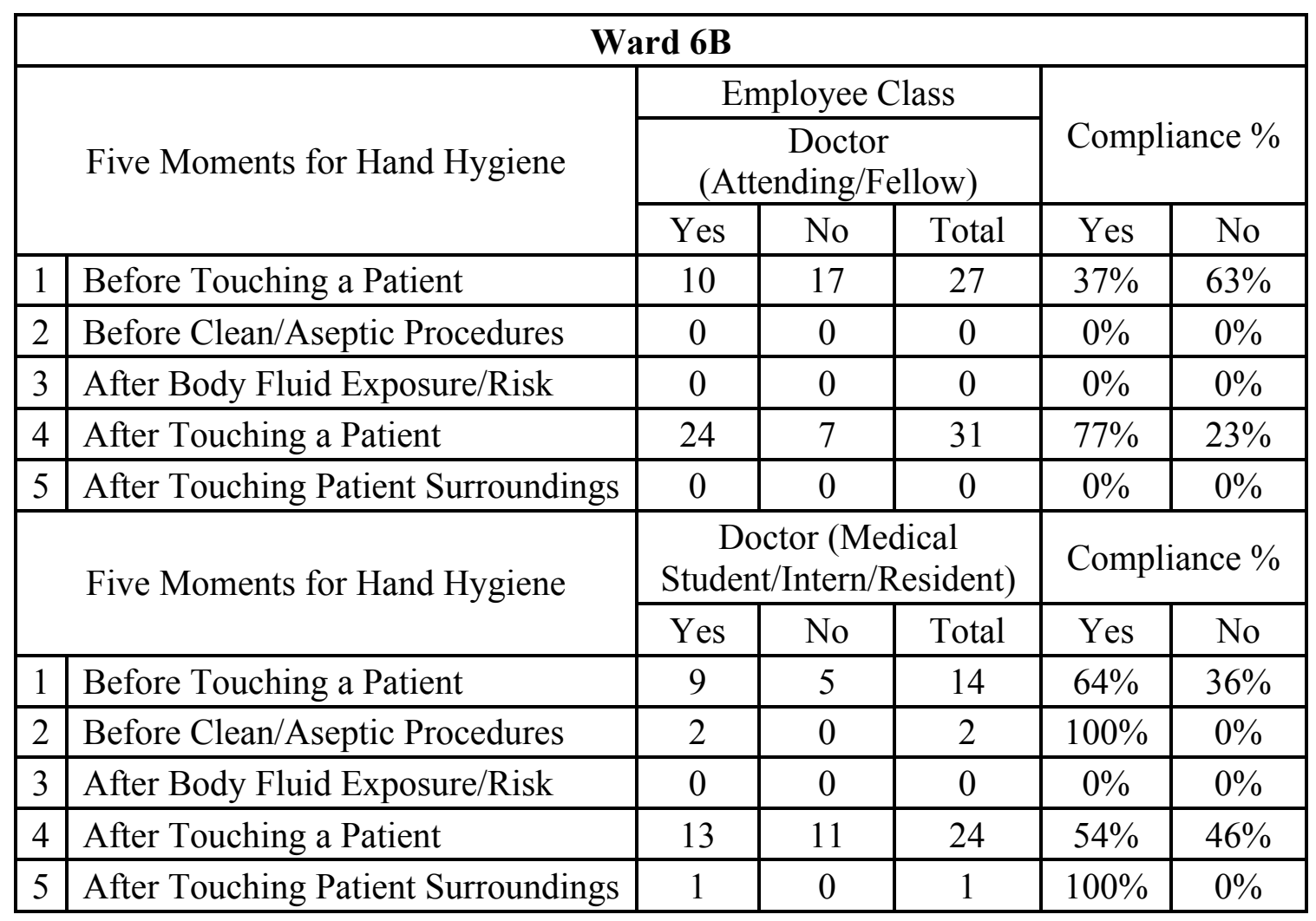

Table 5.9 (A) 6B Inpatient-Ward Hand Hygiene Rate Summarized by Employee Class and My Five Moments 


\begin{tabular}{|c|c|c|c|c|c|c|}
\hline & \multirow{2}{*}{ Five Moments for Hand Hygiene } & \multicolumn{3}{|c|}{ Nurse Practitioner (NP) } & \multicolumn{2}{|c|}{ Compliance $\%$} \\
\hline & & \multirow{2}{*}{$\begin{array}{c}\text { Yes } \\
0\end{array}$} & \multirow{2}{*}{$\frac{\text { No }}{0}$} & \multirow{2}{*}{$\begin{array}{c}\text { Total } \\
0\end{array}$} & \multirow{2}{*}{$\begin{array}{l}\text { Yes } \\
0 \%\end{array}$} & \multirow{2}{*}{$\begin{array}{l}\text { No } \\
0 \%\end{array}$} \\
\hline 1 & Before Touching a Patient & & & & & \\
\hline 2 & Before Clean/Aseptic Procedures & 0 & 0 & 0 & $0 \%$ & $0 \%$ \\
\hline 3 & After Body Fluid Exposure/Risk & 0 & 0 & 0 & $0 \%$ & $0 \%$ \\
\hline 4 & After Touching a Patient & 0 & 0 & 0 & $0 \%$ & $0 \%$ \\
\hline 5 & After Touching Patient Surroundings & 0 & 0 & 0 & $0 \%$ & $0 \%$ \\
\hline \multirow{2}{*}{\multicolumn{2}{|c|}{ Five Moments for Hand Hygiene }} & \multicolumn{3}{|c|}{ Nurse (RN/LPN) } & \multicolumn{2}{|c|}{ Compliance $\%$} \\
\hline & & Yes & No & Total & Yes & No \\
\hline 1 & Before Touching a Patient & 76 & 37 & 113 & $67 \%$ & $33 \%$ \\
\hline 2 & Before Clean/Aseptic Procedures & 2 & 3 & 5 & $40 \%$ & $60 \%$ \\
\hline 3 & After Body Fluid Exposure/Risk & 9 & 0 & 9 & $100 \%$ & $0 \%$ \\
\hline 4 & After Touching a Patient & 137 & 20 & 157 & $87 \%$ & $13 \%$ \\
\hline 5 & After Touching Patient Surroundings & 116 & 2 & 118 & $98 \%$ & $2 \%$ \\
\hline \multirow{2}{*}{\multicolumn{2}{|c|}{ Five Moments for Hand Hygiene }} & \multicolumn{3}{|c|}{ Nurse (Students) } & \multicolumn{2}{|c|}{ Compliance $\%$} \\
\hline & & Yes & No & Total & Yes & No \\
\hline 1 & Before Touching a Patient & 0 & 0 & 0 & $0 \%$ & $0 \%$ \\
\hline 2 & Before Clean/Aseptic Procedures & 0 & 0 & 0 & $0 \%$ & $0 \%$ \\
\hline 3 & After Body Fluid Exposure/Risk & 0 & 0 & 0 & $0 \%$ & $0 \%$ \\
\hline 4 & After Touching a Patient & 0 & 0 & 0 & $0 \%$ & $0 \%$ \\
\hline 5 & After Touching Patient Surroundings & 0 & 0 & 0 & $0 \%$ & $0 \%$ \\
\hline \multirow{2}{*}{\multicolumn{2}{|c|}{ Five Moments for Hand Hygiene }} & \multicolumn{3}{|c|}{ EMS } & \multicolumn{2}{|c|}{ Compliance $\%$} \\
\hline & & Yes & No & Total & Yes & No \\
\hline 1 & Before Touching a Patient & 1 & 9 & 10 & $10 \%$ & $90 \%$ \\
\hline 2 & Before Clean/Aseptic Procedures & 1 & 0 & 1 & $100 \%$ & $0 \%$ \\
\hline 3 & After Body Fluid Exposure/Risk & 0 & 0 & 0 & $0 \%$ & $0 \%$ \\
\hline 4 & After Touching a Patient & 3 & 1 & 4 & $75 \%$ & $25 \%$ \\
\hline 5 & After Touching Patient Surroundings & 4 & 12 & 16 & $25 \%$ & $75 \%$ \\
\hline
\end{tabular}

Table 5.10 (B) 6B Inpatient-Ward Hand Hygiene Rate Summarized by Employee Class and My Five Moments 


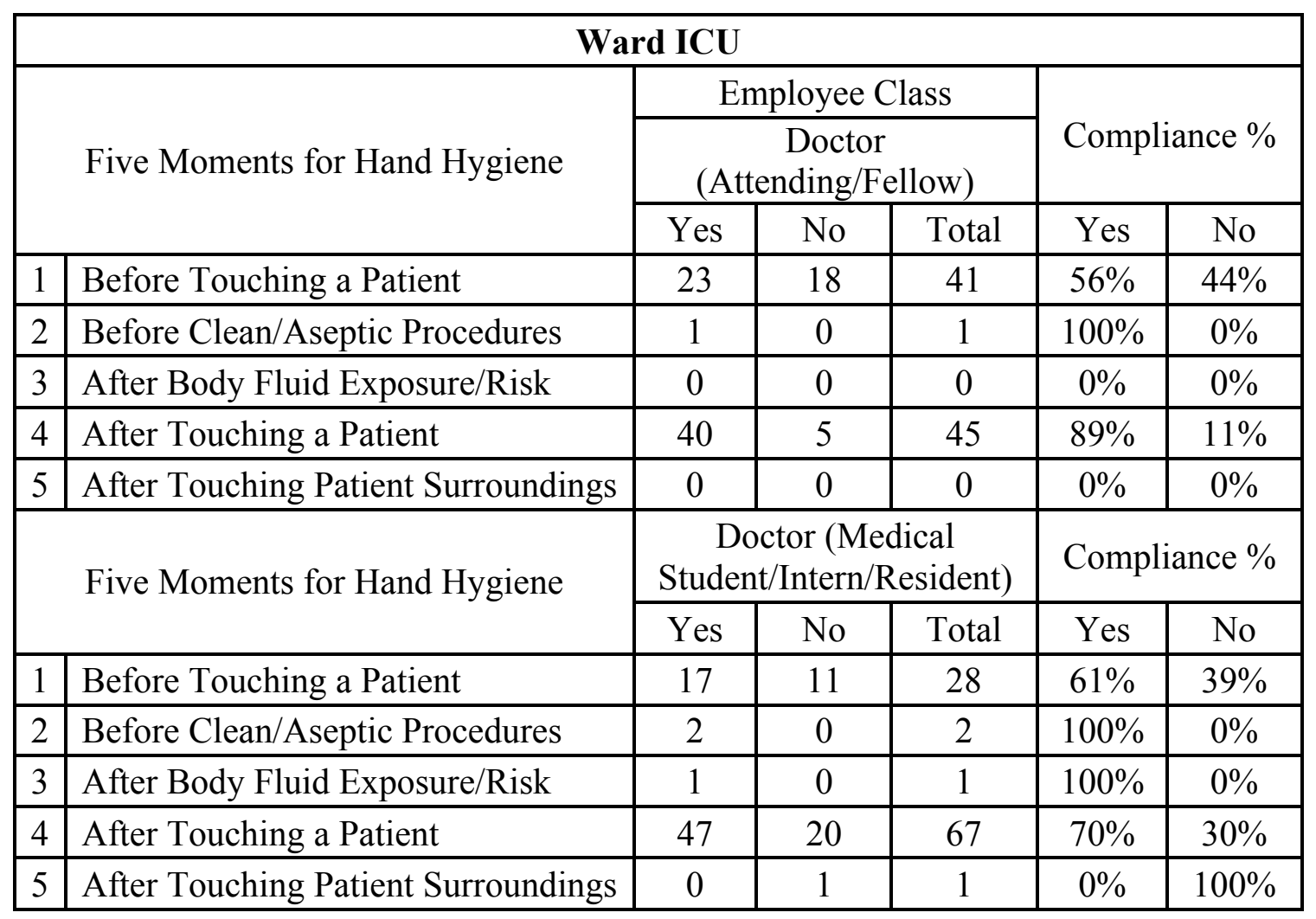

Table 5.11 (A) ICU Inpatient-Ward Hand Hygiene Rate Summarized by Employee Class and My Five Moments 


\begin{tabular}{|c|c|c|c|c|c|c|}
\hline & \multirow{2}{*}{ Five Moments for Hand Hygiene } & \multicolumn{3}{|c|}{ Nurse Practitioner (NP) } & \multicolumn{2}{|c|}{ Compliance $\%$} \\
\hline & & \multirow{2}{*}{$\begin{array}{c}\text { Yes } \\
0\end{array}$} & \multirow{2}{*}{$\frac{\text { No }}{0}$} & \multirow{2}{*}{$\frac{\text { Total }}{0}$} & \multirow{2}{*}{$\begin{array}{l}\text { Yes } \\
0 \%\end{array}$} & \multirow{2}{*}{$\begin{array}{l}\text { No } \\
0 \%\end{array}$} \\
\hline 1 & Before Touching a Patient & & & & & \\
\hline 2 & Before Clean/Aseptic Procedures & 0 & 0 & 0 & $0 \%$ & $0 \%$ \\
\hline 3 & After Body Fluid Exposure/Risk & 0 & 0 & 0 & $0 \%$ & $0 \%$ \\
\hline 4 & After Touching a Patient & 2 & 0 & 2 & $100 \%$ & $0 \%$ \\
\hline 5 & After Touching Patient Surroundings & 0 & 0 & 0 & $0 \%$ & $0 \%$ \\
\hline \multirow{2}{*}{\multicolumn{2}{|c|}{ Five Moments for Hand Hygiene }} & \multicolumn{3}{|c|}{ Nurse (RN/LPN) } & \multicolumn{2}{|c|}{ Compliance $\%$} \\
\hline & & Yes & No & Total & Yes & No \\
\hline 1 & Before Touching a Patient & 119 & 12 & 131 & $91 \%$ & $9 \%$ \\
\hline 2 & Before Clean/Aseptic Procedures & 3 & 0 & 3 & $100 \%$ & $0 \%$ \\
\hline 3 & After Body Fluid Exposure/Risk & 14 & 0 & 14 & $100 \%$ & $0 \%$ \\
\hline 4 & After Touching a Patient & 282 & 6 & 288 & $98 \%$ & $2 \%$ \\
\hline 5 & After Touching Patient Surroundings & 24 & 4 & 28 & $86 \%$ & $14 \%$ \\
\hline \multirow{2}{*}{\multicolumn{2}{|c|}{ Five Moments for Hand Hygiene }} & \multicolumn{3}{|c|}{ Nurse (Students) } & \multicolumn{2}{|c|}{ Compliance \% } \\
\hline & & Yes & No & Total & Yes & No \\
\hline 1 & Before Touching a Patient & 0 & 0 & 0 & $0 \%$ & $0 \%$ \\
\hline 2 & Before Clean/Aseptic Procedures & 0 & 0 & 0 & $0 \%$ & $0 \%$ \\
\hline 3 & After Body Fluid Exposure/Risk & 0 & 0 & 0 & $0 \%$ & $0 \%$ \\
\hline 4 & After Touching a Patient & 0 & 0 & 0 & $0 \%$ & $0 \%$ \\
\hline 5 & After Touching Patient Surroundings & 0 & 1 & 1 & $0 \%$ & $100 \%$ \\
\hline \multirow{2}{*}{\multicolumn{2}{|c|}{ Five Moments for Hand Hygiene }} & \multicolumn{3}{|c|}{ EMS } & \multicolumn{2}{|c|}{ Compliance $\%$} \\
\hline & & Yes & No & Total & Yes & No \\
\hline 1 & Before Touching a Patient & 1 & 10 & 11 & $9 \%$ & $91 \%$ \\
\hline 2 & Before Clean/Aseptic Procedures & 0 & 0 & 0 & $0 \%$ & $0 \%$ \\
\hline 3 & After Body Fluid Exposure/Risk & 0 & 0 & 0 & $0 \%$ & $0 \%$ \\
\hline 4 & After Touching a Patient & 0 & 9 & 9 & $0 \%$ & $100 \%$ \\
\hline 5 & After Touching Patient Surroundings & 7 & 1 & 8 & $88 \%$ & $13 \%$ \\
\hline
\end{tabular}

Table 5.12 (B) ICU Inpatient-Ward Hand Hygiene Rate Summarized by Employee Class and My Five Moments

Hand hygiene rates per inpatient-wards by employee class, hand hygiene moments and hygiene products is depicted in Figure 5.23 and summarized in Table 5.13, Table 5.14 and Table 5.15. In general, Purell outweighs Soap for all hand 
hygiene moments at 5B, 6B and ICU except for the nursing group (RN and LPN) at ICU for all hand hygiene moments except for before touching a patient.

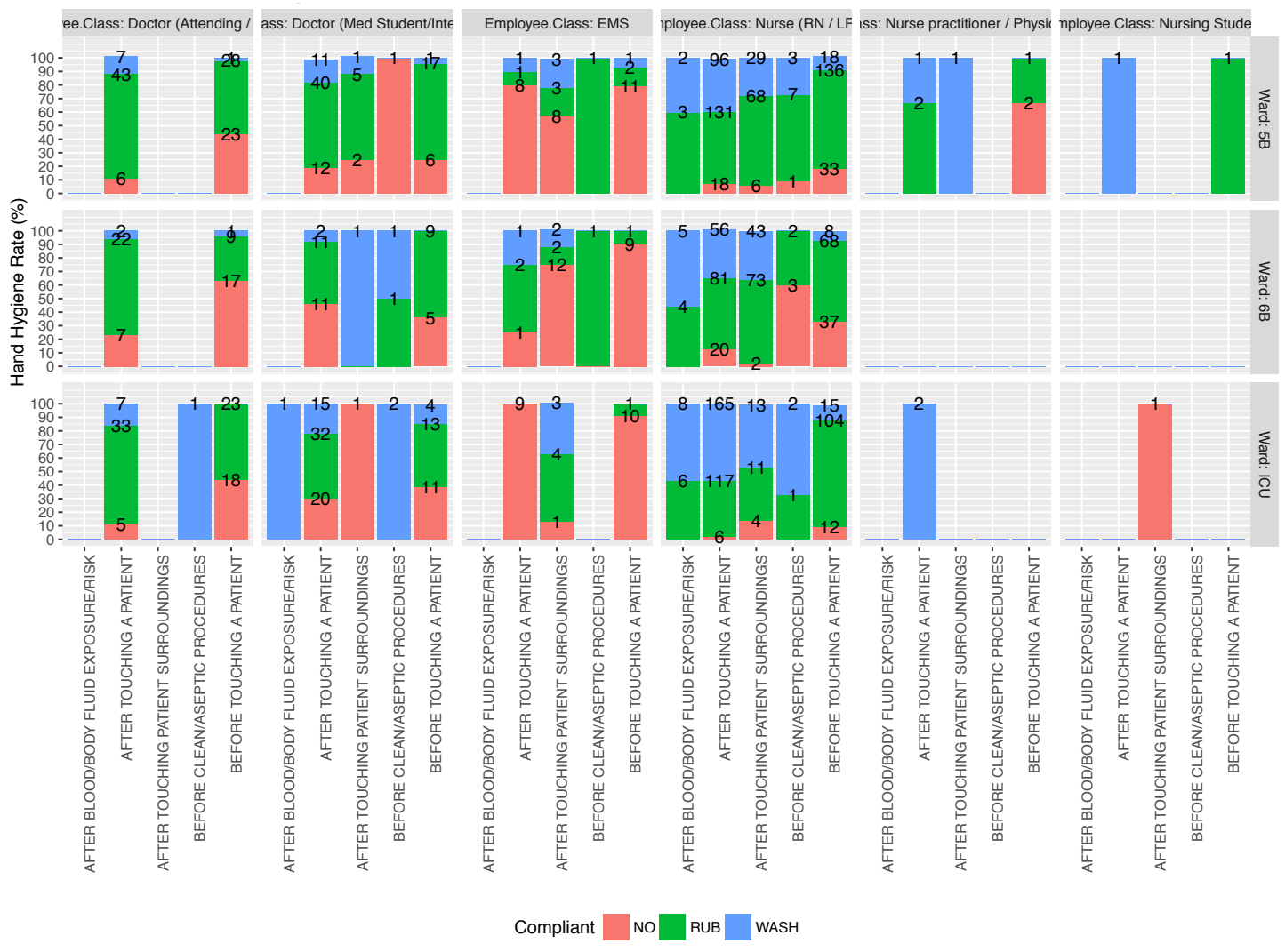

Figure 5.23 Inpatient-Wards Hand Hygiene Observations and Compliance Summarized by Employee Class, My Five Moments and Hygiene Products 


\begin{tabular}{|c|c|c|c|c|c|c|c|}
\hline \multicolumn{8}{|c|}{ Ward 5B } \\
\hline & \multirow{3}{*}{ Five Moments for Hand Hygiene } & \multirow{2}{*}{\multicolumn{3}{|c|}{$\begin{array}{c}\text { Employee Class } \\
\text { Doctor } \\
\text { (Attending/Fellow) }\end{array}$}} & \multirow{2}{*}{\multicolumn{3}{|c|}{ Compliance $\%$}} \\
\hline & & & & & & & \\
\hline & & No & Purell & Soap & No $\%$ & $\begin{array}{c}\text { Purell } \\
\%\end{array}$ & $\begin{array}{c}\text { Soap } \\
\%\end{array}$ \\
\hline 1 & Before Touching a Patient & 23 & 28 & 1 & $44 \%$ & $54 \%$ & $2 \%$ \\
\hline 2 & $\begin{array}{l}\text { Before Clean/Aseptic } \\
\text { Procedures }\end{array}$ & 0 & 0 & 0 & $0 \%$ & $0 \%$ & $0 \%$ \\
\hline 3 & After Body Fluid Exposure/Risk & 0 & 0 & 0 & $0 \%$ & $0 \%$ & $0 \%$ \\
\hline 4 & After Touching a Patient & 6 & 43 & 7 & $11 \%$ & $77 \%$ & $13 \%$ \\
\hline 5 & $\begin{array}{l}\text { After Touching Patient } \\
\text { Surroundings }\end{array}$ & 0 & 0 & 0 & $0 \%$ & $0 \%$ & $0 \%$ \\
\hline \multirow{2}{*}{\multicolumn{2}{|c|}{ Five Moments for Hand Hygiene }} & \multicolumn{3}{|c|}{$\begin{array}{c}\text { Doctor (Medical } \\
\text { Student/Intern/Resid } \\
\text { ent) }\end{array}$} & \multicolumn{3}{|c|}{ Compliance $\%$} \\
\hline & & No & Purell & Soap & No $\%$ & $\begin{array}{c}\text { Purell } \\
\%\end{array}$ & $\begin{array}{c}\text { Soap } \\
\%\end{array}$ \\
\hline 1 & Before Touching a Patient & 6 & 17 & 1 & $25 \%$ & $71 \%$ & $4 \%$ \\
\hline 2 & $\begin{array}{l}\text { Before Clean/Aseptic } \\
\text { Procedures }\end{array}$ & 1 & 0 & 0 & $100 \%$ & $0 \%$ & $0 \%$ \\
\hline 3 & After Body Fluid Exposure/Risk & 0 & 0 & 0 & $0 \%$ & $0 \%$ & $0 \%$ \\
\hline 4 & After Touching a Patient & 12 & 40 & 11 & $19 \%$ & $63 \%$ & $17 \%$ \\
\hline 5 & $\begin{array}{l}\text { After Touching Patient } \\
\text { Surroundings }\end{array}$ & 2 & 5 & 1 & $25 \%$ & $63 \%$ & $13 \%$ \\
\hline
\end{tabular}

Table 5.13 (A) 5B Inpatient-Ward Hand Hygiene Rate Summarized by Employee Class, My Five Moments and Hygiene Products 


\begin{tabular}{|c|c|c|c|c|c|c|c|}
\hline \multirow{2}{*}{\multicolumn{2}{|c|}{ Five Moments for Hand Hygiene }} & \multicolumn{3}{|c|}{$\begin{array}{c}\text { Nurse Practitioner } \\
(\mathrm{NP})\end{array}$} & \multicolumn{3}{|c|}{ Compliance \% } \\
\hline & & No & Purell & Soap & $\begin{array}{l}\text { No } \\
\%\end{array}$ & $\begin{array}{c}\text { Purell } \\
\%\end{array}$ & $\begin{array}{c}\text { Soap } \\
\%\end{array}$ \\
\hline 1 & Before Touching a Patient & 11 & 2 & 1 & $79 \%$ & $14 \%$ & $7 \%$ \\
\hline 2 & Before Clean/Aseptic Procedures & 0 & 1 & 0 & $0 \%$ & $100 \%$ & $0 \%$ \\
\hline 3 & After Body Fluid Exposure/Risk & 0 & 0 & 0 & $0 \%$ & $0 \%$ & $0 \%$ \\
\hline 4 & After Touching a Patient & 8 & 1 & 1 & $80 \%$ & $10 \%$ & $10 \%$ \\
\hline 5 & $\begin{array}{l}\text { After } \\
\text { Surrot }\end{array}$ & 8 & 3 & 3 & $57 \%$ & $21 \%$ & $21 \%$ \\
\hline \multirow{2}{*}{\multicolumn{2}{|c|}{ Five Moments for Hand Hygiene }} & \multicolumn{3}{|c|}{ Nurse (RN/LPN) } & \multicolumn{3}{|c|}{ Compliance $\%$} \\
\hline & & No & Purell & Soap & $\begin{array}{l}\text { No } \\
\%\end{array}$ & $\begin{array}{l}\text { Purell } \\
\%\end{array}$ & $\begin{array}{c}\text { Soap } \\
\%\end{array}$ \\
\hline 1 & Before Tou & 33 & 136 & 18 & $18 \%$ & $73 \%$ & $10 \%$ \\
\hline 2 & Aseptic Procedures & 1 & 7 & 3 & $9 \%$ & $64 \%$ & $27 \%$ \\
\hline 3 & After Body $\mathrm{F}$ & 0 & 3 & 2 & $0 \%$ & $60 \%$ & $40 \%$ \\
\hline 4 & After Tol & 18 & 131 & 96 & $7 \%$ & 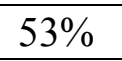 & $39 \%$ \\
\hline 5 & $\begin{array}{l}\text { After T } \\
\text { Surrou }\end{array}$ & 6 & 68 & 29 & $6 \%$ & $66 \%$ & $28 \%$ \\
\hline \multirow{2}{*}{\multicolumn{2}{|c|}{ Five Moments for Hand Hygiene }} & \multicolumn{3}{|c|}{ Nurse (Students) } & \multicolumn{3}{|c|}{ Compliance $\%$} \\
\hline & & No & ell & Soap & $\begin{array}{l}\text { No } \\
\%\end{array}$ & $\begin{array}{c}\text { Purell } \\
\%\end{array}$ & $\begin{array}{c}\text { Soap } \\
\%\end{array}$ \\
\hline 1 & Befor & 2 & 1 & 0 & $67 \%$ & $33 \%$ & $0 \%$ \\
\hline 2 & Before Cle & 0 & 0 & 0 & $0 \%$ & $0 \%$ & $0 \%$ \\
\hline 3 & After Body Fluid Exposure/Risk & 0 & 0 & 0 & $0 \%$ & $0 \%$ & $0 \%$ \\
\hline 4 & After Touching a Patient & 0 & 2 & 1 & $0 \%$ & $67 \%$ & $33 \%$ \\
\hline 5 & $\begin{array}{l}\text { After Touching Patient } \\
\text { Surroundings }\end{array}$ & 0 & 0 & 1 & $0 \%$ & $0 \%$ & $100 \%$ \\
\hline \multirow{2}{*}{\multicolumn{2}{|c|}{ Five Moments for Hand Hygiene }} & \multicolumn{3}{|c|}{ EMS } & \multicolumn{3}{|c|}{ Compliance $\%$} \\
\hline & & No & Purell & Soap & $\begin{array}{l}\text { No } \\
\%\end{array}$ & $\begin{array}{l}\text { Purell } \\
\%\end{array}$ & $\begin{array}{c}\text { Soap } \\
\%\end{array}$ \\
\hline 1 & Before Touching a Patient & 0 & 1 & 0 & $0 \%$ & $100 \%$ & $0 \%$ \\
\hline 2 & Before Clean/Aseptic Procedures & 0 & 0 & 0 & $0 \%$ & $0 \%$ & $0 \%$ \\
\hline 3 & After Body Fluid Exposure/Risk & 0 & 0 & 0 & $0 \%$ & $0 \%$ & $0 \%$ \\
\hline 4 & After Touching a Patient & 0 & 0 & 1 & $0 \%$ & $0 \%$ & $100 \%$ \\
\hline 5 & $\begin{array}{l}\text { After Touching Patient } \\
\text { Surroundings }\end{array}$ & 0 & 0 & 0 & $0 \%$ & $0 \%$ & $0 \%$ \\
\hline
\end{tabular}

Table 5.14 (B) 5B Inpatient-Ward Hand Hygiene Rate Summarized by Employee Class, My Five Moments and Hygiene Products 


\begin{tabular}{|c|c|c|c|c|c|c|c|}
\hline \multicolumn{8}{|c|}{ Ward 6B } \\
\hline & \multirow{3}{*}{ Five Moments for Hand Hygiene } & \multirow{2}{*}{\multicolumn{3}{|c|}{$\begin{array}{c}\text { Employee Class } \\
\text { Doctor } \\
\text { (Attending/Fellow) }\end{array}$}} & \multirow{2}{*}{\multicolumn{3}{|c|}{ Compliance $\%$}} \\
\hline & & & & & & & \\
\hline & & No & Purell & Soap & $\begin{array}{l}\text { No } \\
\%\end{array}$ & $\begin{array}{c}\text { Purell } \\
\%\end{array}$ & $\begin{array}{c}\text { Soap } \\
\%\end{array}$ \\
\hline 1 & Before Touching a Patient & 17 & 9 & 1 & $63 \%$ & $33 \%$ & $4 \%$ \\
\hline 2 & $\begin{array}{l}\text { Before Clean/Aseptic } \\
\text { Procedures }\end{array}$ & 0 & 0 & 0 & $0 \%$ & $0 \%$ & $0 \%$ \\
\hline 3 & After Body Fluid Exposure/Risk & 0 & 0 & 0 & $0 \%$ & $0 \%$ & $0 \%$ \\
\hline 4 & After Touching a Patient & 7 & 22 & 2 & $23 \%$ & $71 \%$ & $6 \%$ \\
\hline 5 & $\begin{array}{l}\text { After Touching Patient } \\
\text { Surroundings }\end{array}$ & 0 & 0 & 0 & $0 \%$ & $0 \%$ & $0 \%$ \\
\hline \multirow{2}{*}{\multicolumn{2}{|c|}{ Five Moments for Hand Hygiene }} & \multicolumn{3}{|c|}{$\begin{array}{c}\text { Doctor (Medical } \\
\text { Student/Intern/Reside } \\
\text { nt) }\end{array}$} & \multicolumn{3}{|c|}{ Compliance $\%$} \\
\hline & & No & Purell & Soap & $\begin{array}{l}\text { No } \\
\%\end{array}$ & $\begin{array}{c}\text { Purell } \\
\%\end{array}$ & $\begin{array}{c}\text { Soap } \\
\%\end{array}$ \\
\hline 1 & Before Touching a Patient & 5 & 9 & 0 & $36 \%$ & $64 \%$ & $0 \%$ \\
\hline 2 & $\begin{array}{l}\text { Before Clean/Aseptic } \\
\text { Procedures }\end{array}$ & 0 & 1 & 1 & $0 \%$ & $50 \%$ & $50 \%$ \\
\hline 3 & After Body Fluid Exposure/Risk & 0 & 0 & 0 & $0 \%$ & $0 \%$ & $0 \%$ \\
\hline 4 & After Touching a Patient & 11 & 11 & 2 & $46 \%$ & $46 \%$ & $8 \%$ \\
\hline 5 & $\begin{array}{l}\text { After Touching Patient } \\
\text { Surroundings }\end{array}$ & 0 & 0 & 1 & $0 \%$ & $0 \%$ & $100 \%$ \\
\hline
\end{tabular}

Table 5.15 (A) 6B Inpatient-Ward Hand Hygiene Rate Summarized by Employee Class, My Five Moments and Hygiene Products 


\begin{tabular}{|c|c|c|c|c|c|c|c|}
\hline \multirow{2}{*}{\multicolumn{2}{|c|}{ Five Moments for Hand Hygiene }} & \multicolumn{3}{|c|}{$\begin{array}{l}\text { Nurse Practitioner } \\
\text { (NP) }\end{array}$} & \multicolumn{3}{|c|}{ Compliance \% } \\
\hline & & No & Purell & Soap & $\begin{array}{l}\text { No } \\
\%\end{array}$ & $\begin{array}{c}\text { Purell } \\
\%\end{array}$ & $\begin{array}{c}\text { Soap } \\
\%\end{array}$ \\
\hline 1 & Before Touching a Patient & 9 & 1 & 0 & $90 \%$ & $10 \%$ & $0 \%$ \\
\hline 2 & Before Clean/Aseptic Procedures & 0 & 1 & 0 & $0 \%$ & $100 \%$ & $0 \%$ \\
\hline 3 & After Body Fluid Exposure/Risk & 0 & 0 & 0 & $0 \%$ & $0 \%$ & $0 \%$ \\
\hline 4 & After Touching a Patient & 1 & 2 & 1 & $25 \%$ & $50 \%$ & $25 \%$ \\
\hline 5 & $\begin{array}{l}\text { After T } \\
\text { Surrou }\end{array}$ & 12 & 2 & 2 & $75 \%$ & $13 \%$ & $13 \%$ \\
\hline \multirow{2}{*}{\multicolumn{2}{|c|}{ Five Moments for Hand Hygiene }} & \multicolumn{3}{|c|}{ Nurse (RN/LPN) } & \multicolumn{3}{|c|}{ Compliance $\%$} \\
\hline & & No & Purell & Soap & $\begin{array}{c}\text { No } \\
\%\end{array}$ & $\begin{array}{c}\text { Purell } \\
\%\end{array}$ & $\begin{array}{c}\text { Soap } \\
\%\end{array}$ \\
\hline 1 & Before Tou & 37 & 68 & 8 & $33 \%$ & $60 \%$ & $7 \%$ \\
\hline 2 & Before Clean/Aseptic Procedures & 3 & 2 & 0 & $60 \%$ & $40 \%$ & $0 \%$ \\
\hline 3 & After Body Fluid Exposure/Risk & 0 & 4 & 5 & $0 \%$ & $44 \%$ & $56 \%$ \\
\hline 4 & After Touching a Patient & 20 & 81 & 56 & $13 \%$ & $520 \%$ & $36 \%$ \\
\hline 5 & $\begin{array}{l}\text { After To } \\
\text { Surrounc }\end{array}$ & 2 & 73 & 43 & $2 \%$ & $62 \%$ & $36 \%$ \\
\hline \multirow{2}{*}{\multicolumn{2}{|c|}{ Five Moments for Hand Hygiene }} & \multicolumn{3}{|c|}{ Nurse (Students) } & \multicolumn{3}{|c|}{ Compliance $\%$} \\
\hline & & No & Purell & soap & $\begin{array}{c}\text { No } \\
\%\end{array}$ & $\begin{array}{c}\text { Purell } \\
\%\end{array}$ & $\begin{array}{c}\text { Soap } \\
\%\end{array}$ \\
\hline 1 & Before Tol & 0 & 0 & 0 & $0 \%$ & $0 \%$ & $0 \%$ \\
\hline 2 & Before Clean/Aseptic Procedures & 0 & 0 & 0 & $0 \%$ & $0 \%$ & $0 \%$ \\
\hline 3 & After Body Fluid Exposure/Risk & 0 & 0 & 0 & $0 \%$ & $0 \%$ & $0 \%$ \\
\hline 4 & After Touching a Patient & 0 & 0 & 0 & $0 \%$ & $0 \%$ & $0 \%$ \\
\hline 5 & $\begin{array}{l}\text { After Touching Patient } \\
\text { Surroundings }\end{array}$ & 0 & 0 & 0 & $0 \%$ & $0 \%$ & $0 \%$ \\
\hline \multirow{2}{*}{\multicolumn{2}{|c|}{ Five Moments for Hand Hygiene }} & \multicolumn{3}{|c|}{ EMS } & \multicolumn{3}{|c|}{ Compliance $\%$} \\
\hline & & No & Purell & Soap & $\begin{array}{l}\text { No } \\
\%\end{array}$ & $\begin{array}{l}\text { Purell } \\
\%\end{array}$ & $\begin{array}{c}\text { Soap } \\
\%\end{array}$ \\
\hline 1 & Before Touching a Patient & 0 & 0 & 0 & $0 \%$ & $0 \%$ & $0 \%$ \\
\hline 2 & Before Clean/Aseptic Procedures & 0 & 0 & 0 & $0 \%$ & $0 \%$ & $0 \%$ \\
\hline 3 & After Body Fluid Exposure/Risk & 0 & 0 & 0 & $0 \%$ & $0 \%$ & $0 \%$ \\
\hline 4 & After Touching a Patient & 0 & 0 & 0 & $0 \%$ & $0 \%$ & $0 \%$ \\
\hline 5 & $\begin{array}{l}\text { After Touching Patient } \\
\text { Surroundings }\end{array}$ & 0 & 0 & 0 & $0 \%$ & $0 \%$ & $0 \%$ \\
\hline
\end{tabular}

Table 5.16 (B) 6B Inpatient-Ward Hand Hygiene Rate Summarized by Employee Class, My Five Moments and Hygiene Products 


\begin{tabular}{|c|c|c|c|c|c|c|c|}
\hline \multicolumn{8}{|c|}{ Ward ICU } \\
\hline & \multirow{3}{*}{ Five Moments for Hand Hygiene } & \multirow{2}{*}{\multicolumn{3}{|c|}{$\begin{array}{c}\text { Employee Class } \\
\text { Doctor } \\
\text { (Attending/Fellow) }\end{array}$}} & \multirow{2}{*}{\multicolumn{3}{|c|}{ Compliance $\%$}} \\
\hline & & & & & & & \\
\hline & & No & Purell & Soap & No $\%$ & $\begin{array}{c}\text { Purell } \\
\%\end{array}$ & $\begin{array}{c}\text { Soap } \\
\%\end{array}$ \\
\hline 1 & Before Touching a Patient & 18 & 23 & 0 & $44 \%$ & $56 \%$ & $0 \%$ \\
\hline 2 & $\begin{array}{l}\text { Before Clean/Aseptic } \\
\text { Procedures }\end{array}$ & 0 & 0 & 1 & $0 \%$ & $0 \%$ & $100 \%$ \\
\hline 3 & After Body Fluid Exposure/Risk & 0 & 0 & 0 & $0 \%$ & $0 \%$ & $0 \%$ \\
\hline 4 & After Touching a Patient & 5 & 33 & 7 & $11 \%$ & $73 \%$ & $16 \%$ \\
\hline 5 & $\begin{array}{l}\text { After Touching Patient } \\
\text { Surroundings }\end{array}$ & 0 & 0 & 0 & $0 \%$ & $0 \%$ & $0 \%$ \\
\hline \multirow{2}{*}{\multicolumn{2}{|c|}{ Five Moments for Hand Hygiene }} & \multicolumn{3}{|c|}{$\begin{array}{c}\text { Doctor (Medical } \\
\text { Student/Intern/Resi } \\
\text { dent) }\end{array}$} & \multicolumn{3}{|c|}{ Compliance $\%$} \\
\hline & & No & Purell & Soap & No $\%$ & $\begin{array}{c}\text { Purell } \\
\%\end{array}$ & $\begin{array}{c}\text { Soap } \\
\%\end{array}$ \\
\hline 1 & Before Touching a Patient & 11 & 13 & 4 & $39 \%$ & $46 \%$ & $14 \%$ \\
\hline 2 & $\begin{array}{l}\text { Before Clean/Aseptic } \\
\text { Procedures }\end{array}$ & 0 & 0 & 2 & $0 \%$ & $0 \%$ & $100 \%$ \\
\hline 3 & After Body Fluid Exposure/Risk & 0 & 0 & 1 & $0 \%$ & $0 \%$ & $100 \%$ \\
\hline 4 & After Touching a Patient & 20 & 32 & 15 & $30 \%$ & $48 \%$ & $22 \%$ \\
\hline 5 & $\begin{array}{l}\text { After Touching Patient } \\
\text { Surroundings }\end{array}$ & 1 & 0 & 0 & $100 \%$ & $0 \%$ & $0 \%$ \\
\hline
\end{tabular}

Table 5.17 (A) ICU Inpatient-Ward Hand Hygiene Rate Summarized by Employee Class, My Five Moments and Hygiene Products 


\begin{tabular}{|c|c|c|c|c|c|c|c|}
\hline \multirow{2}{*}{\multicolumn{2}{|c|}{ Five Moments for Hand Hygiene }} & \multicolumn{3}{|c|}{$\begin{array}{l}\text { Nurse Practitioner } \\
\text { (NP) }\end{array}$} & \multicolumn{3}{|c|}{ Compliance \% } \\
\hline & & No & Purell & Soap & No $\%$ & Purell \% & Soap \% \\
\hline 1 & Before Touching a Patient & 10 & 1 & 0 & $91 \%$ & $9 \%$ & $0 \%$ \\
\hline 2 & $\begin{array}{l}\text { Before Clean/Aseptic } \\
\text { Procedures }\end{array}$ & 0 & 0 & 0 & $0 \%$ & $0 \%$ & $0 \%$ \\
\hline 3 & $\begin{array}{l}\text { After Body Fluid } \\
\text { Exposure/Risk }\end{array}$ & 0 & 0 & 0 & $0 \%$ & $0 \%$ & $0 \%$ \\
\hline 4 & After Touching a Patient & 9 & 0 & 0 & $100 \%$ & $0 \%$ & $0 \%$ \\
\hline 5 & $\begin{array}{l}\text { After Touching Patient } \\
\text { Surroundings }\end{array}$ & 1 & 4 & 3 & $13 \%$ & $50 \%$ & $38 \%$ \\
\hline \multirow{2}{*}{\multicolumn{2}{|c|}{ Five Moments for Hand Hygiene }} & \multicolumn{3}{|c|}{ Nurse (RN/LPN) } & \multicolumn{3}{|c|}{ Compliance $\%$} \\
\hline & & No & Purell & Soap & No $\%$ & Purell \% & Soap $\%$ \\
\hline 1 & Before Touching a Patient & 12 & 104 & 15 & $9 \%$ & $79 \%$ & $11 \%$ \\
\hline 2 & $\begin{array}{l}\text { Before Clean/Aseptic } \\
\text { Procedures }\end{array}$ & 0 & 1 & 2 & $0 \%$ & $33 \%$ & $67 \%$ \\
\hline 3 & $\begin{array}{l}\text { After Body Fluid } \\
\text { Exposure/Risk }\end{array}$ & 0 & 6 & 8 & $0 \%$ & $43 \%$ & $57 \%$ \\
\hline 4 & After Touching a Patient & 6 & 117 & 165 & $2 \%$ & $41 \%$ & $57 \%$ \\
\hline 5 & $\begin{array}{l}\text { After Touching Patient } \\
\text { Surroundings }\end{array}$ & 4 & 11 & 13 & $14 \%$ & $39 \%$ & $46 \%$ \\
\hline \multirow{2}{*}{\multicolumn{2}{|c|}{ Five Moments for Hand Hygiene }} & \multicolumn{3}{|c|}{ Nurse (Students) } & \multicolumn{3}{|c|}{ Compliance $\%$} \\
\hline & & No & Purell & Soap & No $\%$ & Purell \% & Soap \% \\
\hline 1 & Before Touching a Patient & 0 & 0 & 0 & $0 \%$ & $0 \%$ & $0 \%$ \\
\hline 2 & $\begin{array}{l}\text { Before Clean/Aseptic } \\
\text { Procedures }\end{array}$ & 0 & 0 & 0 & $0 \%$ & $0 \%$ & $0 \%$ \\
\hline 3 & $\begin{array}{l}\text { After Body Fluid } \\
\text { Exposure/Risk }\end{array}$ & 0 & 0 & 0 & $0 \%$ & $0 \%$ & $0 \%$ \\
\hline 4 & After & 0 & 0 & 2 & $0 \%$ & $0 \%$ & $100 \%$ \\
\hline 5 & $\begin{array}{l}\text { After Touching Patient } \\
\text { Surroundings }\end{array}$ & 0 & 0 & 0 & $0 \%$ & $0 \%$ & $0 \%$ \\
\hline \multirow{2}{*}{\multicolumn{2}{|c|}{ Five Moments for Hand Hygiene }} & \multicolumn{3}{|c|}{ EMS } & \multicolumn{3}{|c|}{ Compliance $\%$} \\
\hline & & No & Purell & Soap & No $\%$ & Purell \% & Soap \% \\
\hline 1 & Before Touching a Patient & 0 & 0 & 0 & $0 \%$ & $0 \%$ & $0 \%$ \\
\hline 2 & $\begin{array}{l}\text { Before Clean/Aseptic } \\
\text { Procedures }\end{array}$ & 0 & 0 & 0 & $0 \%$ & $0 \%$ & $0 \%$ \\
\hline 3 & $\begin{array}{l}\text { After Body Fluid } \\
\text { Exposure/Risk }\end{array}$ & 0 & 0 & 0 & $0 \%$ & $0 \%$ & $0 \%$ \\
\hline 4 & After Touching a Patient & 0 & 0 & 0 & $0 \%$ & $0 \%$ & $0 \%$ \\
\hline 5 & $\begin{array}{l}\text { After Touching Patient } \\
\text { Surroundings }\end{array}$ & 1 & 0 & 0 & $100 \%$ & $0 \%$ & $0 \%$ \\
\hline
\end{tabular}

Table 5.18 (B) ICU Inpatient-Ward Hand Hygiene Rate Summarized by Employee Class, My Five Moments and Hygiene Products 


\subsubsection{Retrospective Hand Hygiene and PPE Compliance}

Assessing and evaluating hand hygiene and PPE compliance together during patient care on precautions (contact, droplet or airborne precautions) is inevitable. The PVAMC receives patients with clinically approved infections and MRSA colonization almost on a daily basis. Thus, complying with hand hygiene and PPE during patient care is impervious toward infectious prevention.

Recall that the hand hygiene management tool at the PVAMC consists of three different forms:

- Option One is Only Hand Hygiene Compliance Observations: There is no classification between isolation and non-isolation observations. Observations could belong to one or the other.

- Option Two is Only Isolation Precautions: There is no classification between isolation and non-isolation observations. Observations could belong to one or the other since gloves, for example, are sometimes required during nonisolation care.

- Option Three is Both Hand Hygiene Compliance Observations and Isolation Precautions: There is no classification between isolation and non-isolation observations since observing both hand hygiene and PPE compliance together could also occur during non-isolation events.

Under this section, data associated to (Option Three) Both Hand Hygiene Compliance Observations and Isolation Precautions is analyzed. Facility wide, there are 440 PPE and hand hygiene isolation observations in total which all belong to isolation events. The breakdown of the data by year and isolation types is seen in 
Table 5.19 and depicted in Figure 5.24. Of note, the graph resembles the true colors of isolation precautions used at the PVAMC, orange for contact, pink for droplet and blue for airborne. Contact precautions represent the largest portion of the collected observations of PPE and hand hygiene together with 405 observations. There is an increase in the total observations per year as year increases. Unfortunately, when observations are taken and entered on the hand hygiene management tool system, the associated infectious diseases with the precautions are not reported. Thus, there is no way to distinguish between an illnesses that requires contact precautions. Such as MRSA, MRSA colonization or C.Diff or any other illnesses that are not of interest to this research study. Also IPEC does not tell the true BDOC for isolations. In general, the PVAMC experiences patients on contact precautions more than other patients who require other types of precautions. Droplet precautions comes next after contact precautions as the second, in terms of the collected observations of PPE and hand hygiene together with 31 observations though there is huge difference in the sample size. Similarly, there is an increase in the total observations per year as year increases. Airborne precautions are last with very low numbers of observations (only 4) and no observations in year 2013. Airborne cases are expected to be very low. Thus, very low PPE and hand hygiene opportunities are expected as well. 


\begin{tabular}{|c|c|c|c|c|}
\hline \multirow{2}{*}{ Year } & \multicolumn{2}{|c|}{ Hand Hygiene and PPE Observations } & \multirow{2}{*}{ Total } \\
\cline { 2 - 4 } & Contact & Droplet & Airborne & \\
\hline 2012 & 67 & 3 & 1 & 71 \\
\hline 2013 & 91 & 6 & 0 & 97 \\
\hline 2014 & 93 & 8 & 2 & 103 \\
\hline 2015 & 154 & 14 & 1 & 169 \\
\hline Total & 405 & 31 & 4 & 440 \\
\hline
\end{tabular}

Table 5.19 Facility-Wide Hand Hygiene and PPE Observations Summarized by Precaution Types and Year

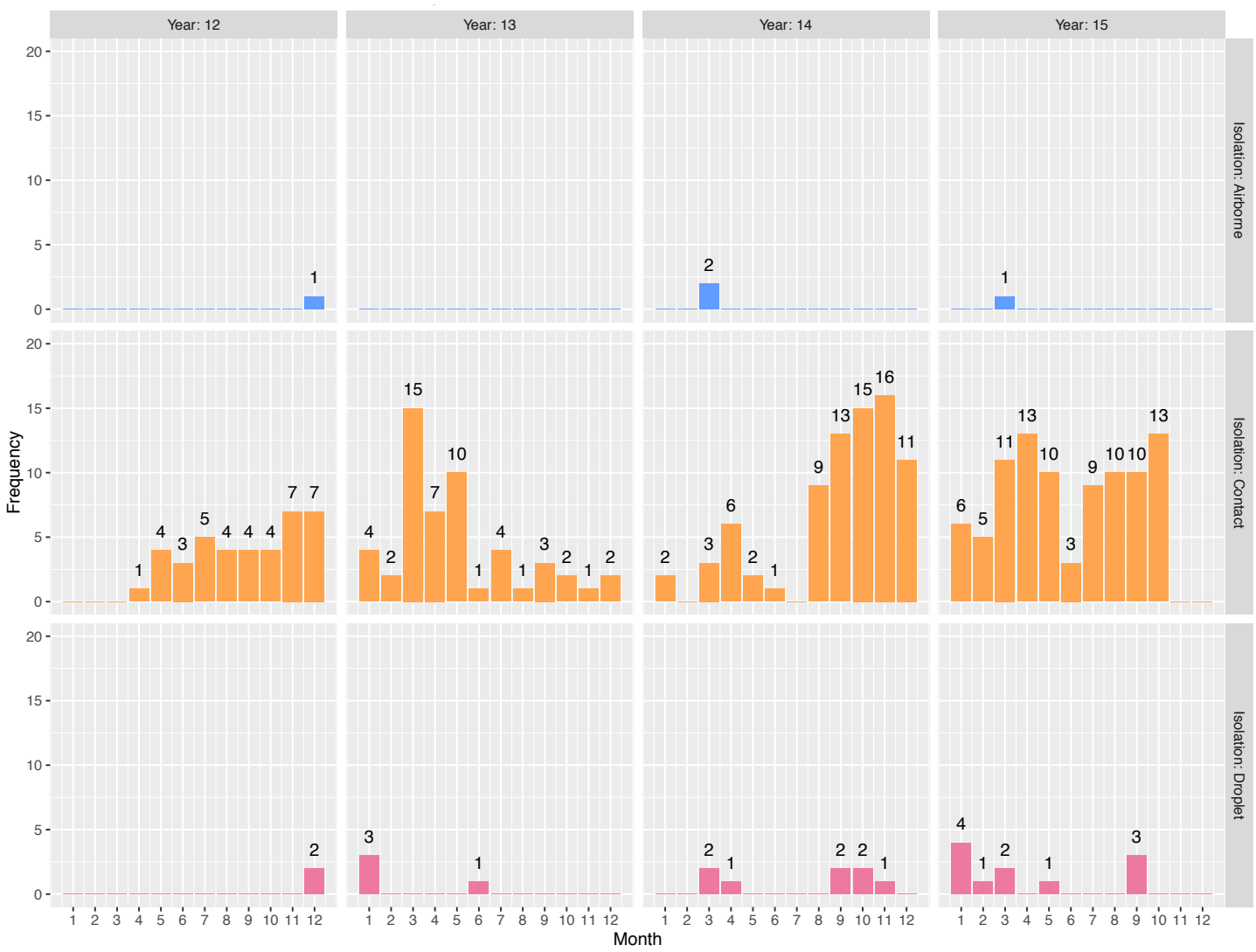

Figure 5.24 Facility Wide Hand Hygiene and PPE Observations by Precaution Types and Year

After removing data entry errors, the data was filtered to include only employee classes of interest (attending, fellow, resident, intern, medical student, nurse practitioner, registered and licensed practical nurses, nurse student and environmental management service), hand hygiene moments of only before and after touching a 
patient and inpatient locations $(5 \mathrm{~B}, 6 \mathrm{~B}$ and $\mathrm{IC})$, the number of observations drops down from 440 to 288 . The data breakdown based on the isolation type, hand hygiene moments and inpatient ward is seen in Table 5.20.

\begin{tabular}{|c|l|c|c|c|c|}
\hline \multirow{2}{*}{ Isolation } & Moments & \multicolumn{3}{|c|}{ Ward } & \multirow{2}{*}{ Total } \\
\cline { 3 - 6 } & & $5 \mathrm{~B}$ & $6 \mathrm{~B}$ & ICU & \\
\hline \multirow{2}{*}{ Contact } & Before Touching a Patient & 53 & 5 & 39 & 97 \\
\cline { 2 - 5 } & After Touching a Patient & 59 & 12 & 91 & 162 \\
\hline \multirow{2}{*}{ Total Contact Precautions Observations } & 112 & 17 & 130 & 259 \\
\hline \multirow{2}{*}{ Droplet } & Before Touching a Patient & 5 & 0 & 3 & 8 \\
\cline { 2 - 6 } & After Touching a Patient & 6 & 3 & 8 & 17 \\
\hline \multirow{2}{*}{ Total Droplet Precautions Observations } & 11 & 3 & 11 & 25 \\
\hline \multirow{2}{*}{ Airborne } & Before Touching a Patient & 1 & 0 & 0 & 1 \\
\cline { 2 - 6 } & After Touching a Patient & 1 & 0 & 2 & 3 \\
\hline \multicolumn{2}{|c|}{ Total Airborne Precautions Observations } & 2 & 0 & 2 & 4 \\
\hline \multicolumn{2}{|c|}{ Total Observations per Ward } & 125 & 20 & 143 & 88 \\
\hline
\end{tabular}

Table 5.20 Inpatient-Wards Hand Hygiene and PPE Observations Summarized by Precaution Types

The analysis is carried out into four phases. First, data is broken down per ward, per precautions and PPE and hand hygiene compliance where all PPE items are included: gloves, gowns and masks. Second, data is also broken down per ward, per precautions and PPE and hand hygiene compliance where only gloves and gowns are included and this is only done for contact precautions. Third, data is also broken down per ward, per precautions, per employee class and PPE and hand hygiene compliance where only gloves and gowns are included and this is also only done for contact precautions. Fourth, data is then broken down per ward, per precautions, per employee class and PPE and hand hygiene compliance where all PPE items are included (gloves, gowns and masks) and this is done for both droplet and airborne. 
The total combinations, for all types of precautions including contact precautions with masks, for having three PPE products, gloves, gowns and masks plus hand hygiene, each with three possible outcomes yes, no and not applicable (NA), except for hand hygiene with only two possible outcomes yes or no, are 54:

$$
\begin{gathered}
\text { Gloves (Yes, No, NA) * Gowns (Yes, No, NA) } * \text { Masks (Yes, No, NA) } \\
* \text { Hand Hygiene (Yes, No) }=3 * 3 * 3 * 2=54
\end{gathered}
$$

On the other hand, for contact precautions with no masks, the total combinations, for having two PPE products, gloves and gowns plus hand hygiene, each with three possible outcomes yes, no and not applicable (NA), except for hand hygiene with only two possible outcomes yes or no, are 18:

$$
\begin{gathered}
\text { Gloves }(\text { Yes, No, NA) } * \text { Gowns }(\text { Yes, No, NA) } * \text { Hand Hygiene }(\text { Yes, No })=3 * 3 * 2 \\
=18
\end{gathered}
$$

Based on the available data, the 54 possible scenarios drops down to 16 scenarios for contact precautions when masks item is included, 6 scenarios for droplet precautions and only 2 scenarios for airborne precautions. On the other hand, the 18 possible scenarios drops down to 9 scenarios for contact precautions when masks item is excluded. 


\subsubsection{Contact Precautions}

Patients who are on contact precautions could have MRSA, MRSA colonization or C.Diff or other infections not listed on the study. For complete lists, please see the back of contact precautions sign in Appendix DD. The contact precautions sign states that gloves and gowns are required all of the time when providing care. Additionally, hand hygiene has to be practiced before and after wearing gloves. Masks and eye goggles are required as needed such as in situations where the HCW expects to be at risk of blood or fluid exposure, Appendix DD.

The first data analysis is when masks are included and employee class is not included. Figure 5.25 shows that not all wards per hand hygiene moments have the sixteen possible scenarios. Some scenarios show masks are required during patients care on contact precautions. But, it is rare. Accounting for all 16 possible scenarios, combinations are insignificant because there are $\left(\begin{array}{c}16 \\ 2\end{array}\right)=\frac{16 !}{2 ! *(16-2) !}$, or 120 possible combinations out of 16 scenarios and some of the combinations do not have data and do not make sense for comparison. However, there are possible interesting scenarios such as when gloves and gowns are practiced, masks are not applicable and hand hygiene is and is not practiced. This scenario shows how many times HCWs practice hand hygiene during the use of PPE when providing care to patients who are on contact precautions (Light Silver vs. Dark Silver Bars). Simple adherence calculation shows that HCWs are $95 \%\left[\frac{36+42}{36+42+4+0} * 100\right], 100 \%\left[\frac{3+4}{3+4+0+0} * 100\right]$ and $85 \%$ $\left[\frac{27+61}{27+61+5+10} * 100\right]$ compliant with practicing hand hygiene during the use of PPE before and after touching a patient combined, and $90 \%\left[\frac{36}{36+4} * 100\right], 100 \%\left[\frac{3}{3+0} *\right.$ 
$100]$ and $84 \%\left[\frac{27}{27+5} * 100\right]$ before touching a patient, and $100 \%\left[\frac{42}{42+0} * 100\right]$, $100 \%\left[\frac{4}{4+0} * 100\right]$ and $86 \%\left[\frac{61}{61+10} * 100\right]$ after touching a patient for $5 \mathrm{~B}, 6 \mathrm{~B}$ and ICU respectively. Clearly HCWs value practicing hand hygiene after touching a patient more than before touching a patient when gloves are worn. Another interesting scenario, which is the opposite of the previous one, is when gloves and gowns are not practiced, masks are not applicable and hand hygiene is and is not practiced. This scenario shows how many times HCWs practice hand hygiene during the absence of PPE when providing care to patients who are on contact precautions (Green vs. Dark Blue Bars). Simple adherence calculation shows that HCWs are $25 \%\left[\frac{0+3}{0+3+3+6} * 100\right]$, $50 \%\left[\frac{0+1}{0+1+0+1} * 100\right]$ and $0 \%\left[\frac{0+0}{0+0+2+1} * 100\right]$ compliant with practicing hand hygiene during the absence of PPE before and after touching a patient combined, and $0 \%$ $\left[\frac{0}{0+3} * 100\right], \mathrm{NA}\left[\frac{0}{0+0} * 100\right]$ and $0 \%\left[\frac{0}{0+2} * 100\right]$ before touching a patient, and $33.33 \%$ $\left[\frac{3}{3+6} * 100\right], 50 \%\left[\frac{1}{1+1} * 100\right]$ and $0 \%\left[\frac{0}{0+1} * 100\right]$ after touching a patient for $5 \mathrm{~B}, 6 \mathrm{~B}$ and ICU respectively. The compliance is very low; however, the samples are very low too. But, though the samples are very low, observing some HCWs with no PPE at all during the care of patients who are on contact precautions is considered too risky not to mention the fact that hand hygiene was not practiced. 


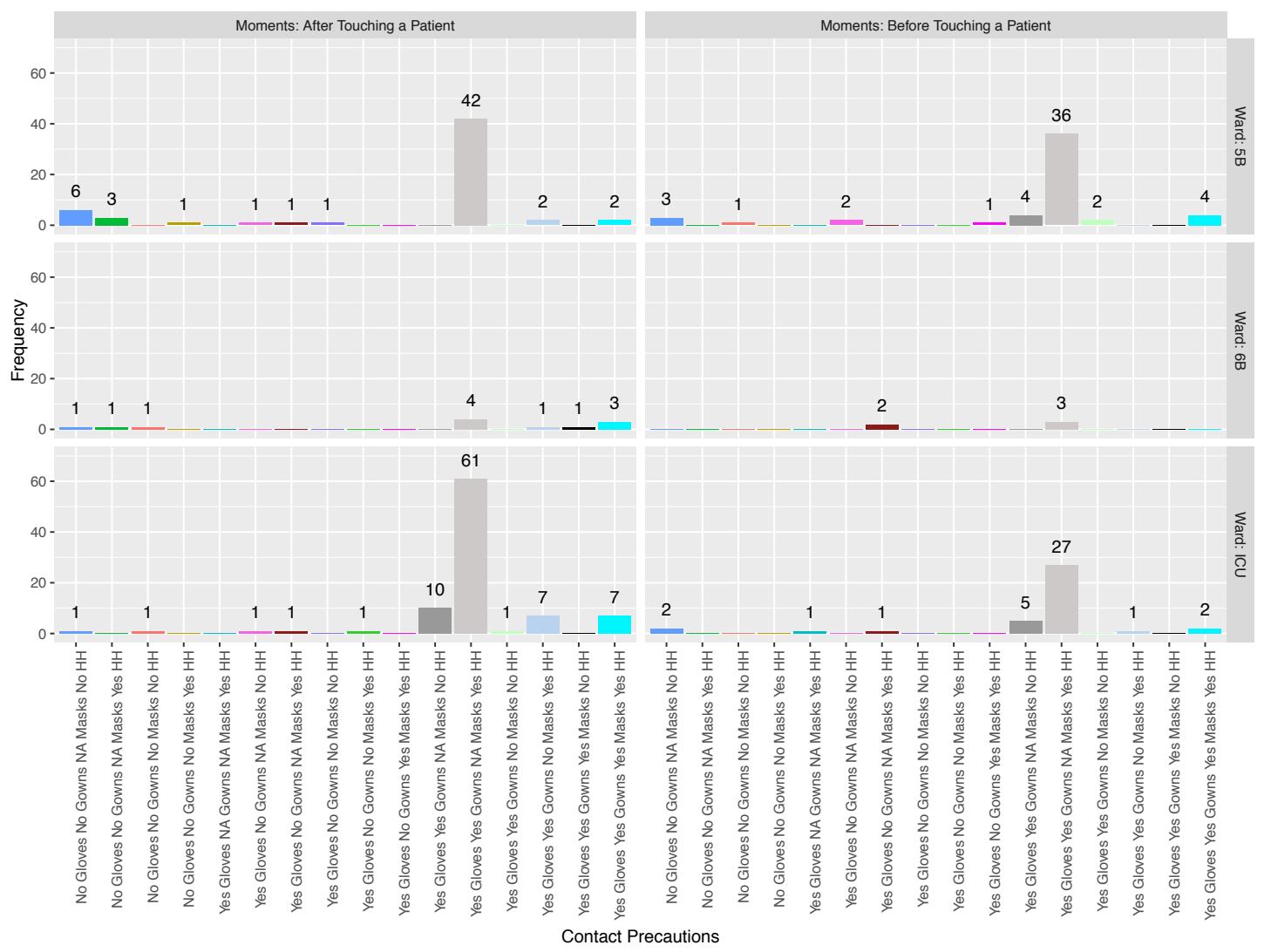

Figure 5.25 Inpatient-Wards Hand Hygiene and PPE Observations and Compliance for Contact Precautions Including Masks

Next is the data analysis when masks and employee class are not added. Figure 5.26 shows that not all wards per hand hygiene moments have the complete nine possible scenarios of the combinations among gloves (yes, no, NA), gowns (yes, no, NA) or hand hygiene (yes, no). One scenario shows gowns are not required during patients care on contact precautions, which is impossible since gloves and gowns are required together. The other opposite scenario is when gloves are not required, which is not reported based on the data, though it could occur. Accounting for all 9 possible scenarios, combinations are insignificant because there are $\left(\begin{array}{l}9 \\ 2\end{array}\right)=\frac{9 !}{2 !^{*}(9-2) !}$, or 36 possible combinations out of 9 scenarios and some of the combinations do not make sense for comparison. However, there are possible interesting scenarios such as when 
gloves and gowns are practiced and hand hygiene is and is not practiced. This scenario shows how many times HCWs practice hand hygiene during the use of PPE when providing care to patients who are on contact precautions (Green vs. Purple Bars). Simple adherence calculation shows that HCWs are $94 \%\left[\frac{40+46}{40+6+46+0} * 100\right]$, $92 \%\left[\frac{3+8}{3+0+8+1} * 100\right]$ and $87 \%\left[\frac{30+75}{30+5+75+11} * 100\right]$ compliant with practicing hand hygiene during the use of PPE before and after touching a patient combined, and $87 \%$ $\left[\frac{40}{40+6} * 100\right], 100 \%\left[\frac{3}{3+0} * 100\right]$ and $86 \%\left[\frac{30}{30+5} * 100\right]$ before touching a patient, and $100 \%\left[\frac{46}{46+0} * 100\right], 89 \%\left[\frac{8}{8+1} * 100\right]$ and $87 \%\left[\frac{75}{75+11} * 100\right]$ after touching a patient for $5 \mathrm{~B}, 6 \mathrm{~B}$ and ICU respectively. Clearly $\mathrm{HCWs}$ value practicing hand hygiene after touching a patient more than before touching a patient when gloves are worn except for $6 \mathrm{~B}$. However, sample size is significantly different between the two hand hygiene moments, before and after. Another interesting scenario, which is the opposite of the previous one, is when gloves and gowns are not practiced, and hand hygiene is and is not practiced. This scenario shows how many times HCWs practice hand hygiene during the absence of PPE when providing care to patients who are on contact precautions (Orange vs. Red Bars). Simple adherence calculation shows that HCWs are $29 \%\left[\frac{0+4}{0+4+4+6} * 100\right], 33.33 \%\left[\frac{0+1}{0+0+1+2} * 100\right]$ and $0 \%\left[\frac{0+0}{0+2+0+2} * 100\right]$ compliant with practicing hand hygiene during the absence of PPE before and after touching a patient combined, and $0 \%\left[\frac{0}{0+4} * 100\right], \mathrm{NA}\left[\frac{0}{0+0} * 100\right]$ and $0 \%\left[\frac{0}{0+2} * 100\right]$ before touching a patient, and $40 \%\left[\frac{4}{4+6} * 100\right], 33.33 \%\left[\frac{1}{1+2} * 100\right]$ and $0 \%\left[\frac{0}{0+2} * 100\right]$ after touching a patient for 5B, 6B and ICU respectively. The compliance is very low; 
however, the samples are very low too. But, though the samples are very low, observing some HCWs with no PPE at all during the care of patients who are on contact precautions is considered too risky not to mention the fact that hand hygiene is not practiced.

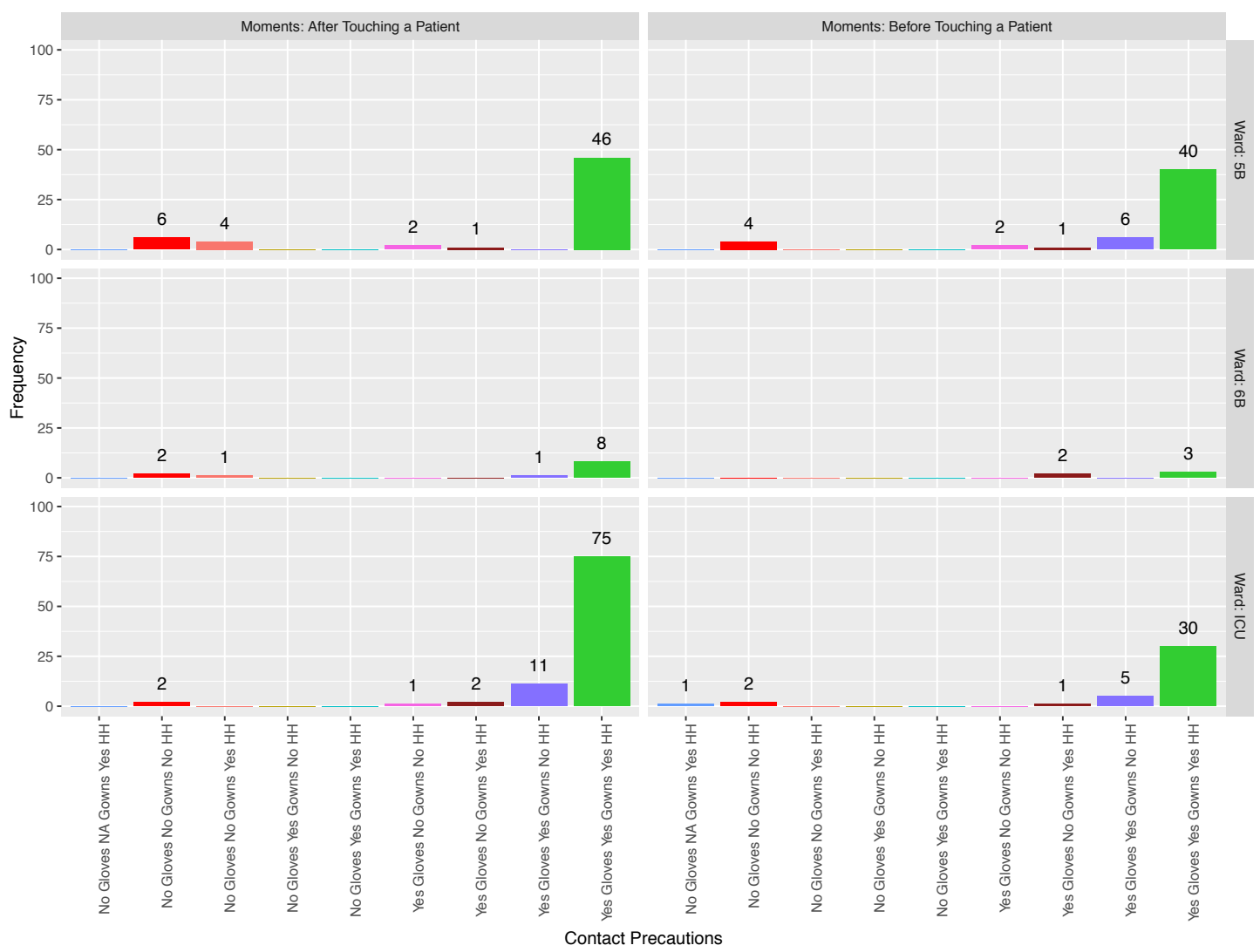

Figure 5.26 Inpatient-Wards Hand Hygiene and PPE Observations and Compliance for Contact Precautions Not Including Masks

For the data analysis when masks are not included, employee class and hand hygiene moments are included. In Figure 5.27, a higher number of observations are captured on RN and LPN, and residents, interns and medical students. The two most interesting scenarios are analyzed when gloves and gowns are used while hand hygiene is practiced and is not, and gloves and gowns are not used while hand hygiene is practiced and is not. The formal scenario, based on Figure 5.27, shows that RN and 
LPN adherence rates are $91 \%\left[\frac{29+35}{29+6+35+0} * 100\right], 91 \%\left[\frac{2+8}{2+0+8+1} * 100\right]$ and $98 \%$ $\left[\frac{23+59}{23+1+59+1} * 100\right]$ compliant with practicing hand hygiene during the absence of PPE before and after touching a patient combined, and $83 \%\left[\frac{29}{29+6} * 100\right], 100 \%\left[\frac{2}{2+0} *\right.$ $100]$ and $96 \%\left[\frac{23}{23+1} * 100\right]$ before touching a patient, and $100 \%\left[\frac{35}{35+0} * 100\right]$, $89 \%\left[\frac{8}{8+1} * 100\right]$ and $98 \%\left[\frac{59}{59+1} * 100\right]$ after touching a patient for $5 \mathrm{~B}, 6 \mathrm{~B}$ and ICU, respectively. In general, RN and LPN show high adherence rate of PPE and hand hygiene compliance. In addition, RN and LPN value practicing hand hygiene after touching a patient more than before touching a patient when gloves are worn except for $6 \mathrm{~B}$. However, sample size is significantly different between the two hand hygiene moments, before and after. Attending and fellow adherence rates are $100 \%$ $\left[\frac{2+3}{2+0+3+0} * 100\right], \quad \mathrm{NA}\left[\frac{0+0}{0+0+0+0} * 100\right]$ and $89 \%\left[\frac{4+4}{4+0+4+1} * 100\right]$ compliant with practicing hand hygiene during the absence of PPE before and after touching a patient combined, and $100 \%\left[\frac{2}{2+0} * 100\right], \mathrm{NA}\left[\frac{0}{0+0} * 100\right]$ and $100 \%\left[\frac{4}{4+0} * 100\right]$ before touching a patient, and $100 \%\left[\frac{3}{3+0} * 100\right], \mathrm{NA}\left[\frac{0}{0+0} * 100\right]$ and $80 \%\left[\frac{4}{4+1} * 100\right]$ after touching a patient for 5B, 6B and ICU, respectively. In general, attending and fellow show high adherence rate of PPE and hand hygiene compliance. In addition, attending and fellow equally value practicing hand hygiene before and after touching a patient at $5 \mathrm{~B}$ and value practicing hand hygiene before touching a patient more than after touching a patient at ICU. However, sample size is significantly low for both hand hygiene moments, before and after, for all wards. Residents, interns and medical students adherence rates are $100 \% \quad\left[\frac{7+6}{7+0+6+0} * 100\right], \quad 100 \%\left[\frac{1+0}{1+0+0+0} * 100\right]$ and $50 \%$ 
$\left[\frac{3+10}{3+4+10+9} * 100\right]$ compliant with practicing hand hygiene during the absence of PPE before and after touching a patient combined, and $100 \%\left[\frac{7}{7+0} * 100\right], 100 \%\left[\frac{1}{1+0} *\right.$ $100]$ and $43 \%\left[\frac{3}{3+4} * 100\right]$ before touching a patient, and $100 \%\left[\frac{6}{6+0} * 100\right]$, NA $\left[\frac{0}{0+0} * 100\right]$ and $53 \%\left[\frac{10}{10+9} * 100\right]$ after touching a patient for $5 \mathrm{~B}, 6 \mathrm{~B}$ and ICU respectively. Huge variability is present in the residents, interns and medical students adherence rate between wards. Usually residents, interns and medical students are assigned to patients in all inpatient wards. It is expected to have residents, interns and medical students highly comply with PPE and hand hygiene especially at ICU because patients who are on precautions at ICU have severe health status. Thus, though sample size is very low, higher PPE and hand hygiene compliance is at the top priority to exist at ICU.

The latter scenario, which is when PPE is not present and hand hygiene is and is not practiced, based on Figure 5.27, shows that RN and LPN adherence rates are $12.5 \%\left[\frac{0+1}{0+2+1+5} * 100\right], 33.33 \%\left[\frac{0+1}{0+0+1+2} * 100\right]$ and $0 \%\left[\frac{0+0}{0+1+0+0} * 100\right]$ compliant with practicing hand hygiene during the absence of PPE before and after touching a patient combined, and $0 \%\left[\frac{0}{0+2} * 100\right], \mathrm{NA}\left[\frac{0}{0+0} * 100\right]$ and $0 \%\left[\frac{0}{0+1} * 100\right]$ before touching a patient, and $17 \%\left[\frac{1}{1+5} * 100\right], 33.33 \%\left[\frac{1}{1+2} * 100\right]$ and NA $\left[\frac{0}{0+0} * 100\right]$ after touching a patient for $5 \mathrm{~B}, 6 \mathrm{~B}$ and ICU, respectively. In general, $\mathrm{RN}$ and LPN show very low adherence rate of hand hygiene compliance when PPE is and is not present. However, cases of such scenarios are low. Low samples prevent analyzing such a scenario for the medical group (attending and fellow, resident, intern and 
medical student). Other employee classes such as EMS, NP and NS are left without analysis due to their very low sample sizes and due to missing values for the comparison scenarios for both hand hygiene moments.

It is necessary that the total samples when PPE is and is not present with or without practicing hand hygiene is analyzed across inpatient wards. Based on Figure 5.27, there are (before and after moments combined) $87 \%\left[\frac{92}{106} * 100\right], 80 \%\left[\frac{12}{15} * 100\right]$ and $97 \%\left[\frac{121}{125} * 100\right]$ PPE compliance with no perception to hand hygiene for $5 \mathrm{~B}, 6 \mathrm{~B}$ and ICU respectively. PPE compliance is not acceptable and considered low for 5B and 6B wards, but is very high for ICU. PPE compliance is not evaluated per hand hygiene moments because PPE is required before touching a patient and entering the room and PPE compliance means whether PPE is present or not at the moment of care. This is analyzed in depth when the form Only Precautions data is analyzed in section 5.3.4. 


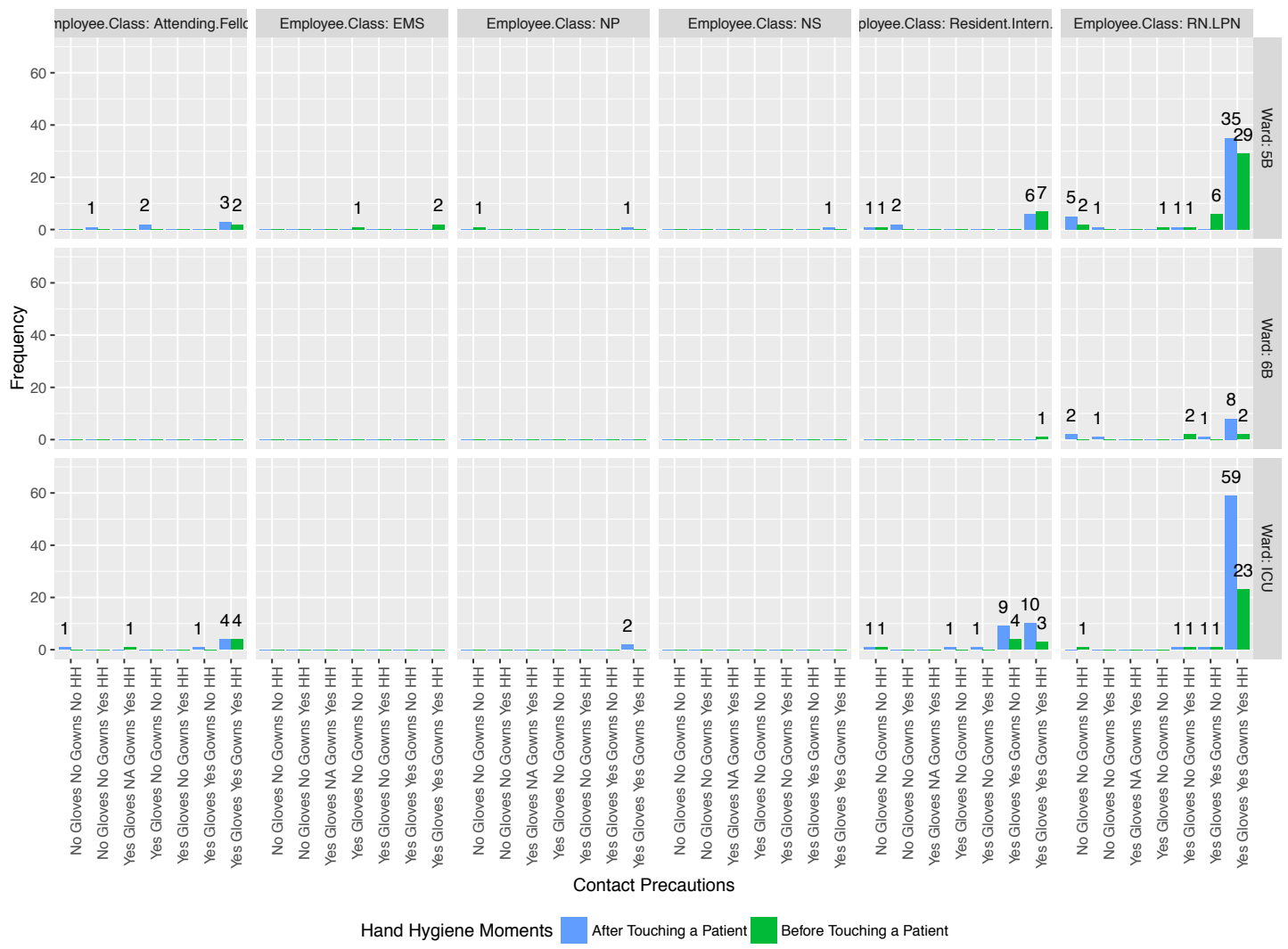

Figure 5.27 Inpatient-Wards Hand Hygiene and PPE Observations and Compliance for Contact Precautions Not Including Masks by Employee Class

\subsubsection{Droplet Precautions}

Patients who are on droplet precautions could have, for example, Flu or Mumps. For complete lists, please see the back of the droplet precautions sign in Appendix EE. The droplet precautions sign states that masks are required all of the time when providing care. Gloves, gowns and goggles for eye protection are required as needed. Additionally, hand hygiene has to be practiced before and after touching a patient and before and after wearing gloves if gloves are required.

Following the lead of the contact precautions analysis, first is with the analysis when employee class is not included. Figure 5.28 shows that not all wards per moments have the six possible scenarios. Accounting for all 6 possible scenario 
combinations is insignificant because there are $\left(\begin{array}{l}6 \\ 2\end{array}\right)=\frac{6 !}{2 !^{*}(6-2) !}$, or 15 possible combinations out of 6 scenarios and some of the combinations do not have data and do not make sense for comparison. All 6 scenarios include practicing hand hygiene, which means based on the collected data there are no observations where PPE is present and hand hygiene is not practiced. Thus, there is no PPE and hand hygiene adherence compliance calculation to be attempted. However, a comparison could be made when masks are and are not present. There are (Brown \& Orange \& Green Bars vs. Light Blue \& Pink \& Dark Blue Bars for before and after moments combined) $91 \%\left[\frac{4+1+0+3+1+1}{4+1+0+3+1+1+0+0+0+0+0+1} * 100\right], 100 \%\left[\frac{0+0+0+3+0+0}{0+0+0+3+0+0+0+0+0+0+0+0} * 100\right]$ and $82 \%\left[\frac{2+0+0+7+0+0}{2+0+0+7+0+0+1+0+0+0+1+0} * 100\right]$ masks compliance with no perception to hand hygiene for 5B, $6 \mathrm{~B}$ and ICU respectively. Masks compliance is considered high for 5B and 6B wards except for ICU. Masks compliance is not evaluated per hand hygiene moments because masks is required before touching a patient and entering the room and masks compliance means whether it is present or not at the moment of care. This is analyzed in depth when the form Only Precautions data is analyzed in the next section. Of note, there is one incident reported where all PPE are assumed to be not applicable even masks, which is totally against the droplet precautions requirements. 


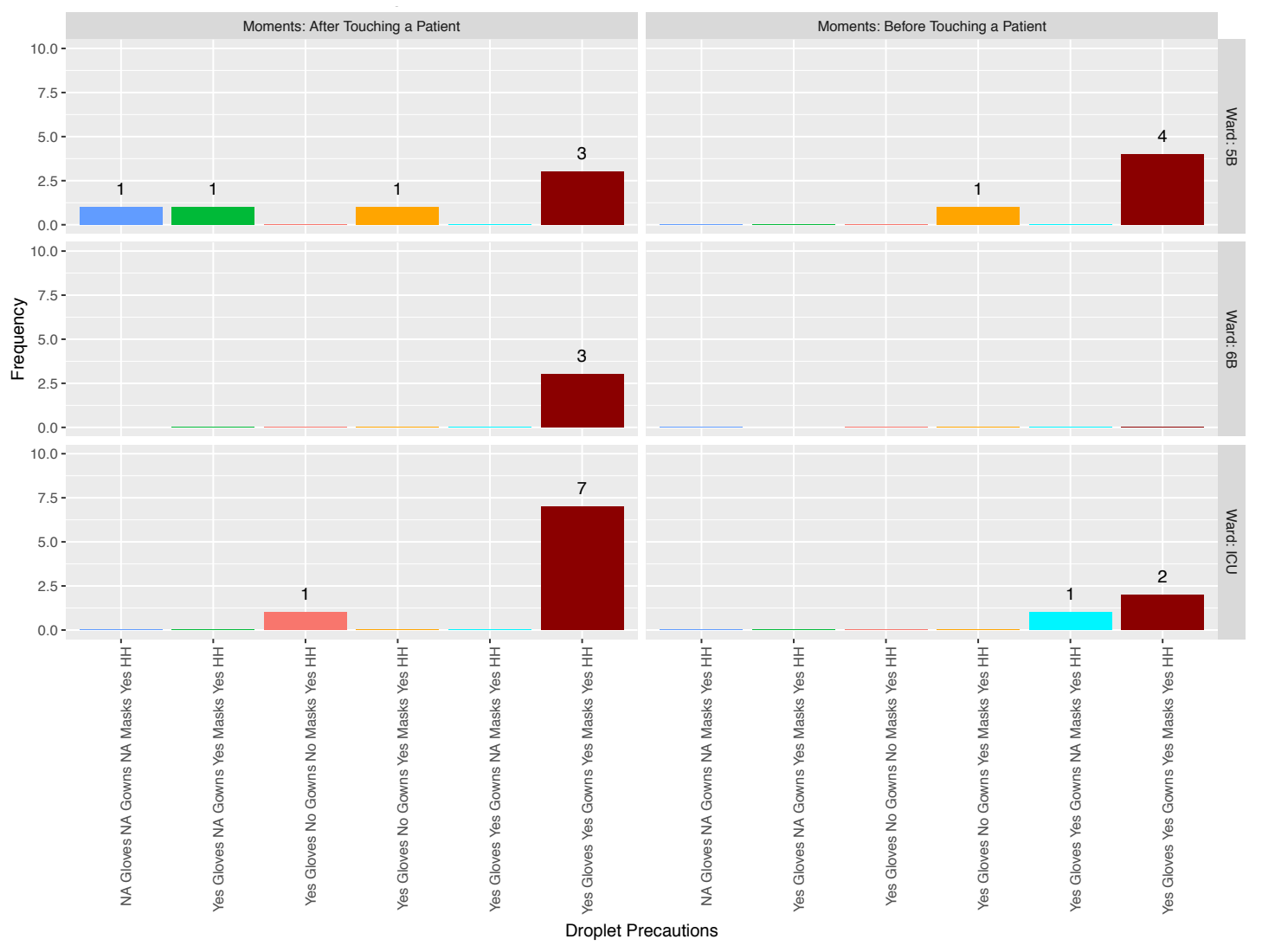

Figure 5.28 Inpatient-Wards Hand Hygiene and PPE Observations for Droplet Precautions

Next the data is analyzed for when employee class is included. Figure 5.29 shows that only RN and LPN and the medical group (attending and fellow, resident, intern and medical student) are observed based on the data. It is hard to report PPE and hand hygiene compliance for the same reason where all data are captured with hand hygiene being practiced. However, cases could be reported in terms of how many times masks are and are not present per employee class per inpatient ward. RN and LPN mask adherence rates are $100 \%\left[\frac{4+3+1}{4+3+1} * 100\right], 100 \%\left[\frac{3}{3} * 100\right]$ and $86 \%$ $\left[\frac{5+1}{5+1+1} * 100\right]$ compliance for $5 \mathrm{~B}, 6 \mathrm{~B}$ and ICU, respectively. Attending and fellow masks adherence rates are NA $\left[\frac{0}{0} * 100\right], \mathrm{NA}\left[\frac{0}{0} * 100\right]$ and $100 \%\left[\frac{2+1}{2+1} * 100\right]$ mask 
compliance for 5B, 6B and ICU, respectively. Resident, intern and medical student mask adherence rates are $67 \%\left[\frac{1+1}{1+1+1} * 100\right], \mathrm{NA}\left[\frac{0}{0} * 100\right]$ and $0 \%\left[\frac{0}{1+0} * 100\right]$ mask compliance for 5B, 6B and ICU, respectively. Samples are very low and rates could be misleading. However, the fact that one incident is reported where masks are not worn by resident, intern and medical student group at ICU is concerning.

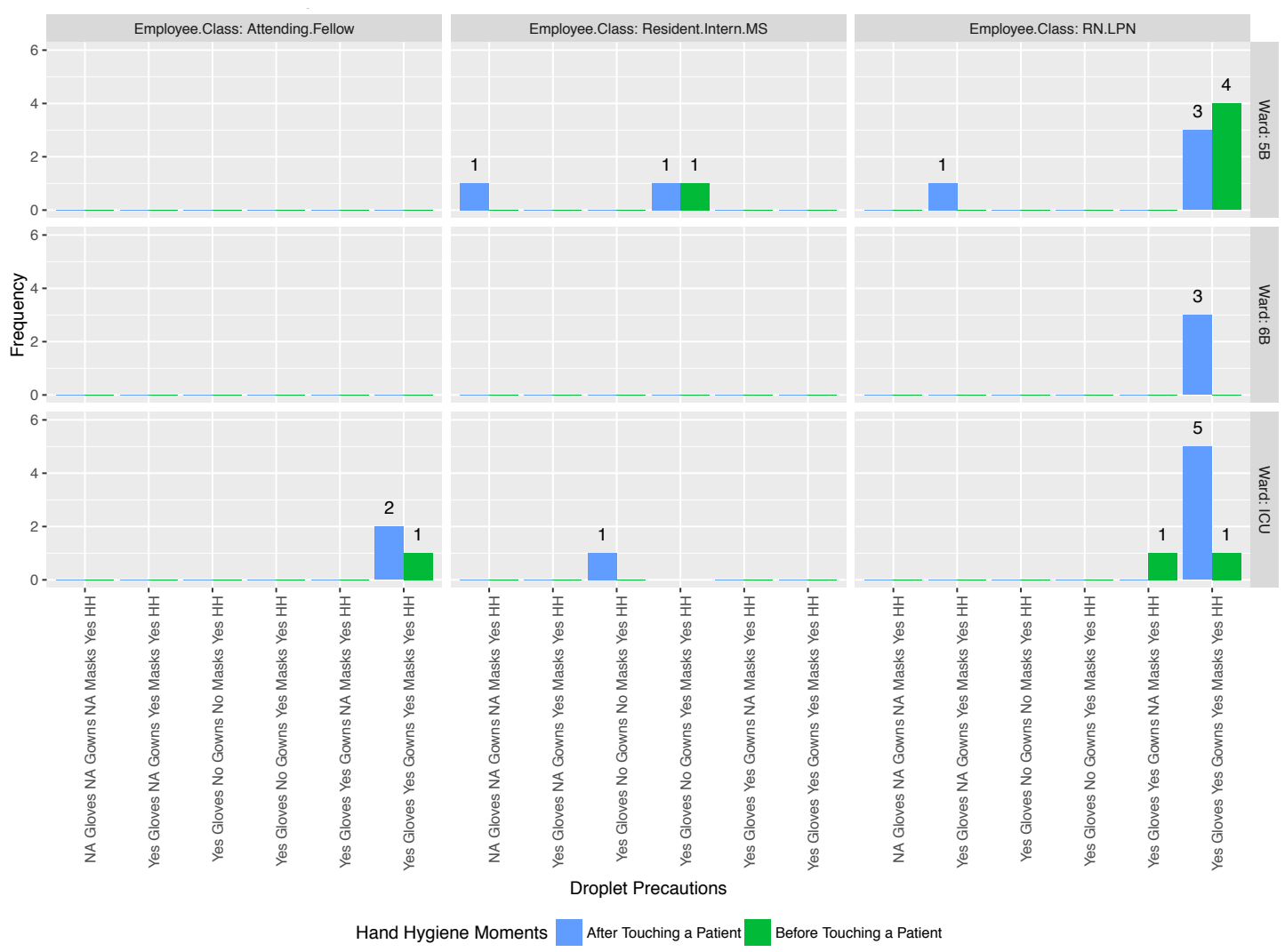

Figure 5.29 Inpatient-Wards Hand Hygiene and PPE Observations for Droplet Precautions by Employee Class 


\subsubsection{Airborne Precautions}

Patients who are on airborne precautions could have, for example, Tuberculosis (TB) or Measles Rubella. For complete lists, please see the back of the airborne precautions sign in Appendix FF. Most illnesses requiring airborne precautions require contact precautions as well. However, airborne cases are very rare. Airborne precautions sign states that N95 masks are required all of the time when providing care and wearing gloves and gowns are highly recommended. N95 is different from surgical masks that are required for droplet precautions. Additionally, hand hygiene has to be practiced before and after wearing gloves.

Following the lead of contact and droplet precautions analysis, first is the analysis when employee class is not included. Figure 5.30 shows that not all wards per hand hygiene moments have the two possible scenarios. There 2 possible scenarios, combinations are $\left(\begin{array}{l}2 \\ 2\end{array}\right)=\frac{2 !}{2 !^{*}(2-2) !}$, or 1 possible combination out of 2 scenarios, especially when there is a ward found with no data, 6B. The two available scenarios are when all PPE are and are not present when hand hygiene is and is not practiced. They are the best and worst case scenarios for such precautions. Thus, the adherence

rates are for before and after moments combined 50\% $\left.\frac{1}{1+1} * 100\right], \mathrm{NA}\left[\frac{0}{0+0} * 100\right]$ and $100 \%\left[\frac{2}{2+0} * 100\right]$ when PPE is present and hand hygiene is practiced versus PPE is not present and hand hygiene is not practiced for $5 \mathrm{~B}, 6 \mathrm{~B}$ and ICU respectively. Though airborne precautions observations are extremely low, the presence of at least one observation where PPE and hand hygiene are not present is a concern. 


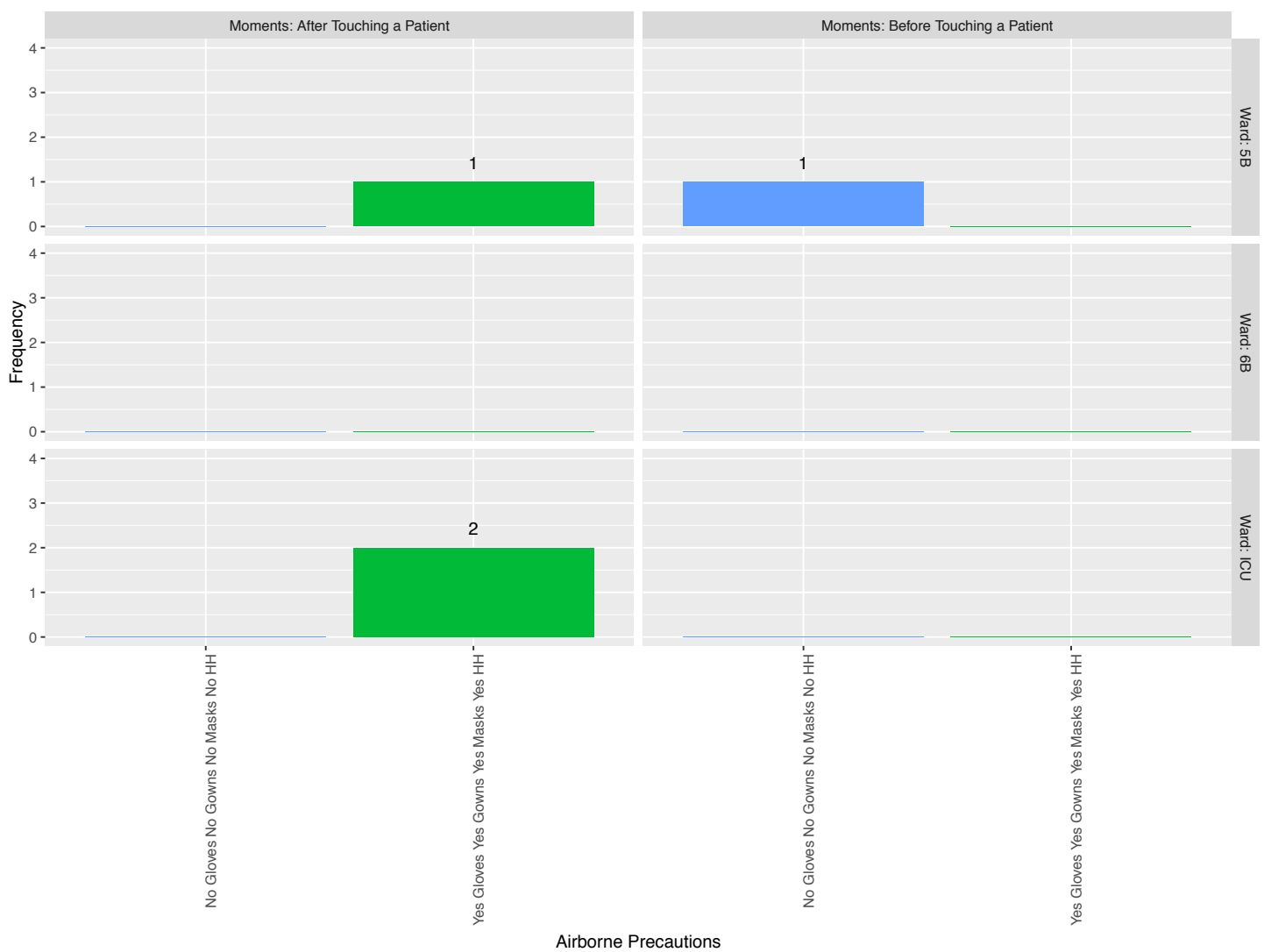

Figure 5.30 Inpatient-Wards Hand Hygiene and PPE Observations for Airborne Precautions

Breaking down the analysis based on employee class reveals that based on Figure 5.31, the resident, intern and medical student group is observed with that disappointing incident. On the other hand, the remaining of the airborne precautions samples are for $\mathrm{RN}$ and LPN group with 100\% compliance for practicing hand hygiene and PPE is present.

Of note, sometimes the PVAMC is in the process of ruling out the infection and presumably HCWs believe patients are free from illness requiring droplet or airborne precautions and thus do not comply with certain precautions requirements. 


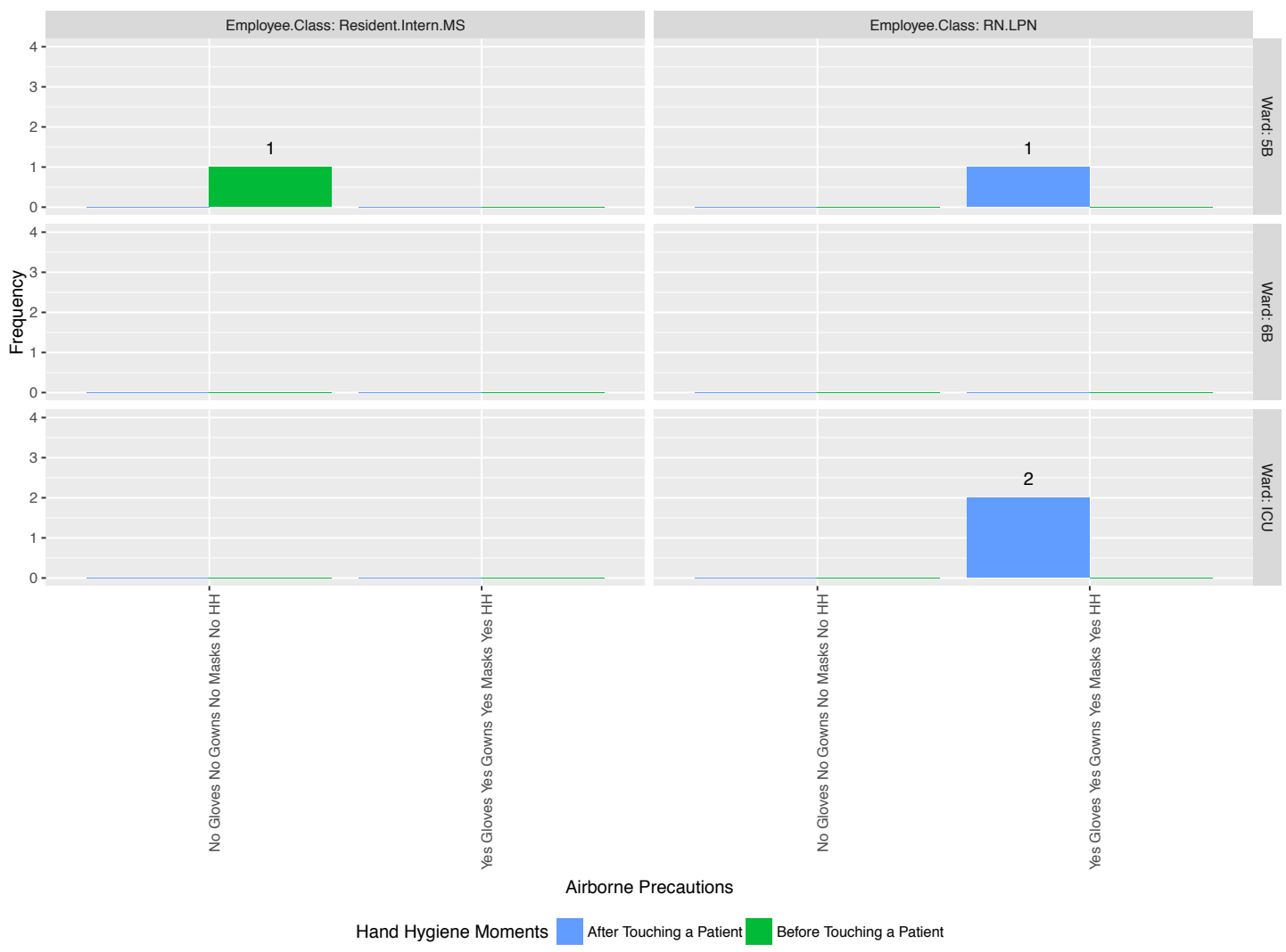

Figure 5.31 Inpatient-Wards Hand Hygiene and PPE Observations for Airborne Precautions by Employee Class

\subsubsection{Retrospective PPE Compliance}

Sometimes HCWs are observed during patient care based on whether they wear the right PPE at the moment of care without evaluating hand hygiene compliance. Such observations are used to report PPE compliance alone. Under this section, data associated to the only Isolation Precautions form of the hand hygiene management tool is analyzed. Facility wide, there are 964 PPE isolation observations in total. The breakdown of the data by year and isolation types is seen in Table 5.21 and depicted in Figure 5.32. Of note, the graph resembles the true colors of isolation precautions used at the PVAMC, orange for contact, pink for droplet and blue for airborne. Contact precautions have the largest number of the collected observations of PPE with 911 
observations. Droplet precautions come next with 45 observations. Airborne precautions are last with only 8 observations. There is a significant difference between the total samples of contact versus droplet and airborne precautions combined.

\begin{tabular}{|c|c|c|c|c|}
\hline \multirow{2}{*}{ Year } & \multicolumn{3}{|c|}{ PPE Observations } & \multirow{2}{*}{ Total } \\
\cline { 2 - 4 } & Contact & Droplet & Airborne & \\
\hline 2012 & 176 & 9 & 3 & 188 \\
\hline 2013 & 436 & 19 & 4 & 459 \\
\hline 2014 & 188 & 10 & 1 & 199 \\
\hline 2015 & 111 & 7 & 0 & 118 \\
\hline Total & 911 & 45 & 8 & 964 \\
\hline
\end{tabular}

Table 5.21 Facility-Wide PPE Observations Summarized by Precaution Types per Year

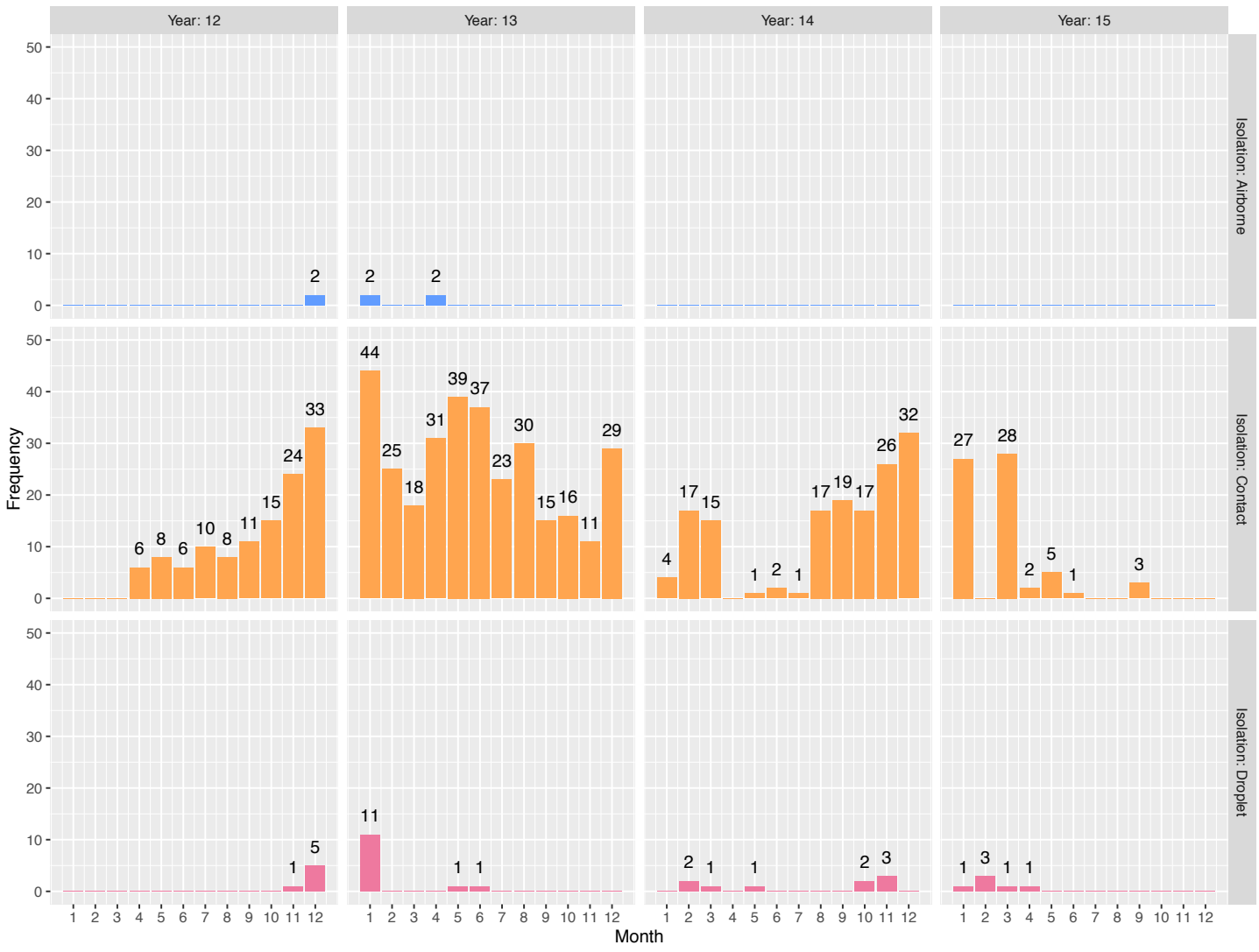

Figure 5.32 Facility-Wide PPE Observations Summarized by All Types of Precaution per Month and Year 
After removing data entry errors, the data was filtered to include only employee classes of interest (attending, fellow, resident, intern, medical student, nurse practitioner, registered and licensed practical nurses, nurse student and environmental management service) and locations of inpatient wards (5B, $6 \mathrm{~B}$ and IC), and then the data drops down from 964 to 696 . The data breakdown based on year, inpatient wards and isolation type is seen in Table 5.22.

\begin{tabular}{|c|c|c|c|c|c|c|c|}
\hline \multirow{2}{*}{ Ward } & \multirow{2}{*}{ Isolation } & \multicolumn{4}{|c|}{ Year } & \multirow{2}{*}{$\begin{array}{c}\text { Total per } \\
\text { Ward }\end{array}$} & \multirow{2}{*}{ Total per Isolation } \\
\hline & & 2012 & 2013 & 2014 & 2015 & & \\
\hline $5 \mathrm{~B}$ & \multirow{3}{*}{ Contact } & 65 & 142 & 72 & 37 & 316 & \multirow{3}{*}{656} \\
\hline $6 \mathrm{~B}$ & & 37 & 72 & 22 & 12 & 143 & \\
\hline ICU & & 19 & 104 & 57 & 17 & 197 & \\
\hline $5 B$ & \multirow{3}{*}{ Droplet } & 4 & 9 & 3 & 1 & 17 & \multirow{3}{*}{34} \\
\hline $6 \mathrm{~B}$ & & 2 & 3 & 1 & 3 & 9 & \\
\hline ICU & & 0 & 1 & 5 & 2 & 8 & \\
\hline $5 \mathrm{~B}$ & \multirow{3}{*}{ Airborne } & 0 & 1 & 0 & 0 & 1 & \multirow{3}{*}{6} \\
\hline $6 \mathrm{~B}$ & & 2 & 0 & 0 & 0 & 2 & \\
\hline ICU & & 0 & 3 & 0 & 0 & 3 & \\
\hline \multicolumn{2}{|c|}{ Total } & 129 & 335 & 160 & 72 & \multicolumn{2}{|c|}{696} \\
\hline
\end{tabular}

Table 5.22 Inpatient-Wards PPE Observations Summarized by Precaution Types per Year

Most contact precaution observations are captured at $5 \mathrm{~B}$, ICU and $6 \mathrm{~B}$, respectively. Contact precautions total observations per ward are 316, 143 and 197 for 5B, 6B and ICU respectively. Droplet precautions total observations per ward are 17, 9 and 8 for 5B, 6B and ICU, respectively. Airborne precautions total observations per ward are 1, 2 and 3 for 5B, 6B and ICU, respectively.

The analysis is carried out in four phases. First, data is broken down per ward, per precautions and PPE compliance where all PPE items are included: gloves, gowns and masks. Second, data is also broken down per ward, per precautions and PPE 
compliance where only gloves and gowns are included and this is only done for contact precautions. Third, data is also broken down per ward, per precautions, per employee class and PPE compliance where only gloves and gowns are included and this is also only done for contact precautions. Fourth, data is then broken down per ward, per precautions, per employee class and PPE compliance where all PPE items are included (gloves, gowns and masks) and this is done for both droplet and airborne.

Now the total combinations, for all types of precautions including contact precautions with masks, for having three PPE products (gloves, gowns and masks), each with three possible outcomes of yes, no and not applicable (NA), are 27:

Gloves (Yes, No, NA) * Gowns (Yes, No, NA) $*$ Masks $\left(\right.$ Yes, No, NA) $=3^{3}=27$

On the other hand, for contact precautions with no masks, the total combinations, for having two PPE products, gloves and gowns, each with three possible outcomes yes, no and not applicable (NA), are 9:

$$
\text { Gloves }(\text { Yes, No, NA) } * \text { Gowns }(\text { Yes, No, NA) }=3 * 3=9
$$

Based on the available data, the 27 possible scenarios drops down to 11 scenarios for contact precautions when masks item is included, 7 scenarios for droplet precautions and only 3 scenarios for airborne precautions. On the other hand, 9 possible scenarios drops down to 5 scenarios for contact precautions when masks item is excluded.

\subsubsection{Contact Precautions}

The first analysis is when masks are included and employee class is not included. Figure 5.33 shows that not all wards have the eleven possible scenarios. Some scenarios show masks are required during care for patient who are on contact 
precautions and it is quite a lot. Accounting for all 11 possible scenario combinations is insignificant because there are $\left(\begin{array}{c}11 \\ 2\end{array}\right)=\frac{11 !}{2 !^{*}(11-2) !}$, or 55 possible combinations out of 11 scenarios and some of the combinations do not have data and do not make sense for comparison. However, there are possible interesting scenarios such as when gloves and gowns are used, masks are not applicable versus gloves and gowns are not used and masks are not applicable. This scenario shows how many times HCWs use PPE when providing care to patients who are on contact precautions when masks are unnecessary (Green vs. Blue Bars). Simple adherence calculation shows that HCWs are $96 \%\left[\frac{250}{250+10} * 100\right], 97 \%\left[\frac{117}{117+4} * 100\right]$ and $94 \%\left[\frac{167}{167+8} * 100\right]$ PPE compliance for $5 \mathrm{~B}, 6 \mathrm{~B}$ and $\mathrm{ICU}$, respectively. High PPE compliance crosses inpatient wards, which means that HCWs value the use of PPE during care of patients on contact precautions. Another interesting scenario is when gloves, gowns and masks are and are not used. This scenario shows how many times masks are required but are not used by HCWs (Silver vs. Red Bars). Simple adherence calculation shows that HCWs are $90 \%\left[\frac{28}{28+3} * 100\right], 100 \%\left[\frac{8}{8+0} * 100\right]$ and $89 \%\left[\frac{8}{8+1} * 100\right]$ PPE compliance for 5B, 6B and ICU, respectively. Certainly, HCWs are not $100 \%$ PPE compliance when masks are required during providing care to patients on contact precautions. 


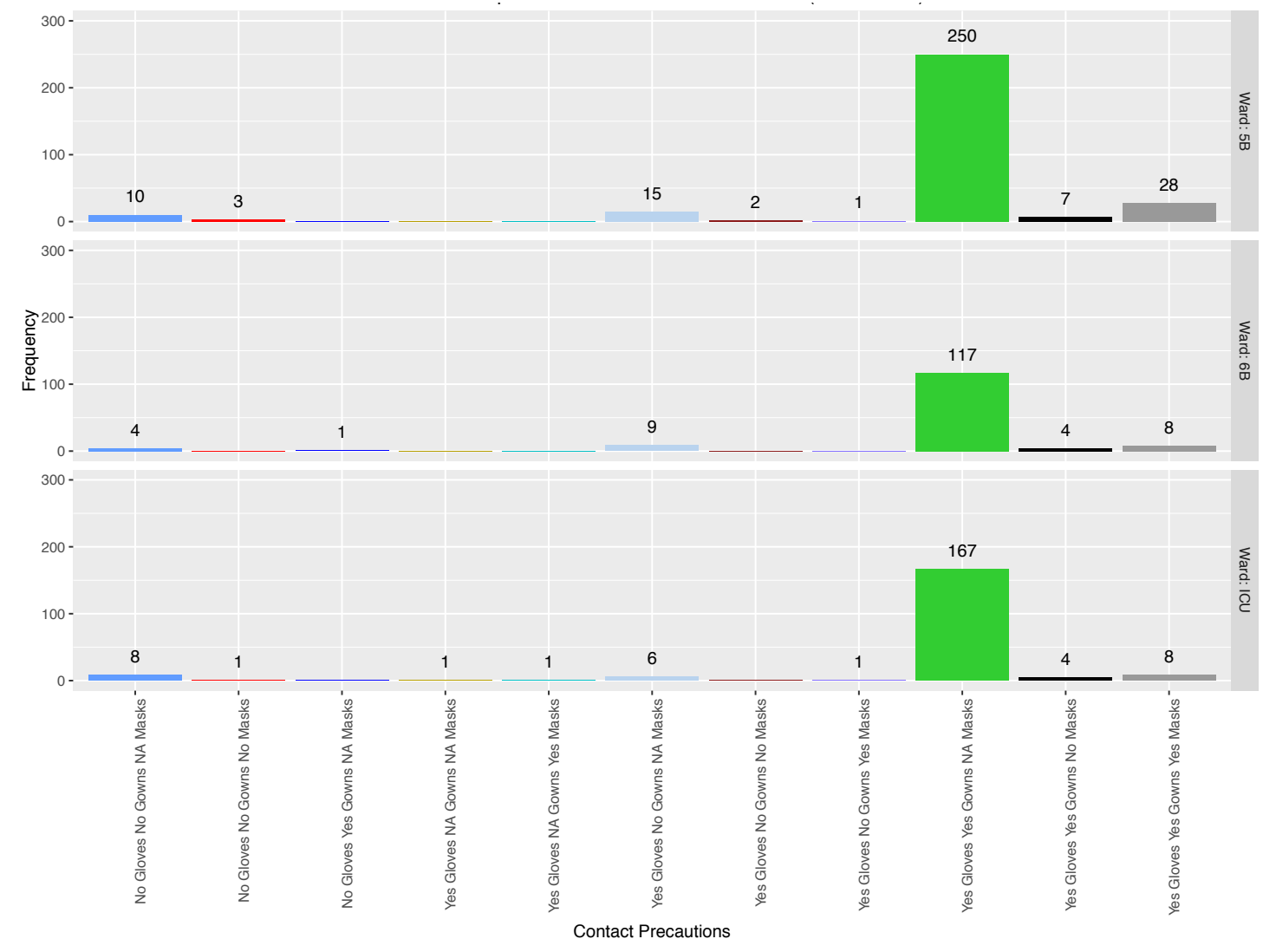

Figure 5.33 Inpatient-Wards PPE Observations for Contact Precautions Including Masks

Next is the analysis when masks and employee class are not included. Figure 5.34 shows that not all wards have the complete 5 possible scenarios of the combinations between gloves (yes, no, NA) and gowns (yes, no, NA). One scenario shows gowns are not required during care for patients who are on contact precautions, which is impossible since gloves and gowns are required together. The other opposite scenario is when gloves are not required, which is not reported based on the data, though it could occur as the formal occurred. Accounting for all 5 possible scenarios, combinations are insignificant because there are $\left(\begin{array}{l}5 \\ 2\end{array}\right)=\frac{5 !}{2 !^{*}(5-2) !}$, or 10 possible combinations out of 5 scenarios and some of the combinations do not make sense for comparison. However, there are possible interesting scenarios such as when gloves 
and gowns are and are not practiced. This scenario shows how many times HCWs practice PPE when providing care to patients who are on contact precautions (Green vs. Red Bars). Simple adherence calculation shows that HCWs are $96 \%\left[\frac{285}{285+13} * 100\right]$, $97 \%\left[\frac{129}{129+4} * 100\right]$ and $95 \%\left[\frac{179}{179+9} * 100\right]$ PPE compliance for 5B, 6B and ICU, respectively. Another scenarios is when gloves are used and gowns are not (Dark Blue Bars). The data shows that there are 18, 9 and 7 observations captured for 5B, $6 \mathrm{~B}$ and ICU respectively when HCWs use gloves and do not use gowns. Clearly HCWs believe that gloves are more important than wearing gowns. In contrast, the opposite scenario to the last one is when gloves are not used and gowns are used (Light Blue Bars). There is only one observation captured for such scenario. Overall, HCWs value practicing PPE during care for patients who are on contact precautions. 


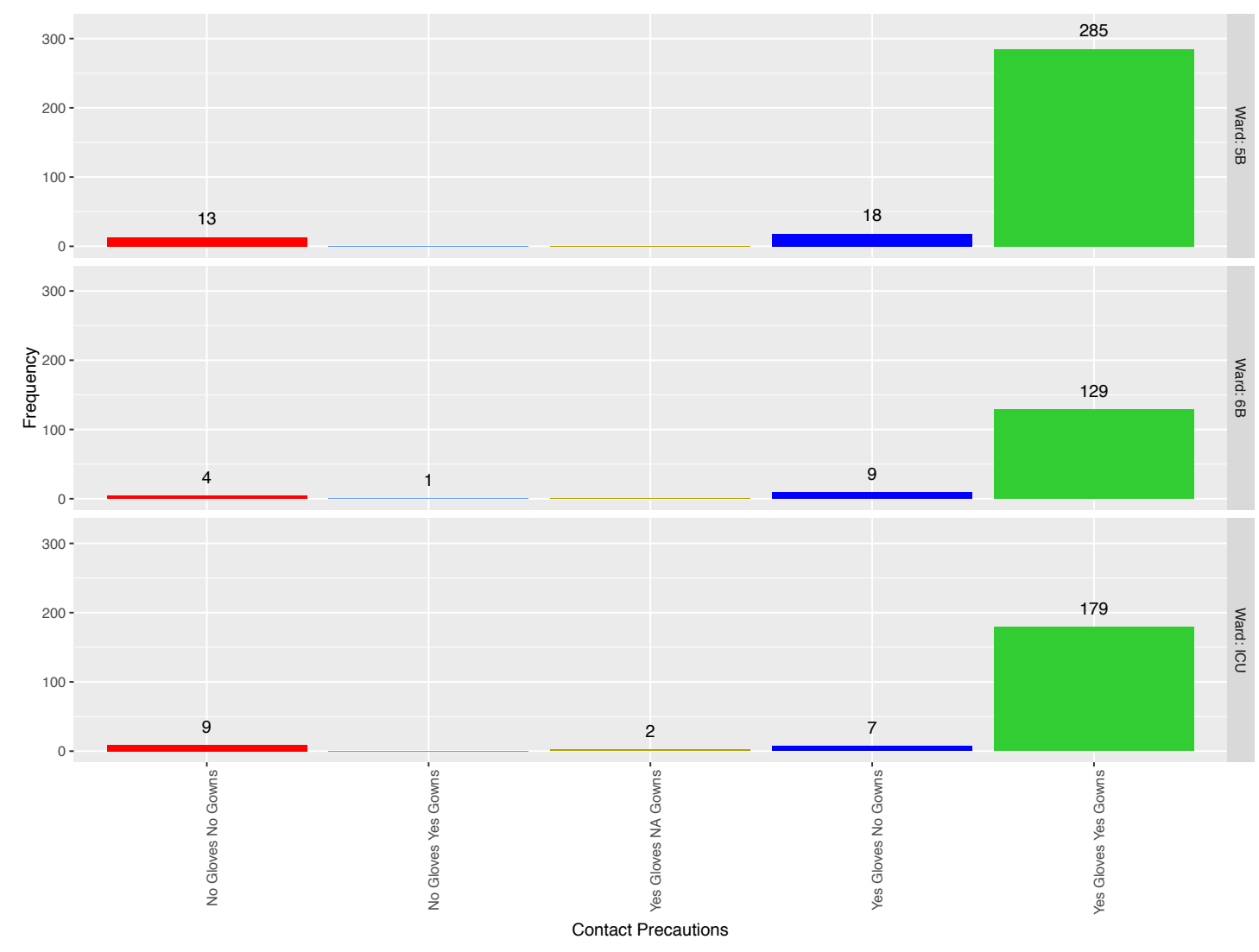

Figure 5.34 Inpatient-Wards PPE Observations for Contact Precautions Not Including Masks

Next is the analysis of data when employee class is included and masks are not included. Figure 5.35 shows that higher observations are captured on RN and LPN, residents, interns and medical students, and interestingly EMS and then attending and fellow, in a descending order. The most interesting scenario is when gloves and gowns are and are not used (Green vs. Red Bars). Figure 5.35 shows that RN and LPN PPE adherence rates are $96 \%\left[\frac{233}{233+9} * 100\right], 97 \%\left[\frac{92}{92+3} * 100\right]$ and $96 \%\left[\frac{155}{155+7} * 100\right]$ for $5 \mathrm{~B}, 6 \mathrm{~B}$ and $\mathrm{ICU}$, respectively. Considering the size of the samples per inpatient ward, RN and LPN show high PPE adherence rate. Attending and fellow PPE adherence rates are $100 \%\left[\frac{1}{1+0} * 100\right], 100 \%\left[\frac{3}{3+0} * 100\right]$ and $75 \%\left[\frac{3}{3+1} * 100\right]$ for $5 \mathrm{~B}, 6 \mathrm{~B}$ and ICU, respectively. Considering the size of the samples per inpatient ward, attending 
and fellow PPE adherence rates cannot be judged. However, attending and fellow are expected to have high PPE adherence rates. Resident, intern and medical student PPE adherence rates are $90 \%\left[\frac{27}{27+3} * 100\right], 97 \%\left[\frac{29}{29+1} * 100\right]$ and $94 \%\left[\frac{16}{16+1} * 100\right]$ for $5 \mathrm{~B}$, 6B and ICU, respectively. Resident, intern and medical student PPE adherence rates are high; but samples are considered very low. EMS PPE adherence rates per inpatient wards are $96 \%\left[\frac{22}{22+1} * 100\right], 100 \%\left[\frac{4}{4+0} * 100\right]$ and $100 \%\left[\frac{5}{5+0} * 100\right]$ for $5 \mathrm{~B}, 6 \mathrm{~B}$ and ICU, respectively. Considering the size of the samples per inpatient wards, EMS PPE adherence rates also cannot be judged.

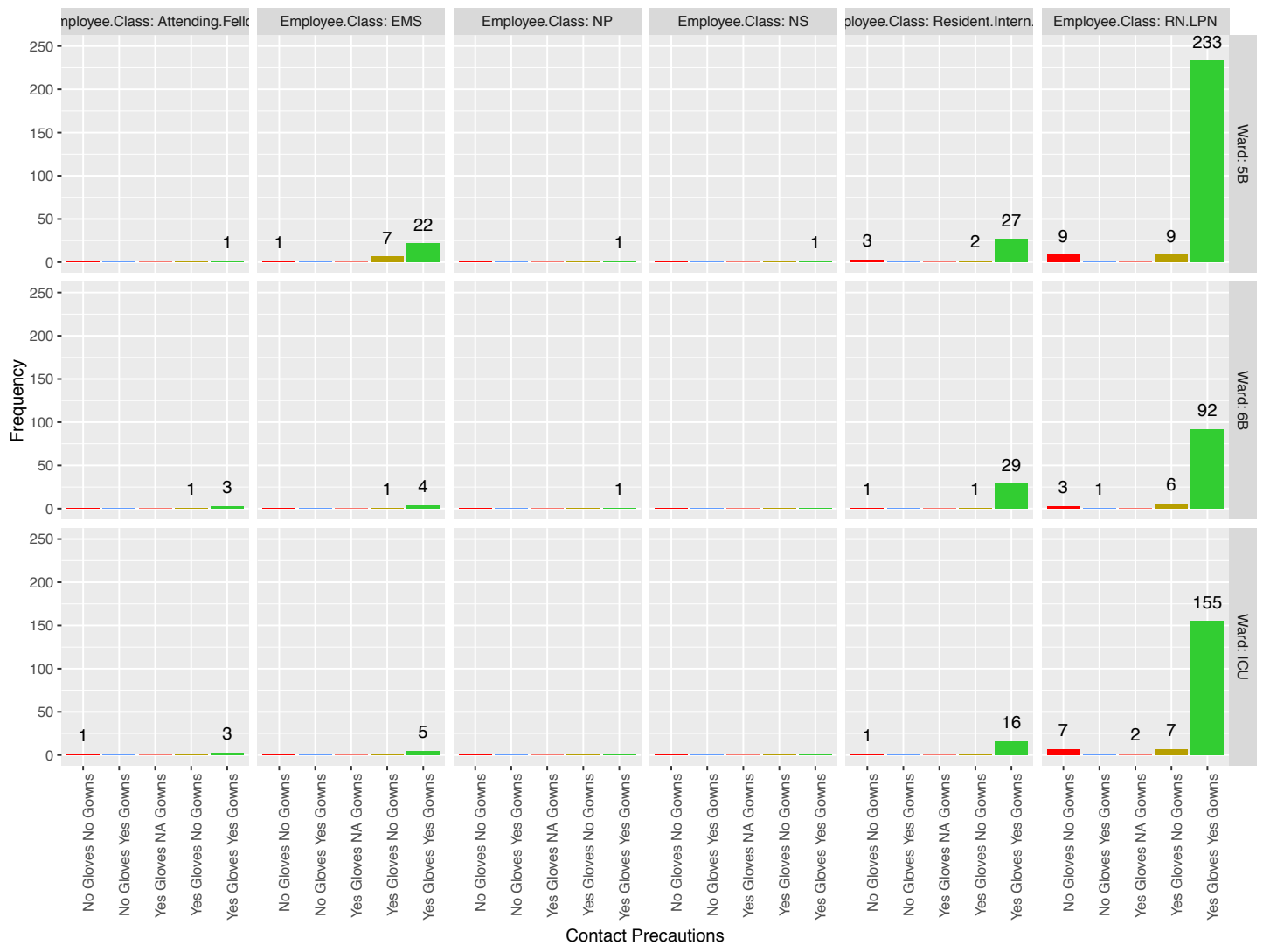

Figure 5.35 Inpatient-Wards PPE Observations for Contact Precautions Not Including Masks by Employee Class 


\subsubsection{Droplet Precautions}

Following the lead of contact precautions analysis, first is the analysis when employee class is not included. Figure 5.36 shows that not all wards have the seven possible scenarios. Accounting for all 7 possible scenario combinations is insignificant because there are $\left(\begin{array}{l}7 \\ 2\end{array}\right)=\frac{7 !}{2 ! *(7-2) !}$, or 21 possible combinations out of 7 scenarios and some of the combinations do not have data and do not make sense for comparison. All 7 scenarios include the three possible outcomes of masks (yes, no or not applicable), which means based on the collected data there are times when masks are not worn and thought of not being applicable during patient care on droplet precautions. There are 6 versus 1 observation for the formal and the latter respectively. Even though the observations are very low for such scenarios, masks are required all the time for droplet precautions and underestimating the risk of ignoring masks are consequently unappreciated. It would be very interesting to compare a scenario where masks are required and gloves and gowns are not. However, the data does not allow that since there are only 4 observations in total when gloves and gowns are not required and masks are used while 0 observations for the opposite scenario when gloves and gowns are not required and masks are not used.

There are possible interesting scenarios such as when gloves and gowns are used and masks are and are not used. This scenario shows how many times HCWs wear and do not wear masks when providing care to patients who are on droplet precautions when gloves and gowns together are necessary (Green vs. Red Bars). HCWs

adherence rates per inpatient wards are $64 \%\left[\frac{7}{7+4} * 100\right], 80 \%\left[\frac{4}{4+1} * 100\right]$ and $100 \%$ 
$\left[\frac{6}{6+0} * 100\right]$ for $5 \mathrm{~B}, 6 \mathrm{~B}$ and ICU, respectively. There is large variability in PPE compliance across inpatient wards. However, masks compliance with the necessity of wearing gloves and gowns cannot be judged because of the low sample size.

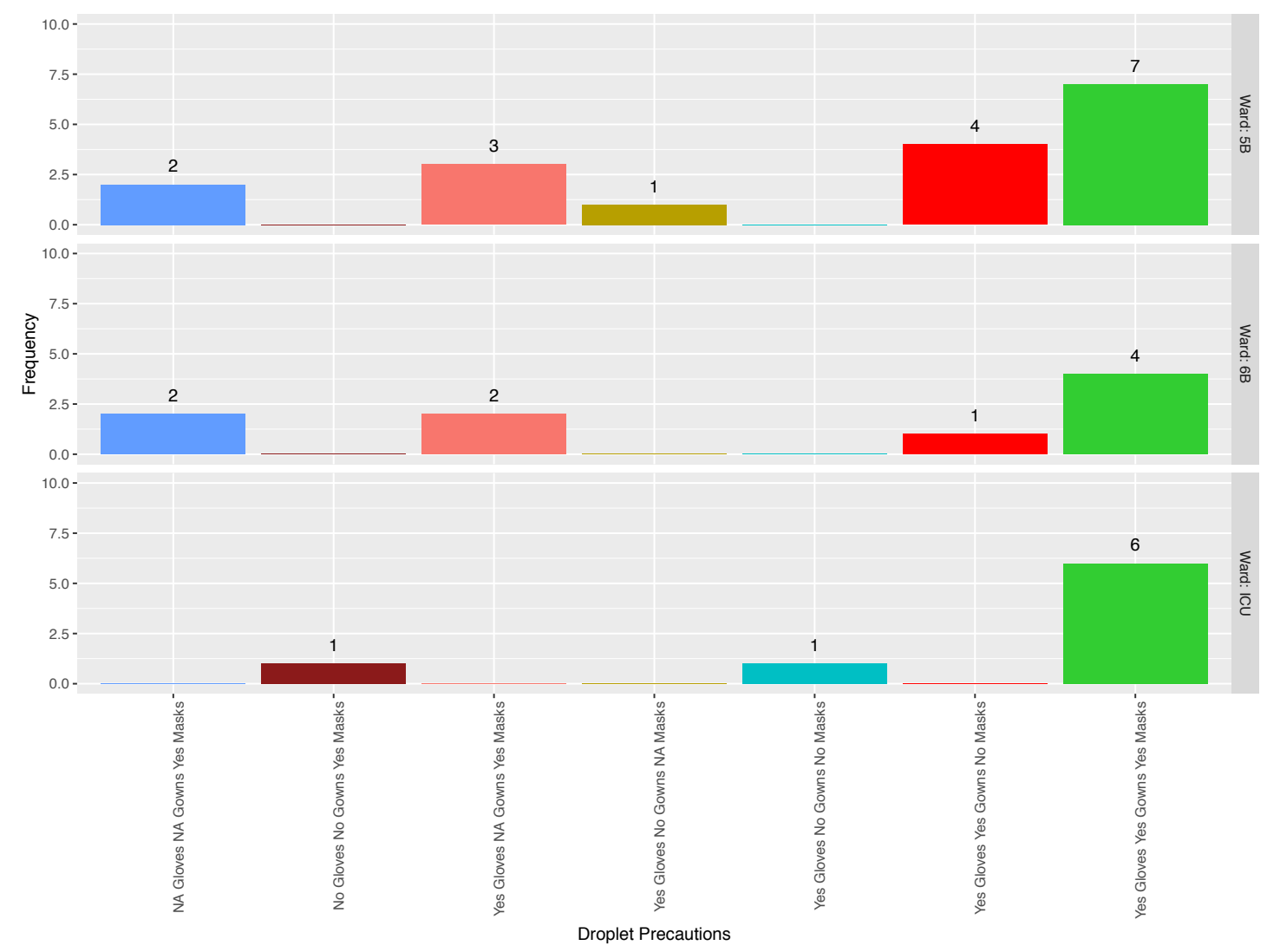

Figure 5.36 Inpatient-Wards PPE Observations for Droplet Precautions

The nexst step is to analyze the data when employee class is included. Figure 5.37 shows that only RN and LPN, the medical group (attending and fellow, resident, intern and medical student) and EMS are observed based on the data. It is hard to report the medical groups and EMS PPE compliance due to their sample size. However, RN and LPN PPE compliance shows an interesting scenario, which is when all PPE are used versus masks are not used. RN and LPN PPE adherence rates per inpatient wards are $64 \%\left[\frac{7}{7+4} * 100\right], 80 \%\left[\frac{4}{4+1} * 100\right]$ and $100 \%\left[\frac{6}{6+0} * 100\right]$ for $5 \mathrm{~B}$, 
6B and ICU, respectively. There is large variability in RN and LPN PPE compliance across inpatient wards. However, mask compliance with the necessity of wearing gloves and gowns cannot be judged because of the low sample size.

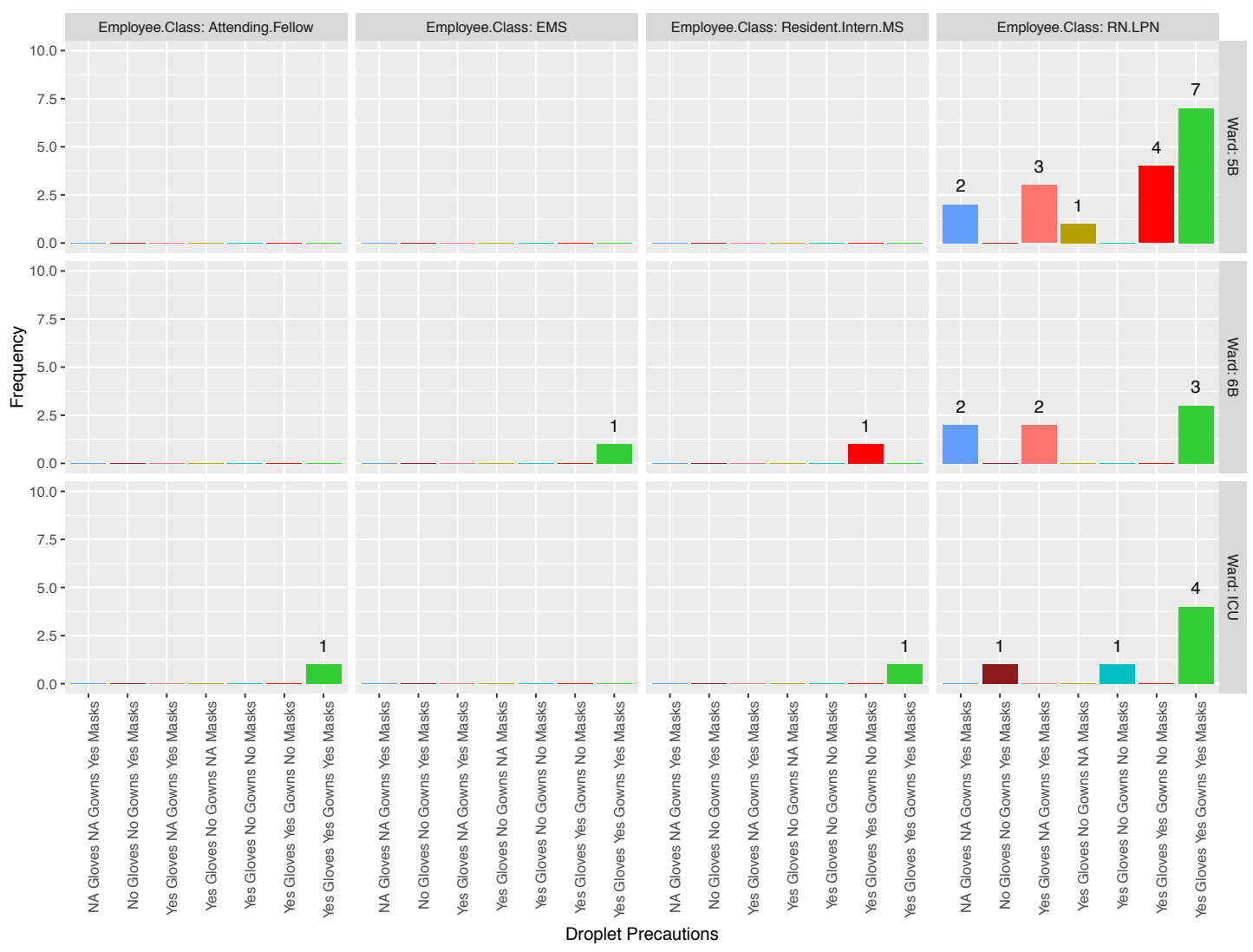

Figure 5.37 Inpatient-Wards PPE Observations for Droplet Precautions by Employee Class

\subsubsection{Airborne Precautions}

Following the lead of contact and droplet precautions analysis, next is the analysis when employee class is and is not included. Figure 5.38 shows that only RN and LPN is observed based on the data. Thus, the analysis is carried out by including employee class since all data is reported on RN and LPN only. Figure 5.38 shows that not all wards have the three possible scenarios. Accounting for all 3 possible scenarios 
combinations is possible because there are $\left(\begin{array}{l}3 \\ 2\end{array}\right)=\frac{3 !}{2 !^{*}(3-2) !}$, or 3 possible combinations out of 3 scenarios. However, all combinations do not have a scenario where either masks or gloves or gowns are not worn (No Gloves, No Gowns, No Masks). Thus, PPE compliance is hard to calculate. The three existing combinations are:

- When gloves, gowns and masks are used with 2 observations.

- When gloves and masks are used and gowns are not applicable with 2 observations.

- When gloves and gowns are not applicable and masks are used with 2 observations.

Logically, RN and LPN PPE compliance could be calculated by considering all available cases for masks being used and there are no single observations or cases where masks are not used. However, due to the low sample sizes per inpatient wards, this is neglected. 


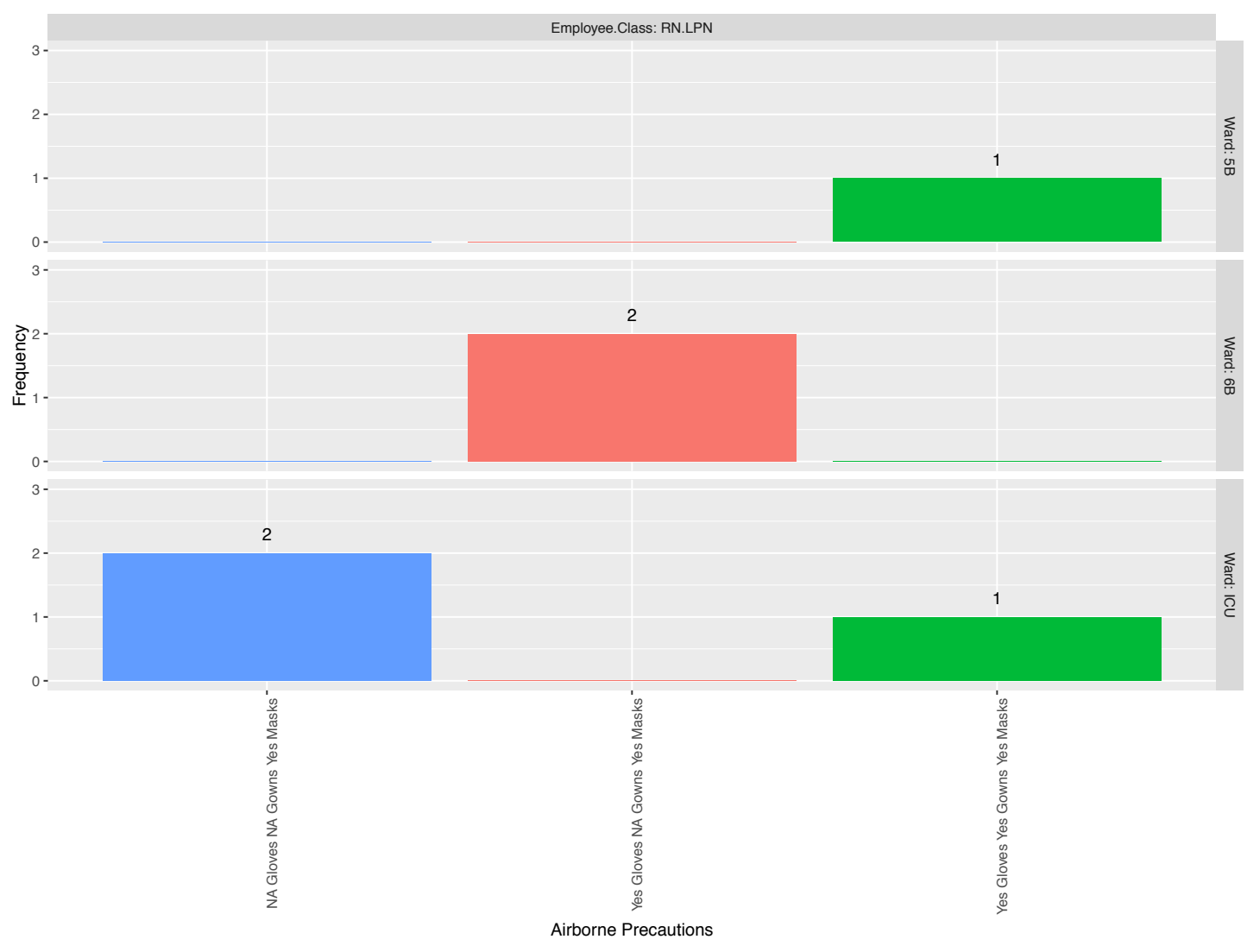

Figure 5.38 Inpatient-Wards PPE Observations for Airborne Precautions by Employee Class 


\subsection{Observational Study of Hand Hygiene Compliance}

After conducting a retrospective analysis of the direct hand hygiene observations method based on the PVAMC data, an analysis of the direct hand hygiene observations method based on data collected by the URI research team is conducted. Summary statistics are reported and the researchable questions the hypotheses testing are addressed.

\subsubsection{Descriptive Statistics}

The URI research team looked for 40 secret observers to participate in directly observing hand hygiene behavior of $\mathrm{HCW}$ at $5 \mathrm{~B}, 6 \mathrm{~B}$ and ICU. Potential participants were any PVAMC employee who is neither a nurse nor a doctor who works in any of the inpatient wards, 5B, 6B and ICU. In addition, a secret observer should have secret observing experience, if possible. Out of the desired 40, 25 secret observers were recruited plus the URI research member. Among the 25 participants, 6 do not have any collected observations by chance. Observations are randomly collected and sometimes a participant does not have the opportunity to capture any hand hygiene observations while doing their job. Over the two months of study, September and October, only five days of October did not have observations collected and they are weekends. Of note, some of the secret observers are experts and some are not. Discrepancy is expected between their observations and 95\% agreement accuracy should be conducted.

The secret observers including the URI research member were able to collect 2432 hand hygiene observations, in total, during September and October as seen in 
Figure 5.39. There are $1456(60 \%)$ hand hygiene observations versus $976(40 \%)$ hand hygiene observations for the URI research member and the PVAMC participants respectively. The hand hygiene compliance observations, in total, are 1264 (52\%) versus 1168 (48\%) for hand hygiene non-compliance observations.

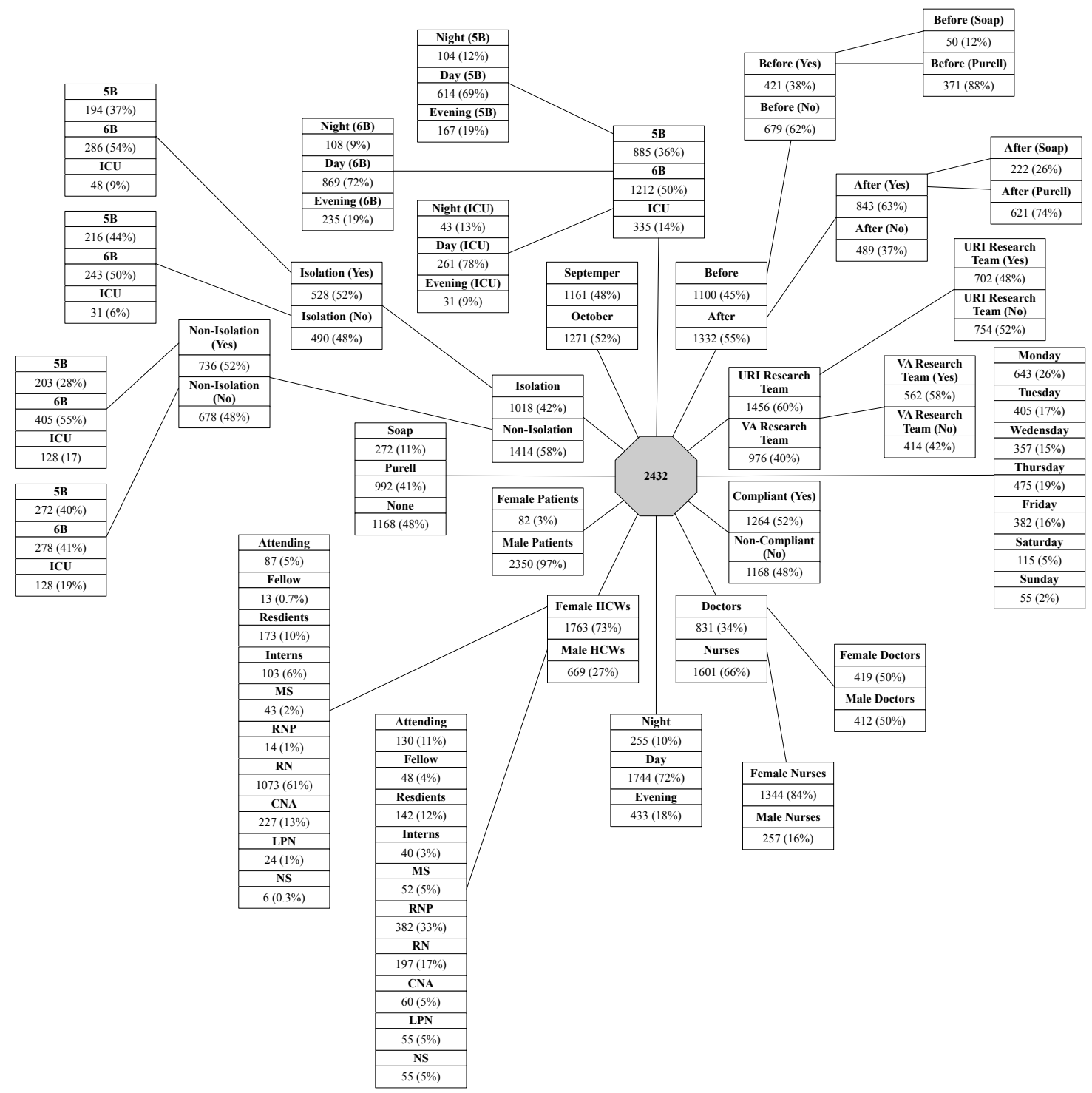

Figure 5.39 Summary Statistics of Hand Hygiene Observations (Total Samples, Hand Hygiene Compliance and Non-Compliance and Percentages) 
For any Hawthorne Effect determination effort, the hand hygiene compliance is calculated based on a separation of both observations, the ones that are collected by the URI research team versus the PVAMC participants. It is important to recognize that hand hygiene compliance improvement could be due to such a Hawthorne effect if the healthcare workers recognize the URI research team member and realize that they are associated with hand hygiene studies, and then make changes in their hand hygiene behavior. Thus, the following hypothesis test is statistically evaluated using a test for two proportions using a level of significance of $5 \%$.

$$
\begin{aligned}
& H_{0}: P_{U R I}=P_{P V A M C} \\
& H_{1}: P_{U R I}<P_{P V A M C}
\end{aligned}
$$

The null hypothesis states that the hand hygiene compliance based on the URI research team and the PVAMC team data collections are the same versus the alternative hypothesis that states the URI research team's hand hygiene compliance is smaller than the PVAMC team's. The URI research team hand hygiene compliance and non-compliance are $702(48 \%)$ and 754 (52\%). On the other hand, the PVAMC participants' hand hygiene compliance and non-compliance are $562(58 \%)$ and 414 $(42 \%)$. Based on the result of the test for two sample proportions with a p-value that is smaller than $\alpha=0.05$, the null hypothesis, $H_{0}$, is rejected. Consequently, the hand hygiene compliance based on the URI research team's data is smaller than the PVAMC's.

There are 1161 (48\%) hand hygiene observations collected in September versus 1271 (52\%) hand hygiene observations collected in October as seen in Figure 5.39. The hand hygiene compliance and non-compliance of September are 648 (56\%) and 
$513(44 \%)$. On the other hand, the hand hygiene compliance and non-compliance of October are $616(49 \%)$ and $655(51 \%)$. In total, there are $1100(45 \%)$ hand hygiene observations collected for Before Touching a Patient hand hygiene moment versus 1332 (55\%) hand hygiene observations collected for After Touching a Patient hand hygiene moment. The hand hygiene compliance and non-compliance for Before Touching a Patient are $421(38 \%)$ and 679 (62\%). On the other hand, the hand hygiene compliance and non-compliance for After Touching a Patient are $843(63 \%)$ and 489 (37\%). It seems that HCWs at the PVAMC value hand wash after touching a patient more than before touching a patient. Yet, both moments' hand hygiene compliances are considered low. In terms of hygiene products, soap is used 272 (11\%) while Purell is used 992 (41\%). Classifying Before and After Touching a Patient hand hygiene moments based on hygiene products, soap is used $50(12 \%)$ for Before Touching a Patient while soap is used 222 (26\%) for After Touching a Patient. On the other hand, Purell is used 371 (88\%) for Before Touching a Patient while Purell is used 621 (74\%) for After Touching a Patient.

In terms of inpatient wards, the hand hygiene collected observations are 885 (36\%), $1212(50 \%)$ and 335 (14\%) for 5B, 6B and ICU, respectively as seen in Figure 5.39. The hand hygiene compliance and non-compliance are 397 (45\%) and 488 (55\%), $691(57 \%)$ and $521(43 \%), 176(53 \%)$ and $159(47 \%)$ for 5B, 6B and ICU respectively. The hand hygiene collected observations per working shift are 255 (10\%), 1744 (72\%) and $433(18 \%)$ for night, day and evening shift, respectively. At 5B, there are $104(12 \%), 614(69 \%)$ and $167(19 \%)$ hand hygiene observations for night, day and evening shift, respectively. At 6B, there are 108 (9\%), 869 (72\%) and 
235 (19\%) hand hygiene observations for night, day and evening shift, respectively. At ICU, there are 43 (13\%), $261(78 \%)$ and $31(9 \%)$ hand hygiene observations for night, day and evening shift, respectively. In terms of patient gender, the hand hygiene collected observations are $82(3 \%)$ and $2350(97 \%)$ for female and male patients respectively. The hand hygiene compliance and non-compliance are $57(70 \%)$ and 25 (30\%), 1207 (51\%) and 1143 (49\%) for female and male patients, respectively. The hand hygiene compliance for female patients is higher than the male patients' and the hand hygiene compliance for male patients is considered low. Of note, most of the PVAMC patients are male and rarely there are female patients and most of the HCWs, both medical and nursing groups, are female.

In terms of isolation status, the hand hygiene collected observations are 1018 (42\%) and $1414(58 \%)$ for isolation and non-isolation respectively as seen in Figure 5.39. The hand hygiene compliance is $528(52 \%)$ and $736(52 \%)$ for isolation and nonisolation, respectively. The hand hygiene compliance for isolation and non-isolation are identical. In total, the hand hygiene collected observations are 831 (34\%) and 1601 (66\%) for medical and nursing groups, respectively. This is expected because the nursing group outweighs the medical group in both numbers of HCWs and in patient contact frequency. Thus, there are more hand hygiene opportunities to capture. The hand hygiene compliance is $444(53 \%)$ and $820(51 \%)$ for the medical and nursing groups, respectively. Their hand hygiene compliances are almost identical but very low. In total, the hand hygiene collected observations are 1763 (73\%) and $669(27 \%)$ for female and male HCWs respectively. The hand hygiene compliance is 920 (52\%) and $344(51 \%)$ for female and male HCWs, respectively. Their hand hygiene 
compliances are almost identical but very low. However, female HCWs outweigh male HCWs in terms of number of employees. There are 99 female HCWs (18 in the medical group versus 81 in the nursing group) and only 22 male HCWs (10 in the medical group versus 12 in the nursing group). In total, the hand hygiene collected observations are $419(50 \%)$ and $412(50 \%)$ for female and male HCWs in the medical group respectively. The hand hygiene compliance and non-compliance are 216 (52\%) and 203 (48\%), $228(55 \%)$ and 184 (45\%) for female and male HCWs in the medical group, respectively. The female and male medical group hand hygiene compliance is almost identical but low. However, the female medical group is almost double the male ones in terms of number of employees, 18 versus 10. In total, the hand hygiene collected observations are $1344(84 \%)$ and 257 (16\%) for female and male HCWs in the nursing group, respectively. The hand hygiene compliance is $704(52 \%)$ and 116 (45\%) for female and male HCWs in the nursing group, respectively. The female and male nursing group hand hygiene compliance are almost identical but low. However, the female nursing group is almost 7 times the male ones in terms of number of employees, 81 versus 12 .

In terms of days of the week, the hand hygiene collected observations are 643 (26\%), $405(17 \%), 357(15 \%), 475(19 \%), 382(16 \%), 115(5 \%), 55(2 \%)$, for Monday, Tuesday, Wednesday, Thursday, Friday, Saturday and Sunday respectively as seen in Figure 5.39. Very low numbers of hand hygiene observations are collected during weekends versus weekdays. 


\subsubsection{Hand Hygiene Population and Compliance}

After providing summary statistics of the hand hygiene compliance, the next is to provide hand hygiene compliance estimation and to report a $95 \%$ confidence interval per inpatient ward and all inpatient wards combined.

Based on patient contact data in Chapter 4 as seen in Figure 4.3, there are in total $5863,7578,2014$ and 15455 patient contacts for 5B, 6B, ICU and all inpatient wards combined respectively for both hand hygiene moments, Before and After. However, these numbers are broken down per weekday in order to precisely and accurately calculate how many patient contacts in total there are in September and October as seen in Figure 5.40. September (30 days) and October (31 days) have different numbers of days and they start on a different day of the week, September starts on Thursday and October starts on Saturday. It would be misleading to just multiply the total patient contacts shown in Figure 4.3 by 4 weeks for both months though one week of October will have the same exact values of patient contact since on that week the patient contact study was conducted. Total patient contact is calculated per weekday per inpatient ward and then the calculation is carried out per month by weekdays. The results are depicted in Figure 5.41. Total patient contacts for September are $25345,32588,8428$ and 66415 for 5B, 6B, ICU and all inpatient wards combined. Total patient contacts for October are 25778, 33484, 9006 and 68268 for 5B, 6B, ICU and all inpatient wards combined. These values are then multiplied by 2 to obtain the total patient contacts for both hand hygiene moments, Before and After.

After calculating total patient contacts per month, the hand hygiene compliance sample proportion (mean) and standard error of the sample proportion (standard 
deviation) of inpatient wards and inpatient wards combined are calculated and depicted per week and month. For September, the 30 days are grouped into four weeks ( 7 days, 7 days, 8 days and 8 days). For October, the 31 days are also grouped into four weeks ( 7 days, 8 days, 8 days and 8 days). Then, the weekly and monthly mean (sample proportion) $\mu_{P S}$ and the standard deviation (the standard error of the sample proportion) $\sigma_{P S}$ of the hand hygiene compliance are calculated and depicted together per inpatient wards and all wards combined as depicted in Figure 5.42. Of note, days with missing or no samples are excluded in the calculation of the hand hygiene compliance sample proportion and standard deviation because hand hygiene compliance cannot be assumed to equal zero. They are unknown.

The monthly mean of hand hygiene compliance for September and October respectively is $45 \%$ and $45 \%$ for $5 \mathrm{~B}, 64 \%$ and $50 \%$ for $6 \mathrm{~B}, 49 \%$ and $57 \%$ for ICU and $55 \%$ and $48 \%$ for all inpatient wards combined. The monthly standard deviation of hand hygiene compliance for September and October respectively is 3\% and $2 \%$ for $5 \mathrm{~B}, 2 \%$ and $3 \%$ for $6 \mathrm{~B}, 4 \%$ and $4 \%$ for ICU and $1 \%$ and $1 \%$ for all inpatient wards combined. The sample size of hand hygiene observations for September and October, respectively, is 350 and 535 for $5 \mathrm{~B}, 620$ and 592 for $6 \mathrm{~B}, 191$ and 144 for ICU and 1161 and 1271 for all inpatient wards combined. Interestingly, the weekly sample proportion (mean) of hand hygiene compliance on September drops from week one to week two for $5 \mathrm{~B}, 6 \mathrm{~B}$ and all wards combined. ICU is a special case because of its sample size as can be seen by the large variability. There might be evidence of a Hawthorne effect because of the high level of hand hygiene compliance during the 
first week of the study. In this study, September is better in hand hygiene compliance than October.

In September, by comparing the monthly total sample size in Figure 5.42 with the monthly total patient contact in Figure 5.41, combining both Before and After moments, for each inpatient ward and all wards combined, the monthly sample size only captures $0.7 \%(350 /[25345 * 2] * 100)$ with $498 \mathrm{BDOC}, 1 \%(620 /[32588 * 2] *$ 100) with 532 BDOC, $1.1 \%(191 /[8482 * 2] * 100)$ with 87 BDOC and $0.9 \%$ $(1161 /[66415 * 2] * 100)$ with 1117 BDOC for 5B, 6B, ICU and all inpatient wards combined, respectively. In October, by comparing the monthly total sample size in Figure 5.42 with the monthly total patient contact in Figure 5.41, combining both Before and After moments, for each inpatient ward and all wards combined, the monthly sample size only captures $1 \%(535 /[25778 * 2] * 100)$ with 448 BDOC, $0.9 \%$ $(592 /[33484 * 2] * 100)$ with 514 BDOC, $0.8 \%(144 /[9006 * 2] * 100)$ with 58 BDOC and $0.9 \%(1271 /[68268 * 2] * 100)$ with $1020 \mathrm{BDOC}$ for 5B, 6B, ICU and all inpatient wards combined, respectively. 


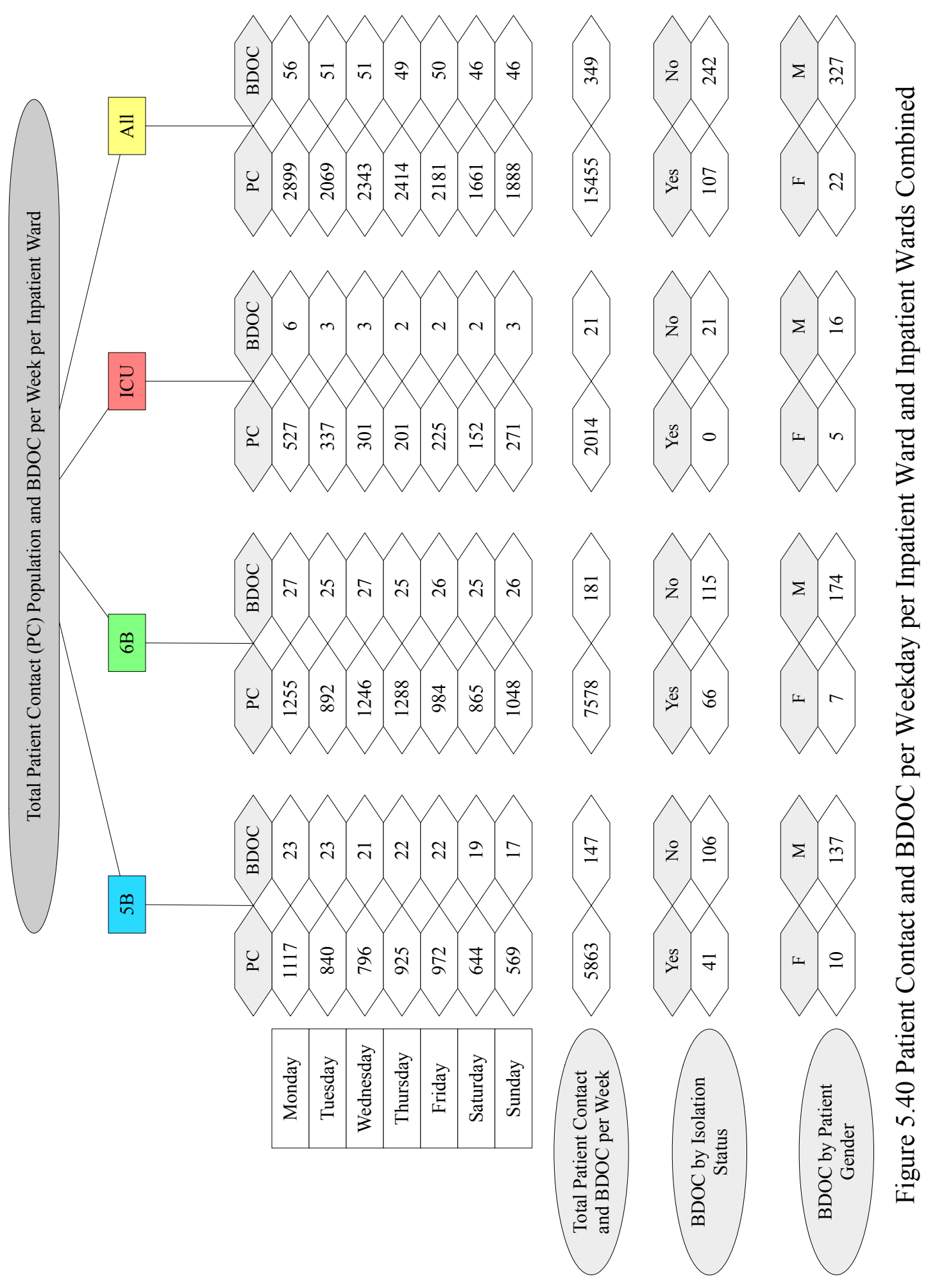



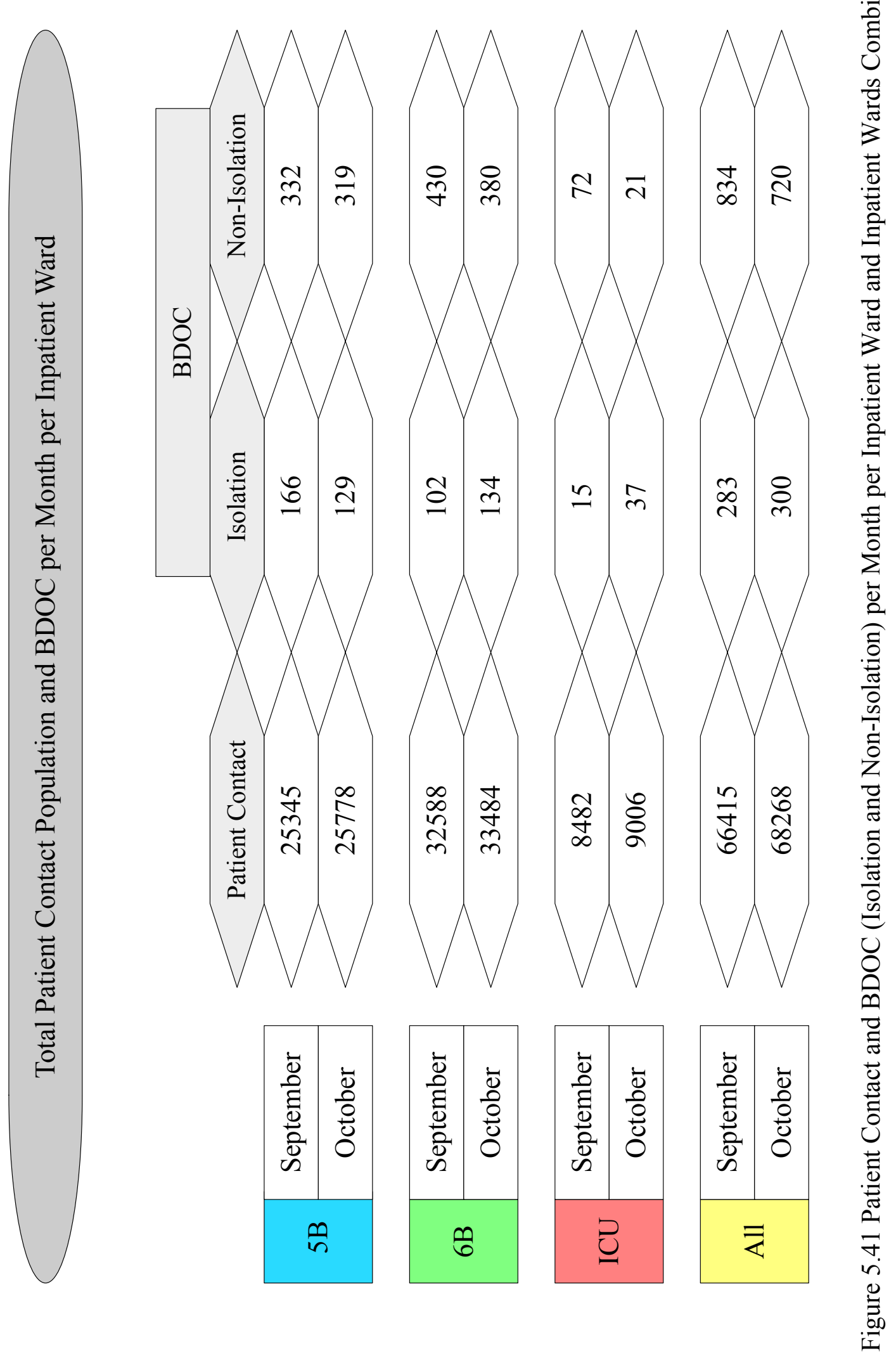


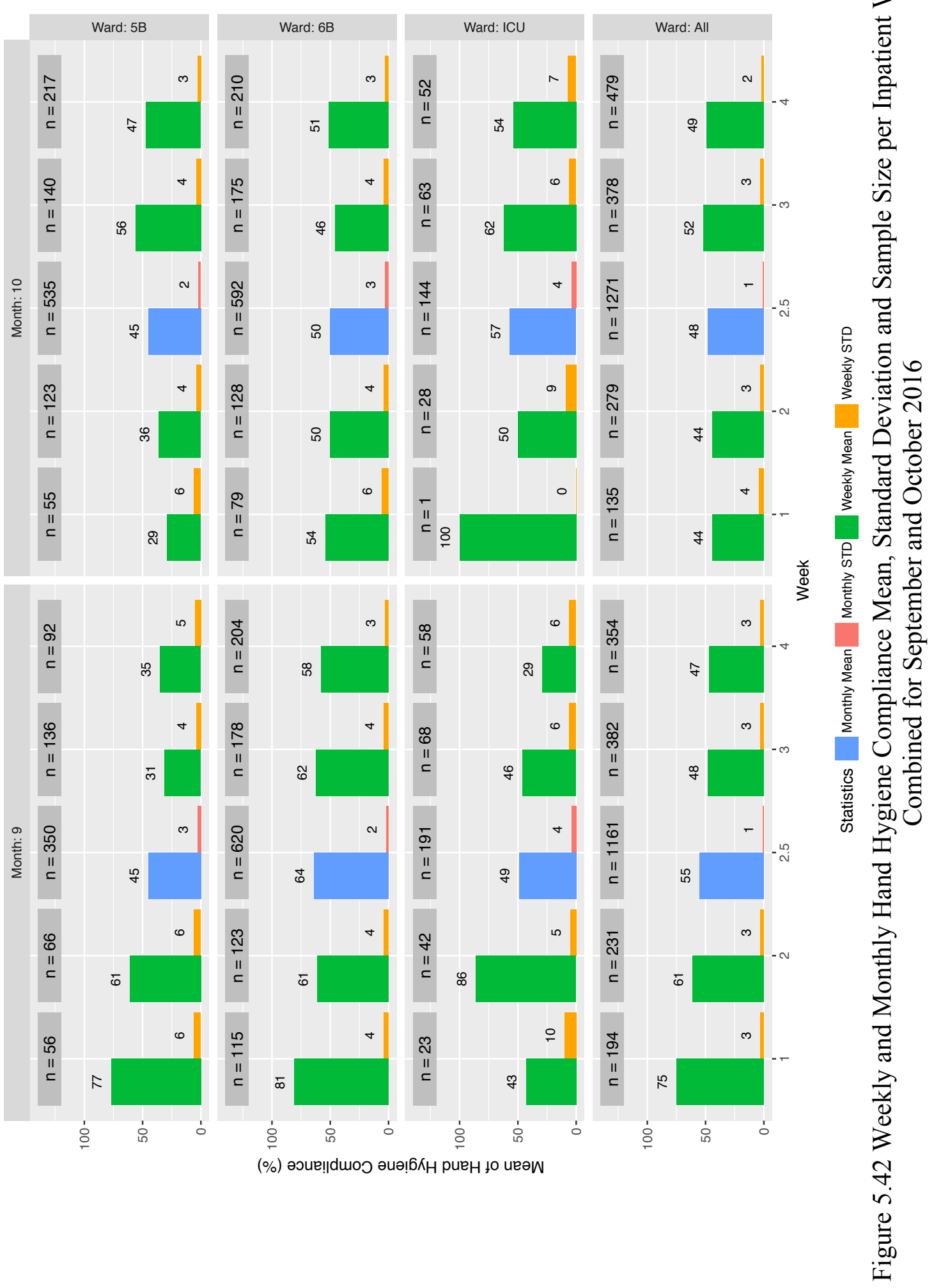


Though BDOC on September is higher than in October, total patient contacts are smaller in September than in October. An explanation could be that there were a higher number of $\mathrm{HCW}$ employees and a higher number of isolation BDOC in October than in September. In the week of patient contact data collection, there were $35 \mathrm{HCWs}$ in the nursing group at 5B, $40 \mathrm{HCWs}$ in the nursing group at $6 \mathrm{~B}, 18 \mathrm{HCWs}$ in the nursing group at ICU and $93 \mathrm{HCWs}$ in the nursing group at all inpatient wards combined across all working shifts as seen in Figure 4.1. In the same week, there were $28 \mathrm{HCWs}$ in the medical group who visited patients across all inpatient wards during all working shifts as seen in Figure 4.1. Unfortunately, there is no way to know exactly how many HCWs there were in September and in October. Based on Figure 5.41, Isolation BDOC is higher in October than in September except for 5B. Isolation BDOC could also be equal or higher than non-isolation BDOC since there are other infections that require patient isolation, which are not part of the research study.

In calculating and developing confidence interval estimates for the population parameter hand hygiene compliance, the type of population has to be determined first. The type of population can affect the width of the confidence interval and therefore, it can increase the accuracy of the hand hygiene compliance mean estimation. In addition, the type of population can determine how large the sample size is (power analysis and sample size). In statistics, according to Kozak (2008), there are two types of population, which are finite or infinite population. "Each population the elements of which exist in a particular time is finite" (Kozak, 2008, p.60). In the case of finite population, an adjustment of the standard error of the confidence interval is made by using the finite population correction (fpc) factor $\sqrt{\frac{N-n}{N-1}}$ (Berenson, Levine and 
Krehbiel, 2002) where $\mathrm{N}$ is population size and $\mathrm{n}$ is sample size. The criteria to decide whether fpc has a significant or insignificant effect on the confidence interval width is as follows (Berenson, Levine and Krehbiel, 2002):

- Significant Effect if: $\frac{n}{N}>5 \%$.

- Insignificant Effect if: $\frac{n}{N}<5 \%$.

With two assumptions exist, "the population variance $\sigma^{2}$ is unknown and Population is normally distributed or the sample size is large" (Berenson, Levine and Krehbiel, 2002, p.119), the right confidence interval formula including fpc, according to Berenson, Levine and Krehbiel (2002) is:

$$
\hat{p}_{s} \pm Z_{\frac{\alpha}{2}} \sqrt{\frac{\hat{p}_{s}\left(1-\hat{p}_{s}\right)}{n}} \sqrt{\frac{(N-n)}{(n-1)}}
$$

where $p_{s}$ is the sample proportion of success, $\mathrm{n}$ is the sample size or the total trials, $\mathrm{N}$ is the population size and $Z_{\frac{\alpha}{2}}$ is the value corresponding to a cumulative area of (1$\alpha$ ) from the standard normal distribution (Berenson, Levine and Krehbiel, 2002).

In the current research study, though the population of the patient contact is considered finite because it is countable and exits in a particular time, for one complete week of October, the collected sample size (n) at any one of the inpatient wards or at all wards combined divided by their population size $(\mathrm{N})$ is not even close to $2 \%$. Thus, the criteria above states that any correction made to the confidence interval is insignificant. As a result, the regular monthly confidence interval is calculated instead for each inpatient ward and all wards combined based on the following equation using $\alpha=0.05$ and the results are reported in Table 5.23. 


$$
\hat{p}-Z_{\frac{\alpha}{2}} \sqrt{\frac{\hat{p}(1-\hat{p})}{n}}<p<\hat{p}+Z_{\frac{\alpha}{2}} \sqrt{\frac{\hat{p}(1-\hat{p})}{n}}
$$

\begin{tabular}{|c|c|c|c|c|c|c|}
\hline \multirow{2}{*}{ Ward } & \multicolumn{3}{|c|}{ Sept } & \multicolumn{3}{c|}{ Oct } \\
\cline { 2 - 7 } & Mean & sd & $95 \%$ CI & Mean & sd & $95 \%$ CI \\
\hline 5B & 0.45 & 0.03 & $(0.40,0.50)$ & 0.45 & 0.02 & $(0.41,0.49)$ \\
\hline 6B & 0.64 & 0.02 & $(0.60,0.68)$ & 0.50 & 0.03 & $(0.46,0.54)$ \\
\hline ICU & 0.49 & 0.04 & $(0.42,0.56)$ & 0.57 & 0.04 & $(0.49,0.65)$ \\
\hline All & 0.55 & 0.01 & $(0.52,0.58)$ & 0.48 & 0.01 & $(0.45,0.51)$ \\
\hline
\end{tabular}

Table 5.23 Monthly Mean, Standard Deviation and 95\% Confidence Interval for Hand Hygiene Compliance per Inpatient Ward and All Wards Combined

By looking at Figure 5.43, the hand hygiene compliance mean could go as low as $40 \%$, which belongs to $5 \mathrm{~B}$ during September, and as high as $68 \%$, which belongs to 6B during September. The Hand hygiene compliance mean at 5B stays the same but with higher variability during September. On the other hand, there is a huge drop at 6B from September to October; but the lower hand hygiene compliance of $6 \mathrm{~B}$ is still higher than both 5B's. There is an increase in hand hygiene compliance mean at ICU from September to October; however, ICU has the highest variability in the study due to the small sample size. Based on all inpatient wards combined, the PVAMC's hand hygiene compliance mean is considered very low during the months of the study, $55 \%$ and $48 \%$ for September and October, respectively. When correlating the weekly hand hygiene compliance mean with the weekly BDOC per inpatient ward and all wards combined during September and October as seen in Figure 5.44, correlation is found except at all wards combined. There is a strong negative correlation between the weekly hand hygiene compliance mean and BDOC at 5B $(-0.90), 6 \mathrm{~B}(-0.55)$ and ICU (-0.59). As BDOC increased the hand hygiene compliance decreased. 
Now, two important scenarios are created and statistically tested, which lead to a future recommendation for the PVAMC:

- First: The PVAMC claims that their hand hygiene compliance is at its desired level, acceptably high, and their belief is entirely based on the very low number of HAIs in addition to the direct method observations that were collected over five years since April 2012. These observations, however, are actually a very small sample. The question was asked to determine how large the sample size should be to detect an effect of either $5 \%$ to reject such a claim. A $90 \%$ hand hygiene compliance mean is assumed.

- Second: The investigator or the URI research team believes that the PVAMC's HCWs take a 50-50 chance of practicing hand hygiene and would like to assess whether the hand hygiene compliance mean is either higher or lower than $50 \%$. Is there evidence of statistically higher or lower hand hygiene compliance as compared to the study's hand hygiene compliance results? How large should the sample size be to detect an effect of either $5 \%$ to reject such a null hypothesis though the samples that were already collected?

Though the study's results already show that the PVAMC's hand hygiene compliance is not at its best and is not very close to $90 \%$, statistical evidence has to be shown for the two assumptions, $90 \%$ and $50 \%$, separately. The following hypothesis tests are created for all inpatient wards combined and per month, September and October as seen in Table 5.24 for $90 \%$ for the first scenario and in Table 5.25 for $50 \%$ for the second scenario. The total trials in September are 1161 with number of success of 648 and the total trials in October are 1271 with number of success of 616 . Based 
on the p-value in Table 5.24, the null hypotheses of the two-tailed and the lower-tailed tests are rejected based on a p-value that is lower than $\alpha=0.05$ and failed to reject the null hypothesis of the upper-tailed test based on a p-value that is larger than $\alpha=0.05$ for September and October. There is statistically significant evidence at $\alpha=0.05$ to show that the monthly hand hygiene compliance mean at the PVAMC is not equal to $90 \%$ and indeed is lower than that.

\begin{tabular}{|c|c|c|c|}
\hline \multicolumn{2}{|c|}{ One Sample Hypotheses Test } & September (0.55) & October $(0.48)$ \\
\cline { 2 - 4 } Two-Tailed Test & $\begin{array}{l}H_{0}: p=0.9 \\
H_{1}: p \neq 0.9\end{array}$ & $\sim 0$ & $\sim 0$ \\
\hline \multirow{2}{*}{ Lower-Tailed Test } & $\begin{array}{l}H_{0}: p=0.9 \\
H_{1}: p<0.9\end{array}$ & $\sim 0$ & $\sim 0$ \\
\hline \multirow{2}{*}{ Upper-Tailed Test } & $\begin{array}{l}H_{0}: p=0.9 \\
H_{1}: p>0.9\end{array}$ & $\sim 1$ & $\sim 1$ \\
\hline
\end{tabular}

Table 5.24 One Sample Hypotheses Test (90\%) for Monthly Hand Hygiene Compliance Mean for All Inpatient Wards Combined

Based on the p-value in Table 5.25 for September, the null hypotheses of the two-tailed and the upper-tailed tests are rejected based on a p-value that is lower than $\alpha=0.05$ and failed to reject the null hypothesis of the lower-tailed test based on a pvalue that is larger than $\alpha=0.05$. There is statistically significant evidence at $\alpha=0.05$ to show that the monthly hand hygiene compliance mean for September at the PVAMC is not equal to $50 \%$ and indeed is larger than that. This conclusion was already proved based on the collected sample size during September (55\%).

Based on the p-value in Table 5.25 for October, all null hypotheses are failed to reject based on a p-value that is larger than $\alpha=0.05$. There is statistically insignificant evidence at $\alpha=0.05$ to show that the monthly hand hygiene compliance 
mean for October at the PVAMC is not equal to, larger or lower than $50 \%$. However, it is marginal insignificantly for a lower-tailed test with a p-value of 0.07 , which is not that much larger than $\alpha=0.05$. This conclusion is already proved based on the collected sample size during October (48\%).

\begin{tabular}{|c|c|c|c|}
\hline \multirow{2}{*}{\multicolumn{2}{|c|}{ One Sample Hypotheses Test }} & September $(0.55)$ & October $(0.48)$ \\
\hline & & \multicolumn{2}{|c|}{$\mathrm{p}$-value } \\
\hline Two-Tailed Test & $\begin{array}{l}H_{0}: p=0.5 \\
H_{1}: p \neq 0.5\end{array}$ & 0.0006559815 & 0.1538558 \\
\hline Lower-Tailed Test & $\begin{array}{l}H_{0}: p=0.5 \\
H_{1}: p<0.5\end{array}$ & 0.999672 & 0.0769279 \\
\hline Upper-Tailed Test & $\begin{array}{l}H_{0}: p=0.5 \\
H_{1}: p>0.5\end{array}$ & 0.0003279908 & 0.9230721 \\
\hline
\end{tabular}

Table 5.25 One Sample Hypotheses Test (50\%) for Monthly Hand Hygiene Compliance Mean for All Inpatient Wards Combined

There are two ways to determine how large of a sample is needed to have $95 \%$ confidence that an estimate of hand hygiene compliance is within a certain amount of error percentage of the true value of the hand hygiene compliance. The first method is to use a preliminary sample such as the samples that were collected already during September and October. The second method is to use no preliminary sample in case of starting a new study investigation. In the first method the following formula is used $\left(\frac{z_{\frac{\alpha}{2} * p *(1-p)}^{2}}{e^{2}}\right)$. In the second method the following formula is used $\left(\frac{Z_{\frac{\alpha}{2}}^{2}}{4 * e^{2}}\right)$. In the first method, the required sample is 380 and 383 for September and October respectively. Such samples are required to be in a $95 \%$ confidence that the monthly hand hygiene compliance mean estimate for September (0.55) and October $(0.48)$ is within $5 \%$ of the true value. In the second method, however, the required sample is 384 for each month. Such samples are required to be at least at $95 \%$ confidence that the monthly 
hand hygiene compliance mean estimate for September (0.55) and October (0.48) is within $5 \%$ of the true value. As the $5 \%$ margin error increases the required sample size decreases. Fortunately, both samples from the research study that were already collected for September (1161) and October (1271) are large enough to detect an error of size 3\% from the true mean. From the first method the required samples are 1056 and 1065 for September and October respectively and from the second method the required samples are 1067 for each month. 


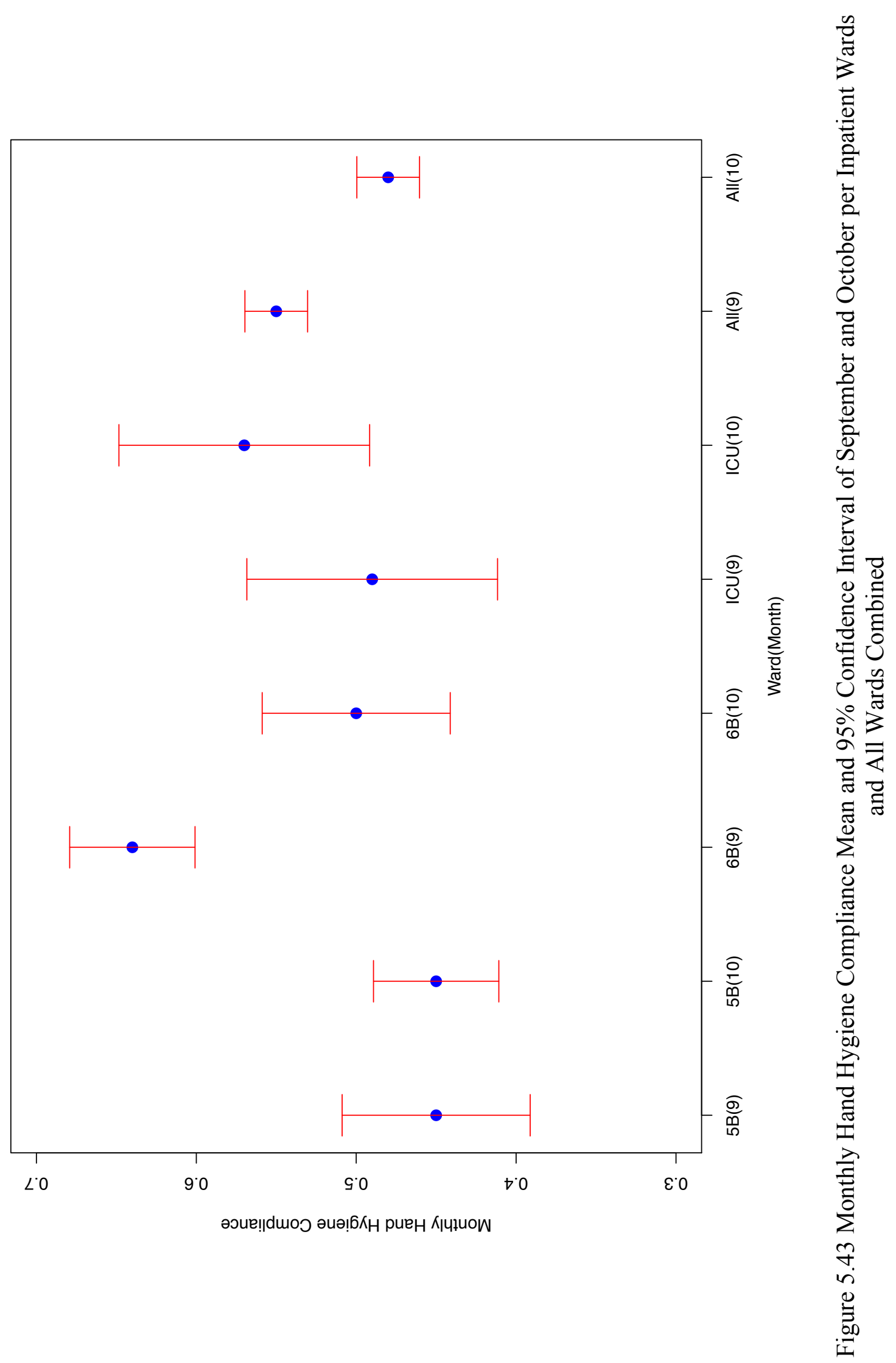



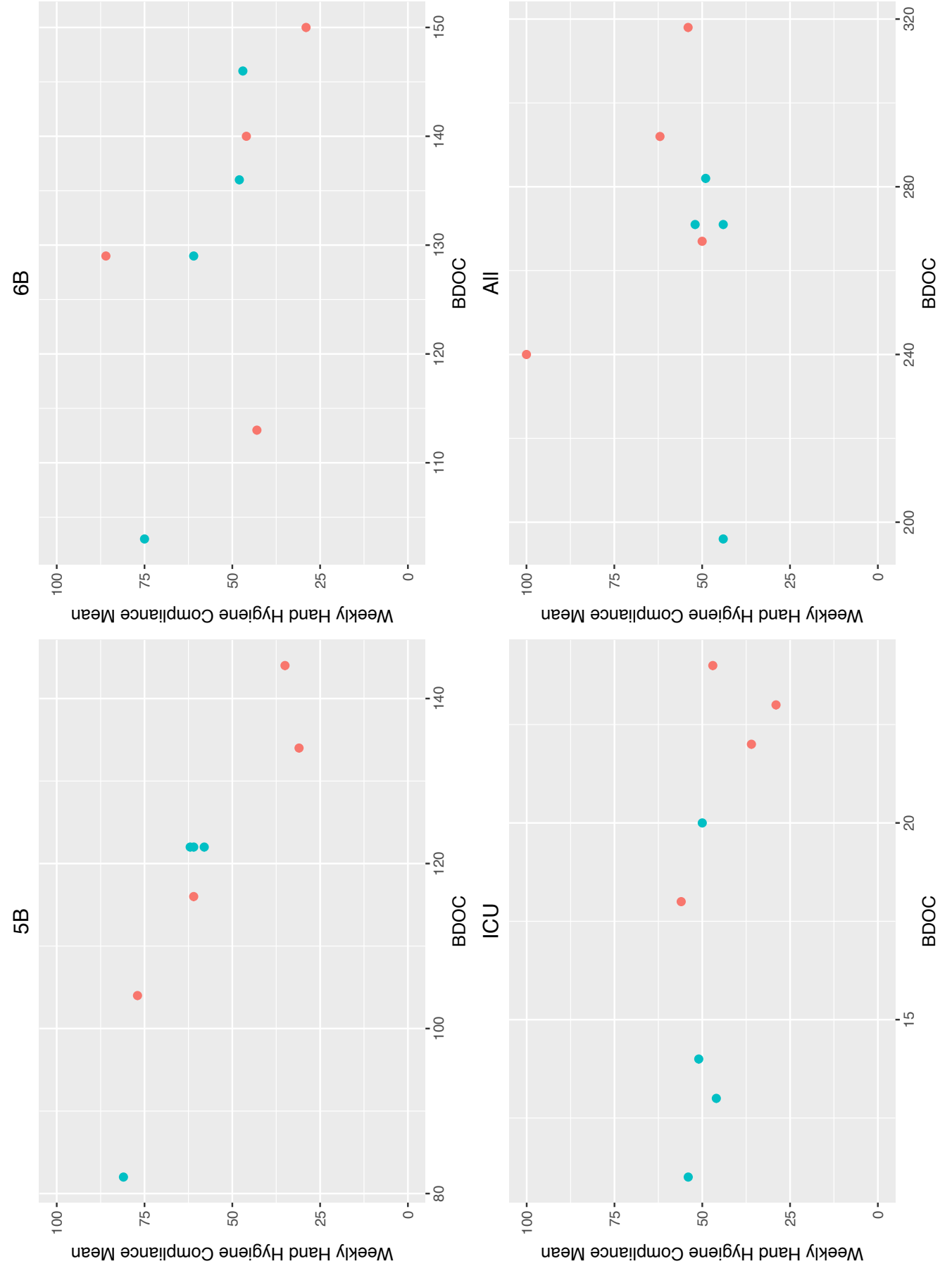


\subsubsection{Hypothesis Testing}

This subsection includes results from the hypotheses testing that was described in the methodology section in Chapter 3. These eight hypotheses deal with identifying any risk factor(s) for poor adherence to recommended hand-hygiene practices. Each hypothesis is tested individually, its sample proportion and p-value of the test are reported and a conclusion is drawn based on p-value as follows.

Hypotheses number 1, 2, 5, 6 and 8 are tested using a test for two proportions. Results for the hypothesis test for two proportions (Hypotheses number 1, 2, 5, 6 and 8), with a 95\% confidence interval and p-value are summarized together in Table 5.34. Additionally, hypotheses number 3,4 and 7 are tested using a test for several proportions, which is based on the Chi-Squared Statistic test. Results for the hypothesis test for several proportions (Hypotheses number 3, 4 and 7) and p-value are summarized together in Table 5.35.

Hypothesis 1: There is no difference in hand hygiene compliance rate by employee gender.

$$
\begin{aligned}
& H_{0}: p_{\text {Female }}-p_{\text {Male }}=0 \\
& H_{1}: p_{\text {Female }}-p_{\text {Male }} \neq 0
\end{aligned}
$$

\begin{tabular}{|c|c|c|c|}
\hline Group of Comparison & Female HCWs & Male HCWs & Total \\
\hline $\begin{array}{c}\text { Practicing Hand Hygiene } \\
\text { (Compliant) }\end{array}$ & 920 & 344 & 1264 \\
\hline $\begin{array}{c}\text { Non-Practicing Hand Hygiene } \\
\text { (Non-Compliant) }\end{array}$ & 843 & 325 & 1168 \\
\hline Total & 1763 & 669 & 2432 \\
\hline
\end{tabular}

Table 5.26 First Hypothesis Test: Proportion of Hand Hygiene Compliance by HCWs Gender 
Female's and males' compliant and non-compliant and total samples are reported in Table 5.26. The first hypothesis test is to estimate the difference between the proportion of subjects practicing hand hygiene by female HCWs and male HCWs, $H_{0}: p_{\text {Female }}-p_{\text {Male }}$. In other words, the hypothesis tests whether the hand hygiene compliance of female HCWs and male HCWs is different or not. Based on Table 5.34, $H_{0}$ is failed to be rejected and thus there is statistically insignificant evidence at $\alpha=0.05$ to show that the hand hygiene compliance differs by HCWs' gender, female versus male, based on a p-value that is equal to 0.736 as seen in Table 5.34. Based on such a result, HCWs' gender is not identified as a risk factor for poor adherence to recommended hand-hygiene practices at the PVAMC. However, the hand hygiene compliance mean for both HCWs genders are considered low, 52\% and 51\% for female and male HCWs, respectively.

Hypothesis 2: There is no difference in hand hygiene compliance rate by professional category, doctors vs. nurses.

$$
\begin{aligned}
& H_{0}: p_{\text {Doctors }}-p_{\text {Nurses }}=0 \\
& H_{1}: p_{\text {Doctors }}-p_{\text {Nurses }} \neq 0
\end{aligned}
$$

\begin{tabular}{|c|c|c|c|}
\hline Group of Comparison & Doctors & Nurses & Total \\
\hline $\begin{array}{c}\text { Practicing Hand Hygiene } \\
\text { (Compliant) }\end{array}$ & 44 & 820 & 1264 \\
\hline $\begin{array}{c}\text { Non-Practicing Hand Hygiene } \\
\text { (Non-Compliant) }\end{array}$ & 387 & 781 & 1168 \\
\hline Total & 831 & 1601 & 2432 \\
\hline
\end{tabular}

Table 5.27 Second Hypothesis Test: Proportion of Hand Hygiene Compliance by HCWs' Job Category

Doctor's and nurse's compliant and non-compliant and total samples are reported in Table 5.27. The second hypothesis test is to estimate the difference 
between the proportion of subjects practicing hand hygiene by doctors and nurses, $H_{0}: p_{\text {Doctors }}-p_{\text {Nurses }}$. In other words, the hypothesis tests whether the hand hygiene compliance of doctors and nurses is different or not. Based on Table 5.34, $H_{0}$ is failed to be rejected and thus there is statistically insignificant evidence at $\alpha=0.05$ to show that the hand hygiene compliance differs by HCWs' job category, doctors versus nurses, based on a p-value that is equal to 0.300 . Based on such a result, HCWs' job category is not identified as a risk factor for poor adherence to recommended handhygiene practices at the PVAMC. However, the hand hygiene compliance mean for both doctors and nurses are considered low, 53\% and 51\% for doctors and nurses respectively.

Hypothesis 3: There is no difference in hand hygiene compliance rate by type of unit, $5 \mathrm{~B}$ vs. $6 \mathrm{~B}$ vs. ICU.

$$
H_{0}: p_{5 B}=p_{6 B}=p_{I C U}
$$

$H_{1}: p_{5 B}, p_{6 B}$ and $p_{I C U}$ are not all equal

\begin{tabular}{|c|c|c|c|c|}
\hline Group of Comparison & $5 \mathrm{~B}$ & $6 \mathrm{~B}$ & ICU & Total \\
\hline $\begin{array}{c}\text { Practicing Hand Hygiene } \\
\text { (Compliant) }\end{array}$ & 397 & 691 & 176 & 1264 \\
\hline $\begin{array}{c}\text { Non-Practicing Hand Hygiene } \\
\text { (Non-Compliant) }\end{array}$ & 488 & 521 & 159 & 1168 \\
\hline Total & 885 & 1212 & 335 & 2432 \\
\hline
\end{tabular}

Table 5.28 Third Hypothesis Test: Proportion of Hand Hygiene Compliance by Inpatient Wards

5B's, 6B's and ICU's compliant and non-compliant and total samples are reported in Table 5.28. The third hypothesis test is to estimate whether the proportions of subjects practicing hand hygiene at the inpatient wards, 5B, 6B and ICU, $H_{0}: p_{5 B}=p_{6 B}=p_{I C U}$, are the same or whether the hand hygiene compliance for at 
least one of the wards is different. Based on Table 5.35, $H_{0}$ is rejected and thus there is statistically significant evidence at $\alpha=0.05$ to show that the proportion of hand hygiene compliance differs by inpatient ward, 5B, $6 \mathrm{~B}$ and ICU, based on a p-value that is equal to 0.00000026 . Based on such a result, inpatient ward is identified as a risk factor for poor adherence to recommended hand-hygiene practices at the PVAMC. However, the hand hygiene compliance mean for all inpatient wards are considered low, $45 \%, 57 \%$ and 53\% for 5B, $6 \mathrm{~B}$ and ICU respectively. In addition, 5B is statistically significantly lower, but $6 \mathrm{~B}$ and ICU are not statistically different. This is backed up with the correlation finding on Figure 5.44 and reported earlier where 5B $(-0.90), 6 \mathrm{~B}(-0.55)$ and ICU $(-0.59)$ have decreasing compliance with increasing BDOC. Additionally, based on the Marascuilo procedure for multiple proportions comparisons outcome on Table 5.29, since the absolute difference of 5B-6B is only greater than its critical range, and the absolute difference of 5B-ICU is marginally smaller than its critical range, the proportion of $5 \mathrm{~B}$ is significantly different from the proportion of $6 \mathrm{~B}$ and is marginally significantly different from the proportion of ICU.

\begin{tabular}{|c|c|c|c|}
\hline Proportions & Absolute Differences & Critical Range & Significance \\
\hline$\left|p_{5 B}-p_{6 B}\right|$ & 0.121 & 0.054 & Yes \\
\hline$\left|p_{5 B}-p_{I C U}\right|$ & 0.076 & 0.078 & No \\
\hline$\left|p_{6 B}-p_{I C U}\right|$ & 0.045 & 0.075 & No \\
\hline
\end{tabular}

Table 5.29 Hypothesis Three: The Marascuilo Procedure for Multiple Proportions Comparisons of Inpatient Wards

Hypothesis 4: There is no difference in hand hygiene compliance rate by days of the week, Monday vs. Friday.

$$
\begin{gathered}
H_{0}: p_{\text {Mon }}=p_{\text {Tues }}=p_{\text {Wed }}=p_{\text {Thurs }}=p_{\text {Fri }}=p_{\text {Sat }}=p_{\text {Sun }} \\
H_{1}: p_{\text {Mon }}, p_{\text {Tues }}, p_{\text {Wed }}, p_{\text {Thurs }}, p_{\text {Fri }}, p_{\text {Sat }} \text { and } p_{\text {Sun }} \text { are not all equal }
\end{gathered}
$$




\begin{tabular}{|c|c|c|c|c|c|c|c|c|}
\hline Group of Comparison & Mon & Tues & Wed & Thurs & Fri & Sat & Sun & Total \\
\hline $\begin{array}{c}\text { Practicing Hand Hygiene } \\
\text { (Compliant) }\end{array}$ & 312 & 202 & 176 & 254 & 204 & 73 & 43 & 1264 \\
\hline $\begin{array}{c}\text { Non-Practicing Hand Hygiene } \\
\text { (Non-Compliant) }\end{array}$ & 331 & 203 & 181 & 221 & 178 & 42 & 12 & 1168 \\
\hline Total & 643 & 405 & 357 & 475 & 382 & 115 & 55 & 2432 \\
\hline
\end{tabular}

Table 5.30 Fourth Hypothesis Test: Proportion of Hand Hygiene Compliance by Days of the Week

Days of the week's compliant and non-compliant and total samples are reported in Table 5.29. The fourth hypothesis test is to estimate whether the proportions of subjects practicing hand hygiene during the days of the week, Monday through Sunday, $H_{0}: p_{\text {Mon }}=p_{\text {Tues }}=p_{\text {Wed }}=p_{\text {Thurs }}=p_{\text {Fri }}=p_{\text {Sat }}=p_{\text {Sun }}$, are the same. In other words, the hypothesis tests whether the hand hygiene compliance on all days of the week is the same or if at least one day is different. Based on Table 5.35, $H_{0}$ is rejected and thus there is statistically significant evidence at $\alpha=0.05$ to show that the proportion of hand hygiene compliance differs by days of the week, based on a pvalue that is equal to 0.0001594 . Based on such a result, days of the week are identified as a risk factor for poor adherence to recommended hand-hygiene practices at the PVAMC. However, the hand hygiene compliance mean for all days of the week are considered low, 49\%, 50\%, 49\%, 53\%, 53\%, 63\% and 78\% for Monday, Tuesday, Wednesday, Thursday, Friday, Saturday and Sunday, respectively. Weekends seem to have better hand hygiene compliance than weekdays. Additionally, based on the Marascuilo procedure for multiple proportions comparisons outcome on Table 5.31, since the absolute difference of Sunday with all weekdays except Saturday are greater than the critical range, the proportion of Sunday is significantly different from the proportion of all weekdays except the proportion of Saturday. 


\begin{tabular}{|c|c|c|c|}
\hline Proportions & Absolute Differences & Critical Range & Significance \\
\hline$\left|p_{\text {Mon }}-p_{\text {Tues }}\right|$ & 0.014 & 0.113 & No \\
\hline$\left|p_{\text {Mon }}-p_{\text {Wed }}\right|$ & 0.008 & 0.117 & No \\
\hline$\left|p_{\text {Mon }}-p_{\text {Thurs }}\right|$ & 0.05 & 0.107 & No \\
\hline$\left|p_{\text {Mon }}-p_{\text {Fri }}\right|$ & 0.049 & 0.114 & No \\
\hline$\left|p_{\text {Mon }}-p_{\text {Sat }}\right|$ & 0.15 & 0.174 & No \\
\hline$\left|p_{\text {Mon }}-p_{\text {Sun }}\right|$ & 0.297 & 0.21 & Yes \\
\hline$\left|p_{\text {Tues }}-p_{\text {Wed }}\right|$ & 0.006 & 0.129 & No \\
\hline$\left|p_{\text {Tues }}-p_{\text {Thurs }}\right|$ & 0.036 & 0.12 & No \\
\hline$\left|p_{\text {Tues }}-p_{\text {Fri }}\right|$ & 0.035 & 0.126 & No \\
\hline$\left|p_{\text {Tues }}-p_{\text {Sat }}\right|$ & 0.136 & 0.182 & No \\
\hline$\left|p_{\text {Tues }}-p_{\text {Sun }}\right|$ & 0.283 & 0.216 & Yes \\
\hline$\left|p_{\text {Wed }}-p_{\text {Thurs }}\right|$ & 0.042 & 0.124 & No \\
\hline$\left|p_{\text {Wed }}-p_{\text {Fri }}\right|$ & 0.041 & 0.13 & No \\
\hline$\left|p_{\text {Wed }}-p_{\text {Sat }}\right|$ & 0.142 & 0.185 & No \\
\hline$\left|p_{\text {Wed }}-p_{\text {Sun }}\right|$ & 0.289 & 0.219 & Yes \\
\hline$\left|p_{\text {Thurs }}-p_{\text {Fri }}\right|$ & 0.001 & 0.122 & No \\
\hline$\left|p_{\text {Thurs }}-p_{\text {Sat }}\right|$ & 0.1 & 0.179 & No \\
\hline$\left|p_{\text {Thurs }}-p_{\text {Sun }}\right|$ & 0.247 & 0.214 & Yes \\
\hline$\left|p_{\text {Fri }}-p_{\text {Sat }}\right|$ & 0.101 & 0.183 & No \\
\hline$\left|p_{\text {Fri }}-p_{\text {Sun }}\right|$ & 0.248 & 0.217 & Yes \\
\hline$\left|p_{\text {Sat }}-p_{\text {Sun }}\right|$ & 0.147 & 0.254 & No \\
\hline
\end{tabular}

Table 5.31 Hypothesis Four: The Marascuilo Procedure for Multiple Proportions Comparisons of Days of the Week

Hypothesis 5: There is no difference in hand hygiene compliance rate by "My 5 Moments for Hand Hygiene" (WHO, 2009), such as Before and After Contacting a Patient.

$$
\begin{aligned}
& H_{0}: p_{\text {Before }}-p_{\text {After }}=0 \\
& H_{1}: p_{\text {Before }}-p_{\text {After }} \neq 0
\end{aligned}
$$




\begin{tabular}{|c|c|c|c|}
\hline Group of Comparison & $\begin{array}{c}\text { Before Touching a } \\
\text { Patient }\end{array}$ & $\begin{array}{c}\text { After Touching a } \\
\text { Patient }\end{array}$ & Total \\
\hline $\begin{array}{c}\text { Practicing Hand Hygiene } \\
\text { (Compliant) }\end{array}$ & 421 & 843 & 1264 \\
\hline $\begin{array}{c}\text { Non-Practicing Hand Hygiene } \\
\text { (Non-Compliant) }\end{array}$ & 679 & 489 & 1168 \\
\hline Total & 1100 & 1332 & 2432 \\
\hline
\end{tabular}

Table 5.32 Fifth Hypothesis Test: Proportion of Hand Hygiene Compliance by Hand Hygiene Moments

Before's and after's touching a patient compliant and non-compliant and total samples are reported in Table 5.30. The fifth hypothesis test is to estimate the difference between the proportion of subjects practicing hand hygiene before touching a patient and after touching a patient, $H_{0}: p_{\text {Before }}-p_{\text {After. }}$ In other words, the hypothesis tests whether the hand hygiene compliance of before or after touching a patient is different or not. Based on Table 5.34, $H_{0}$ is rejected and thus there is statistically significant evidence at $\alpha=0.05$ to show that the hand hygiene compliance differs by hand hygiene moment, before and after touching a patient, based on a p-value that is almost equal to 0 . Based on such a result, hand hygiene moment is identified as a risk factor for poor adherence to recommended handhygiene practices at the PVAMC. In addition, the hand hygiene compliance mean for before touching a patient is very low, 38\% and for after touching a patient is considered low too, $63 \%$.

Hypothesis 6: There is no difference in hand hygiene compliance rate by patient gender.

$$
\begin{aligned}
& H_{0}: p_{\text {Female Patients }}-p_{\text {Male Patients }}=0 \\
& H_{1}: p_{\text {Female Patients }}-p_{\text {Male Patients }} \neq 0
\end{aligned}
$$




\begin{tabular}{|c|c|c|c|}
\hline Group of Comparison & Female Patient & Male Patient & Total \\
\hline $\begin{array}{c}\text { Practicing Hand Hygiene } \\
\text { (Compliant) }\end{array}$ & 57 & 1207 & 1264 \\
\hline $\begin{array}{c}\text { Non-Practicing Hand Hygiene } \\
\text { (Non-Compliant) }\end{array}$ & 25 & 1143 & 1168 \\
\hline Total & 82 & 2350 & 2432 \\
\hline
\end{tabular}

Table 5.33 Sixth Hypothesis Test: Proportion of Hand Hygiene Compliance by Patient's Gender

Female Patient's and male Patient's compliant and non-compliant and total samples are reported in Table 5.31. The sixth hypothesis test is to estimate the difference between the proportion of subjects practicing hand hygiene when caregiving is provided for female patients versus male patients, $H_{0}: p_{\text {Femal Patient }}-$ $p_{\text {Mal Patient }}$. In other words, the hypothesis tests whether the hand hygiene compliance when caregiving is provided for female patients versus male patients is different or not. Based on Table 5.34, $H_{0}$ is rejected and thus there is statistically significant evidence at $\alpha=0.05$ to show that the hand hygiene compliance differs when caregiving is provided for female patients versus male patients, based on a p-value that is almost equal to 0 . Based on such a result, patient gender is identified as a risk factor for poor adherence to recommended hand-hygiene practices at the PVAMC. In addition, the hand hygiene compliance mean based on male patients is low, $51 \%$ and based on female patients is much better but still not at a good level, $70 \%$.

Hypothesis 7: There is no difference in hand hygiene compliance rate by working shift, Night, Day and Evening.

$$
\begin{gathered}
H_{0}: p_{\text {Night }}=p_{\text {Day }}=p_{\text {Evening }} \\
H_{1}: p_{\text {Night }}, p_{\text {Day }} \text { and } p_{\text {Evening }} \text { are not all equal }
\end{gathered}
$$




\begin{tabular}{|c|c|c|c|c|}
\hline Group of Comparison & Night & Day & Evening & Total \\
\hline $\begin{array}{c}\text { Practicing Hand Hygiene } \\
\text { (Compliant) }\end{array}$ & 97 & 939 & 228 & 1264 \\
\hline $\begin{array}{c}\text { Non-Practicing Hand Hygiene } \\
\text { (Non-Compliant) }\end{array}$ & 158 & 805 & 205 & 1168 \\
\hline Total & 255 & 1744 & 433 & 2432 \\
\hline
\end{tabular}

Table 5.34 Seventh Hypothesis Test: Proportion of Hand Hygiene Compliance by Working Shift

Night's, day's and evening's compliant and non-compliant and total samples are reported in Table 5.32. The seventh hypothesis test is to estimate whether the proportions of subjects practicing hand hygiene during the three different shifts, Night, Day and Evening, $H_{0}: p_{\text {Night }}=p_{\text {Day }}=p_{\text {Evening }}$, are the same. In other words, the hypothesis tests whether the hand hygiene compliance of all working shifts is the same or at least one shift is different. Based on Table 5.35, $H_{0}$ is rejected and thus there is statistically significant evidence at $\alpha=0.05$ to show that the proportion of hand hygiene compliance differs by working shift, Night, Day and Evening, based on a pvalue that is equal to 0.000014 . Based on such a result, shift is identified as a risk factor for poor adherence to recommended hand-hygiene practices at the PVAMC. However, the hand hygiene compliance mean for all working shifts are considered low, 38\%, 54\% and 53\% for Night, Day and Evening, respectively. Additionally, based on the Marascuilo procedure for multiple proportions comparisons outcome on Table 5.35, since the absolute differences of Night with Day and Evening are greater than the critical range, the proportion of Night is significantly different from the proportion of Day and Evening. 


\begin{tabular}{|c|c|c|c|}
\hline Proportions & Absolute Differences & Critical Range & Significance \\
\hline$\left|p_{\text {Night }}-p_{\text {Day }}\right|$ & 0.158 & 0.080 & Yes \\
\hline$\left|p_{\text {Night }}-p_{\text {Evening }}\right|$ & 0.147 & 0.095 & Yes \\
\hline$\left|p_{\text {Day }}-p_{\text {Evening }}\right|$ & 0.011 & 0.066 & No \\
\hline
\end{tabular}

Table 5.35 Hypothesis Seven: The Marascuilo Procedure for Multiple Proportions Comparisons of Working Shift

Hypothesis 8: There is no difference in hand hygiene compliance rate by patient status, such as patients who are on isolation vs. those who are not on isolation.

$$
\begin{aligned}
& H_{0}: p_{\text {Isolation }}-p_{\text {Non-Isolation }}=0 \\
& H_{1}: p_{\text {Isolation }}-p_{\text {Non-Isolation }} \neq 0
\end{aligned}
$$

\begin{tabular}{|c|c|c|c|}
\hline Group of Comparison & $\begin{array}{c}\text { Patients on } \\
\text { Isolation }\end{array}$ & $\begin{array}{c}\text { Patients on } \\
\text { Non-Isolation }\end{array}$ & Total \\
\hline $\begin{array}{c}\text { Practicing Hand Hygiene } \\
\text { (Compliant) }\end{array}$ & 528 & 736 & 1264 \\
\hline $\begin{array}{c}\text { Non-Practicing Hand Hygiene } \\
\text { (Non-Compliant) }\end{array}$ & 490 & 678 & 1168 \\
\hline Total & 1018 & 1414 & 2432 \\
\hline
\end{tabular}

Table 5.36 Eighth Hypothesis Test: Proportion of Hand Hygiene Compliance by Patient's Isolation Status

Isolation status's compliant and non-compliant and total samples are reported in Table 5.33. The eighth hypothesis test is to estimate the difference between the proportion of subjects practicing hand hygiene when caregiving is provided for patients on isolation versus on non-isolation, $H_{0}$ : $p_{\text {Isolation }}-p_{\text {Non-Isolation. }}$ In other words, the hypothesis tests whether the hand hygiene compliance when caregiving is provided based on patient's isolation status is different or not. Based on Table 5.34, $H_{0}$ is failed to be rejected and thus there is statistically insignificant evidence at $\alpha=0.05$ to show that the hand hygiene compliance differs when caregiving is provided based on patient's isolation status, based on a p-value that is almost equal to 
0.799. Based on such a result, patient's isolation status is not identified as a risk factor for poor adherence to recommended hand-hygiene practices at the PVAMC. However, the hand hygiene compliance mean based on patient's isolation status is low, $52 \%$ and $51 \%$ for isolation and non-isolation, respectively. 


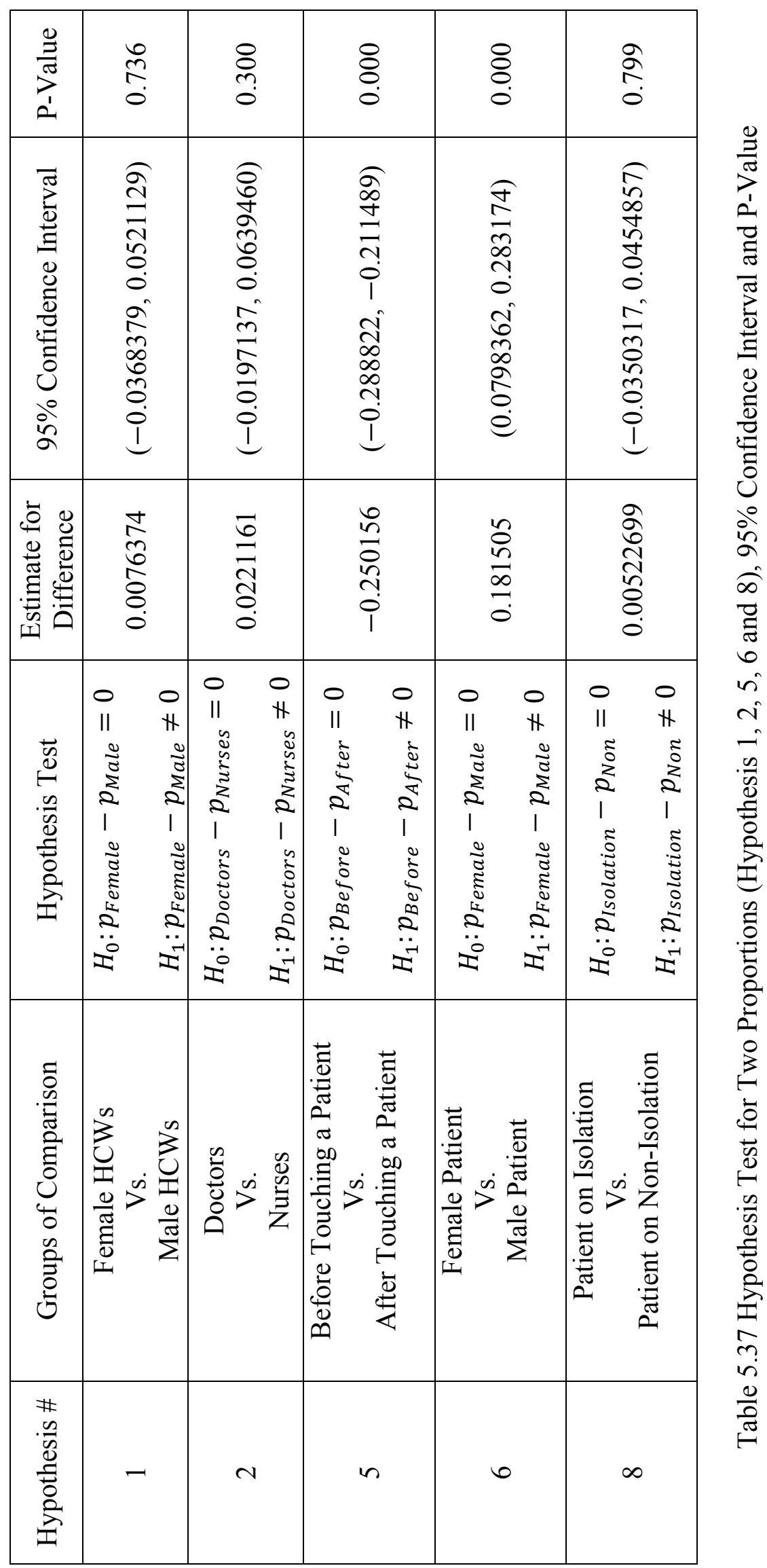




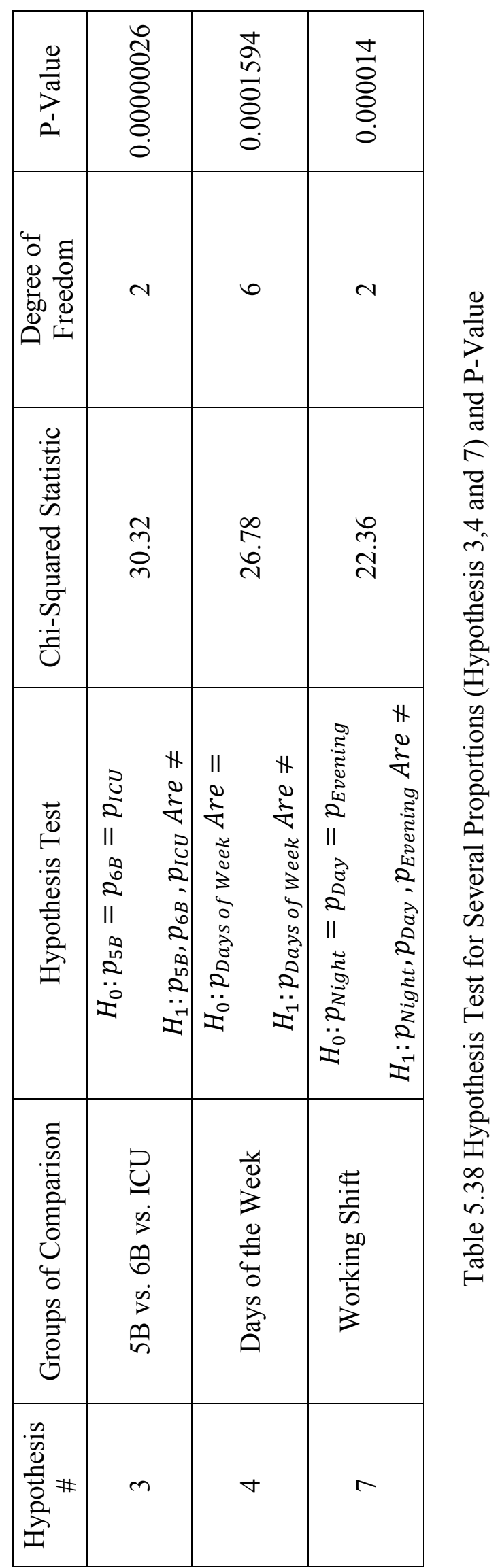




\subsection{The Correlation of HAI and Hand Hygiene Compliance}

It is important to study the relationship between hand hygiene compliance and HAIs. It is expected that HAIs go down as hand hygiene compliance goes up. Simply, the more HCWs adhere to recommended hand hygiene practices, the less likely HAI incidents will be. At the PVAMC, there were about 76 HAIs incidents between 2012 and 2015 combining all inpatient wards. There were 0 MRSA, 11 CAUTI, 1 CLABSI, 4 VAP and 60 C.Diff as seen in Table 5.39. During the study time in September and October 2016, there were zero HAIs in total.

\begin{tabular}{|c|c|c|c|c|c|c|}
\hline Year & MRSA & CAUTI & CLABSI & VAP & C.Diff & Total HAIs \\
\hline 2012 & 0 & 8 & 1 & 2 & 19 & 30 \\
\hline 2013 & 0 & 2 & 0 & 1 & 18 & 21 \\
\hline 2014 & 0 & 0 & 0 & 1 & 13 & 14 \\
\hline 2015 & 0 & 1 & 0 & 0 & 10 & 11 \\
\hline Total HAI & 0 & 11 & 1 & 4 & 60 & 76 \\
\hline
\end{tabular}

Table 5.39 Healthcare-Associated Infections (HAI) at the PVAMC for year 2012-2015

When plotting monthly hand hygiene compliance versus monthly HAI for year 2012-2015, as seen in Figure 5.45, it is hard to identify any correlation. However, the correlation coefficient is -0.50 . Considering having zero HAIs during the two month of the study and the low hand hygiene compliance, September (55\%) and October (48\%) 2016, hand hygiene cannot be associated with the HAIs reduction. However, it is plausible that these HAIs incidents could have been occurred for patients with colonization and were not incidents that occurred by cross-transmission between patients. Thus, other infection prevention factors should also be measured and tracked besides hand hygiene compliance. The PVAMC is recommended to track and report 
HAIs by colonization status and see how many HAIs occur by cross-transmission versus colonization.

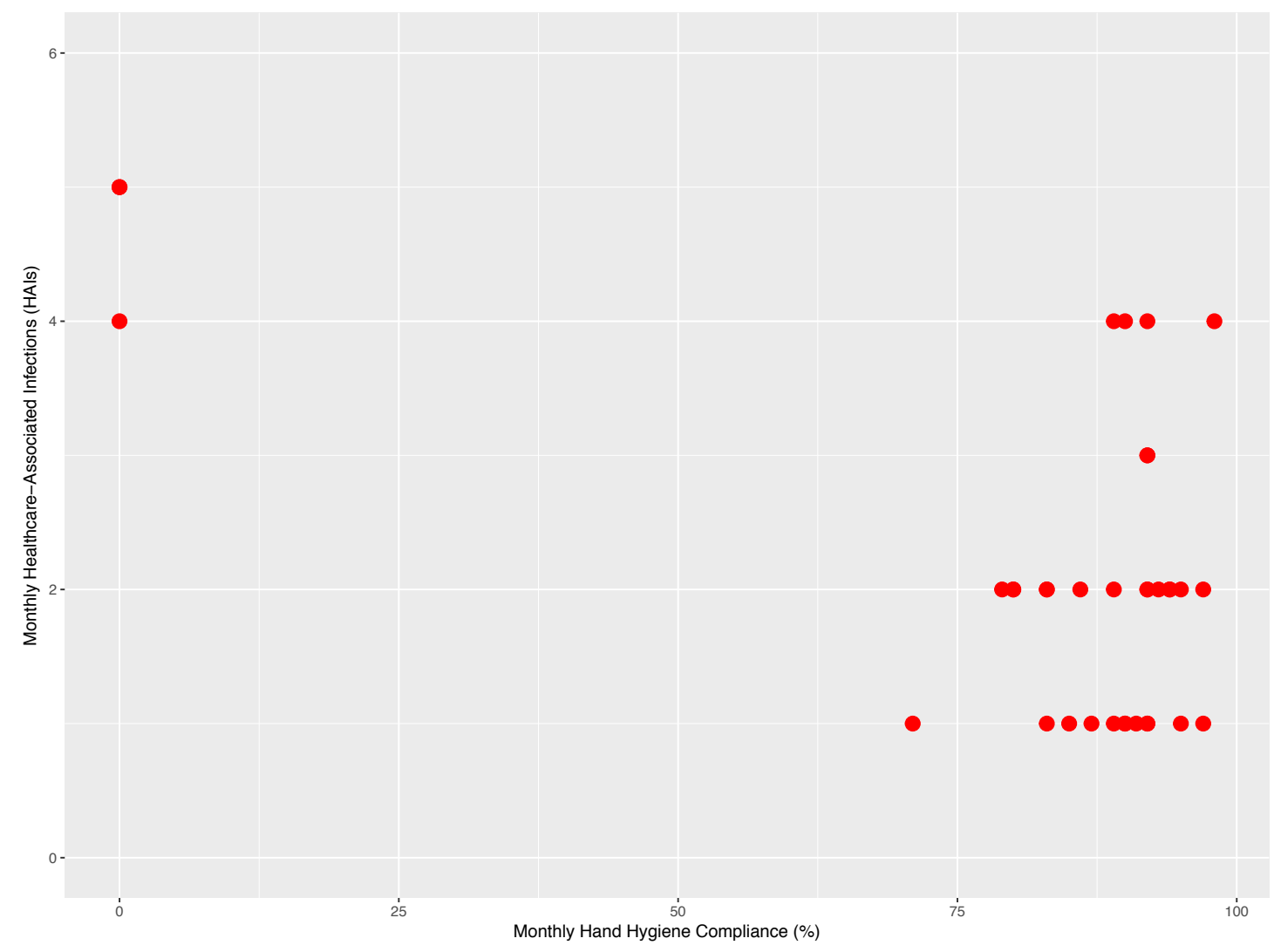

Figure 5.45 Correlation of Healthcare-Associated Infection (HAI) and Hand Hygiene Compliance (\%) for Year 2012-2015 at the PVAMC. 


\section{CHAPTER 6}

\section{INDIRECT HAND HYGIENE COMPLIANCE METHOD}

\subsection{Introduction}

Though there are several hand hygiene-monitoring methods developed in the past and current centuries, some still have to be improved to give more meaningful outcomes, while others cannot be implemented by some healthcare organizations due to cost limitations. According to WHO (2009) manual monitoring of hand hygiene by measuring the hygiene product consumption such as towels, hand soap and alcoholbased handrub is considered a less expensive monitoring approach among the other approaches. Despite the cost factor, bias does not exist (the observation bias or Hawthorne effect, the observer bias and the selection bias) when this approach is implemented. However, it does not identify the true hand hygiene moments or actions. Thus, the denominator for the calculation of hand hygiene compliance is missing. However, some studies used some surrogate denominators to make this approach more reliable such as using patient-days or workload measures. Another drawback is that breaking down hand hygiene opportunities by professional categories is almost impossible since different people use the hygiene products, especially hand soap and alcohol-based handrub on the floor, such as patients and patients' families (WHO, 2009). A list of advantages and disadvantages of the indirect hand hygiene monitoring methods by measuring the consumption of the hygiene products is available in Table 6.1 . 


\begin{tabular}{|c|c|}
\hline Advantages & Disadvantages \\
\hline $\begin{array}{l}\text { - } \text { Inexpensive } \\
\text { - Reflects overall hand hygiene } \\
\text { activity (no selection bias) } \\
\text { - Validity may be improved by } \\
\text { surrogate denominators for the need } \\
\text { for hand hygiene (patient-days, } \\
\text { workload measures, etc.) }\end{array}$ & $\begin{array}{l}\text { - } \begin{array}{l}\text { Does not reliably measure the need for } \\
\text { hand hygiene (denominator) }\end{array} \\
\text { - No information about the appropriate } \\
\text { timing of hand hygiene actions } \\
\text { - Prolonged stocking of products at ward } \\
\text { level complicates and might jeopardize } \\
\text { the validity } \\
\text { - Validity threatened by increased patient } \\
\text { and visitor usage } \\
\text { No possibility to discriminate between } \\
\text { individuals or professional groups }\end{array}$ \\
\hline
\end{tabular}

Table 6.1 Pros and Cons of Indirect Monitoring of Hand Hygiene Performance by Measuring the Consumption of Product Use (WHO, 2009, Page 161 and 162)

\subsection{Objectives}

Developing a method for estimating hand hygiene compliance based on the consumption of hygiene products used in three inpatient wards at the PVAMC is the objective of this portion of the study by determining a correlation between bed days of care (BDOC) and the consumption of products used, Soap and Purell. As a secondary measurement, the consumption of hygiene products leads to an estimate of the break down of hygiene products used in the inpatient wards, 5B, 6B and ICU at PVAMC, which then is compared with the monthly purchase and sales data of Purell and Soap from the warehouse.

\subsection{Setting}

The three-inpatient units 5B, 6B and ICU at the PVAMC have 26 Soap dispensers and 29 Purell dispensers, 27 Soap dispensers and 22 Purell dispensers and 
11 Soap dispensers and 12 Purell dispensers respectively and 64 Soap dispensers and 63 Purell dispensers collectively. From January 14th, 2015 to October 31st, 2015, the consumption of hygiene products used was measured at each inpatient ward.

\subsection{Design and Method}

A study is conducted to estimate hand hygiene compliance based on the consumption of hygiene products used at each inpatient ward individually and collectively. Each Soap and Purell dispenser at each ward is assigned with a unique integer number written on a sticker that is inside the dispenser to avoid any scratching or dropping off when cleaning walls and more importantly complying with the PVAMC rules of no wall-mounting. A Purell and Soap log sheet (Appendices U, V and $\mathrm{W}$ ) was created to track the replacement of each Purell and Soap cartridge during the 10-month study on a daily basis. A log sheet is supplied for each single month. The log sheet is placed in each housekeeper closet at each ward. Every time a cartridge is replaced, the housekeeper who is in charge marks an (x) in front of the appropriate cell on the log sheet. At the end of the 10 months, the following data were collected from the daily Soap and Purell cartridge replacement observations and the IPEC system at the PVAMC to calculate the hand hygiene compliance and test for correlation between product consumption and BDOC for each ward:

- Total number of Purell and Soap bottles replaced per month and total volume in milliliters $(\mathrm{mL})$ of these replaced cartridges.

- Total number of Purell and Soap aliquots or hits generated from the total replaced cartridges. 
- Bed days of care (BDOC) and admissions of patients at each ward from IPEC system for 2012-15 years.

The following data are collected to estimate the break down use of hygiene products of each ward:

- Purell-and-Soap purchase and sales data from the warehouse for the same 10 months of the study (the first 10 months of 2015).

\subsection{Data Analysis}

\subsubsection{Inpatient-Wards Bed Days of Care vs. Patient Admission}

The analysis starts by identifying correlation between patient admissions and bed days of care (BDOC) for the three-inpatient units individually and collectively and also by aggregating the data for all years by ward. The objective from testing the correlation is that it is expected to have an increase in the consumption of hygiene products as BDOC increases. Once the correlation is determined, BDOC is correlated to the consumption of hygiene products used to evaluate the hand hygiene compliance. The following hypothesis is evaluated first:

$\mathrm{H}_{0}$ : Patient's Admission Does Not Have an Impact on BDOC

$\mathrm{H}_{1}$ : Patient's Admission Does Have an Impact on BDOC

In summary, the objective of statistically investigating the correlation of admission with BDOC is to explore whether the inpatient wards' BDOC increases as admission of patients increases.

At the beginning, it is better to identify the best distribution(s) that fit the response (depend) variable, BDOC. BDOC is an unbounded positive count-depended variable 
that its variance (595102.8 BDOC $^{2}$ ) exceeds its mean (1499.833BDOC). A Negative Binomial distribution rather than a Poisson distribution would better handle such overdispersion by having one extra parameter that takes care of adjusting the variance independently of the mean. From the histogram, Figure 6.1, the negative binomial distribution is the best-fit distribution for BDOC.

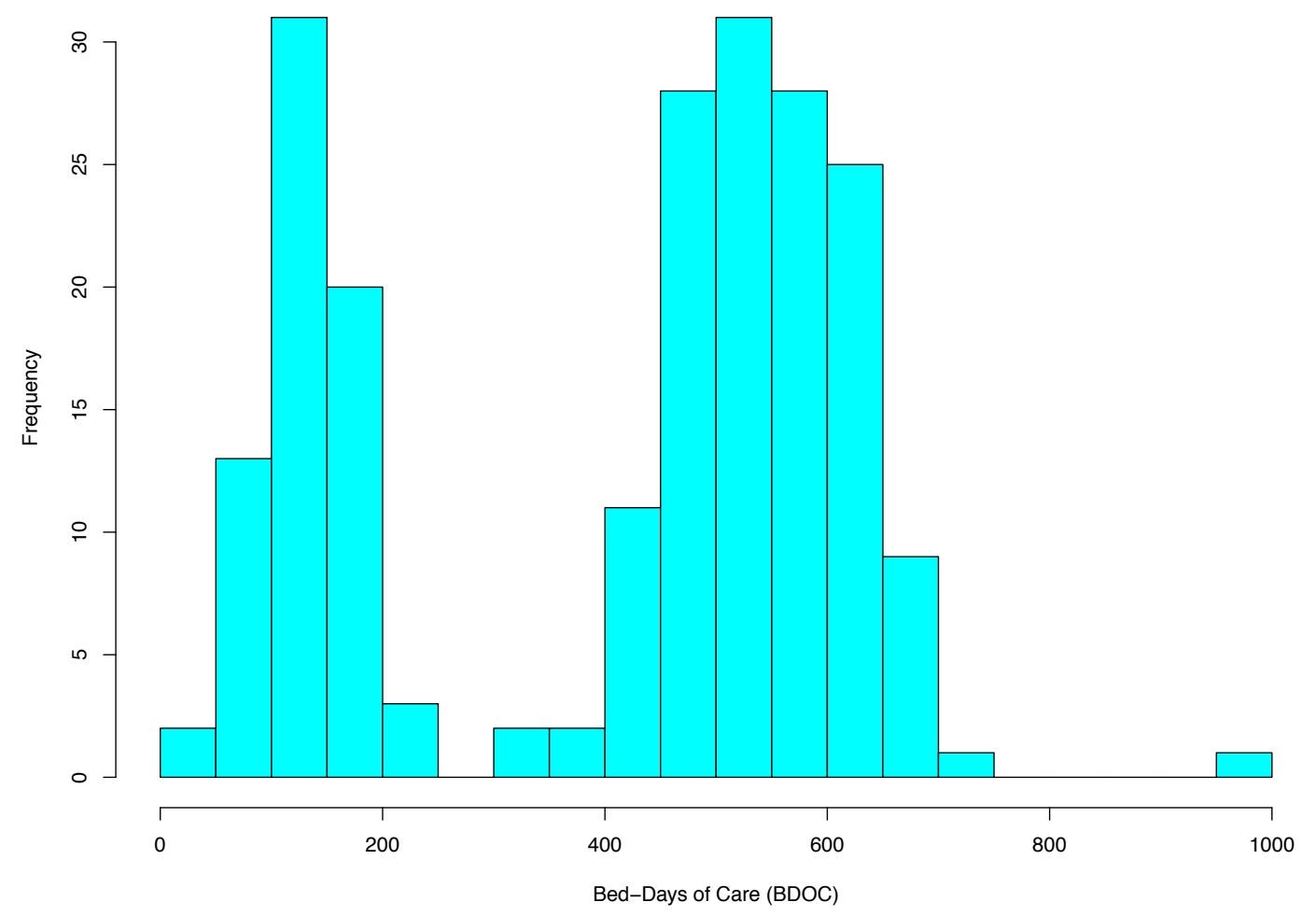

Figure 6.1 Histogram of BDOC of Inpatient Wards

The two peaks appearing in the histogram are due to the fact that ICU has far less BDOC than 5B and 6B because of the difference in the bed capacity of each ward. The easiest way to identify any correlation between two variables is via graphic visualization as seen in the scatter plot in Figure 6.2. 


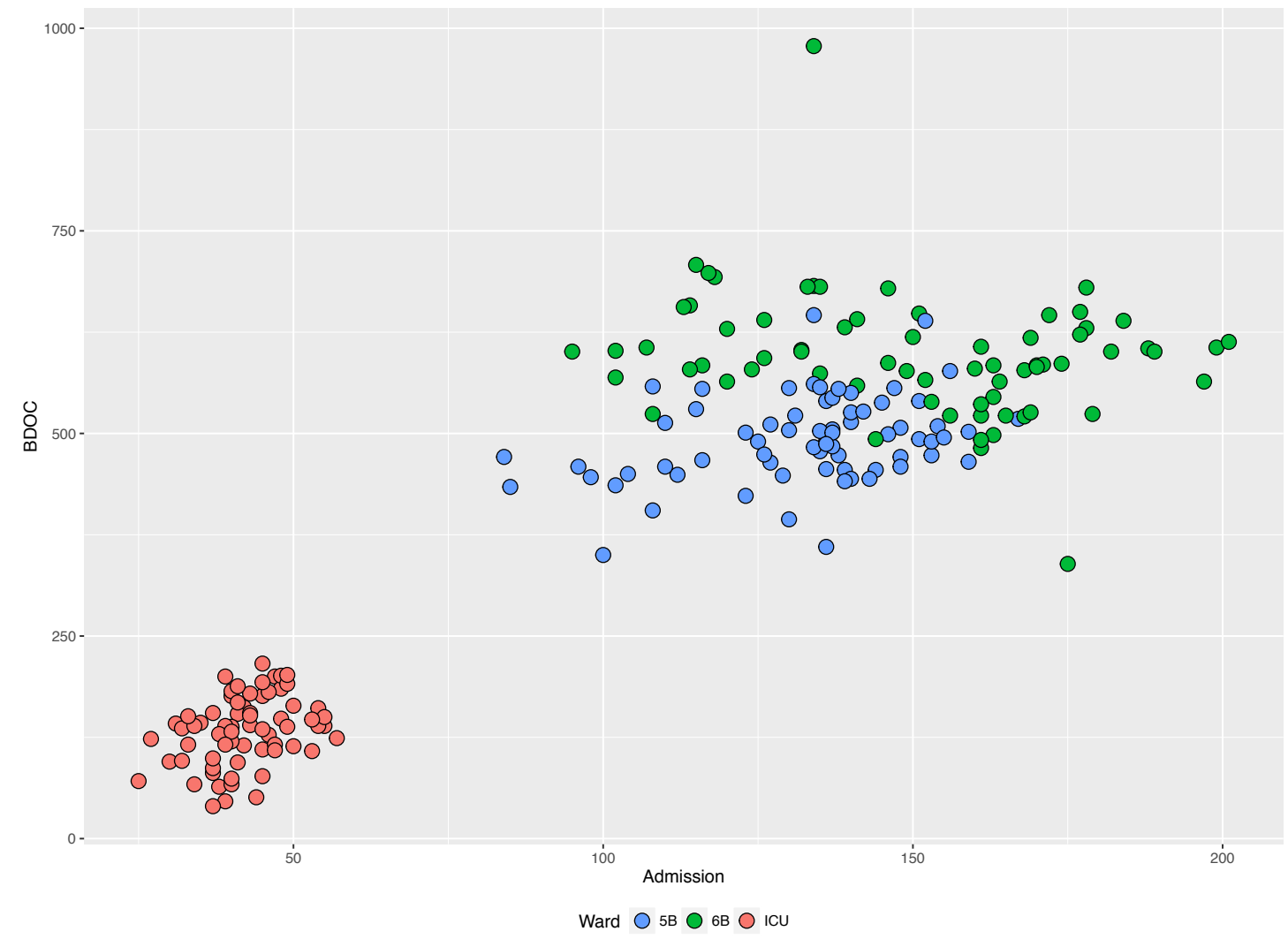

Figure 6.2 Admission versus BDOC Aggregated (2011-2016) per Inpatient Ward

Figure 6.2 shows that there is a correlation between patient admission to the floor and BDOC for each ward separately and wards combined for the nearly six years from 2011 to September 2016. If a negative binomial with a linear deterministic function is used to explain the correlation between the two variables, the following fit is shown, as seen in Figure 6.3. 


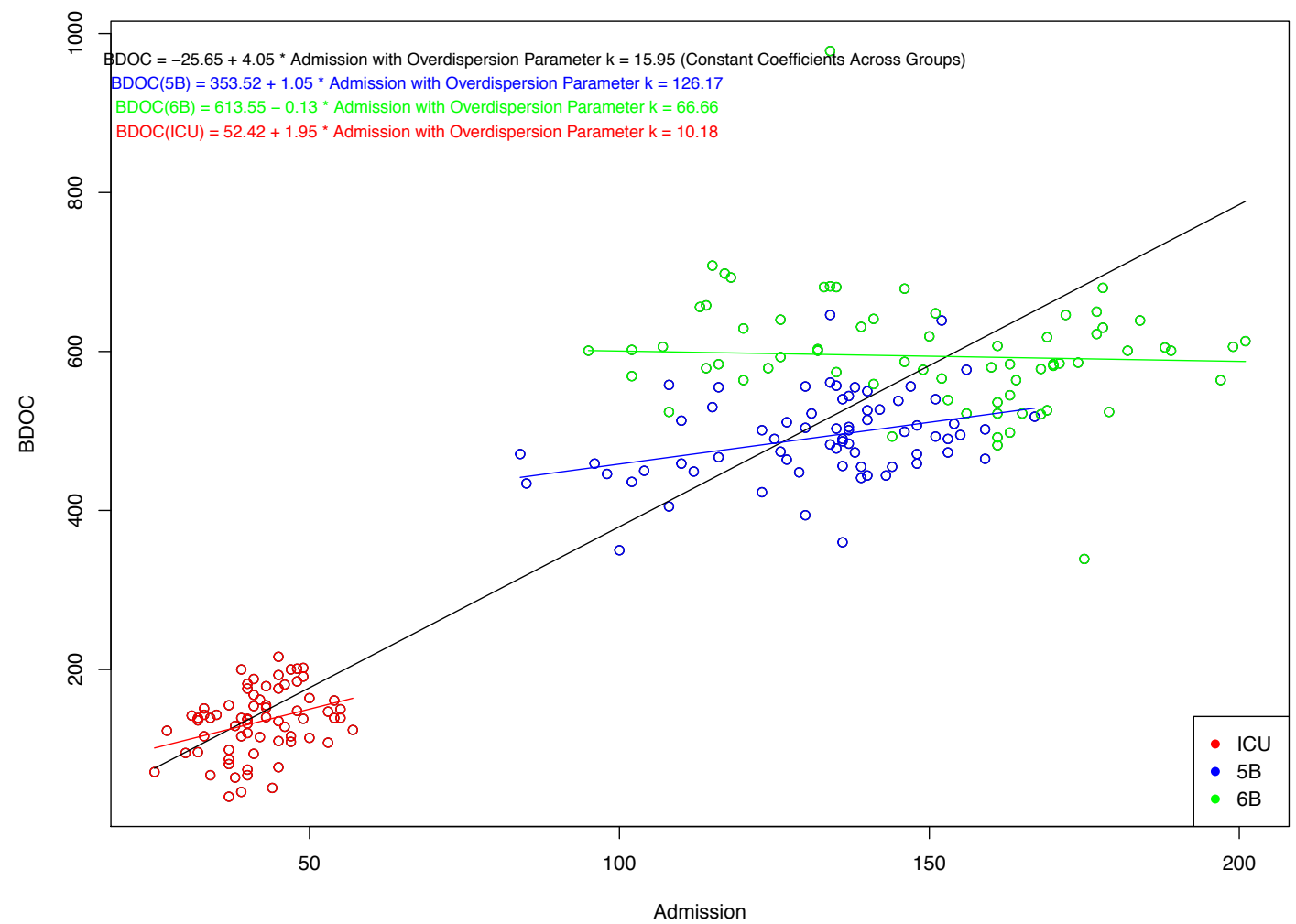

Figure 6.3 Modeling Admission versus BDOC with Negative Binomial Distribution and a Linear Deterministic Function

Several models are fitted first and based on Akaike's An Information and Bayesian Information Criteria (AIC and BIC) for model selection, the smaller the better, the model with varying the three parameters, the intercept, the rate (slope) and the dispersion parameters across groups (wards) is chosen as the best-fitted model. In addition, a model with constant coefficients across groups, wards, is also fitted, which is the black fitted line in Figure 6.3. Based on the model coefficients, BDOC increases as admissions increase by the same rate for all wards except $6 \mathrm{~B}$, which is the opposite, as admission increases BDOC decreases with a rate of 0.13 but with a large intercept parameter among the wards that offsets such declining. The correlation coefficient between BDOC and admissions are $0.34,-0.24$, and 0.32 for $5 \mathrm{~B}, 6 \mathrm{~B}$ and ICU respectively. One explanation for such a negative or inverse moderate correlation 
coefficient for $6 \mathrm{~B}$ is that higher admissions means higher discharges, transfers or deaths and less length of stay (LOS). Whoever gets to 6B does not stay long enough or at least the majority behaves like that.

\subsubsection{Soap-and-Purell Cartridge Replacement BDOC}

Then, the analysis is carried out based on summing all Purell-and-Soap cartridges replaced during each month of the 10 month study per ward in 2015. Then, the total number of Purell and Soap cartridges replaced over time for each inpatient ward and all wards combined are plotted versus BDOC that are associated with each ward. Then, the total number of hygiene products replaced is correlated with BDOC for each ward and all wards combined. It is expected that there is an increase in total number of Soap and Purell cartridges replaced at each ward as BDOC increases since HCWs have to consume more hygiene products. The hypothesis testing of interest is as follows:

$\mathrm{H}_{0}$ : BDOC Does Not Have an Impact on the Number of Hygiene Products Replaced $\mathrm{H}_{1}$ : BDOC Does Have an Impact on the Number of Hygiene Products Replaced

Before testing the hypothesis, Soap-and-Purell cartridge replacements per wards over the 10 months of the study are depicted as seen in Figure 6.4 and their replacement frequency in the histograms, Figure 6.5. In general, Figure 6.5 shows that there is more Purell replacement than Soap. In addition, there are no patterns identified for wards nor for the hygiene products with BDOC. 


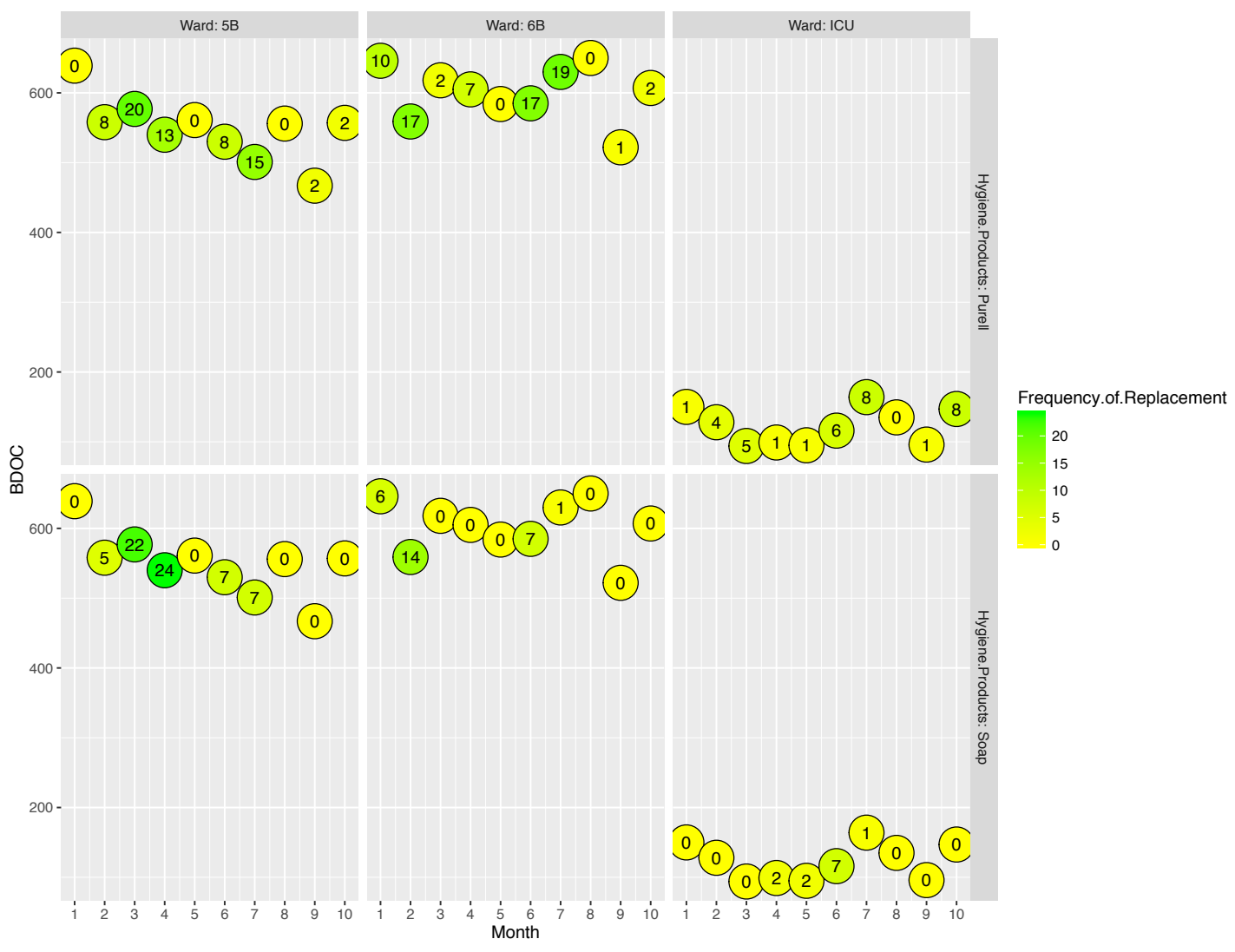

Figure 6.4 Consumption of Hygiene Products per Inpatient Ward 


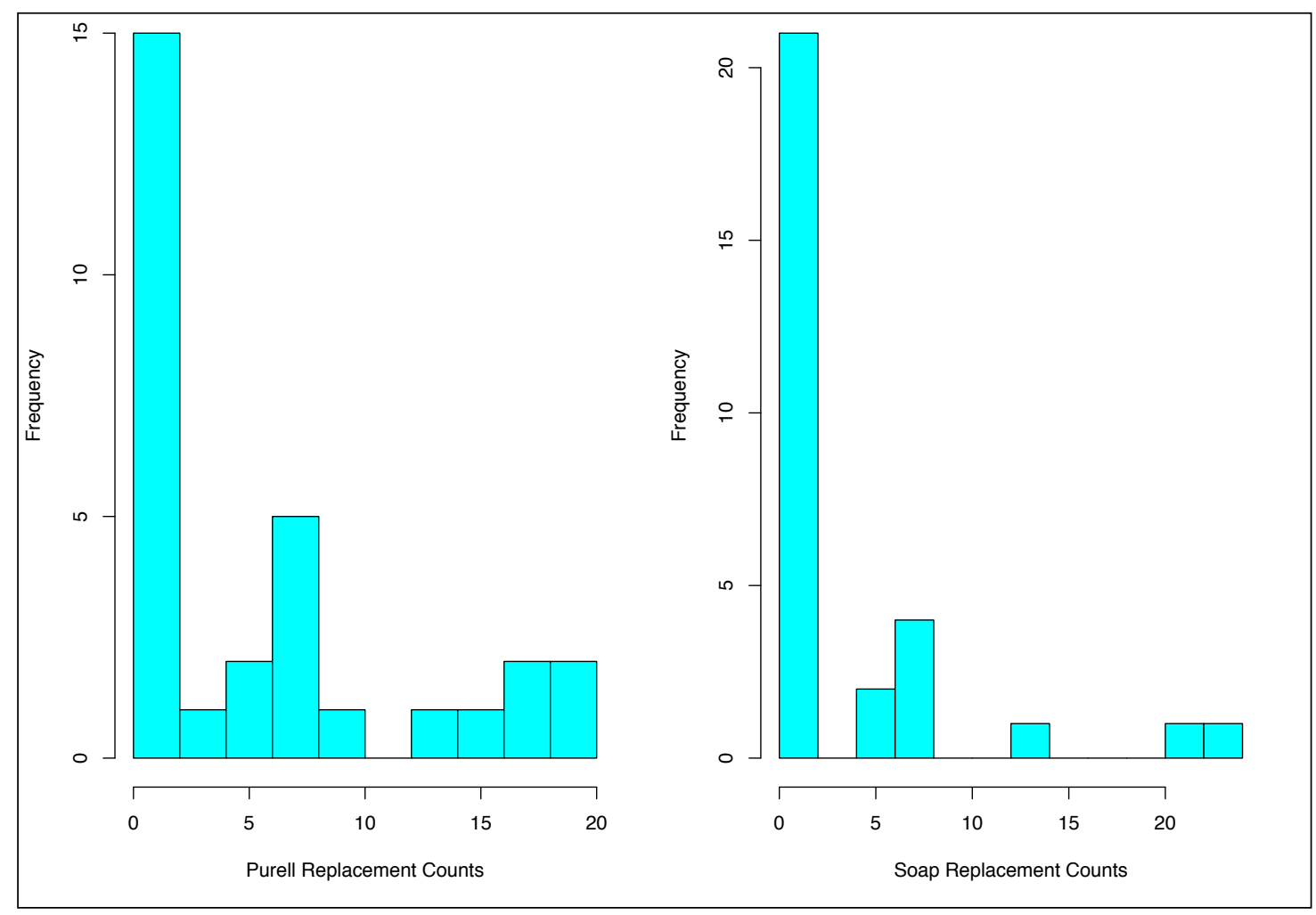

Figure 6.5 Histograms of Purell and Soap Replacement Inpatient Wards Combined

Based on the total number of Soap and Purell replacements combined, Figure 6.6, which is a Pie chart, and Table 6.2 and Table 6.3, show that assuming every hit either from Purell or Soap dispensers is used for hand hygiene opportunities, more replacements occurred at $5 \mathrm{~B}$ than $6 \mathrm{~B}$. However, though ICU is not comparable with the other two wards in terms of the total number of beds available at the ward, the number of patient admissions to the floor and BDOC, ICU contributes to almost $35 \%$ $\left([35+12] /[68+65]^{*} 100\right)$ of total replacements of Soap and Purell combined at $5 \mathrm{~B}$ and about $46 \%\left([35+12] /[75+28]^{*} 100\right)$ of the total replacement of Soap and Purell combined at $6 \mathrm{~B}$. Reasons could be that the fact that patients at ICU are in riskier health status and need more care than patients at $6 \mathrm{~B}$ and $5 \mathrm{~B}$, respectively. Ward $5 \mathrm{~B}$ is a step-down unit while 6B and ICU are considered acute care. 


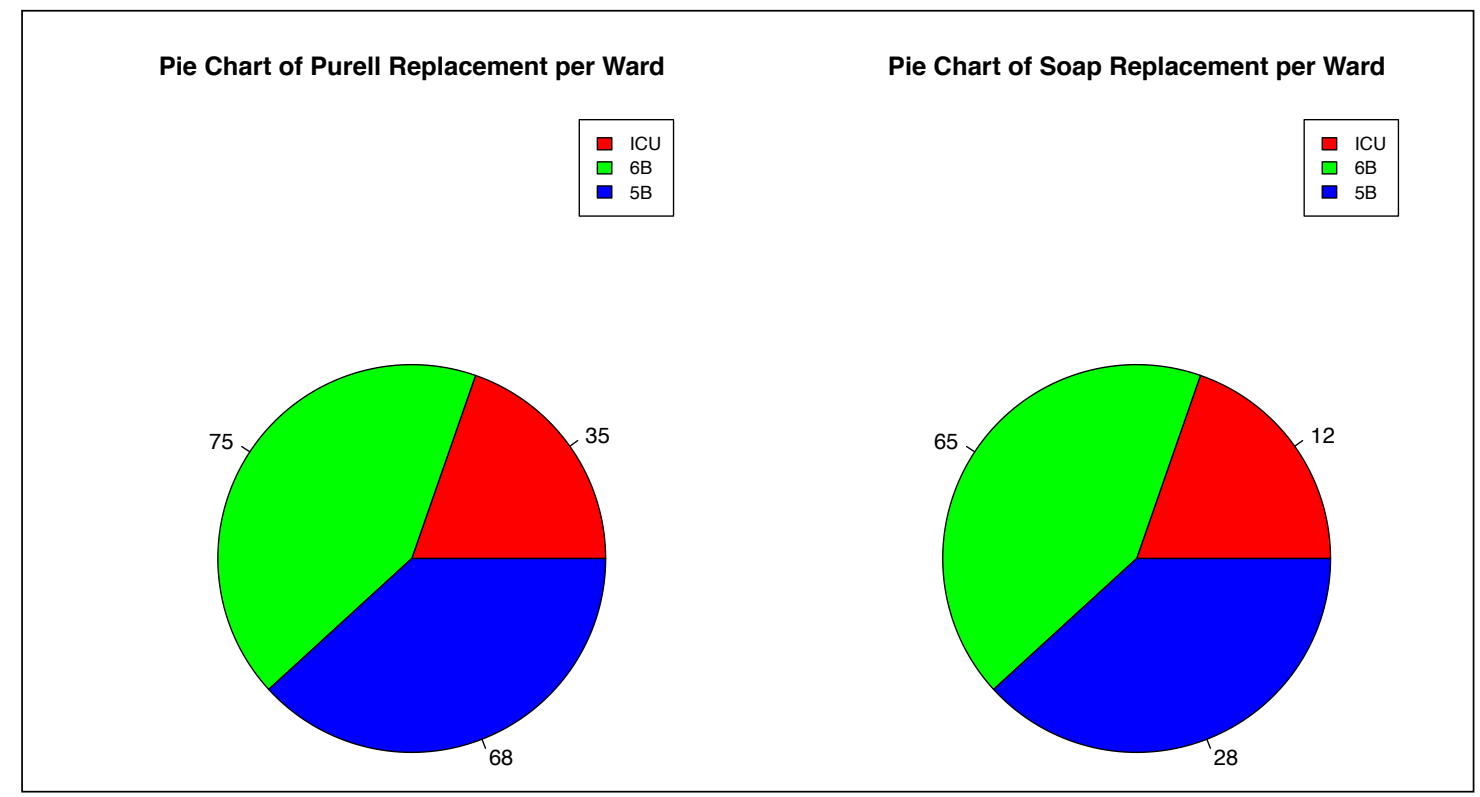

Figure 6.6 Consumption of Hygiene Products per Inpatient Ward

\begin{tabular}{|c|c|c|c|c|c|}
\hline Month & Year & Admission & $\begin{array}{c}\text { BDOC Wards } \\
\text { Combined }\end{array}$ & $\begin{array}{c}\text { Purell Replaced } \\
\text { Wards Combined }\end{array}$ & $\begin{array}{c}\text { Soap Replaced } \\
\text { Wards Combined }\end{array}$ \\
\hline 1 & 2015 & 379 & 1435 & 11 & 6 \\
\hline 2 & 2015 & 295 & 1245 & 29 & 19 \\
\hline 3 & 2015 & 366 & 1289 & 27 & 22 \\
\hline 4 & 2015 & 361 & 1244 & 21 & 26 \\
\hline 5 & 2015 & 334 & 1240 & 1 & 21 \\
\hline 6 & 2015 & 319 & 1231 & 31 & 9 \\
\hline 7 & 2015 & 365 & 1295 & 42 & 0 \\
\hline 8 & 2015 & 369 & 1341 & 0 & 0 \\
\hline 9 & 2015 & 313 & 1085 & 4 & 0 \\
\hline 10 & 2015 & 349 & 1311 & 12 & \\
\hline
\end{tabular}

Table 6.2 Aggregated Soap and Purell for Inpatient Wards Combined 


\begin{tabular}{|c|c|c|c|c|c|c|c|}
\hline Month & Year & Ward & Admission & BDOC & $\begin{array}{c}\text { Purell } \\
\text { Replaced } \\
\text { per Ward }\end{array}$ & $\begin{array}{c}\text { Soap } \\
\text { Replaced } \\
\text { per Ward }\end{array}$ & $\begin{array}{c}\text { Purell } \\
\text { plus } \\
\text { Soap } \\
\text { per } \\
\text { Ward }\end{array}$ \\
\hline 1 & 2015 & ICU & 55 & 150 & 1 & 0 & 1 \\
\hline 2 & 2015 & ICU & 46 & 128 & 4 & 0 & 4 \\
\hline 3 & 2015 & ICU & 41 & 94 & 5 & 0 & 5 \\
\hline 4 & 2015 & ICU & 37 & 99 & 1 & 2 & 3 \\
\hline 5 & 2015 & ICU & 30 & 95 & 1 & 2 & 3 \\
\hline 6 & 2015 & ICU & 33 & 116 & 6 & 7 & 13 \\
\hline 7 & 2015 & ICU & 50 & 164 & 8 & 1 & 9 \\
\hline 8 & 2015 & ICU & 45 & 135 & 0 & 0 & 0 \\
\hline 9 & 2015 & ICU & 32 & 96 & 1 & 0 & 1 \\
\hline 10 & 2015 & ICU & 53 & 147 & 8 & 0 & 8 \\
\hline 1 & 2015 & $5 \mathrm{~B}$ & 152 & 639 & 0 & 0 & 0 \\
\hline 2 & 2015 & $5 \mathrm{~B}$ & 108 & 558 & 8 & 5 & 13 \\
\hline 3 & 2015 & $5 \mathrm{~B}$ & 156 & 577 & 20 & 22 & 42 \\
\hline 4 & 2015 & $5 \mathrm{~B}$ & 136 & 540 & 13 & 24 & 37 \\
\hline 5 & 2015 & $5 \mathrm{~B}$ & 134 & 561 & 0 & 0 & 0 \\
\hline 6 & 2015 & $5 \mathrm{~B}$ & 115 & 530 & 8 & 7 & 15 \\
\hline 7 & 2015 & $5 \mathrm{~B}$ & 137 & 501 & 15 & 7 & 22 \\
\hline 8 & 2015 & $5 \mathrm{~B}$ & 147 & 556 & 0 & 0 & 0 \\
\hline 9 & 2015 & $5 B$ & 116 & 467 & 2 & 0 & 2 \\
\hline 10 & 2015 & $5 \mathrm{~B}$ & 135 & 557 & 2 & 0 & 2 \\
\hline 1 & 2015 & $6 \mathrm{~B}$ & 172 & 646 & 10 & 6 & 16 \\
\hline 2 & 2015 & $6 \mathrm{~B}$ & 141 & 559 & 17 & 14 & 31 \\
\hline 3 & 2015 & $6 \mathrm{~B}$ & 169 & 618 & 2 & 0 & 2 \\
\hline 4 & 2015 & $6 \mathrm{~B}$ & 188 & 605 & 7 & 0 & 7 \\
\hline 5 & 2015 & $6 \mathrm{~B}$ & 170 & 584 & 0 & 0 & 0 \\
\hline 6 & 2015 & $6 \mathrm{~B}$ & 171 & 585 & 17 & 7 & 24 \\
\hline 7 & 2015 & $6 \mathrm{~B}$ & 178 & 630 & 19 & 1 & 20 \\
\hline 8 & 2015 & $6 \mathrm{~B}$ & 177 & 650 & 0 & 0 & 0 \\
\hline 9 & 2015 & $6 \mathrm{~B}$ & 165 & 522 & 1 & 0 & 1 \\
\hline 10 & 2015 & $6 \mathrm{~B}$ & 161 & 607 & 2 & 0 & 2 \\
\hline
\end{tabular}

Table 6.3 Individual and Combined Replacement of Soap and Purell as One Hygiene Product per Inpatient Ward 
Based on Purell and Soap purchases and sales data from the warehouse at the PVAMC, as seen in Table 6.4, a Purell cartridge costs 7 times $\left(\frac{23.36}{3.49} * 100\right)$ more than a Soap cartridge based on an average unit cost from the 10 months of 2015 sales data.

$$
\begin{gathered}
\text { Average Unit Cost of a Purell Cartridge }=\$ 23.36 \\
\text { Average Unit Cost of a Soap Cartridge }=\$ 3.49
\end{gathered}
$$

\begin{tabular}{|c|c|c|c|c|}
\hline Ward & Purell & Purell Cost & Soap & Soap Cost \\
\hline ICU & 35 & $\$ 817.35$ & 12 & $\$ 42.18$ \\
\hline 5B & 68 & $\$ 1,587.94$ & 65 & $\$ 226.35$ \\
\hline 6B & 75 & $\$ 1,752.37$ & 28 & $\$ 97.71$ \\
\hline
\end{tabular}

Table 6.4 Total Purell and Soap Replacement and the Associated Total Cost of each Hygiene Product per Inpatient Ward

It can be seen that there is almost similar behavior for both Soap and Purell cartridges replaced; however, the number of Soap dispensers are far less than the number of Purell dispensers for each ward; yet, Purell is replaced more than Soap. There are 5 Soap dispensers at 5 sink locations for $5 \mathrm{~B}$ and $6 \mathrm{~B}$ and there are 2 Soap dispensers at 2 sink locations for ICU ignoring sinks inside patients' rooms. This could mean that HCWs favor practicing hand hygiene with Purell more than Soap, at least for this time period. This result is inconclusive, however, because in this type of indirect hand hygiene monitoring method, it is hard to identify who practices hand hygiene or during which hand hygiene moments. Additionally, a Purell cartridge generates about $800(1200 \mathrm{~mL} / 1.5 \mathrm{~mL})$ hits versus $667(1000 \mathrm{~mL} / 1.5 \mathrm{~mL})$ hits for a Soap cartridge; that means a ratio of 0.8:1 between Soap and Purell. Also sometimes Purell is not available, so HCWs switch to Soap and vice versa. Thus, it is hard to say which hygiene products are more favorable in terms of use by caregivers. 
In an effort to identify patterns or correlation between BDOC and Soap and Purell replacement counts per ward, Figure 6.7, Figure 6.8 and Figure 6.9 are generated.
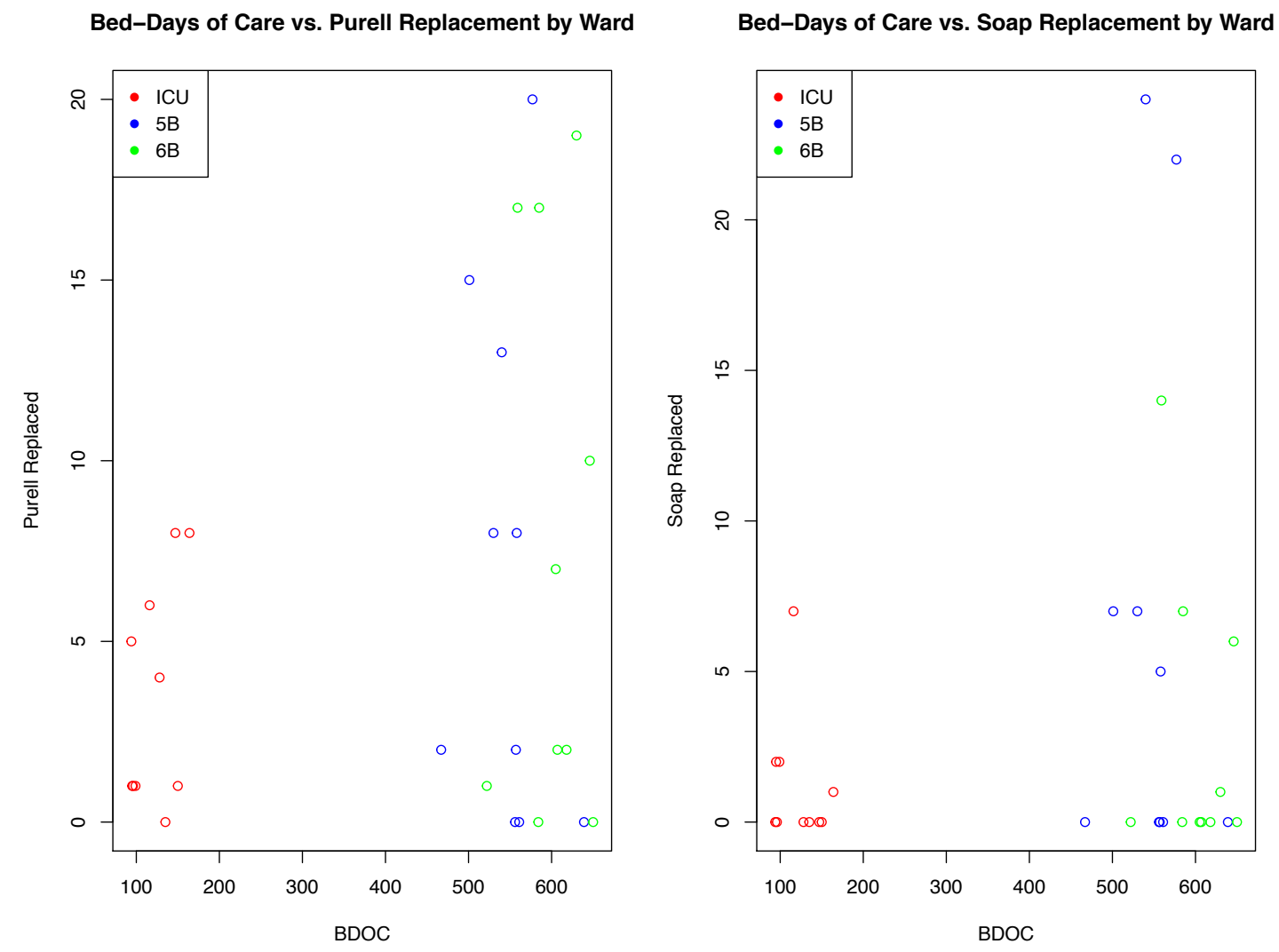

Figure 6.7 BDOC versus Purell and Soap Replacement 

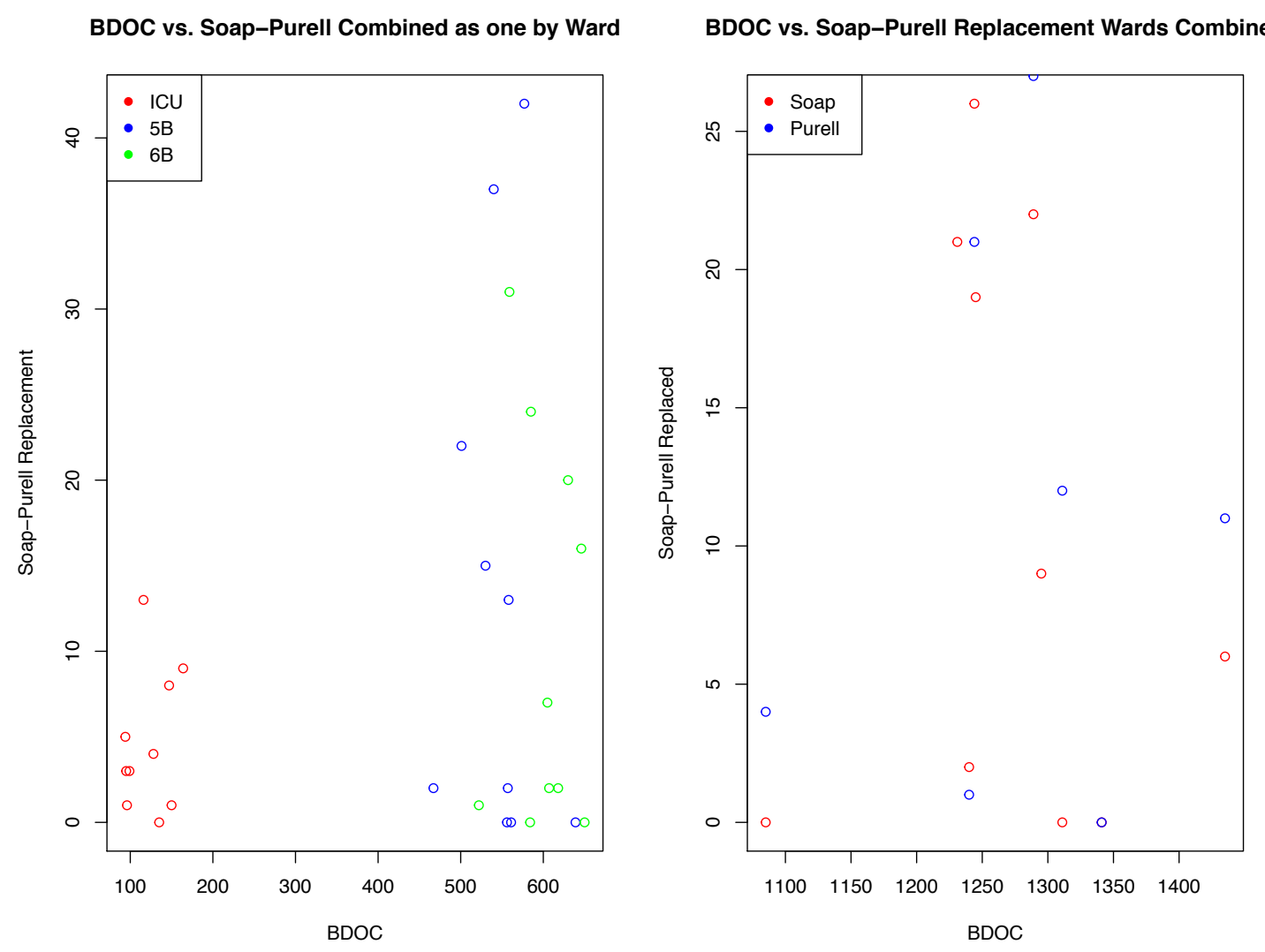

Figure 6.8 BDOC versus Purell and Soap Replacement during 2015

As can be seen there are no patterns in any of the plots for Soap and Purell separately nor combined by wards. Correlation coefficients are very weak and sometimes negative except for ICU where soap replacement counts are slightly strong and positive though the plot reveals none as seen in Table 6.5.

\begin{tabular}{|c|c|c|}
\hline \multirow{2}{*}{ Ward } & \multicolumn{2}{|c|}{ Correlation Coefficients } \\
\cline { 2 - 3 } & Soap & Purell \\
\hline 5B & -0.1616 & 0.0005 \\
\hline 6B & 0.0145 & -0.2296 \\
\hline ICU & 0.4422 & -0.2027 \\
\hline Wards Combined & 0.22 & 0.27 \\
\hline
\end{tabular}

Table 6.5 Correlation Coefficients of BDOC versus Hygiene Products Replacement 


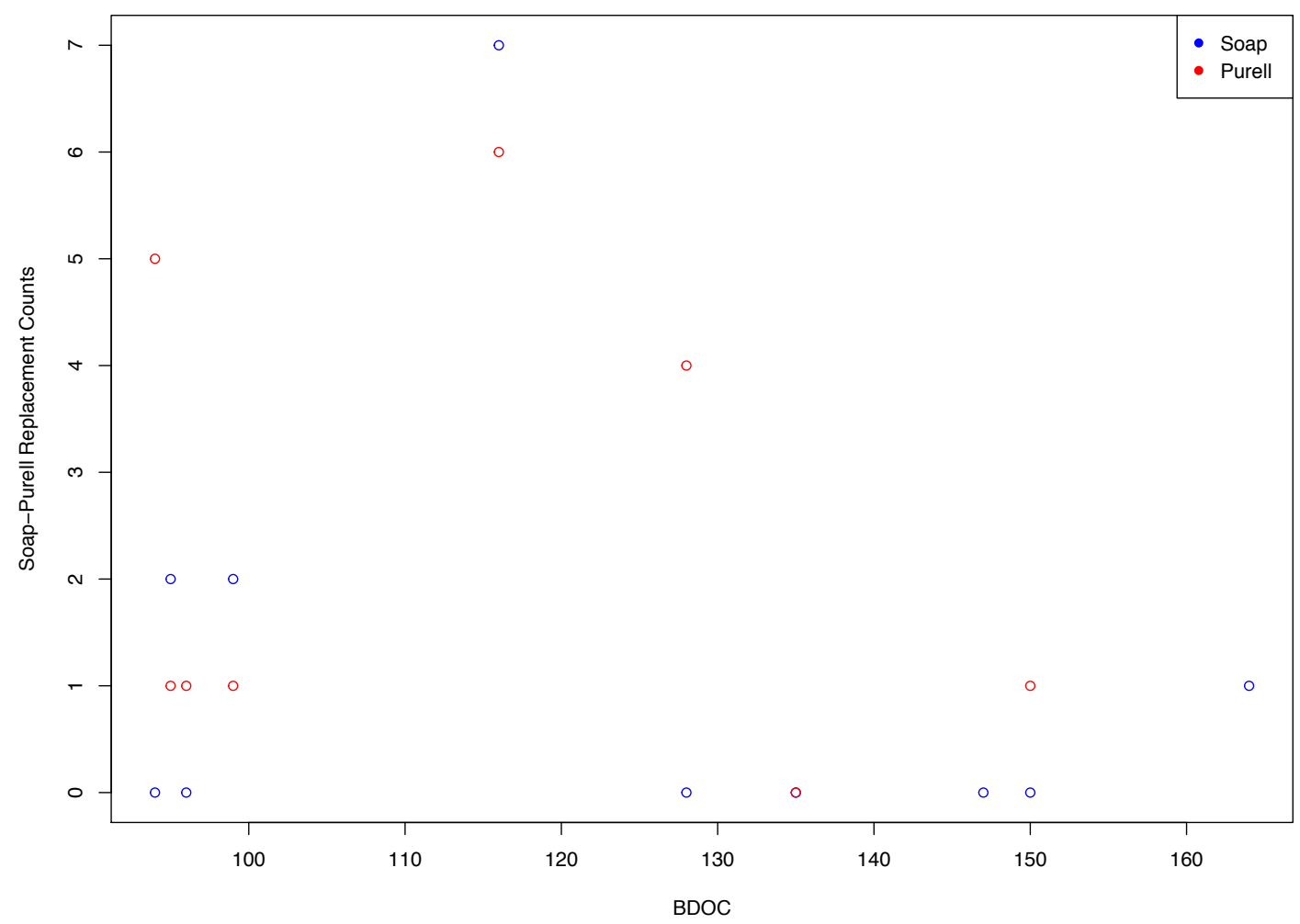

Figure 6.9 BDOC versus Soap and Purell Replacement at ICU

Based on the results, $\mathrm{p}$-value that is greater than 0.05 , of the likelihood ratio test for the Negative Binomial distribution in Table 6.6, the test fails to reject the null hypothesis that states BDOC does not have an impact on the number of hygiene products replaced per ward. In this case, it is highly likely that hand hygiene compliance rates over the 10 months of the study are not as expected as it should be based on the following calculations and estimates.

\begin{tabular}{|c|c|c|c|c|}
\hline Coefficients: & Estimate & Std. Error & $\mathrm{z}$ value & $\operatorname{Pr}(>|\mathrm{z}|)$ \\
\hline (Intercept) & 4.988026 & 5.234055 & 0.953 & 0.341 \\
\hline BDOC & -0.004389 & 0.009518 & -0.461 & 0.645 \\
\hline Ward6B & -0.870993 & 8.422534 & -0.103 & 0.918 \\
\hline WardICU & -4.378119 & 5.663404 & -0.773 & 0.439 \\
\hline BDOC:Ward6B & 0.001409 & 0.014523 & 0.097 & 0.923 \\
\hline BDOC:WardICU & 0.011909 & 0.019699 & 0.605 & 0.545 \\
\hline
\end{tabular}

Table 6.6 The Likelihood Ratio Test for Negative Binomial GLM Model 
In this indirect hand hygiene monitoring method, the denominator for total hand hygiene opportunities does not exist, so an estimate of the denominator is calculated based on the following steps:

1. Find $X_{i}$ : where $X$ represents the total BDOC in a month and $i$ represents each inpatient ward, 5B, 6B and ICU.

2. Find $Y_{i}$ : which is the average number of times a patient (Isolation versus nonIsolation) is seen by a nurse of doctor per day per ward during all shifts combined. Based on patient contact data in Chapter 4, for this study $Y$ is the average patient contacs per day of 50,58 and 111 for $5 \mathrm{~B}, 6 \mathrm{~B}$ and ICU respectively, and $i$ represents the inpatient ward, 5B, 6B and ICU.

3. Find $Z_{i}$ : which is the sum of all device-associated care days in a particular month. This value was found for each month during the time of the study, which was the first 10 months of 2015 , where each bed day of care with a device provides hand hygiene opportunities before and after installation of a Central Line, Catheter of Ventilator. $Z$ represents the aggregation of central line days, catheter days an ventilator days, and $i$ represents the inpatient ward, $5 \mathrm{~B}, 6 \mathrm{~B}$ and ICU.

RNs and CNAs from the nursing group and attending, residents, interns and medical students from the medical group are only included because they are the groups of interest in this research study. There is an assumption that all hygiene products consumed or replaced are consumed by HCWs, in the numerator. On the other hand, the denominator is the expected hand hygiene opportunities that take place 
on the floor by the HCWs of interest, from the nursing group (RN, CNA) and the medical group (Attending, resident, intern and medical student) only.

$$
=\frac{\begin{array}{c}
\text { Monthly Hand Hygiene Compliance } \% \\
\text { Tot. Hits Generated from Purell and Soap Replacement }
\end{array}}{\text { Hand Hygiene Estimated Moments (Before and After) }}
$$

\section{Calculation of the Numerator:}

Tot. Hits Generated from Purell and Soap Replacement

$=[800$ hits generated per a Purell Cartridge

* How Many Purell Replaced]

$+[667$ hits generated per a Soap Cartridge

* How Many Soap Replaced]

Estimate of the Denominator $=X_{i} * Y_{i} * 2_{(\text {Before\&After })}+Z_{i} * 2_{\text {(Before\&After })}$

$$
\begin{gathered}
{\left[\begin{array}{c}
\text { BDOC }_{5 B} \\
\text { BDOC }_{6 B} \\
\text { BDOC }_{I C U}
\end{array}\right] \cdot\left[\begin{array}{l}
\text { Average Patient Contact } \\
\text { Average Patient Contact } \\
6 B \\
\text { Average Patient Contact }
\end{array}\right] \cdot\left[\begin{array}{l}
2_{(B \text { Before\&After })} \\
2_{(\text {Before\&After })} \\
2_{(\text {Before\&After })}
\end{array}\right]} \\
+ \\
{\left[\begin{array}{l}
\text { Device_Invasive } \\
\text { Device_Invasive }_{6 B} \\
\text { Device_Invasive }_{I C U}
\end{array}\right] \cdot\left[\begin{array}{l}
2_{(\text {Before\&After })} \\
2_{(\text {Before\&After })} \\
2_{(\text {Before\&After })}
\end{array}\right]}
\end{gathered}
$$

Before jumping into the analysis, the sources of error are listed, which could increase or decrease the error in both the numerator and denominator of hand hygiene compliance adherence rate found with this indirect method.

\section{Sources of Error in the Numerator and Denominator:}

- These hand hygiene replacement data do not include partial use of cartridges of Purell and Soap on the floor during the time of the study.

- Some of the replacements may not have been reported by mistake. 
- Some of the replacements may not have been done by people who did not report it because they did not know about the study.

- Other groups such as EMS or patients and family members use some of Purell and Soap.

- Use of Purell and Soap for hand hygiene true moments versus non-related practices of hand hygiene cannot be distinguished.

The results are shown in Table 6.7. Plotting the hand hygiene adherence rates by BDOC over the 10 months reveals that the rates are very low as expected from the total number of hygiene products replacement per month per ward as seen in Figure 6.10. Based on Figure 6.10, the BDOC versus hand hygiene adherence rate scatter plot, there is hardly any correlation or pattern between BDOC and the rate of hand hygiene.

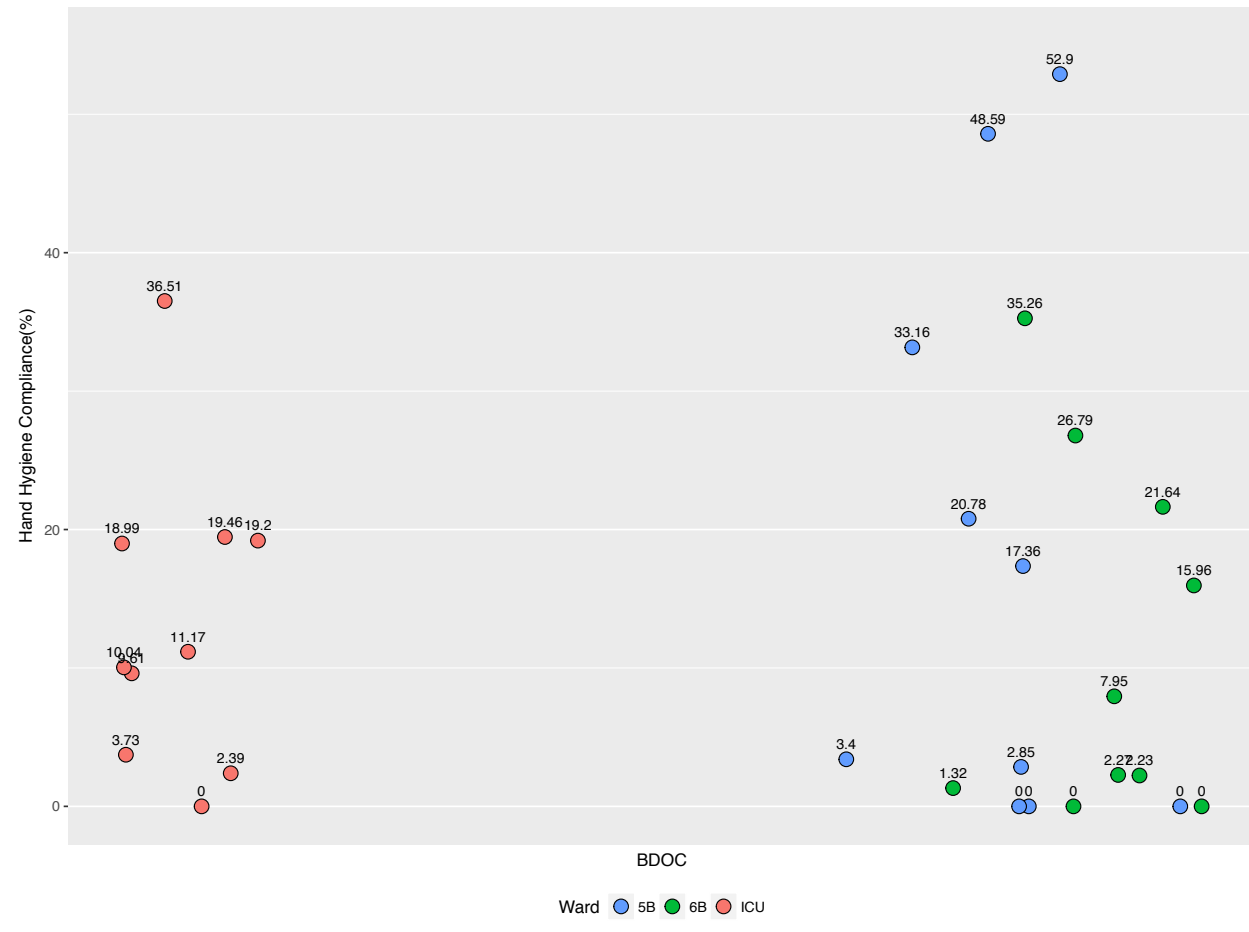

Figure 6.10 BDOC versus Hand Hygiene Compliance Rate per Inpatient Ward Based on Indirect Method 


\begin{tabular}{|c|c|c|c|c|c|c|c|c|c|}
\hline Month & Year & Ward & BDOC & $\begin{array}{c}\text { Device } \\
- \\
\text { Counts }\end{array}$ & $\begin{array}{c}\text { Purell } \\
\text { Repla } \\
\text { ced } \\
\text { per } \\
\text { Ward }\end{array}$ & $\begin{array}{l}\text { Soap } \\
\text { Replac } \\
\text { ed per } \\
\text { Ward }\end{array}$ & $\begin{array}{c}\text { HH } \\
\text { Numer } \\
\text { ator }\end{array}$ & $\begin{array}{c}\mathrm{HH} \\
\text { Deno } \\
\text { minato } \\
\mathrm{r}\end{array}$ & $\mathrm{HH} \%$ \\
\hline 1 & 2015 & $\mathrm{ICU}$ & 150 & 87 & 1 & 0 & 800 & 33474 & 2.39 \\
\hline 2 & 2015 & $\mathrm{ICU}$ & 128 & 111 & 4 & 0 & 3200 & 28638 & 11.17 \\
\hline 3 & 2015 & ICU & 94 & 99 & 5 & 0 & 4000 & 21066 & 18.99 \\
\hline 4 & 2015 & $\mathrm{ICU}$ & 99 & 109 & 1 & 2 & 2134 & 22196 & 9.61 \\
\hline 5 & 2015 & ICU & 95 & 85 & 1 & 2 & 2134 & 21260 & 10.04 \\
\hline 6 & 2015 & ICU & 116 & 92 & 6 & 7 & 9469 & 25936 & 36.51 \\
\hline 7 & 2015 & $\mathrm{ICU}$ & 164 & 198 & 8 & 1 & 7067 & 36804 & 19.20 \\
\hline 8 & 2015 & $\mathrm{ICU}$ & 135 & 83 & 0 & 0 & 0 & 30136 & 0.00 \\
\hline 9 & 2015 & $\mathrm{ICU}$ & 96 & 61 & 1 & 0 & 800 & 21434 & 3.73 \\
\hline 10 & 2015 & $\mathrm{ICU}$ & 147 & 129 & 8 & 0 & 6400 & 32892 & 19.46 \\
\hline 1 & 2015 & $5 \mathrm{~B}$ & 639 & 142 & 0 & 0 & 0 & 64184 & 0.00 \\
\hline 2 & 2015 & $5 B$ & 558 & 134 & 8 & 5 & 9735 & 56068 & 17.36 \\
\hline 3 & 2015 & $5 B$ & 577 & 145 & 20 & 22 & 30674 & 57990 & 52.90 \\
\hline 4 & 2015 & $5 B$ & 540 & 175 & 13 & 24 & 26408 & 54350 & 48.59 \\
\hline 5 & 2015 & $5 B$ & 561 & 180 & 0 & 0 & 0 & 56460 & 0.00 \\
\hline 6 & 2015 & $5 B$ & 530 & 129 & 8 & 7 & 11069 & 53258 & 20.78 \\
\hline 7 & 2015 & $5 B$ & 501 & 86 & 15 & 7 & 16669 & 50272 & 33.16 \\
\hline 8 & 2015 & $5 B$ & 556 & 189 & 0 & 0 & 0 & 55978 & 0.00 \\
\hline 9 & 2015 & $5 \mathrm{~B}$ & 467 & 202 & 2 & 0 & 1600 & 47104 & 3.40 \\
\hline 10 & 2015 & $5 B$ & 557 & 260 & 2 & 0 & 1600 & 56220 & 2.85 \\
\hline 1 & 2015 & $6 \mathrm{~B}$ & 646 & 143 & 10 & 6 & 12002 & 75222 & 15.96 \\
\hline 2 & 2015 & $6 \mathrm{~B}$ & 559 & 102 & 17 & 14 & 22938 & 65048 & 35.26 \\
\hline 3 & 2015 & $6 \mathrm{~B}$ & 618 & 111 & 2 & 0 & 1600 & 71910 & 2.23 \\
\hline 4 & 2015 & $6 \mathrm{~B}$ & 605 & 110 & 7 & 0 & 5600 & 70400 & 7.95 \\
\hline 5 & 2015 & $6 \mathrm{~B}$ & 584 & 137 & 0 & 0 & 0 & 68018 & 0.00 \\
\hline 6 & 2015 & $6 \mathrm{~B}$ & 585 & 167 & 17 & 7 & 18269 & 68194 & 26.79 \\
\hline 7 & 2015 & $6 \mathrm{~B}$ & 630 & 113 & 19 & 1 & 15867 & 73306 & 21.64 \\
\hline 8 & 2015 & $6 \mathrm{~B}$ & 650 & 127 & 0 & 0 & 0 & 75654 & 0.00 \\
\hline 9 & 2015 & $6 \mathrm{~B}$ & 522 & 95 & 1 & 0 & 800 & 60742 & 1.32 \\
\hline 10 & 2015 & $6 \mathrm{~B}$ & 607 & 90 & 2 & 0 & 1600 & 70592 & 2.27 \\
\hline
\end{tabular}

Table 6.7 Hand Hygiene Adherence Rates per Inpatient Ward per Month Based on Indirect Method 
Figure 6.11 shows the hand hygiene rates per inpatient wards per month per year 2015. The rate goes as low as $1.32 \%$ and as high as $52.9 \%$. Zeros that are shown on the plot are associated with months with no Purell or Soap replacement. However, if more HCWs and other people who are not in the study who regularly use the hygiene products during hand hygiene opportunities were added to the denominator, if possible, hand hygiene adherence rate would dramatically drop further.

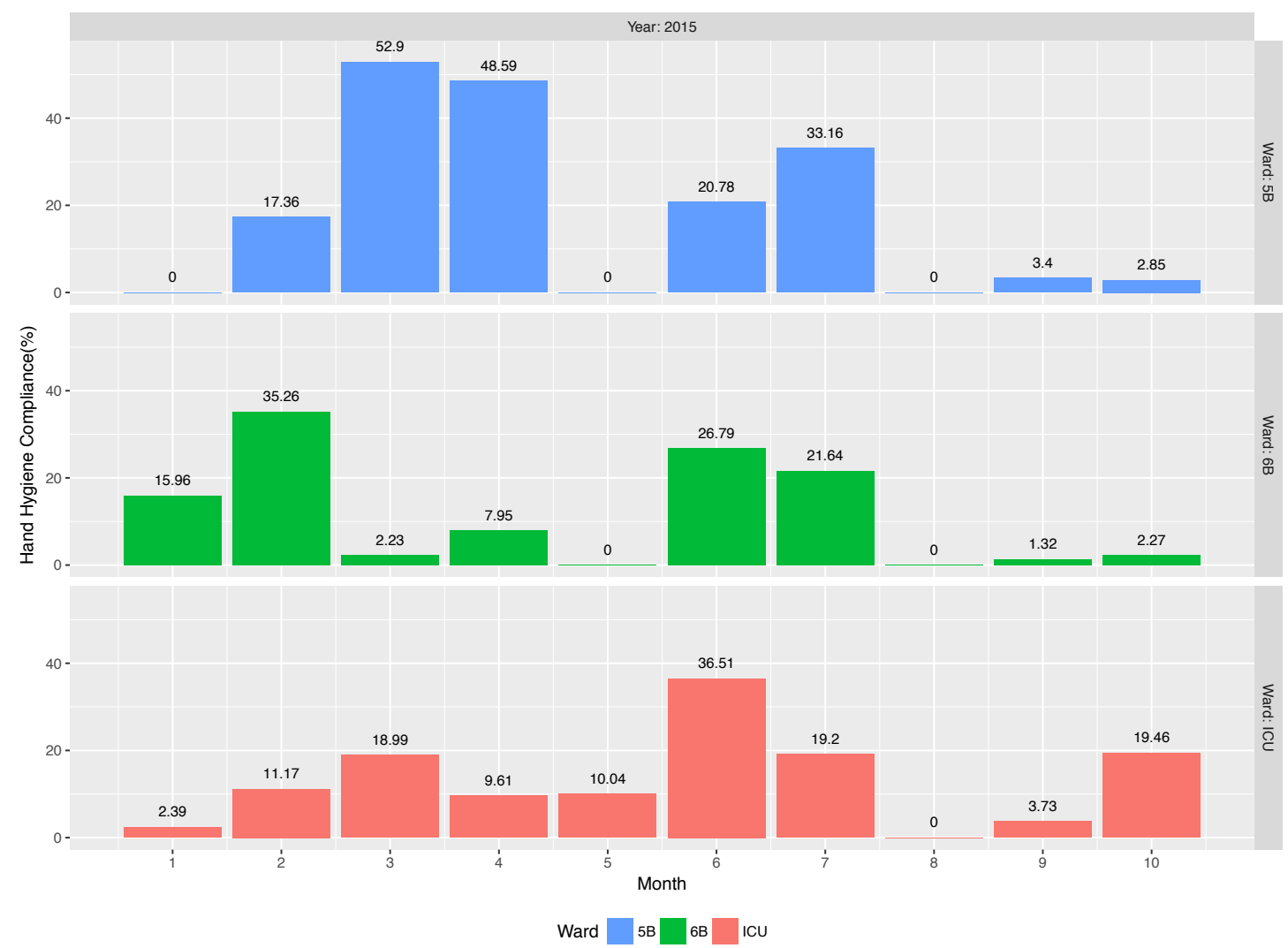

Figure 6.11 Hand Hygiene Adherence Rate per Inpatient Ward per Month (2015)

The indirect hand hygiene monitoring method based on the measurement of the consumption of the hygiene products is still a valid proposed approach. However, the following considerations should be kept in mind for better judgment of hand hygiene compliance rate:

- Patients' contacts differ based on patient's health status and needs per ward. 
- Counting the cartridges should be done per ward per shift, time of installation and replacement should be noted, and any cartridges with remaining volume should be labeled.

- The frequency of each dispenser should be tracked and compared with BDOC of the room associated to that dispenser for a comparison between isolation and non-isolation patients.

Correlating the replaced hygiene products with $\mathrm{BDOC}$ and plotting that over time seems enough to assess hand hygiene compliance without going further into estimating the denominator and calculating hand hygiene adherence rate.

Finally, the inpatient wards are ranked based on hand hygiene compliance in ascending order, 5B, ICU, and 6B with a hand hygiene compliance mean of $17.9 \%$, $13.11 \%$ and $11.34 \%$ respectively, as seen in Table 6.8 .

\begin{tabular}{|c|c|c|c|}
\hline \multirow{2}{*}{ Ward } & \multicolumn{3}{|c|}{ Hand Hygiene \% } \\
\cline { 2 - 4 } & Mean & Variance & SD \\
\hline 5B & 17.9 & 422.92 & 20.57 \\
\hline 6B & 11.34 & 163.63 & 12.79 \\
\hline ICU & 13.11 & 117.75 & 10.85 \\
\hline
\end{tabular}

Table 6.8 Descriptive Statistics of Hand Hygiene Adherence Rate Based on Indirect Method of Hygiene Products Replacement per Inpatient Ward per Month 2015 


\subsubsection{Actual vs. Target Replacement of Soap and Purell}

Another way of assessing hand hygiene compliance without estimating the denominator and calculating the adherence rate is by estimating the target replacement of the combination of Soap and Purell together. This is accomplished by estimating the total hits per month by multiplying BDOC by an estimated number of patient contacts by nurses (RN and CNA) and doctors (Attending, Resident, Intern and Medical Student) and dividing that by total hits per Purell and Soap cartridge ( 800 hits per $1200 \mathrm{~mL}$ of Purell and 667 hits per $1000 \mathrm{~mL}$ of Soap), as seen in Table 6.9. BDOC is multiplied by $[5 \mathrm{~B}=50,6 \mathrm{~B}=58$ and $\mathrm{ICU}=11]$ visits per patient per month per ward and then that is divided by 800 hits per Purell and 667 hits per Soap together to get the target replacement.

At first glance of Figure 6.12, targeted replacement of the hygiene products (Green Bars) per ward per month is almost steady (flat). It could be different if other groups of people who are on the floor and use the hygiene products for non-hand hygiene purposes are included. Imposing the actual hygiene products replacement method reveals that hand hygiene is very low, in general, for all wards. 


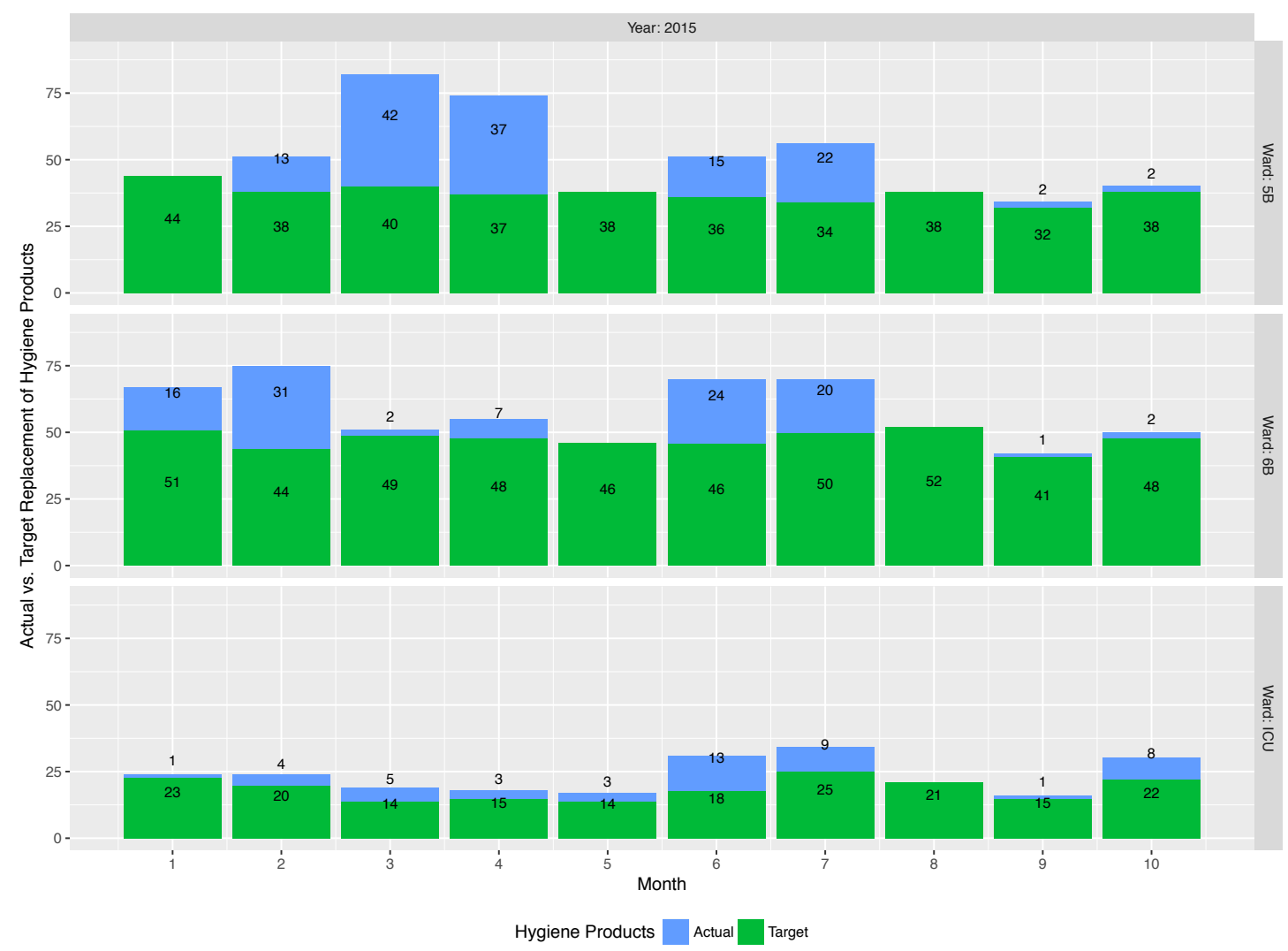

Figure 6.12 Actual (Purple) versus Target (Green) Replacement of Hygiene Products per Inpatient Ward per Month (2015)

The limitation of such a method appears by having some bars of actual replacement (Purple Bars) higher than target replacement (Green Bars) as in March 2015 at 5B (42 Actual versus 40 Target), which means the hand hygiene compliance rate could exceed $100 \%$. 


\begin{tabular}{|c|c|c|c|c|c|c|}
\hline Month & Year & Ward & BDOC & $\begin{array}{c}\text { Actual } \\
\text { Replacement } \\
(\mathrm{S}+\mathrm{P})\end{array}$ & Tot. Hits & $\begin{array}{c}\text { Target } \\
\text { Replacement } \\
(\mathrm{S}+\mathrm{P})\end{array}$ \\
\hline 1 & 2015 & ICU & 150 & 1 & 33474 & 23 \\
\hline 2 & 2015 & ICU & 128 & 4 & 28638 & 20 \\
\hline 3 & 2015 & ICU & 94 & 5 & 21066 & 14 \\
\hline 4 & 2015 & ICU & 99 & 3 & 22196 & 15 \\
\hline 5 & 2015 & ICU & 95 & 3 & 21260 & 14 \\
\hline 6 & 2015 & $\mathrm{ICU}$ & 116 & 13 & 25936 & 18 \\
\hline 7 & 2015 & ICU & 164 & 9 & 36804 & 25 \\
\hline 8 & 2015 & ICU & 135 & 0 & 30136 & 21 \\
\hline 9 & 2015 & ICU & 96 & 1 & 21434 & 15 \\
\hline 10 & 2015 & ICU & 147 & 8 & 32892 & 22 \\
\hline 1 & 2015 & $5 B$ & 639 & 0 & 64184 & 44 \\
\hline 2 & 2015 & $5 B$ & 558 & 13 & 56068 & 38 \\
\hline 3 & 2015 & $5 B$ & 577 & 42 & 57990 & 40 \\
\hline 4 & 2015 & $5 B$ & 540 & 37 & 54350 & 37 \\
\hline 5 & 2015 & $5 B$ & 561 & 0 & 56460 & 38 \\
\hline 6 & 2015 & $5 B$ & 530 & 15 & 53258 & 36 \\
\hline 7 & 2015 & $5 B$ & 501 & 22 & 50272 & 34 \\
\hline 8 & 2015 & $5 B$ & 556 & 0 & 55978 & 38 \\
\hline 9 & 2015 & $5 B$ & 467 & 2 & 47104 & 32 \\
\hline 10 & 2015 & $5 B$ & 557 & 2 & 56220 & 38 \\
\hline 1 & 2015 & $6 \mathrm{~B}$ & 646 & 16 & 75222 & 51 \\
\hline 2 & 2015 & $6 B$ & 559 & 31 & 65048 & 44 \\
\hline 3 & 2015 & $6 \mathrm{~B}$ & 618 & 2 & 71910 & 49 \\
\hline 4 & 2015 & $6 \mathrm{~B}$ & 605 & 7 & 70400 & 48 \\
\hline 5 & 2015 & $6 \mathrm{~B}$ & 584 & 0 & 68018 & 46 \\
\hline 6 & 2015 & $6 \mathrm{~B}$ & 585 & 24 & 68194 & 46 \\
\hline 7 & 2015 & $6 \mathrm{~B}$ & 630 & 20 & 73306 & 50 \\
\hline 8 & 2015 & $6 \mathrm{~B}$ & 650 & 0 & 75654 & 52 \\
\hline 9 & 2015 & $6 \mathrm{~B}$ & 522 & 1 & 60742 & 41 \\
\hline 10 & 2015 & $6 \mathrm{~B}$ & 607 & 2 & 70592 & 48 \\
\hline
\end{tabular}

Table 6.9 Actual versus Target Replacement of Hygiene Products per Ward per Month 


\subsubsection{Inpatient Ward Sales Data and Hygiene Products Use}

During 2015 at the PVAMC, most of the purchased hygiene products are soap compared to Purell. An average unit cost of one soap cartridge is about $\$ 3.49$ compared to an average unit cost of $\$ 23.36$ for a Purell cartridge. However, looking at the replacement dataset for 5B, 6B and ICU from the previous section and Figure 6.13, most of the replacement cartridges are Purell, though most of the purchased product is soap. Purell gives more hits than Soap, 800 and 667 hits, respectively. Either soap is being used more frequently in locations different than these three locations $(5 \mathrm{~B}, 6 \mathrm{~B}$ and ICU) or there are errors in recording and entering the replacement dataset at these three locations.
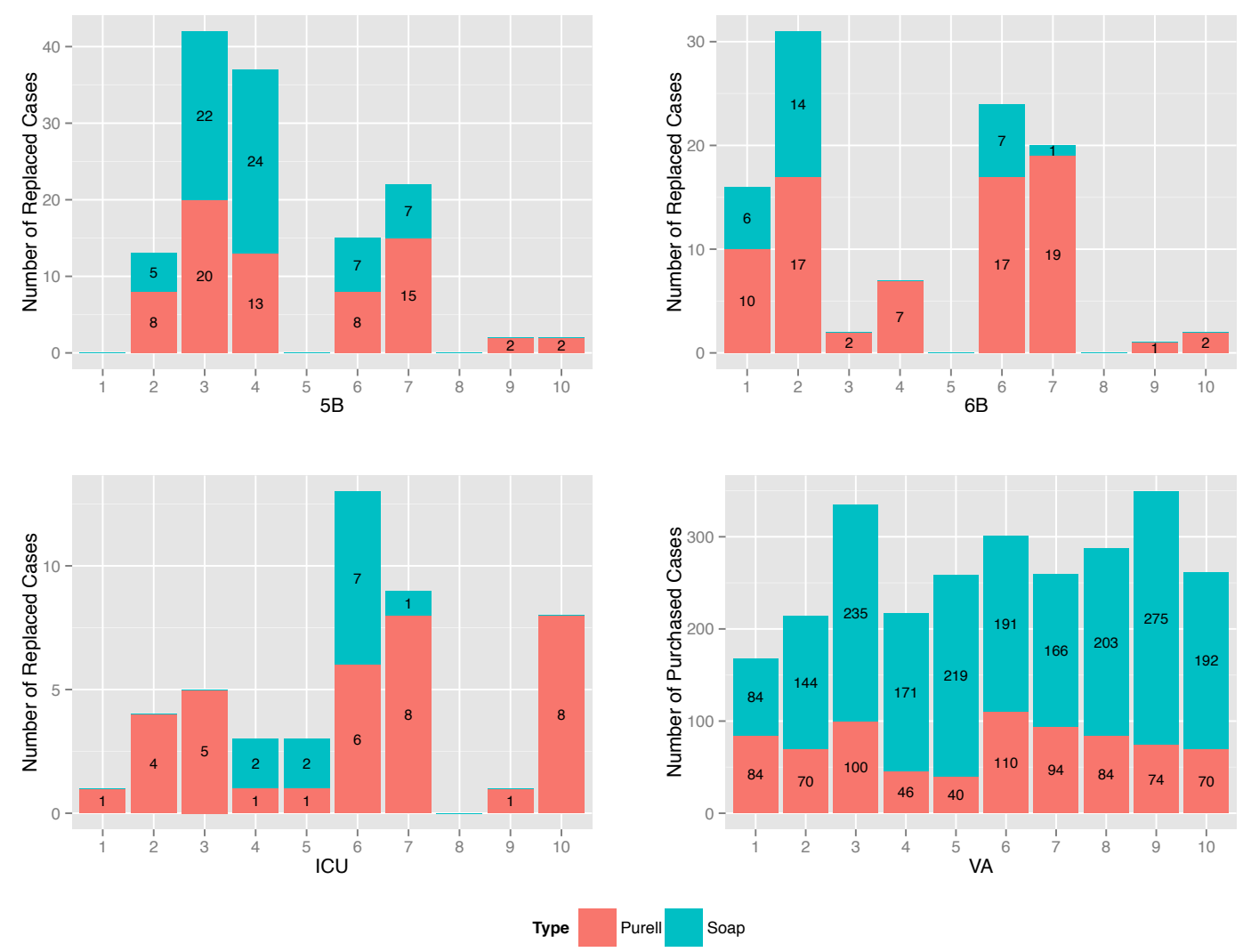

Figure 6.13 Total Number of Soap and Purell Cartridges Replaced During 2015 at 5B, $6 \mathrm{~B}$ and ICU Compared with Sales Data for Overall Facility at the PVAMC 
Based on the tracking dataset for replacing Soap and Purell cartridges and purchased dataset during 2015 , the breakdown of product use at $5 \mathrm{~B}, 6 \mathrm{~B}$ and ICU is shown in Figure 6.14. There are 1880 soap cartridges and 772 Purell cartridges purchased during 2015. Ward 5B contributes to about $3.5 \%$ and $9 \%$ of product use of Soap and Purell, respectively. Ward 6B contributes to about $1.5 \%$ and $10 \%$ of product use of Soap and Purell, respectively. Ward ICU contributes to about $0.6 \%$ and $5 \%$ of product use of Soap and Purell, respectively.
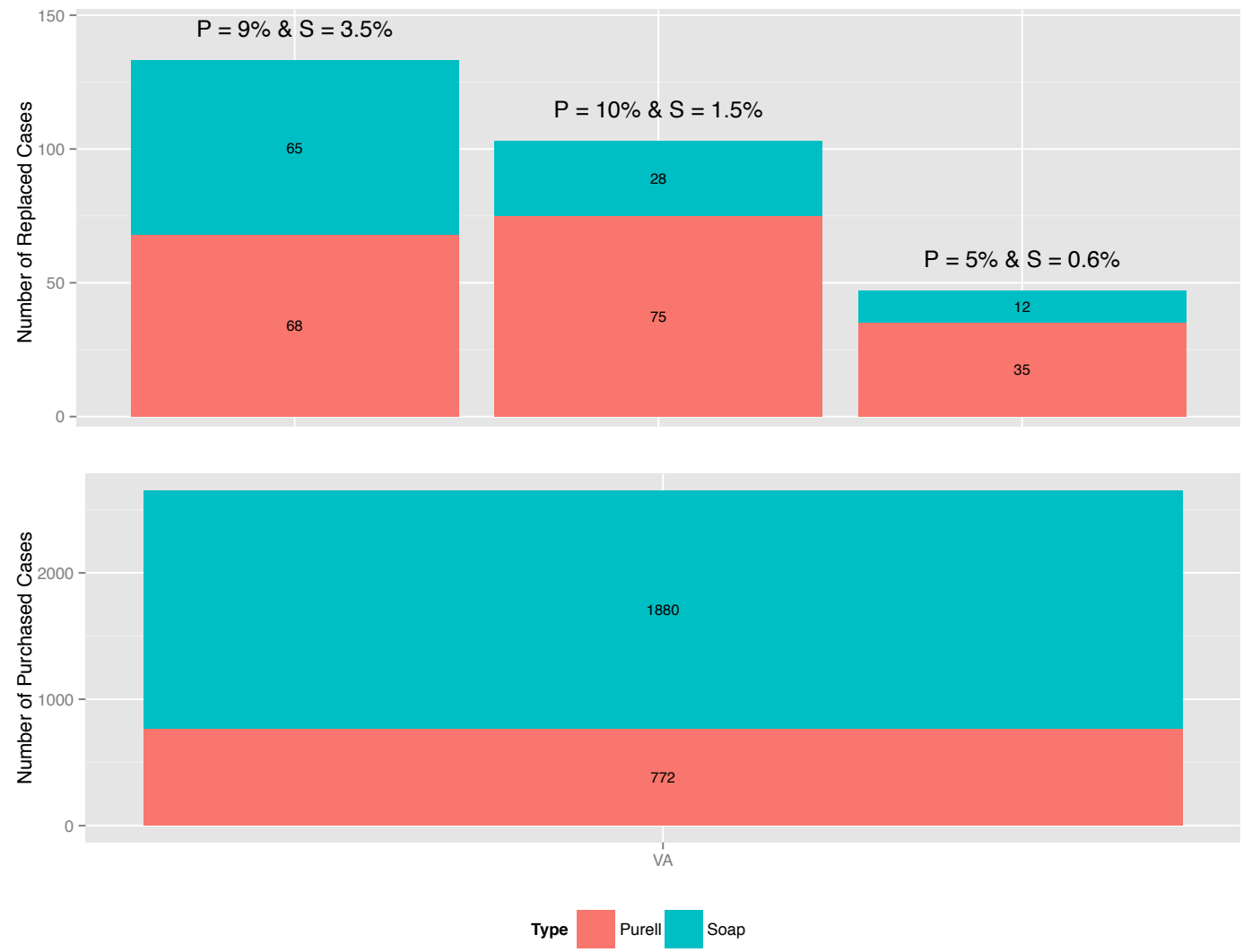

Figure 6.14 Breakdown of Product Use of Soap and Purell Cartridges Compared to the Replaced Units During 2015 


\section{CHAPTER 7}

\section{INDIRECT PPE COMPLIANCE METHOD}

\subsection{Introduction}

In healthcare settings, monitoring and reporting hand hygiene compliance is the only concern. Therefore, several hand hygiene-monitoring methods were developed and continuously improved in the past. Yet, monitoring and reporting personal protective equipment (PEE) compliance is no less important. Monitoring and reporting PPE compliance sometimes accompanies reporting with a hand hygiene direct observation method. However, PPE compliance is still considered as a secondary measurement and sometimes is not targeted for reporting.

Indirect manual monitoring of PPE compliance by using the primary inventory PPE data at the PVAMC is inspired by the indirect manual monitoring of hand hygiene by measuring the product consumption. Thus, it resembles some of the indirect manual monitoring of hand hygiene benefits and drawbacks summarized by WHO (2009). The indirect manual monitoring of PPE compliance is inexpensive and the Hawthorne effect (bias) is avoided. However, as in the indirect manual monitoring of hand hygiene, it does not capture the true PPE moments. Therefore, PPE adherence rate is unreported unless the denominator is estimated. A surrogate denominator is used to add some reliability to such an approach by using an average of how frequently a patient is seen by a HCW, how many transmission versus standard BDOC and how many invasive-device days per month per inpatient ward. Similar to the indirect manual monitoring of hand hygiene, the indirect manual monitoring of PPE 
compliance lacks the breakdown of compliance by healthcare professional categories since unlimited groups use PPE for health purposes or personal use.

The WHO (2009 WHO, 2009, Page 161 and 162) presents the pros and cons of indirect hand hygiene methods. The proposed indirect PPE measurement method will also have similar advantages and disadvantages, as shown in Table 7.1.

\begin{tabular}{|l|l|}
\hline \multicolumn{1}{|c|}{ Advantages } & \multicolumn{1}{c|}{ Disadvantages } \\
\hline - Inexpensive. & $\begin{array}{l}\text { Does not capture when the PPE is } \\
\text { suppose to be used. }\end{array}$ \\
$\begin{array}{l}\text { Reports all PPE use, to avoid } \\
\text { selection bias. }\end{array}$ & $\begin{array}{l}\text { Improves validity through using PPE } \\
\text { inventory data to develop the } \\
\text { denominator. }\end{array}$ \\
each inpatient wards.
\end{tabular}

Table 7.1 Pros and Cons of Indirect Manual Monitoring of PPE Performance by Measuring Inventory Consumption

\subsection{Objectives}

Developing a method for estimating PPE compliance based on the inventory consumption of PPE products used in three inpatient wards at the PVAMC is the objective of this study by determining a correlation between bed days of care and the consumption of PPE products use, Gloves and Gowns.

\subsection{Setting}

The three-inpatient units 5B, 6B and ICU at the PVAMC collectively have 49 wall-mounted slots of gloves which each fits four different sizes of gloves cases (small, medium, large and x-large) and each gloves case comes with 100 individual 
gloves. There are also 19 wall-mounted slots of gowns each fits three gowns cases and each gowns case comes with 10 individual gowns and 29 wall-mounted slots of masks each fits one masks case and each masks case comes with 100 individual masks.

In 5B, there are 13 wall-mounted slots of gloves, 7 wall-mounted slots of gowns and 13 wall-mounted slots of masks. In 6B, there are 24 wall-mounted slots of gloves, 7 wall-mounted slots of gowns and 10 wall-mounted slots of masks. In ICU, there are 12 wall-mounted slots of gloves, 5 wall-mounted slots of gowns and 6 wall-mounted slots of masks. These numbers do not include PPE products stored in the supply closet at each inpatient ward.

The primary inventory at the PVAMC has two different groups of supplies, which are stocked inventory in the Primary locations and special ordered items or nonstocked. The PPE products such as gloves, gowns and masks are medical and surgical items that are considered stocked items and are ordered through an auto-generated generic inventory package (G.I.P) system (L. Renaud, Personal Communication, September 26, 2016; The VHA Handbook, 2016). PPE products and their stock and reorder levels are as follows, and shown in Figure 7.1:

- Nitrile Examination Gloves: come in four different sizes (Small, Medium, Large and X-Large) and is powder-\&-latex free and textured and for single use only. Small size has a level of 212 cases and a reorder level of 106. Medium size has a level of 404 cases and a reorder level of 202. Large size has a level of 257 cases and a reorder level of 128. X-Large size has a level of 129 cases and a reorder level of 65 . Medium size is the most common size to be worn by HCW. 
- Masks: come in one universal size and is for single use only.

- Gowns: come in one universal size and is for single use only. Its stocking level is 292 and its reorder level is 146.

The primary inventory is manually checked three days per week, on Monday, Wednesday and Friday, for restocking.

From January $1^{\text {st }}, 2014$ to September $30^{\text {th }}, 2016$, the primary inventory consumption of PPE products orders is reported for each inpatient ward via VistA System. VistA is "The Veterans Health Information Systems and Technology Architecture Information System - is a Health Information Technology (HIT) system created and used by the Veterans Health Administration (VHA) of the U.S. Department of Veterans Affairs (VA) in serving America's Veterans through the provision of exceptional-quality health care which enhances our Veterans' health and well-being.” (U.S. Department of Veterans Affairs, 2013, Page 13).

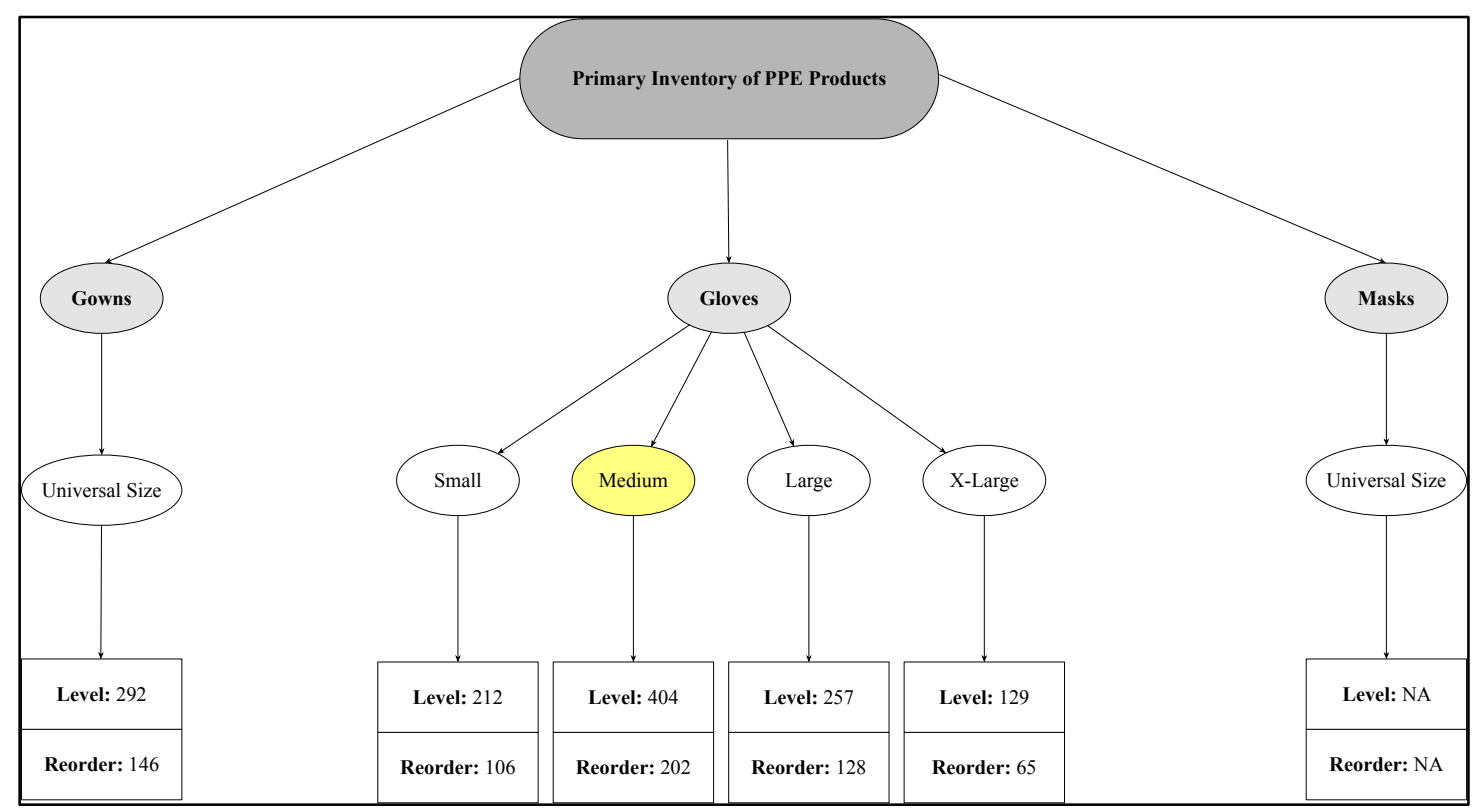

Figure 7.1 Primary Inventory PPE Products and Their Stock and Reorder Levels 


\subsection{Design and Method}

A study is conducted to estimate PPE compliance based on the inventory consumption of PPE product orders at each inpatient ward individually and collectively. At the end of each calendar year, 2014, 2015 and 2016, the following data are collected from VistA and IPEC system at the PVAMC to calculate PPE compliance and test for correlation between PPE products and BDOC for each inpatient ward:

- The Inventory Consumption of PPE Products per inpatient ward.

- Bed days of care (BDOC) and admissions of patients at each inpatient ward from IPEC system for complete calendar year of 2014 and 2015 and 9 months of 2016.

- $\quad$ HAIs cases such as MRSA, CAUTI, CLABSI and VAP from IPEC system for complete calendar year of 2014 and 2015 and 9 months of 2016.

- Invasive-devices days for HAI such as CAUTI, CLABSI and VAP from IPEC system for complete calendar year of 2014 and 2015 and 9 months of 2016.

- Average patient visits per HCW per inpatient ward are driven from patient contact data in Chapter $4(5 \mathrm{~B}=50,6 \mathrm{~B}=58$ and $\mathrm{ICU}=111)$.

- An observational data of PPE products usage of other groups at the PVAMC such as environmental management service (EMS) and food and nutrition services who frequently consume PPE for their regular work. 


\subsection{Data Analysis}

\subsubsection{Inpatient-Wards Bed Days of Care vs. PPE Use}

Practicing hand hygiene during key moments is considered the first protection step taken by HCWs to prevent and control the transmission of infectious diseases. Sometimes a patient's health status requires $\mathrm{HCW}$ s to take extra precaution to reduce the risk of transmission of the infectious diseases. The extra precaution is to comply with the isolation precautions (standard precautions and transmission-based precautions) hung on the room door of patients with known infectious diseases or being colonized with any important pathogens (VAMHCS, 2012).

The PPE of interest to this study are:

- Gloves,

- Gowns, and

- Masks.

Since practicing hand hygiene before and after wearing hand gloves, wearing gowns and masks before having contacting patients is mandatory, identifying patterns or correlation between:

- $\quad$ BDOC versus PPE and

- HAIs incidents and Colonization Moments versus PPE

is worth attempting.

Five HAIs of interest to this study, MRSA, CAUTI, CLABSI, VAP and MRSA Colonization are considered for modeling. C.Diff is not reported per ward at the PVAMC. The objective of this modeling is not to study those HCWs who wear 
different sizes of hand gloves. Thus, different sizes, small, medium, large and x-large of hand gloves are aggregated as one size.

PPE for fiscal year of 2015 is missing. Thus, year 2015 PPE is found by conducting the following steps:

- Several statistical models using negative binomial distribution are fitted to explain the relationship between BDOC and each PPE individually (gloves and gowns) using only year 2014 .

- The best-fit model is then chosen to predict PPE for 2016, which exists already.

- Mean Square Prediction Error (MSPE) or Mean Absolute Prediction Error (MAPE) is used to evaluate the prediction model of 2016.

- The best-fit model then is used to predict PPE for 2015.

- Mask compliance are not calculated and not included since it is almost impossible to identify when masks are required except when there are huge data related to patients who are on either droplet or airborne precautions. Nevertheless, masks are predicted for year 2015.

The hypothesis testing for modeling the dependence of PPE counts based on BDOC for year 2014 only is as follows:

$\mathrm{H}_{0}$ : BDOC Does Not Have an Impact on the PPE used $\mathrm{H}_{1}$ : BDOC Does Have an Impact on the PPE used

By nature, HCWs are expected to use more PPE as BDOC increases especially if patients are on precautions. The more precautions signs on the floor, the more PPE used. The summary of the descriptive statistics, Table 7.2, shows that the response 
variable PPE could be modeled based on BDOC, because the mean value of the outcome appears to vary by BDOC. The unconditional mean of the response variable is much lower than its variance as seen in Table 7.2 and the conditional variances within each level (ward) of BDOC are higher than the conditional means within each level (ward). These differences suggest that a Negative Binomial model with varying parameters across wards, in which these values are assumed not to be equal, could be appropriate to this data.

\begin{tabular}{|c|c|c|c|c|c|c|c|c|c|}
\hline PPE & \multicolumn{3}{|c|}{ Gloves } & \multicolumn{3}{c|}{ Gowns } & \multicolumn{3}{c|}{ Masks } \\
\hline Ward & Mean & Variance & SD & Mean & Variance & SD & Mean & Variance & SD \\
\hline 5B & 118.17 & 9972.52 & 99.86 & 61.92 & 6447.72 & 80.3 & 5.25 & 29.66 & 5.45 \\
\hline 6B & 248.67 & 16696.97 & 129.22 & 396.33 & 11718.24 & 108.25 & 13.17 & 23.24 & 4.82 \\
\hline ICU & 94 & 1081.82 & 32.89 & 218.42 & 17797.54 & 133.41 & 7.17 & 67.97 & 8.24 \\
\hline Combined & 153.61 & 13468.82 & 116.06 & 225.56 & 30500.65 & 174.64 & 8.53 & 49.68 & 7.05 \\
\hline
\end{tabular}

Table 7.2 Mean, Variance and Standard Deviation of PPE per Inpatient Ward and Inpatient Wards Combined for Year 2014

Looking at the scatter plot of BDOC versus each PPE (gloves, gowns and masks) in Figure 7.2, it can be seen that there is evidence of a proportional relationship between BDOC and each PPE, because as one increases the other increases too. Correlation coefficients can be seen in Table 7.3 per ward and wards combined.

\begin{tabular}{|c|c|c|c|}
\hline \multirow{2}{*}{ Ward } & \multicolumn{3}{|c|}{ Correlation Coefficients } \\
\cline { 2 - 4 } & Gloves & Gowns & Masks \\
\hline 5B & 0.62 & 0.37 & 0.41 \\
\hline 6B & 0.74 & 0.68 & 0.69 \\
\hline ICU & 0.66 & 0.36 & 0.49 \\
\hline Wards Combined & 0.55 & 0.24 & 0.30 \\
\hline
\end{tabular}

Table 7.3 Correlation Coefficients of BDOC versus PPE per Inpatient Ward 

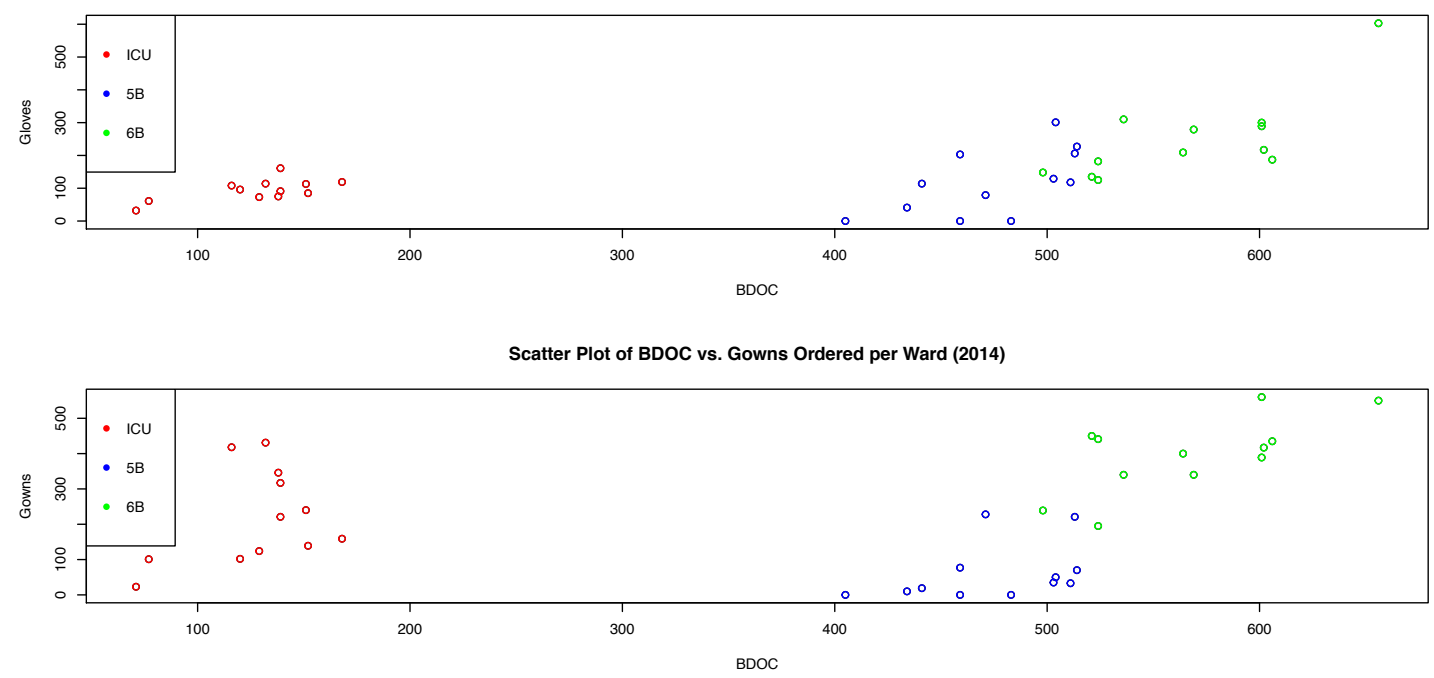

Scatter Plot of BDOC vs. Masks Ordered per Ward (2014)

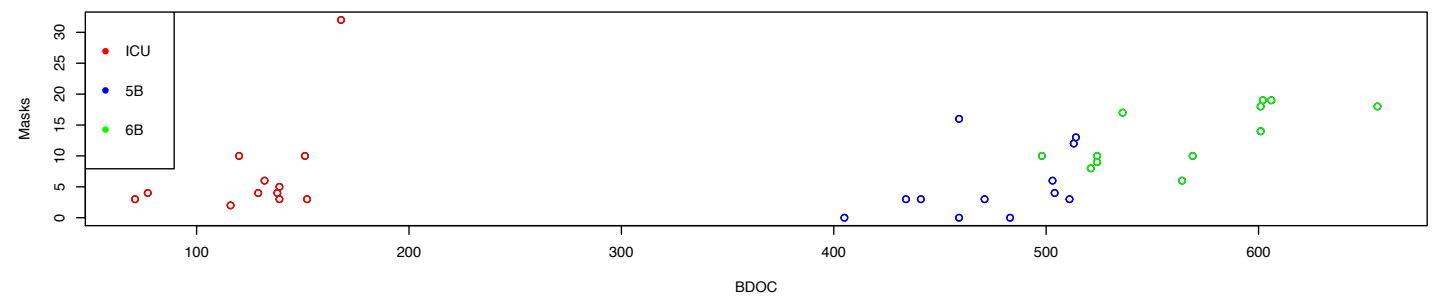

Figure 7.2 BDOC versus PPE (Gloves, Gowns and Masks) per Ward for Year 2014

For gloves, a Negative Binomial distribution with a Power deterministic function is used to fit a model with constant coefficients across wards and a model with parameters varying across wards since ward factor by itself is significant, which means that each ward is different from the other in terms of gloves quantity ordered as depicted in Figure 7.3. Two of the three parameters of the Negative Binomial distribution are different for each ward the intercept and the dispersion with constant slope (rate) across wards. Using Akaike information criterion (AIC) and Bayesian information criterion (BIC) criteria for model selection, the model with parameters varying across wards is the best-fitted model for modeling gloves counts based on BDOC. 


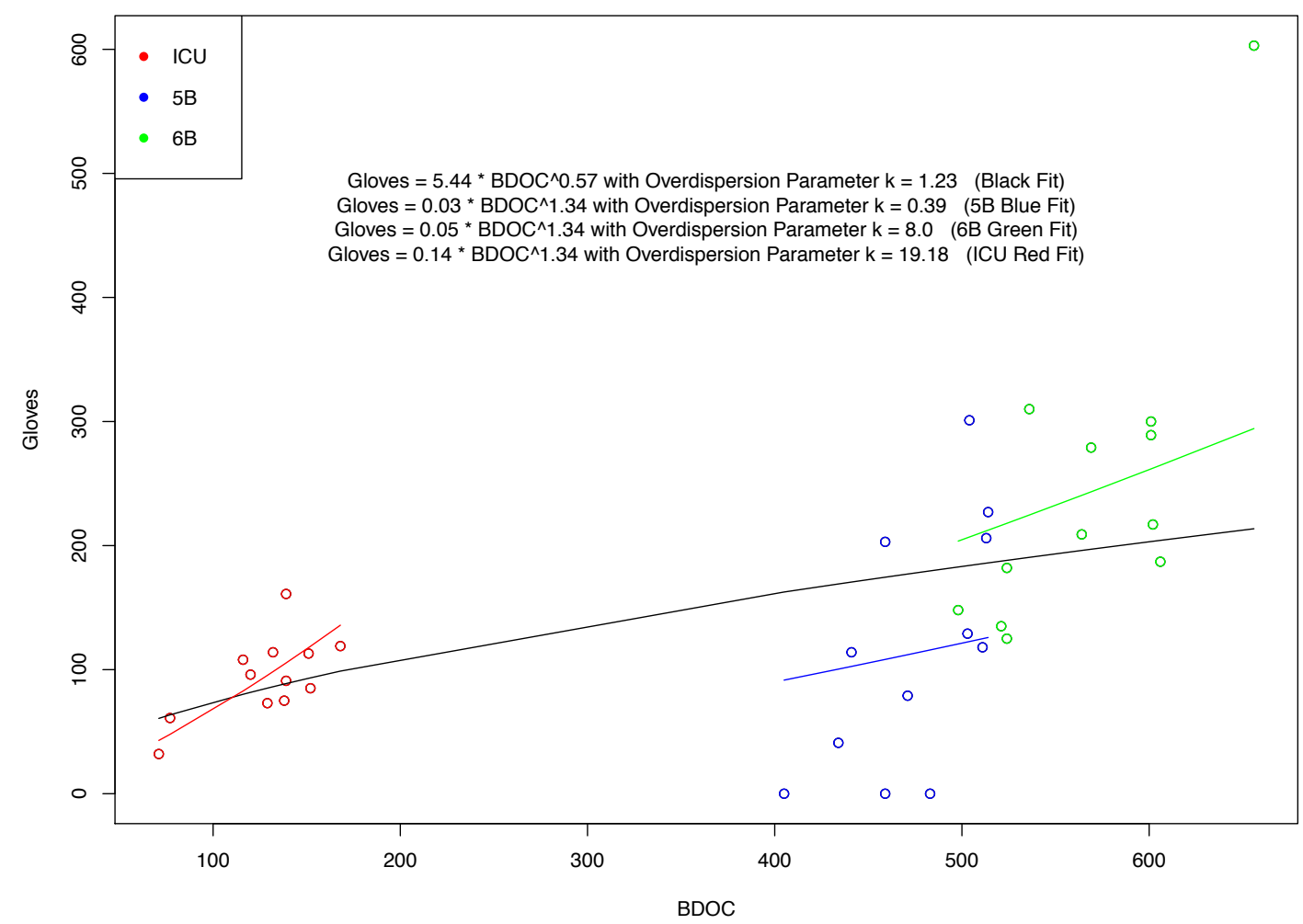

Figure 7.3 Negative Binomial Distribution with a Power Deterministic Function Fitted Model for BDOC versus Gloves per Ward for Year 2014

For gowns, a Negative Binomial distribution with a Power deterministic function is used to fit a model with constant coefficients across wards and a model with a Power deterministic function with parameters varying across wards since ward factor by itself is significant, which means that each ward is different from the other in terms of gowns quantity ordered as depicted in Figure 7.4. Two of the three parameters of the Negative Binomial distribution are different for each ward the slope (rate) and the dispersion with constant intercept across wards. Using AIC and BIC criteria for model selection, the model with parameters varying across wards is the best-fitted model for modeling gowns counts based on BDOC. 


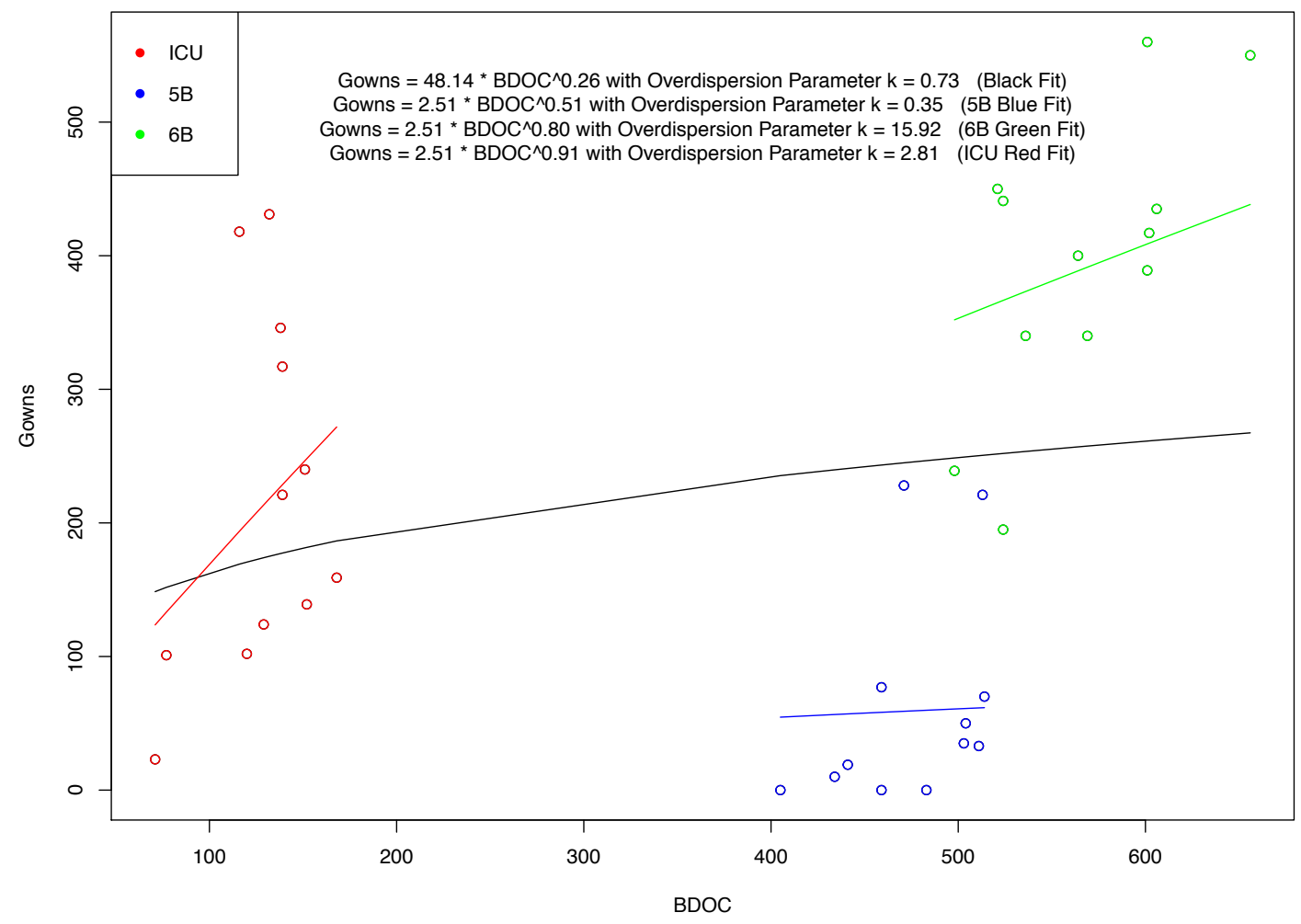

Figure 7.4 Negative Binomial Distribution with a Power Deterministic Function Fitted Model for BDOC versus Gowns per Ward for Year 2014

For masks, a Negative Binomial distribution with a Linear deterministic function is used to fit a model with constant coefficients across wards and a model with parameters varying across wards since ward factor by itself is significant, means each ward is different from the other in terms of masks quantity ordered as depicted in Figure 7.5. Two of the three parameters of the Negative Binomial distribution are different for each ward the slope (rate) and the dispersion with constant intercept across wards. Using AIC and BIC criteria for model selection, the model with parameters varying across wards is the best-fitted model for modeling masks counts based on BDOC. 


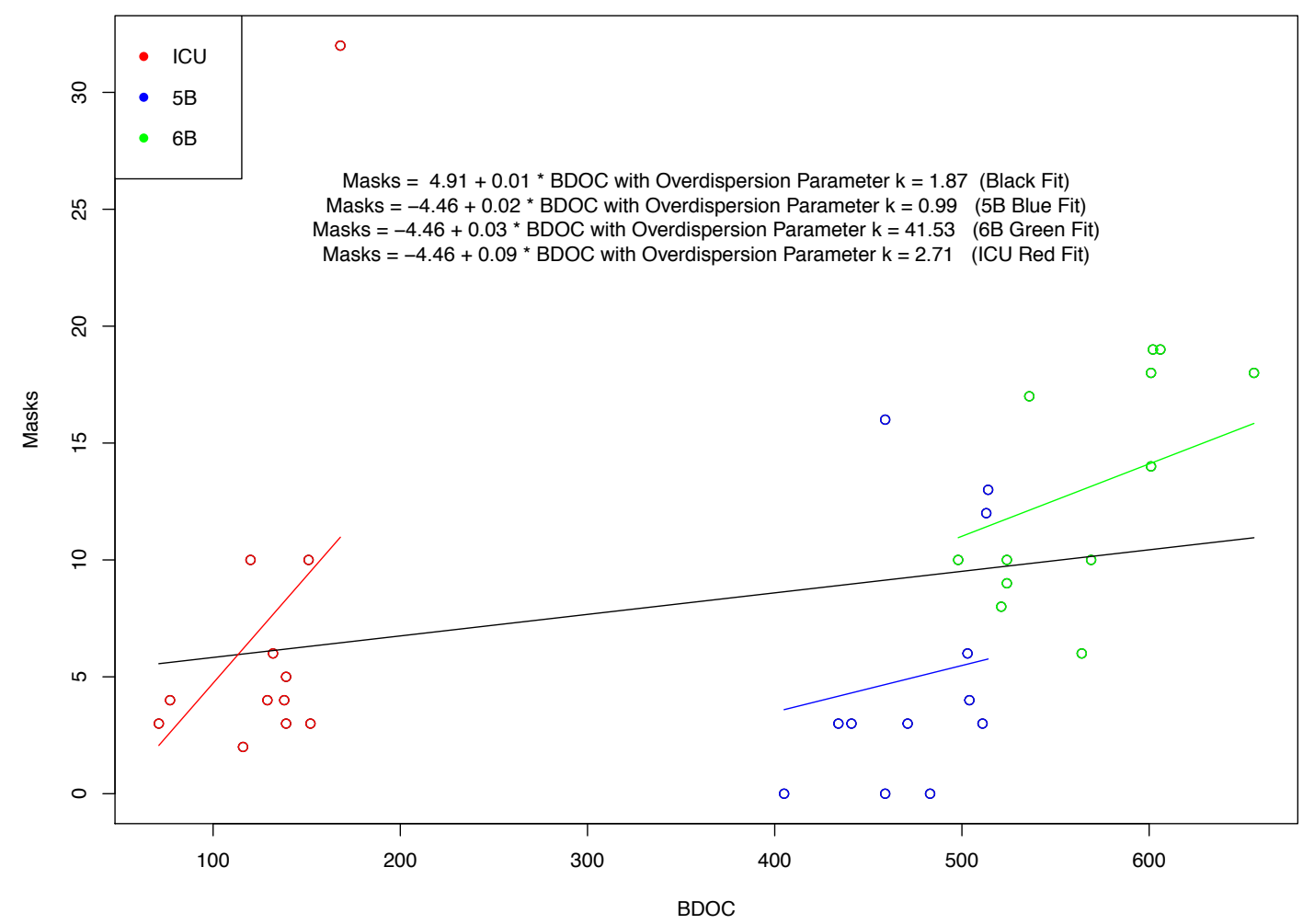

Figure 7.5 Negative Binomial Distribution with a Linear Deterministic Function Fitted Model for BDOC versus Masks per Ward for Year 2014

For model validation, the best-fit models for gloves, gowns and masks from year 2014 are used to predict gloves, gowns and masks of year 2016. Then, the predicted values of year 2016 are compared to the true values of gloves, gowns and masks of the same year 2016 using the following criteria:

$$
\begin{aligned}
\text { Mean Square Prediction Error }(\mathrm{MSPE}) & =\frac{\sum_{\mathrm{i}=1}^{\mathrm{n}}\left(\mathrm{y}_{\mathrm{i}}-\tilde{\mathrm{y}}_{\mathrm{i}}\right)^{2}}{\mathrm{n}} \\
\text { Mean Absolute Prediction Error (MAPE) } & =\frac{\sum_{\mathrm{i}=1}^{\mathrm{n}}\left|\frac{\left(\mathrm{y}_{\mathrm{i}}-\tilde{\mathrm{y}}_{\mathrm{i}}\right)}{\mathrm{y}_{\mathrm{i}}}\right|}{\mathrm{n}}
\end{aligned}
$$

where $y_{i}$ is the PPE true values of year 2016, $\tilde{y}_{i}$ is the PPE predicted values of year 2016 and $\mathrm{n}$ is the total number of observations. 
Table 7.4 shows the prediction values for each PPE for year 2016. Of note, there are only 9 months of year 2016 provided from the PVAMC. The true and prediction values are depicted on Figure 7.6, Figure 7.7 and Figure 7.8 for gloves, gowns and masks respectively. MSPE and MAPE values are also written on each figure. MSPE values are 22852, 18642 and 52 for gloves, gowns and masks, respectively. MAPE values are $42 \%, 51 \%$ and $56 \%$ for gloves, gowns and masks, respectively. MAPE is preferred to be used since it is easy to interpret and understand. MAPE values are considered low but acceptable considering the small sample size. Only one year is used which is 2014, and not implementing a time series model are two important factors to explain such low percentages. 


\begin{tabular}{|c|c|c|c|c|c|c|c|c|}
\hline Year & Month & Ward & $\begin{array}{c}\text { Gloves } \\
\text { (True) }\end{array}$ & $\begin{array}{c}\text { Gloves } \\
\text { (Predicted) }\end{array}$ & $\begin{array}{c}\text { Gowns } \\
\text { (True) }\end{array}$ & $\begin{array}{c}\text { Gowns } \\
\text { (Predict } \\
\text { ed) }\end{array}$ & $\begin{array}{c}\text { Masks } \\
\text { (True) }\end{array}$ & $\begin{array}{c}\text { Masks } \\
\text { (Predi } \\
\text { cted) }\end{array}$ \\
\hline 2016 & 1 & ICU & 77 & 90 & 150 & 206 & 5 & 7 \\
\hline 2016 & 2 & ICU & 83 & 76 & 110 & 183 & 4 & 6 \\
\hline 2016 & 3 & ICU & 101 & 75 & 99 & 181 & 11 & 5 \\
\hline 2016 & 4 & ICU & 109 & 81 & 60 & 191 & 4 & 6 \\
\hline 2016 & 5 & ICU & 110 & 45 & 160 & 128 & 3 & 2 \\
\hline 2016 & 6 & ICU & 59 & 24 & 86 & 83 & 3 & 0 \\
\hline 2016 & 7 & ICU & 76 & 20 & 69 & 73 & 1 & -1 \\
\hline 2016 & 8 & ICU & 94 & 28 & 59 & 91 & 1 & 0 \\
\hline 2016 & 9 & ICU & 70 & 40 & 72 & 117 & 0 & 2 \\
\hline 2016 & 1 & 5B & 216 & 130 & 269 & 62 & 14 & 6 \\
\hline 2016 & 2 & 5B & 199 & 120 & 295 & 61 & 9 & 5 \\
\hline 2016 & 3 & 5B & 250 & 130 & 249 & 63 & 16 & 6 \\
\hline 2016 & 4 & 5B & 249 & 117 & 234 & 60 & 18 & 5 \\
\hline 2016 & 5 & 5B & 223 & 136 & 260 & 64 & 13 & 6 \\
\hline 2016 & 6 & 5B & 235 & 140 & 265 & 64 & 9 & 7 \\
\hline 2016 & 7 & 5B & 240 & 171 & 250 & 69 & 5 & 8 \\
\hline 2016 & 8 & 5B & 251 & 138 & 250 & 64 & 2 & 6 \\
\hline 2016 & 9 & 5B & 216 & 140 & 337 & 64 & 9 & 7 \\
\hline 2016 & 1 & 6B & 439 & 274 & 395 & 420 & 13 & 15 \\
\hline 2016 & 2 & 6B & 475 & 250 & 567 & 397 & 13 & 13 \\
\hline 2016 & 3 & 6B & 428 & 269 & 425 & 415 & 34 & 15 \\
\hline 2016 & 4 & 6B & 507 & 251 & 407 & 399 & 32 & 14 \\
\hline 2016 & 5 & 6B & 361 & 200 & 475 & 349 & 8 & 11 \\
\hline 2016 & 6 & 6B & 439 & 240 & 571 & 389 & 24 & 13 \\
\hline 2016 & 7 & 6B & 477 & 253 & 427 & 401 & 23 & 14 \\
\hline 2016 & 8 & 6B & 728 & 248 & 340 & 396 & 15 & 13 \\
\hline 2016 & 9 & $6 B$ & 254 & 217 & 330 & 365 & 13 & 12 \\
\hline
\end{tabular}

Table 7.4 PPE True and Prediction Values of Year 2016 per Inpatient Ward 


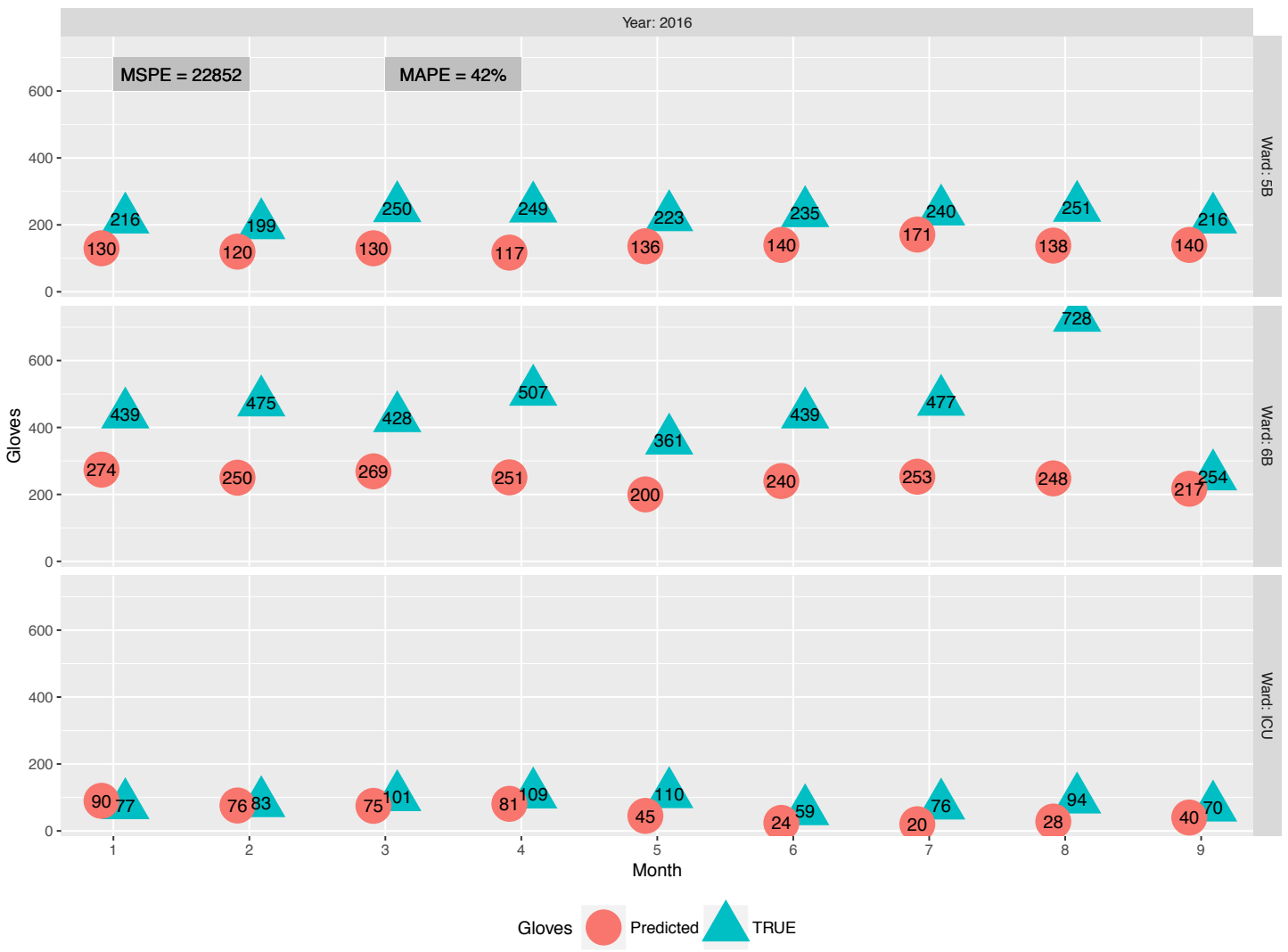

Figure 7.6 Gloves True (Green) and Prediction (Red) Values per Month per Year 2016 per Ward with MSPE and MAPE Values 


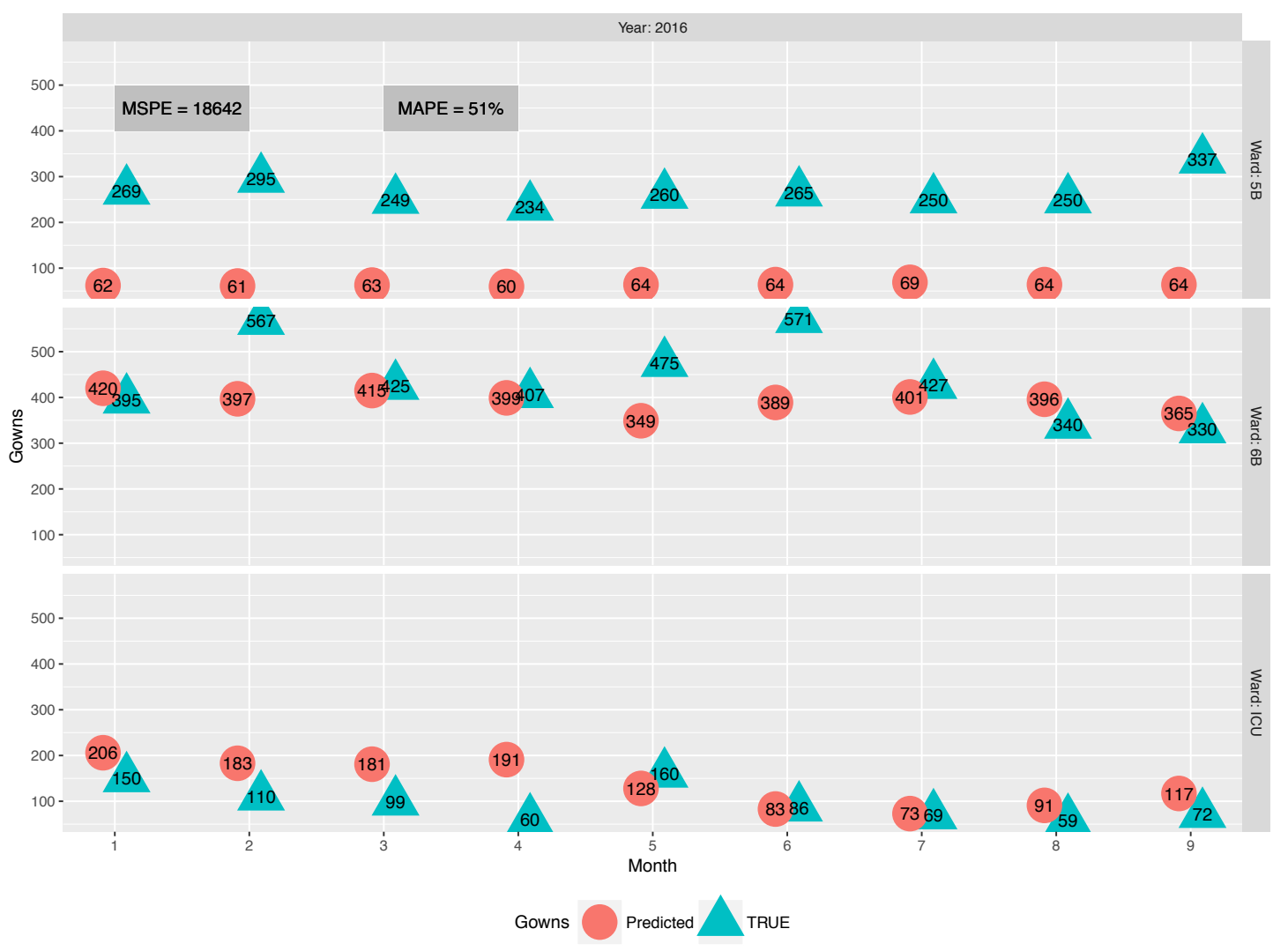

Figure 7.7 Gowns True (Green) and Prediction (Red) Values per Month per Year 2016 per Ward with MSPE and MAPE Values 


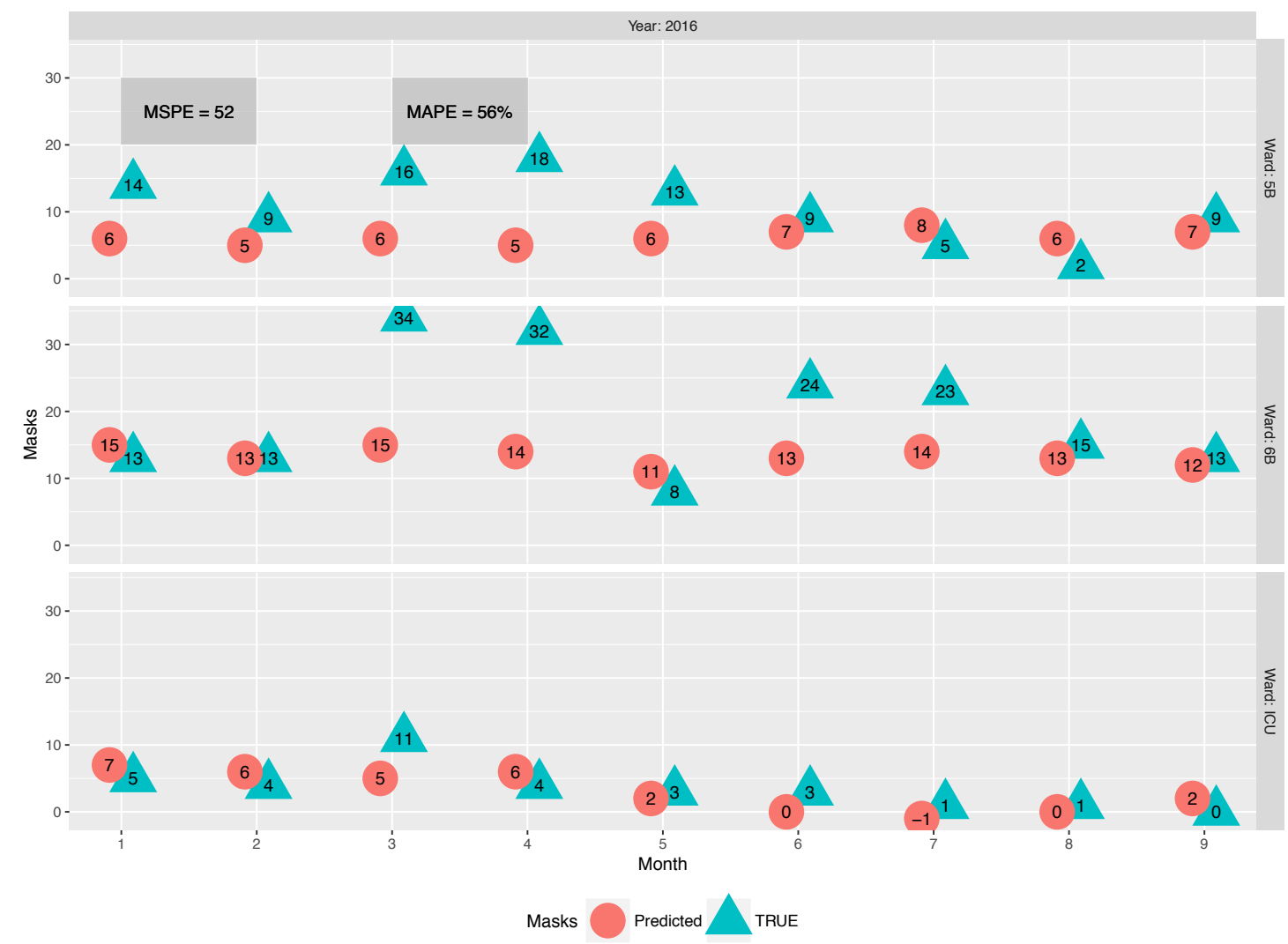

Figure 7.8 Masks True (Green) and Prediction (Red) Values per Month per Year 2016 per Ward with MSPE and MAPE Values

After validating the models, PPE for year 2015 is predicted. The predicted values are shown in Table 7.5. Statistical models that are depicted in Figure 7.3, Figure 7.4 and Figure 7.5 are used for the prediction calculation. BDOC for year 2015 is substituted into these best fit models to produce the prediction values. Of note, gloves prediction values are calculated for all sizes combined. 


\begin{tabular}{|c|c|c|c|c|c|}
\hline Year & Month & Ward & Gloves (Predicted) & Gowns (Predicted) & Masks (Predicted) \\
\hline 2015 & 1 & ICU & 117 & 245 & 9 \\
\hline 2015 & 2 & ICU & 94 & 212 & 7 \\
\hline 2015 & 3 & ICU & 62 & 160 & 4 \\
\hline 2015 & 4 & ICU & 67 & 168 & 5 \\
\hline 2015 & 5 & ICU & 63 & 161 & 4 \\
\hline 2015 & 6 & ICU & 83 & 194 & 6 \\
\hline 2015 & 7 & ICU & 132 & 266 & 11 \\
\hline 2015 & 8 & ICU & 101 & 223 & 8 \\
\hline 2015 & 9 & ICU & 64 & 163 & 4 \\
\hline 2015 & 10 & ICU & 114 & 241 & 9 \\
\hline 2015 & 11 & $\mathrm{ICU}$ & 40 & 117 & 2 \\
\hline 2015 & 12 & ICU & 83 & 194 & 6 \\
\hline 2015 & 1 & 5B & 168 & 69 & 8 \\
\hline 2015 & 2 & $5 \mathrm{~B}$ & 141 & 64 & 7 \\
\hline 2015 & 3 & $5 \mathrm{~B}$ & 147 & 65 & 7 \\
\hline 2015 & 4 & 5B & 135 & 63 & 6 \\
\hline 2015 & 5 & $5 \mathrm{~B}$ & 142 & 65 & 7 \\
\hline 2015 & 6 & 5B & 131 & 63 & 6 \\
\hline 2015 & 7 & $5 \mathrm{~B}$ & 122 & 61 & 6 \\
\hline 2015 & 8 & $5 \mathrm{~B}$ & 140 & 64 & 7 \\
\hline 2015 & 9 & $5 \mathrm{~B}$ & 111 & 59 & 5 \\
\hline 2015 & 10 & $5 \mathrm{~B}$ & 140 & 64 & 7 \\
\hline 2015 & 11 & $5 \mathrm{~B}$ & 140 & 64 & 7 \\
\hline 2015 & 12 & $5 \mathrm{~B}$ & 134 & 63 & 6 \\
\hline 2015 & 1 & $6 \mathrm{~B}$ & 288 & 433 & 16 \\
\hline 2015 & 2 & $6 \mathrm{~B}$ & 238 & 386 & 13 \\
\hline 2015 & 3 & $6 \mathrm{~B}$ & 272 & 418 & 15 \\
\hline 2015 & 4 & $6 \mathrm{~B}$ & 264 & 411 & 14 \\
\hline 2015 & 5 & $6 \mathrm{~B}$ & 252 & 400 & 14 \\
\hline 2015 & 6 & $6 \mathrm{~B}$ & 253 & 400 & 14 \\
\hline 2015 & 7 & 6B & 279 & 424 & 15 \\
\hline 2015 & 8 & $6 \mathrm{~B}$ & 291 & 435 & 16 \\
\hline 2015 & 9 & $6 \mathrm{~B}$ & 217 & 365 & 12 \\
\hline 2015 & 10 & $6 \mathrm{~B}$ & 265 & 412 & 14 \\
\hline 2015 & 11 & 6B & 219 & 368 & 12 \\
\hline 2015 & 12 & $6 \mathrm{~B}$ & 262 & 409 & 14 \\
\hline
\end{tabular}

Table 7.5 PPE Prediction Values per Ward for Year 2015 
The dependence of PPE use at the inpatient wards at the PVAMC based on the true moments of PPE is modeled. The data considered for this study are:

- $\quad$ BDOC for year 2014 and 2015 (12 months) and 2016 (first 9 months).

- PPE for year 2014 (12 months), predicted PPE for 2015 (12 months) and 2016 (first 9 months).

- HAIs and MRSA colonization for year 2014 and 2015 (12 months) and 2016 (first 9 months).

The analysis is carried per ward and wards combined except for C.Diff since C.Diff is not reported per ward by IPEC system at the PVAMC. One issue with this kind of data reporting at the PVAMC is that, for example, when reporting patients with MRSA or MRSA colonization, BDOC associated to those patients are not reported. The number of admitted patients with MRSA or MRSA colonization out of the total number of admission per month per ward is recorded. Thus, either the analysis is carried out by replacing BDOC with the true admission number and using the counts of those patients with MRSA and MRSA colonization or by calculating the percentage of patients with MRSA and MRSA colonization, dividing that by the total number of admission and then the result is multiplied by BDOC. The latter is used to avoid any misleading interpretation when correlating the PPE use with total number of MRSA or MRSA colonization. All HAIs incidents (MRSA, CAUTI, CLABSI and VAP) are summed up with MRSA colonization per month per ward during 2014, 2015 and 2016.

Before getting into modeling, descriptive statistics of PPE are reported. Figure 7.9 shows the quantity of gloves ordered at each ward each month. Though the size of 
the ICU ward is significantly different from $5 \mathrm{~B}$ and $6 \mathrm{~B}$, where $5 \mathrm{~B}$ and $6 \mathrm{~B}$ have the same physical size of the unit but different bed capacity, ICU's gloves quantity ordered are not that different from 5B. Mean gloves for each ward per year can be seen in Table 7.6 and Table 7.7. It can be seen that the mean gets larger from one year to the next for 5B and 6B; however, it gets smaller for ICU.

At $6 \mathrm{~B}$, there are 24 wall-mounted slots which each fit four different size of gloves cases (small, medium, large and x-large). There are 96 (24*4 different sizes) cases of gloves that could be available at the floor with $9600(96 * 100$ gloves per case) individual gloves and $4800(9600$ / 2) pairs of gloves. At 5B, there are 13 wallmounted slots similarly fit 2600 pairs of gloves. At ICU, there are 12 wall-mounted slots which then fit 2400 pairs of gloves.

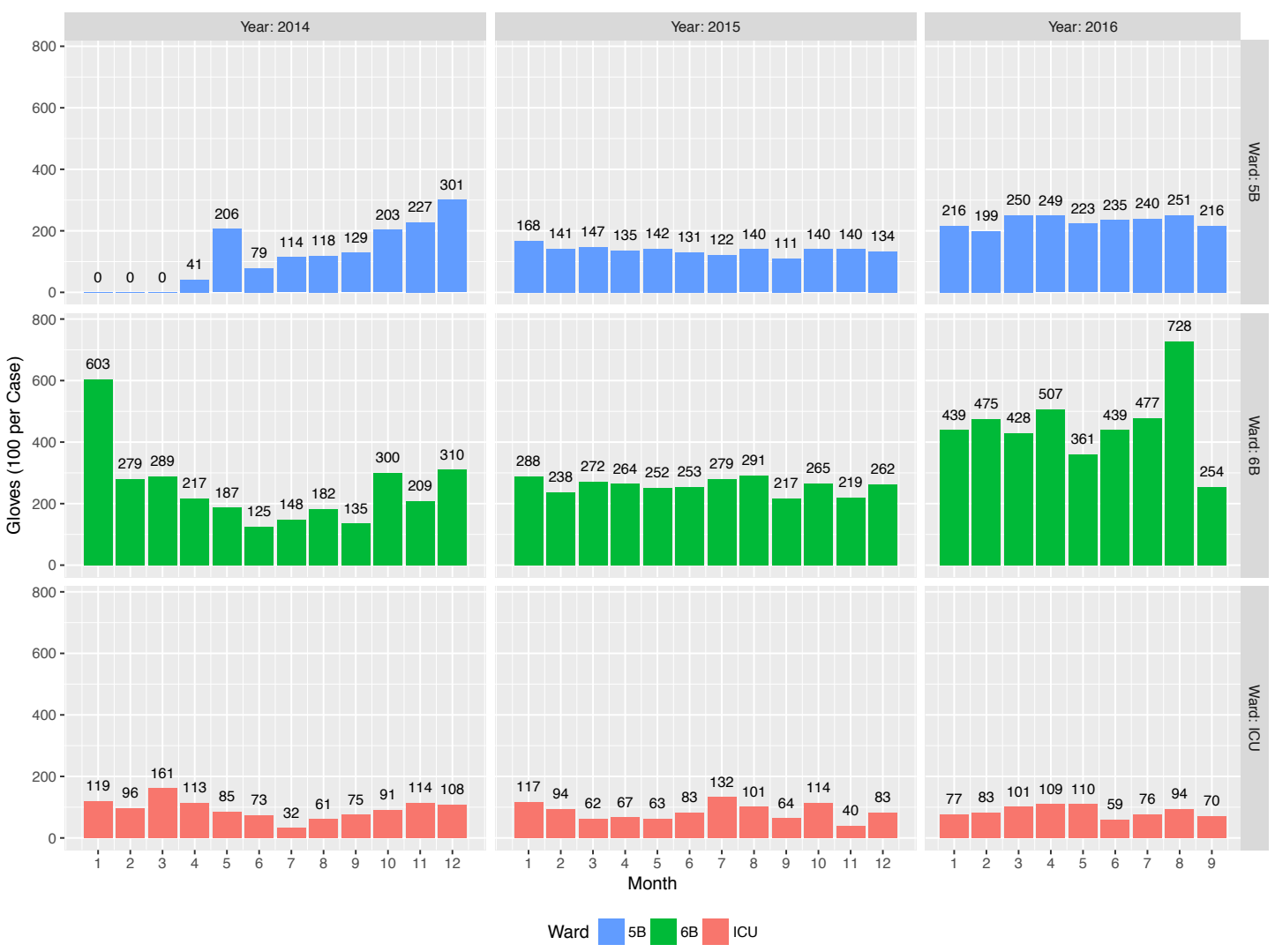

Figure 7.9 Quantity of Gloves Ordered per Ward per Month per Year 


\begin{tabular}{|c|c|c|c|c|c|c|}
\hline PPE & \multicolumn{2}{|c|}{ Gloves } & \multicolumn{2}{c|}{ Gowns } & \multicolumn{2}{c|}{ Masks } \\
\hline Ward & Mean & SD & Mean & SD & Mean & SD \\
\hline 5B & 156 & 76.31 & 118.67 & 105.1 & 7.18 & 4.67 \\
\hline 6B & 308.85 & 135.64 & 410.73 & 79.83 & 15.21 & 6.07 \\
\hline ICU & 88.7 & 26.94 & 176.67 & 98.66 & 5.85 & 5.53 \\
\hline Combined & 184.52 & 129.30 & 235.35 & 158.01 & 9.41 & 6.81 \\
\hline
\end{tabular}

Table 7.6 Mean, Variance and Standard Deviation of PPE per Ward and Wards Combined

\begin{tabular}{|c|c|c|c|c|c|}
\hline \multirow{2}{*}{ PPE } & \multirow{2}{*}{ Year } & \multirow{2}{*}{ Summary of Statistics } & \multicolumn{3}{|c|}{ Ward } \\
\hline & & & $5 B$ & $6 \mathrm{~B}$ & ICU \\
\hline \multirow{6}{*}{ Gloves } & \multirow{2}{*}{2014} & Mean & 118.17 & 248.67 & 94 \\
\hline & & SD & 99.86 & 129.22 & 32.89 \\
\hline & \multirow{2}{*}{2015} & Mean & 137.58 & 258.33 & 85 \\
\hline & & SD & 13.73 & 24.1 & 27.34 \\
\hline & \multirow{2}{*}{2016} & Mean & 231 & 456.44 & 86.56 \\
\hline & & SD & 18.44 & 126.78 & 17.91 \\
\hline \multirow{6}{*}{ Gowns } & \multirow{2}{*}{2014} & Mean & 61.92 & 396.33 & 218.42 \\
\hline & & SD & 80.3 & 108.25 & 133.41 \\
\hline & \multirow{2}{*}{2015} & Mean & 63.67 & 405.08 & 195.33 \\
\hline & & SD & 2.39 & 22.75 & 43.64 \\
\hline & \multirow{2}{*}{2016} & Mean & 267.67 & 437.44 & 96.11 \\
\hline & & SD & 31.08 & 86.65 & 37.56 \\
\hline \multirow{6}{*}{ Masks } & \multirow{2}{*}{2014} & Mean & 5.25 & 13.17 & 7.17 \\
\hline & & SD & 5.45 & 4.82 & 8.24 \\
\hline & \multirow{2}{*}{2015} & Mean & 6.58 & 14.08 & 6.25 \\
\hline & & SD & 0.79 & 1.31 & 2.63 \\
\hline & \multirow{2}{*}{2016} & Mean & 10.56 & 19.44 & 3.56 \\
\hline & & SD & 5.17 & 9.21 & 3.24 \\
\hline
\end{tabular}

Table 7.7 Mean and Standard Deviation of PPE per Ward per Year 
Figure 7.10 shows the gowns quantity ordered at each ward each month. Though the size of the ICU ward is significantly different from 5B and $6 \mathrm{~B}$, ICU's gowns quantity ordered are significantly higher than 5B for most of years 2014 and 2015 and significantly smaller than 5B for year 2016. Gowns mean for each ward per year, as can be seen in Table 7.6 and Table 7.7. It can be seen that mean gets larger from year to the next for $5 \mathrm{~B}$ and $6 \mathrm{~B}$; however, it gets smaller for ICU.

For each ward, $6 \mathrm{~B}$ and $5 \mathrm{~B}$, there are 7 wall-mounted slots each fits 3 gowns cases with 10 individual gowns per case. There are $210(7 * 3 * 10)$ cases of gowns that could be available at the floor. Similarly, at ICU, there are 5 wall-mounted slots with $150(5 * 3 * 10)$ cases of gowns that could be available at the floor. This number does not include gowns stored in the supply closet at the ward.

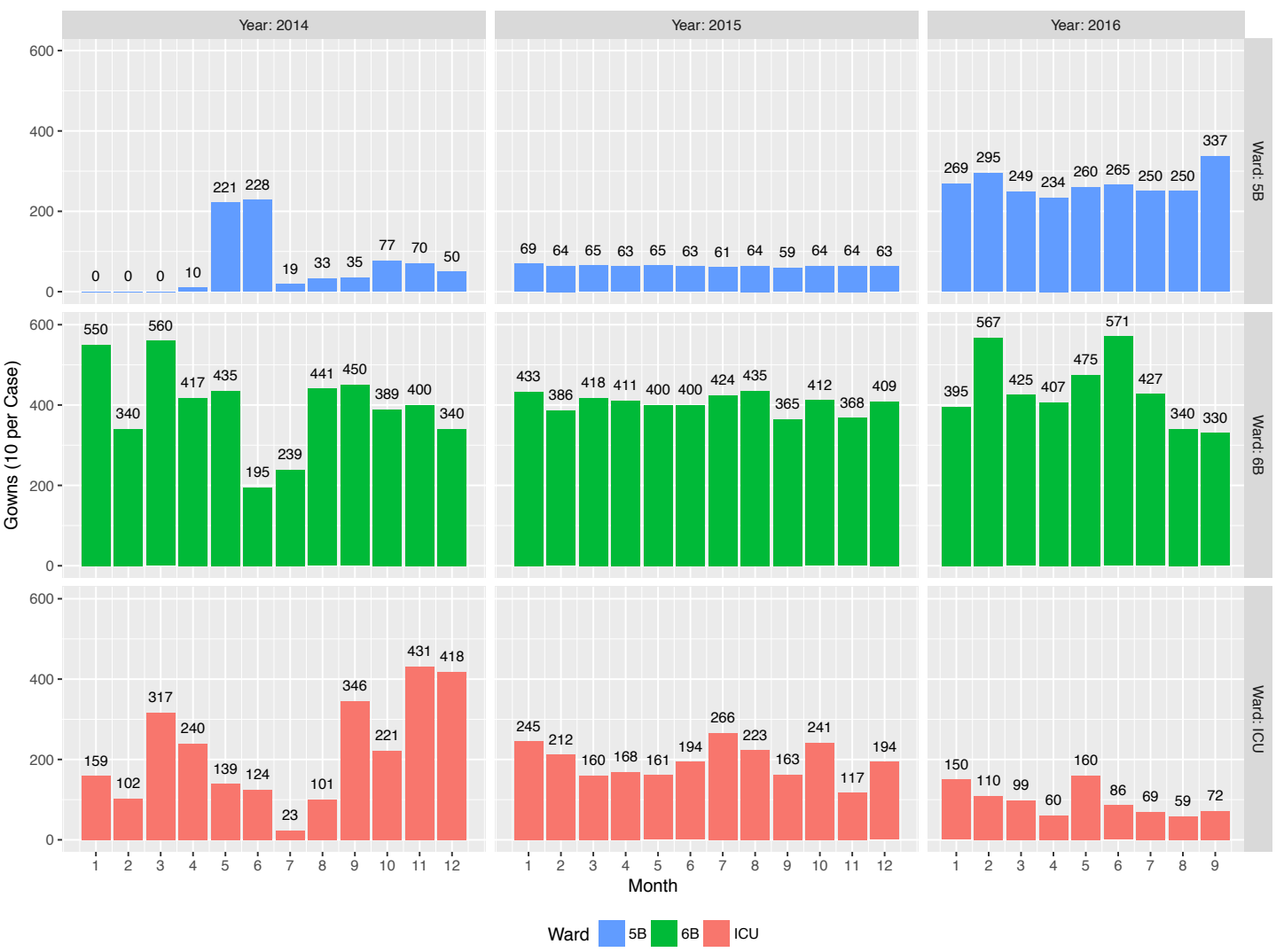

Figure 7.10 Quantity of Gowns Ordered per Ward per Month per Year 
Figure 7.11 shows quantity of masks ordered in each inpatient ward each month for 2014-2016. All wards have similar masks quantity ordered regardless of the physical size of each ward in terms of the bed capacity. Masks mean for each ward per year, can be seen in Table 7.6 and Table 7.7. It can be seen that mean gets larger from one year to the next for 5B and 6B; however, it gets smaller for ICU.

For $6 \mathrm{~B}$, there are 13 wall-mounted slots which each fit 1 masks case with 100 individual masks per case. For a total of $1300(13 * 100)$ cases of masks that could be available at the floor. For 5B, there are 10 wall-mounted slots with 1000 . For ICU, there are 6 wall-mounted slots with 600 . This number does not include masks stored in the supply closet at the ward.

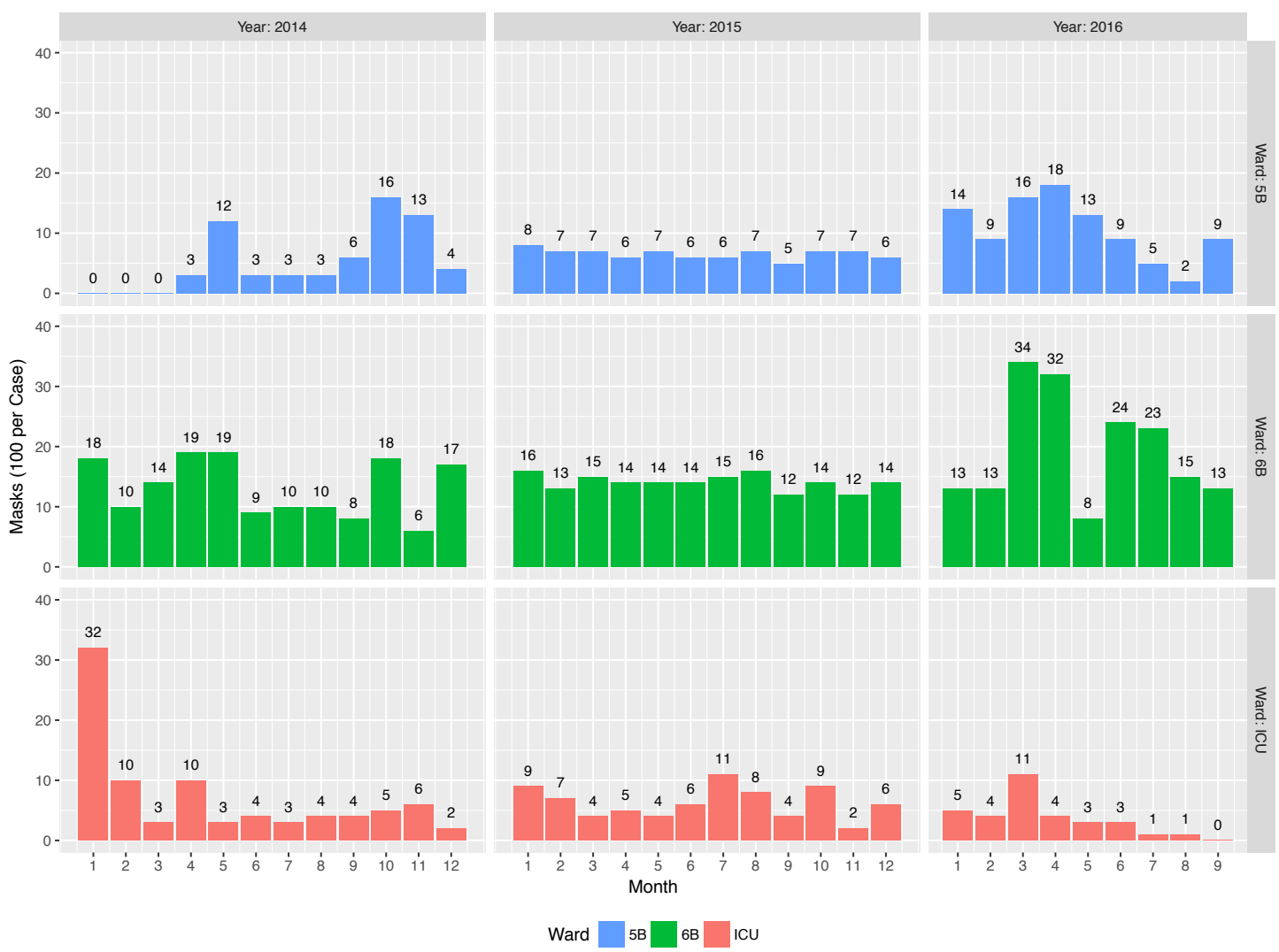

Figure 7.11 Quantity of Masks Ordered per Ward per Month per Year 
Gloves and gowns are ordered a lot during the last three years while masks are not. Reasons could be that the inpatient wards treat patients who are on contact precautions, which requires gloves and gowns, and have very few cases of patients who are on droplet precautions, which requires wearing masks and if necessary gloves and gowns, and only rare cases of patients who are on airborne precautions, which requires wearing special masks (N-95 or HEPA mask) accompanied with gloves and gowns. Unfortunately this result is inconclusive because the PVAMC does not report isolation data by type of isolation precautions in the IPEC system (admission and BDOC). In addition, PPE are used frequently not only by HCWs but also by housekeepers to clean patients' rooms and make beds on a daily basis, and by phlebotomists to draw blood and by other groups of healthcare professionals or even visitors.

In summary, Figure 7.12 shows that $6 \mathrm{~B}$ has the largest share of gloves, gowns and masks ordered for all years. 5B outweighs ICU for 2014 and 2015 in terms of gloves. But, interestingly, ICU outweighs 5B during 2014 for gowns and masks products. 


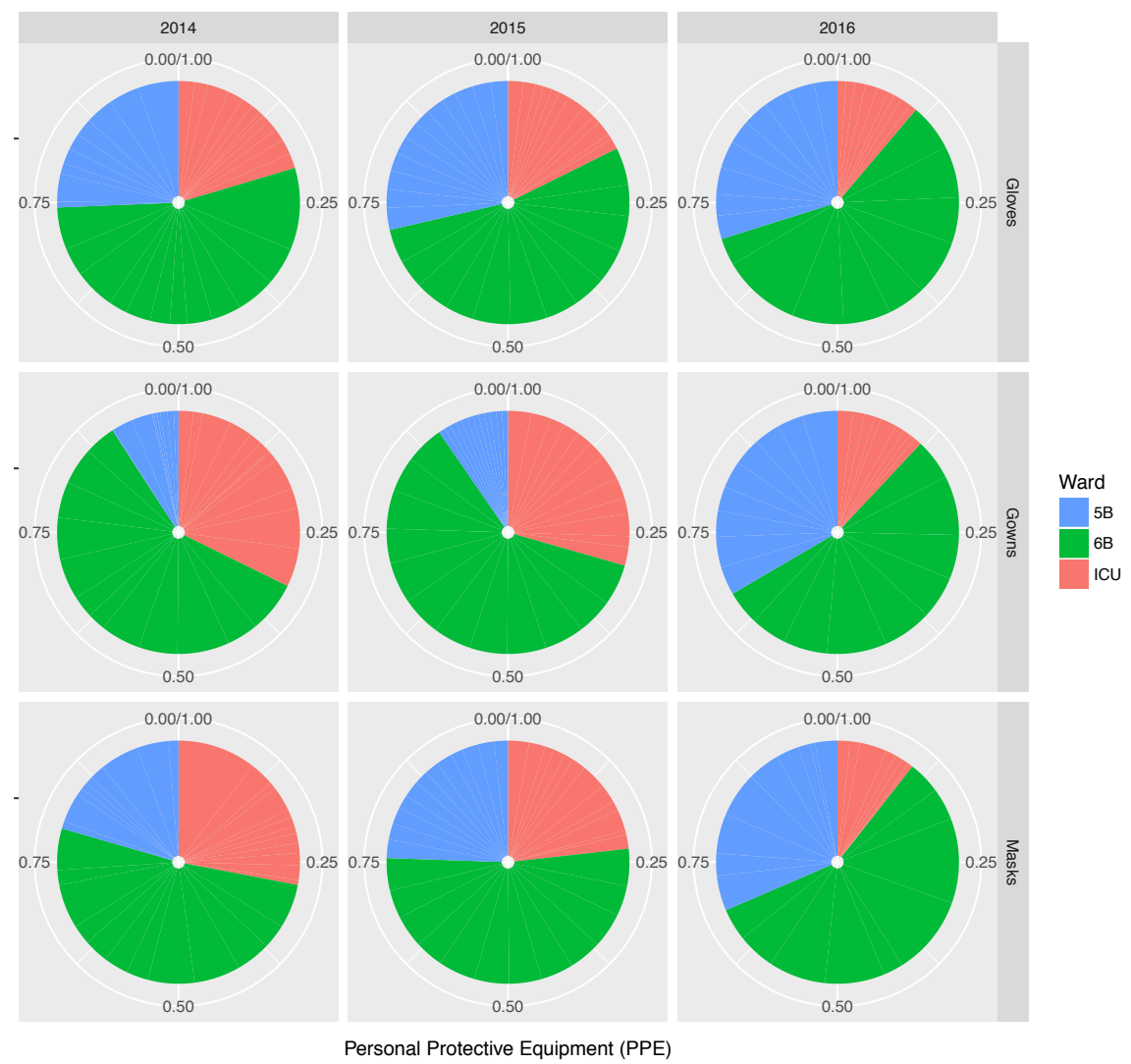

Figure 7.12 PPE Ratios per Ward per Year

Now, looking at BDOC per ward per year in Figure 7.13 and the Table 7.8, BDOC are almost similar for 5B during all years 2014, 2015 and 2016 with year 2014 being the smallest with mean of $474.75,548.25$ and 542.89 for each year respectively. BDOC are almost similar for 6B during all years 2014, 2015 and 2016 with year 2014 with mean of 566.83, 594.42 and 570.89 for each year respectively. BDOC are similar for ICU for some parts of the three years 2014, 2015 and 2016 with a decline in the second half of year 2016 with a mean of $127.67,117.25$ and 81.44 for each year respectively. 


\begin{tabular}{|c|c|c|c|c|c|}
\hline \multirow{10}{*}{$\mathrm{BDOC}$} & \multirow{2}{*}{ Year } & \multirow{2}{*}{ Summary of Statistics } & \multicolumn{3}{|c|}{ Ward } \\
\hline & & & $5 \mathrm{~B}$ & $6 \mathrm{~B}$ & ICU \\
\hline & \multirow{2}{*}{2014} & Mean & 474.75 & 566.83 & 127.67 \\
\hline & & $\mathrm{SD}$ & 35.95 & 47.22 & 28.79 \\
\hline & \multirow{2}{*}{2015} & Mean & 548.25 & 594.42 & 117.25 \\
\hline & & SD & 41.39 & 41.85 & 28.48 \\
\hline & \multirow{2}{*}{2016} & Mean & 542.89 & 570.89 & 81.44 \\
\hline & & SD & 46.04 & 41.11 & 32.6 \\
\hline & \multirow{2}{*}{ Years Combined } & Mean & 520.06 & 577.97 & 111.27 \\
\hline & & SD & 52.7 & 44.19 & 34.55 \\
\hline
\end{tabular}

Table 7.8 Descriptive Statistics of BDOC per Ward and Wards Combined per Year
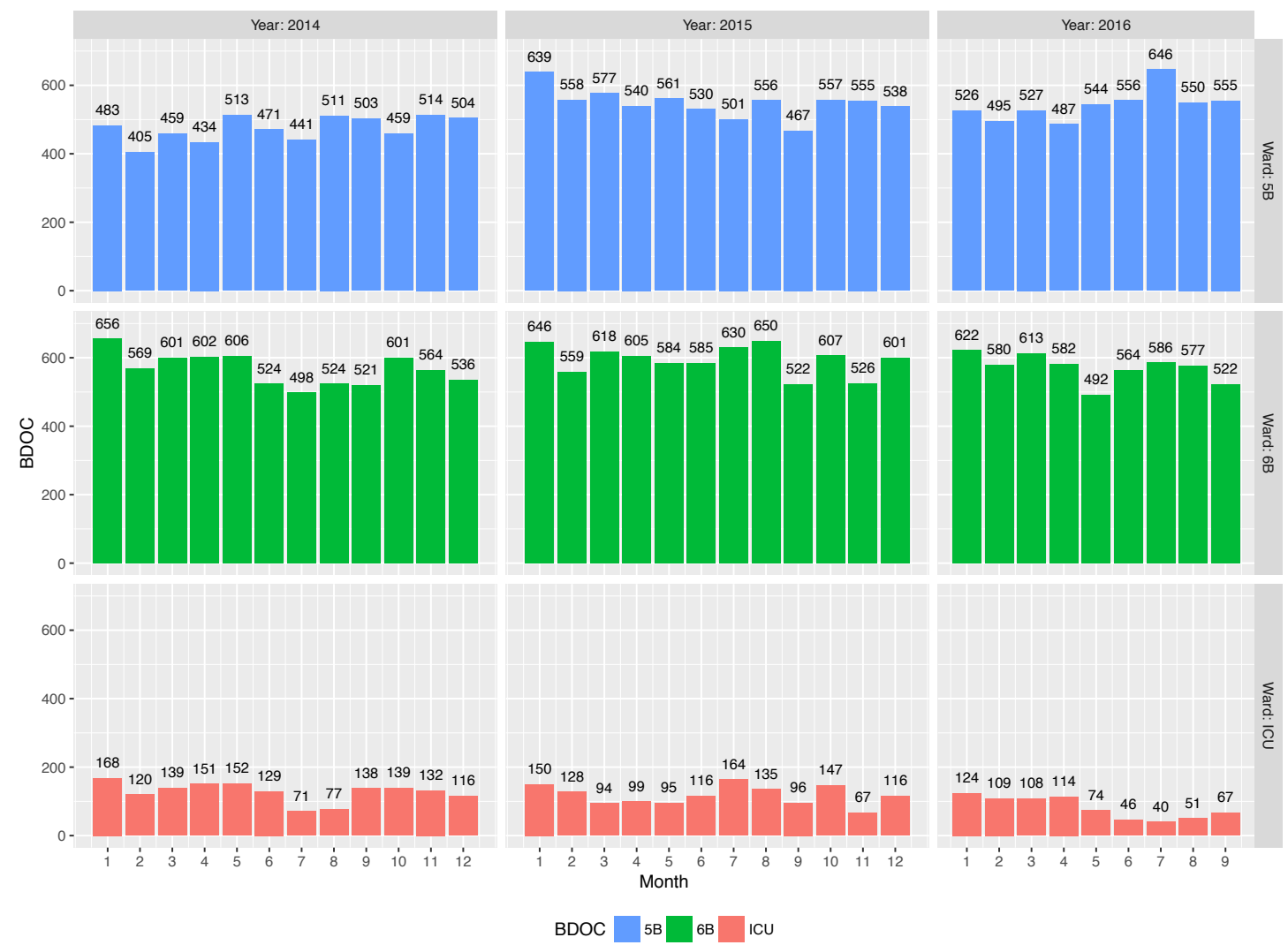

Figure 7.13 BDOC per Ward per Month per Year

Combining the analysis of PPE quantities ordered and BDOC, it can be concluded that though BDOC are almost similar during all years for each ward, PPE quantities ordered are not. An investigation can be made by statistically modeling the 
dependence of PPE based on BDOC before modeling the dependence of PPE at the inpatient wards at the PVAMC based on the true moments of PPE use.

The hypothesis testing for modeling the dependence of PPE based on BDOC for all years combined $(2014,2015$ and 2016) is as follows:

$\mathrm{H}_{0}$ : BDOC Does Not Have an Impact on the PPE used

$\mathrm{H}_{1}$ : BDOC Does Have an Impact on the PPE used

By nature, HCWs are expected to use more PPE as BDOC increases especially if patients are on precautions. The more precautions signs on the floor, the more PPE used. Looking at the scatter plot of BDOC versus each PPE (gloves, gowns and masks) in Figure 7.14, it can be seen that there is evidence of a proportional relationship between $\mathrm{BDOC}$ and each PPE; one increases the other increases too. Correlation coefficients can be seen in Table 7.9 per ward and wards combined. 

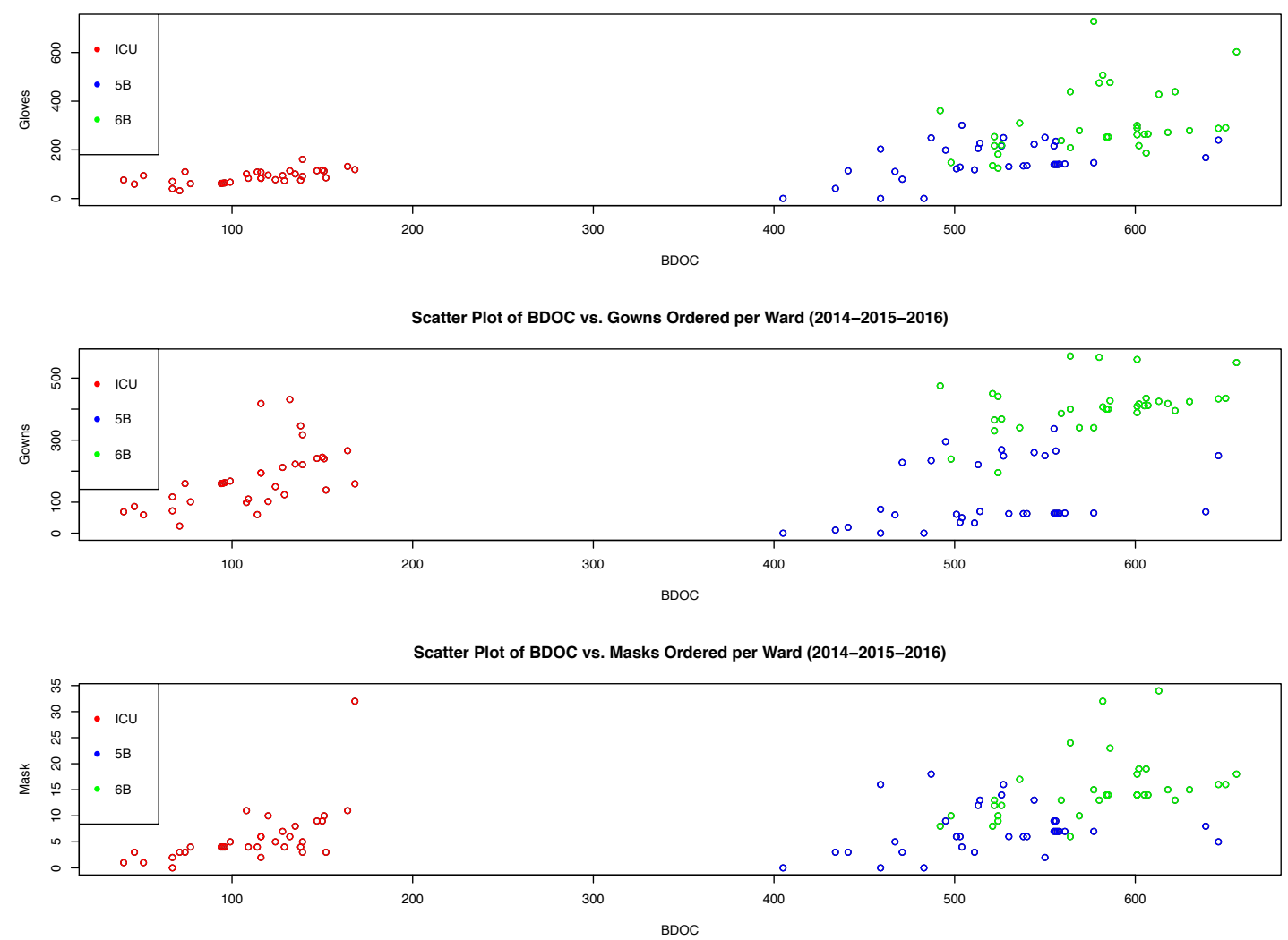

Figure 7.14 BDOC versus PPE per Ward for Year 2014-2016

\begin{tabular}{|c|c|c|c|}
\hline \multirow{2}{*}{ Ward } & \multicolumn{3}{|c|}{ Correlation Coefficients } \\
\cline { 2 - 4 } & Gloves & Gowns & Masks \\
\hline 5B & 0.48 & 0.31 & 0.20 \\
\hline 6B & 0.35 & 0.41 & 0.43 \\
\hline ICU & 0.62 & 0.59 & 0.58 \\
\hline Wards Combined & 0.62 & 0.39 & 0.48 \\
\hline
\end{tabular}

Table 7.9 Correlation Coefficients of BDOC versus PPE per Ward and Wards Combined

For gloves, the Negative Binomial distribution with a Linear deterministic function is used to fit a model with constant coefficients across wards and a model with parameters varying across wards since ward factor by itself is significant, and thus each ward is different from the other in terms of gloves quantity ordered. Two of the three parameters of the Negative Binomial distribution are different for each ward the slope (rate) and the dispersion with constant intercept across wards. Using AIC 
and BIC criteria for model selection, the model with parameters varying across wards is the best-fit model for gloves counts based on BDOC, can be seen in Figure 7.15.

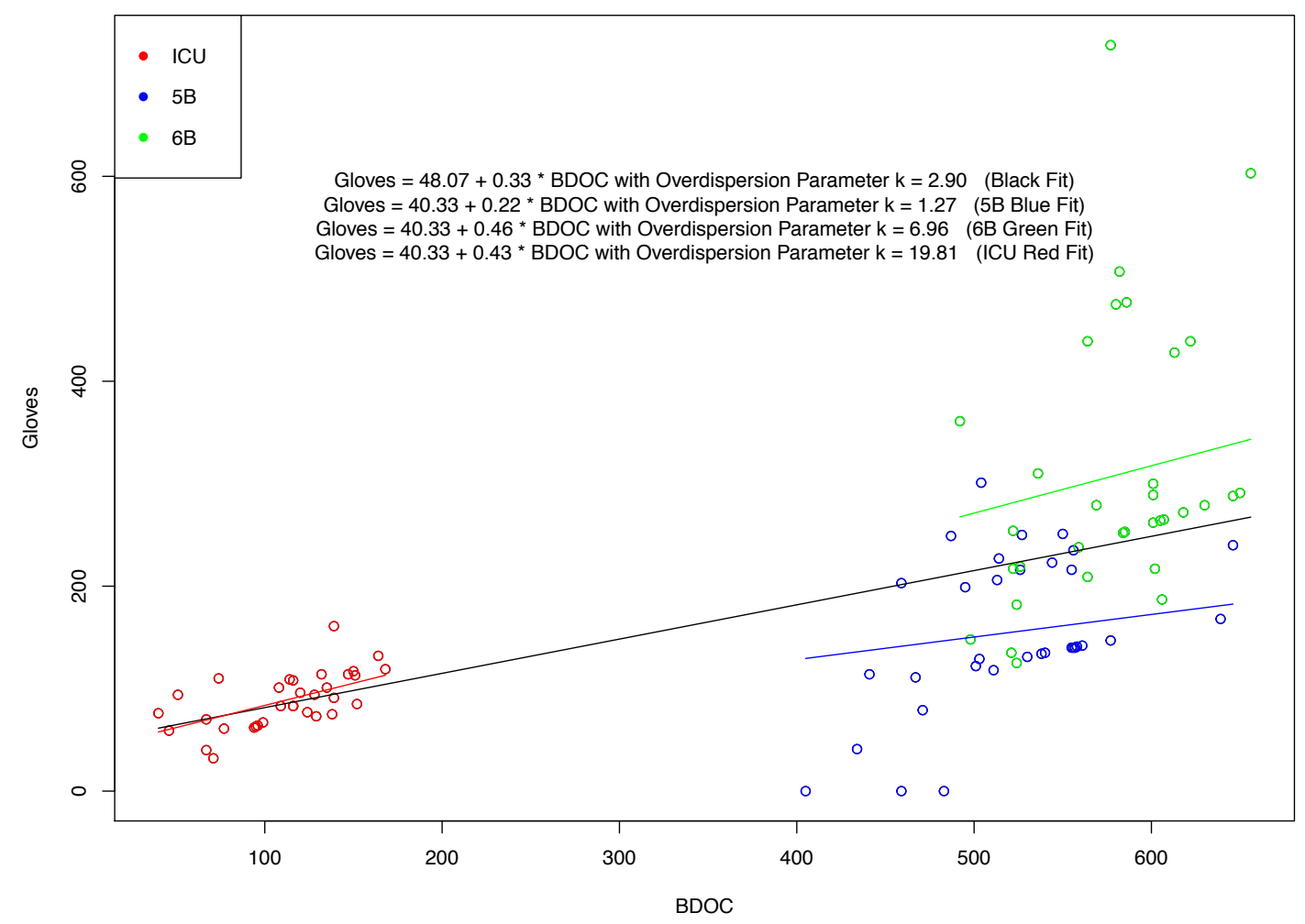

Figure 7.15 Negative Binomial Distribution with a Linear Deterministic Function Fitted Model for BDOC versus Gloves per Ward for Year 2014-2016

Similarly, the best-fit model for gowns counts based on BDOC can be seen in Figure 7.16 and the best-fit model for masks counts based on BDOC can be seen in Figure 7.17. 


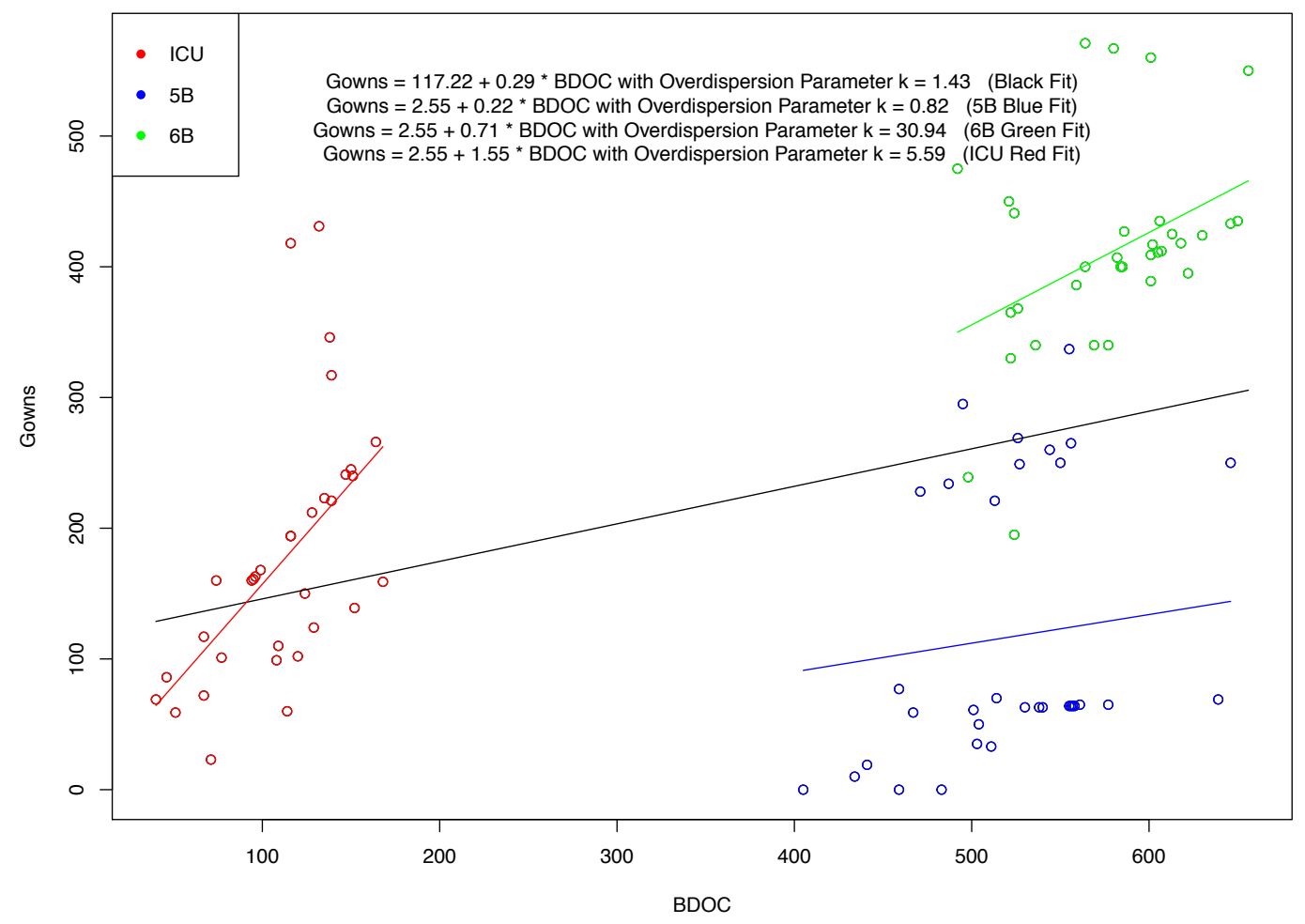

Figure 7.16 Negative Binomial Distribution with a Linear Deterministic Function Fitted Model for BDOC versus Gowns per Ward for Year 2014-2016

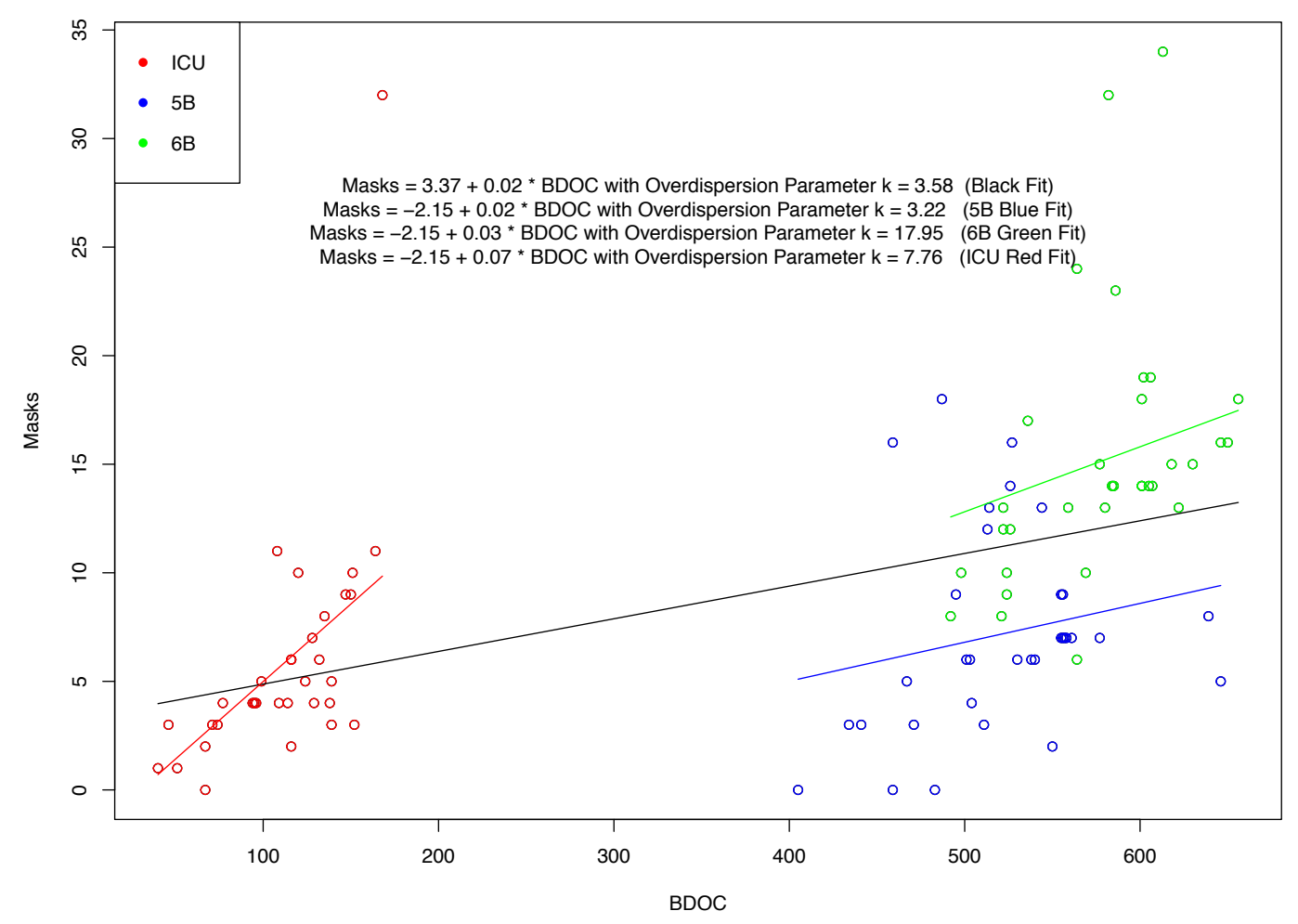

Figure 7.17 Negative Binomial Distribution with a Linear Deterministic Function Fitted Model for BDOC versus Masks per Ward for Year 2014-2016 
In conclusion, based on the models outputs, the null hypothesis $H_{0}$ that states BDOC does not have an impact on PPE quantity ordered is rejected. Consequently, as BDOC increases, indeed PPE quantity ordered increases too.

In summary, the objective of statistically investigating the relationship between BDOC versus PPE quantity ordered is found.

\subsubsection{PPE Ordering and PPE True Moments}

Now, after determining the relationship between BDOC and PPE, the hypothesis testing for modeling the dependence of PPE quantity ordered based on PPE true moments of use is as follows:

$\mathrm{H}_{0}$ : PPE Quantity Ordered Does Not Depend on PPE True Moments of Use on the Floor. $\mathrm{H}_{1}$ : PPE Quantity Ordered Does Depend on PPE True Moments of Use on the Floor.

The assumption states that PPE quantities ordered are assumed to be equal to the amount consumed on the floor during required moments such as wearing gloves before providing care to patients who are on precautions, although defects such as damaged gloves could occur. The objective of this model is to indirectly estimate the PPE compliance based on PPE inventory data for PPE moments in which HCWs should use gloves, gowns and masks during caregiving to patients who are on precautions. Basically, a patient could be on precautions, which requires wearing gloves, a gown for contact precautions plus surgical mask for droplet precaution or N95 mask for airborne precautions. In addition, all other patients are considered to be on standard precautions, where there is no isolation sign hung up on the room door, but a HCW may need to wear gloves for certain kinds of medical or non-medical treatment. Now, instead of using BDOC as a predictor, an attempt is made to estimate how many 
times PPE are required during caregiving, which is called PPE Moments, named after hand hygiene five moments. PPE quantities ordered are used as the numerator. However, in an indirect method of measuring PPE compliance, attempts are made to create a denominator from an estimate that leads to a compliance percentage. Essentially, the denominator represents how many PPE are supposed to be used versus the numerator which represents how many PPE are actually used during key moments of PPE use. The following steps summarize the estimate of the denominator calculations for both gloves and gowns:

$$
\text { PPE (Gloves, Gowns) Compliance } \%=\frac{\text { How Many You Have Used }}{\text { How Many You Are Suppose to Use }}
$$

1) Find HAIm: which is the sum of all HAIs (MRSA, MRSA Colonization, CAUTI, CLABSI and VAP) per month per year (2014-2015-2016) per inpatient wards.

2) Find $H A I \% m$ by dividing $H A I m$ by monthly admission and multiplying by $100 \%$.

3) Find $T_{i}$ : Multiply BDOC by HAIm to get an estimate of Transmission Isolation BDOC. $T$ represents the transmission BDOC per inpatient ward and $i$ represents each inpatient ward, 5B, 6B and ICU.

4) Find $S_{i}$ : Subtract $T_{i}$ from BDOC to get an estimate of Standard Isolation BDOC. $S$ represents the standard BDOC per inpatient ward and $i$ represents each inpatient ward, 5B, 6B and ICU.

5) Find $Z_{i}$ : which is the sum of all device-associated care days in a particular month. This value was found for each month during the time of the study. The Summation $Z_{i}$ is then multiplied by 2 for the number of gloves used. This 
gives the first part of the gloves compliance denominator. However, gowns and/or masks could be required during any of these device-associated days. Unfortunately, IPEC does not distinguish which days belong isolation and which do not. Thus, these data are used toward gloves compliance only. $Z$ represenst the aggregation of central line days, catheter days and ventilator days, and $i$ represents the inpatient ward, 5B, 6B and ICU.

6) Find $Y_{i}$ : which is the average number of times a patient (Isolation versus nonIsolation) is seen by a nurse or doctor per day per ward during all shifts combined. Based on patient contact data in Chapter 4, for this study $Y$ is the average patient contacts per day of 50,58 and 111 for $5 \mathrm{~B}, 6 \mathrm{~B}$ and $\mathrm{ICu}$ respectivelym and $i$ represents the inpatient ward, 5B, 6B and ICU.

7) Based on contact, droplet and airborne precautions (Appendices DD, EE, FF) gloves are required during care for patients who are on precautions. Thus, gloves moments of Transmission Isolation BDOC are equal to the average daily patient contact $\left(Y_{i}\right)$ multiplied by 2 gloves multiplied by $\left(T_{i}\right)$. This gives the second part of the gloves compliance denominator. In addition, gowns moments of Transmission Isolation BDOC are equal to the average daily patient contacts $\left(Y_{i}\right)$ multiplied by 1 gowns multiplied by $\left(T_{i}\right)$. This gives the first and only part of the gowns compliance denominator.

8) For patients who are on non-precautions, an assumption is made that at least one out of the daily patient contact visits $\left(Y_{i}\right)$ require gloves during care. Thus, gloves moments of Standard Isolation BDOC are equal to 1 out of the DPC 
$\left(Y_{i}\right)$ multiplied by 2 gloves multiplied by $\left(Z_{i}\right)$. This gives the third and last part of the gloves compliance denominator.

9) The Gloves Quantity Ordered, which is the gloves compliance numerator, and is found by taking the number of cases of gloves ordered in the month $\left(C_{g l}\right)$, multiplied by 100 gloves in the box, and divided by 2 because each healthcare worker must wear a pair of gloves. The Gowns Quantity Ordered, which is the gowns compliance numerator, is found by taking the number of cases of gowns ordered in the month, multiplied by 10 gowns per case.

The denominator is estimated to find the compliance of only those employee groups who are of interest to this study (medical groups such as attending, residents, interns and medical students and nursing group such as RN, LPN and CNA). Thus, one source of error in the numerator can be addressed by removing the known portions of PPE used by other groups on the floor such as housekeepers from EMS and nutrition and food services. The following steps are made to reduce errors imposed by the earlier assumption stating that PPE quantities ordered are assumed to be equal to the amount consumed on the floor:

1) Housekeepers are required to clean patients' rooms and make beds at least twice a day. One type of cleaning is called terminal clean, which is upon patient discharge and labeled on Environmental Checklist as Step 1 as seen in Appendix CC, and another clean is called regular clean, which does not appear in Appendix CC. Thus, the housekeepers' gloves consumption is calculated by multiplying 2 gloves times 2 cleans times total BDOC. There is no need to distinguish between transmission precautions BDOC and standard precautions 
$\mathrm{BDOC}$ since housekeepers are required to wear gloves for every room clean. However, an extra clean is required for rooms with C.Diff based on the environmental checklist on Appendix CC. Then, this number is subtracted from the gloves quantities ordered in the numerator. For gowns, it is necessary to distinguish between isolation versus non-isolation $\mathrm{BDOC}$ since gowns are required during cleaning patients' rooms and making beds in rooms with precautions signs. Thus, the housekeepers' gowns consumption is calculated by multiplying 1 gown times 2 cleans times transmission precautions BDOC, which is $T_{i}$. Then, this number is subtracted from the gowns quantities ordered in the numerator.

2) Nutrition and food services deliver three meals to patients in inpatient wards on a daily basis. Thus, they are required to wear gloves at least once per meal delivery per day. They are not allowed to enter isolation rooms. Thus, there is no need to distinguish between transmission precautions BDOC and standard precautions BDOC. The nutrition and food services' gloves consumption is calculated by multiplying 2 gloves times 3 meals times days per month, which varies. Then, this number is subtracted from the quantity of gloves ordered in the numerator.

Shifts are important; however, PPE quantities ordered are not broken down by shift. In addition, HCWs have to comply with PPE use when providing care for patients who are on precautions; however, HCWs are not required to use PPE when providing care for patients who are not on precautions except for certain procedure such as drawing blood or inserting intravenous lines (IV). CLABSI Line Days, 
CAUTI Catheter Days and Ventilator Days are not reported on an isolations and nonisolations basis. Thus, an assumption is made that these practices are made on nonisolation patients that do not require gowns. Masks are not used in such a calculation because it is hard to determine or estimate a true moment of using masks.

The derived PPE compliance equation is as follows:

$$
\begin{gathered}
\text { PPE (Gloves) Compliance } \%=\frac{\text { Gloves Quantity Ordered }}{\text { Estimated Gloves Moments }} \\
\text { Gloves Quantity Ordered (Numerator) }=\frac{C_{g l} * \mathbf{1 0 0}_{(\text {Gloves per Case })}}{2_{(\text {Gloves per Case })}}
\end{gathered}
$$

\section{Estimated Gloves Moments (Denominator) $=$}

$$
\begin{aligned}
& Z_{i} * 2_{(\text {Gloves })}+\mathrm{S}_{i} * 1_{(\text {Gloves Required once a Day })} * 2_{(\text {Gloves })}+\mathrm{T}_{i} * \mathrm{Y}_{i} * 2_{(\text {Gloves })} \\
& =\left[\begin{array}{l}
\text { Device_Invasive }_{5 B} \\
\text { Device_Invasive }_{6 B} \\
\text { Device_Invasive }_{I C U}
\end{array}\right] \cdot\left[\begin{array}{l}
\left.2_{(G l o v e s}\right) \\
2_{(\text {Gloves })} \\
2_{(\text {Gloves })}
\end{array}\right] \\
& + \\
& {\left[\begin{array}{l}
\text { Standard_BDOC } \\
\text { Standard_BDOC } \\
\text { Standard_BDOC } \\
\text { StCU }
\end{array}\right] \cdot\left[\begin{array}{l}
1_{(\text {Gloves Required Once a Day })} \\
1_{(\text {Gloves Required Once a Day })} \\
1_{(\text {Gloves Required Once a Day })}
\end{array}\right] \cdot\left[\begin{array}{l}
2_{(\text {Gloves })} \\
2_{(\text {Gloves })} \\
2_{(\text {Gloves })}
\end{array}\right]} \\
& + \\
& {\left[\begin{array}{l}
\text { Transmission_BDOC } \\
\text { Transmission_BDOC } \\
\text { TraB } \\
\text { Transmission_BDOC } \\
I C U
\end{array}\right] \cdot\left[\begin{array}{l}
\text { Average Patient Contact } \\
\text { Average Patient Contact } \\
6 B \\
\text { Average Patient Contact } \\
\text { ACU }
\end{array}\right] \cdot\left[\begin{array}{l}
2_{(\text {Gloves })} \\
2_{(\text {Gloves })} \\
2_{(\text {Gloves })}
\end{array}\right]}
\end{aligned}
$$




$$
\text { PPE (Gowns) Compliance } \%=\frac{\text { Gowns Quantity Ordered }}{\text { Estimated Gowns Moments }}
$$

\section{Gowns Quantity Ordered (Numerator) $=C_{g} * 10_{(\text {Gowns per Case })}$}

$$
\text { Gowns Quantity Ordered (Numerator) }=C_{g} * 10_{(\text {Gowns per Case })}
$$$$
\text { Estimated Gowns Moments (Denominator) }=\mathrm{T}_{i} * \mathrm{Y}_{i} * 1_{(\text {Gown })}
$$$$
=\left[\begin{array}{l}
\text { Transmission_BDOC } \\
\text { Transmission_BDOC }_{6 B} \\
\text { Transmission_BDOC }_{I C U}
\end{array}\right] \cdot\left[\begin{array}{l}
\text { Average Patient Contact } \\
\text { Average Patient Contact } \\
\text { AB } \\
\text { Average Patient Contact } \\
\text { ACU }
\end{array}\right] \cdot\left[\begin{array}{l}
1_{(\text {Gown })} \\
1_{(\text {Gown })} \\
1_{(\text {Gown })}
\end{array}\right]
$$

Sources of error could increase or decrease the numerator and/or the denominator of PPE Compliance. Subsequently, the percentage could go beyond $100 \%$.

\section{Sources of Error in the Numerator could originate from:}

- The uncertainty that comes from the assumption that PPE data are inventory ordered data.

- The fact that some PPE are discarded, misused and damaged (defect).

- A hypothesis test could be tested later for the percentage of patient contacts relative to isolation patients vs. non-isolation patients per inpatient wards to reduce the error imposed by such average visits in which this average visits could be different per inpatients ward per patients isolation classification.

- Not distinguishing between using PPE with Hand Hygiene or not: Standard Precautions or Transmission Precautions.

- The fact that it is hard to quantify how many are used in the floor or how many are left on the floor per month because they are unknown for sure. 


\section{Sources of Error in the Denominator could originate from:}

- The fact that the average visits of isolation patients versus non-isolation patients vary per HCWs groups on a daily basis per ward.

- The missing isolations information in IPEC system for other HAIs such as C.Diff, which is not reported by ward and other isolations precautions for other diseases such as droplet (Flu) or airborne (TB) or Neutropenic Precautions (Protective Environment) (Appendix GG).

- The missing isolations information from device-associated, which is not reported by isolation versus non-isolation. That could affect gowns compliance since all device-associated events are assumed to occur for non-isolation patients and thus no gowns are required.

- The assumption that states HCWs wear gloves once per day for non-isolations patients for all visits. It could be more than that or zero for some cases.

- The way transmission precautions BDOC are calculated. Transmission precautions BDOC could be less, equal or more than standard precautions BDOC. This calculation affects the denominator for both gloves and gowns compliance and the numerator for gowns compliance.

All PPE moments calculations are carried out in Microsoft Excel. Figure 7.18 and Figure 7.19 show that for all wards during all years (2014, 2015 and 2016), numbers of patients who are in non-isolation (Standard Precautions "Blue") are more than numbers of patients who are in isolation (Transmission Precautions "Green"). 


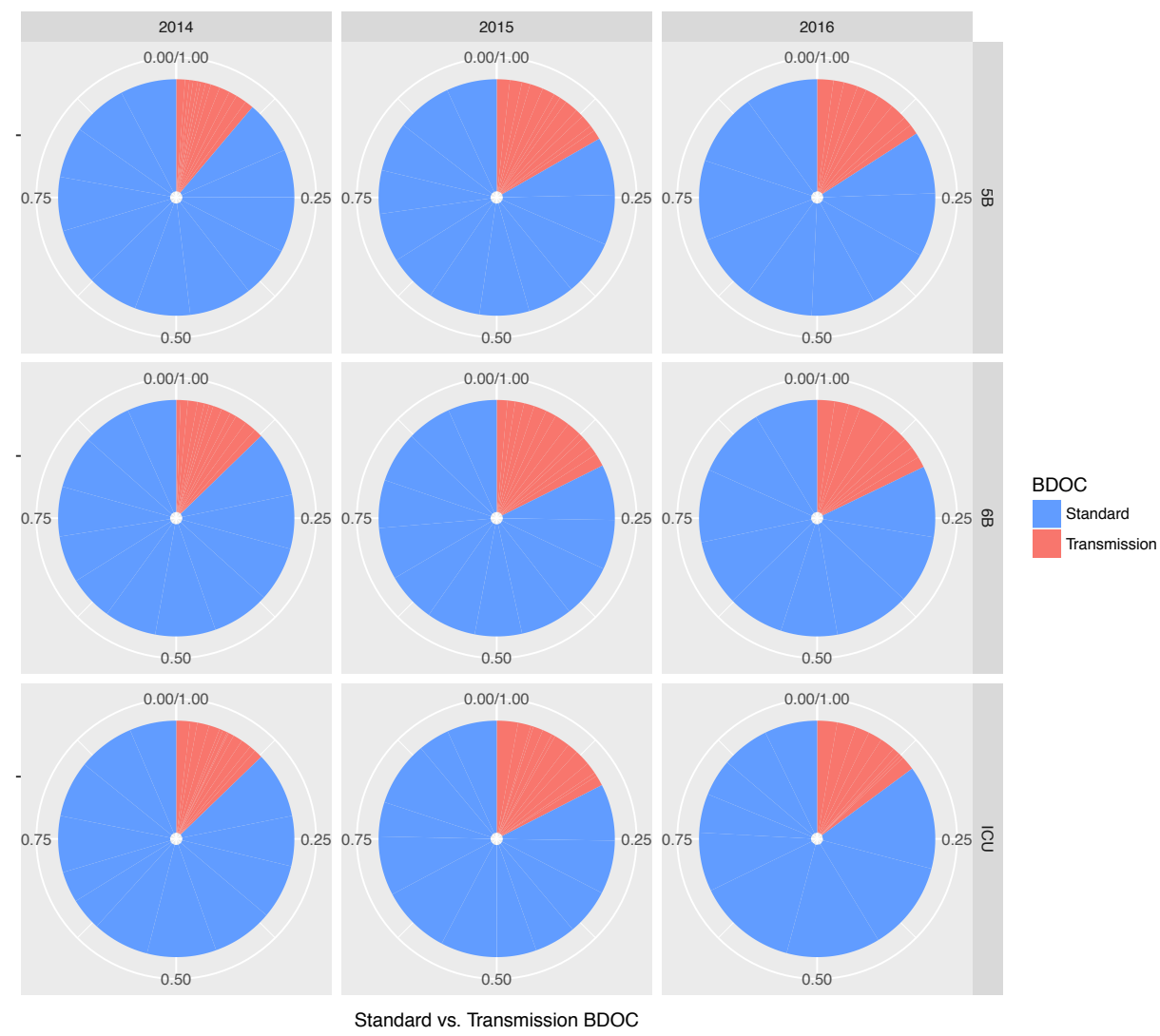

Figure 7.18 Standard (Purple) BDOC versus Transmission (Red) BDOC Ratio per Ward per Year 

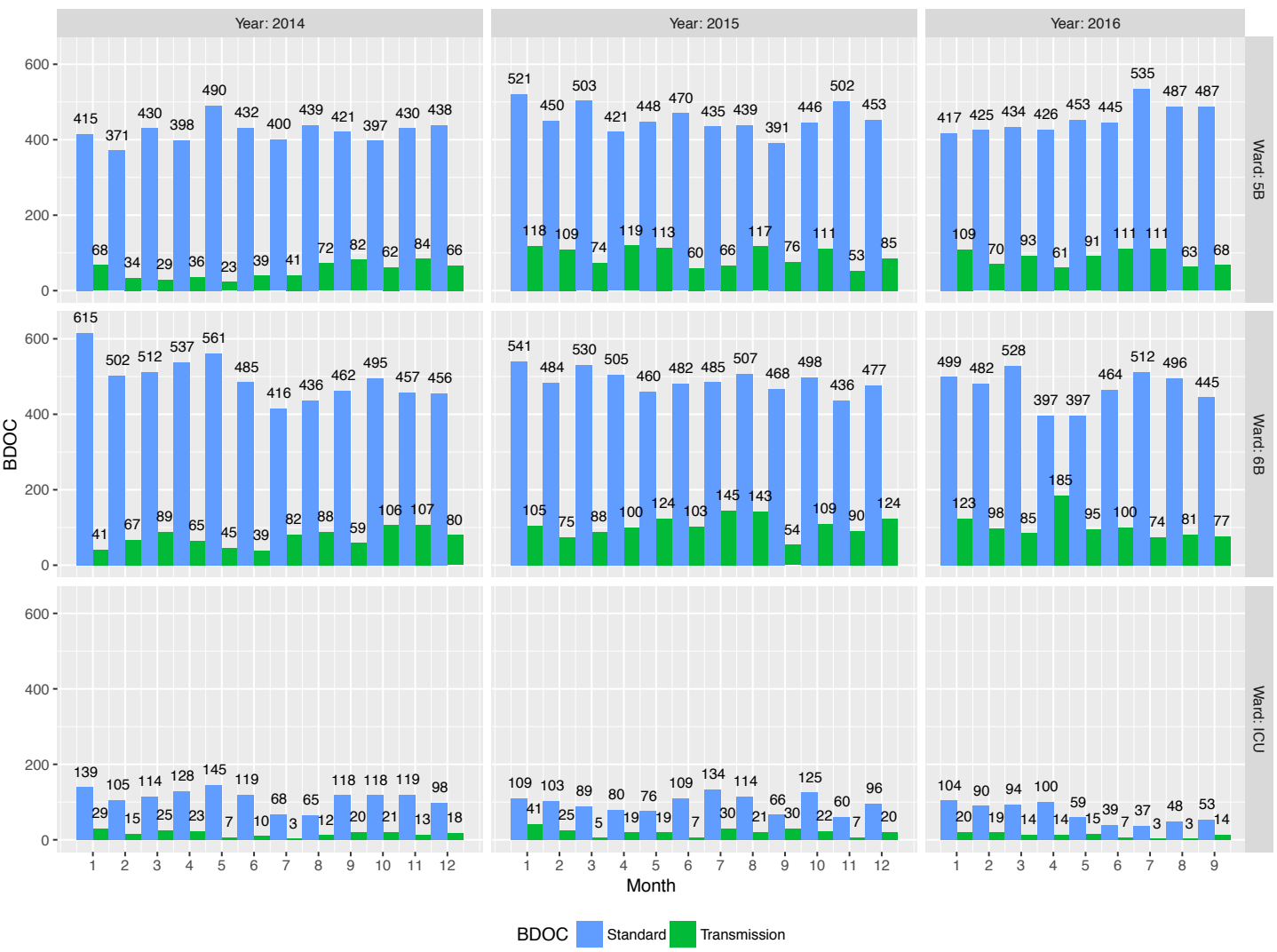

Figure 7.19 Standard (Purple) BDOC versus Transmission (Green) BDOC per Ward per Month per Year

A scatter plot, Figure 7.20 for gloves and Figure 7.21 for gowns examines the relationships between PPE moments (cases) for gloves and gowns separately versus PPE quantities ordered (cases), and a positive linear relationship is revealed, but with more scatter in the gowns plot. 


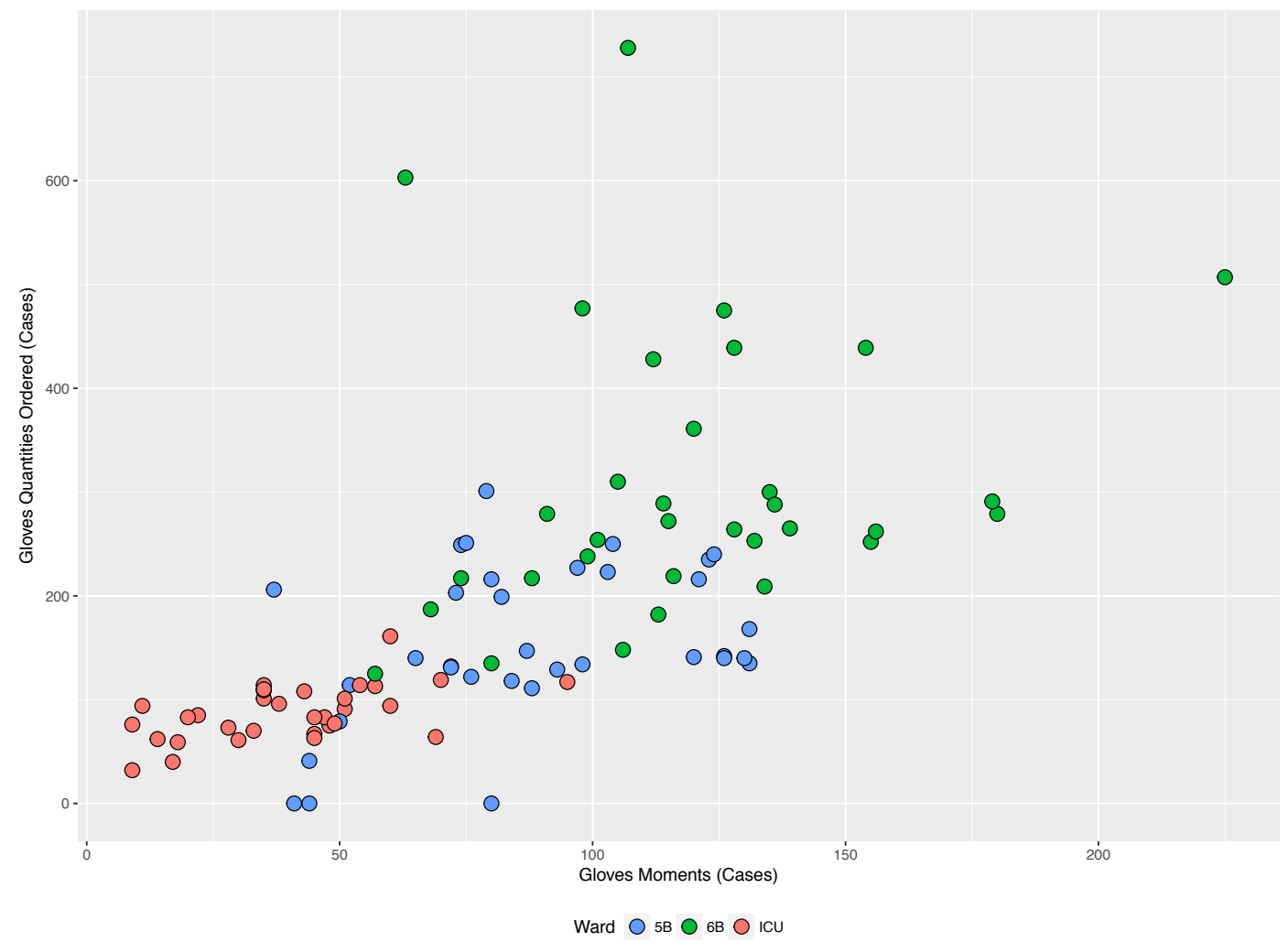

Figure 7.20 Gloves Moments versus Gloves Quantity Ordered per Ward (2014-2016)

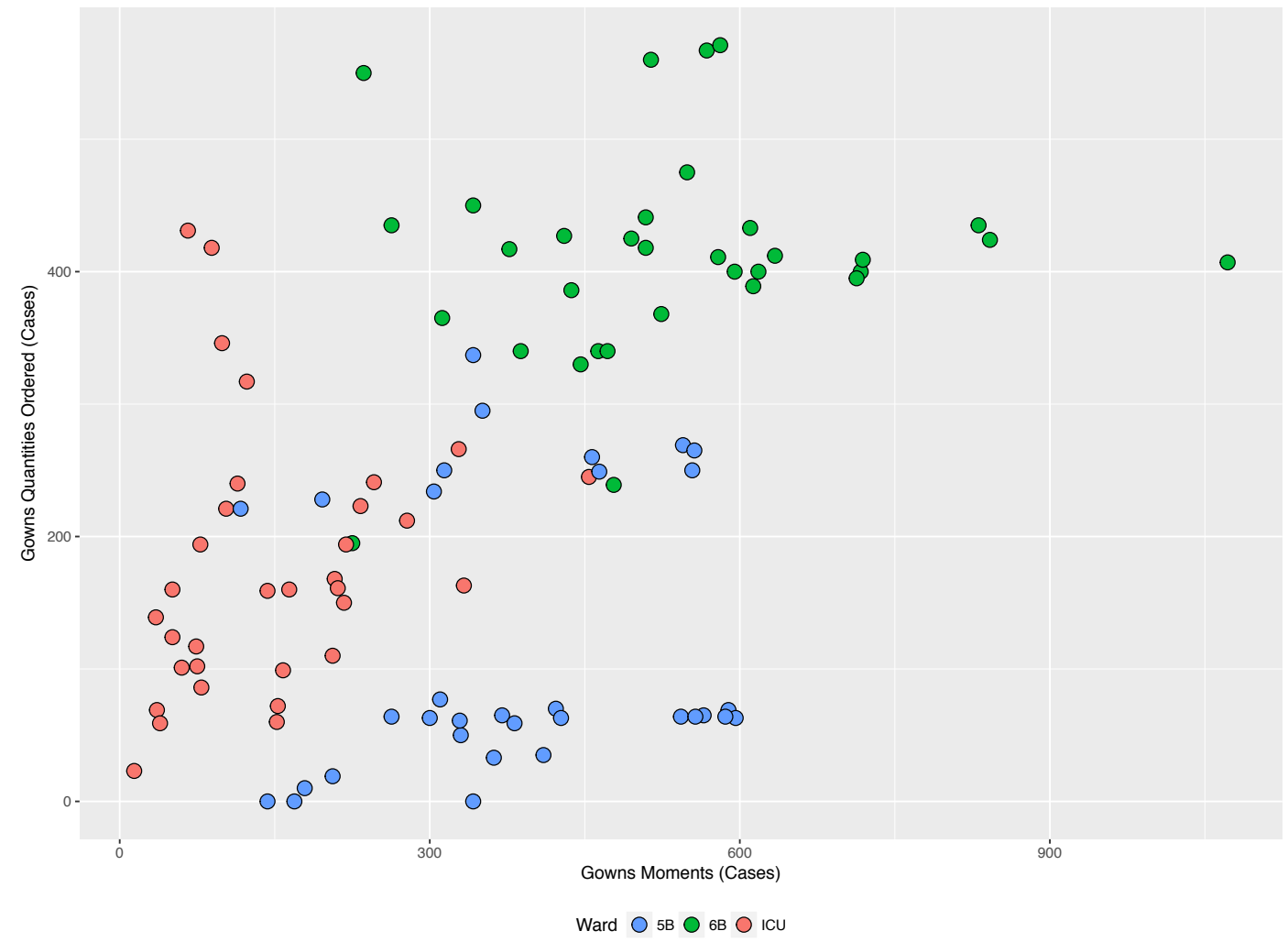

Figure 7.21 Gowns Moments versus Gowns Quantity Ordered per Ward (2014-2016) 
The same procedure that was used for gloves and gowns vs. BDOC is now used to find the best fit model for gloves moments vs. gloves ordered, as seen in Figure 7.22. The procedure is repeated to find the best fit model for gowns moments vs. gowns ordered, as seen in Figure 7.23.

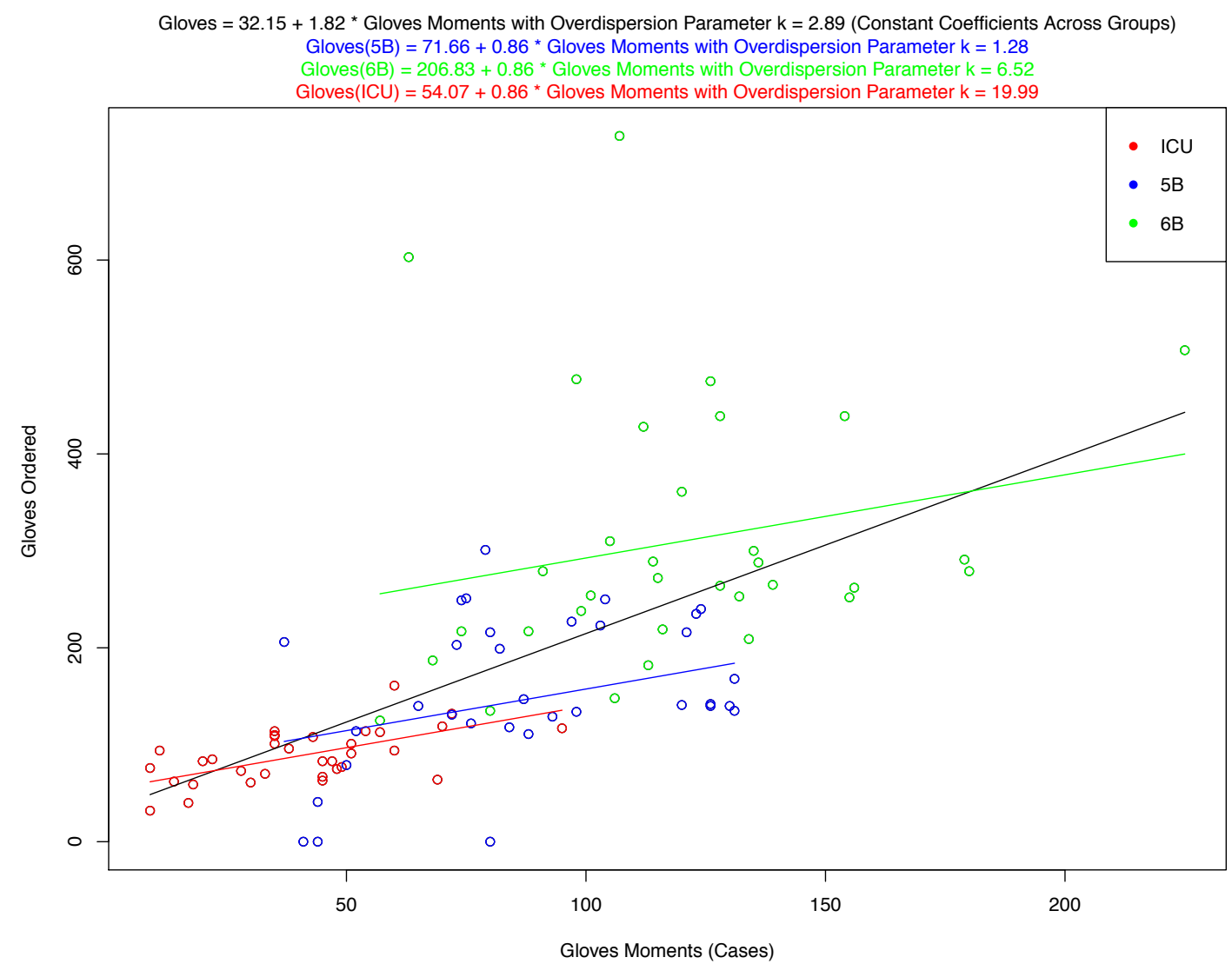

Figure 7.22 Negative Binomial Distribution with a Power Deterministic Function Fitted Model for Gloves Moments versus Gloves Quantity Ordered per Ward (20142016) 


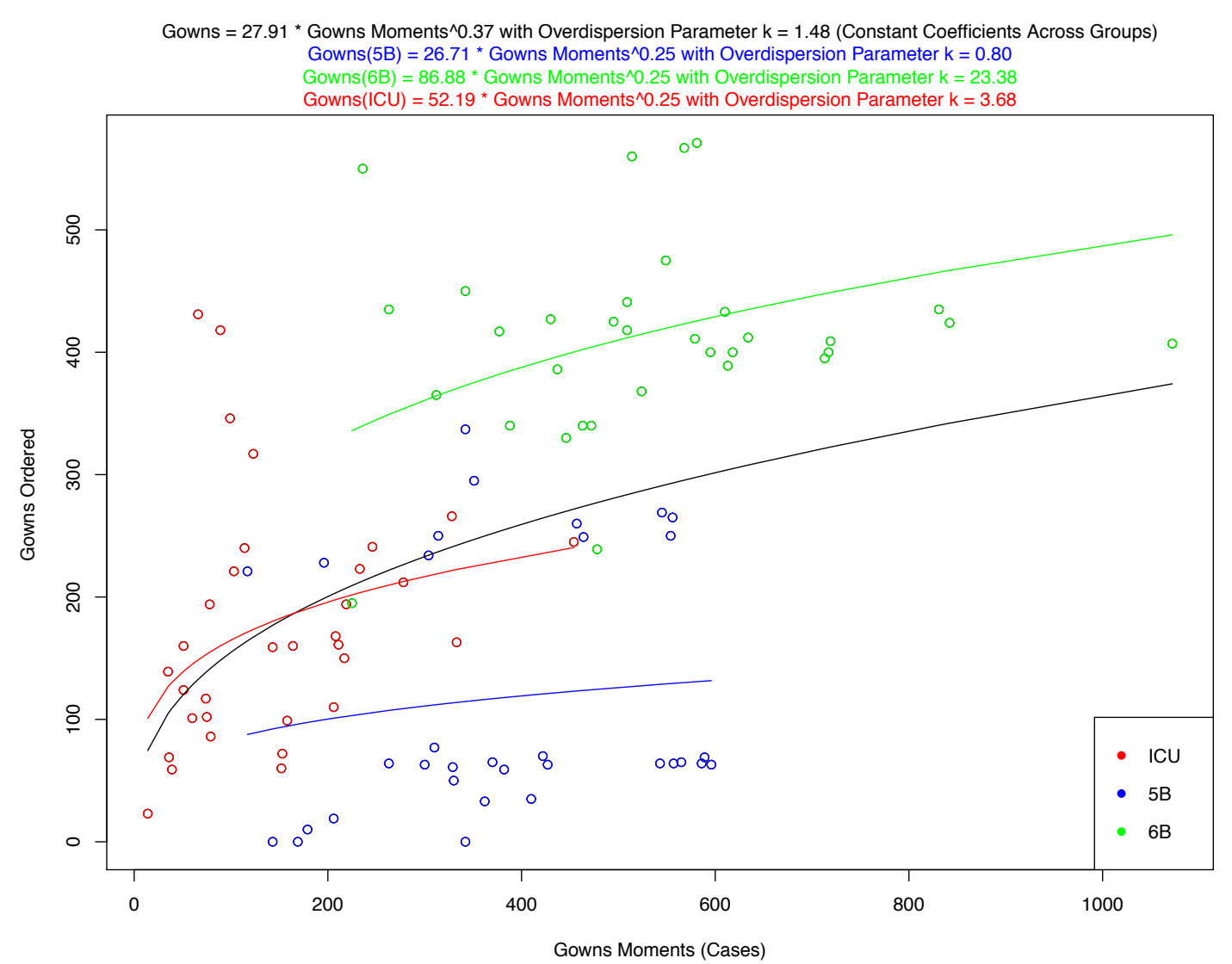

Figure 7.23 Negative Binomial Distribution with a Power Deterministic Function Fitted Model for Gowns Moments versus Gowns Quantity Ordered per Ward (20142016)

In summary, the objective of statistically investigating the relationship between PPE true moments versus PPE quantities ordered is found.

Now after revealing how the interested groups of HCWs at the PVAMC behave in terms of consuming PPE per PPE moments, PPE compliance \% is calculated and a bar plot is generated for gloves and gowns, as can be seen in Figure 7.24 and Figure 7.25 , respectively.

For gloves compliance, it can be seen in Figure 7.24 that there are some bars with compliance exceeding $100 \%$. This is expected because of the source of errors explained earlier. The highest compliance is $436 \%$ and the lowest is $3 \%$. Across all 
years, 5B's gloves compliance is very low. $6 \mathrm{~B}$ 's gloves compliance is mostly in a good range. That might be based on the fact that $5 \mathrm{~B}$ is considered a step-down unit while $6 \mathrm{~B}$ is considered an acute care unit. However, ICU's gloves compliance is very high. Several months of 2014 and 2015 and most of 2016 exceed $100 \%$. ICU is a special case since surgeries are sometimes done on a daily basis because of the health condition of patients. The sample means of the gloves compliance per wards and years combined is seen in Table 7.10. Based on the standard deviations of gloves compliance per wards and years combined, variability is large, which is also revealed on the scatter plots in Figure 7.20 and Figure 7.22. If a comparison between 2014 and 2016 is done, neglecting 2015 for now since gloves for 2015 is predicted, an increase in the compliance for all wards can be seen. Of note, the standard deviation of 2014 gloves compliance at 5B exceeds its mean and that is because for the first three months of 2014 the ward had zero orders. Expectantly, the sample mean of gloves compliance in 2015 is the smallest for all wards because variability from 2014 is added to the predicted values of gloves. 


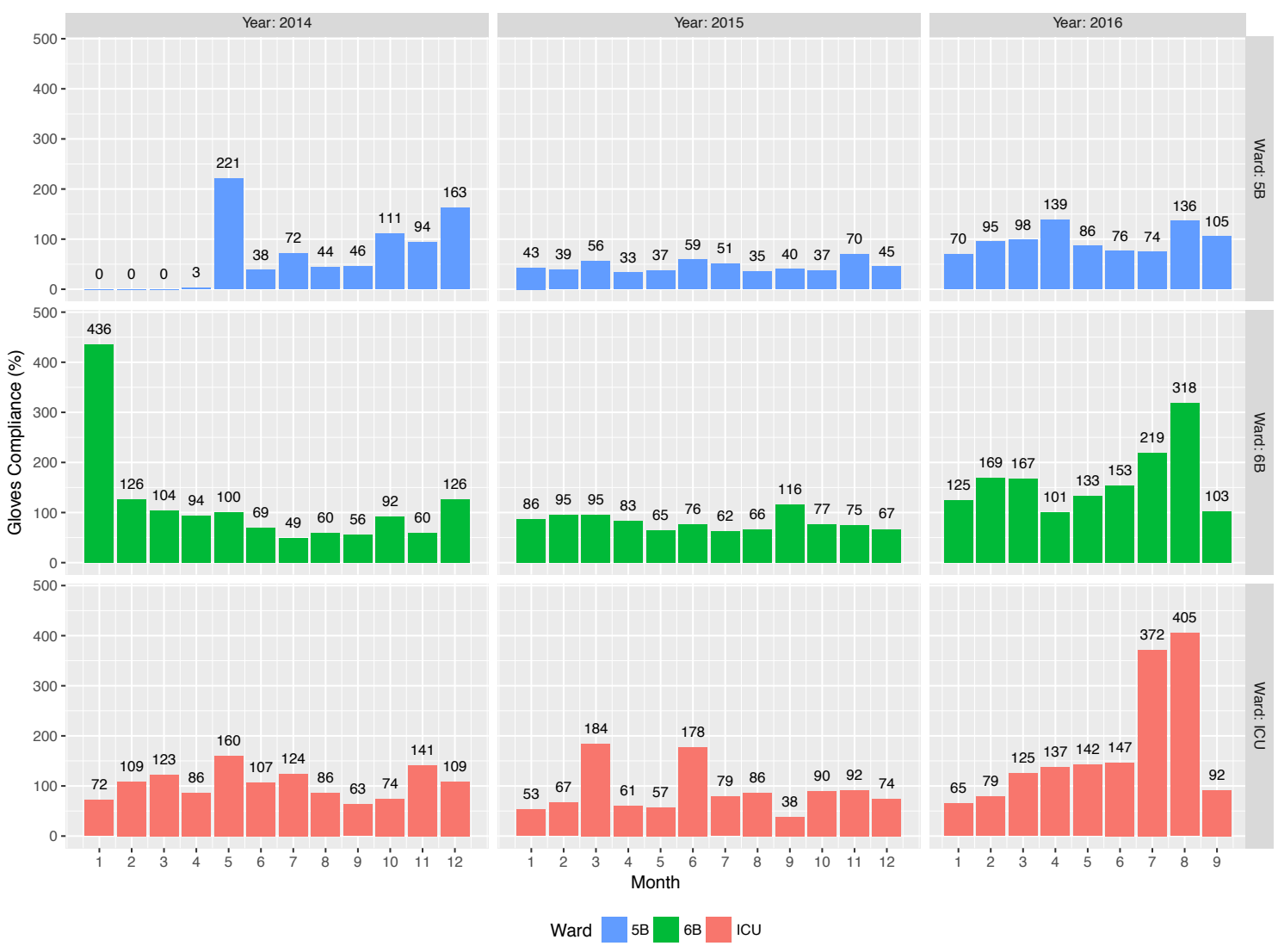

Figure 7.24 Gloves Compliance (\%) per Ward (2014-2016)

\begin{tabular}{|c|c|c|c|c|c|}
\hline \multirow{10}{*}{ Gloves \% } & \multirow{2}{*}{ Year } & \multirow{2}{*}{ Summary of Statistics } & \multicolumn{3}{|c|}{ Ward } \\
\hline & & & $5 B$ & $6 \mathrm{~B}$ & ICU \\
\hline & \multirow{2}{*}{2014} & Mean & 66 & 114.33 & 104.5 \\
\hline & & SD & 70.7 & 104.71 & 29.5 \\
\hline & \multirow{2}{*}{2015} & Mean & 45.42 & 80.25 & 88.25 \\
\hline & & SD & 11.33 & 15.82 & 46.19 \\
\hline & \multirow{2}{*}{2016} & Mean & 97.67 & 165.33 & 173.78 \\
\hline & & SD & 25.42 & 68.06 & 125.36 \\
\hline & \multirow{2}{*}{$\begin{array}{c}\text { Years } \\
\text { Combined }\end{array}$} & Mean & 67.15 & 115.85 & 117.48 \\
\hline & & SD & 48.62 & 78.6 & 78.97 \\
\hline
\end{tabular}

Table 7.10 Summary of Descriptive Statistic of Gloves Compliance per Ward and Ward Combined (2014-2016)

For gowns compliance \%, it can be seen in Figure 7.25 that there are some bars with compliance exceeding $100 \%$. This is expected because of the source of errors 
explained earlier. The highest compliance is $312 \%$ and the lowest is $2 \%$. 5B's gowns compliance is relatively low. In general, 6B's gowns compliance is in a good range for most of the time. However, ICU's gowns compliance exceeds $100 \%$ for most of the months of all three years. The sample means of gowns compliance per wards and years combined is seen in Table 7.11. Based on the standard deviations of gowns compliance per wards and years combined, variability is large for ICU and $5 \mathrm{~B}$, which is revealed on the scatter plots Figure 7.21 and Figure 7.23. 6B's gowns compliance standard deviation is the smallest. If a comparison between 2014 and 2016 is done, neglecting 2015 for now since gowns for 2015 is predicted, a decrease in the compliance for all wards can be seen except 5B, where an increase occurs. In 2014, the mean of gowns compliance is $30 \%, 101.75 \%$ and $124.58 \%$ for $5 \mathrm{~B}, 6 \mathrm{~B}$ and ICU respectively. In 2016 , the mean of gowns compliance is $61.89 \%, 75.33 \%$ and $89.78 \%$ for $5 \mathrm{~B}, 6 \mathrm{~B}$ and ICU, respectively. The sample mean of gowns compliance in 2015 is the smallest for all wards except at ICU, which is larger than that of 2016 but smaller than that of 2014, because variability from 2014 is added to the predicted values of gowns. 

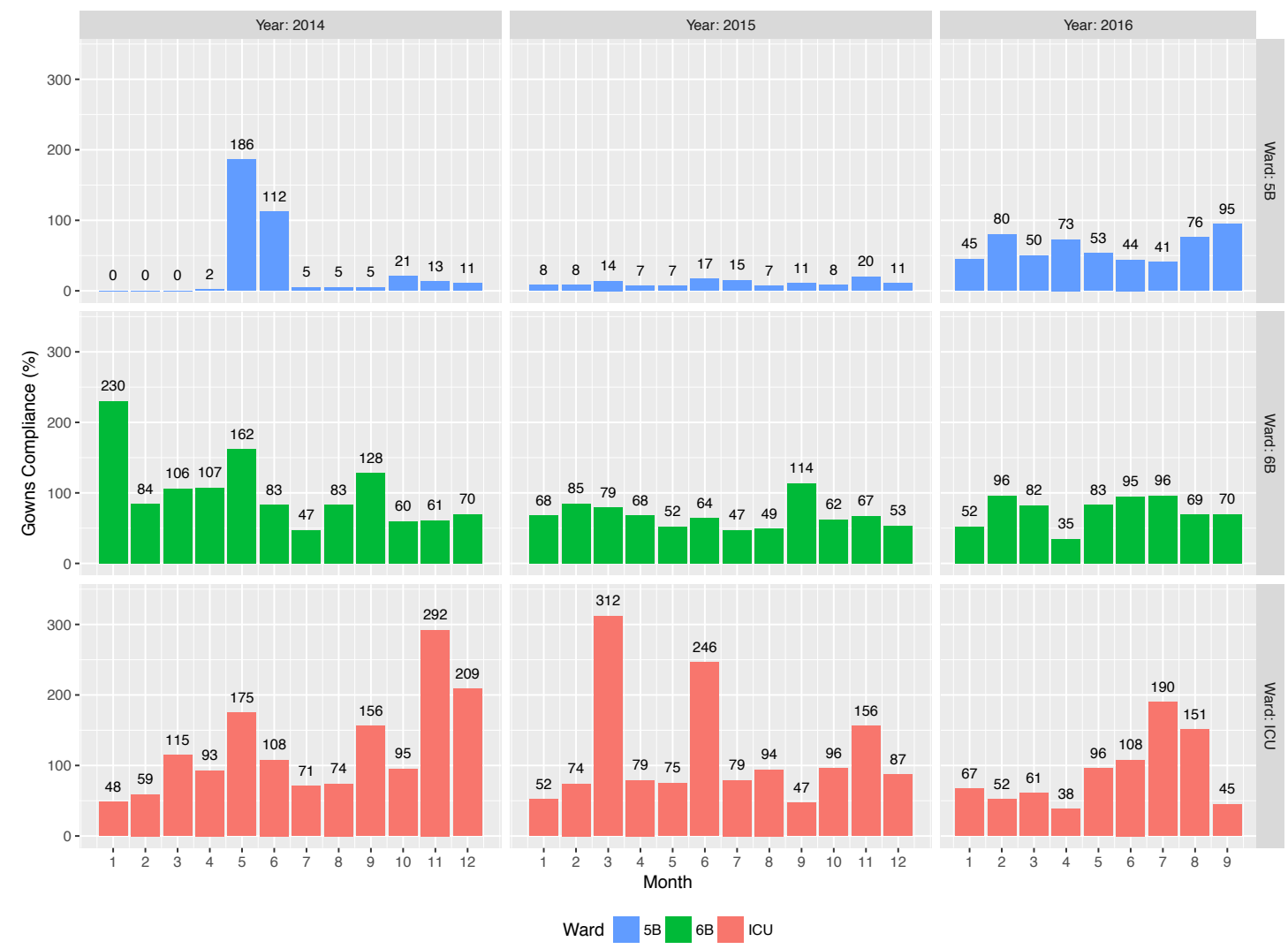

Figure 7.25 Gowns Compliance (\%) per Ward (2014-2016)

\begin{tabular}{|c|c|c|c|c|c|}
\hline \multirow{4}{*}{ Year } & \multirow{3}{*}{ Summary of Statistics } & \multicolumn{3}{|c|}{ Ward } \\
\cline { 3 - 6 } & & & $5 \mathrm{~B}$ & $6 \mathrm{~B}$ & ICU \\
\cline { 2 - 6 } & \multirow{3}{*}{2014} & Mean & 30 & 101.75 & 124.58 \\
\cline { 2 - 6 } & \multirow{2}{*}{2015} & SD & 58.11 & 51.56 & 71.68 \\
\cline { 3 - 6 } & & Mean & 11.08 & 67.33 & 116.42 \\
\cline { 2 - 6 } & \multirow{2}{*}{2016} & SD & 4.44 & 18.72 & 81.84 \\
\cline { 2 - 6 } & & Mean & 61.89 & 75.33 & 89.78 \\
\cline { 2 - 6 } & \multirow{2}{*}{ Years Combined } & SD & 19.39 & 21.17 & 51.99 \\
\cline { 2 - 6 } & Mean & 31.82 & 82.03 & 112.12 \\
\cline { 2 - 6 } & SD & 40.97 & 37.23 & 70.36 \\
\hline
\end{tabular}

Table 7.11 Summary of Descriptive Statistic of Gowns Compliance per Ward and Ward Combined (2014-2016)

Now every time a $\mathrm{HCW}$ is about to enter a patient room who is on contact precautions, two gloves and one gown have to be worn, and before that hand hygiene has to be practiced. Thus, any time a compliance percentage of gloves or gowns 
outweighs each other means there are PPE moments where a HCW either wears gloves and no gown, or gown and no gloves. In both cases, this would impose a risk of transmitting HAIs from or to other patients and HCWs. A critical question to be asked here is which would be too dangerous to forget, gloves or gowns?

Looking at Figure 7.26, ignoring the first three months of 2014 for 5B since no PPE orders are made at all, it can be seen that most of the time gowns are missing during caregiving at $5 \mathrm{~B}$ and $6 \mathrm{~B}$ and during 2016 at ICU. In contrast, gloves are missing during caregiving at ICU. There are some months across wards and years where the missing PPE (gloves or gowns) percentages are very low, which is a positive sign of higher gloves and gowns compliance being worn together. However, it is crucial to keep in mind that gowns compliance is only calculated based on transmission precautions BDOC while gloves compliance is calculated based on both standard and transmission precautions BDOC. Clearly, there is an impact on the PPE non-compliance from the way the PPE compliance originally is calculated. To have more meaningful representation of gloves and gowns non-compliance, gloves compliance has to be calculated separately for standard and transmission precautions, which is currently impossible because it is difficult to separate the numerator. 


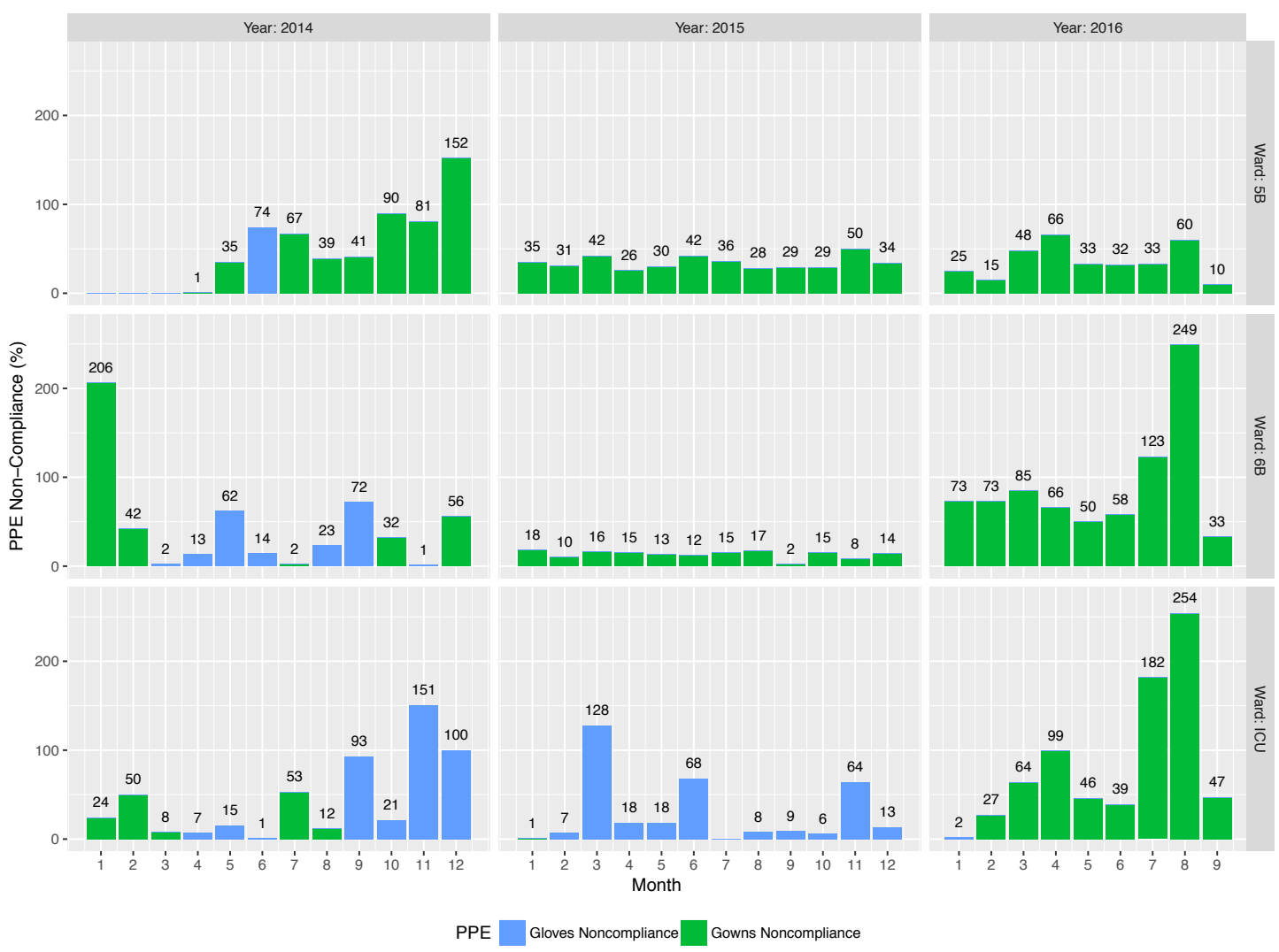

Figure 7.26 PPE Non-Compliance (\%) per Ward (2014-2016) 


\section{CHAPTER 8}

\section{CONCLUSIONS}

This is an intensive, statistically based hand hygiene study that was conducted at a VA medical center, the PVAMC. There are about 168 medical centers nationwide. The patient contact study encompasses all working shifts and all inpatient wards. In the direct hand hygiene observation method, the correlation between HAI and hand hygiene compliance was conducted and several hypotheses were tested to identify risk factors. In the indirect hand hygiene monitoring of product use method, a better estimate of hand hygiene opportunities (the denominator) is developed. The indirect method of measuring PPE compliance is a brand new method of its own where PPE inventory data is used for PPE compliance estimation.

\subsection{Summary of Results}

The direct hand hygiene observation method conducted by the URI research team was able to obtain a better estimate of hand hygiene compliance. The reported hand hygiene compliance is, thus, reliable and can be used to represent the PVAMC. Further precision and accuracy on hand hygiene compliance was attempted by approximating the patient contact population (hand hygiene opportunities). Though the population was approximated, the population was considered infinite and thus, confidence interval correction was not conducted. The PVAMC claim of having high hand hygiene adherence was statistically rejected and the opposite was proven. Relating such claims to the number of infectious disease cases that occur, however, is unreliable because some could occur by chance. However, having zero HAIs (CDI, 
MRSA, CAUTI, CLABSI and VAE) during the two months of the study, September and October 2016, and the efforts that were put together by the PVAMC to prevent and control such infectious diseases are positively enormous.

The monthly hand hygiene compliance mean and $95 \%$ confidence interval of September was $45 \%(40 \%, 50 \%), 64 \%(60 \%, 68 \%), 49 \%(42 \%, 56 \%)$ and $55 \%(52 \%$, $58 \%$ ) for $5 \mathrm{~B}, 6 \mathrm{~B}$, ICU and all inpatient wards combined. The monthly hand hygiene compliance mean of October was 45\% (41\%, 49\%), 50\% (46\%, 54\%), 57\% (49\%, $65 \%)$ and $48 \%(45 \%, 51 \%)$ for $5 \mathrm{~B}, 6 \mathrm{~B}, \mathrm{ICU}$ and all inpatient wards combined. These percentages represented hand hygiene behavior of the medical and nursing groups who worked at the inpatient wards only. The three inpatient wards represent about $80 \%$ of the total operating beds at the PVAMC. Thus, the monthly hand hygiene compliance mean that was calculated based on the combination of these three wards was representative of the PVAMC facility.

Based on patient contact outcomes, the nursing group, on average, has a significantly higher number of patient contacts than the medical group. On average, a patient at $5 \mathrm{~B}$ was seen by the nursing group 43 times and by the medical group 7 times. On average, a patient at $6 \mathrm{~B}$ was seen by the nursing group 51 times and by the medical group 7 times. On average, a patient at ICU was seen by the nursing group 103 times and by the medical group 8 times. On average, a patient was seen by the nursing group 54 times and by the medical group 7 times by combining all inpatient wards together. As expected, RN (63.69\%) and CNA (29.99\%) contributed to the most number of patient contacts. 
For four years in total, the PVAMC collected about 6249 observations with $88 \%$ hand hygiene compliance. The hand hygiene compliance seemed high. However, these 6249 observations encompassed all HCWs categories and subcategories, EMS employees, social workers, and others. Such a sample did not represent the PVAMC true hand hygiene compliance. In contrast, the sample that was collected by the URI research team during the two month study was about $40 \%(2432 / 6249)$ of the four year sample size collected by the PVAMC. In addition, such a sample was mainly collected on the most important groups who had the highest patient contact, the medical and nursing groups.

The direct hand hygiene observation method showed a high hand hygiene compliance mean in the first week of the study at 5B, $6 \mathrm{~B}$ and at all wards combined. The URI research team believed that could be due to a Hawthorne Effect. The hand hygiene compliance mean of the remaining seven weeks of September and October did not reach the first week level. The monthly hand hygiene compliance mean at the inpatient wards individually and collectively from September to October did not significantly change in either direction. Yet, the weekly hand hygiene compliance mean experienced some fluctuation.

There were zero cases or incidents of healthcare-associated infections (HAI) at all inpatient wards during the two months of the study. There were some cases of community-acquired infections (CAI) such as CDI, MRSA, MRSA colonization and VAE. Thus, correlation between HAI and hand hygiene compliance seemed unreasonable. However, there was a strong negative correlation between the weekly hand hygiene compliance mean and BDOC at 5B (-0.90), 6B (-0.55) and ICU (-0.59). 
As BDOC increased, the hand hygiene compliance decreased. However, when correlating HAIs with the hand hygiene compliance using the PVAMC's four year direct hand hygiene observations, a moderate correlation was found with a correlation coefficient of about -0.50 .

Hypothesis testing revealed that healthcare worker's gender (female vs. male), healthcare worker's job category (doctors vs. nurses) and patient's isolation status were not identified as statistically significant risk factors for poor adherence to hand hygiene recommended practices. In contrast, hypothesis testing revealed that hand hygiene moments (Before and After touching a patient), patient's gender (female vs. male), inpatient wards (5B, 6B and ICU), days of the week and working shift (night, day and evening) were statistically significant risk factors for poor adherence to hand hygiene recommended practices. From a patient's perspective, HCWs ought to be notified to practice hand hygiene before providing care. A male patient is at higher risk than a female patient though more male patients are admitted to the PVAMC. A patient would be less worried to be admitted to $6 \mathrm{~B}$ and ICU than to $5 \mathrm{~B}$ though $5 \mathrm{~B}$ is a step-down ward. A patient would be less worried to stay at the PVAMC during weekends rather than weekdays and during day and evening shifts rather than night shift.

The monthly hand hygiene compliance over a 10 month study monitoring Purell and Soap usage as an indirect hand hygiene method revealed that compliance was as low as $1.53 \%$ and as high as $80.35 \%$. Huge variability was seen over the 10 -month period across all inpatient wards. Correlation was not found between the monthly hand hygiene compliance and the monthly BDOC based on such a method. This method 
revealed that the actual replacement of Purell and Soap cartridges was far below the targeted replacement. During the same study period, the indirect method of measuring PPE compliance revealed that PPE compliance was overestimated for certain months because of the source of error on both the numerator and denominator. However, gloves compliance could go as low as $3 \%$, which was observed at 5B. Gown compliance, on the other hand, seemed reasonably estimated for 5B and 6B. ICU was a special case in this method because of patients' health status. PPE has to be overstocked at ICU for emergency cases. Correlation was not found between BDOC and gloves or gowns compliance except at ICU. A medium negative correlation $(-0.53)$ was identified between BDOC and gloves compliance. As BDOC increased, the gloves compliance decreased.

In conclusion, the study findings confirmed that though HAI cases were very rare, hand hygiene compliance was statistically proven to also be very low. The rare incidents of HAIs at the PVAMC were either caused by another factor or by chance.

\subsection{Limitations and Future Work}

In conducting a direct hand hygiene observation method, randomization is always a concern. The study was conducted at a single facility, in three inpatient wards and the focus was on two important groups, the medical and nursing groups only. The Hawthorne Effect always exists in such a method. Such bias was seen during the first week of the study in September, at 5B and 6B though these two wards are larger than ICU and numbers of HCWs are higher as well. Though larger sample size was collected during the two months study, breaking down the sample by subgroups such as registered nurse practitioner (NP), licensed practical nurse (LPN) 
and nursing student (NS) disabled the detection of any statistically significant changes in hand hygiene compliance. Though the medical and nursing groups are the most important HCWs in terms of patient contact, limiting the study to them affects the hand hygiene compliance representation of the PVAMC. These two groups are subgroups of a larger list of HCWs and other professional categories at the PVAMC. In addition, the study focused on the two most important hand hygiene moments, before and after touching a patient. In conducting patient contact, one complete week is not enough for average patient contact estimation. Hand hygiene and PPE compliance estimations in the indirect hand hygiene monitoring of product use and in the indirect method of measuring PPE compliance are affected by averaging patient contact over one week. Finally, implementing different models to measure and estimate hand hygiene and PPE compliance over different periods of time was not beneficial. The direct hand hygiene observation method was conducted during September and October 2016. The indirect hand hygiene monitoring of product use method was conducted during the first 10 months of 2015. The indirect method of measuring PPE compliance was conducted during 2014, 2015 and 2016, but 2015 data had to be estimated. The indirect hand hygiene and PPE compliance methods overlapped for 10 months of 2015 while the direct hand hygiene observation method did not overlap. In the direct hand hygiene monitoring of product use method, any remaining Purell or Soap volume was not measured or tracked, because that would have been extra work for the EMS employees at the PVAMC. In the indirect method of measuring PPE compliance, the PPE inventory assumption ought to be revised. Sources of error on both the numerator and denominator must be reduced by collecting 
or observing other groups, such as other medical specialists, who utilize PPE along with the medical and nursing groups. Similarly, other groups should be included in the patient contact study including specialists and surgeons. Sources of error on the denominator of the estimate of gloves moments also could be reduced by observing how many times gloves are used when care is provided to patients who are on nonisolation.

The major limitation was that the patient contact study was conducted once and not as was planned to be repeated three times over the year. In addition, the PVAMC IRB approval and the participation of the PVAMC employees for patient contact and direct hand hygiene observation methods prevented overlapping all methods in one single period of time. The patient contact method was only approved for one complete week at a time. The medical and nursing groups at the PVAMC suggested the patient contact self-report during the first week of October. The recruited secret observers at the PVAMC agreed to collect hand hygiene observations over two months. Regardless, if the PVAMC is convinced with the current findings of the study, the study could be conducted in the future over at least 6 months. RFID would be a more preferable method to record patient contact data for accuracy, rather than selfreporting. More secret observers should be recruited and since discrepancy is expected, 95\% agreement accuracy should be conducted, and a housekeeper would be assigned per inpatient ward per working shift to track Purell and Soap replacement for the indirect hand hygiene manual monitoring of product use. 


\subsection{Recommendations}

The PVAMC has made an important initiative toward measuring precise and accurate hand hygiene compliance at the inpatient ward level. The PVAMC leaders and quality management have to consider implementing a hand hygiene intervention that could lead to an increase in hand hygiene compliance. The PVAMC has to collect the right sample size to ensure detecting such a shift in the hand hygiene compliance mean. Based on the study findings, a sample size of about 380 per month in each inpatient ward would be enough to be able to have a confidence of $95 \%$ to detect an error of about $5 \%$ from the true hand hygiene compliance. Combining these sample sizes to represent the PVAMC would enable the PVAMC to have about 1140 sample size that would be enough to be able to have a confidence of $95 \%$ to detect an error of about 3\% from the true hand hygiene compliance. About 95 observations should be collected weekly to have a better representation of hand hygiene compliance behavior of HWCs at each inpatient ward. Definitely, the more observations the better the estimate of hand hygiene compliance. Otherwise, it would be a waste to implement any intervention. A gradual or incremental improvement on hand hygiene compliance could lead to more stable and sustainable result. The PVAMC should have a hand hygiene campaign showing the real results of hand hygiene compliance and the PVAMC's HCWs have to be empowered to participate in such improvement. Though measuring hand hygiene compliance is just the beginning of continuous improvement for further HAIs reduction and superior quality of the PVAMC environment, other important factors have to be considered for future studies such as room cleaning, food delivery, patient transfer, admission and discharge. The EMS employees and food and 
nutrition services are very interesting groups to study hand hygiene compliance behavior as well as patient contact. Other doctors such as surgeons, psychiatrists and respiratory therapists are encouraged for hand hygiene compliance and patient contact participation.

The PVAMC should consider implementing a hand hygiene compliance monitoring system that includes both direct and indirect methods. PPE compliance is not less important than hand hygiene since HCWs are recommended to comply with PPE guidelines along with practicing hand hygiene during patient care for various patient isolation status. Consequently, tracking Purell and Soap replacement and PPE inventory data becomes important to the PVAMC. The tracking of Purell and Soap use should be at the level of inpatient ward or in general per division. The PPE inventory data should no longer be purged for future sake of indirectly measuring PPE compliance. These incremental changes could improve data reporting and lead to systems improvement. The infectious disease and quality management department should consider reporting CDI per inpatient ward as other HAIs are reported. In addition, isolation and non-isolation BDOC should be reported and separated by patient gender per inpatient ward. Invasive-device days and lines for CAUTI, CLABSI and VAE (VAP) should also be reported based on isolation and non-isolation status. Hand hygiene, BDOC and HAIs should be tracked weekly. For the ongoing direct hand hygiene observation method, weekly sample size should be no less than 10 observations per secret observer. The current 63 secret observers, and more who are recruited, should all participate in reaching such number. Total observations per month should not be less than 1140 observations to statistically be able to detect a $3 \%$ shift in 
the mean. Such a sample size is recommended for all three inpatient wards combined and per the medical and nursing groups.

The PVAMC leaders should consider using RFID technology for patient contact over at least three months, if not permanently. Moreover, if there is any chance Purell and Soap cartridge replacement could be tracked via RFID, that chance should be taken seriously. Finally, the PVAMC leaders should encourage the hand hygiene program director and infection disease preventionist to build their statistical knowledge beyond reporting hand hygiene compliance by percentages on a monthly basis. Test statistics, power analysis and sample size, correlation and statistical models and statistical programming languages (R or Minitab) or Excel are powerful tools and knowledge for statistical analysis.

Though hand hygiene is a simple practice to prevent infectious disease prevalence, it is a hot topic for investigation. Consequently, hand hygiene is important for improving healthcare to help patients and reduce costs. Several tracking and reporting methods have been developed and continually improved. However, it depends on the healthcare institute or hospitals to choose the appropriate ones.

As the way HCWs interact with patients at a VA medical center is interesting to study and investigate the behavior of hand hygiene, it is hard to choose a better tracking method. That was seen when the patient contact method was conducted and the results showed that there were about 15,455 entries in only one week. Direct and indirect hand hygiene methods were implemented. However, the PVAMC could find the indirect method to be a better alternative. Secret observing in the direct hand hygiene observation method is voluntary and hard to cover all working shifts with the 
same sample size. Thus, the PVAMC could continually improve the indirect hand hygiene manual monitoring of product use (Soap and Purell) and the indirect method of measuring personal protective equipment (PPE) compliance.

The advantages of implementing the direct hand hygiene observation method is that such a method enables the PVAMC to identify risk factors for poor adherence to hand hygiene recommended practices. The $\mathrm{HCW}$ and patient gender imbalance, the isolation status and working shift are worth investigating further. However, for both desires, reliable direct hand hygiene observation method and risk factors identification, a large sample size is a must. The PVAMC has been tracking hand hygiene compliance since April 2012 and they continually are doing that. Thus, a more reliable direct and indirect hand hygiene observation method are better to be implemented especially if the national VA intends to have hand hygiene compliance as a quality measure. 


\section{APPENDICES}

\section{APPENDIX AAA: Research Time Plan}

\begin{tabular}{|l|c|}
\hline \multicolumn{1}{|c|}{ Activity } & Timeline \\
\hline $\begin{array}{l}\text { Understanding Healthcare, Hand Hygiene and } \\
\text { Healthcare-Associated Infections (HAIs) }\end{array}$ & May 2014 \\
\hline Establishment of a Research Committee by URI & January $7^{\text {th }} 2015$ \\
\hline Program of Study Approval by URI & January $7^{\text {th }} 2015$ \\
\hline Developing Purell \& Soap Log Form & January 2015 \\
\hline Developing Patient-Contact Form & March 2016 \\
\hline Developing Hand Hygiene Monitoring Form & March 2016 \\
\hline Developing Infections per Operating Bed per Unit Form & April 2016 \\
\hline $\begin{array}{l}\text { Preparing and Getting IRB and R\&D Approvals at the } \\
\text { PVAMC and URI }\end{array}$ & April - July 2016 \\
\hline Dissertation Proposal Approval by URI & May $10^{\text {th }} 2016$ \\
\hline IRB Protocol Approval by the PVAMC & July 2016 \\
\hline R\&D Protocol Approval by the PVAMC & July 2016 \\
\hline $\begin{array}{l}\text { Pilot Study } \mathbf{s}^{\text {st }} \text { Part: } \\
\text { Conducting Hand Hygiene Secret Observers Data } \\
\text { Collection }\end{array}$ & September - October 2016 \\
\hline $\begin{array}{l}\text { Pilot Study 2 } \\
\text { Conducting Part: }\end{array}$ & \\
\hline Analyzing Data Collected and Reporting to Management & Oct $3^{\text {rd }}-$ Oct $9^{\text {th }} 2016$ \\
\hline Written Doctoral Comprehensive Examination & December 2016 \\
\hline Oral Doctoral Comprehensive Examination & January $17^{\text {th }} 2017$ \\
\hline Oral Defense Examination of Doctoral Dissertation & January $18^{\text {th }} 2017$ \\
\hline Full Dissertation Submission to URI & April $14^{\text {th }} 2016$ \\
\hline Full Report Submission to the PVAMC & April $21^{\text {st }} 2016$ \\
\hline 131 $1^{\text {st }}$ Commencement Ceremony at URI & May 2016 \\
\hline & May $20^{\text {th }} 2017$ \\
\hline
\end{tabular}




\title{
APPENDIX BBB: PVAMC IRB Approval
}

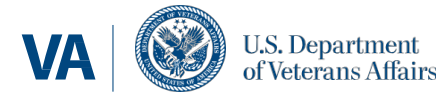

\section{Memorandum}

\author{
Date: July 26, 2016 \\ From: Aisling Caffrey, Ph.D., Chair, Institutional Review Board \\ VA Medical Center, Providence, RI \\ Subj: Approval of responses to conditions of approval \\ To: Valerie Maier-Speredelozzi, Ph.D. \\ Melissa Gaitanis, M.D.
}

Review and approval was given this date of the investigator's responses dated July 19,2016 , to the IRB Committee's conditions of approval for the minimal risk new protocol [2016-010] "Assessing Hand Hygiene Compliance in Healthcare Workers to Reduce Infectious Disease" submitted by Valerie Maier-Speredelozzi, Ph.D. This protocol now has full IRB approval.

\section{The period of approval is from July 13, 2016, through July 12, 2017.}

This study meets the following criteria for non-applicability of requirements for HIPAA Authorization: Use of a Limited Data Set and Data Collected on VHA Employees.

The currently approved protocol documents are:

1) Protocol, version $7 / 19 / 2016$

2) Informed consent form (ICF) PC, version $7 / 26 / 2016$

3) Informed consent form (ICF) SO, version $7 / 26 / 2016$

This request meets the following criteria for expedited review and approval: Approval is requested for change(s) specifically requested by the IRB as condition(s) of approval.

If you have any questions, please call the Research Office at (401) 457-3066.

Aisling Caffrey, Ph.D.

IRB Chair 


\section{APPENDIX CCC: PVAMC R\&D Approval}

Department of Veterans Affairs

Date: July 27,2016

From: Providence VAMC Research \& Development Committee

Subj: Review of Research Proposal

To: $\quad$ Valerie Maier-Speredelozzi, M.D. and Melissa Gaitanis, M.D.

Your protocol entitled [2016-010] "Assessing Hand Hygiene Compliance in Healthcare Workers to Reduce Infectious Disease" received the following review:

This new protocol was conditionally approved at the July 13,2016 , IRB meeting.

PI met conditions on July 26, 2016.

R\&D approval was granted at its meeting held on July 27, 2016.

You may now initiate your research.

The period of approval is from July 13,2016 through July 12, 2017.

A request for Continued Approval with a Progress Report must be fully approved by the appropriate committee(s) prior to the end of the project approval period. Due to the scheduling of a committee meetings and because some requests are not always approved at the initial meeting, we recommend you submit your Request for Continued Approval two months prior to expiration of your approval date.

If you have any questions, concerns, or would like additional information, please call the Research Office at (401) 457-3066.

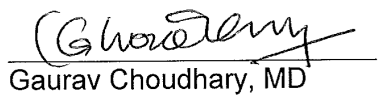

Associate Chief of Staff (Research) 
APPENDIX A: Employee Class at PVAMC

\begin{tabular}{|l|l|}
\hline$\#$ & \multicolumn{1}{|c|}{ Employee Class } \\
\hline 1 & Administrative Assistant / Medical Support \\
\hline 2 & Chaplain \\
\hline 3 & Dental Assistant / Nurse \\
\hline 4 & Dentist \\
\hline 5 & Dietary / Food Service \\
\hline 6 & Doctor (Attending / Fellow) \\
\hline 7 & Doctor (Med Student / Intern / Resident) \\
\hline 8 & EMS \\
\hline 9 & Escort \\
\hline 10 & IV Nurse \\
\hline 11 & Laboratory Service \\
\hline 12 & Nurse (RN / LPN) \\
\hline 13 & Nurse Practitioner / Physician Assistant \\
\hline 14 & Nursing Assistant Health Tech \\
\hline 15 & Nursing Student \\
\hline 16 & Nutritionist / Dietician \\
\hline 17 & Pharmacist / Pharm Tech \\
\hline 18 & Phlebotomist \\
\hline 19 & Psychiatrist / Psychologist \\
\hline 20 & Radiology Service \\
\hline 21 & Recreation Therapist \\
\hline 22 & Rehab (PT / OT / KT) \\
\hline 23 & Respiratory Service \\
\hline 24 & Social Worker \\
\hline 25 & Speech Therapy \\
\hline 26 & Voluntary Service \\
\hline 27 & Other \\
\hline & \\
\hline
\end{tabular}


APPENDIX B: Location and Service Type at PVAMC

\begin{tabular}{|c|l|l|c|l|c|}
\hline$\#$ & \multicolumn{1}{|c|}{ Location } & \multicolumn{1}{|c|}{ Service Type } & $\#$ & \multicolumn{1}{c|}{ Location } & Service Type \\
\hline 1 & Occupational Therapy & Clinical Support & 20 & Cardiology & Outpatient Clinic \\
\hline 2 & OR & Clinical Support & 21 & Dental Clinic & Outpatient Clinic \\
\hline 3 & Physical Therapy & Clinical Support & 22 & Dermatology Clinic & Outpatient Clinic \\
\hline 4 & Radiology - CT Scan & Clinical Support & 23 & Dialysis & Outpatient Clinic \\
\hline 5 & Radiology - General & Clinical Support & 24 & ER / Urgent Care & Outpatient Clinic \\
\hline 6 & Radiology - Interventional & Clinical Support & 25 & Eye Clinic & Outpatient Clinic \\
\hline 7 & Radiology - MRI & Clinical Support & 26 & GI Endoscopy & Outpatient Clinic \\
\hline 8 & Radiology - Nurclear Med & Clinical Support & 27 & HBPC & Outpatient Clinic \\
\hline 9 & Rec Therapy & Clinical Support & 28 & Medical SS Clinic & Outpatient Clinic \\
\hline 10 & 4 A & Inpatient Ward & 29 & Mental Health Clinic & Outpatient Clinic \\
\hline 11 & $4 B$ & Inpatient Ward & 30 & Ortho Clinic & Outpatient Clinic \\
\hline 12 & 5B & Inpatient Ward & 31 & Podiatry Clinic & Outpatient Clinic \\
\hline 13 & 6A & Inpatient Ward & 32 & Primary Care & Outpatient Clinic \\
\hline 14 & 6B & Inpatient Ward & 33 & Pulmonary/Resp Clinic & Outpatient Clinic \\
\hline 15 & ICU & Inpatient Ward & 34 & Speech Pathology & Outpatient Clinic \\
\hline 16 & PACU & Inpatient Ward & 35 & Surg Clinics & Outpatient Clinic \\
\hline 17 & Escort & Non-Clinical Support & 36 & Urology & Outpatient Clinic \\
\hline 18 & Laboratory & Non-Clinical Support & 37 & Women's Health Clinic & Outpatient Clinic \\
\hline 19 & Occupational Health & Non-Clinical Support & 38 & Primary Care & Outpatient Clinic \\
\hline
\end{tabular}




\section{APPENDIX C: 6B Patient-Contact Day Shift Data Sheet UNIT: 6B ONLY}

Instructions:-

Please mark a tally ( / ) every time you Contact your patient in the correct cell. For example, if you need to contact a patient who is a Male in room 669, bed (A) at 10:20 a.m., mark a tally (/) on that cell below Time 10:00 a.m. and in row Room 669 A. $\begin{array}{lll}\text { Feet-to-Feet Patient Room: } & \text { Window-to-Door Patient Room: } & \text { Note: If a new patient is } \\ \text { Bed (A): Left } & \text { Bed (A): Door } & \text { admitted, please draw a star }\end{array}$ Bed (B): Right

Bed (B): Window (*). Add $\mathrm{M}$ or $\mathrm{F}$ if gender is different $(* \mathrm{M})$ or $(* \mathrm{~F})$.

Employee Code:

Employee Gender: $\square$ M $\square$ F

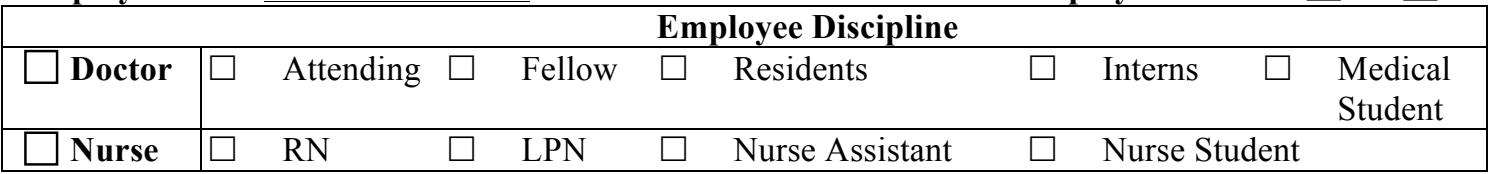

Day: $\square \mathrm{M} \square \mathrm{T} \square \mathrm{w} \square$ Th $\square \mathrm{F} \square \mathrm{Sa} \square \mathrm{Su}$.

Date (mm/dd):

12016.

\begin{tabular}{|c|c|c|c|c|c|c|c|c|c|}
\hline \multirow{2}{*}{$\begin{array}{l}\text { Patient } \\
\text { Gender }\end{array}$} & \multirow[b]{2}{*}{ Room } & \multicolumn{8}{|c|}{ Patient Contacts for Day Shift $\left(1^{\text {st }}\right)$ ONLY } \\
\hline & & $\begin{array}{l}\text { 8-9 } \\
\text { am }\end{array}$ & $\begin{array}{c}9-10 \\
\text { am }\end{array}$ & $\begin{array}{c}10-11 \\
\text { am }\end{array}$ & $\begin{array}{c}11-12 \\
\mathrm{pm}\end{array}$ & $\begin{array}{c}12-1 \\
\mathrm{pm}\end{array}$ & $\begin{array}{l}1-2 \\
\mathrm{pm}\end{array}$ & $\begin{array}{l}2-3 \\
\mathrm{pm}\end{array}$ & $\begin{array}{l}\text { 3-4 } \\
\text { pm }\end{array}$ \\
\hline$\checkmark \mathrm{M} \quad \square \mathrm{F}$ & Sample & $/ / / /$ & & $/ / /$ & 1 & $/ / / /$ & $* \mathrm{~F} / / /$ & & $/ /$ \\
\hline$\square \mathrm{M} \square \mathrm{F}$ & $669 \mathrm{~A}$ & & & & & & & & \\
\hline$\square \mathrm{M} \square \mathrm{F}$ & $669 \mathrm{~B}$ & & & & & & & & \\
\hline$\square \mathrm{M} \square \mathrm{F}$ & $670 \mathrm{~A}$ & & & & & & & & \\
\hline$\square \mathrm{M} \square \mathrm{F}$ & $670 \mathrm{~B}$ & & & & & & & & \\
\hline$\square \mathrm{M} \square \mathrm{F}$ & $671 \mathrm{~A}$ & & & & & & & & \\
\hline$\square \mathrm{M} \square \mathrm{F}$ & $671 \mathrm{~B}$ & & & & & & & & \\
\hline$\square \mathrm{M} \square \mathrm{F}$ & $672 \mathrm{~A}$ & & & & & & & & \\
\hline$\square \mathrm{M} \square \mathrm{F}$ & $672 \mathrm{~B}$ & & & & & & & & \\
\hline$\square \mathrm{M} \square \mathrm{F}$ & $673 \mathrm{~A}$ & & & & & & & & \\
\hline$\square \mathrm{M} \square \mathrm{F}$ & $673 \mathrm{~B}$ & & & & & & & & \\
\hline$\square \mathrm{M} \square \mathrm{F}$ & $674 \mathrm{~A}$ & & & & & & & & \\
\hline$\square \mathrm{M} \square \mathrm{F}$ & $674 \mathrm{~B}$ & & & & & & & & \\
\hline$\square \mathrm{M} \square \mathrm{F}$ & $675 \mathrm{~A}$ & & & & & & & & \\
\hline$\square \mathrm{M} \square \mathrm{F}$ & $679 \mathrm{~A}$ & & & & & & & & \\
\hline$\square \mathrm{M} \square \mathrm{F}$ & $680 \mathrm{~A}$ & & & & & & & & \\
\hline$\square \mathrm{M} \square \mathrm{F}$ & $680 \mathrm{~B}$ & & & & & & & & \\
\hline$\square \mathrm{M} \square \mathrm{F}$ & $681 \mathrm{~A}$ & & & & & & & & \\
\hline$\square \mathrm{M} \square \mathrm{F}$ & $681 \mathrm{~B}$ & & & & & & & & \\
\hline$\square \mathrm{M} \square \mathrm{F}$ & $682 \mathrm{~A}$ & & & & & & & & \\
\hline$\square \mathrm{M} \square \mathrm{F}$ & $682 \mathrm{~B}$ & & & & & & & & \\
\hline$\square \mathrm{M} \square \mathrm{F}$ & $683 \mathrm{~A}$ & & & & & & & & \\
\hline$\square \mathrm{M} \square \mathrm{F}$ & $683 \mathrm{~B}$ & & & & & & & & \\
\hline$\square \mathrm{M} \square \mathrm{F}$ & $684 \mathrm{~A}$ & & & & & & & & \\
\hline$\square \mathrm{M} \square \mathrm{F}$ & $685 \mathrm{~A}$ & & & & & & & & \\
\hline$\square \mathrm{M} \square \mathrm{F}$ & $685 \mathrm{~B}$ & & & & & & & & \\
\hline$\square \mathrm{M} \square \mathrm{F}$ & $686 \mathrm{~A}$ & & & & & & & & \\
\hline$\square \mathrm{M} \square \mathrm{F}$ & $686 \mathrm{~B}$ & & & & & & & & \\
\hline
\end{tabular}




\section{APPENDIX D: 6B Patient-Contact Evening Shift Data Sheet UNIT: 6B ONLY}

Instructions:-

Please mark a tally (/) every time you Contact your patient in the correct cell. For example, if you need to contact a patient who is a Male in room 669, bed (A) at 7:20 p.m., mark a tally (/) on that cell below Time 7:00 p.m. and in row Room 669 A.
Feet-to-Feet Patient Room:
Window-to-Door Patient Room:
Bed (A): Left
Bed (A): Door
Note: If a new patient is
Bed (B): Right
Bed (B): Window admitted, please draw a star (*). Add $\mathrm{M}$ or $\mathrm{F}$ if gender is different $(* \mathrm{M})$ or $\left({ }^{*} \mathrm{~F}\right)$.

Employee Code:

Employee Gender: $\square$ M $\square$ F

\begin{tabular}{|c|c|c|c|c|c|c|c|c|c|}
\hline \multicolumn{10}{|c|}{ Employee Discipline } \\
\hline$\square$ Doctor & $\square$ & Attending & $\square$ & Fellow & $\square$ & Residents & $\square$ & Interns & $\begin{array}{l}\text { Medical } \\
\text { Student }\end{array}$ \\
\hline$\square$ Nurse & $\square$ & $\mathrm{RN}$ & $\square$ & LPN & $\square$ & Nurse Assistant & $\square$ & Nurse Student & \\
\hline
\end{tabular}

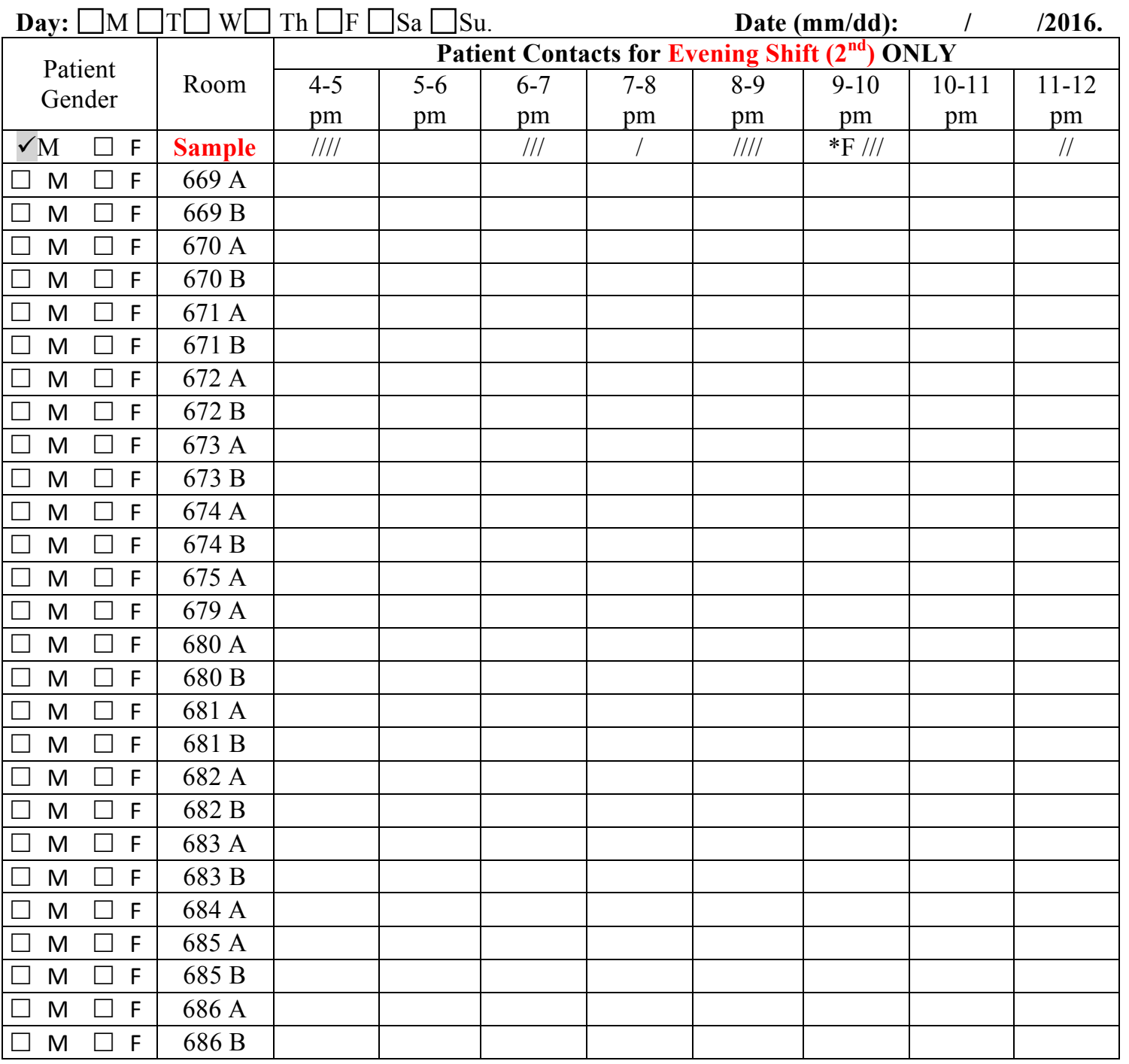




\section{APPENDIX E: 6B Patient-Contact Night Shift Data Sheet UNIT: 6B ONLY}

Instructions:-

Please mark a tally (/) every time you Contact your patient in the correct cell. For example, if you need to contact a patient who is a Male in room 669, bed (A) at 1:20 a.m., mark a tally (/) on that cell below Time 1:00 a.m. and in row Room 669 A.
Feet-to-Feet Patient Room:
Window-to-Door Patient Room:
Bed (A): Left
Bed (A): Door
Bed (B): Window
Note: If a new patient is admitted, please draw a star
Bed (B): Right
(*). Add $\mathrm{M}$ or $\mathrm{F}$ if gender is different $(* \mathrm{M})$ or $(* \mathrm{~F})$.

Employee Code:

Employee Gender: $\square$ M $\square$ F

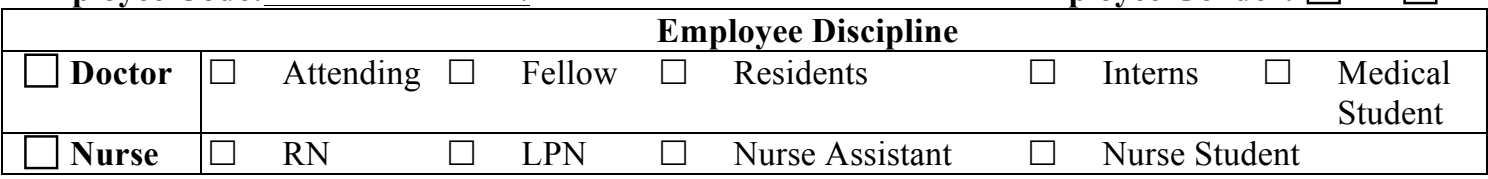

Day: $\square \mathrm{M} \square \mathrm{T} \square \mathrm{W} \square$ Th $\square \mathrm{F} \square \mathrm{Sa} \square$ Su. $\quad$ Date (mm/dd): $\quad / \quad 12016$.

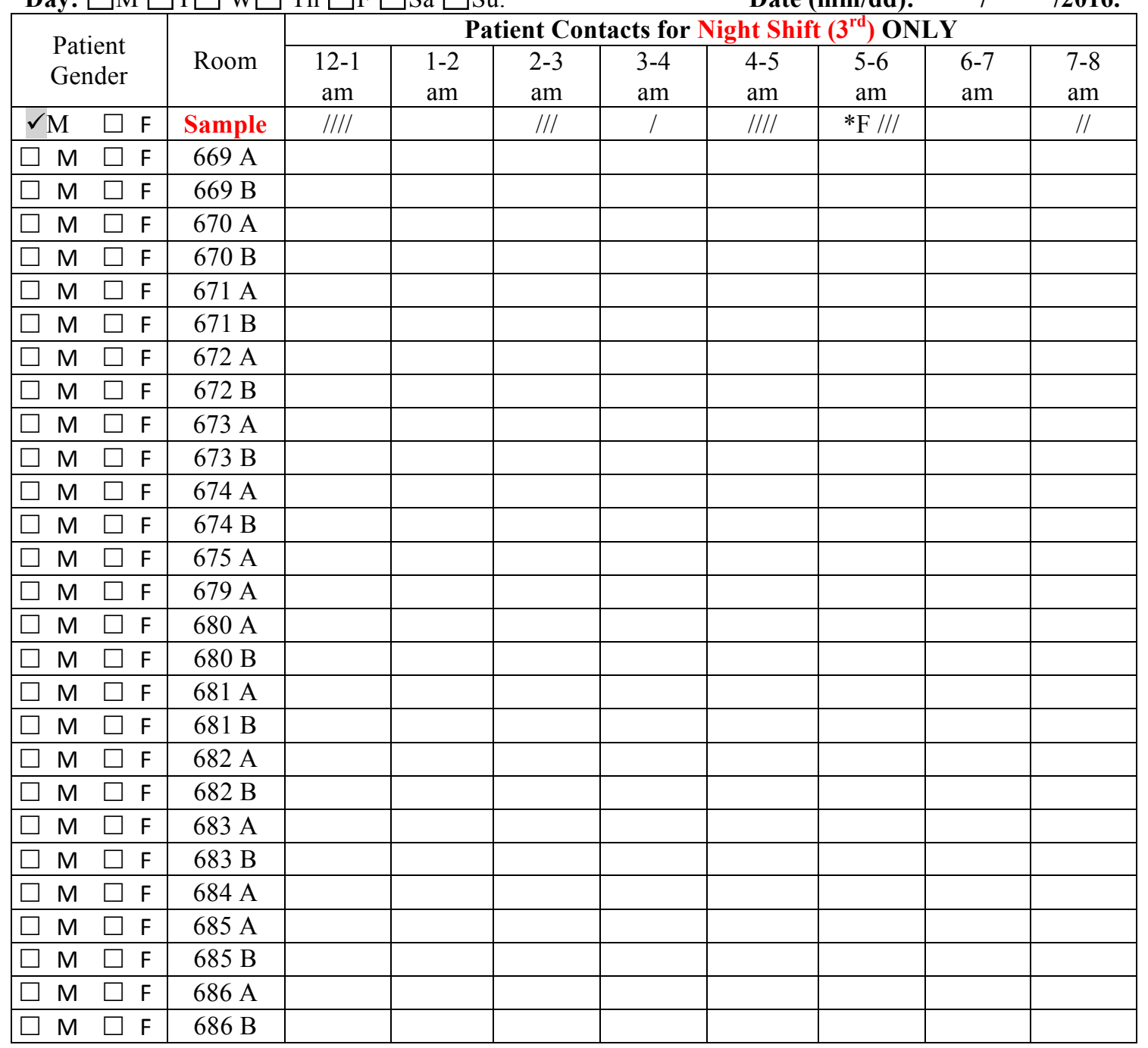




\section{APPENDIX F: 5B Patient-Contact Day Shift Data Sheet UNIT: 5B ONLY}

Instructions:-

Please mark a tally (/) every time you Contact your patient in the correct cell. For example, if you need to contact a patient who is a Male in room 569, bed (A) at 10:20 a.m., mark a tally (/) on that cell below Time 10:00 a.m. and in row Room 569 A.
Feet-to-Feet Patient Room:
Window-to-Door Patient Room:
Bed (A): Left
Bed (A): Door
Bed (B): Right
Bed (B): Window
Note: If a new patient is admitted, please draw a star (*). Add $\mathrm{M}$ or $\mathrm{F}$ if gender is different $(* \mathrm{M})$ or $\left({ }^{*} \mathrm{~F}\right)$.

Employee Code:

Employee Gender: $\square$ M $\square$ F

\begin{tabular}{|l|lllllllll|}
\hline \multicolumn{10}{|c|}{ Employee Discipline } \\
\hline$\square$ Doctor & $\square$ & Attending & $\square$ & Fellow & $\square$ & Residents & $\square$ & Interns & $\begin{array}{l}\text { Medical } \\
\text { Student }\end{array}$ \\
\hline$\square$ Nurse & $\square$ & RN & $\square$ & LPN & $\square$ & Nurse Assistant & $\square$ & Nurse Student \\
\hline
\end{tabular}

Day: $\square \mathrm{M} \square \mathrm{T} \square \mathrm{W} \square$ Th $\square \mathrm{F} \square \mathrm{Sa} \square \mathrm{Su} . \quad$ Date (mm/dd): / / $/ 2016$

\begin{tabular}{|c|c|c|c|c|c|c|c|c|c|}
\hline \multirow{2}{*}{$\begin{array}{l}\text { Patient } \\
\text { Gender }\end{array}$} & \multirow[b]{2}{*}{ Room } & \multicolumn{8}{|c|}{ Patient Contacts for Day Shift $\left(1^{\text {st }}\right)$ ONLY } \\
\hline & & $\begin{array}{l}8-9 \\
\text { am }\end{array}$ & $\begin{array}{c}9-10 \\
\text { am }\end{array}$ & $\begin{array}{c}10-11 \\
\text { am }\end{array}$ & $\begin{array}{c}11-12 \\
\mathrm{pm}\end{array}$ & $\begin{array}{c}12-1 \\
\mathrm{pm}\end{array}$ & $\begin{array}{l}1-2 \\
\mathrm{pm}\end{array}$ & $\begin{array}{l}2-3 \\
\mathrm{pm}\end{array}$ & $\begin{array}{l}\text { 3-4 } \\
\text { pm }\end{array}$ \\
\hline$\checkmark \mathrm{M} \quad \square \mathrm{F}$ & Sample & $/ / / /$ & & $/ / /$ & l & $/ / / /$ & $* \mathrm{~F} / / /$ & & $/ /$ \\
\hline$\square \mathrm{M} \square \mathrm{F}$ & $569 \mathrm{~A}$ & & & & & & & & \\
\hline$\square \mathrm{M} \square \mathrm{F}$ & $569 \mathrm{~B}$ & & & & & & & & \\
\hline$\square \mathrm{M} \square \mathrm{F}$ & $570 \mathrm{~A}$ & & & & & & & & \\
\hline$\square \mathrm{M} \square \mathrm{F}$ & $570 \mathrm{~B}$ & & & & & & & & \\
\hline$\square \mathrm{M} \square \mathrm{F}$ & $571 \mathrm{~A}$ & & & & & & & & \\
\hline$\square \mathrm{M} \square \mathrm{F}$ & $571 \mathrm{~B}$ & & & & & & & & \\
\hline$\square \mathrm{M} \square \mathrm{F}$ & $572 \mathrm{~A}$ & & & & & & & & \\
\hline$\square \mathrm{M} \square \mathrm{F}$ & $572 \mathrm{~B}$ & & & & & & & & \\
\hline$\square \mathrm{M} \square \mathrm{F}$ & $573 \mathrm{~A}$ & & & & & & & & \\
\hline$\square \mathrm{M} \square \mathrm{F}$ & $573 \mathrm{~B}$ & & & & & & & & \\
\hline$\square \mathrm{M} \square \mathrm{F}$ & $575 \mathrm{~A}$ & & & & & & & & \\
\hline$\square \mathrm{M} \square \mathrm{F}$ & $583 \mathrm{~A}$ & & & & & & & & \\
\hline$\square \mathrm{M} \square \mathrm{F}$ & $584 \mathrm{~A}$ & & & & & & & & \\
\hline$\square \mathrm{M} \square \mathrm{F}$ & $584 \mathrm{~B}$ & & & & & & & & \\
\hline$\square \mathrm{M} \square \mathrm{F}$ & $585 \mathrm{~A}$ & & & & & & & & \\
\hline$\square \mathrm{M} \square \mathrm{F}$ & $585 \mathrm{~B}$ & & & & & & & & \\
\hline$\square \mathrm{M} \square \mathrm{F}$ & $586 \mathrm{~A}$ & & & & & & & & \\
\hline$\square \mathrm{M} \square \mathrm{F}$ & $586 \mathrm{~B}$ & & & & & & & & \\
\hline$\square \mathrm{M} \square \mathrm{F}$ & $588 \mathrm{~A}$ & & & & & & & & \\
\hline$\square \mathrm{M} \square \mathrm{F}$ & $589 \mathrm{~A}$ & & & & & & & & \\
\hline$\square \mathrm{M} \square \mathrm{F}$ & $589 \mathrm{~B}$ & & & & & & & & \\
\hline$\square \mathrm{M} \square \mathrm{F}$ & $590 \mathrm{~A}$ & & & & & & & & \\
\hline$\square \mathrm{M} \square \mathrm{F}$ & $590 \mathrm{~B}$ & & & & & & & & \\
\hline
\end{tabular}




\section{APPENDIX G: 5B Patient-Contact Evening Shift Data Sheet UNIT: 5B ONLY}

Instructions:-

Please mark a tally (/) every time you Contact your patient in the correct cell. For example, if you need to contact a patient who is a Male in room 569, bed (A) at 7:20 p.m., mark a tally (/) on that cell below Time 7:00 p.m. and in row Room 569 A.
Feet-to-Feet Patient Room:
Window-to-Door Patient Room:
Bed (A): Left
Bed (A): Door
Bed (B): Right
Bed (B): Window
Note: If a new patient is admitted, please draw a star (*). Add $\mathrm{M}$ or $\mathrm{F}$ if gender is different $(* \mathrm{M})$ or $\left({ }^{*} \mathrm{~F}\right)$.

Employee Code:

Employee Gender: $\square$ M $\square$ F

\begin{tabular}{|c|c|c|c|c|c|c|c|c|}
\hline \multicolumn{9}{|c|}{ Employee Discipline } \\
\hline$\square$ Doctor & $\square$ & Attending $\square$ & Fellow & $\square \quad$ Residents & $\square$ & Interns & $\square$ & $\begin{array}{l}\text { Medical } \\
\text { Student }\end{array}$ \\
\hline$\square$ Nurse & $\square$ & $\mathrm{RN}$ & LPN & Nurse Assistant & $\square$ & Nurse $S$ & lent & \\
\hline
\end{tabular}

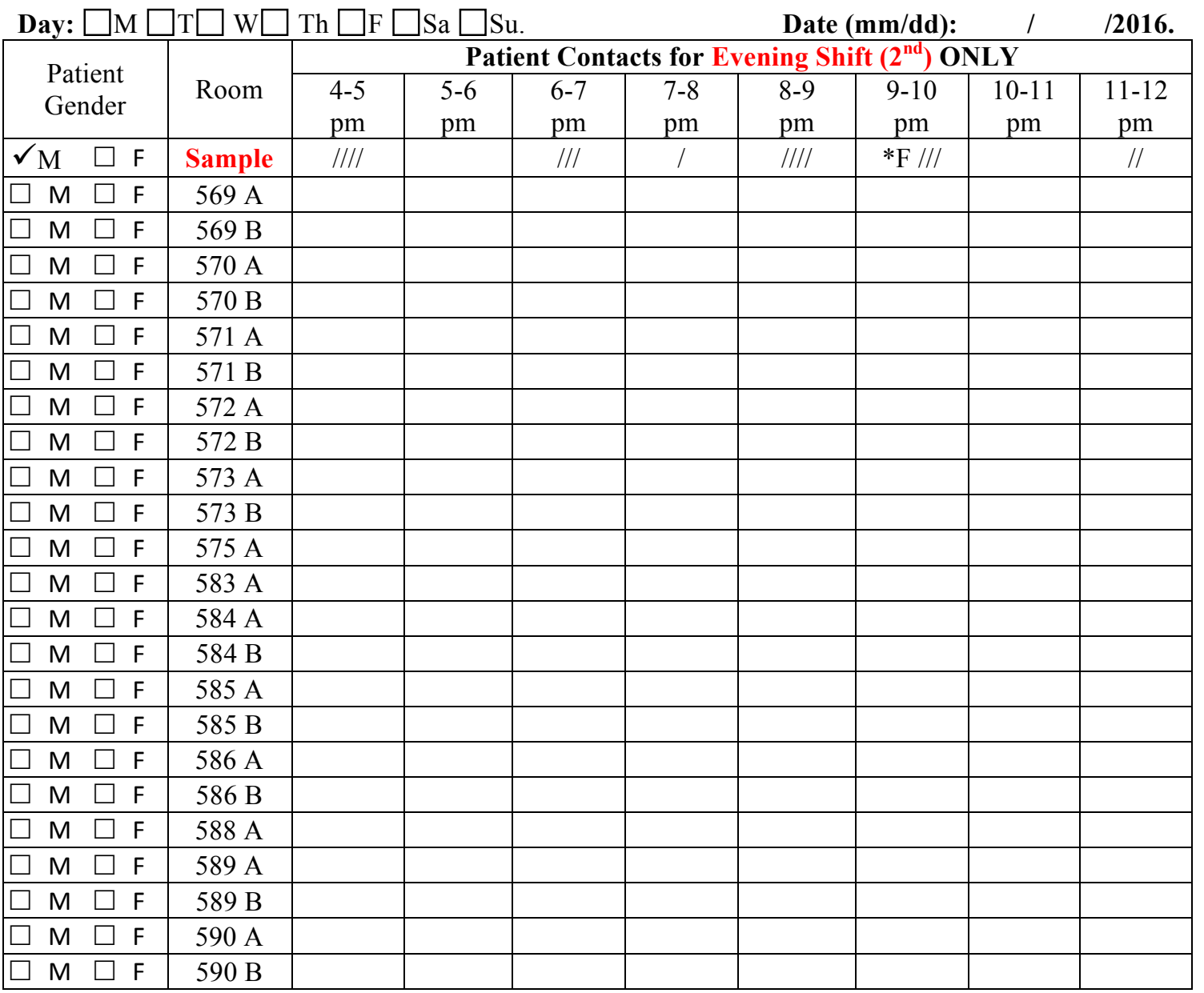




\section{APPENDIX H: 5B Patient-Contact Night Shift Data Sheet UNIT: 5B ONLY}

Instructions:-

Please mark a tally (/) every time you Contact your patient in the correct cell. For example, if you need to contact a patient who is a Male in room 569, bed (A) at 1:20 a.m., mark a tally (/) on that cell below Time 1:00 a.m. and in row Room 569 A.
Feet-to-Feet Patient Room: Window-to-Door Patient Room: Note: If a new patient is
Bed (A): Left $\quad$ Bed (A): Door
Bed (B): Right Bed (B): Window

Employee Code:

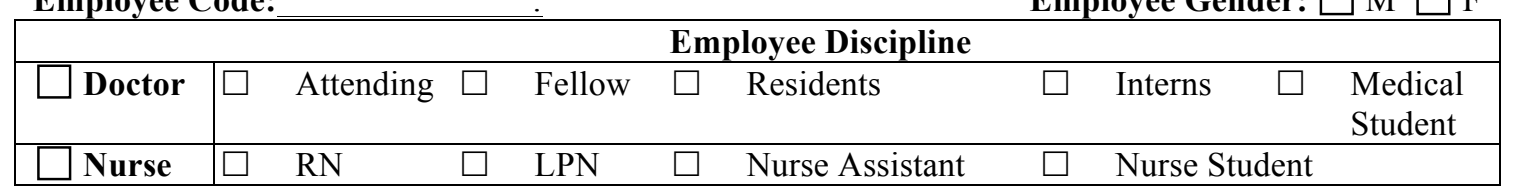

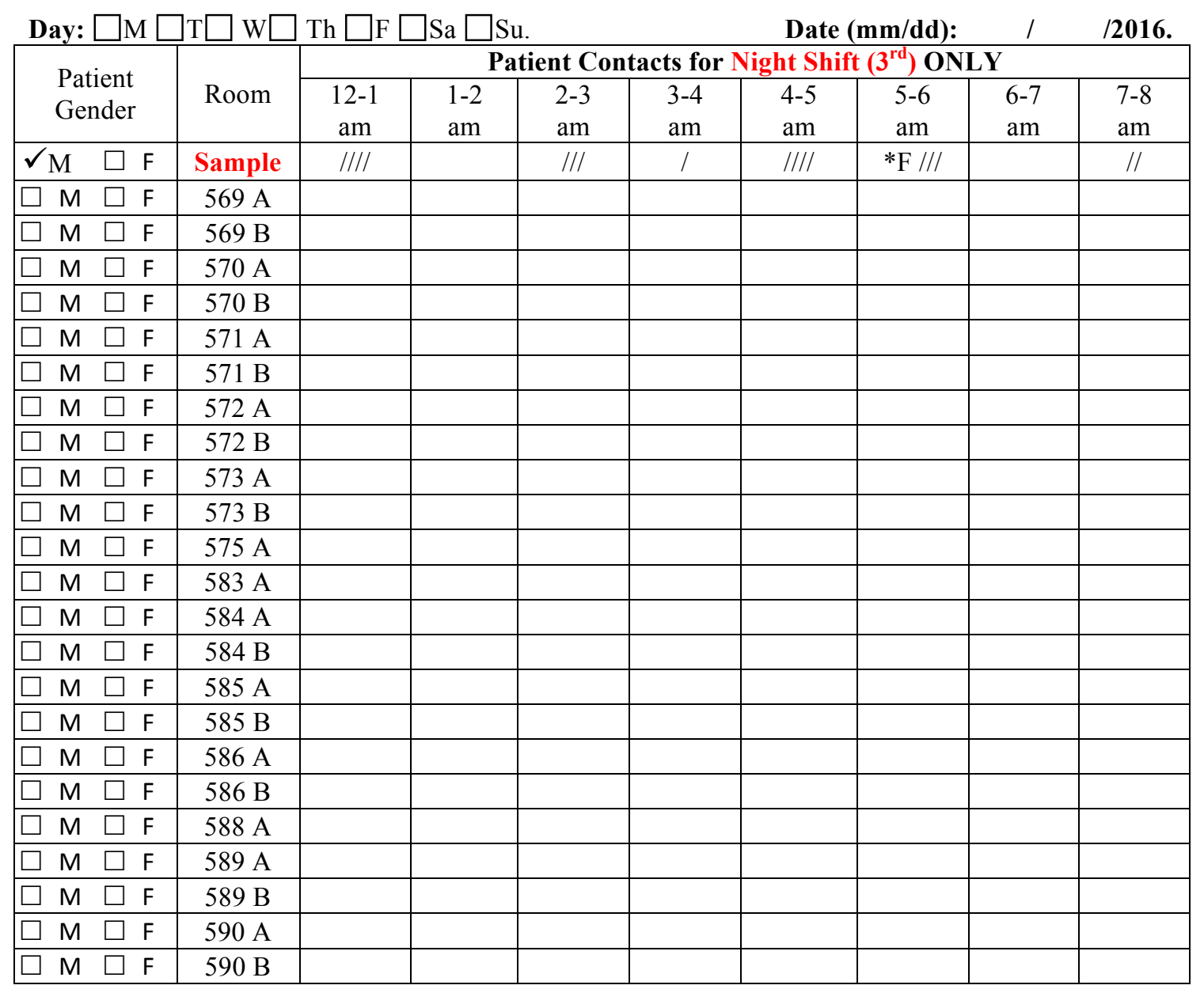




\section{APPENDIX I: ICU Patient-Contact Day Shift Data Sheet UNIT: ICU ONLY}

Instructions:-

Please mark a tally (/) every time you Contact your patient in the correct cell. For example, if you need to contact a patient who is a Male in bed 624-1 at 10:20 a.m., mark a tally ( $/$ ) on that cell below Time 10:00 a.m. and in row Bed 624-1.

$\begin{array}{lll}\text { Feet-to-Feet Patient Room: } & \text { Window-to-Door Patient Room: } & \text { Note: If a new patient is } \\ \text { Bed (A): Left } & \text { Bed (A): Door } & \text { admitted, please draw a star } \\ \text { Bed (B): Right } & \text { Bed (B): Window } & (*) \text {. Add M or F if gender is } \\ & & \text { different }(* \mathrm{M}) \text { or }(* \mathrm{~F}) .\end{array}$

Employee Code:

Employee Gender: $\square$ M $\square$ F

\begin{tabular}{|l|lllllllll|}
\hline \multicolumn{10}{|c|}{ Employee Discipline } \\
\hline$\square$ Doctor & $\square$ & Attending & $\square$ & Fellow & $\square$ & Residents & $\square$ & Interns & $\begin{array}{l}\text { Medical } \\
\text { Student }\end{array}$ \\
\hline$\square$ Nurse & $\square$ & RN & $\square$ & LPN & $\square$ & Nurse Assistant & $\square$ & Nurse Student \\
\hline
\end{tabular}

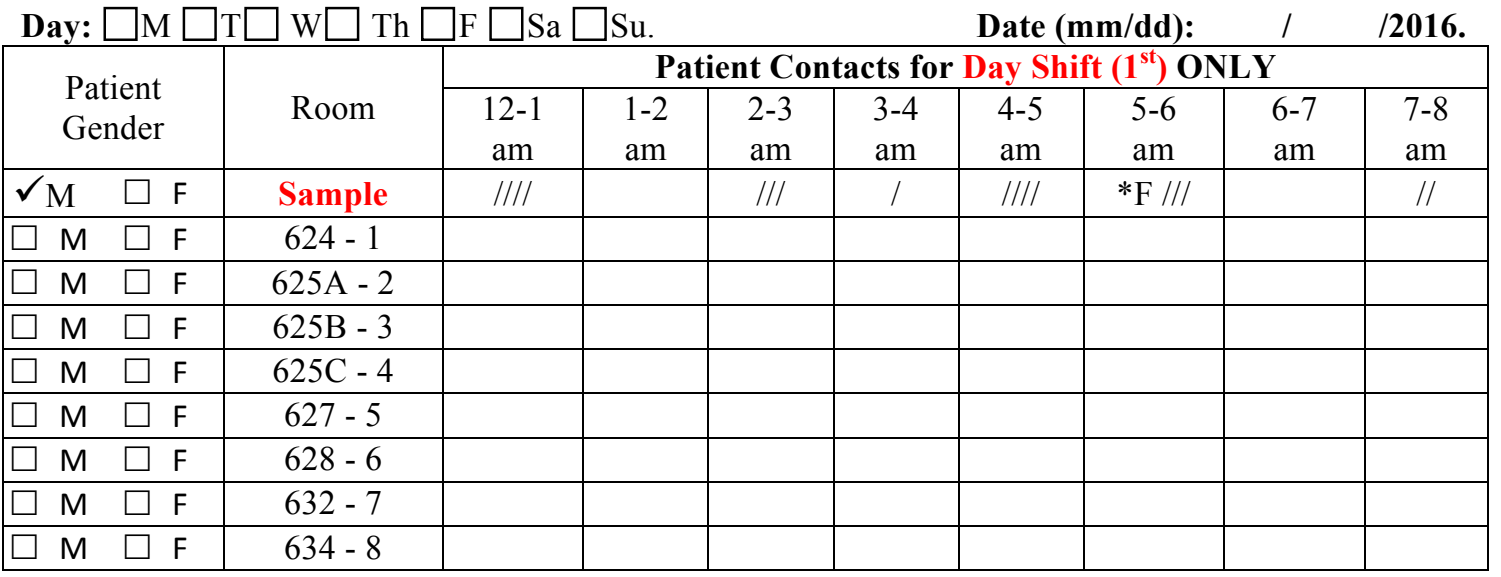




\section{APPENDIX J: ICU Patient-Contact Evening Shift Data Sheet UNIT: ICU ONLY}

Instructions:-

Please mark a tally ( / ) every time you Contact your patient in the correct cell. For example, if you need to contact a patient who is a Male in bed 624-1 at 7:20 p.m., mark a tally (/) on that cell below Time 7:00 p.m. and in row Bed 624-1.

Feet-to-Feet Patient Room: Window-to-Door Patient Room: Note: If a new patient is Bed (A): Left

Bed (A): Door

Bed (B): Right admitted, please draw a star

(*). Add $\mathrm{M}$ or $\mathrm{F}$ if gender is different $(* \mathrm{M})$ or $\left({ }^{*} \mathrm{~F}\right)$.

Employee Code:

Employee Gender: $\square$ M $\square$ F

\begin{tabular}{|c|c|c|c|c|c|c|c|c|c|}
\hline \multicolumn{10}{|c|}{ Employee Discipline } \\
\hline$\square$ Doctor & $\square$ & Attending $\square$ & Fellow & $\square$ & Residents & $\square$ & Interns & $\square$ & $\begin{array}{l}\text { Medical } \\
\text { Student }\end{array}$ \\
\hline$\square$ Nurse & $\square$ & $\mathrm{RN}$ & $\mathrm{LPN}$ & $\square$ & Nurse Assistant & $\square$ & Nurse & dent & \\
\hline
\end{tabular}

Day: $\square \mathrm{M} \square \mathrm{T} \square \mathrm{W} \square \mathrm{Th} \square \mathrm{F} \square \mathrm{Sa} \square \mathrm{Su} . \quad$ Date (mm/dd): $\quad / \quad / 2016$.

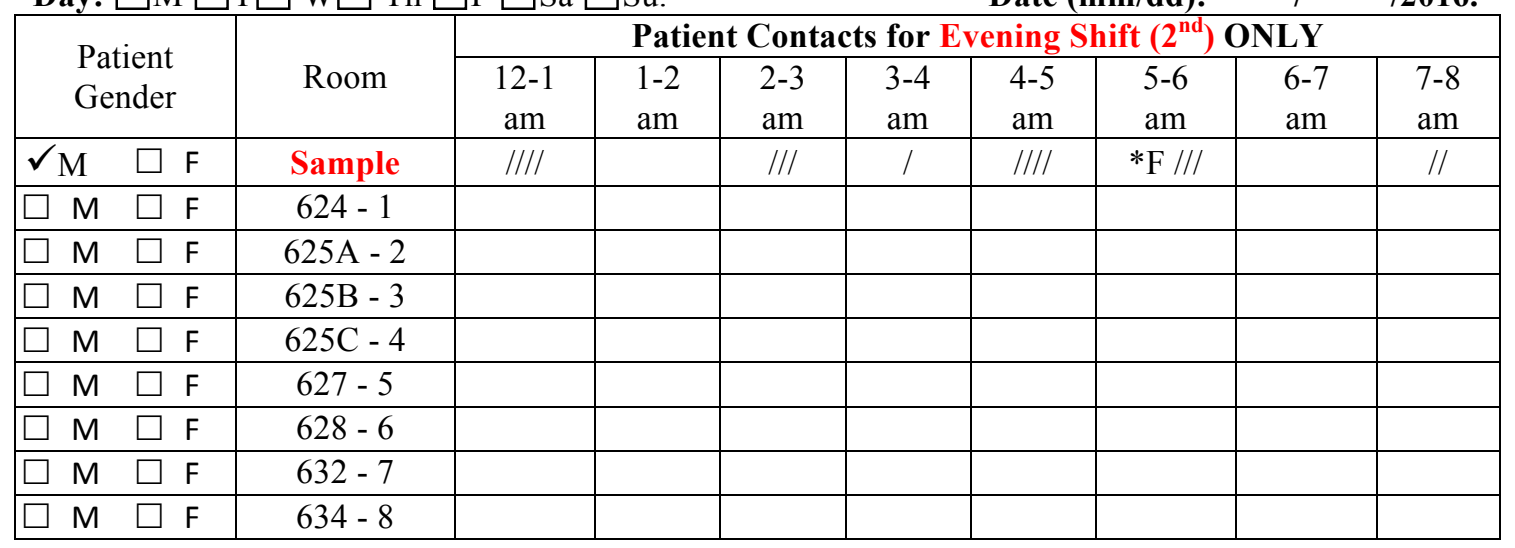




\section{APPENDIX K: ICU Patient-Contact Night Shift Data Sheet UNIT: ICU ONLY}

Instructions:-

Please mark a tally (/) every time you Contact your patient in the correct cell. For example, if you need to contact a patient who is a Male in bed 624-1 at 1:20 a.m., mark a tally ( /) on that cell below Time 1:00 a.m. and in row Bed 624-1.

Feet-to-Feet Patient Room: Window-to-Door Patient Room: Note: If a new patient is Bed (A): Left

Bed (A): Door

Bed (B): Right
Bed (B): Window admitted, please draw a star

(*). Add $\mathrm{M}$ or $\mathrm{F}$ if gender is different $(* \mathrm{M})$ or $\left({ }^{*} \mathrm{~F}\right)$.

Employee Code:

Employee Gender: $\square$ M $\square$ F

\begin{tabular}{|c|c|c|c|c|c|c|c|c|}
\hline \multicolumn{9}{|c|}{ Employee Discipline } \\
\hline$\square$ Doctor & $\square$ & Attending & $\square$ & Fellow & Residents & $\square$ & Interns & $\begin{array}{l}\text { Medical } \\
\text { Student }\end{array}$ \\
\hline$\square$ Nurse & $\square$ & $\mathrm{RN}$ & $\square$ & $\mathrm{LPN}$ & Nurse Assistant & $\square$ & Nurse Student & \\
\hline
\end{tabular}

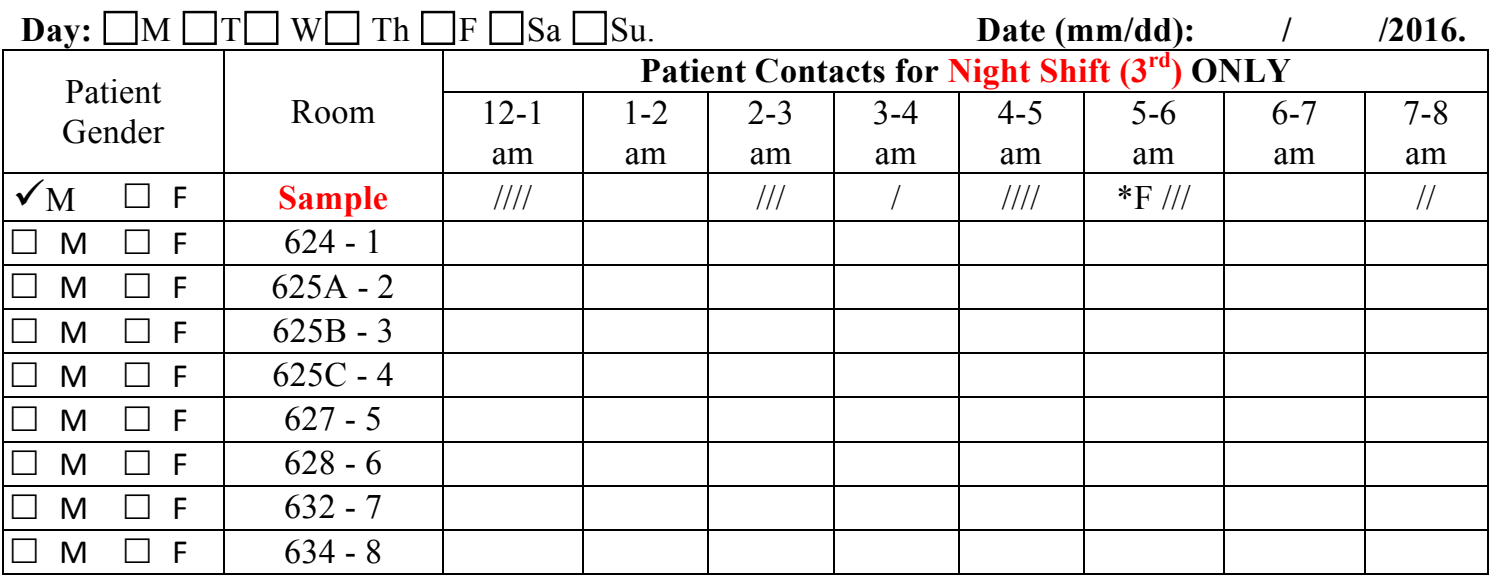




\section{APPENDIX L: Patient Contact Consent Form}

\begin{tabular}{l}
$\begin{array}{l}\text { RESEARCH CONSENT FORM } \\
\text { Providence VA Medical Center } \\
\text { IRB \# } 00001402\end{array}$ \\
$\begin{array}{l}\text { Subject Name: } \\
\text { Title of Study: Assessing Hand Hygiene Compliance in Healthcare Workers to Reduce Infectious Disease- Pt Contact } \\
\text { Principal Investigator: Associate Prof. Valerie Maier-Speredelozzi } \\
\text { Study Sponsor (if applicable): }\end{array}$ \\
\hline
\end{tabular}

1. Purpose of study and how long it will last:

The purpose of this research study is to obtain a more accurate estimate of the total number of times per week that a doctor or a nurse contacts different patients at the Providence Veterans Affairs Medical Center.

\section{Description of the study including procedures to be used:}

If you decide to take part in this study, you will be asked to complete a patient-contact form for the inpatient unit $(5 \mathrm{~B}, 6 \mathrm{~B}$ and ICU) where you work. Each time you enter a patient room, you will be asked to put a tally mark (/) on a data collection sheet, for the room number, bed, and patient gender. This form should take approximately 1-2 minutes per patient-contact. You will be asked to document each patient contact during your entire shift for 1 entire week, and for an additional 1-3 weeks later in the year.

3. Description of any procedures that may result in discomfort or inconvenience: There are not any foreseeable discomforts associated with the study.

\section{Expected risks of study:}

There are not any foreseeable risks associated with the study. The decision to take part in this study is entirely voluntary and your employer will not know what you decide. Your responses will not be reported with your name or any identifying information other than your workgroup code. All form fields should be completed. If you decide to take part in the study, you may quit at any time.

\section{Expected benefits of study:}

Although there is no direct benefit to you for taking part in this study, the researcher may learn more about assessing hand hygiene. Thus, the research findings may benefit the hospital in general.

\section{Other treatment(s) available:}

There are not alternative treatments associated with this study.

\section{Costs to participants and compensation:}

Costs to Participants: There is no cost to participate in the study, other than the time needed to collect the required dataset.

Compensation Offered for Participation: There are no incentives, payment or gift certificates offered for completing the study forms.

\section{Use of research results:}


Title of Study: Assessing Hand Hygiene Compliance in Healthcare Workers to Reduce Infectious Disease- Pt Contact

Principal Investigator: Associate Prof. Valerie Maier-Speredelozzi

Study Sponsor (if applicable):

Your participation in this study is confidential. The spreadsheet that contains names and unique codes of all participants, and data collected will be electronically stored in a restricted folder on the secure server at the PVAMC. Only the research team will have access to the restricted folder. All physical forms will be kept secured in a double locked cabinet at the Providence VA Medical Center after the study is done and are only accessible by the research team.

RESEARCH PARTICIPANT'S RIGHTS: I have read or have had read to me all of the above.

The Study Staff has explained the study to me and answered all of my questions. I have been told of the risks or discomforts and possible benefits of the study. I have been told of other choices of treatment available to me.

I have been told that I do not have to take part in this study, and my refusal to participate will involve no penalty or loss of rights to which I am entitled. I may withdraw from this study at any time without penalty or loss of VA or other benefits to which I am entitled.

The results of this study may be published, but my records will not be revealed unless required by law. The Institutional Review Board at the Providence VA Medical Center or other federal oversight offices may monitor my records for quality assurance purposes. Federal agencies including, but not limited to, the Food and Drug Administration (FDA), the Office for Human Research Protection (OHRP), the Office for Research Oversight (ORO), the Office of the Inspector General for Human Research Protection (OHRP), the Office for Research Oversight (ORO), the Office of the Inspector Gener
$(\mathrm{OIG})$ and the Government Accounting Office (GAO) may have access to the records as allowed by law. If an FDA(OIG) and the Government Accounting Office (GAO) may have access to the records as allowed by law. If an FDA-
regulated test article is part of this study, the FDA may choose to inspect research records that include research subject's individual medical records. Records will be maintained in accordance with the Department of Veterans Affairs Record Control Schedule 10-1.

If I experience a side effect or adverse (bad or unexpected) reaction as a result of my involvement in this study, I will report these to the study investigator Associate Professor Valerie Maier-Speredelozzi at (401) 874-5187 who will arrange for any medical treatment that is necessary. After hours, I will call the operator at (401) 273-7100 and ask to speak to the infectious disease physician on call.

In case there are medical problems or questions, I have been told I can call Dr. Melissa Gaitanis at (401) 273-7100 extension $\underline{3609}$ during the day. or After hours, I will call the operator at (401) 273-7100 and ask to speak to the infectious disease physician on call. If any medical problems occur in connection with this study the VA will provide emergency care.

The VA has the authority to provide medical treatment to participants (veterans and non-veterans) injured by participation in a VA study. If you are injured as a result of being in this study, the VA will provide the necessary medical treatment in accordance with federal law. If you want to make a legal claim against the VA or anyone who works for the VA, special laws may apply. The Federal Tort Claims Act (28 U.S.C. 1346(b), 2671-2680) is a federal law that controls when and how a person can bring a claim against the U.S. Government. If you sign this document you are not giving up your right to make a legal claim against the United States.

I can call the IRB Coordinator at (401) 273-7100 ext. 3470, the Research Administrative Officer at (401) 273-7100 ext. 3478 or the Providence VAMC Patient Advocate at (401) 273-7100 ext. 3093 while I am a participant or after my

Approval Date: July 26, 2016 Expiration Date: July 12, 2017 $\quad$ Page $\mathbf{2}$ of 3

Form Version: 4/10/2015 
Date:

Title of Study: Assessing Hand Hygiene Compliance in Healthcare Workers to Reduce Infectious Disease- Pt Contact

Principal Investigator: Associate Prof. Valerie Maier-Speredelozzi

Study Sponsor (if applicable):

participation is over for the following: 1) concerns, 2) complaints, 3) problems, 4) suggestions, 5) more information, 6) questions about my rights as a research participant or 7) verifying the validity of the study and authorized contacts.

I voluntarily consent to participate in this study. I confirm that I have read this consent form or it has been read to me and I agree it explains what this study is about and how and why it is being done. I will receive a signed copy of the consent form document after I sign it.

Participant's Signature

Signature of Person Obtaining Consent
Participant (printed)

Person Obtaining Consent (printed)
Date

Date

Version Date: 7/26/2016

Approval Date: July 26, 2016

Form Version: 4/10/2015
Expiration Date: July 12, 2017

Page $\mathbf{3}$ of $\mathbf{3}$ 


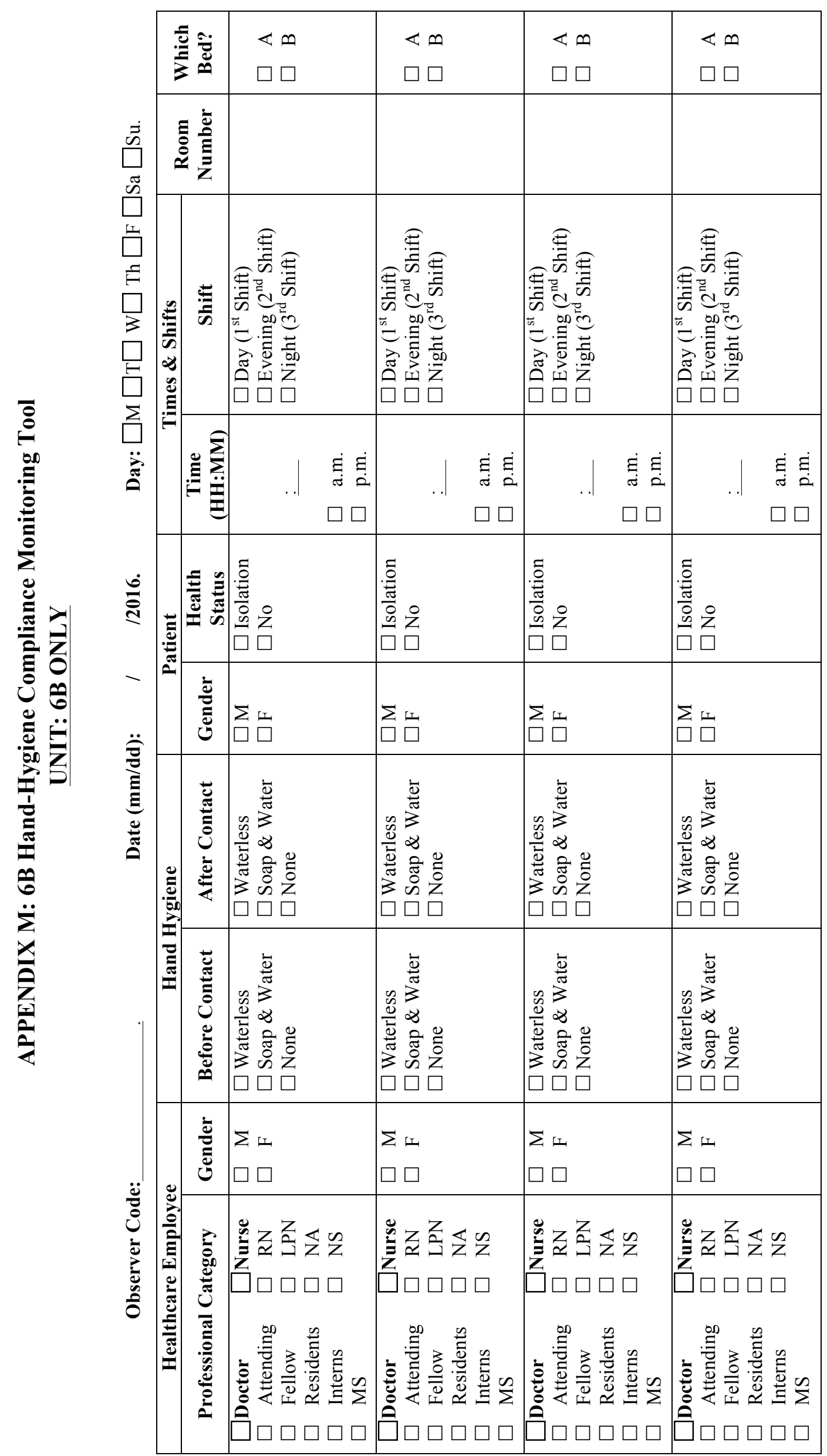




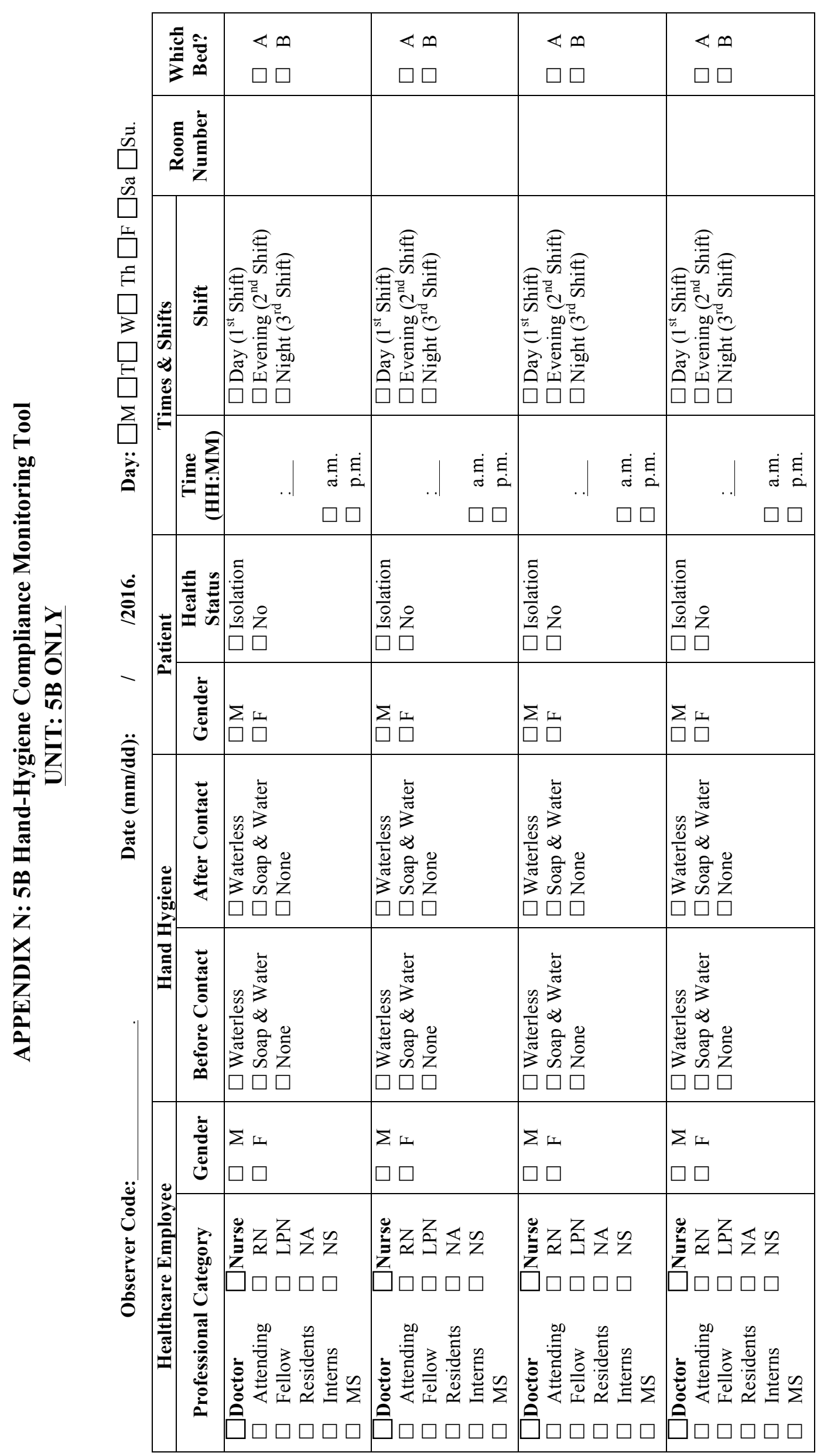




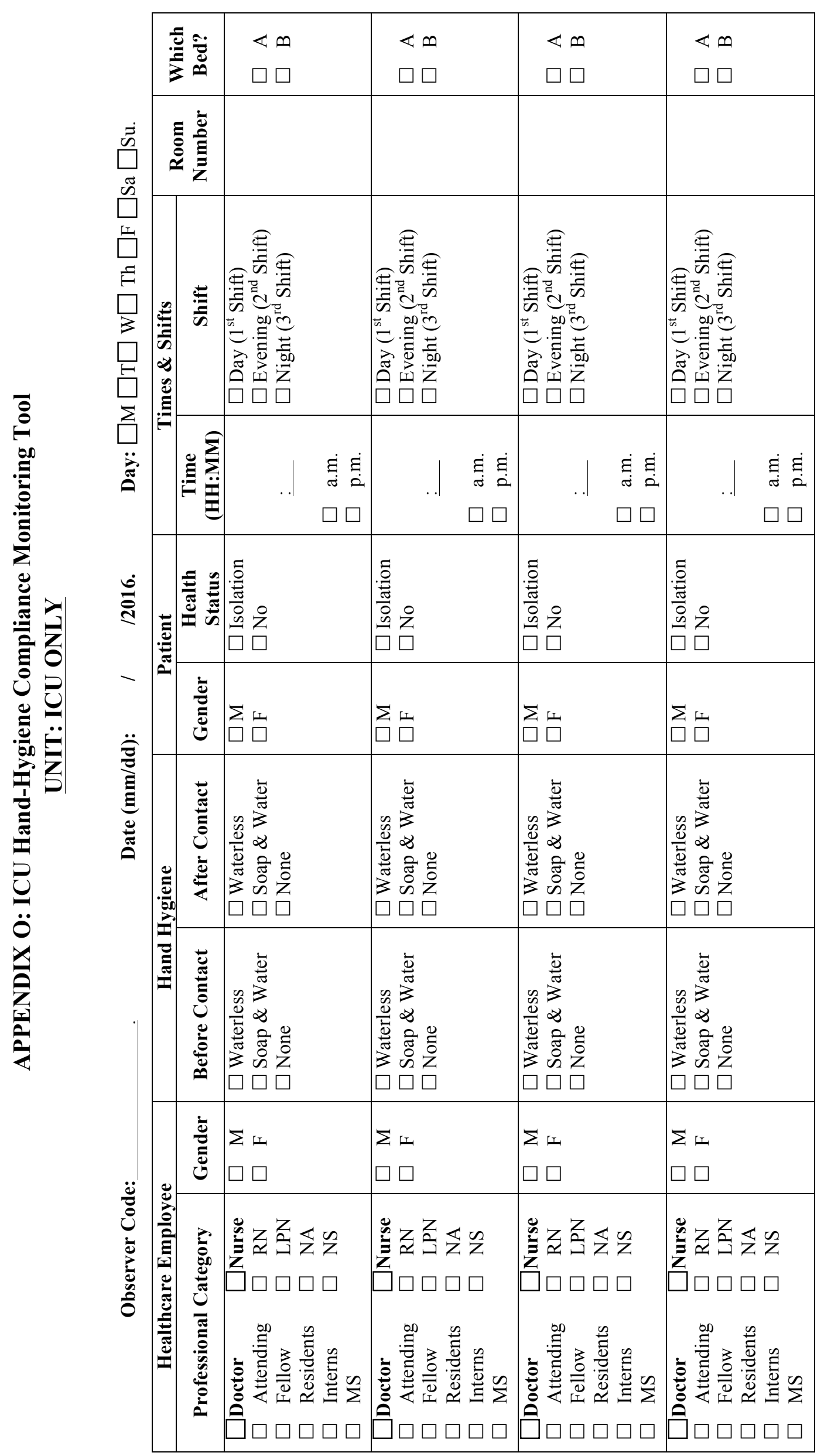




\section{APPENDIX P: Hand Hygiene and Precautions Compliance Monitoring Tool}

Hand Hygiene and Precautions Compliance Monitoring Tool

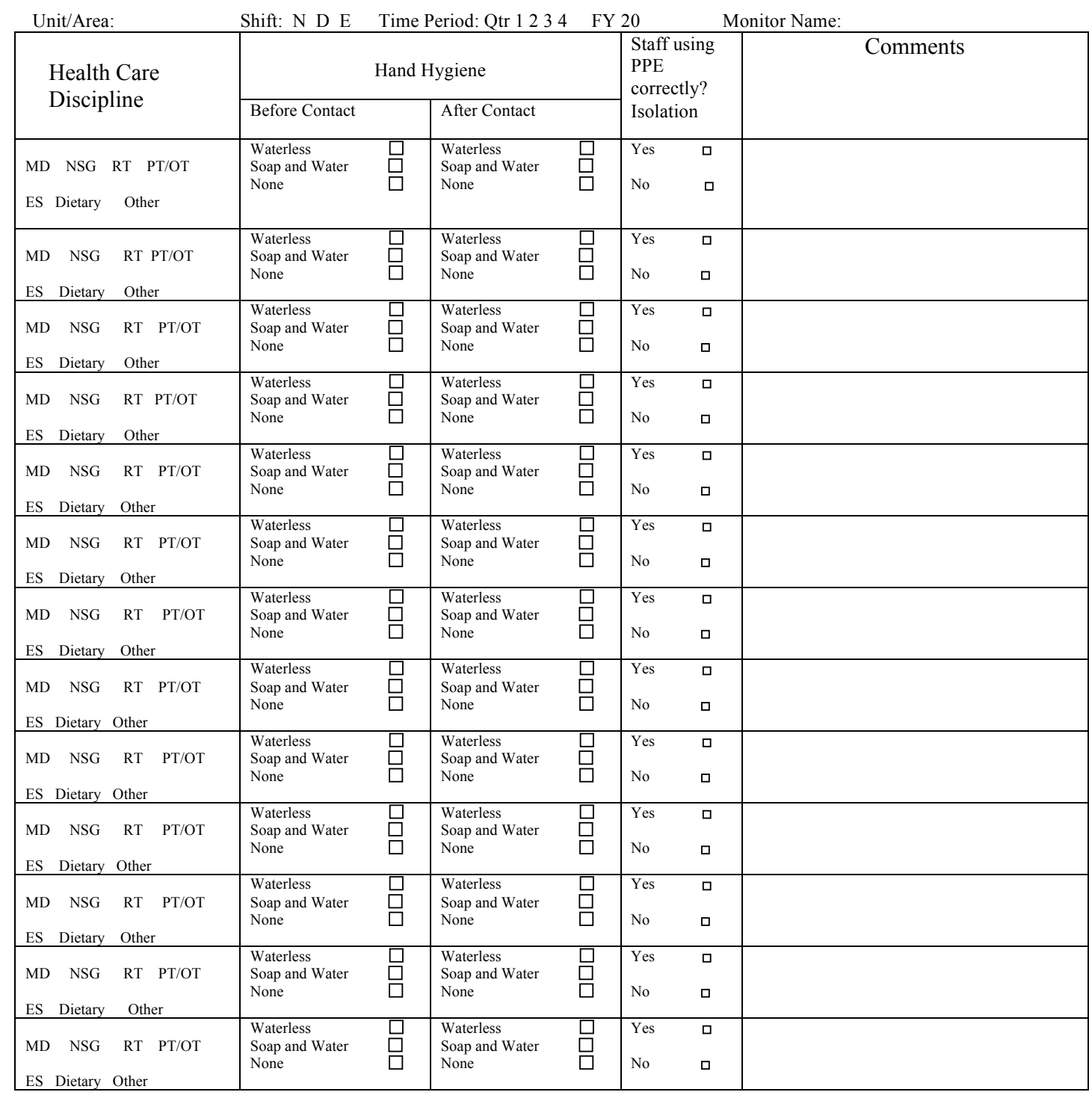

No. HH Contacts Performed Correctly: Total Hand Hygiene Observations: Recommendations:
No. Correct Precaution Episodes: Total Precaution Episodes Observed:

Report sent to:

Date: 


\section{APPENDIX Q: Hand Hygiene and Precautions Compliance Monitoring Tool with Stethoscope}

Hand Hygiene and Precautions Compliance Monitoring Tool

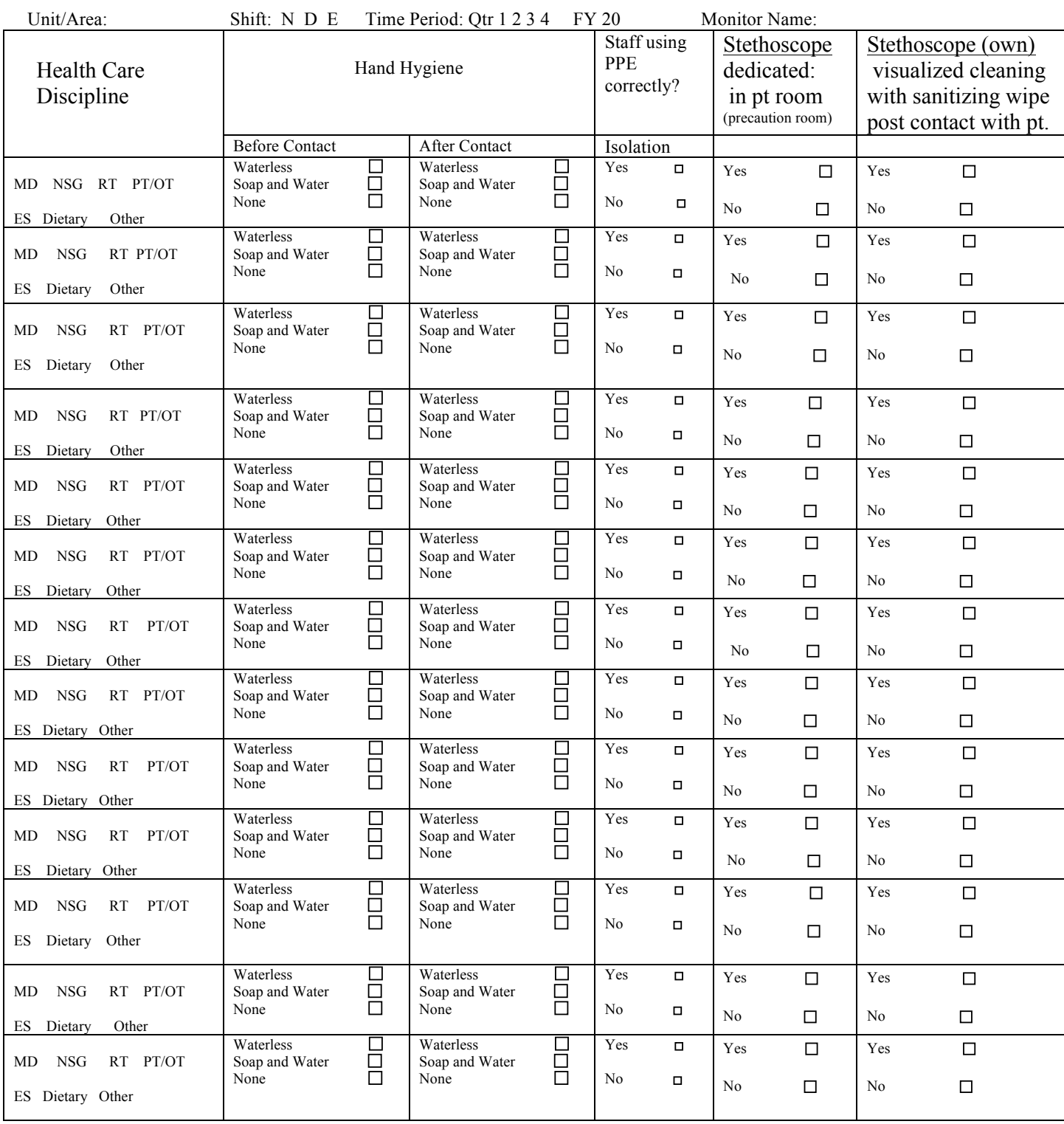

No. HH Contacts Performed Correctly:

Total Hand Hygiene Observations:

Recommendations:
No. Correct Precaution Episodes:

Total Precaution Episodes Observed:

Report sent to:

Date: 
APPENDIX R: Hand Hygiene Management Site

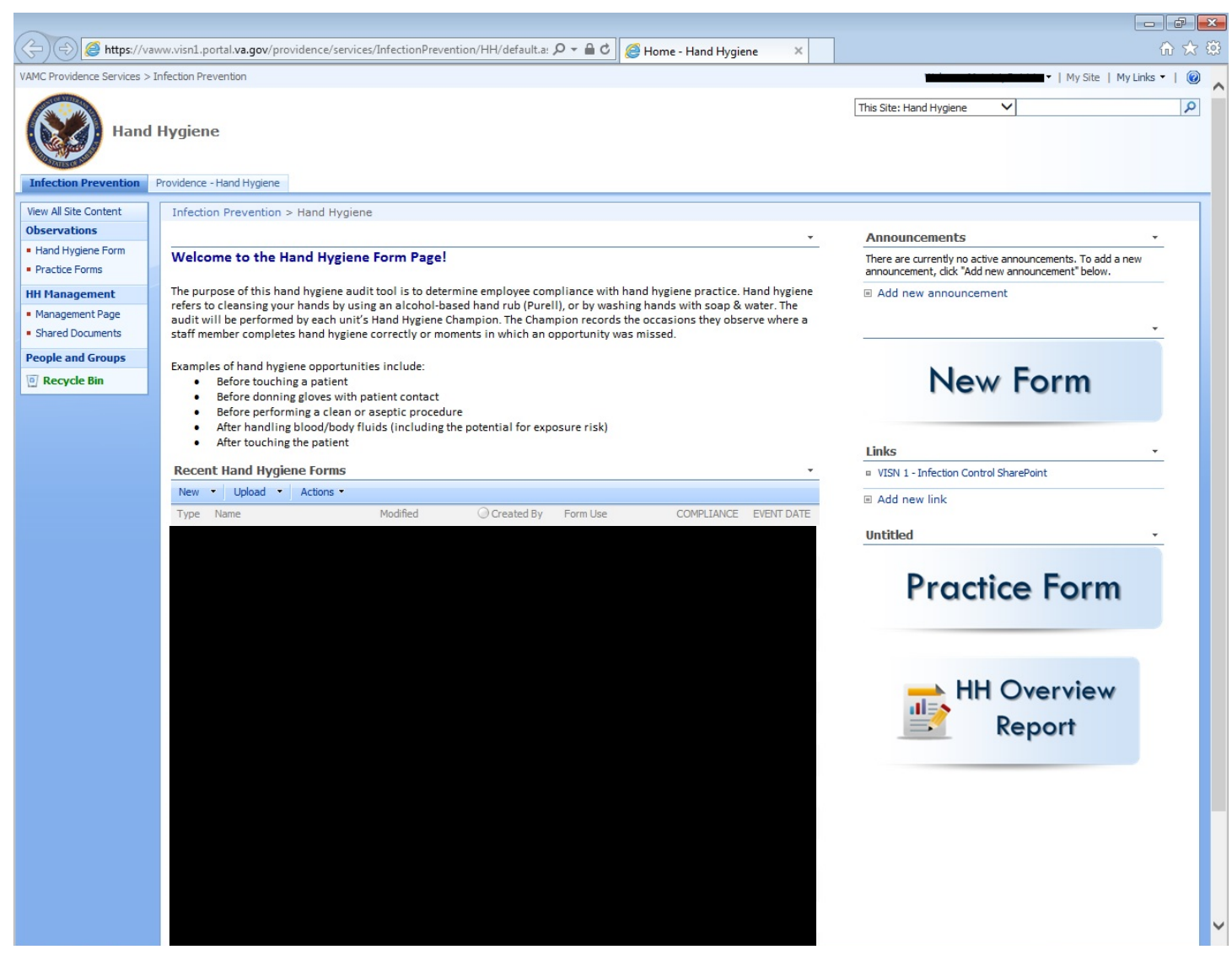


APPENDIX S: Hand Hygiene Event Data Input Form

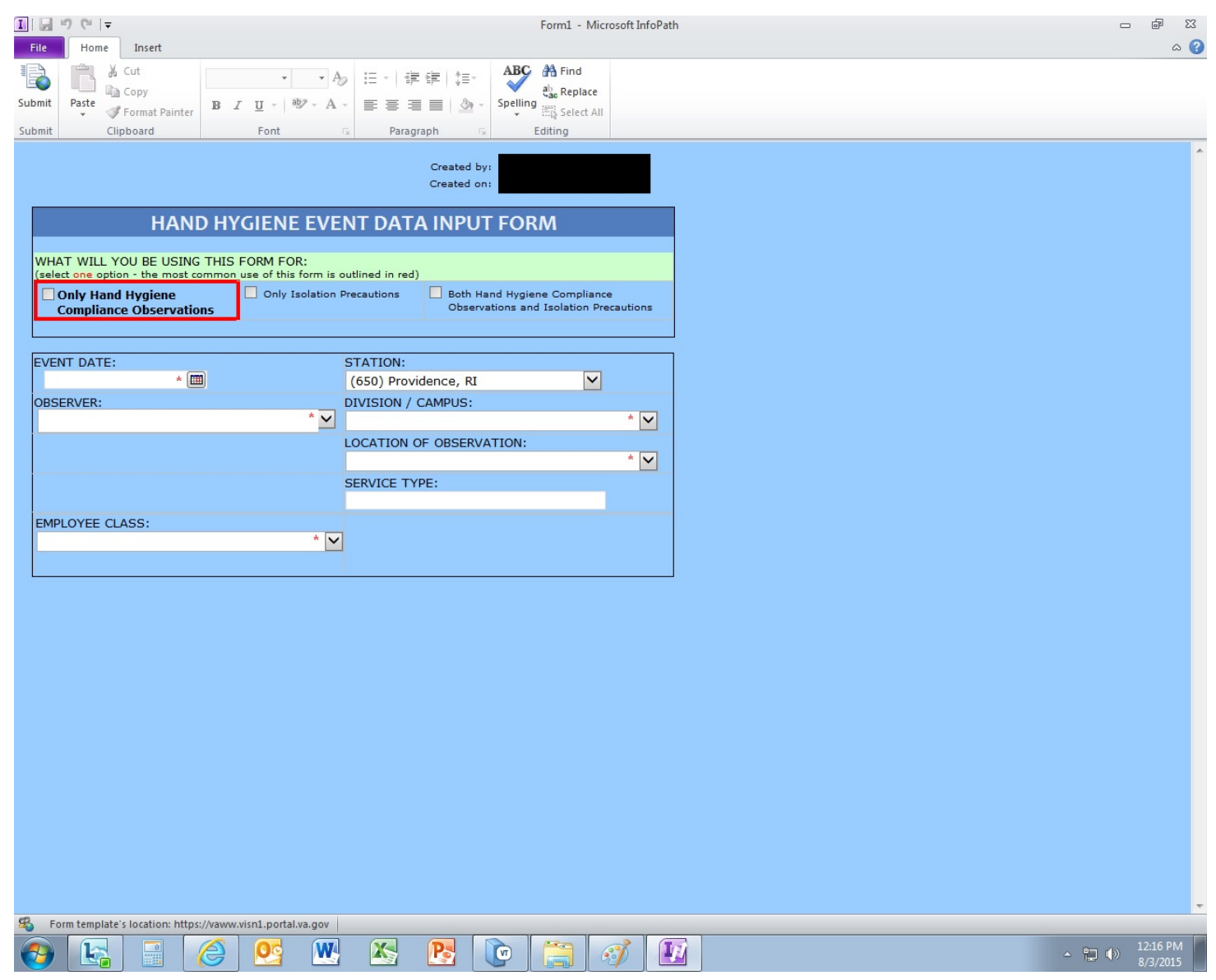




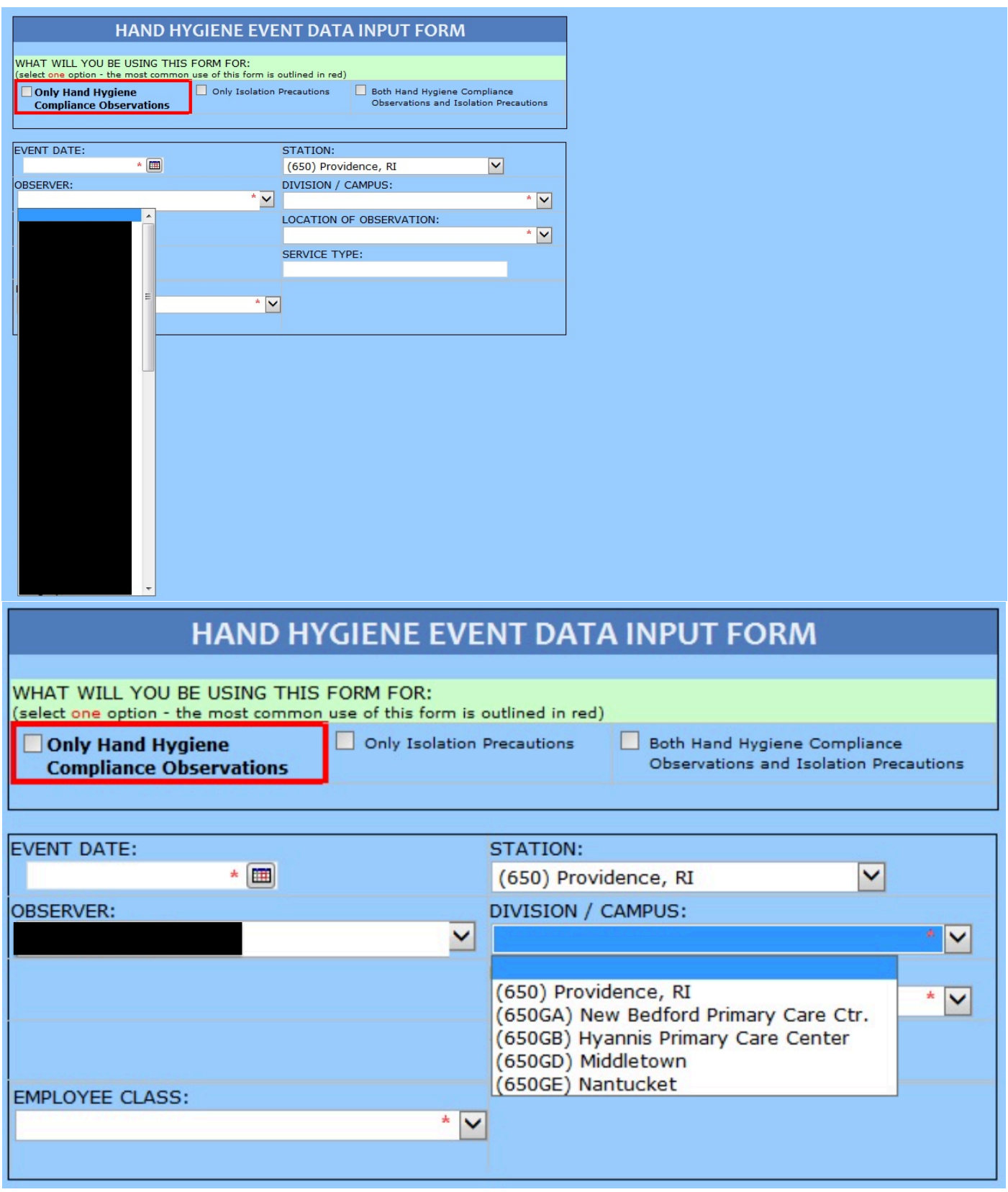


Administrative Assistant / Medical Support Sub Anesthesiology Staff

Chaplain

Dental Assistant / Nurse

Dentist

Dietary / Food Service

Doctor (Attending / Fellow)

Doctor (Med Student/Intern/Resident)

EMS

Escort

IV Nurse

Laboratory Service

Nurse (RN / LPN)

Nurse practitioner / Physician Assistant

Nursing Assistant Health Tech

Nursing Student

Nutritionist / Dietician

Other

Pharmacist / Pharm Tech

Phlebotomist

Psychiatrist / Psychologist

Radiology Service

Recreation Therapist

Rehab (PT/OT/KT)

Respiratory Service

Social Worker

Speech Therapy

Voluntary Service

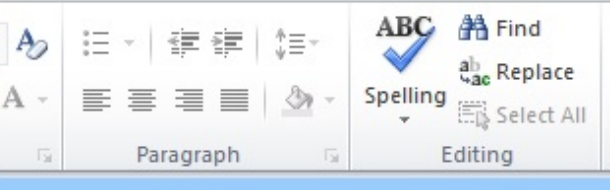

Created by: Created on:

\section{VENT DATA INPUT FORM}

is outlined in red)

ion Precautions

$\square$ Both Hand Hygiene Compliance Observations and Isolation Precautions

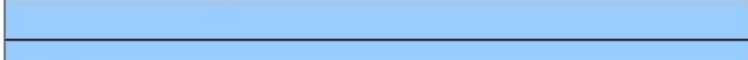

\section{STATION:}

(650) Providence, RI

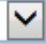
DIVISION / CAMPUS:

$\checkmark$

LOCATION OF OBSERVATION:

$* v$ 


\section{HAND HYGIENE EVENT DATA INPUT FORM}

WHAT WILL YOU BE USING THIS FORM FOR:

(select one option - the most common use of this form is outlined in red)

\begin{tabular}{|c|c|c|}
\hline \begin{tabular}{|l}
$\square$ Only Hand Hygiene \\
Compliance Observations
\end{tabular} & $\square$ Only Isolation Precautions & $\begin{array}{l}\square \text { Both Hand Hygiene Compliance } \\
\text { Observations and Isolation Precautions }\end{array}$ \\
\hline
\end{tabular}

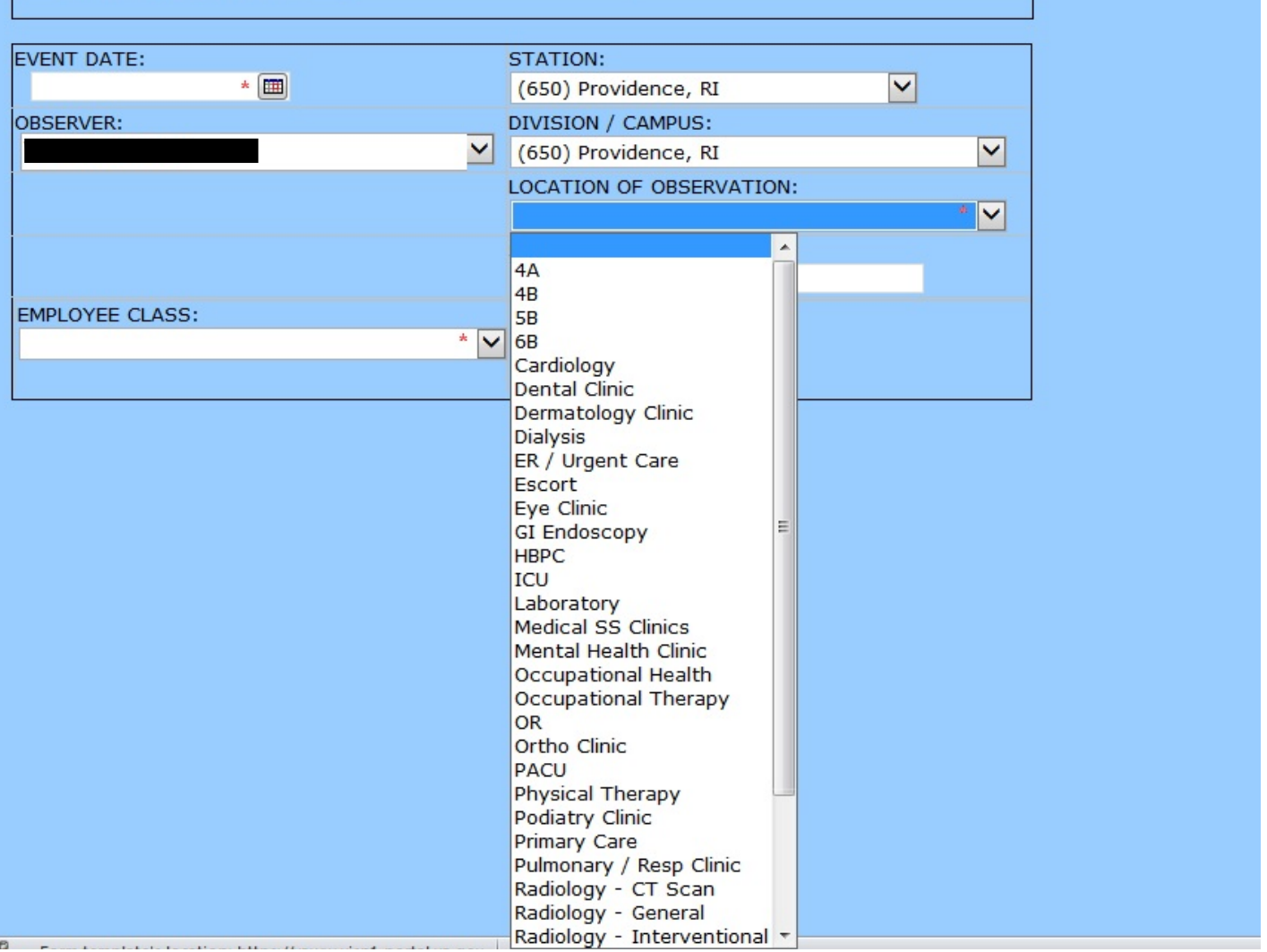




\section{HAND HYGIENE EVENT DATA INPUT FORM}

WHAT WILL YOU BE USING THIS FORM FOR:

(select one option - the most common use of this form is outlined in red)

\begin{tabular}{l}
$\begin{array}{l}\square \\
\text { Only Hand Hygiene } \\
\text { Compliance Observations }\end{array}$ \\
\hline Only Isolation Precautions
\end{tabular} $\begin{aligned} & \square \text { Both Hand Hygiene Compliance } \\
& \text { Observations and Isolation Precautions }\end{aligned}$

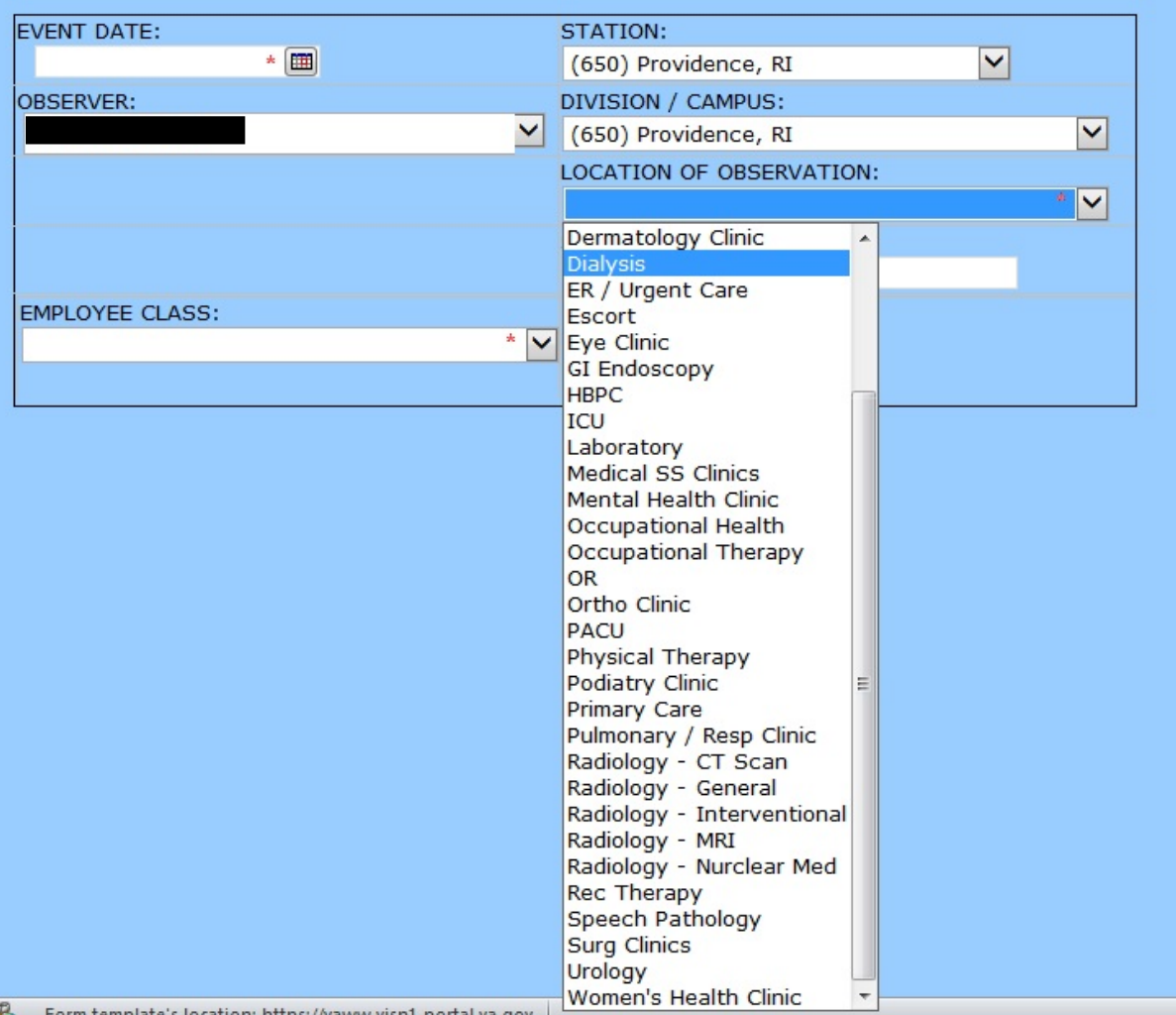




\section{HAND HYGIENE EVENT DATA INPUT FORM}

WHAT WILL YOU BE USING THIS FORM FOR:

U

Compliance Observations

Only Isolation Precaution

$\checkmark$ Both Hand Hygiene Compliance

Observations and Isolation Precautions

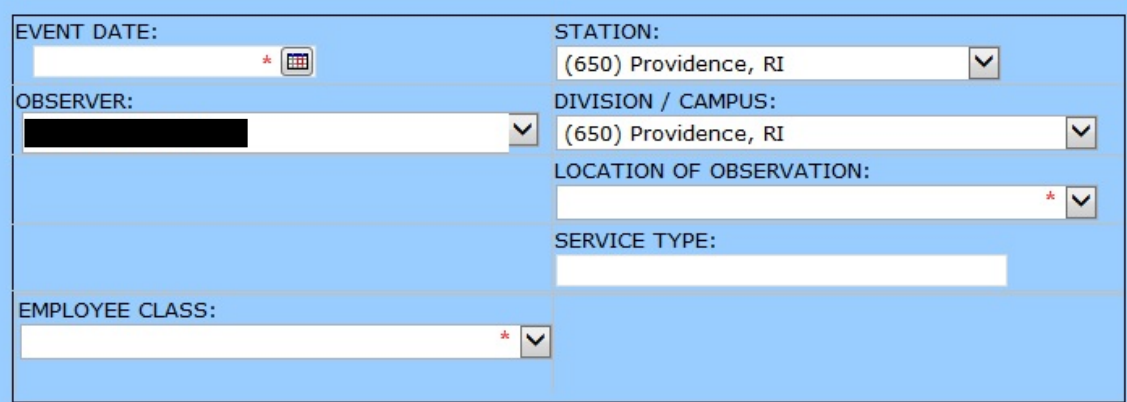

\begin{tabular}{l|r|r|}
\hline MOMENT: & $\checkmark$ COMPLIANCE: & \\
& $\checkmark$ & $\checkmark$ \\
& GLOVES USED? & $\checkmark$ \\
\hline
\end{tabular}

BARRIERS

$\square$ Dispenser Broken/Empty $\square$ Forgot or Distracted $\quad \square$ Hands Full of Supplies

$\square$ Improper Use of Gloves $\quad \square$ Perception $\mathrm{HH}$ Not Needed $\quad \square$ Perception of Skin Irritation

Please describe any

additional barriers:

\begin{tabular}{|c|c|c|}
\hline \multicolumn{3}{|c|}{ PRECAUTIONS } \\
\hline Isolation Precaution Type & $\begin{array}{l}\text { PPE/Notifications in Place - } \\
\text { If Indicated }\end{array}$ & Identified Infection Control Barriers \\
\hline$\square$ Contact & $\checkmark$ Appropriate Signage & $\square$ Hand Hygiene supplies not accessible \\
\hline$\square$ Special Contact & $\checkmark$ Glove Use & $\square$ PPE supplies not accessible \\
\hline$\square$ Enhanced Contact & $\checkmark$ Gown Use & \\
\hline$\square$ Droplet & $\checkmark$ Facemask / goggles & \\
\hline$\square$ Airborne & & \\
\hline
\end{tabular}

*you must provide an answer to all PPE/Notification answers, including if N/A. COMMENTS:

\section{Be sure and click "SUBMIT". Thanks.}

*If the button is disabled:

- check to make sure you selected only one option in the first question (not two)

- check that a moment and compliance have been selected

- if you used the form for precautions, check that all PPE / Notification items have been answered 


\section{HAND HYGIENE EVENT DATA INPUT FORM}

WHAT WILL YOU BE USING THIS FORM FOR:

(select one option - the most common use of this form is outlined in red)

Only Hand Hygiene

$\square$ Only Isolation Precautions

$\checkmark$ Both Hand Hygiene Compliance

Observations and Isolation Precautions

Compliance Observations

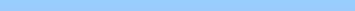

\begin{tabular}{|c|c|c|c|c|c|}
\hline EVENT DATE: & & STATION: & & & \\
\hline *回 & & (650) Providence, RI & $\checkmark$ & & \\
\hline OBSERVER: & & DIVISION / CAMPUS: & & & \\
\hline & $\checkmark$ & (650) Providence, RI & & & $\checkmark$ \\
\hline & & LOCATION OF OBSERV & & & \\
\hline & & & & & $\checkmark$ \\
\hline & & SERVICE TYPE: & & & \\
\hline |EMPLOYEE CLASS: & & & & & \\
\hline & $* \checkmark$ & & & & \\
\hline
\end{tabular}

MOMENT:

COMPLIANCE

BEFORE TOUCHING A PATIENT

BEFORE CLEAN/ASEPTIC PROCEDURES

AFTER TOUCHING A PATIENT

AFTER TOUCHING PATIENT SURROUNDINGS

AFTER BLOOD/BODY FLUID EXPOSURE/RISK stracted

$\square$ Improper Use of Gloves $\square$ Perception HH Not Needed

Please describe any

additional barriers:

GLOVES USED? $\quad \checkmark$

BARRIERS

$\square$ Hands Full of Supplies

$\square$ Perception of Skin Irritation

PRECAUTIONS

\begin{tabular}{|l|c|c|}
\hline & \multicolumn{2}{c}{ PRECAUTIONS } \\
\hline Isolation Precaution Type & $\begin{array}{l}\text { PPE/Notifications in Place - } \\
\text { If Indicated }\end{array}$ & Identified Infection Control Barriers \\
\hline$\square$ Contact & $\checkmark$ Appropriate Signage & $\square$ Hand Hygiene supplies not accessible \\
\hline$\square$ Special Contact & $\checkmark$ Glove Use & $\square$ PPE supplies not accessible \\
\hline$\square$ Enhanced Contact & $\checkmark$ Gown Use & \\
\hline
\end{tabular}




\section{HAND HYGIENE EVENT DATA INPUT FORM}

WHAT WILL YOU BE USING THIS FORM FOR:

(select one option - the most common use of this form is outlined in red)

$\square$ Only Hand Hygiene $\quad \square$ Only Isolation Precautions

Compliance Observations

Both Hand Hygiene Compliance Observations and Isolation Precautions

EVENT DATE:

STATION

OBSERVER:

*䧃

(650) Providence, RI

$\checkmark$

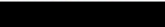

DIVISION / CAMPUS:

$\checkmark$ (650) Providence, RI

LOCATION OF OBSERVATION:

SERVICE TYPE:

EMPLOYEE CLASS:

* $\checkmark$

\section{MOMENT:}

COMPLIANCE:
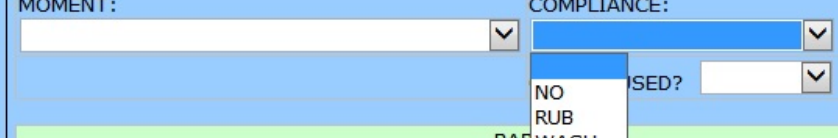

BARWASH

\begin{tabular}{|c|c|c|}
\hline$\square$ Dispenser Broken/Empty & $\square$ Forgot or Distracted & $\square$ Hands Full of Supplies \\
\hline$\square$ Improper Use of Gloves & $\square$ Perception $\mathrm{HH}$ Not Needed & $\square$ Perception of Skin Irritation \\
\hline
\end{tabular}
additional barriers:

PRECAUTIONS

\begin{tabular}{|c|c|c|}
\hline \multicolumn{3}{|c|}{ PRECAUTIONS } \\
\hline Isolation Precaution Type & $\begin{array}{l}\text { PPE/Notifications in Place - } \\
\text { If Indicated }\end{array}$ & Identified Infection Control Barriers \\
\hline$\square$ Contact & $\checkmark$ Appropriate Signage & $\square$ Hand Hygiene supplies not accessible \\
\hline$\square$ Special Contact & $\checkmark$ Glove Use & $\square$ PPE supplies not accessible \\
\hline$\Pi$ Enhanced Contact & $\checkmark$ Gown Use & \\
\hline
\end{tabular}




\section{HAND HYGIENE EVENT DATA INPUT FORM}

WHAT WILL YOU BE USING THIS FORM FOR:

(select one option - the most common use of this form is outlined in red)
$\square$ Only Hand Hygiene
Compliance Observations
$\square$ Only Isolation Precautions
$\checkmark$ Both Hand Hygiene Compliance Observations and Isolation Precautions

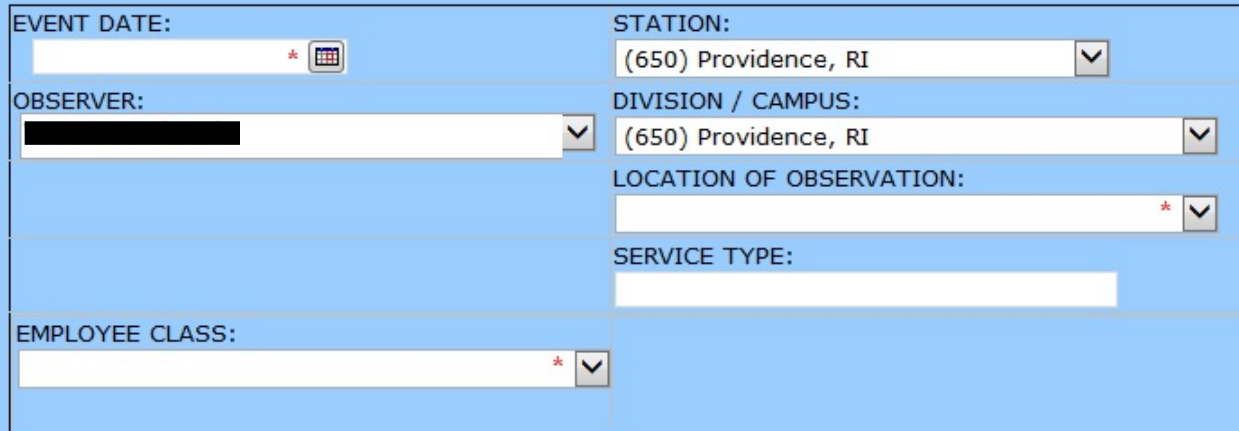

MOMENT:

COMPLIANCE:

$\checkmark$

GLOVES USED?

BARRIERS

Yes

\begin{tabular}{|c|c|c|}
\hline & BARRIERS & Yes \\
\hline$\square$ Dispenser Broken/Empty & $\square$ Forgot or Distracted & Hands Full of Supplies \\
\hline$\square$ Improper Use of Gloves & $\square$ Perception $\mathrm{HH}$ Not Needed & $\square$ Perception of Skin Irritation \\
\hline
\end{tabular}

PRECAUTIONS

\begin{tabular}{|l|c|c|}
\hline & \multicolumn{2}{c}{ PRECAUTIONS } \\
\hline Isolation Precaution Type & $\begin{array}{l}\text { PPE/Notifications in Place - } \\
\text { If Indicated }\end{array}$ & Identified Infection Control Barriers \\
\hline$\square$ Contact & $\checkmark$ Appropriate Signage & $\square$ Hand Hygiene supplies not accessible \\
\hline$\square$ Special Contact & $\checkmark$ Glove Use & $\square$ PPE supplies not accessible \\
\hline$\square$ Enhanced Contact & $\checkmark$ Gown Use &
\end{tabular}




\section{APPENDIX T: Secret Observer Consent Form}

$\begin{aligned} & \text { RESEARCH CONSENT FORM } \\ & \text { Providence VA Medical Center } \\ & \text { IRB \# } 00001402\end{aligned}$
Subject Name:
Title of Study: Assessing Hand Hygiene Compliance in Healthcare Workers to Reduce Infectious Disease -Observer
Principal Investigator: Associate Prof. Valerie Maier-Speredelozzi
Study Sponsor (if applicable):

1. Purpose of study and how long it will last:

The purpose of this research is to study hand hygiene compliance by directly observing healthcare workers at the Providence Veterans Affairs Medical Center during identified hand hygiene moments (before/after contacting a patient).

2. Description of the study including procedures to be used:

If you decide to take part in this study, you will be asked to observe doctors or nurses in inpatient units $(5 \mathrm{~B}, 6 \mathrm{~B}$ and ICU) and complete a hand-hygiene compliance monitoring form. Each time you observe a doctor or a nurse, you will be asked to put a mark (x) on a data collection sheet for the room number, bed, patient gender and health status (isolation vs. no isolation) and then record hand hygiene methods that were used (soap, Purell, etc.). This form should take approximately 1-2 minutes per observation. You will be asked to document each observation you make over a 2 month period, which may be repeated 1-3 times later in the year.

3. Description of any procedures that may result in discomfort or inconvenience: There are not any foreseeable discomforts associated with the study.

\section{Expected risks of study:}

There are not any foreseeable risks associated with the study. The decision to take part in this study is entirely voluntary and your employer will not know what you decide. Your responses will not be reported with your name or any identifying information other than your workgroup code. All form fields should be completed. If you decide to take part in the study, you may quit at any time.

\section{Expected benefits of study:}

Although there is no direct benefit to you for taking part in this study, the researcher may learn more about assessing hand hygiene. Thus, the research findings may benefit the hospital in general.

6. Other treatment(s) available:

There are not alternative treatments associated with this study.

\section{Costs to participants and compensation:}

Costs to Participants: There is no cost to participate in the study, other than the time needed to collect the required dataset.

Compensation Offered for Participation: There are no incentives, payment or gift certificates offered for completing the study forms.

8. Use of research results:

Approval Date: July 26, 2016

Expiration Date:

July 12,2017

Page 1 of 3

Form Version: 4/10/2015 


\section{RESEARCH CONSENT FORM}

Providence VA Medical Center

IRB \# 00001402

Subject Name:

Date:

Title of Study: Assessing Hand Hygiene Compliance in Healthcare Workers to Reduce Infectious Disease -Observer

Principal Investigator: Associate Prof. Valerie Maier-Speredelozzi

Study Sponsor (if applicable):

Your participation in this study is confidential. The spreadsheet that contains names and unique codes of all participants, and data collected will be electronically stored in a restricted folder on the secure server at the PVAMC. Only the research team will have access to the restricted folder. All physical forms will be kept secured in a double locked cabinet at Providence VA Medical Center after the study is done and are only accessible to the research team.

RESEARCH PARTICIPANT'S RIGHTS: I have read or have had read to me all of the above.

The Study Staff has explained the study to me and answered all of my questions. I have been told of the risks or discomforts and possible benefits of the study. I have been told of other choices of treatment available to me.

I have been told that I do not have to take part in this study, and my refusal to participate will involve no penalty or loss of rights to which I am entitled. I may withdraw from this study at any time without penalty or loss of VA or other benefits to which I am entitled.

The results of this study may be published, but my records will not be revealed unless required by law. The Institutional Review Board at the Providence VA Medical Center or other federal oversight offices may monitor my records for quality assurance purposes. Federal agencies including, but not limited to, the Food and Drug Administration (FDA), the Office for Human Research Protection (OHRP), the Office for Research Oversight (ORO), the Office of the Inspector General (OIG) and the Government Accounting Office (GAO) may have access to the records as allowed by law. If an FDAregulated test article is part of this study, the FDA may choose to inspect research records that include research subject's individual medical records. Records will be maintained in accordance with the Department of Veterans Affairs Record Control Schedule 10-1.

If I experience a side effect or adverse (bad or unexpected) reaction as a result of my involvement in this study, I will report these to the study investigator Associate Professor Valerie Maier-Speredelozzi at (401) 874-5187 who will arrange for any medical treatment that is necessary. After hours, I will call the operator at (401) 273-7100 and ask to speak to the infectious disease physician on call.

In case there are medical problems or questions, I have been told I can call Dr. Melissa Gaitanis at (401) 273-7100 extension $\underline{3609}$ during the day. After hours, I will call the operator at (401) 273-7100 and ask to speak to the infectious disease physician on call. If any medical problems occur in connection with this study the VA will provide emergency care.

The VA has the authority to provide medical treatment to participants (veterans and non-veterans) injured by participation in a VA study. If you are injured as a result of being in this study, the VA will provide the necessary medical treatment in accordance with federal law. If you want to make a legal claim against the VA or anyone who works for the VA, special laws may apply. The Federal Tort Claims Act (28 U.S.C. 1346(b), 2671-2680) is a federal law that controls when and how a person can bring a claim against the U.S. Government. If you sign this document you are not giving up your right to make a legal claim against the United States.

I can call the IRB Coordinator at (401) 273-7100 ext. 3470, the Research Administrative Officer at (401) 273-7100 ext. 3478 or the Providence VAMC Patient Advocate at (401) 273-7100 ext. 3093 while I am a participant or after my 


\section{RESEARCH CONSENT FORM \\ Providence VA Medical Center}

IRB \# 00001402

Subject Name:

Date:

Title of Study: Assessing Hand Hygiene Compliance in Healthcare Workers to Reduce Infectious Disease -Observer

Principal Investigator: Associate Prof. Valerie Maier-Speredelozzi

Study Sponsor (if applicable):

participation is over for the following: 1) concerns, 2) complaints, 3) problems, 4) suggestions, 5) more information, 6 ) questions about my rights as a research participant or 7 ) verifying the validity of the study and authorized contacts.

I voluntarily consent to participate in this study. I confirm that I have read this consent form or it has been read to me, and I agree it explains what this study is about and how and why it is being done. I will receive a signed copy of the consent form document after I sign it.

Participant's Signature

Participant (printed)

Date

Signature of Person Obtaining Consent

Person Obtaining Consent (printed)

Date

Version Date: $07 / 26 / 2016$

Approval Date: _July 26, 2016

Expiration Date: _ July 12, 2017

Page 3 of $\mathbf{3}$

Form Version: 4/10/2015 


\section{APPENDIX U: 6B Purell \& Soap Log Sheet}

\section{UNIT: 6B ONLY}

\section{Instructions:-}

Please mark a tally (/) every time you replace a cartridge of Purell or Soap and its replacement date under the correct product and shift cell.

\begin{tabular}{|c|c|c|c|c|c|c|}
\hline Product & \multicolumn{3}{|c|}{$\begin{array}{l}\text { Purell Cartridges } \\
\text { Replaced }\end{array}$} & \multicolumn{3}{|c|}{$\begin{array}{c}\text { Hand Soap Cartridges } \\
\text { Replaced }\end{array}$} \\
\hline Shift & Shift 1 & Shift 2 & Shift 3 & Shift 1 & Shift 2 & Shift 3 \\
\hline Date/(Sample) & /II & I & // & //I & I & // \\
\hline & & & & & & \\
\hline & & & & & & \\
\hline & & & & & & \\
\hline & & & & & & \\
\hline & & & & & & \\
\hline & & & & & & \\
\hline & & & & & & \\
\hline & & & & & & \\
\hline & & & & & & \\
\hline & & & & & & \\
\hline & & & & & & \\
\hline & & & & & & \\
\hline & & & & & & \\
\hline & & & & & & \\
\hline & & & & & & \\
\hline & & & & & & \\
\hline & & & & & & \\
\hline & & & & & & \\
\hline & & & & & & \\
\hline & & & & & & \\
\hline & & & & & & \\
\hline
\end{tabular}




\section{APPENDIX V: 5B Purell \& Soap Log Sheet}

\section{UNIT: 5B ONLY}

\section{Instructions:-}

Please mark a tally (/) every time you replace a cartridge of Purell or Soap and its replacement date under the correct product and shift cell.

\begin{tabular}{|c|c|c|c|c|c|c|}
\hline Product & \multicolumn{3}{|c|}{$\begin{array}{l}\text { Purell Cartridges } \\
\text { Replaced }\end{array}$} & \multicolumn{3}{|c|}{$\begin{array}{c}\text { Hand Soap Cartridges } \\
\text { Replaced }\end{array}$} \\
\hline Shift & Shift 1 & Shift 2 & Shift 3 & Shift 1 & Shift 2 & Shift 3 \\
\hline Date/(Sample) & /II & I & // & //I & I & // \\
\hline & & & & & & \\
\hline & & & & & & \\
\hline & & & & & & \\
\hline & & & & & & \\
\hline & & & & & & \\
\hline & & & & & & \\
\hline & & & & & & \\
\hline & & & & & & \\
\hline & & & & & & \\
\hline & & & & & & \\
\hline & & & & & & \\
\hline & & & & & & \\
\hline & & & & & & \\
\hline & & & & & & \\
\hline & & & & & & \\
\hline & & & & & & \\
\hline & & & & & & \\
\hline & & & & & & \\
\hline & & & & & & \\
\hline & & & & & & \\
\hline & & & & & & \\
\hline
\end{tabular}




\section{APPENDIX W: ICU Purell \& Soap Log Sheet}

\section{UNIT: ICU ONLY}

\section{Instructions:-}

Please mark a tally (/) every time you replace a cartridge of Purell or Soap and its replacement date under the correct product and shift cell.

\begin{tabular}{|c|c|c|c|c|c|c|}
\hline Product & \multicolumn{3}{|c|}{$\begin{array}{c}\text { Purell Cartridges } \\
\text { Replaced }\end{array}$} & \multicolumn{3}{|c|}{$\begin{array}{c}\text { Hand Soap Cartridges } \\
\text { Replaced }\end{array}$} \\
\hline Shift & Shift 1 & Shift 2 & Shift 3 & Shift 1 & Shift 2 & Shift 3 \\
\hline Date/(Sample) & III & 1 & // & /II & 1 & /1 \\
\hline & & & & & & \\
\hline & & & & & & \\
\hline & & & & & & \\
\hline & & & & & & \\
\hline & & & & & & \\
\hline & & & & & & \\
\hline & & & & & & \\
\hline & & & & & & \\
\hline & & & & & & \\
\hline & & & & & & \\
\hline & & & & & & \\
\hline & & & & & & \\
\hline & & & & & & \\
\hline & & & & & & \\
\hline & & & & & & \\
\hline & & & & & & \\
\hline & & & & & & \\
\hline & & & & & & \\
\hline & & & & & & \\
\hline & & & & & & \\
\hline & & & & & & \\
\hline
\end{tabular}


APPENDIX X: 6B HAIs per Operating Bed per Inpatient Unit Sheet UNIT: 6B ONLY

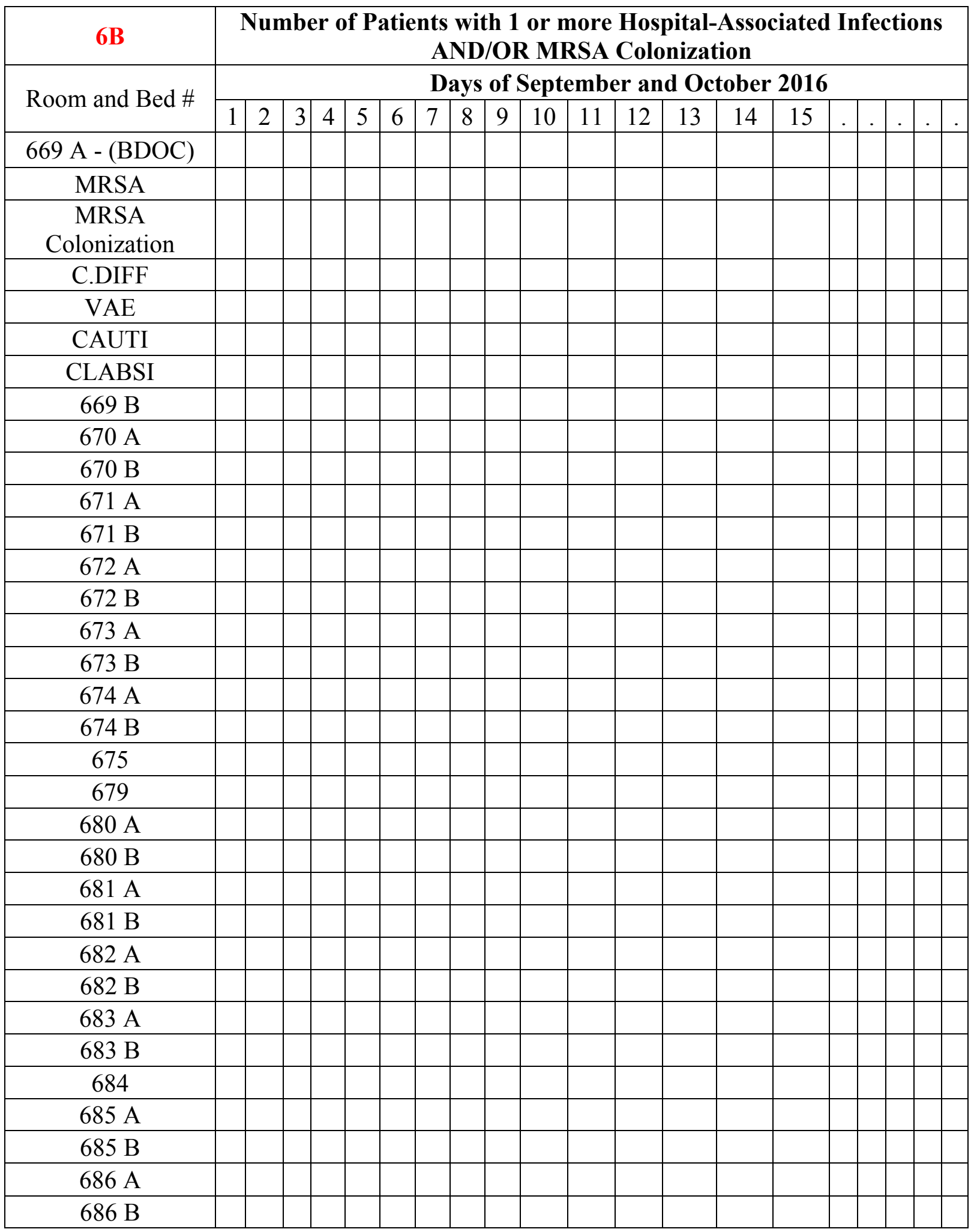


APPENDIX Y: 5B HAIs per Operating Bed per Inpatient Unit Sheet UNIT: 5B ONLY

\begin{tabular}{|c|c|c|c|c|c|c|c|c|c|c|c|c|c|c|c|c|c|}
\hline \multirow{2}{*}{ 5B } & \multicolumn{10}{|c|}{ Number of Patients with 1 or more Hospital-Associated Infections } \\
AND/OR MRSA Colonization \\
\hline Room and Bed \# \\
\hline
\end{tabular}


APPENDIX Z: ICU HAIs per Operating Bed per Inpatient Unit Sheet UNIT: ICU ONLY

\begin{tabular}{|c|c|c|c|c|c|c|c|c|c|c|c|c|c|c|c|c|c|c|c|}
\hline \multirow{3}{*}{ Room and Bed \# } & \multicolumn{19}{|c|}{$\begin{array}{c}\text { Number of Patients with } 1 \text { or more Hospital-Associated } \\
\text { Infections AND/OR MRSA Colonization }\end{array}$} \\
\hline & \multicolumn{19}{|c|}{ Days of September and October 2016} \\
\hline & 1 & 2 & 3 & 4 & 5 & 6 & 7 & 8 & 9 & 10 & 11 & 12 & 13 & 14 & 15 & . & & & \\
\hline \multicolumn{20}{|l|}{$624-1(\mathrm{BDOC})$} \\
\hline \multicolumn{20}{|l|}{ MRSA } \\
\hline \multicolumn{20}{|l|}{$\begin{array}{c}\text { MRSA } \\
\text { Colonization }\end{array}$} \\
\hline \multicolumn{20}{|l|}{ C.DIFF } \\
\hline \multicolumn{20}{|l|}{ VAE } \\
\hline \multicolumn{20}{|l|}{ CAUTI } \\
\hline \multicolumn{20}{|l|}{ CLABSI } \\
\hline \multicolumn{20}{|l|}{$625 \mathrm{~A}-2$} \\
\hline \multicolumn{20}{|l|}{$625 \mathrm{~B}-3$} \\
\hline \multicolumn{20}{|l|}{$625 C-4$} \\
\hline \multicolumn{20}{|l|}{$627-5$} \\
\hline \multicolumn{20}{|l|}{$628-6$} \\
\hline \multicolumn{20}{|l|}{$632-7$} \\
\hline $634-8$ & & & & & & & & & & & & & & & & & & & \\
\hline
\end{tabular}




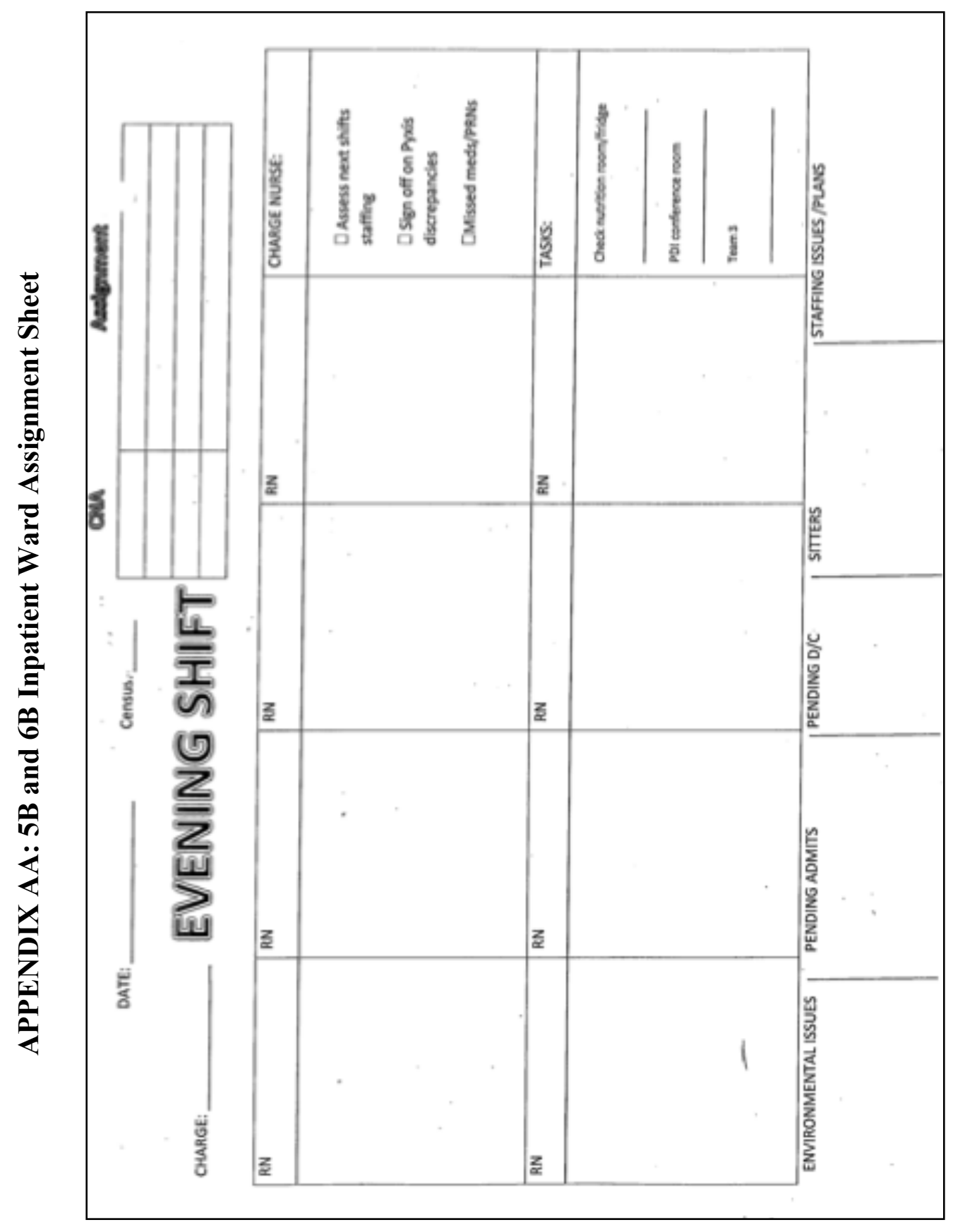




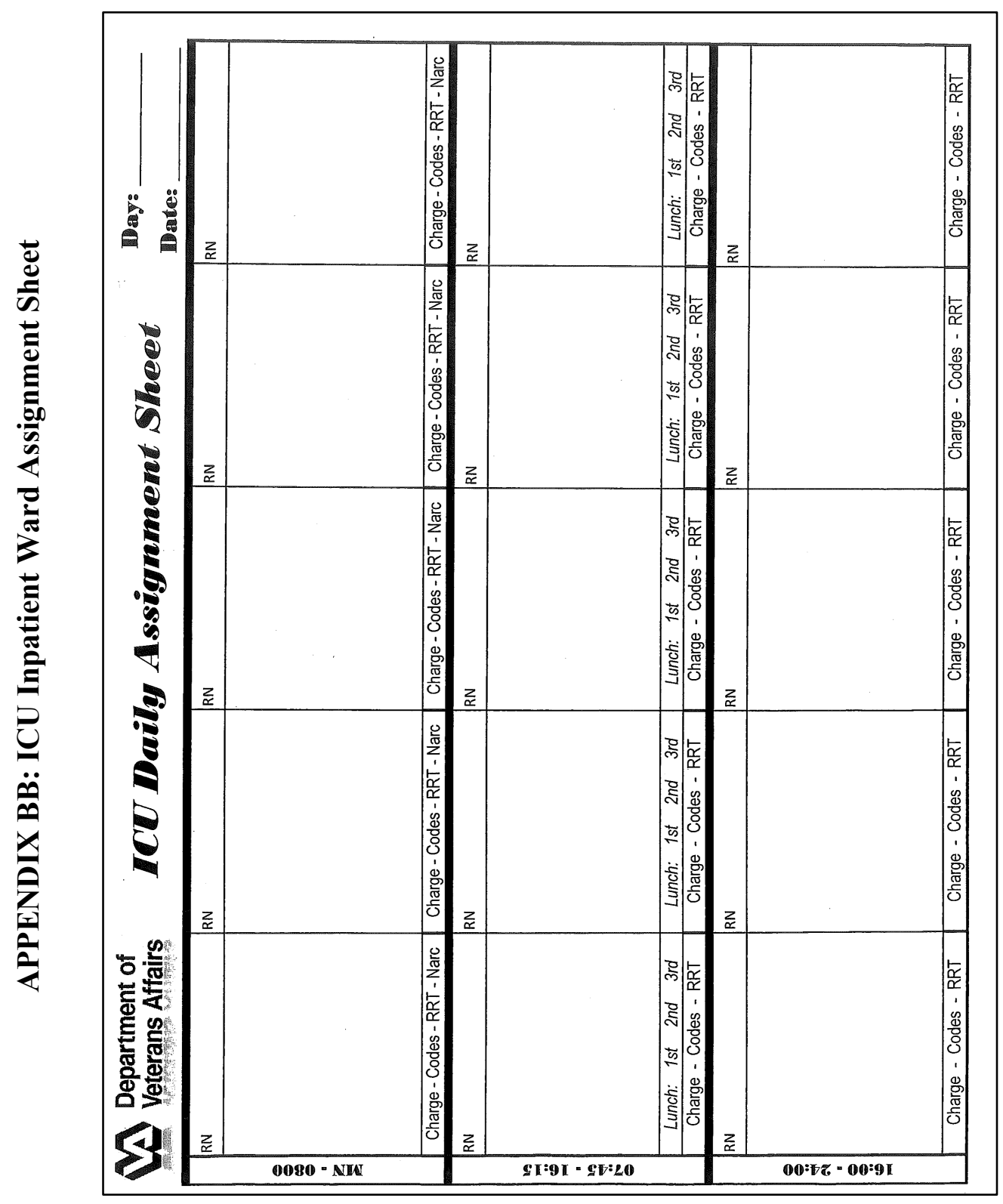




\section{APPENDIX CC: 2-Step Checklist Sheet Page One}

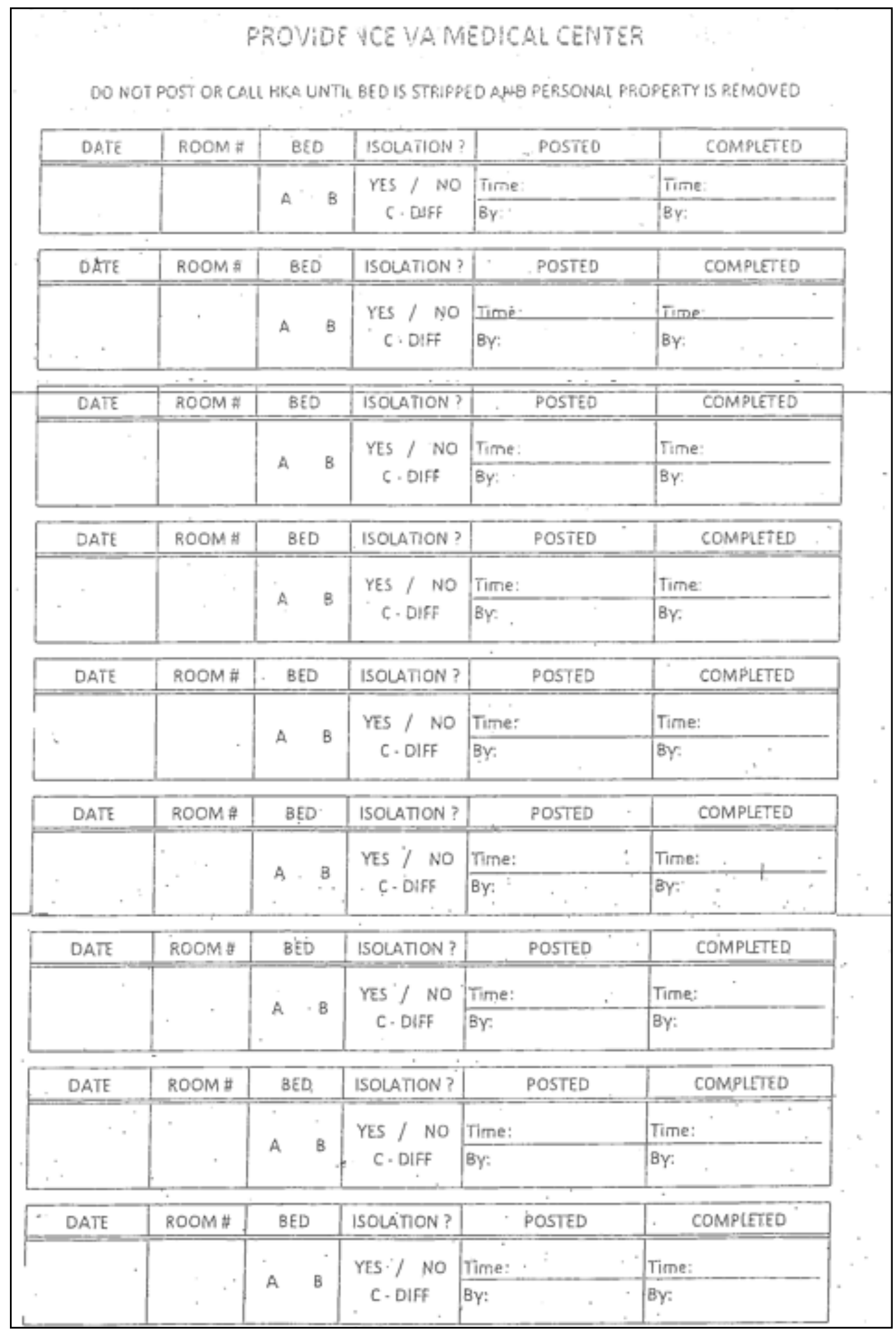




\section{APPENDIX CC: 2-Step Checklist Sheet Page Two}

\section{ENVIRONMENTAL CHECKLIST - 2 STEP PROCESS}

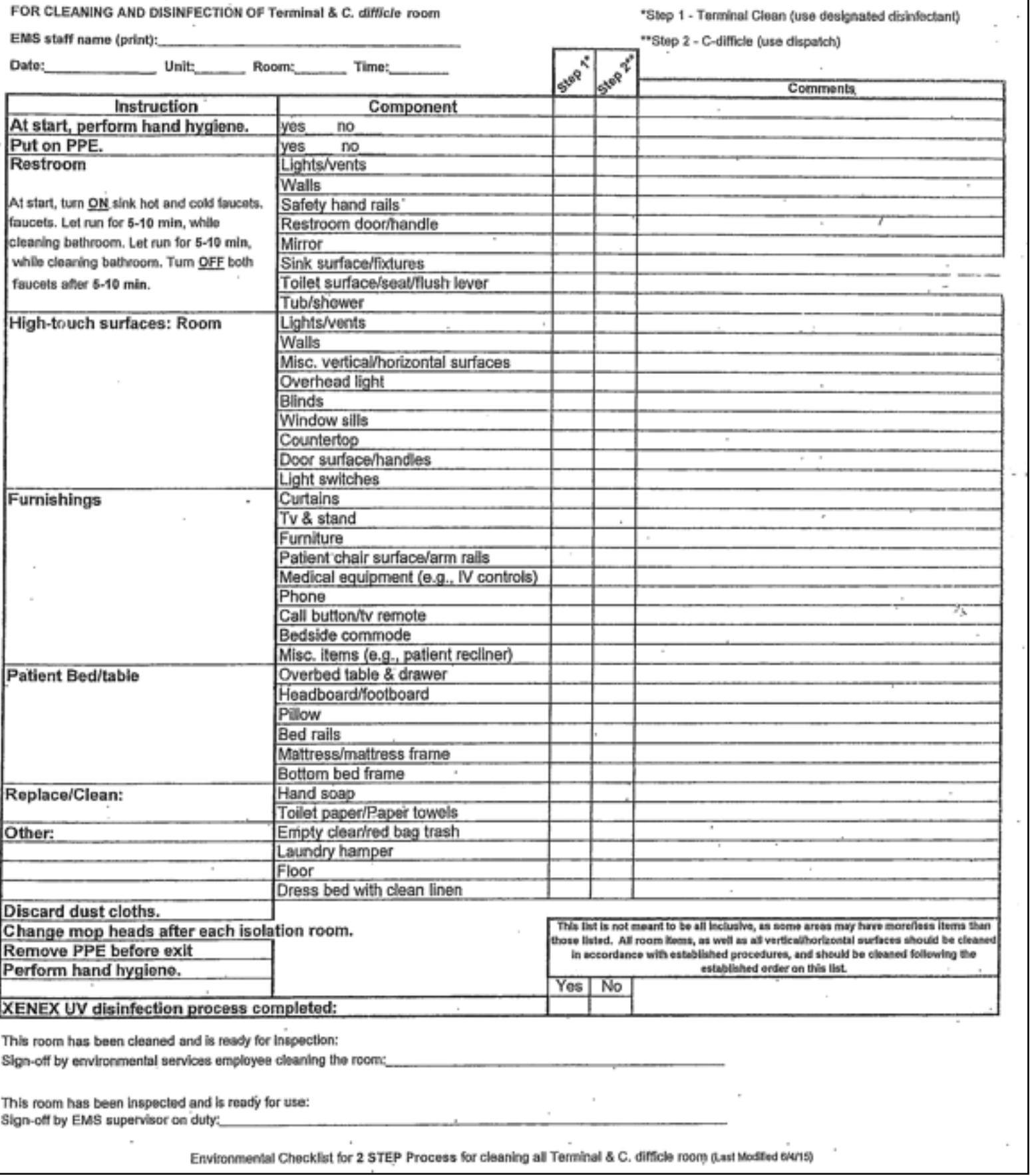




\section{APPENDIX DD: Contact Precautions Sign Page One}

\section{CONTACT PRECAUTIONS}

VISITORS: Report to nurses' station for instructions before entering room

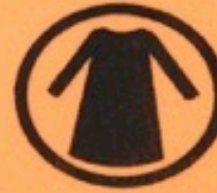

$\Rightarrow$ GOWN REQUIRED

Discard used gown before leaving room

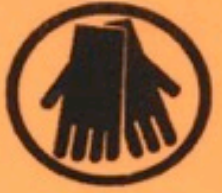

$\Rightarrow$ GLOVES REQUIRED

Discard used gloves before leaving room.

$\rightarrow$ WASH HANDS

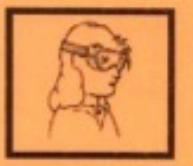

EYE PROTECTION REQUIRED if potential for body fluid splash/spray to eyes

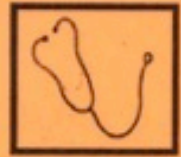

\section{PATIENT CARE EQUIPMENT}

- Dedicate non-critical equipment to patient.

- Clean and disinfect reusable equipment between patients.

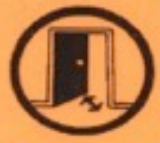

\section{PRIVATE ROOM}

- Or cohort via Infection Control consult.

- Door may be left open.

\section{PATIENT TRANSPORT IS LIMITED!}

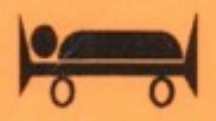

- $O N L Y$ medically necessary procedure(s) that cannot be performed at bedside.

- Patient must wear clean clothes and have clean hands when leaving room.

- Notify area receiving patient of precautions required.

If patient is isolated at discharge, leave sign posted. Environmental Services staff will remove \& diseard sign after terminal eleaning. 


\section{APPENDIX DD: Contact Precautions Sign Page Two}

\section{Illnesses Requiring Contact Precautions:}

\section{Agents/Diseases}

Antibiotic-resistant organisms: MRSA, VRE, or key multi-dnug resistant gram negative"

Adenovinus

Clostridium difficile ("C. diff")

Diarnhea, acute, unknown cause*

Lassa fever

Diphtheria, cutaneous form

Note: pharyngeal diphtheria requires droplet

Ebola fever

Hepatitis A

Lice

Marburg virus

Respirztory Syncytial vinus (RSV)

Rotavinus

Herpes simplex, disseminaled mucocutaneous Slaphylococeal skin/wound burn infection, major

Herpes zoster (shingles) localized in Sircptococcal skin/wound/bum infection, major immunocompetent patient

- Infection Control flags gram negatives of epidemiological significance.

\section{Clinical Syndromes}

Abscess, draining major

Conjunctivitis, viral

Cellulitis, acute (uncontained)

Dianhea, acute, unknown cause**

Decubitus ulcer, major, infected

Enterocolitis, Clostridium difficile

Impetigo

SARS* (also Airbome procautions)

Shingles, localized in immunocompetent patient

Skin/wound or burn infections, with hard to control drainage

Hemorrhagic fevers

Wound infection, major

- Severe Acute Respiratory Syndrome

* Gastroenteritis (acute diarrhea) in incontinent patients caused by any of the followiag: Campylabacter.

Clostridium difficile, Cryptosponidium, Escherichia coli, Giardia, Hepatitis A virus, Norovirus, Rotavinus,

Salmonella, Shigella, Vibrio cholerae (cholera), Vibrio parahacmolyticus, Yersimia enterocolitica -OR - if

the cause of acute diantica is unknown, but an infectious cause is suspected.

\section{CONTACT Definition:}

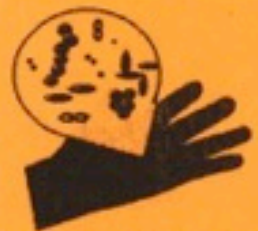

Contact transmission occurs when your hands, gloves of elothing become contaminated with organisms by touching the patient's skin, secretions. excretions, drainage, body fluids or by touching items soiled with these materials. Some organisms (see agents above) readily contaminate the patient's environment and can be found on bedrails, bedside tables, blood pressure cuffs, monitor buttons, IV poles, etc. Carrying the organisms on your hands or clothing can allow for their spread to other patients.

Sandard Precautions must be used with all patients at all times, regardless of whether they are under bolation for a specific disrase/condition. This means:

1. Gloves must be womn for reasonably anticiputed contact with any body fluid (except sweat) or with a patiena's mucous menbranes or noo-intact skin.

2. Gogelesiface visor and a mask muat be wom if there is a risk of splash or speay of body fluids to the mocosa of your eyes, nose and mouth (e.g, while intubating of suetioning patient, accessing a hemodiaiysis fistula, etc.)

3. Govns must be worn if soiling of clothing or skin is likely.

4. Safety-designed share items (eg. shielding or blunting needlex, ete.) should always be used and be discarded into sharps containers IMMEDIATEL $Y$ affer use. 
APPENDIX EE: Droplet Precautions Sign Page One

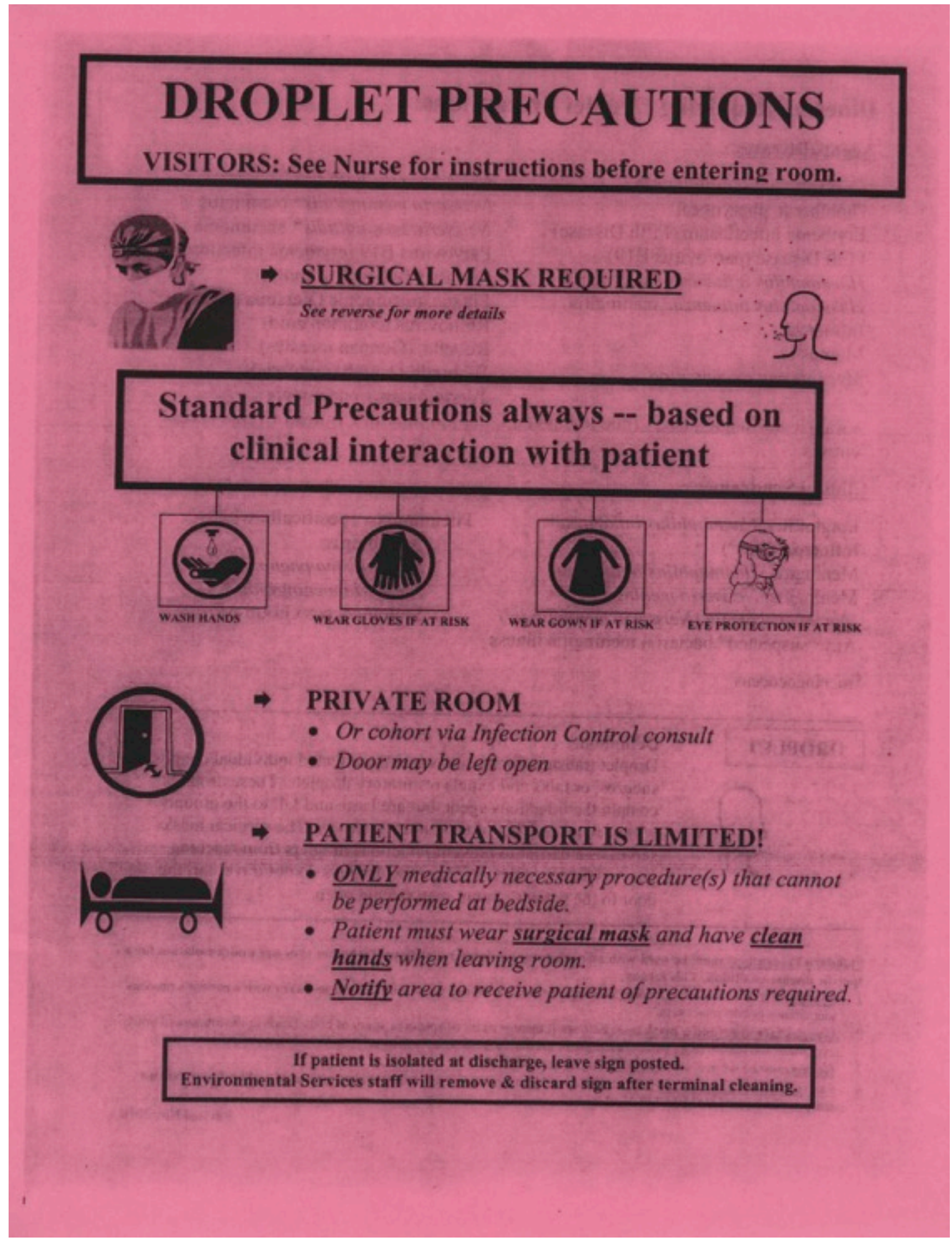


APPENDIX EE: Droplet Precautions Sign Page Two

\section{Illnesses Requiring Droplet Precautions:}

\section{Agents/Diseases}

Corynebacteria diphtheriae

Diphtheria, pharyngeal

Erythema infectiosum (Fifth Disease)

Fifth Disease (parvovinus B19)

Haemophilus influenzae pneumonia

Haemophilus influenzae meningitis

Influenza

Mumps

Mycoplasma pneumoniae
Neisseria meningitidis* bacteremia

Neisseria meningitidis* meningitis

Neisseria meningitidis* pneumonia

Parvovirus B19 (erythema infectiosum)

Pertussis (whooping cough)

Plague, pneumonic (Yersinia pestis)

Rhinovirus (common cold)

Rubella, (German measles)

Whooping cough (pertussis)

Yersinia pestis pneumonia

Viral Hemorrhagic Fevers (due to Lassa, Ebola, Marburg or Crimean-Congo fever viruses

\section{Clinical Syndromes}

Epiglottitis, Haemophilus influenzae

Influenza ("flu")

Meningitis, Haemophilus influenzae

Meningitis, Neisseria meningitidis*

Meningococcemia (Neisseria meningitidis)

Any "suspected" bacterial meningitis illness

Pneumonia - specifically with: Adenovirus

Mycoplasma pneumoniae

Neisseria meningitidis *

Streptococcus Group A

*meningococcus

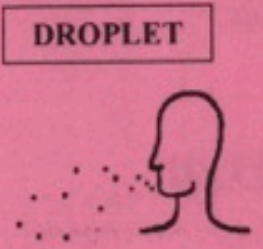

Definition:

Droplet transmission occurs when the infected individual coughs, sneezes, or talks and expels respiratory droplets. These droplets contain the infectious agent, but are large and fall to the ground within about $6-8$ feet of the patient's airway. The surgical mask serves as a barrier to prevent infectious droplets from reaching your respiratory tract. Because the droplets do not travel far, the door to the patient's room may remain open.

Standard Presautions must be used with all patients at all times, regardless of whether they are under isolation for a specific diseaseicondition. This means:

1. Gleves must be wom for reasonably anticipated contact with any body fluid (except sweat) or with a patient's mucous menbranes or non-intact skin.

2. Coesles/face visor and a mask must be wom if there is a risk of splach or spray of body fluids to the mucosa of your eyex, nose and mouth (e.g., while intubating or suetioning patient, accessing a hemodialysis fistula, etc.)

3. Goxns mus be worn if soiling of elothing or skin is likely.

4. Safety-designed share items (e.g., shielding or bluating needles, ete.) should always be used and be discarded into shams containers IMMEDIATELY afler use. 


\section{APPENDIX FF: Airborne Precautions Sign Page One}

\section{AIRBORNE PRECAUTIONS}

VISITORS: Report to nurses' station for instructions before entering room

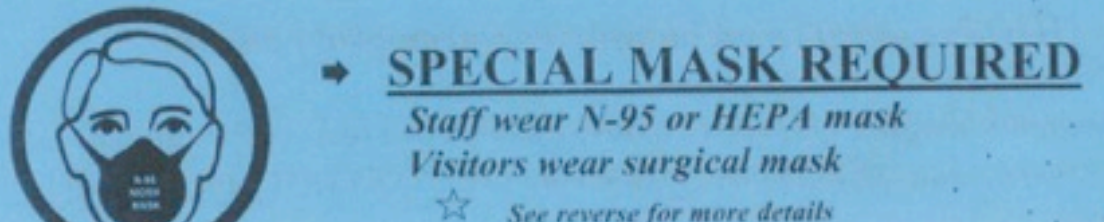

$\hat{i}$ See reverse for more details
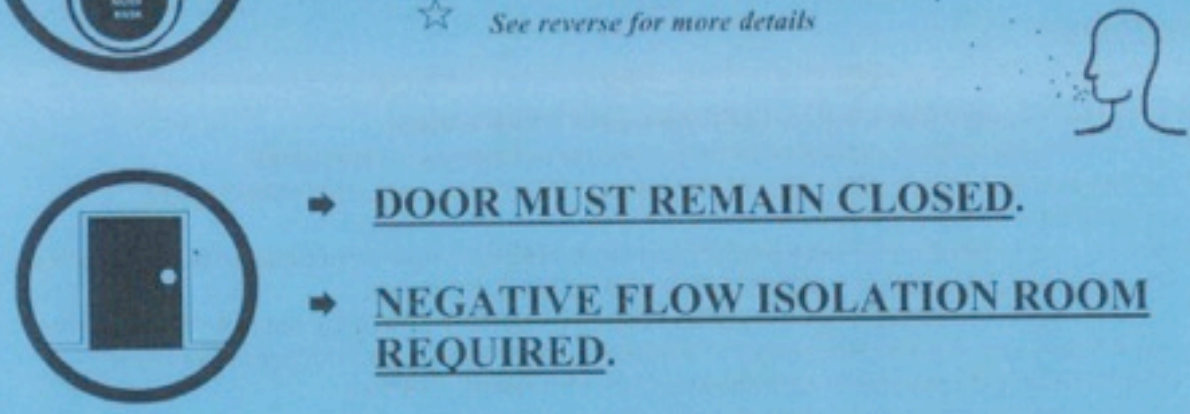

$\Rightarrow$ DOOR MUST REMAIN CLOSED.

$\Rightarrow$ NEGATIVE FLOW ISOLATION ROOM REQUIRED.

\section{Standard Precautions required at all times!}

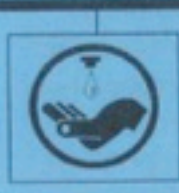

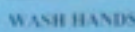

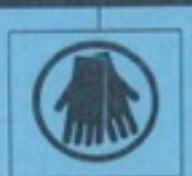

WE AR GLONS If AT HAS

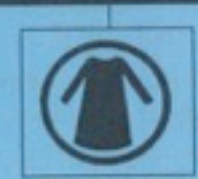

WrAR GOWNIF AT RISh

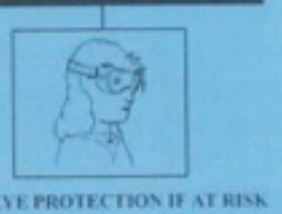

\section{PATIENT TRANSPORT IS LIMITED!}

- ONLY medically necessary procedure(s) that cannot be performed at bedside.

- Patient must wear surgical mask when leaving room.

- Notify area receiving patient of precautions required. 


\section{APPENDIX FF: Airborne Precautions Sign Page Two}

Illnesses Requiring Airborne Precautions:

- Tuberculosis (Mycobacterium tuberculosis complex)

- Chickenpox (Varicella) (also CONTACT precautions)

- Measles (Rubeola)

- Shingles (Herpes zoster) in an immunocompromised patient (also CONTACT precautions)

- Disseminated shingles (Herpes zoster) (also CONTACT precautions)

- SARS - Severe Acute Respiratory Syndrome (also CONTACT precautions)

- Smallpox

A N-95 or HEPA Special Mask is REQUIRED for Staff ENTRY when

$\rightarrow$ Patient has suspected or contimed (active) pulmonary or laryngeal tuberculosis.

$\rightarrow$ Patient has suspected or confirmed extrapulmonary tuberculosis, until active pulmonary or laryngeal tubereulosis is ruled out.

$\rightarrow$ Physician has ordered specimens for AFB (acid fast bacilli) on suspicion of pulmonary, laryngeal or extrapulmonary tuberculosis, until pulmonary tuberculosis is ruled out.

$\rightarrow$ Persons never having chickenpox and not immunized (varicella vaccine) are not permitted to enter the room of a patient with chickenpox or shingles. Also persons never having measies and not immunized are not permitted to enter the room of a patient with measles.

\section{AIRBORNE Definition:}

Airborne transmission occurs when the infected individual coughs or sneezes and expels respiratory droplets that then evaporate. Evaporation of the droplets leave the infectious

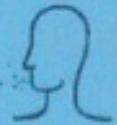
agents suspended in the air, where they may be carried on air currents to reach other people. The patient must be placed in a designated isolation room with negative pressure ventilation. The door must remain closed, and the patient is not permitted to leave the room except for medically essential procedures that cannot be performed at bedside.

Standard Precautions must be used with all patients at all times, regardless of whether they are under isolation for a specific disease/condition. This means:

1. Glove must he wom for reasonably anticipated contact with any body fuid (except sweat) or with a patient's mucous membranes or non-intact skin.

2. Gogeles face viser and a mask must be wom if there is a risk of splash or spray of body fluids to the mucosa of your eyes, nose and mouth (e.g, while intubating or suctioning patient, accessing a hemodialysis fistula, etc.)

3. Gowns must be wom if soiling of clothing of skin is likely.

4. Safety-designed sharp items (e.g., shielding or blunting needles, etc.) should always be used and be discanded into sharps contaliners IMMEDIATHLY after use. 


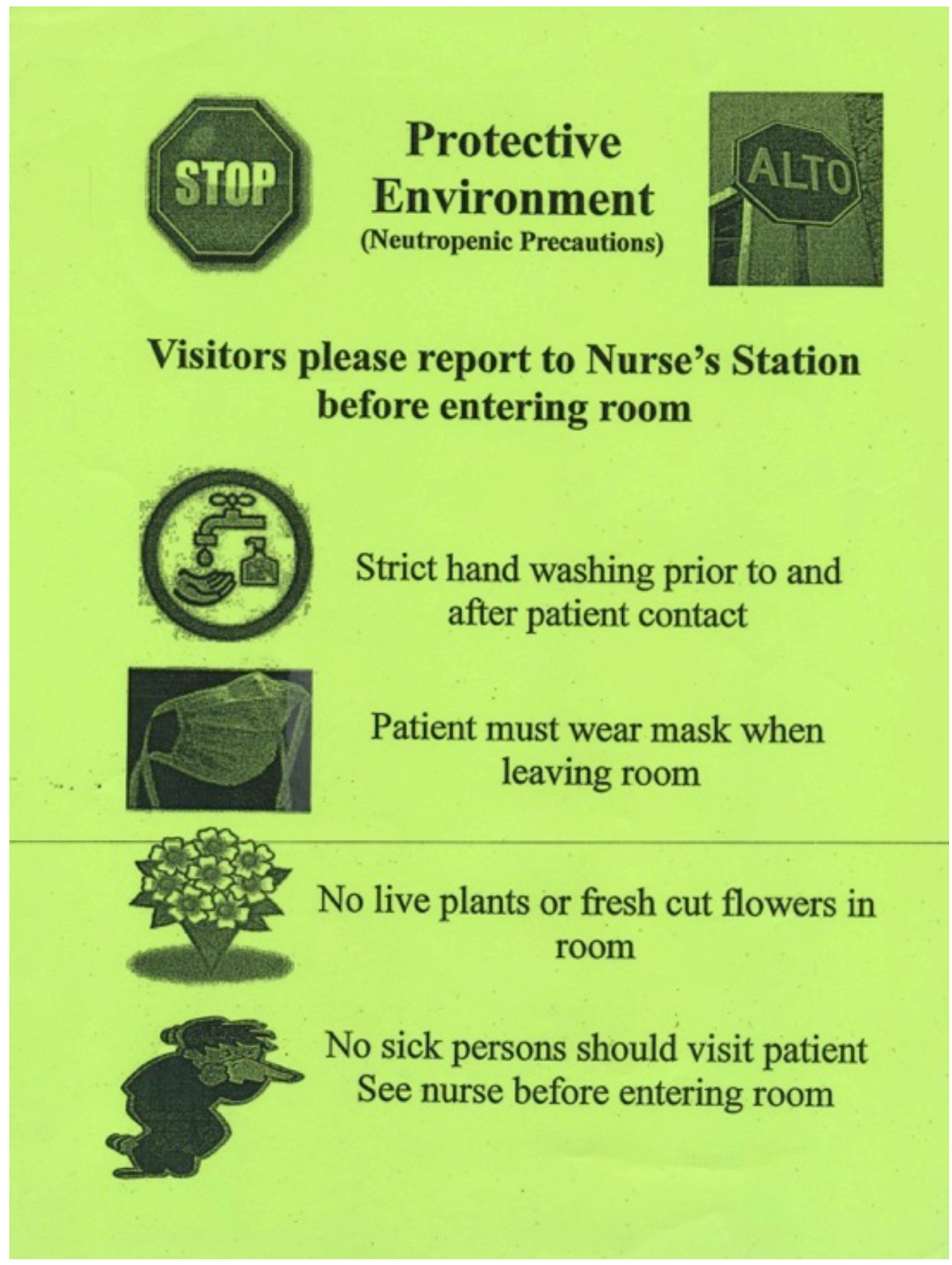


APPENDIX HH: Crown Program Sign

\section{CROWN PROGRAM}

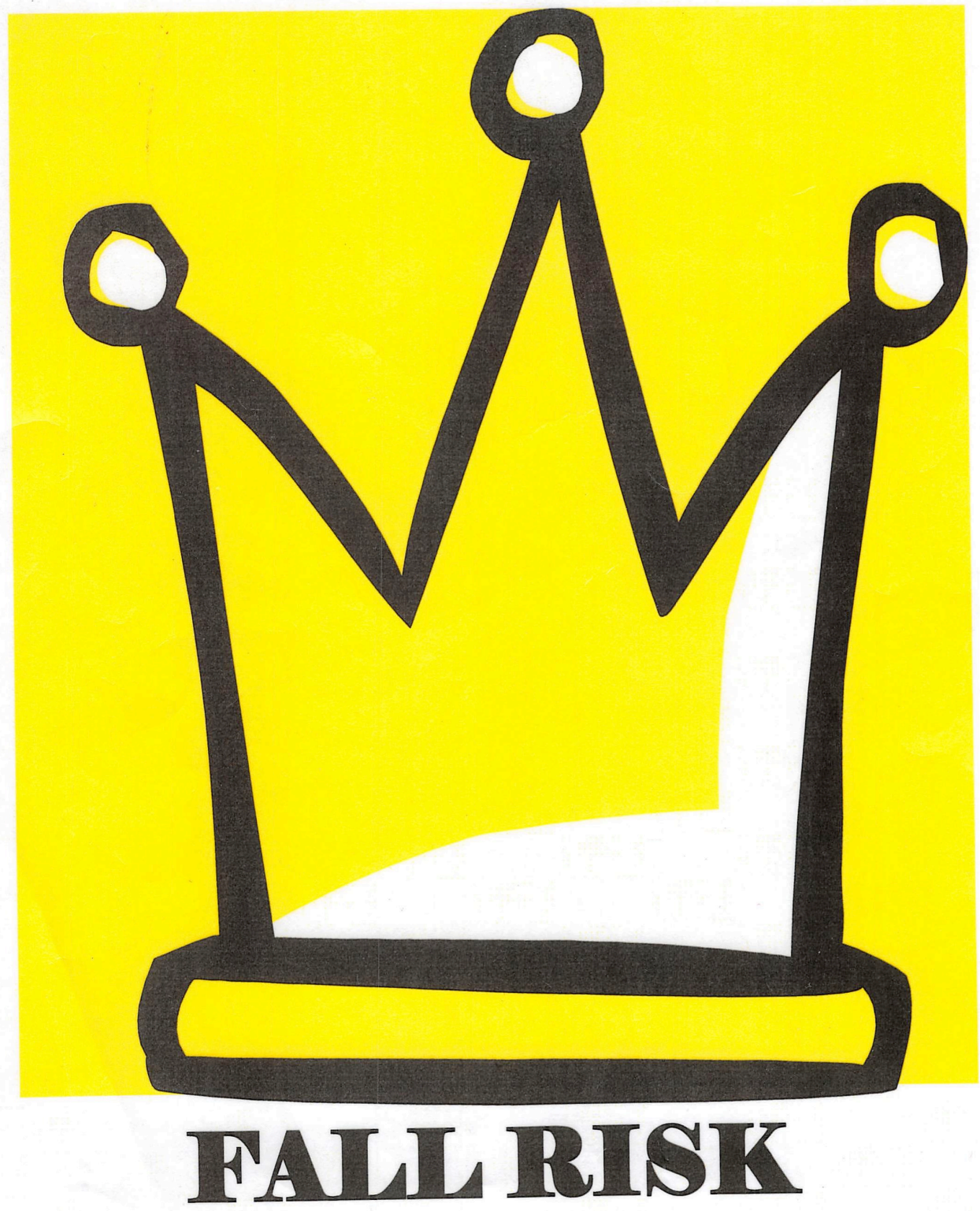




\section{APPENDIX II: Strict I\&O’s Sign}

\section{RM:}

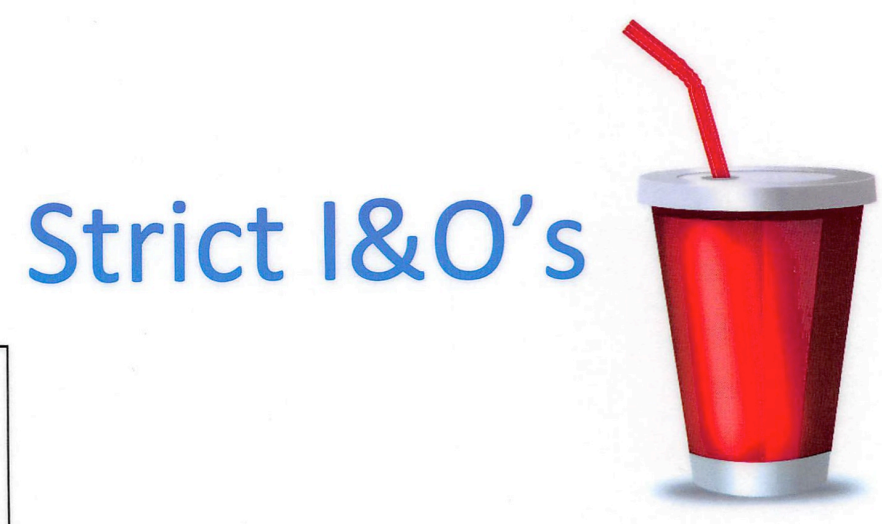

Juice cup...120mL Milk...240 mL Popsicle...90mL Soup... $180 \mathrm{~mL}$ 1 cup...240mL 8 oz....240 mL $10 z \ldots 30 \mathrm{~mL}$

Daily Weight

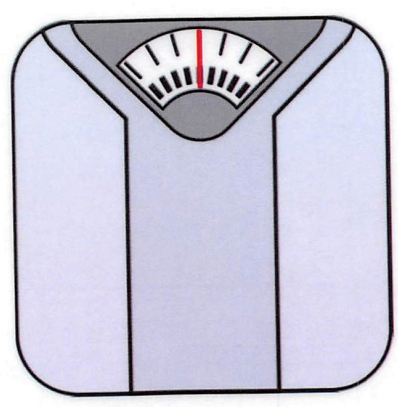




\section{BIBLIOGRAPHY}

1. Ajami, S., Akbari, B., editors. (2012). "RFID in Earthquake Information Management System: Work in progress. In Health system, risk management And Disaster." Isfahan, Iran: Health Management and Economics Research Center, Isfahan University of Medical Sciences; 2012.

2. Ajami, S., and Rajabzadeh, A. (2013). "Radio Frequency Identification (RFID) technology and patient safety." Journal of Research in Medical Sciences: The Official Journal of Isfahan University of Medical Sciences, 18(9), 809-813.

3. Alonso-Echanove, J., Edwards, J.R., Richards, M.J., et al. (2003). "Effect of nurse staffing and antimicrobial-impregnated central venous catheters on the risk for bloodstream infections in intensive care units." Infect Control Hosp Epidemiol. 2003 Dec;24(12):916-25. PMID 14700407.

4. Arenas, M.D., Sánchez-Payá, J., Barril, G., García-Valdecasas, J., Gorriz, J.L., Soriano, A., Antolin, A., Lacueva, J., García, S., Sirvent, A., Espinosa, M., Angoso, M. (2005). "A multicentric survey of the practice of hand hygiene in haemodialysis units: factors affecting compliance." Nephrology, Dialysis, Transplantation, 2005, 20:1164-1171.

5. Armellino, D., Trivedi, M., Law, I., Singh, N., Schilling, M., Hussain, E., Farber, F. (2013). "Replicating changes in hand hygiene in a surgical intensive care unit with remote video auditing and feedback." American Journal of Infection Control, Volume 41, Issue 10, October 2013, Pages 925927, ISSN 0196-6553, http://dx.doi.org/10.1016/j.ajic.2012.12.011.

6. Asif, A., Mandviwalla, M. (2005). "Integrating the supply chain with RFID: A technical and business analysis." Communications of the Association for Information Systems, 15, 393-427.

7. Bensel, P., Gunther, O., Tribowski, C., Vogeler, S. (2008). "Costbenefit sharing in cross-company RFID applications: A case study approach." In: 29th International Conference on Information Systems (ICIS) (pp. 1-17). Paris, France. $\quad$ Retrieved from http://www.icsi.berkeley.edu/pubs/other/costbenefit08.pdf

8. Berild, D., Ringertz, SH., Lelek, M., Fosse, B. (2001). "Antibiotic guidelines lead to reductions in the use and cost of antibiotics in a university hospital." Scandinavian Journal of Infectious Diseases 2001;33:63-67. 
9. Berenson, M. L., Levine, D. M., \& Krehbiel, T. C. (2002). Basic business statistics: concepts and applications (8th ed.). Upper Saddle River, NJ: Prentice-Hall.

10. Bischoff, W.E., Reynolds, T.M., Sessler, C.N., Edmond, M.B., Wenzel, R.P. (2000). "Handwashing compliance by health care workers: the impact of introducing an accessible, alcohol- based hand antiseptic." Archives of Internal Medicine, 2000, 160:1017-1021.

11. Bittner, M.J., Rich, E.C. (1998). "Surveillance of handwashing episodes in adult intensive care units by measuring an index of soap and paper towel consumption." Clinical Performance and Quality Health Care, 4:179182.

12. Bittner, M.J., Rich, E.C., Turner, P.D., Arnold, W.H. (2002). "Limited impact of sustained simple feedback based on soap and paper towel consumption on the frequency of hand washing in an adult intensive care unit." Infection Control and Hospital Epidemiology, 2002, 23:120 -126. Retrieved from

https://www.jstor.org/stable/pdf/10.1086/502020.pdf?acceptTC=true

13. Blot, S.I., Depuydt, P., Annemans, L., Benoit, D., Hoste, E., De Waele, J.J., Decruyenaere, J., Vogelaers, D., Colardyn, F., Vandewoude, K.H. (2005). "Clinical and economic outcomes in critically ill patients with nosocomial catheter-related bloodstream infections." Clin Infect Dis. 2005 Dec 1;41(11):1591-1598. Epub 2005 Oct 25.

14. Boscart, V.M., McGilton, K.S., Levchenko, A., Hufton, G., Holliday, P., Fernie, G.R. (2008). "Acceptability of a wearable hand hygiene device with monitoring capabilities." J Hosp Infect 2008;70:216-222.

15. Boyce, J.M. (2011). "Measuring Healthcare Worker Hand Hygiene Activity: Current Practices and Emerging Technologies." Infection Control \& Hospital Epidemiology, 32, pp 1016-1028.

16. Boyce, J.M., Cooper, T., Dolan, M. (2008). "Evaluation of an electronic device for real-time measurement of use of alcohol-based hand rub." Paper presented at: 18th Annual Scientific Meeting of the Society of Healthcare Epidemiology of America, Orlando, FL, 2008, abstr. 363.

17. Burkhard, R.J., Schooley, B., Dawson, J., Horan, T.A.. (2010). "Information systems and healthcare XXXVII: When your employer provides your personal health record-Exploring employee perceptions of an employersponsored PHR system." Communications of the Association for Information Systems, Vol. 27, 323-338. Retrieved from 
http://citeseerx.ist.psu.edu/viewdoc/download?doi=10.1.1.175.2080\&rep=rep1 \&type $=$ pdf

18. Button, K., Ioannidis, J., Mokrysz, C., Nosek, B., Flint, J., Robinson, E. and Munafo, M. (2013). "Power failure: why small sample size undermines the reliability of neuroscience." Nature Review Neuroscience, 14, 365-376 (May, 2013). Retrieved http://www.nature.com/nrn/journal/v14/n5/pdf/nrn3475.pdf

19. Centers for Disease Control and Prevention (CDC). (2002). "Guideline for Hand Hygiene in Health-Care Settings: Recommendations of the Healthcare Infection Control Practices Advisory Committee and the HICPAC/SHEA/APIC/IDSA Hand Hygiene Task Force." Morbidity and mortality weekly report, 2002;51(No. RR-16):[inclusive page numbers]. Prepared by Boyce, J.M., Pittet, D. Retrieved from http://www.cdc.gov/mmwr/PDF/rr/rr5116.pdf

20. Centers for Disease Control and Prevention (CDC). (2011). "Vital signs: Central line-associated blood stream infections-United States, 2001, 2008, and 2009." MMWR Morb Mortal Wkly Rep. 2011 Mar 4;60(8):243248.

21. Centers for Disease Control and Prevention (CDC). (2012). "Ventilator-associated pneumonia (VAP)." Healthcare-associated Infections (HAIs). Content source: Centers for Disease Control and Prevention, National Center for Emerging and Zoonotic Infectious Diseases (NCEZID), Division of Healthcare Quality Promotion (DHQP) Retrieved from http://www.cdc.gov/HAI/vap/vap.html

22. Centers for Disease Control and Prevention (CDC). (2014). "Hand Hygiene Basics". Retrieved from http://www.cdc.gov/handhygiene/Basics.html

23. Centers for Disease Control and Prevention (CDC). (2015). "Methicillin-resistant Staphylococcus aureus (MRSA) Infections." Retrieved from

http://www.cdc.gov/mrsa/

24. CDC and NHSN. (2010). Centers for Disease Control and Prevention (CDC) and National Healthcare Safety Network (NHSN), National Center for Emerging and Zoonotic Infectious Diseases, Division of Healthcare Quality Promotion. "National and State Healthcare-Associated Infections Standardized Infection Ratio Report Using Data Reported to the National Healthcare Safety Network" Published December 2010, Retrieved from http://www.cdc.gov/HAI/pdfs/SIR/national-SIR-Report_03_29_2012.pdf 
25. CDC and NHSN. (2015). Centers for Disease Control and Prevention CDC and National Healthcare Safety Network (NHSN), The Product and Service Codes (PSC) Manual. (2015). "Bloodstream Infection Event (Central Line-Associated Bloodstream Infection and Non-central line-associated Bloodstream Infection).” Published January 2015 (Modified April 2015), available at http://www.cdc.gov/nhsn/PDFs/pscManual/4PSC_CLABScurrent.pdf

26. Chopra, V., Krein, S.L., Olmsted, R.N., et al. (2013). "Prevention of Central Line-Associated Bloodstream Infections: Brief Update Review." In: Making Health Care Safer II: An Updated Critical Analysis of the Evidence for Patient Safety Practices. Rockville (MD): Agency for Healthcare Research and Quality (US); 2013 Mar. (Evidence Reports/Technology Assessments, No. 211.) Chapter $10 . \quad$ Available from: http://www.ncbi.nlm.nih.gov/books/NBK133364/

27. Collins, A.S. (2008). "Preventing Health Care-Associated Infections. In: Hughes RG, editor. Patient Safety and Quality: An Evidence-Based Handbook for Nurses." Rockville (MD): Agency for Healthcare Research and Quality (US); Chapter 41. Available from: http://www.ncbi.nlm.nih.gov/books/NBK2683/

28. Cosgrove, S.E., Qi, Y., Kaye, K.S., Harbarth, S., Karchmer, A.W., Carmeli, Y. (2005). "The impact of methicillin resistance in Staphylococcus aureus bacteremia on patient outcomes: mortality, length of stay, and hospital charges." Infect Control Hosp Epidemiol 2005; 26: 166-174.

29. Craven, D., Kunches, L., Kilinsky, V., Lichtenberg, D., Make, B. and McCabe, W. (1986). "Risk factors for pneumonia and fatality in patients receiving continuous mechanical ventilation." Am Rev Respir Dis 1986; 133:792-796.

30. Davis, C. (2010). "Infection-free surgery: how to improve handhygiene compliance and eradicate methicillin-resistant Staph- ylococcus aureus from surgical wards." Ann R Coll Surg Engl 2010;92:316-319. Retrieved from http://publishing.rcseng.ac.uk/doi/pdf/10.1308/003588410X12628812459931

31. Delgado-Rodríguez, M., Llorca, J. (2004). "Continuing professional education: Bias.” J Epidemiol Community Health 2004;58:8 635-641 doi:10.1136/jech.2003.008466.

32. Denton, G.W. (1991). "Chlorhexidine [Chapter 16]." In: Block SS, ed. Disinfection, sterilization and reservation. 4th ed. Philadelphia, PA: Lea and Febiger, 1991. 
33. Dubberke, E.R., Butler, A.M., Yokoe, D.S., et al. (2000). "Multicenter study of Clostridium difficile infection rates from 2000 to 2006." Infect Control Hosp Epidemiol 2010;31:1030-7.

34. Dubberke, ER, Olsen MA. (2012). "Burden of Clostridium difficile on the healthcare system." Clin Infect Dis 2012;55:Suppl 2:S88- S92.

35. Dubberke, E.R., Reske, K.A., Olsen, M.A., McDonald, L.C., Fraser, V.J. (2008). "Short- and long-term attributable costs of Clostridium Difficileassociated disease in nonsurgical patients." Clin Infect Dis 2008;46:497-504.

36. Dubberke, E. R., Butler, A. M., Reske, K. A., Agniel, D., Olsen, M. A., D’Angelo, G., McDonald L. C., Fraser, V. J. (2008). "Attributable Outcomes of Endemic Clostridium difficile-associated Disease in Nonsurgical Patients." Emerging Infectious Diseases, 14(7), 1031-1038. http://doi.org/10.3201/eid1407.070867

37. Dubberke, E., Gerding, D., Classen, D., et al. (2008). "Strategies to Prevent Clostridium difficile Infections in Acute Care Hospitals." Infection Control and Hospital Epidemiology. Vol. 29, No. S1, A Compendium of Strategies to Prevent Healthcare-Associated Infections in Acute Care Hospitals (October 2008), pp. S81-S92 Published by: Cambridge University Press on behalf of The Society for Healthcare Epidemiology of America. Retrieved from http://www.jstor.org/stable/10.1086/591065

38. Duggan, J.M., Hensley, S., Khuder, S., Papadimos, T.J., Jacobs, L. (2008). "Inverse correlation between level of professional education and rate of handwashing compliance in a teaching hospital." Infection Control and Hospital Epidemiology, 2008, 29:534-538.

39. Edgeworth, J. (2009). "Intravascular catheter infections." J Hosp Infect. 2009 Dec;73(4):323-330. Epub 2009 Aug 22.

40. Ellis, M., Hospenthal, D., Dooley, D., Gray, P., and Murray, C. (2004). "Natural History of Community-Acquired Methicillin-Resistant Staphylococcus aureus Colonization and Infection in Soldiers." Clin Infect Dis. (2004) 39 (7): 971-979 doi:10.1086/423965

41. Emori, T. and Gaynes, R. (1993). "An overview of nosocomial infections, including the role of the microbiology laboratory." Clin Microbiol Rev 1993; 6(4):428-442.

42. Etikan, I., Musa, S., Alkassim, R. (2016). "Comparison of Convenience Sampling and Purposive Sampling." American Journal of Theoretical and Applied Statistics. Vol. 5, No. 1, 2016, pp. 1-4. doi: 10.11648/j.ajtas.20160501.11 
43. Fekety, R., Kim, K.H., Brown, D., Batts, D.H., Cudmore, M., Silva, J. Jr. (1981). "Epidemiology of antibiotic-associated colitis: isolation of Clostridium difficile from the hospital environment." The American Journal of Medicine, 1981; 70,4:906-908.

44. Fordtran, J.S. (2006). "Colitis due to Clostridium difficile toxins: underdiagnosed, highly virulent, and nosocomial." Proc (Bayl Univ Med Cent) 2006; 19: 3-12.

45. Gastmeier, P., Stamm-Balderjahn, S., Hansen, S., et al. (2005). "How outbreaks can contribute to prevention of nosocomial infection: analysis of 1,022 outbreaks." Infect Control Hosp Epidemiol 2005 Apr;26:357-61.

46. Gerding, D.N., Johnson, S. (2012). "Chapter 129. Clostridium Difficile Infection, Including Pseudomembranous Colitis." In Longo D.L., Fauci A.S., Kasper D.L., Hauser S.L., Jameson J, Loscalzo J (Eds), Harrison's Principles of Internal Medicine, 18e. Retrieved November 06, 2014 from http://accesspharmacy.mhmedical.com.uri.idm.oclc.org/content.aspx?bookid= 331\&Sectionid $=40726873$.

47. Gerding, D.N., Johnson, S., Peterson, L.R., Mulligan, M.E., Silva, J. Jr. (1995). "Clostridium difficile-associated diarrhea and colitis." Infect Control Hosp Epidemiol 1995; 16:459-77.

48. Gerding, D. N., Muto, C. A., and Owens Jr., R. C., (2008). "Measures to Control and Prevent Clostridium difficile Infection." Oxford Journals, Clinical Infectious Diseases, (2008) 46 (Supplement 1): S43-S49 doi:10.1086/521861. Retrieved from http://cid.oxfordjournals.org/content/46/Supplement_1/S43.long

49. Gershenfeld, L. (1962). "Povidone-iodine as a sporicide." Am J Pharm 1962;134:79-81.

50. Gloud, C. and McDonlad, C. (2009). "Clostridium Difficile (CDI) Infections Toolkit Activity C: ELC Prevention Collaboratives." Retrieved from http://www.cdc.gov/hai/pdfs/toolkits/CDItoolkitwhite_clearance_edits.pdf

51. Gould, C., Umscheid, C., Rajender, K., Agarwal, R., Kuntz, G., Pegues, D., and the Healthcare Infection Control Practices Advisory Committee (HICPAC). (2009). "Guideline for prevention of catheterassociated urinary tract infections, 2009." Available from: http://www.cdc.gov/hicpac/cauti/001_cauti.html.

52. Haas, J.P., Larson, E.L. (2007). "Measurement of compliance with hand hygiene." J Hosp Infect 66:6-14, May 2007. 
53. Haley, RW, Bregman, DA. (1982). "The role of understaffing and overcrowding in recurrent outbreaks of staphylococcal infection in a neonatal special-care unit." J Infect Dis 1982;145:875-85.

54. Harbarth, S, Sudre, P, Dharan, S, Cadenas, M, Pittet, D. (1999). "Outbreak of Enterobacter cloacae related to understaffing, overcrowding, and poor hygiene practices.” Infect Control Hosp Epidemiol 1999;20:598-603.

55. Hidron, A., Kourbatova, E., Halvosa, J., Terrell, B., McDougal, L., Tenover, F., Blumberg, H., and King, D. (2005). "Risk Factors for Colonization with Methicillin-Resistant Staphylococcus aureus (MRSA) in Patients Admitted to an Urban Hospital: Emergence of Community-Associated MRSA Nasal Carriage." Clin Infect Dis. (2005) 41 (2): 159-166 doi:10.1086/430910

56. Higuera, F., Rangel-Frausto, M.S., Rosenthal, V.D., Soto, J.M., Castañon, J., Franco, G., Tabal-Galan, N., Ruiz, J., Duarte, P., Graves, N. (2007). "Attributable cost and length of stay for patients with central venous catheter-associated bloodstream infection in Mexico City intensive care units: A prospective, matched analysis." Infect Control Hosp Epidemiol. 2007 Jan;28(1):31-35.

57. Horan, T., Andrus, M., Dudeck, M. (2008). “CDC/NHSN surveillance definition of health care-associated infection and criteria for specific types of infections in the acute care setting." American Journal of Infection Control, Volume 36, Issue 5, June 2008, Pages 309-332

http://www.sciencedirect.com/science/article/pii/S0196655308001673

58. Horan, T., White, J., Jarvis, W. et al. (1984). "Nosocomial infection surveillance." MMWR 1986; 35(SS1):17-29.

http://0-web.a.ebscohost.com.helin.uri.edu/chc/detail?sid=26dd6c5a-daf3-

4254-

9fe168405ec $18082 \% 40$ sessionmgr4003\&vid $=4 \&$ hid $=4106 \&$ bdata $=$ JnNpdGU 9Y2hjLWx dmU\%3d\#db=cmh\&AN=HL165103

59. Hughes, C., Tunney, M., Bradley, M.C. (2013). "Infection control strategies for preventing the transmission of meticillin-resistant Staphylococcus aureus (MRSA) in nursing homes for older people." Cochrane Database of Systematic Reviews 2013, Issue 11. Art. No.: CD006354. DOI: 10.1002/14651858.CD006354.pub4.

60. Hugonnet, S., Perneger, T.V., Pittet, D. (2002). "Alcohol-based handrub improves compliance with hand hygiene in intensive care units." Archives of Internal Medicine, 2002, 162(9):1037-1043. 
61. Jarvis, W., Edwards, J., Culver, D., et al. (1991). "Nosocomial infection rates in adult and pediatric intensive care units in the United States." Am J Med 1991; 91(supp3B):185S-191S.

62. Jevons, M. P. (1961). "Celbenin" - resistant Staphylococci." British Medical Journal, 1(5219), 124-125.

63. Kinsella, G., Thomas, A.N., Taylor, R.J. (2007). "Electronic surveillance of wal--mounted soap and alcohol gel dispensers in an intensive care unit." Journal of Hospital Infection, 2007, 66:34-39.

64. Kozak, M. (2008). "Finite and Infinite Populations in Biological Statistics: Should We Distinguish Them?". The Journal of American Science, 2008, 4(1).

65. Kretzer, EK, Larson, EL. (1998). "Behavioral interventions to improve infection control practices". American Journal of Infection Control, 26:245253.

66. Krohn, R. (2015). "The Standard of Excellence for RTLS." ClinicalGrading Locating. Practical Location Intelligence for Healthcare. Retrieved from http://clinicalgradelocating.com/resources/

67. Kuzu, N et al. (2005). "Compliance with hand hygiene and glove use in a university-affiliated hospital." Infection Control and Hospital Epidemiology, 2005, 26:312-315.

68. Landsberger, H. (1958). "Hawthorne revisited." Ithaca, NY, Cornell Social Science Research Center, Cornell University.

69. Landt, J. (2005). “The history of RFID.” New York: IEEE Institute of Electrical and Electronics Engineers; 2005. Vol. 24. pp. 8-11.

70. Lankford, M.G. et al. (2003). "Influence of role models and hospital design on hand hygiene of healthcare workers." Emerging Infectious Diseases, 2003, 9:217-223.

71. Larson EL, Morton HE. (1991). "Alcohols [Chapter 11]." In: Block SS, ed. Disinfection, sterilization and preservation. 4th ed. Philadelphia, PA: Lea and Febiger, 1991:642-54.

72. Larson, E.L., Albrecht, S., O'Keefe, M. (2005). "Hand hygiene behavior in a pediatric emergency department and a pediatric intensive care unit: Comparison of use of 2 dispenser systems." Am J Crit Care 14:304-310, Jul. $2005 . \quad$ Retrieved from http://ajcc.aacnjournals.org/content/14/4/304.full.pdf + html 
73. Lenth, R.V. (2001). "Some Practical Guidelines for Effective Sample Size Determination.” The American Statistician, Vol.55, No.3. (Aug., 2001), pp. 187-193. Available from

http://www.jstor.org/stable/2685797?seq=1\#page scan tab contents

74. Lessa, F., Mu, Y., Bamberg, W. Beldavs, Z., Dumyati, G., Dunn, J., Farley, M., Holzbauer, S., Meek, J., Phipps, E., Wilson, L., Winston, L., Cohen, J., Limbago, B., Fridkin, S., Gerding, D., and McDonald, L. (2015). "Burden of Clostridium difficile Infection in the United States." New England Journal of Medicine, 372(9), 825-834. http://dx.doi.org/10.1056/NEJMoa1408913.

75. Lieberman, M. and Cunningham, W. (2009). "Tools of the Trade: Type I and Type II error concerns in fMRI research: re-balancing the scale." Oxford Journals, Social Cognitive and Affective Neuroscience. first published online December 24, 2009. Retrieved from http://scan.oxfordjournals.org/content/early/2009/12/24/scan.nsp052.full.pdf + h $\underline{\mathrm{tml}}$

76. Loo, V., Poirier, L., Miller, M., Oughton, M., Libman, M., Michaud, S., Bourgault, A., Nguyen, T., Frenette, C., Kelly, M., Vibien, A., Brassard, P., Fenn, S., Dewar, K., Hudson, T., Horn, R., René, P., Monczak, Y., Dascal, A. (2005). "A predominantly clonal multi- institutional outbreak of Clostridium difficile-associated diarrhea with high morbidity and mortality." New England Journal of Medicine, 353:2442-2449. http://dx.doi.org/10.1056/NEJMoa051639

77. Luteijn, J., Hubben, G., Pechlivanoglou, P., Bonten, M. and Postma, M. (2011). "Diagnostic accuracy of culture-based and PCR-based detection tests for methicillin-resistant Staphylococcus aureus: a meta-analysis." Clinical Microbiology and Infection, Volume 17, Issue 2, February 2011, Pages 146154, ISSN 1198-743X, http://dx.doi.org/10.1111/j.1469-0691.2010.03202.x.

78. Magill, S., Edwards, J., Bamberg, W., Beldavs, Z., Dumyati, G., Kainer, M., Lynfield, R., Maloney, M., McAllister-Hollod, L., Nadle, J., Ray, S., Thompson, D., Wilson, L. and Fridkin, S. (2014). "Multistate PointPrevalence Survey of Health Care-Associated Infections." the New England Journal of Medicine, Volume 370, Issue 13, March 27 2014, Pages 1198-1208, ISSN 0028-4793, DOI 10.1056/NEJMoa1306801, http://www.nejm.org/doi/full/10.1056/NEJMoa1306801.

79. Marshall, C., Wesselingh, S., McDonald, M. and Spelman, D. (2004). "Control of endemic MRSA - what is the evidence? A personal view." Journal of Hospital Infection, Volume 56, Issue 4, April 2004, Pages 253-268, ISSN 0195-6701, http://0dx.doi.org.helin.uri.edu/10.1016/j.jhin.2004.02.001 
80. Mayfield, J.L., Leet, T., Miller, J., Mundy, L.M. (2000). "Environmental control to reduce transmission of Clostridium Difficile." Clin Infect Dis 2000; 31: 995-1000.

81. McCoy, K. (2013). "Methicillin-Resistant Staph Infection: MRSA; Methicillin-Resistant Staphylococcus aureus Infection; Infection, MethicillinResistant; Methicillin-Resistant Staphylococcus aureus Community-Acquired MRSA; CA-MRSA; Methicillin-Resistant Staphylococcus aureus Nosocomial MRSA; Healthcare-Associated MRSA; HA-MRSA." Conditions \& Procedures Inbrief.

82. McCrum-Gardner, E. (2010). "Sample size and power calculations made simple." International Journal of Therapy and Rehabilitation, Vol.17, No.1. (January, 2010), pp. 10-14. Available from http://www.uv.es/uvetica/files/McCrum_Gardner2010.pdf

83. McDonald, L. C., Owings, M., \& Jernigan, D. B. (2006). “Clostridium difficile Infection in Patients Discharged from US Short-stay Hospitals, 19962003.” Emerging Infectious Diseases, 12(3), 409-415.

http://doi.org/10.3201/eid1203.051064.

84. McFarland, L, Mulligan, M., Kwok, R., and Stamm, W. (1989). "Nosocomial Acquisition of Clostridium difficile Infection." N Engl J Med 1989; 320:204-210January 26, 1989. Retreived From http://www.nejm.org/doi/pdf/10.1056/NEJM198901263200402

85. McGuckin, M. Taylor, A., Martin, V., Porten, L., Salcido, R. (2004). "Evaluation of a patient education model for increasing hand hygiene compliance in an inpatient rehabilitation unit." American Journal of Infection Control, 2004, 32:235-238.

86. McGuckin, M., Waterman, R., Storr, J., Bowler, I., Ashby, M., Topley, K., Porten, L. (2001). "Evaluation of a patient-empowering hand hygiene programme in the UK." Journal of Hospital Infection, 2001, 48:222-227.

87. McMaster, J., Booth, M., Smith, A., Hamilton, K. (2015). "Meticillinresistant Staphylococcus aureus in the intensive care unit: its effect on outcome and risk factors for acquisition." Journal of Hospital Infection, Volume 90, Issue 4, August 2015, Pages 327-332, ISSN 0195-6701, http://dx.doi.org/10.1016/j.jhin.2015.04.009. (http://www.sciencedirect.com/science/article/pii/S0195670115001814)

88. Mille, D., Roy, T., Carrere, MO., Ray, I., Ferdjaoui, N., Spath, HM., et al. (2000). "Economic impact of harmonizing medical practices: Compliance with clinical practice guidelines in the follow-up of breast cancer in a French 
Comprehensive Cancer Center." Journal of Clinical Oncology 2000;18:17181724.

89. Montgomery, D.C., (2012). "Introduction to statistical quality control." $6^{\text {th }}$ Edition. Jefferson City, MI: John Wiley \& Sons, Inc.

90. Mortel, V., Francisca, T., Murgo M. (2006). "An examination of covert observation and solution audit as tools to measure the success of hand hygiene interventions." American Journal of Infection Control, 2006, 34:95-99.

91. Nathwani, D., Rubinstein, E., Barlow, G., Davey, P. (2001). "Do guidelines for community-acquired pneumonia improve the cost-effectiveness of hospital care?" Clinical Infectious Diseases 2001;32:728-741.

92. National Clostridium difficile Standards Group. (2004). Report to the Department of Health. J Hosp Infect 2004; 56(Suppl 1):1-38.

93. Nguyen, K. V., Nguyen, P. T. M. and Jones, S. L. (2008). "Effectiveness of an alcohol-based hand hygiene programme in reducing nosocomial infections in the Urology Ward of Binh Dan Hospital, Vietnam." Tropical Medicine \& International Health, 13: 1297-1302. doi: 10.1111/j.1365-3156.2008.02141.x

94. Nicolle, L. (2000). "Urinary tract infection in long-term-care facility residents." Clin Infect Dis 2000;31:757-61.

95. O'Boyle, CA, Henly, SJ, Duckett, LJ. (2001). "Nurses' motivation to wash their hands: a standardized measurement approach." Applied Nursing Research 2001;14:136-45.

96. O'Grady, N., Alexander, M. and et al. (2011). "Guidelines for the Prevention of Intravascular Catheter-Related Infections, 2011." Centers for Disease Control and Prevention (CDC). Retrieved from http://www.cdc.gov/hicpac/pdf/guidelines/bsi-guidelines-2011.pdf

97. Orgeas, M., Timsit, J., Soufir, L., Tafflet, M., Adrie, C., Philippart, F., Zahar, J., Clec'h, C., Goldran-Toledano, D., Jamali, S., Dumenil, A., Azoulay, E., Carlet, J.(2008). "Impact of adverse events on outcomes in intensive care unit patients." Crit Care Med 2008;36:2041-7.

98. Owens, RC. (2006). "Clostridium difficile-associated disease: an emerging threat to patient safety: insights from the Society of Infectious Diseases Pharmacists." Pharmacotherapy 2006; 26:299-311.

99. Patarakul, K, Tan-Khum, A., Kanha, S., Padungpean, D., Jaichaiyapum, O. (2005). "Cross-sectional survey of hand-hygiene compliance 
and attitudes of health care workers and visitors in the intensive care units at King Chulalongkorn Memorial Hospital". Journal of the Medical Association of Thailand, 88(Suppl. 4):S287-S293.

100. Payton, F.C., Pare, G., LeRouge, C., Reddy, M. (2011). Health care IT: Process, people, patients and interdisciplinary considerations. Journal of the Association for Information Systems, Vol. 12 Special Issue (2/3). pp.i-xiii. February 2011. Retrieved from http://aisel.aisnet.org/cgi/viewcontent.cgi?article $=1571 \&$ context $=$ jais

101. Pepin, J., Valiquette, L., Cossette, B. (2005). "Mortality attributable to nosocomial Clostridium difficile-associated disease during an epidemic caused by a hypervirulent strain in Quebec." Canadian Medical Association Journal 2005; 173(7), 1037-1042. http://www.cmaj.ca/content/173/9/1037.full.pdf+html

102. Pittet D, Tarara D, Wenzel RP. (1994). "Nosocomial bloodstream infection in critically ill patients. Excess length of stay, extra costs, and attributable mortality." JAMA. 1994 May 25;271(20):1598-1601.

103. Pittet, D. (2000). "Improving Compliance with Hand Hygiene in Hospitals." Infect Hosp Epidemiol 2000;21:381-6.

104. Pittet, D. (2002). "Promotion of hand hygiene: magic, hype, or scientific challenge?" Infection Control and Hospital Epidemiology, 2002, 23:118-119.

105. Pittet, D. (2003). "Hand hygiene: Improved standards and practice for hospital care." Current Opinion in Infectious Diseases, 16(4), 327-335. doi:10.1097/01.qco.0000083564.72029.27

106. Pittet, D., Allegranzi, B., Sax, H., Dharan, S., Pessoa-Silva, C., Donaldson, L., Boyce, J. (2006). "Evidence-based model for hand transmission during patient care and the role of improved practices." Lancet Infectious Diseases, 2006, 6:641-652.

107. Pittet, D., Mourouga, P., Perneger, TV. (1999). "Compliance with handwashing." Annals of Internal Medicine, 130:126-130.

108. Polgreen, P. M., Hlady, C. S., Severson, M. A., Segre, A. M., \& Herman, T. (2010). "Method for Automated Monitoring of Hand Hygiene Adherence without Radio-Frequency Identification." Infection Control and Hospital Epidemiology: The Official Journal of the Society of Hospital Epidemiologists of America, 31(12), 1294-1297. Retrieved from http://www.ncbi.nlm.nih.gov/pmc/articles/PMC3024851/pdf/nihms262498.pdf 
109. Providence VA Medical Center, Rhode Island (PVAMC). (2015). "About the Providence VA Medical Center, Rhode Island." U.S. Department of Veterans Affairs. http://www.providence.va.gov/about/

110. Rello, J., Ollendorf, D., Osler, G., et al. (2002). "Epidemiology and outcomes of ventilator- associated pneumonia in a large US database." Chest 2002; 122:2115-2121.

111. Richard, M., Edwards, J., Culver, D. and Gaynes R. (2000). "Nosocomial infections in combined medical-surgical intensive care units in the United States." Infect Control Hosp Epidemiol 2000;21:510-5.

112. Rosenthal, V.D., Guzman, S., Migone, O., Crnich, C.J. (2003). "The attributable cost, length of hospital stay, and mortality of central lineassociated bloodstream infection in intensive care departments in Argentina: A prospective, matched analysis." Am J Infect Control. 2003 Dec;31(8):475-480.

113. Russell, A.D. (1991). "Chemical sporicidal and sporostatic agents [Chapter 22]." In: Block SS, ed. Disinfection, sterilization and preservation. 4th ed. Philadelphia, PA: Lea and Febiger, 1991.

114. Safdar, N., Kluger, D.M., Maki, D.G. (2002). "A review of risk factors for catheter-related bloodstream infection caused by percutaneously inserted, noncuffed central venous catheters: implications for preventive strategies." Medicine (Baltimore). 2002 Nov;81(6):466-79. PMID 12441903.

115. Sax, H., Allegranzi, B., Uckay, I., Larson, E., Boyce, J., Pittet, D. (2007). "'My five moments for hand hygiene': a user- centred design approach to understand, train, monitor and report hand hygiene." Journal of Hospital Infection, 67:9-21. Retrieved from http://microbiology.mtsinai.on.ca/coreEducModules/hand-hygiene-aug2007.pdf

116. Scott II, R.D. (2009). "The direct medical costs of healthcareassociated Infections in U.S. hospitals and the benefits of prevention." Atlanta, GA: Division of Healthcare Quality Promotion National Center for Preparedness, Detection, and Control of Infectious Diseases Coordinating Center for Infectious Diseases Centers for Disease Control and Prevention; 2009. Retrieved from http://www.cdc.gov/HAI/pdfs/hai/Scott_CostPaper.pdf

117. Shuman, EK., and Chenoweth, CE. (2010). "Recognition and Prevention of Healthcare-Associated Urinary Tract Infections in the Intensive Care Unit." US National Library of Medicine. 
118. Siegel, J., Rhinehart, E., Jackson, M. and Chiarello, L. (2006). "Management of Multidrug-Resistant Organisms in Healthcare Settings." the Healthcare Infection Control Practices Advisory Committee (HICPAC).

119. Siegel, J., Rhinehart, E., Jackson, M., Chiarello, L. (2007). and the Healthcare Infection Control Practices Advisory Committee, 2007 Guideline for Isolation Precautions: Preventing Transmission of Infectious Agents in Healthcare Settings http://www.cdc.gov/ncidod/dhqp/pdf/isolation2007.pdf

120. Simmons, B., Bryant, J., Neiman, K., Spencer, L., \& Arheart, K.. (1990). "The Role of Handwashing in Prevention of Endemic Intensive Care Unit Infections." Infection Control and Hospital Epidemiology, 11(11), 589594. Retrieved from http://www.jstor.org/stable/30148433

121. Stone, P.W., Braccia, D., Larson, E. (2005). "Systematic review of economic analyses of health care- associated infections." Am J Infect Control. 2005 Nov;33(9):501-9. PMID 16260325.

122. Strymish, J. and Gupta, K. (2012). "Triplett Transition Hand Hygiene." VA Boston Healthcare System (VABHS) Infection Control (IC) and New England Veterans Engineering Resource Center (VERC) Partnership.

123. Sullivan, G. M., \& Feinn, R. (2012). "Using Effect Size—or Why the P Value Is Not Enough." Journal of Graduate Medical Education, 4(3), 279282. http://www.ncbi.nlm.nih.gov/pmc/articles/PMC3444174/pdf/i1949-83574-3-279.pdf

124. Sumitani, Y. and Kobayashi, Y. (2009). "Comparative evaluation of a rapid MRSA detection assay based on multiplex real-time PCR versus MRSA screening cultures containing egg yolk." Journal of Infection and Chemotherapy, Volume 15, Issue 4, 2009, Pages 262-265, ISSN 1341-321X, http://dx.doi.org/10.1007/s10156-009-0685-9.

125. Swoboda, S.M. et al. (2004). "Electronic monitoring and voice prompts improve hand hygiene and decrease nosocomial infections in an intermediate care unit." Critical Care Medicine, 2004, 32:358-363.

126. Swoboda, S.M., Earsing, K., Strauss, K., Lane, S., Lipsett, P. (2007). "Isolation status and voice prompts improve hand hygiene." American Journal of Infection Control, 2007, 35:470-476. Retrieved from

http://www.ajicjournal.org/article/S0196-6553(06)01243-0/pdf

127. Tablan, O., Anderson, L., Besser, R., Bridges, C. and Hajjeh, R. (2004). Healthcare Infection Control Practices Advisory Committee, Centers for Disease Control and Prevention. "Guidelines for preventing health-careassociated pneumonia, 2003: recommendations of the CDC and the Health- 
care Infection Control Practices Advisory Committee." MMWR Recommendations and Reports March, 26, 2004;53(RR-3):1-36.

128. The Joint Commission Center for Transforming Healthcare TJC (2009). "Measuring Hand Hygiene Adherence: Overcoming the Challenges". Retrieved http://www.jointcommission.org/assets/1/18/hh_monograph.pdf

129. The Joint Commission for Transforming Healthcare TJC. (2012). "Preventing Central Line-Associated Bloodstream Infections: A Global Challenge, a Global Perspective." Oak Brook, IL: Joint Commission Resources, May 2012. http://www.PreventingCLABSIs.pdf.

130. The President's Council of Advisory on Science and Technology (PCAST). (2010). "Report to the President realizing the full potential of the health information technology to improve healthcare for Americans: The path forward." President's Council of Advisors on Science and Technology (PCAST): Executive Office of the President. Dec 2010. Retrieved from https://www.whitehouse.gov/sites/default/files/microsites/ostp/pcast-health-itreport.pdf

131. The World Health Organization (WHO). (2009). "The World Health Organization Guidelines on Hand Hygiene in Health Care: First Global Patient Safety Challenge, Clean Care is Safer Care. Geneva [Switzerland]: WHO Press." Retrieved from http://apps.who.int/iris/bitstream/10665/44102/1/9789241597906_eng.pdf

132. The VHA Handbook. (2016). The VHA Procurement \& Logistics Office, U.S. Department of Veterans Affairs. (2016). "Supply Chain Inventory Management". Retrieved from https://www.va.gov/vhapublications/ViewPublication.asp?pub ID=3286\&usg $=$ AFQjCNF9rh00vwbHCexdFPuFvqktA_ax 7w\&sig2=7BBpIIzIwrzOVMkL2 E68Mg\&bvm=bv.139782543,d.eWE

133. Traugott, K. (2013). "Chapter 33. Clostridium Difficile Infections." In Attridge R.L., Miller M.L., Moote R, Ryan L (Eds), Internal Medicine: A Guide to Clinical Therapeutics. Retrieved November 06, 2014 from http://accesspharmacy.mhmedical.com.uri.idm.oclc.org/content.aspx?bookid= 565\&S ctionid $=42003747$.

134. U.S. Department of Veterans Affairs. (2013). Retrieved from http://www.ehealth.va.gov/VistA_Monograph.asp

135. Underwood, S., Stephenson, K., Fawley, W.N., et al. (2005). "Effects of hospital cleaning agents on spore formation by North American and UK 
out- break Clostridium difficile (CD) strains [abstract LB-28-2005]." In: Program and abstracts of the 45th Annual Interscience Conference on Antimicrobials and Chemotherapy (Washington, DC). 2005.

136. US Department of Health and Human Services. (2009). "HHS Action Plan to Prevent Healthcare-Associated Infections: Introduction." Jun 2009. http://www.hhs.gov/ash/initiatives/hai/introduction.html.

137. Vakili, S., Pandit, R., Singman, L., Appelbaum, J. and Boland, M. (2015). "A comparison of commercial and custom-made electronic tracking systems to measure patient flow through an ambulatory clinic." International Journal of Health Geographics. Retrieved from https:/ijhealthgeographics.biomedcentral.com/articles/10.1186/s12942-015-0023-7

138. VAMHCS. (2012). Maryland Health Care System, U.S. Department of Veterans Affairs. "Policy Memorandum 512-11/COS-IC-012, subject: Guidelines for Standard and Transmission Based Precautions (Formerly Isolation)", dated December 2012.

139. Venkatesh, A.K., Lankford, M., Rooney, D., Blachford, T., Watts, C., Noskin, G. (2008). "Use of electronic alerts to enhance hand hygiene compliance and decrease transmission of vancomycin-resistant Enterococcus in a hematology unit." American Journal of Infection Control, 2008, 36:199205. Retrieved from http://www.ajicjournal.org/article/S0196-6553(08)000552/pdf

140. Wade, S. (1995). "Partnership in care: a critical review." Nursing Standard, 1995, 9:29-32.

141. Wamba, S., Anand, A., Carter, L. (2013). "A literature review of RFIDenabled healthcare applications and issues." International Journal of Information Management, Volume 33, Issue 5, October 2013, Pages 875-891, ISSN 0268-4012, http://dx.doi.org/10.1016/j.ijinfomgt.2013.07.005.

142. Wamba, S., Lefebvre, L., Bendavid, Y., Lefebvre, E. (2008). "Exploring the impact of RFID technol- ogy and the EPC network on mobile B 2B ecommerce: A case study in the retail industry." International Journal of Production Economics, 112(2), 614-629.

143. Warren, DK., Quadir, WW., Hollenbeak, CS., Elward, AM., Cox, MJ., Fraser, VJ. (2006). "Attributable cost of catheter-associated bloodstream infections among intensive care patients in a nonteaching hospital." Crit Care Med. 2006 Aug;34(8):2084-2089. 
144. Wertheim, H., Vos, M., Ott, A., Belkum, A., Voss, A., Kluytmans, J., Keulen, P., Vandenbroucke-Grauls, C., Meester, M., and Verbrugh, H. (2004) "Risk and outcome of nosocomial Staphylococcus aureus bacteraemia in nasal carriers versus non-carriers.", The Lancet, Volume 364, Issue 9435, 21-27 August 2004, Pages 703-705, ISSN 0140-6736, http://dx.doi.org/10.1016/S0140-6736(04)16897-9.

(http://www.sciencedirect.com/science/article/pii/S0140673604168979)

145. Williams, T. (2002). "Patient empowerment and ethical decision making: the patient/partner and the right to act." Dimensions of Critical Care Nursing, 2002, 21:100-104. Retrieved from

http://journals.lww.com/dccnjournal/Fulltext/2002/05000/Patient_Empowerme nt_and_Ethical_Decision_Making_.6.aspx

146. Wittrup, K., and Burba, M. (2015). "Case Study: Tracking and Preventing the Spread of C. Diff with an Automated Hand Hygiene System." Biovigil Healthcare Systems. Preventing Infection. The Biovigil Approach. May 4, 2015. Retrieved from http://www.biovigilsystems.com/wpcontent/uploads/2015/05/Biovigil-4-AutomatedHHC-v2b.pdf

147. Wu, Y., Ranasinghe, DC., Sheng, QZ., Zeadally, S., Yu, J. (2011). "RFID enabled traceability networks: A survey." Distrib Parallel Databases. 2011;29:397-443.

148. Wurtz, R., Moye, G., Jovanovic, B. (1994). "Handwashing machines, handwashing compliance, and potential for cross-contamination." Am J Infect Control 22:228-230, Aug. 1994. 